\title{
Geographic Determinants of Malaria Transmission
}

A Case Study from Kossi Province, Burkina Faso

Dissertation zur Erlangung des Doktorgrades der Mathematisch-Naturwissenschaftlichen Fakultäten der Georg-August-Universität zu Göttingen

\author{
vorgelegt von \\ Daniel Karthe
}

aus

Mannheim

Göttingen 2009 
Referent: Prof. Dr. Martin Kappas

Korreferent: Prof. Dr. Gerhard Gerold

Tag der mündlichen Prüfung: 27.10.2009 
«Human malaria has persisted through the development of miracle drugs and insecticides, a global eradication effort, and 30 years of intensive efforts to develop a practical vaccine. Not only does malaria persist; it thrives. ${ }^{1}$

1 Doolan, D.L.; Dobaño, C. \& Baird, J.K. (2009), p. 13. 


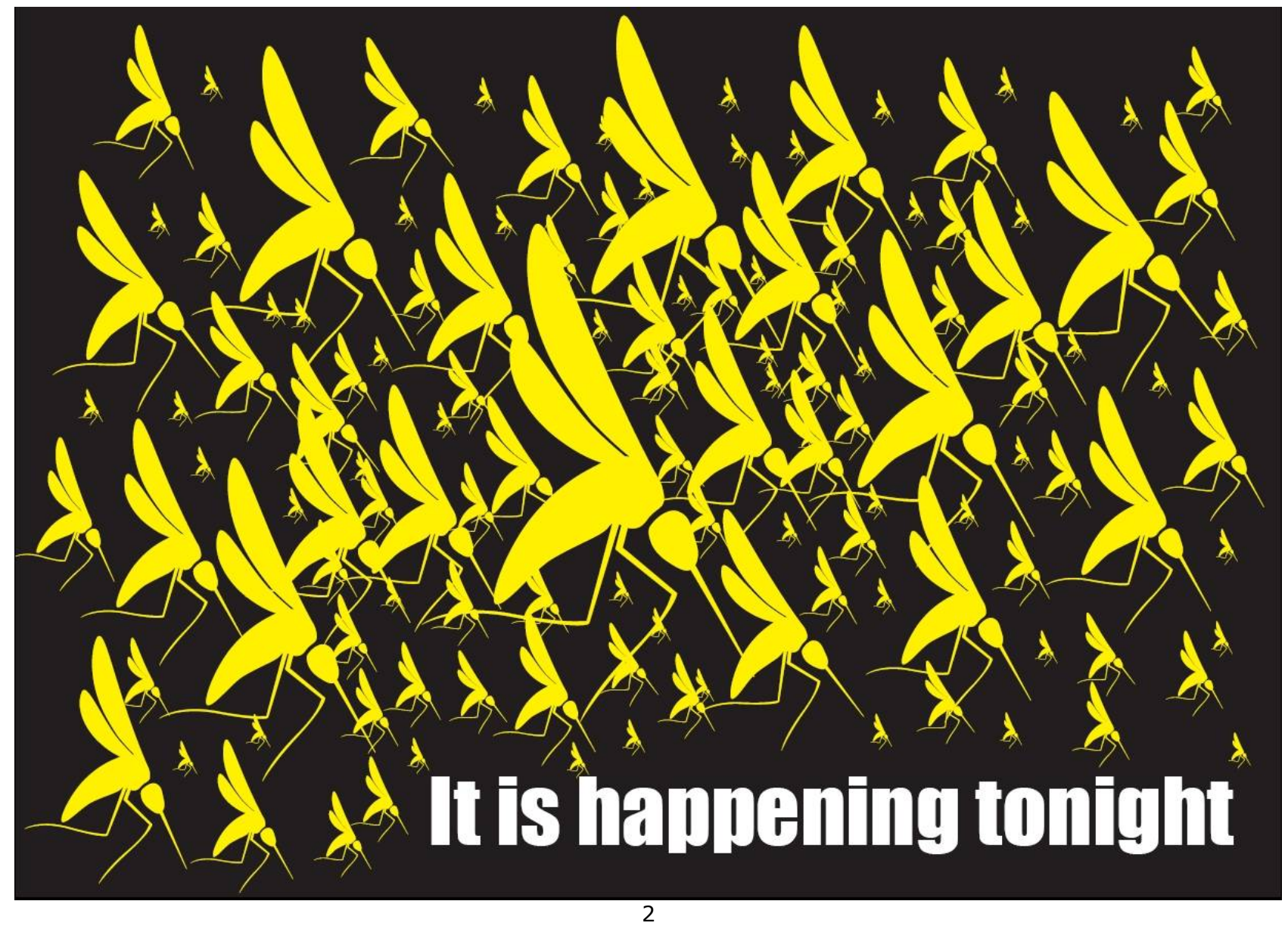

2 http://www.stopmalarianow.org/, accessed 09/09/09. 


\section{Foreword}

Despite massive efforts in the fields of malaria control and eradication, the disease remains one of the most important causes of morbidity and mortality in the developing world. At the same time, the dimension of the malaria burden remains largely unnoticed outside affected regions and the scientific community. The global malaria burden is largely concentrated on the African continent, with the countries between the southern fringe of the Sahara and the equator being the most affected. Even though the importance of malaria in the context of human development has been formally recognized by the United Nations in the framework of their Millennium Development Goals campaign, it remains unclear whether progress in the field of malaria control will significantly reduce the malaria-related public health burden. Experiences from intervention programs in the past have identified malaria risk maps as one of the key prerequisites for the implementation of efficient control programs.

The core of this thesis forms a case study of the spatial pattern and temporal dynamics of malaria incidence and their determinants in Kossi Province, a malaria-endemic region located in the Sahelo-Sudanian savanna zone of Western Burkina Faso. Spatio-temporally heterogeneous levels of transmission in the region are linked to a wide variety of geographical factors ranging from the natural environment to socioeconomic processes. Their combination and dynamics determine the pattern of malaria transmission at the regional and local scale. Moreover, fundamental changes in many of these factors have been observed in recent decades and are likely to continue in the future. This situation is complicated by the fact that these processes take place at both the world-wide scale (e.g. global warming) and in highly localized ways (e.g. micro-climatic changes due to small-scale modifications in land cover), and they do not occur in isolation but are interwoven into complex developments. Irrespective of their scale, all these alterations of the physical and socioeconomic environments have an impact on the transmission dynamics of malaria. Even though a scarcity of scientifically sound long-term data on the multitude of (potential) drivers of malaria transmission mean that this study cannot come up with a precise mathematical quantification of individual causeresult chains, it provides a comprehensive picture of the spatio-temporal pattern of malaria incidence and its geographic determinants for a region characterized by poor availability of such base data.

The multifactorial system of environmental and sociogeographic determinants of malaria transmission means that this study had to make use of multiple geographic methods. While relying largely on passive case detection data on malaria that were provided by rural health centers, geographic data have been compiled from various sources including field studies during the dry and wet seasons of 2007 and 2008, existing topographic and thematic maps and a 
range of remote sensing (RS) products. In many cases, RS and ground survey data had to be used to prepare topographic and thematic maps of the region, many of which are the first of their kind for Kossi Province. Indeed, the production of a systematic set of cartographic resources has been one of the key tasks of this study, and the resulting map compilation may be used as a basic planning tool for future investigations not only in the field of medical geography/malariology. Besides the contribution to the cartography of Kossi Province, this study provides a collection of geographic key data of the region, including an evaluation of their role with regard to malaria transmission.

The actual case study is preceded by a thorough overview of the current state of research in the fields of malariology, vector entomology, geographic determinants of malaria, the application of advanced geographic techniques in the field of medical geography and vector control (chapter 2). This compendium of information does not only provide the theoretical basis for the case study but at the same time forms a unique reference for scientists with an interest in the links geographic environment and vector-borne disease transmission. The case study itself (chapter 3 ) consists of a geographic introduction into the study region, including the physical and sociogeographic environment, the description of spatial and temporal pattern of malaria incidence and an analysis of potential determinants. Finally, the results of the study are summarized and discussed in chapter 4, which also outlines future research perspectives. A glossary located at the end of the study, highlighted key terms in the text and a comprehensive alphabetical index facilitate the use of this study and make it a comprehensive but understandable resource for planning and capacity building purposes.

Last but not least, it should be mentioned that this study is not the very first look at the determinants of malaria in Kossi Province, but that it builds on the works of the CRSN in Nouna and a team of researchers around Dr. Bocar Kouyaté, Prof. Dr. Rainer Sauerborn and Dr. Yazoumé Yé, and Prof. Dr. Martin Kappas' research on the climatology and agroecology of Burkina Faso. At the same time, the intention and focus of this work was different from previous studies, with the geography of disease, including its multiple drivers, forming the center of interest. Even though many aspects have been looked upon, this study is certainly still far from offering a complete picture of the "geography of malaria transmission" in Kossi. Future investigations are certainly needed and outlined in the form of research perspectives in the final chapter. 


\section{Acknowledgments}

This work is the result of a doctoral research program carried out in the Cartography, GIS and Remote Sensing Section of Göttingen University's Department of Geography. First of all, my thanks go to Prof. Dr. Martin Kappas, who guided this project from the beginning to the end. His suggestions regarding advanced remote sensing techniques and his working experience in Burkina Faso impressed not only the local project partners but were important inputs for this study. At the same time, the working atmosphere was characterized by an open exchange of ideas and creative freedom. Prof. Kappas' ideas regarding publications are similarly appreciated. I deeply acknowledge the support of Dr. Stefan Erasmi, who very often proved to be a competent and patient advisor and helped me to find solutions to a multitude of technical problems (including the acquisition of RS imagery). I also have to thank Jobst Augustin and Daniel Langhans for their assistance in GIS-related questions. Not to be forgotten is Prof. Dr. Gerhard Gerold who did not only discuss the structure of this thesis with me, but gave me the much needed support when complications regarding my working contract threatened the continuation of the project. Moreover, I appreciate his advice regarding publications on the project.

A case study on malaria in Burkina Faso would certainly not be possible without the help of local partners. Here my first acknowledgment goes to Dr. Yazoumé Yé, who is now a geographer and malariologist at the African Population Health Research Center in Nairobi, Kenya. Yazoumé Yé introduced me to the fascinating world of Kossi Province before I first set my foot there. Many of the ideas presented in this thesis are founded on discussions with Yazoumé while he was doing his doctoral research at Heidelberg University. Moreover, my first field visit to Kossi Province greatly profited from the Yazoumé's insight and knowledge into both the geographic region and local malaria pattern. In the same context, Prof. Dr. Rainer Sauerborn should not remain unmentioned: he first introduced me to the idea of linking malaria pattern and geographic factors at a meeting around seven years ago, and established the contact to Yazoumé Yé and their project partners in Burkina Faso where I particularly thank Dr. Ali Sié, the director of the health research center (CRSN) at Nouna, and his predecessor, Dr. Bocar Kouyaté (who is now in charge of Burkina Faso's national malaria research center) for fruitful discussions, providing both equipment and data and introducing me to the CRSN's staff, most notably Mr. Séraphin Simboro and the two lab entomologists, Mr. Saïdou Ouédraogo and Mr. François d'Assise Gonro. I sincerely wish that our envisaged collaboration in the framework of a formal project on the role of habitat quality and entomological/epidemiological characteristics of the vector population will one day become true. Issouf Traoré was a humorous, very knowledgeable and reliable partner during the 
field study, and without his help, much of the fieldwork would not have been possible. I am happy that his application for a German Academic Exchange Service scholarship was crowned with success. I wish him good luck for his stay in Germany and look forward to the perspectives of collaboration this opens for us.

Finally, I am grateful to everyone involved in the time-consuming task of proofreading the manuscript. This was done most intensively by Jobst Augustin, who did not only make minor corrections and suggestions but also discussed the fundamental design of the thesis. I thank my friend Matthias Stähle for proofreading several central passages. The expertise of Dr. Stefan Erasmi and Dr. Anne le Mellec who have proofread the sections on remote sensing and entomology is similarly appreciated. Last, but not least, I am deeply obliged to Mrs. Karin Reiss for her thorough and critical review of the vegetation chapter and the provision of hard-to-obtain materials from her private library. 


\section{Table of Contents}

\section{Preface}

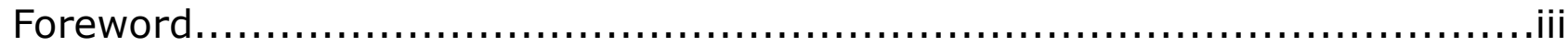

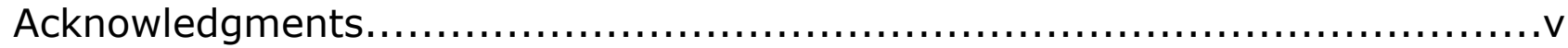

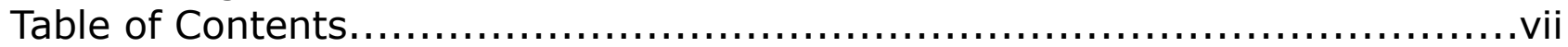

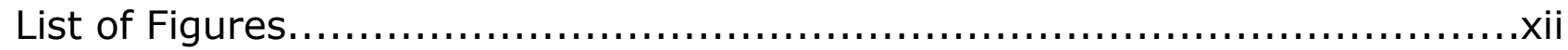

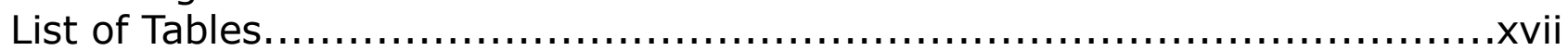

1 Introduction............................................................................1

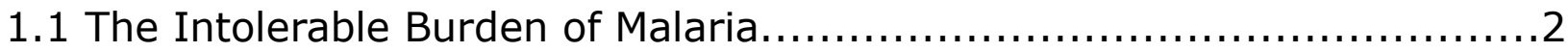

1.1.1 Malaria - A Re-Emerging Threat To Human Health? .............................

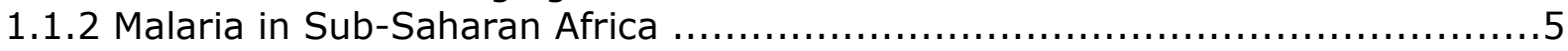

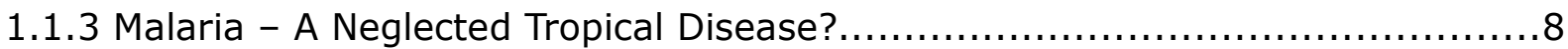

1.2 Geographic Contributions Towards Malaria Control .......................12

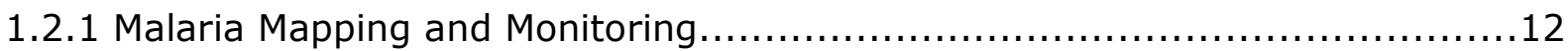

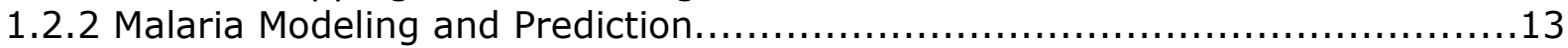

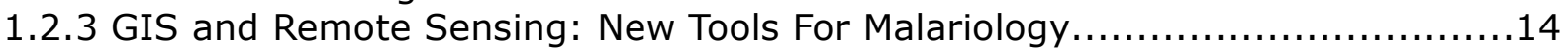

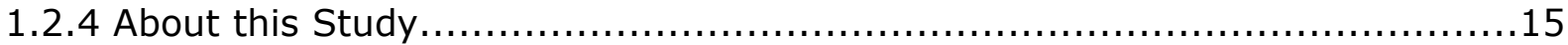

2 Malaria in West Africa: Transmission, Monitoring \& Control............18

2.1 Africa's Malaria Burden............................................. 18

2.1.1 Malaria Morbidity and Mortality in Africa....................................... 19

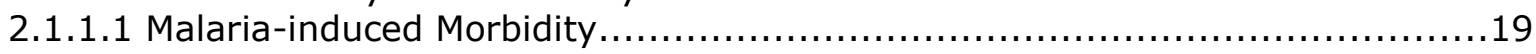

2.1.1.2 Malaria and Other Health Disorders................................................. 20

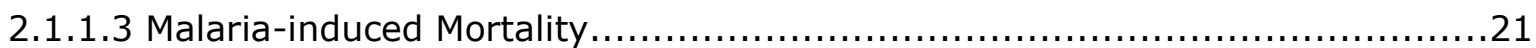

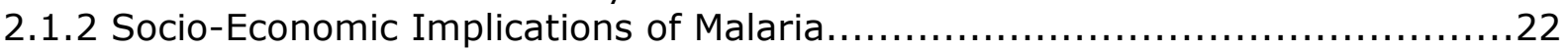

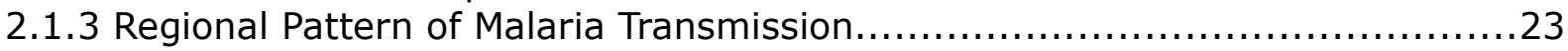

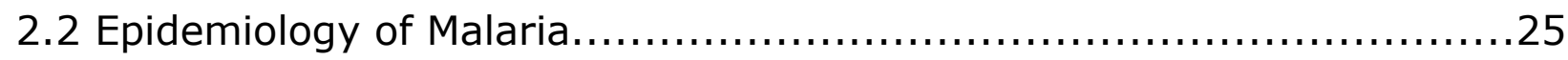

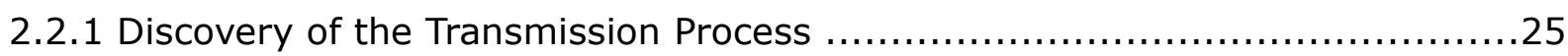

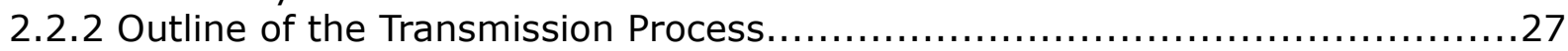

2.2.3 Classifications of Malaria Incidence and Transmission...........................29

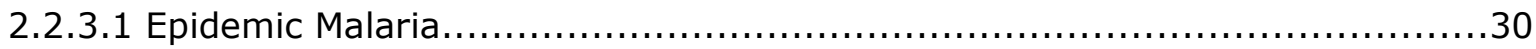

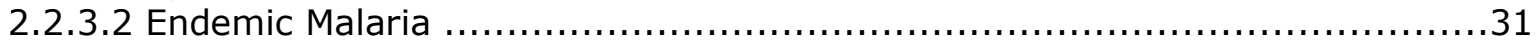

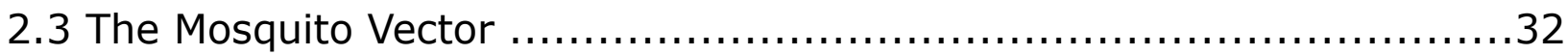

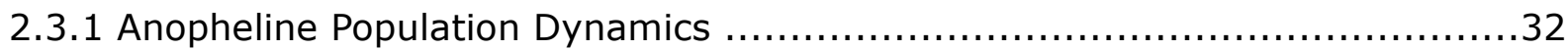

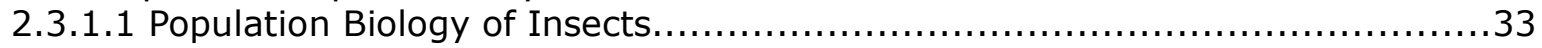

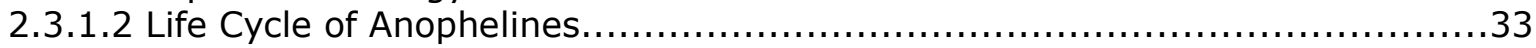

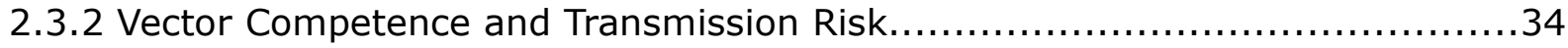

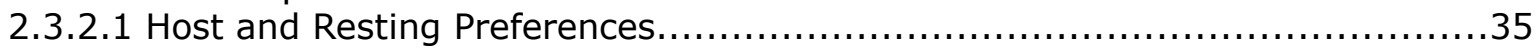

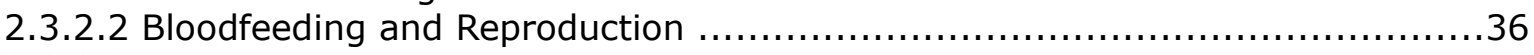

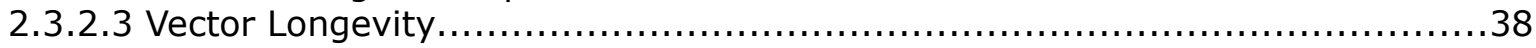




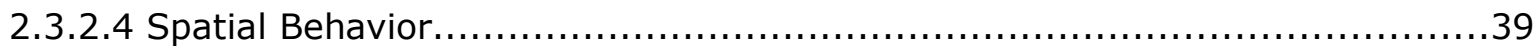

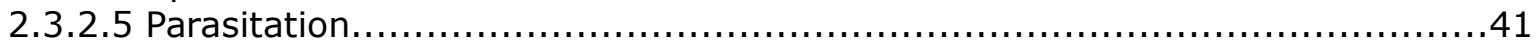

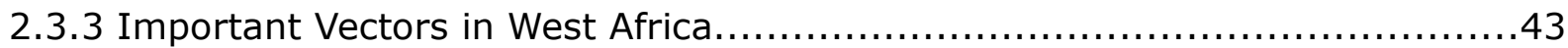

2.3.3.1 The Anopheles gambiae Complex................................................ 43

2.3.3.2 West African Vectors and Their Characteristics...............................44

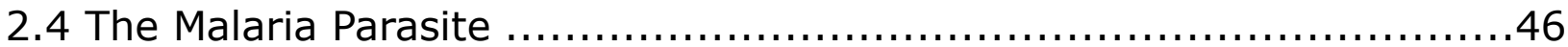

2.4.1 Life Cycle of the Malaria Parasites............................................... 47

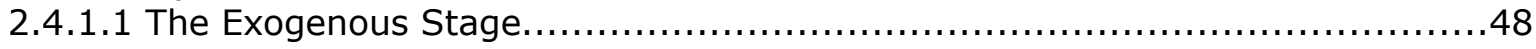

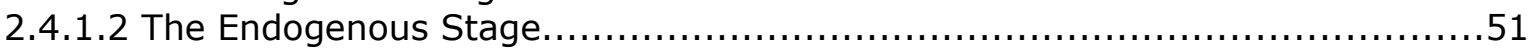

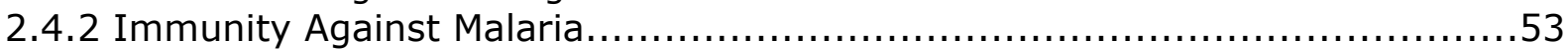

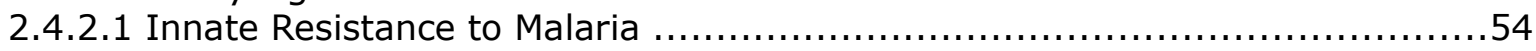

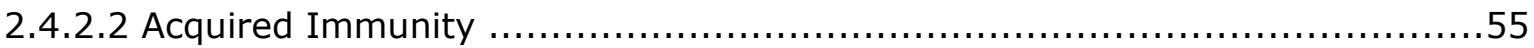

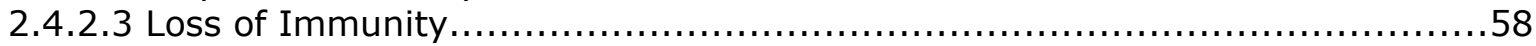

2.5 Determinants of Malaria Transmission................................. 58

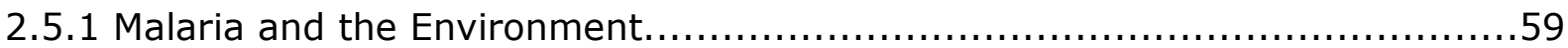

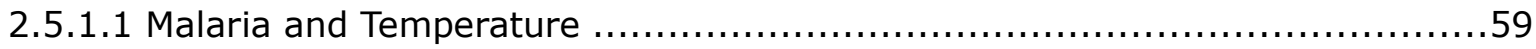

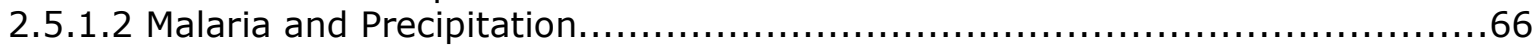

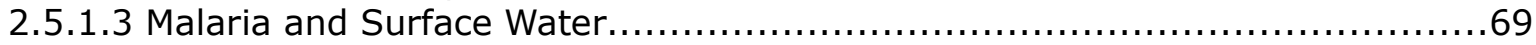

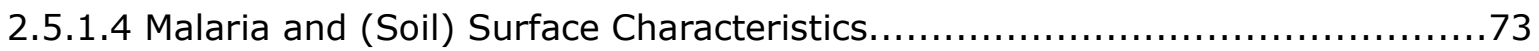

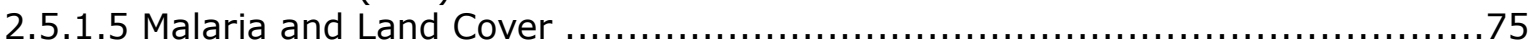

2.5.2 Anthropogenic Determinants of Malaria Transmission........................... 76

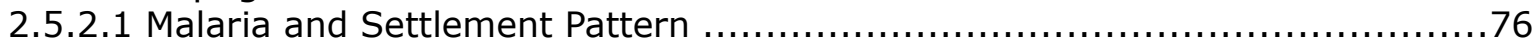

2.5.2.2 Malaria and Land Cover Change.................................................... 79

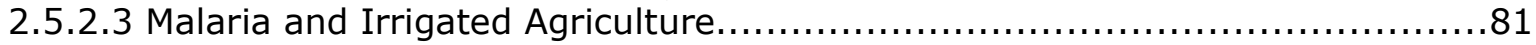

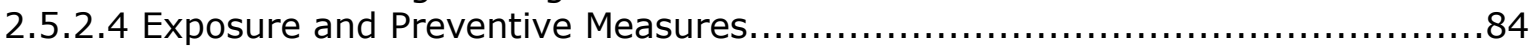

2.6 Monitoring, Mapping and Modeling Malaria Transmission.................85

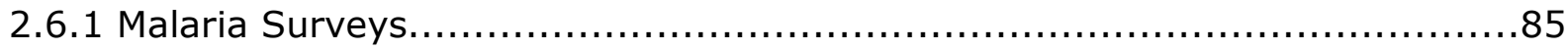

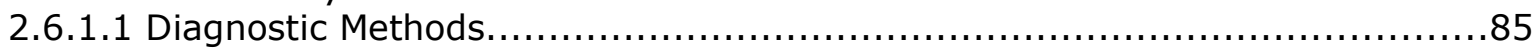

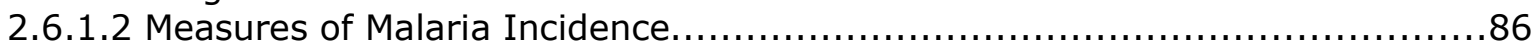

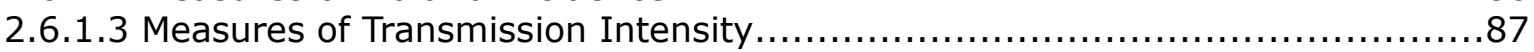

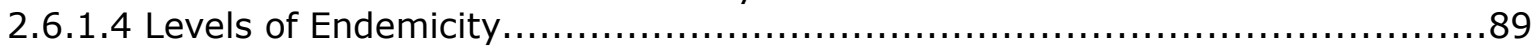

2.6.1.5 Limitations of Malaria Surveys and Statistics...................................... 90

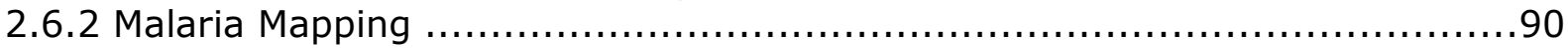

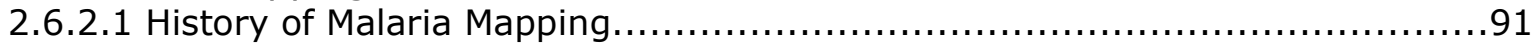

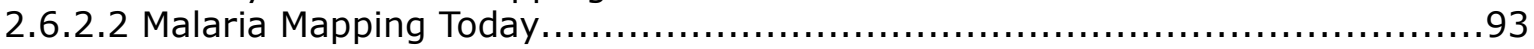

2.6.3 Malaria Modeling and Prediction................................................... 96

2.6.3.1 'Classic Models' by Ross and Macdonald........................................ 97

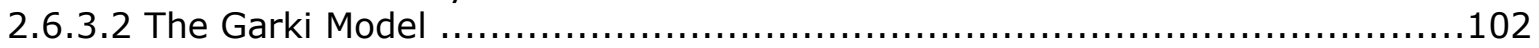

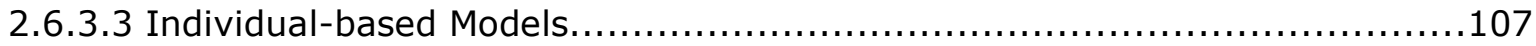

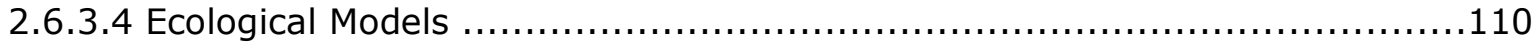

2.6.3.5 Feasibility and Limitations of Malaria Models.................................. 113

2.6.4 Synopsis: Determinants of Malaria Transmission ...............................115

2.7 GIS and Remote Sensing: New Tools For Malariology..................119

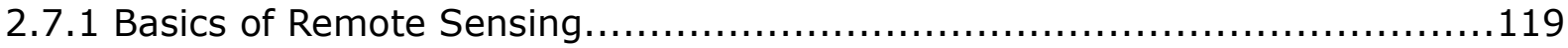

2.7.1.1 Physical Basics of Remote Sensing .............................................. 120

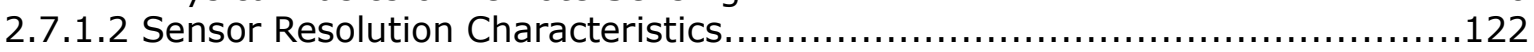

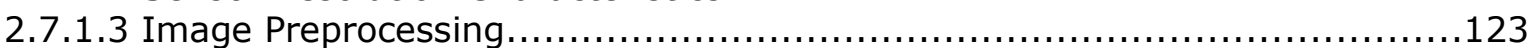

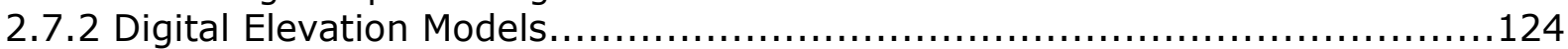

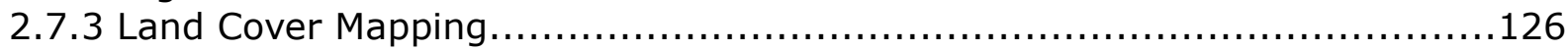


2.7.3.1 Land Use Classifications........................................................ 126

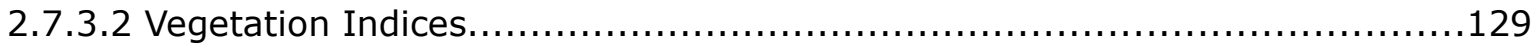

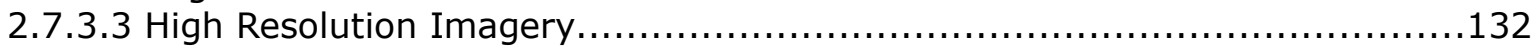

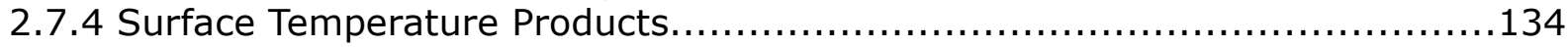

2.7.4.1 Thermal Infrared Remote Sensing............................................. 134

2.7.4.2 MODIS Surface Temperature Products........................................ 136

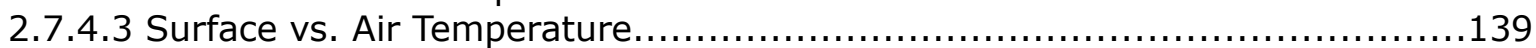

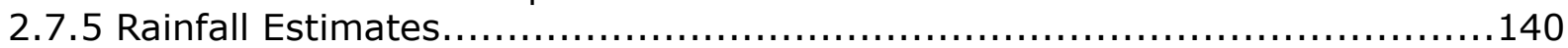

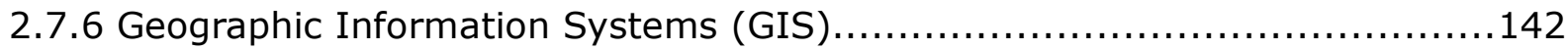

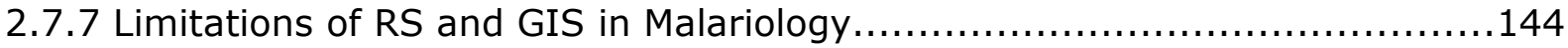

2.8 Malaria Control and Eradication....................................... 146

2.8.1 Principles of Malaria Control and Eradication.................................. 147

2.8.1.1 Vector Control through Insecticides............................................ 149

2.8.1.2 Vector Control through Environmental Management............................ 150

2.8.1.3 Prevention of Vector-Host Contact............................................... 154

2.8.1.4 Treatment and Chemoprophylaxis.............................................. 156

2.8.1.5 Development of Malaria Vaccines............................................... 158

2.8.2 The History of Malaria Control and Eradication.................................. 158

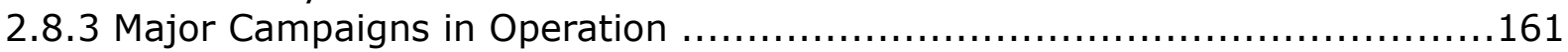

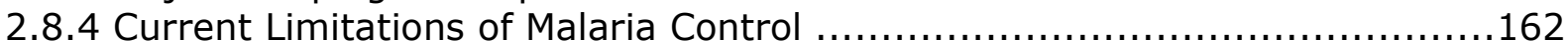

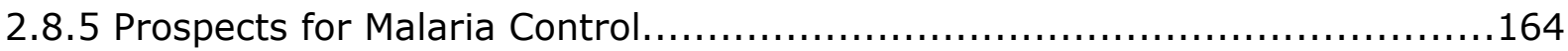

3Case Study: Malaria in Kossi Province......................................166

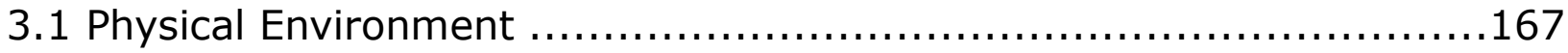

3.1.1 Land Surface Characteristics.................................................

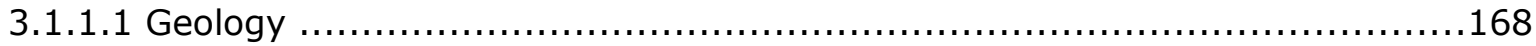

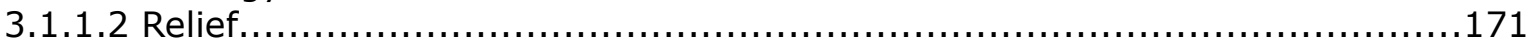

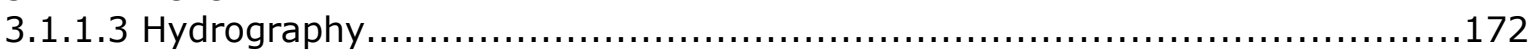

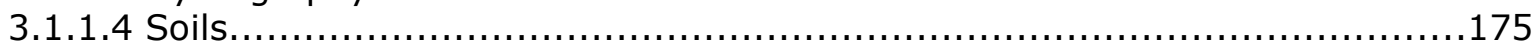

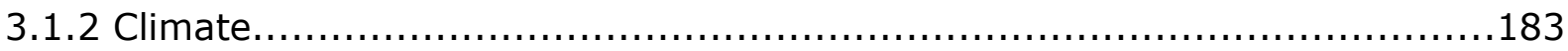

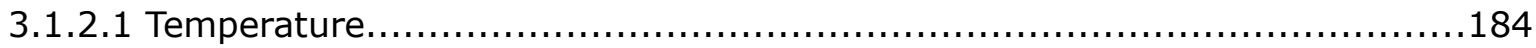

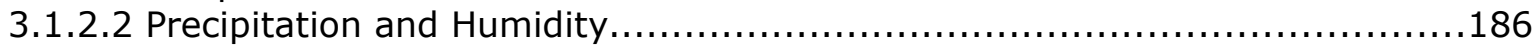

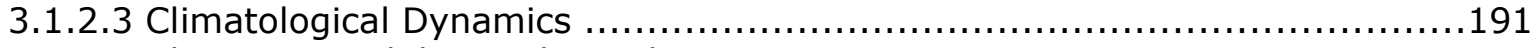

3.1.2.4 Climatic Variability and Trends................................................. 194

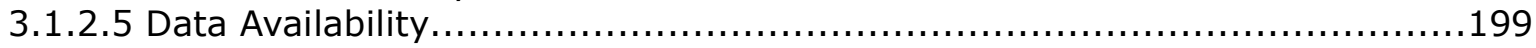

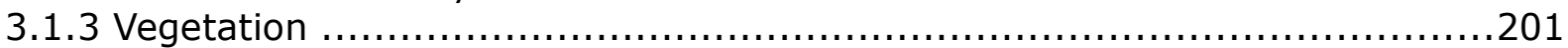

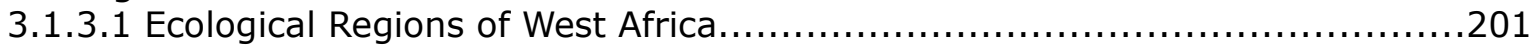

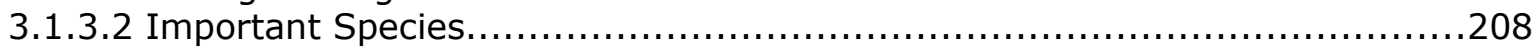

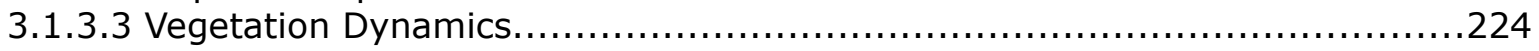

3.2 Sociogeographic Environment and Public Health.......................227

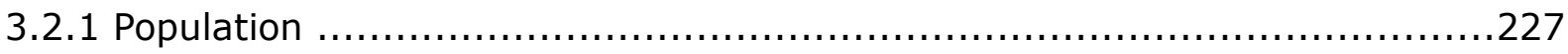

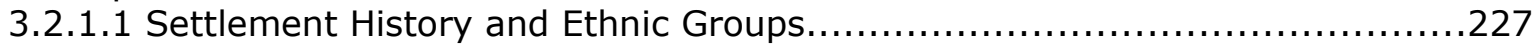

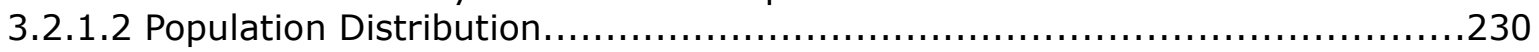

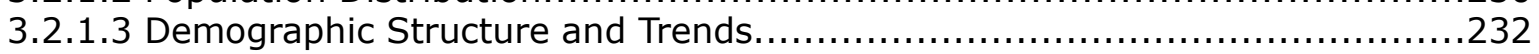

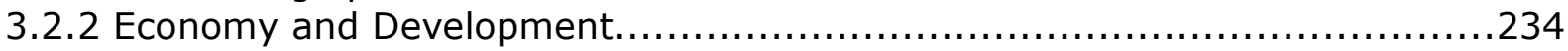

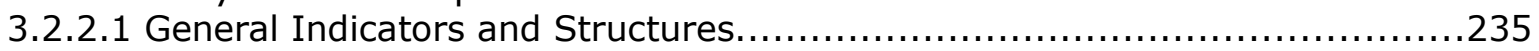

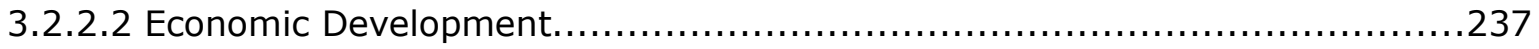

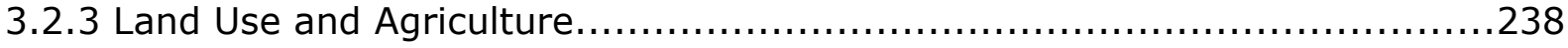

3.2.3.1 Sorghum and Millet: Traditional Subsistence Crops............................242

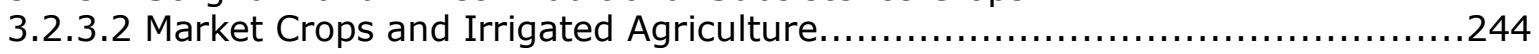


3.2.3.3 Animal Husbandry and Agro-Pastoralism.....................................252

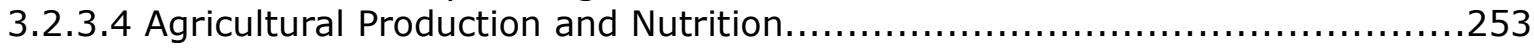

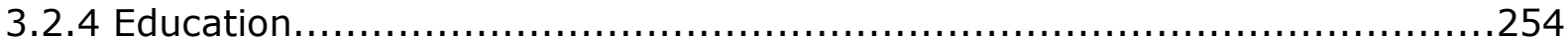

3.2.4.1 Primary, Secondary and Tertiary Education in Burkina Faso.....................254

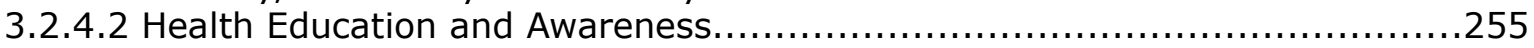

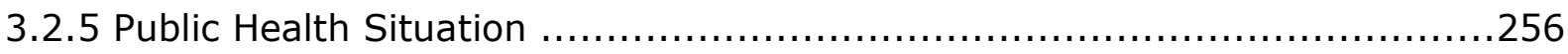

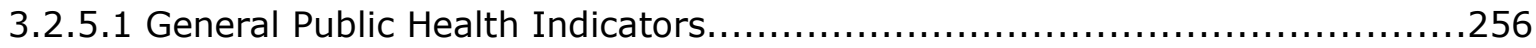

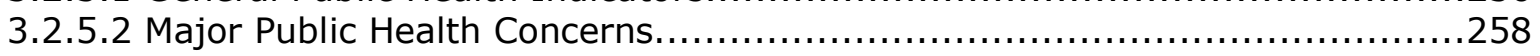

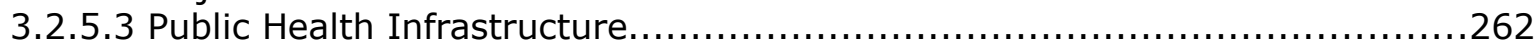

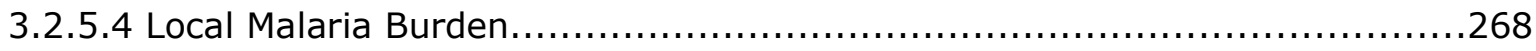

3.3 Geographic Pattern of Malaria Incidence and Risk.....................272

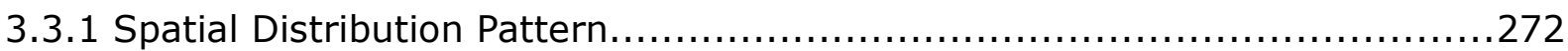

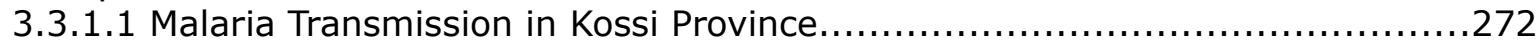

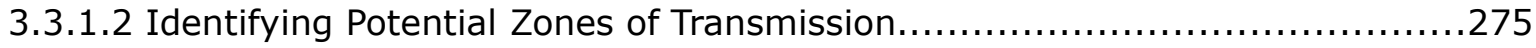

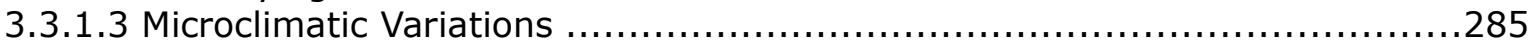

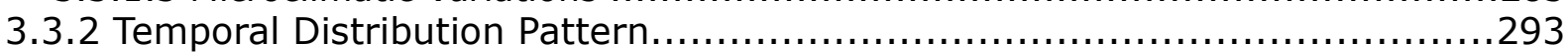

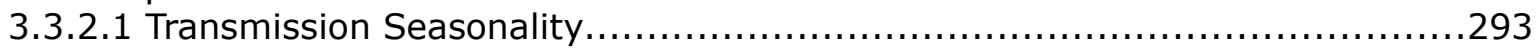

3.3.2.2 Interannual Variations in Malaria Incidence.................................... 298

3.4 Determinants of Malaria in Kossi Province.............................. 302

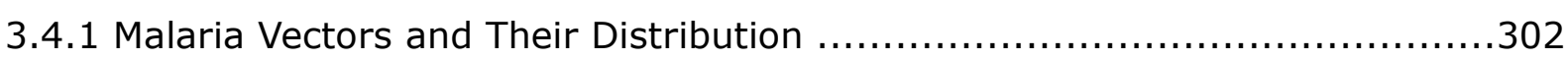

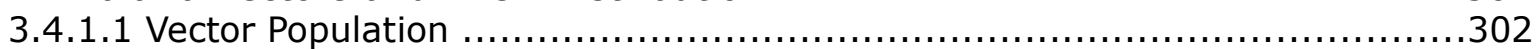

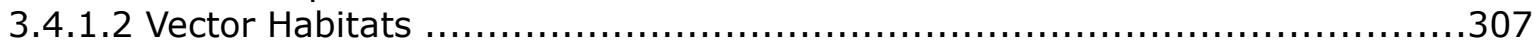

3.4.2 Geographic Determinants of Malaria Transmission............................. 308

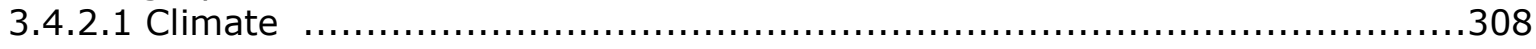

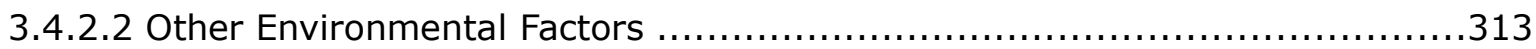

3.4.2.3 Socio-Economic and Socio-Cultural Determinants............................ 318

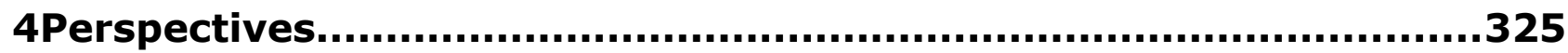

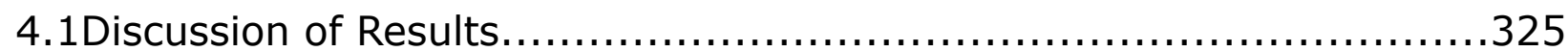

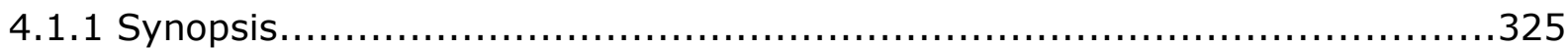

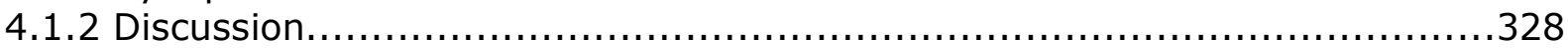

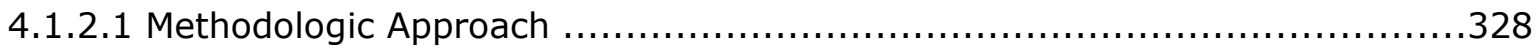

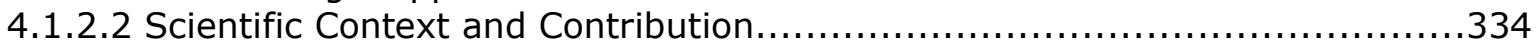

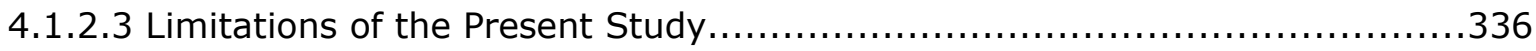

4.2The Prospects: Malaria in The Future................................. 338

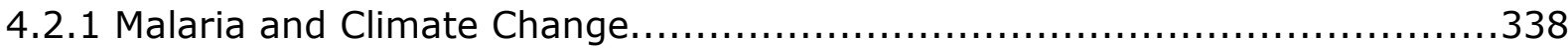

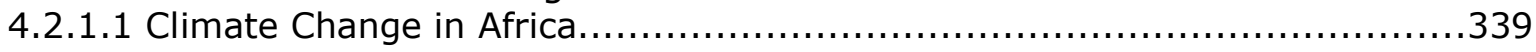

4.2.1.2 Malariological Impacts of Climate Change...................................... 341

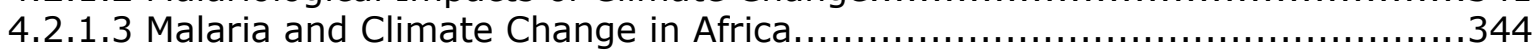

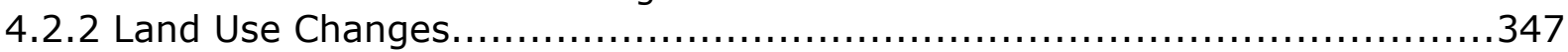

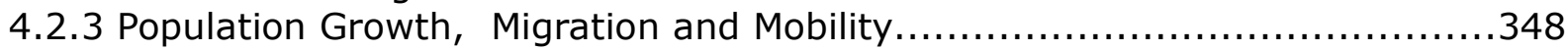

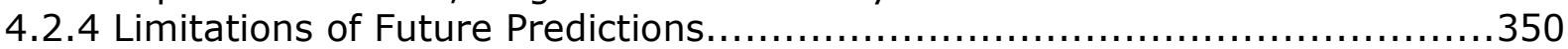

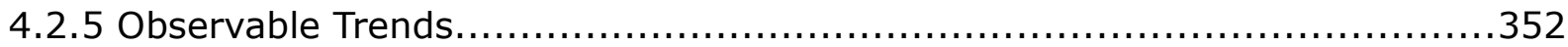

4.3 Research Perspectives................................................. 353

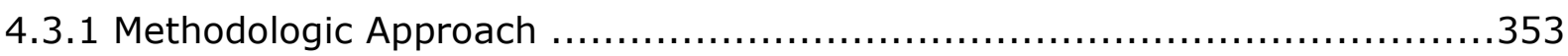

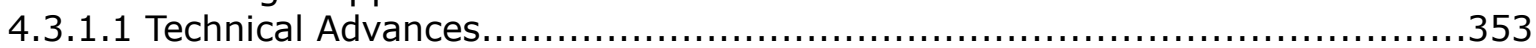

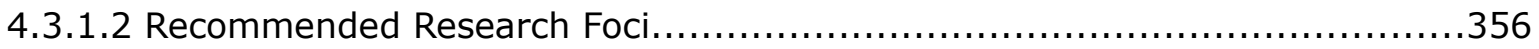

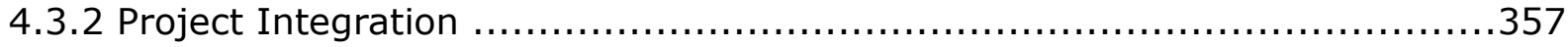


4.3.2.1 Integrated Projects on VBDs.

357

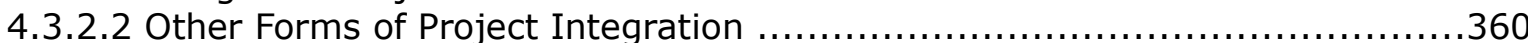

\section{Postscript}

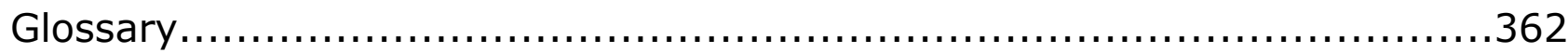

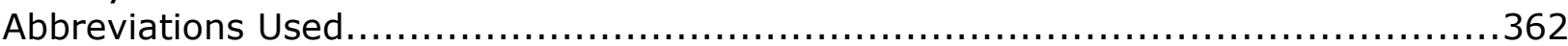

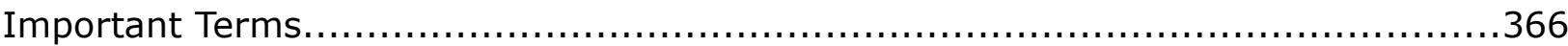

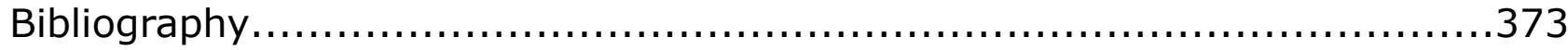

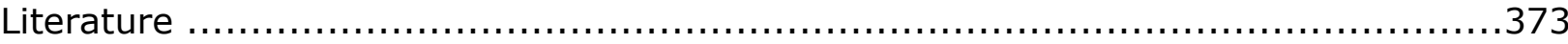

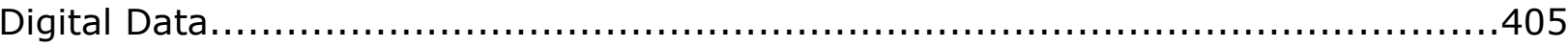

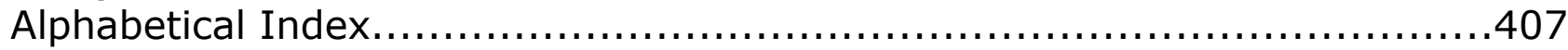

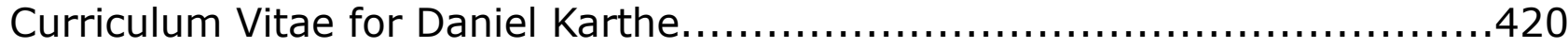

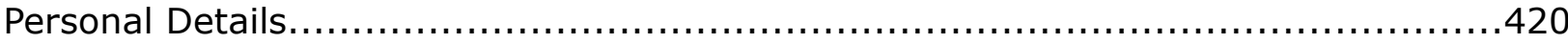

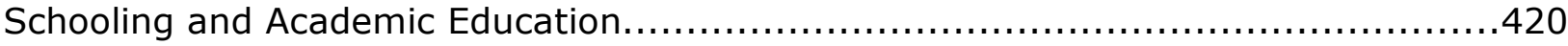

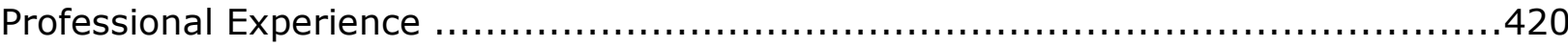

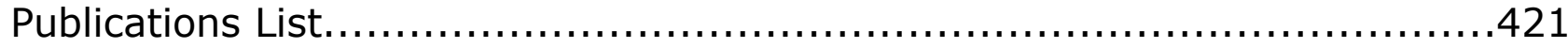

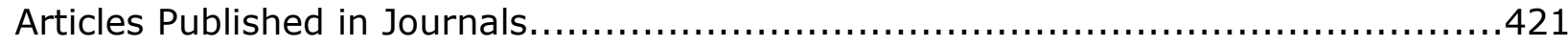

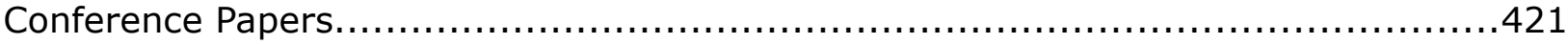

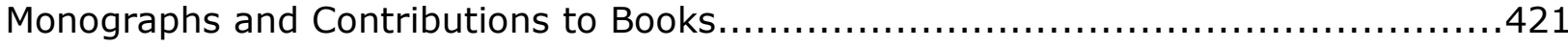




\section{List of Figures}

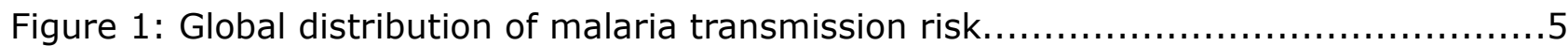

Figure 2: Cartogram of the population at risk of contracting Plasmodium falciparum

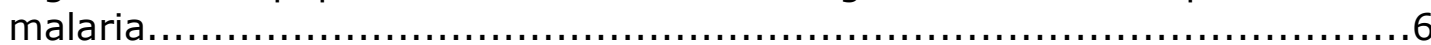

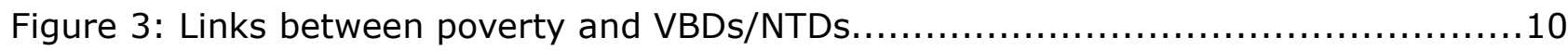

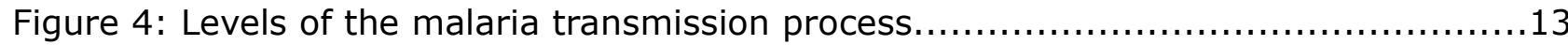

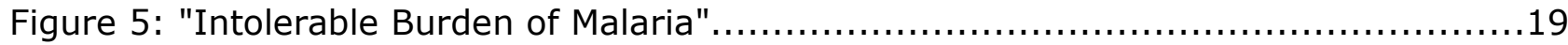

Figure 6: Malaria morbidity and mortality in Africa....................................... 24

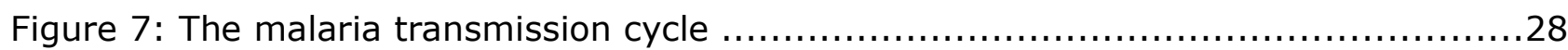

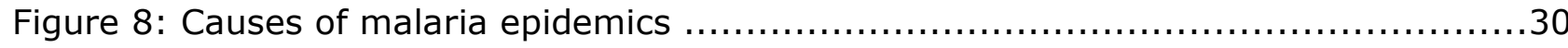

Figure 9: Female Anopheles gambiae during blood meal.................................

Figure 10: Macro-ecological factors influencing mosquito movement ...................40

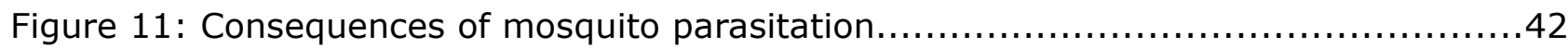

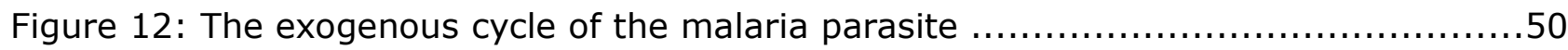

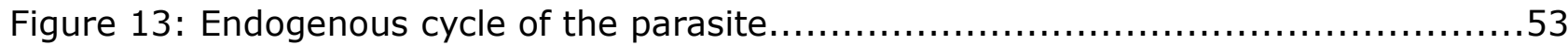

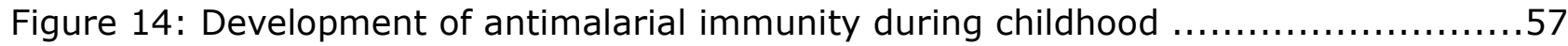

Figure 15: Duration of the extrinsic incubation period and its relation to temperature....60

Figure 16: Effect of temperature on eggs of Anopheles gambiae....

Figure 17: Effect of temperature on the aquatic stage development of Anopheles gambiae

Figure 18:Combined impact of temperature on important epidemiological factors.........64

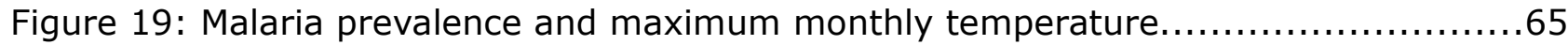

Figure 20: Temperature and Plasmodium falciparum malaria (summary).................66

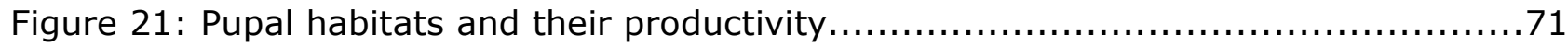

Figure 22: Egg-laying behavior of anophelines depending on soil moisture................74

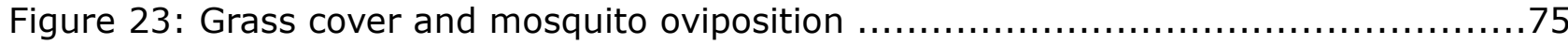

Figure 24: Aquatic vegetation and mosquito larval habitats.............................

Figure 25: Mosquito biting rate / EIR and urban agriculture $\ldots \ldots \ldots \ldots \ldots \ldots \ldots \ldots \ldots \ldots \ldots \ldots$

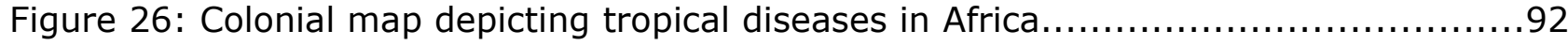

Figure 27: Climate suitability for endemic malaria in sub-Saharan Africa..................94

Figure 28: Malaria distribution in West Africa: incidence rates for children................95 
Figure 29: Ross' model of malaria transmission. 97

Figure 30: Relevance of the vector-to-host contact rate. 99

Figure 31: Impacts of reductions in malaria prevalence and host-to-vector contact......100

Figure 32: States of the human and anopheles population in the Macdonald model......101

Figure 33: States and transitions in the Garki model... 103

Figure 34: Measures of transmission intensity in the Garki model......................106

Figure 35: Symbolic presentation of Martens' individual-based model. 107

Figure 36: Rainfall anomalies in zones of epidemic malaria in West Africa (21 to 31 July 2008).

Figure 37: Logo of the Stop Malaria Now initiative. 146

Figure 38: The mosquito fish Gambusia affinis and larval-stage Anopheles gambiae. ..153

Figure 39: Physical map of Africa 167

Figure 40: Geological map of Burkina Faso. 169

Figure 41: Geological map of western Burkina Faso 170

Figure 42: Physical map of Burkina Faso..... 171

Figure 43: Physical map of Kossi Province. .173

Figure 44: FAO soil resources map of Africa.... 175

Figure 45: Soil map of Kossi Province. 178

Figure 46: Vertisol at the height of the dry season (Toni). 180

Figure 47: Climate in Dédougou (2008 vs. 1961-1990 mean)............ 183

Figure 48: Seasonal and diurnal temperature variation in Dédougou (1983 to 2008 mean)

Figure 49: Diurnal variation of temperature in Nouna. 186

Figure 50: Spatial distribution of precipitation. 187

Figure 51: Precipitation gradient in Kossi Province.... 188

Figure 52: Precipitation in Dédougou: average (1984-2008), 1994, 1996. 189

Figure 53: Precipitation and humidity in Nouna (based on meteo station data, 2004)...190

Figure 54: Precipitation and humidity in Nouna, July 2004. 191

Figure 55: Location of the ITCZ and tropical west wind zone over Africa. 192

Figure 56: Precipitation variability and trend in the Volta Basin (1901-2001)............196

Figure 57: Agro-ecological zones of Burkina Faso.....................................

Figure 58: Dry savanna near Bomborokuy (before a 'winter' shower)..... 206 
Figure 59: Landcover and landuse in Burkina Faso

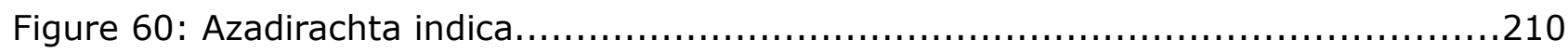

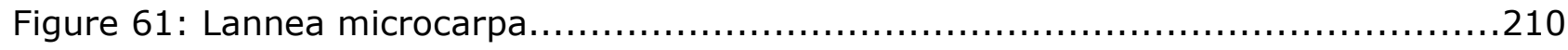

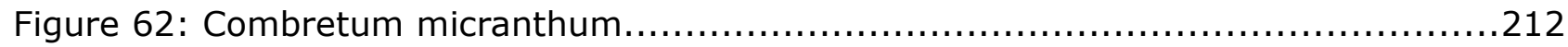

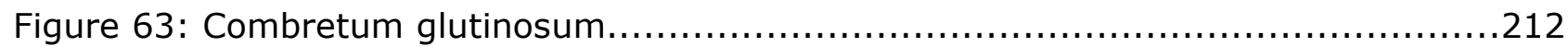

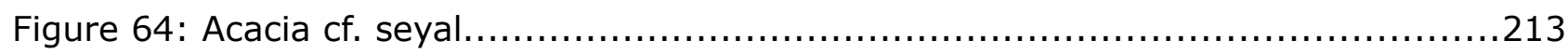

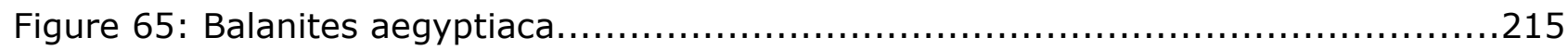

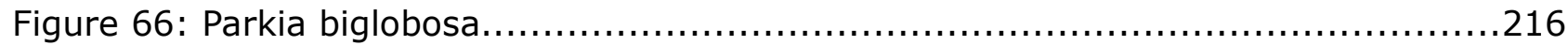

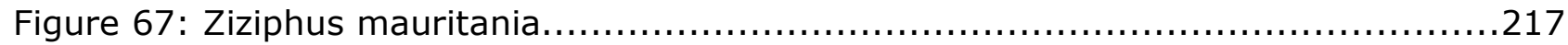

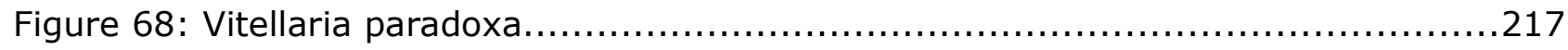

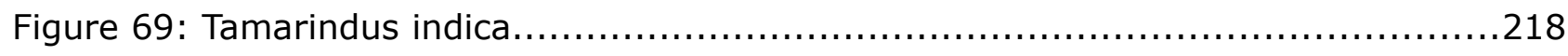

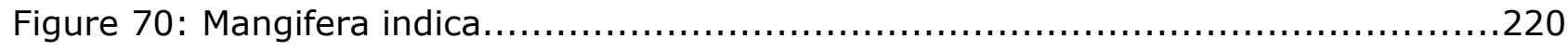

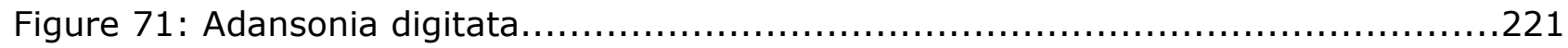

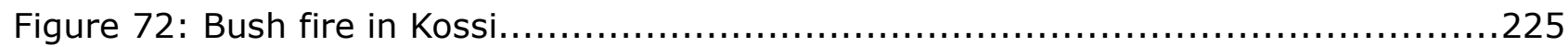

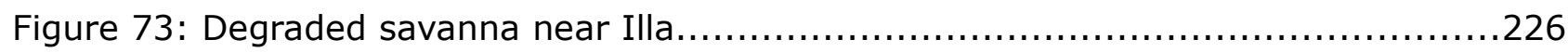

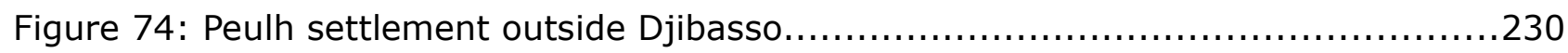

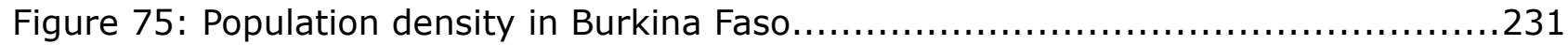

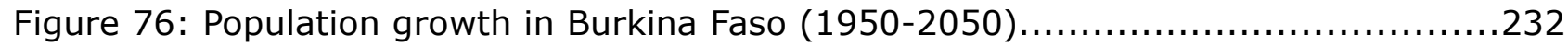

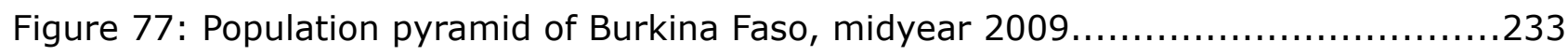

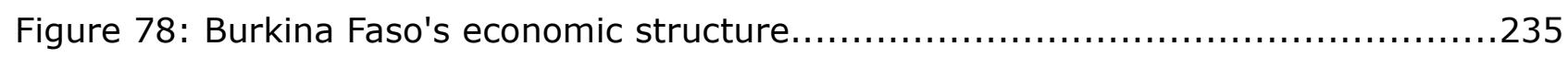

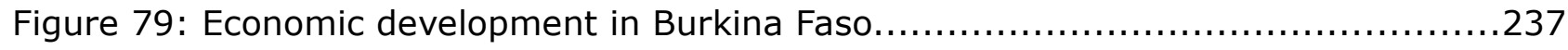

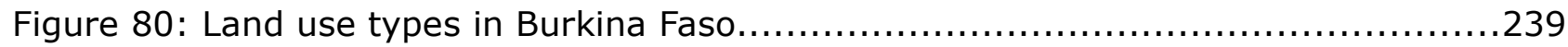

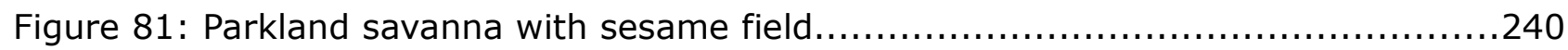

Figure 82: Land use intensity in Burkina Faso..................................... 241

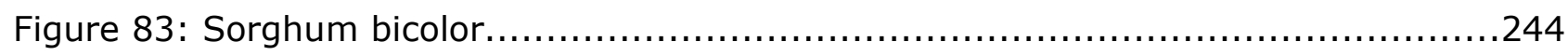

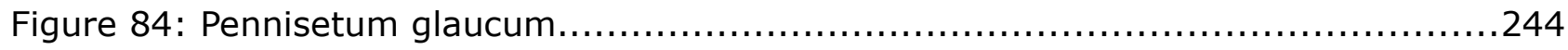

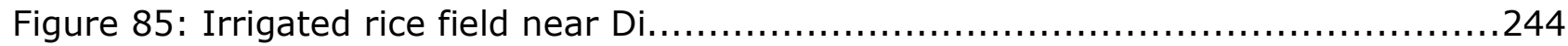

Figure 86: Area under cultivation with important cereal crops........................246

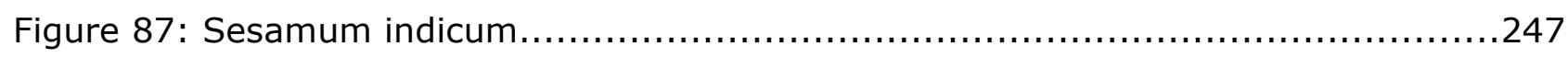

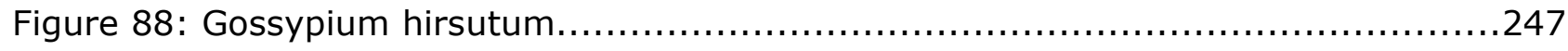

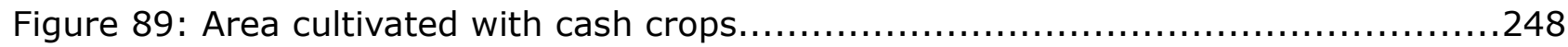


Figure 90: Area cultivated with vegetables. 249

Figure 91: Women grinding pearl millet (Pennisetum glaucum) $\ldots \ldots \ldots \ldots \ldots \ldots \ldots \ldots \ldots \ldots \ldots \ldots \ldots \ldots \ldots \ldots \ldots \ldots$

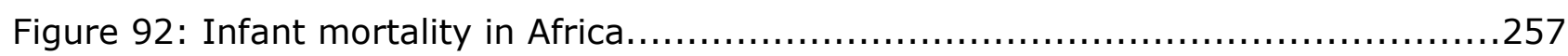

Figure 93: Leading Causes of Death in Nouna DSS (1999-2003)......................258

Figure 94: Signboard at Nouna District Hospital....................................260

Figure 95: Causes of mortality among children under five (Nouna DSS, 1999-2003)...262

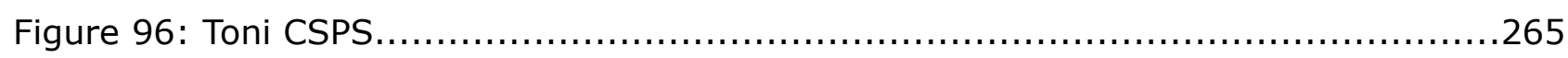

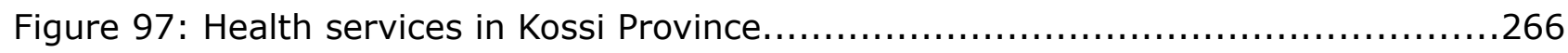

Figure 98: Sale of medicine at a market stall in Djibasso............................268

Figure 99: Spatial pattern of malaria incidence in Burkina Faso.......................270

Figure 100: Malaria as cause of death in Nouna DSS Area (1999-2003).................271

Figure 101: Endemic and epidemic malaria in West Africa..............................273

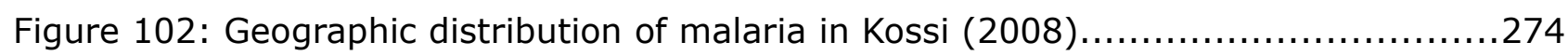

Figure 103: Newly dug irrigation and drainage channels, Illa..........................276

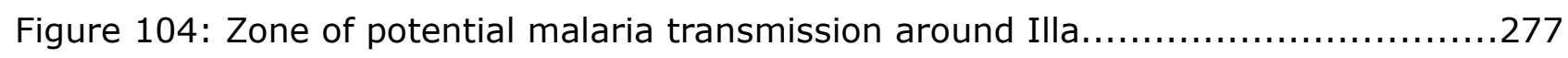

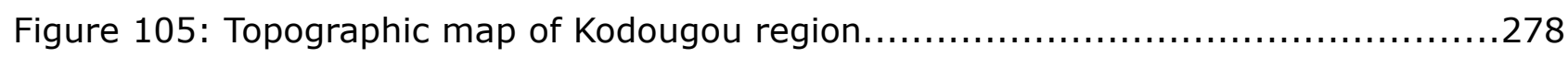

Figure 106: Depression near Kodougou Mossi at the beginning of the dry season .......280

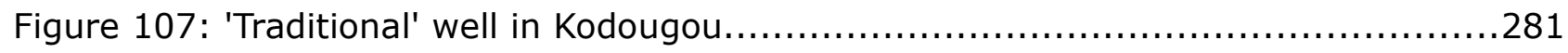

Figure 108: Kodougou region: zone of potential malaria transmission $\ldots \ldots \ldots \ldots \ldots \ldots . \ldots 2$

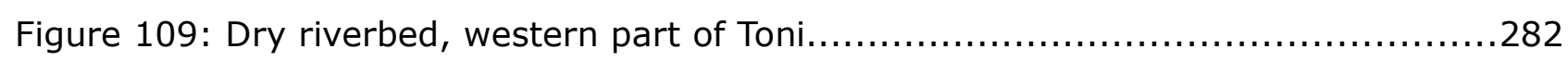

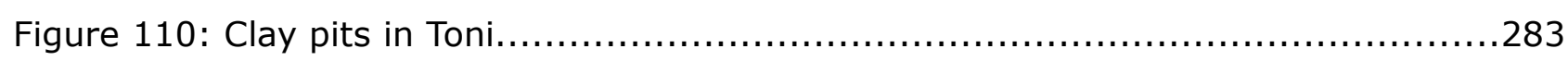

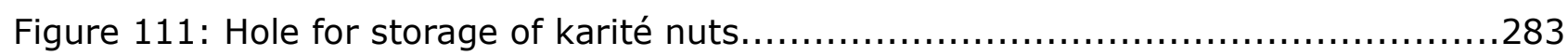

Figure 112: Toni region: zone of potential malaria transmission.........................284

Figure 113: Land surface temperatures in Kossi Province, 17 January 2008............285

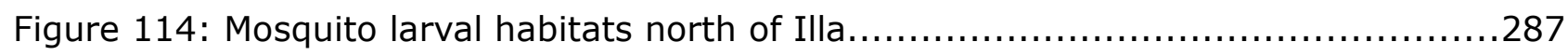

Figure 115: Location of Kodougou meteo station and test sites.......................289

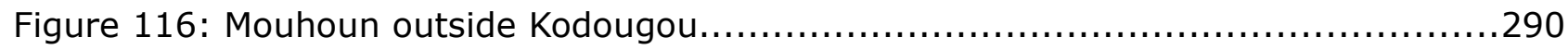

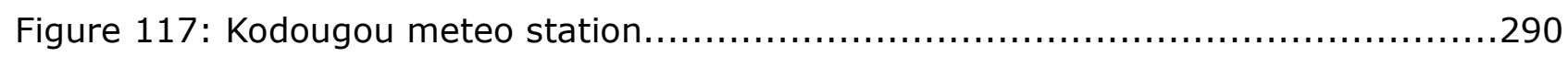

Figure 118: Diurnal course of temperature in Kodougou (08 February 2007).............291

Figure 119: Diurnal course of humidity in Kodougou (08 February 2007)................292

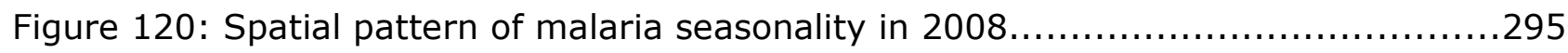




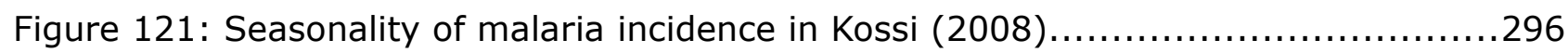

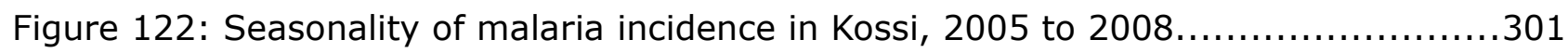

Figure 123: Distribution of important malaria vectors in Burkina Faso...................303

Figure 124: Light trap used for mosquito capture, CRSN Nouna..........................304

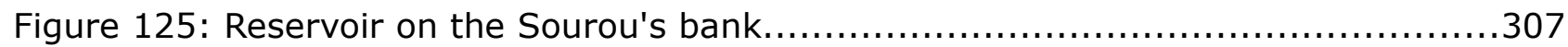

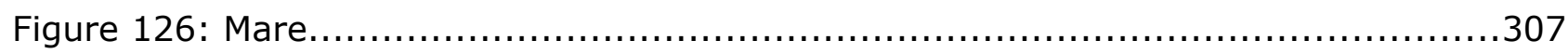

Figure 127: Precipitation, vector density and clinical malaria in Cissé.....................309

Figure 128: Precipitation and malaria incidence in U5 children in Kossi (2004)..........310

Figure 129: Malaria incidence and precipitation in Kossi (2005 to 2008).................311

Figure 130: Precipitation, vector density and clinical malaria in Kodougou (2004)........312

Figure 131: Anopheles gambiae abundance vs. temperature in Nouna (2004)...........313

Figure 132: Malaria incidence and elevation in Kossi................................ 314

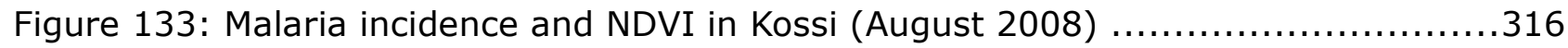

Figure 134: Mosquito abundance vs. NDVI and rainfall in Nouna (2004).................317

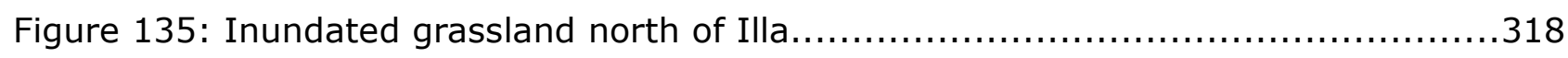

Figure 136: Survey on bednet use and personal protection in Illa......................... 321

Figure 137: Survey on bednet use and personal protection in Toni........................323

Figure 138: Rainfall estimates for West Africa (21 to 31 July 2008).....................335

Figure 139: Malaria transmission risk and climate change in Africa.......................345

Figure 140: Effects of climate change on malaria transmission risk in West Africa.......346 


\section{List of Tables}

Table 1: Mortality and morbidity burden of important infectious diseases..................

Table 2: Population at risk of Plasmodium falciparum malaria in $2007 \ldots \ldots \ldots \ldots \ldots \ldots \ldots . \ldots$

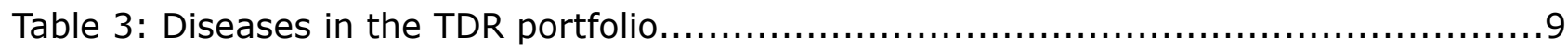

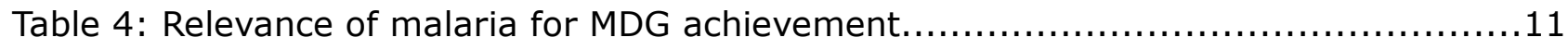

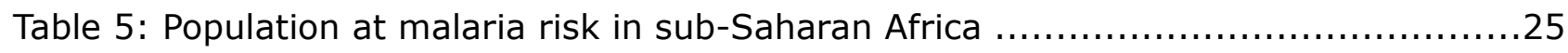

Table 6: Stage-dependent daily survival rates during aestivation..........................39

Table 7: Flight ranges of tropical mosquitoes............................................

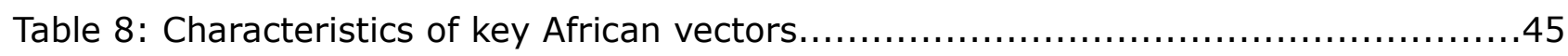

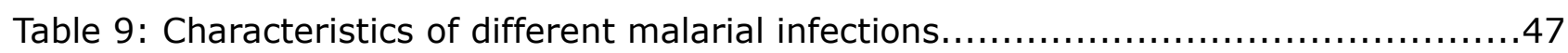

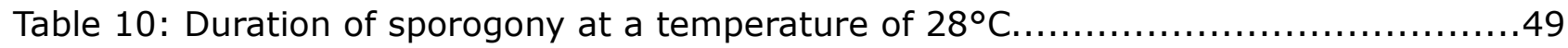

Table 11: Duration of pre-erythrocytic schizogony, pre-patent period and erythrocytic

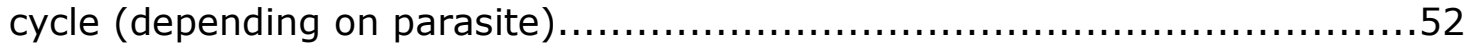

Table 12: Impact of temperature on immature stages of Anopheles gambiae..............62

Table 13: Influence of temperature on the lag between rainfall and malaria incidence....67

Table 14: Soil moisture, precipitation, NDVI as determinants malaria transmission ......75

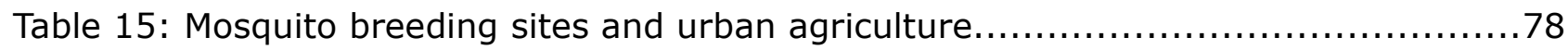

Table 16: Forest cover and mosquito biting rates (in Loreto District, Peru) ...............79

Table 17: Maize cultivation and malaria transmission intensity in Ethiopia...................80

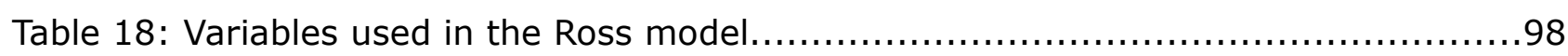

Table 19: States of human individuals in the Garki model.............................. 104

Table 20: Parameters used in the Garki model.......................................... 105

Table 21: Parameters used in Martens' individual-based model...........................109

Table 22: Parameters used in the individual-based model (Gu et al.)...................110

Table 23: Parameters used in the integrated ecological model proposed by Killeen et al.

Table 24: Environmental parameters related to malaria transmission....................116

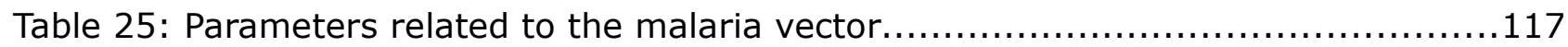

Table 26: Role of the human hosts in malaria transmission..............................118

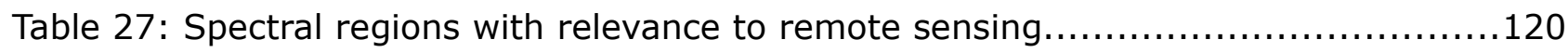


Table 28: Characteristics of Landsat 7 ETM +

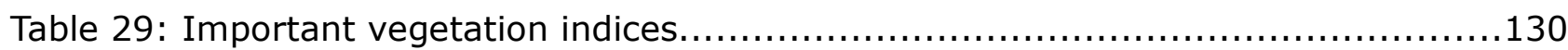

Table 30: Spectral bands used for MODIS vegetation indices..........................

Table 31: Characteristics of the IKONOS and QuickBird satellites.......................132

Table 32: Indices for detection and characterization of ponds..........................

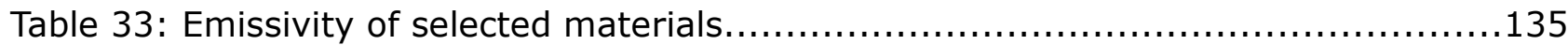

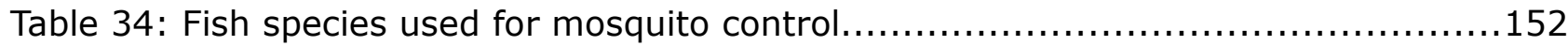

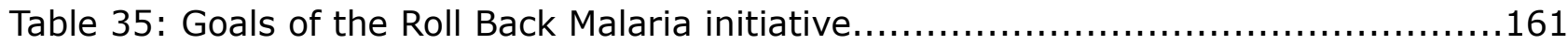

Table 36: Hydrological balance in the Mouhoun subbasin (annual data) $\ldots \ldots \ldots \ldots \ldots \ldots 173$

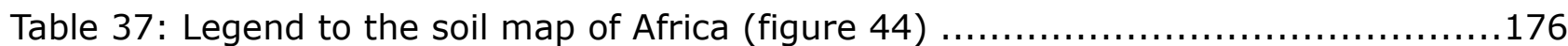

Table 38: Important soil types in West Africa (according to FAO classification)...........177

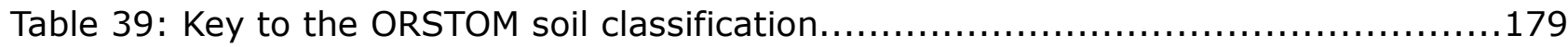

Table 40: Important soil types in Kossi province.......................................

Table 41: Temperature extrema and variation in Dédougou............................. 185

Table 42: Sahelian rainfall trends according to WMO normal periods......................195

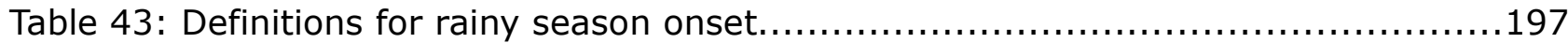

Table 44: Data availability for Dédougou meteo station (2008 vs. 1984).................199

Table 45: Data availability for the meteo stations operated by CRSN Nouna (2004).....200

Table 46: Data availability for the meteo stations operated by CRSN Nouna (2008).....200

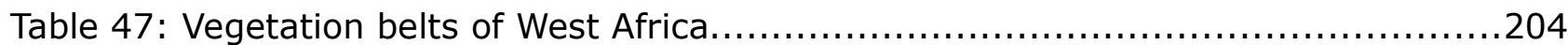

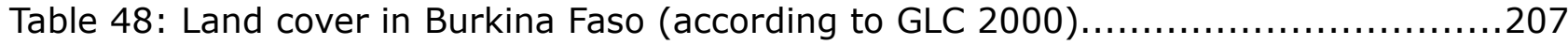

Table 49: Botanic, English and local names of important woody species found in Kossi. 209

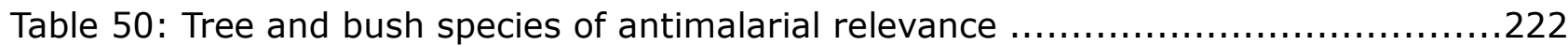

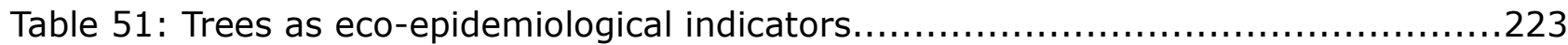

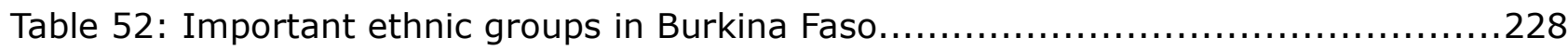

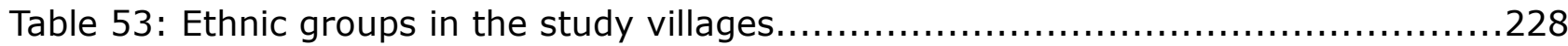

Table 54: Key demographic indicators for selected West African countries................233

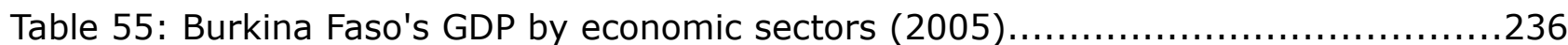

Table 56: Environmental prerequisites for sorghum and millet production...............243

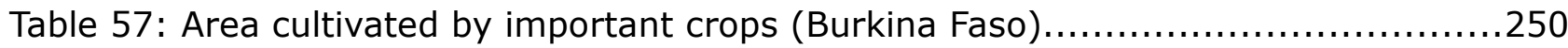

Table 58: Important crops and agro-ecological conditions for their cultivation in Kossi...251 
Table 59: Development of livestock in Burkina Faso. 252

Table 60: Per capita agricultural production of Burkina Faso and Germany...............253

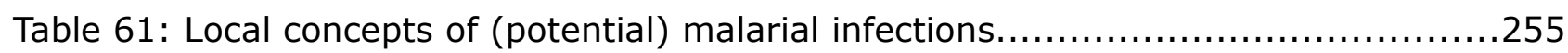

Table 62: Medical coverage in Burkina Faso: regional disparities........................258

Table 63: Mortality and morbidity burden of important infectious diseases in Burkina Faso

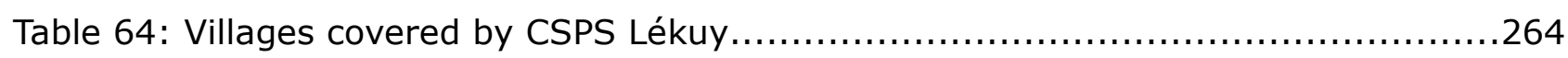

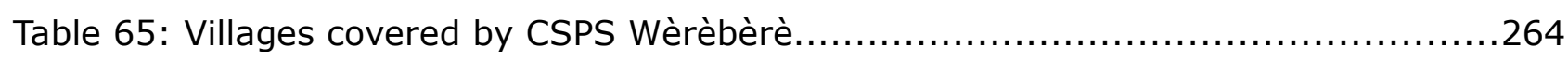

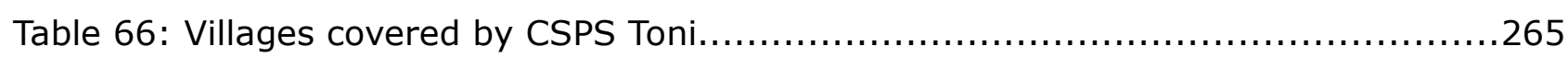

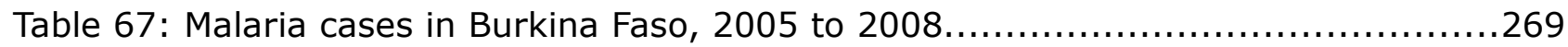

Table 68: Malaria - the leasing cause of death in most age groups in Nouna.............270

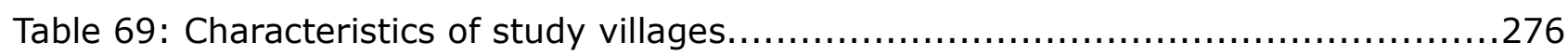

Table 70: Field survey of microclimatic conditions close to Illa.......................288

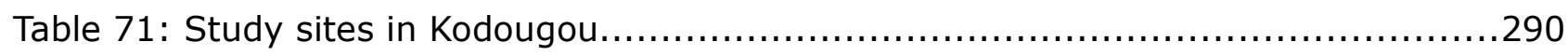

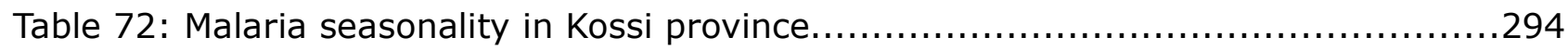

Table 73: Average malaria incidence rates (2005 to 2008) recorded at three CSPS in Kossi

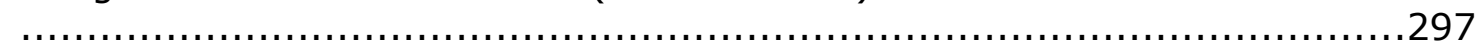

Table 74: Combined malaria cases recorded at Lékuy, Wèrèbèrè and Toni CSPS.........297

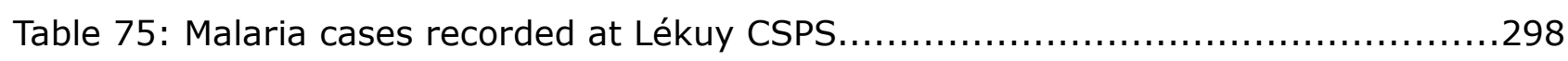

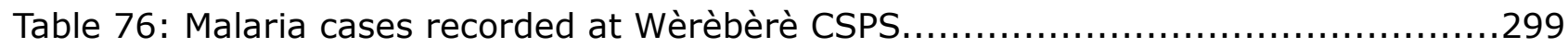

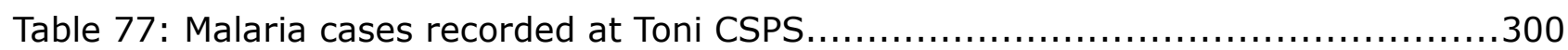

Table 78: Malaria incidence rates at three CSPSs in Kossi $(2005-2008) \ldots \ldots \ldots \ldots \ldots \ldots . \ldots . \ldots . \ldots . \ldots 300$

Table 79: Results of mosquito surveys in the Lékuy CSPS area........................... 305

Table 80: Results of mosquito surveys in the Toni CSPS area...........................306

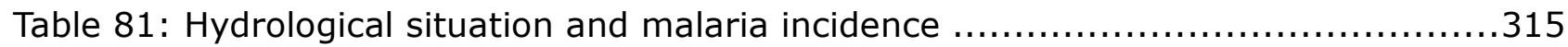

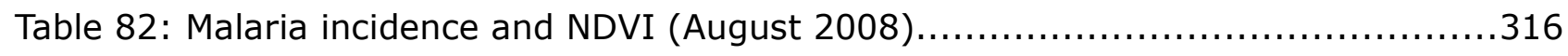

Table 83: Survey on bednet use and personal protection in Illa...........................320

Table 84: Survey on bednet use and personal protection in Toni..........................322

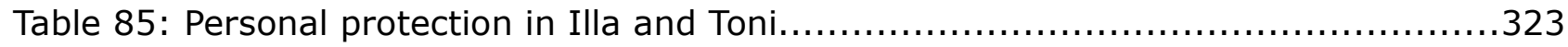

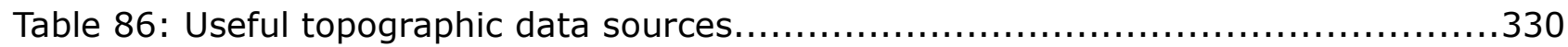

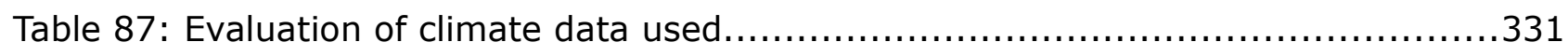

Table 88: Techniques for estimating malaria incidence................................. 332 
Table 89: Techniques for assessing malaria transmission risks.

Table 90: Regional projections for temperature and precipitation changes in Africa, A1B scenario....................................................................... 340

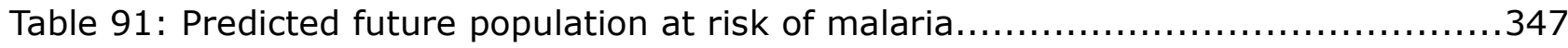

Table 92: Malaria incidence in Burkina Faso and the study villages.......................352

Table 93: Characteristics of the GeoEye-1 and WorldView-2 satellites....................355

Table 94: Vector-borne diseases with a transmission cycle closely resembling malaria. .358

Table 95: Viral diseases transmitted by mosquitoes and other flying insects..............359

Table 96: Diseases transmitted by vectors not belonging to the order Diptera..... 360 


\section{Introduction}

Malaria, one of the main causes of mortality in the world, is essentially a disease of the poor. 99,9\% (!) of the global malaria morbidity and morbidity occurs in low- and medium-income countries, whereas only a very small fraction of the global malaria burden is shouldered by the industrialized nations. ${ }^{3}$ In 2005, the World Health Organization estimated that more than 350 million out of some 400 million infections occurred in Africa, the continent worst affected by the disease. ${ }^{4}$

Malaria transmission -even in the form of epidemic outbreaks- does not occur randomly, but is closely linked to a set of ecological and socio-economic factors. A sound understanding of the connections between these driving forces and the spatial and temporal pattern of malaria transmission are a necessity for the development of reliable malaria risk prediction systems and hence prerequisites for optimizing control and intervention strategies.

The enormous dimension of the global malaria burden (hundreds of millions cases annually), the diversity, complexity and limitations of available control strategies and a shortage of financial resources in many malaria-afflicted regions have created the need for well-planned intervention programs. In the past, the relatively poor availability of differentiated and up-to-data base data often coincided with failures of control programs, including ambitious projects such as the World Health Organization's global campaign to eradicate malaria.

In recent years, new technologies such as remote sensing and geographic information systems have evolved as promising tools for medical geography in general and malariology in particular: While earth observing satellites provide almost real-time data on the physical environment, geographic information systems have become valuable instruments for the analysis of spatio-temporal data. This has already brought about a leap ahead from the static malaria maps of the past - and continuous, rapid progress in the fields of remote sensing and information technology promises further advances in the near future.

This study looks at the potentials and limitations of utilizing geographic information systems for combining remote sensing and ground based data on the environment and anthropogenic factors under the ecological, economic and sociocultural conditions found in a rural region of Sub-Saharan West Africa. Based on a systematic overview on malaria epidemiology, its determinants and the potential of geographic tools and methods for malaria monitoring on the

3 Calculation based on burden of disease data; Lopez, A.; Mathers, C.D.; Ezzati, M. et al. (ed.) (2006), pp. 126, 174, 180, 228.

4 http://www.who.int/evidence/bod/ (accessed 22/11/07) 
one hand and an introduction to the geography of the study region on the other hand, the causes of intraregional and temporal variations in malaria in Kossi Province (Burkina Faso) will be investigated. Different methodological approaches will be presented, followed by an evaluation of their feasibility in the framework of this project. Finally, the results of this study will be assessed in the context of other scientific findings and recommendations for future research be formulated.

\subsection{The Intolerable Burden of Malaria}

At the beginning of the $21^{\text {st }}$ century, malaria remains the most important parasitic disease worldwide. ${ }^{5}$ More than $40 \%$ of the world population and $93 \%$ of the African population are exposed to the risk of contracting malaria ${ }^{6}$, and every 30 seconds, a child dies from malaria. ${ }^{7}$ However, this risk is far from equally distributed, and much of the world's malaria burden falls on low-income countries, particularly on the African continent.

\subsubsection{Malaria - A Re-Emerging Threat To Human Health?}

The World Health Organization (WHO) estimates that around 3 billion people in 109 countries live in malaria risk areas. ${ }^{8}$ Among these, more than 2,3 billion live in areas of Plasmodium falciparum endemicity, the malaria parasite causing the severest form of infection. ${ }^{9}$ Due to global population growth, this number is greater than at any time in history. ${ }^{10}$ About 250 to 500 million people get infected each year, and malaria causes at least 1 million deaths annually ${ }^{11}$, even though this may be an underestimation. Assuming that less than $10 \%$ of all malaria cases are officially reported, Breman et al. (2007) estimated the annual malaria burden to be around 1 billion infections and more than two million deaths. ${ }^{12}$ In Sub-Sahara Africa, malaria accounts for about $18 \%$ of all childhood mortality. ${ }^{13}$ The relative importance of malaria as compared to other important tropical diseases is illustrated in table 1 which broadly categorizes diseases as communicable infectious diseases (i.e. diseases directly spread one from person to another, e.g. by droplet infections), sexually transmitted diseases (i.e. diseases most commonly spread by sexual intercourse), food-

5 KouYaté, B.; Sié, A.; Yé, M. et al. (2007), p. 997.

6 Organisation Mondiale de la Santé (Ed.) (1995), pp. $2 ; 9$.

7 Roll Back Malaria Partnership (2005), p. 3.

8 WHO (2008), p. 1.

9 Hay, S.I.; Guerra, C.A. ; Gethin, P.W. et al. (2009), p. 295.

10 Hay, S.I.; Guerra, C.A.; TAtem, A.J. et al. (2005), p. 81.

11 WHO (2005), p. 11 ; WHO (2008), p. 1.

12 Breman, J.G.; Alilio, M.S. \& White, N. (2007), p. vi.

13 WHO (2005), p. 11. 
and waterborne diseases (i.e. diseases which typically result from the consumption of microbiologically contaminated food and drinks) and vectorborne infections (diseases transmitted from one person to another by some sort of agent, most commonly a mosquito).

\begin{tabular}{|c|c|c|c|c|}
\hline Disease & Category & $\begin{array}{l}\text { Disease } \\
\text { burden } \\
\text { [DALYs] }\end{array}$ & $\begin{array}{l}\text { Annual } \\
\text { number of } \\
\text { deaths }\end{array}$ & $\begin{array}{l}\text { Regions most } \\
\text { affected }^{14}\end{array}$ \\
\hline HIV/AIDS & $\begin{array}{l}\text { Sexually- } \\
\text { transmitted } \\
\text { disease }\end{array}$ & $\begin{array}{c}71,46 \text { mio. } \\
(84,9 \text { mio. })\end{array}$ & 2.574 .000 & $\begin{array}{l}\text { Sub-Saharan } \\
\text { Africa }\end{array}$ \\
\hline Malaria & $\begin{array}{l}\text { Vector-borne } \\
\text { disease }\end{array}$ & $\begin{array}{l}39,97 \text { mio. } \\
(45,6 \text { mio. })\end{array}$ & 1.208 .000 & $\begin{array}{l}\text { Sub-Saharan } \\
\text { Africa }\end{array}$ \\
\hline Tuberculosis & $\begin{array}{l}\text { Communicable } \\
\text { infectious } \\
\text { disease }\end{array}$ & 36,09 mio. & 1.606 .000 & $\begin{array}{l}\text { South Asia, } \\
\text { East Asia, Sub- } \\
\text { Saharan Africa }\end{array}$ \\
\hline Meningitis & $\begin{array}{l}\text { Communicable } \\
\text { infectious } \\
\text { disease }\end{array}$ & 5,61 mio. & 173.000 & $\begin{array}{l}\text { East Asia, Sub- } \\
\text { Saharan } \\
\text { Africa, Latin } \\
\text { America }\end{array}$ \\
\hline Hepatitis B & $\begin{array}{l}\text { Sexually- } \\
\text { transmitted } \\
\text { disease }\end{array}$ & 2,17 mio. & 100000 & $\begin{array}{l}\text { East Asia, } \\
\text { South Asia, } \\
\text { Sub-Saharan } \\
\text { Africa }\end{array}$ \\
\hline $\begin{array}{l}\text { Schisto- } \\
\text { somiasis }\end{array}$ & $\begin{array}{l}\text { Vector-borne } \\
\text { disease }\end{array}$ & 1,52 mio. & $\begin{array}{r}14.000 \\
(280.000) \\
\end{array}$ & $\begin{array}{l}\text { Africa, East } \\
\text { Asia }\end{array}$ \\
\hline $\begin{array}{l}\text { Japanese } \\
\text { encephalitis }\end{array}$ & $\begin{array}{l}\text { Vector-borne } \\
\text { disease }\end{array}$ & 0,60 mio. & 14.000 & $\begin{array}{l}\text { South Asia, } \\
\text { East Asia }\end{array}$ \\
\hline Dengue fever & $\begin{array}{l}\text { Vector-borne } \\
\text { disease }\end{array}$ & 0,53 mio. & 19.000 & $\begin{array}{l}\text { South Asia; } \\
\text { East Asia; } \\
\text { Latin America }\end{array}$ \\
\hline Leprosy & $\begin{array}{l}\text { Communicable } \\
\text { infectious } \\
\text { disease }\end{array}$ & 192.000 & 6.000 & South Asia \\
\hline
\end{tabular}

Table 1: Mortality and morbidity burden of important infectious diseases

14 Lopez, A.; Mathers, C.D.; Ezzati, M. et al. (ed.) (2006), p. 132-162.

15 Lopez, A.; Mathers, C.D.; Ezzati, M. et al. (ed.) (2006), pp. 132-228;

data in brackets: World Health Report 2004. 
Among the world's most deadly infectious diseases, only HIV/AIDS, tuberculosis and the entire group of diarrheal diseases (if all are combined) cause more deaths than malaria. However, in this context it must be kept in mind that simultaneous infections involving malaria and other diseases (such as HIV) are frequent, with health statistics in developing countries often failing to identify such co-infections. Therefore, causes of death are often assigned to a single disease even though lethal consequences may have been the result of a certain combination of diseases. For reasons of comparability, the WHO recommends to express the total disease burden in terms of DALYs or disability-adjusted life years, an index which combines years of life lost due to premature mortality and years of life lost due to time lived in states of less than full health). Measured by DALYs, malaria takes the third rank among the world's most important infectious diseases, behind HIV/AIDS and the diarrheal diseases (but again, only if all of them are combined).

Malaria is or has been found on all continents except for Antarctica, and wherever suitable vector mosquitoes are found, there is a potential risk of malaria transmission. Malaria transmission is usually confined to areas with a tropical or subtropical climate, and the continent most affected by malaria is Africa. However, malaria transmission also occurs in large parts of Latin America, and South and Southeast Asia (see figure 1). ${ }^{16}$

16 Service, M.W. (1993), p. 102. 


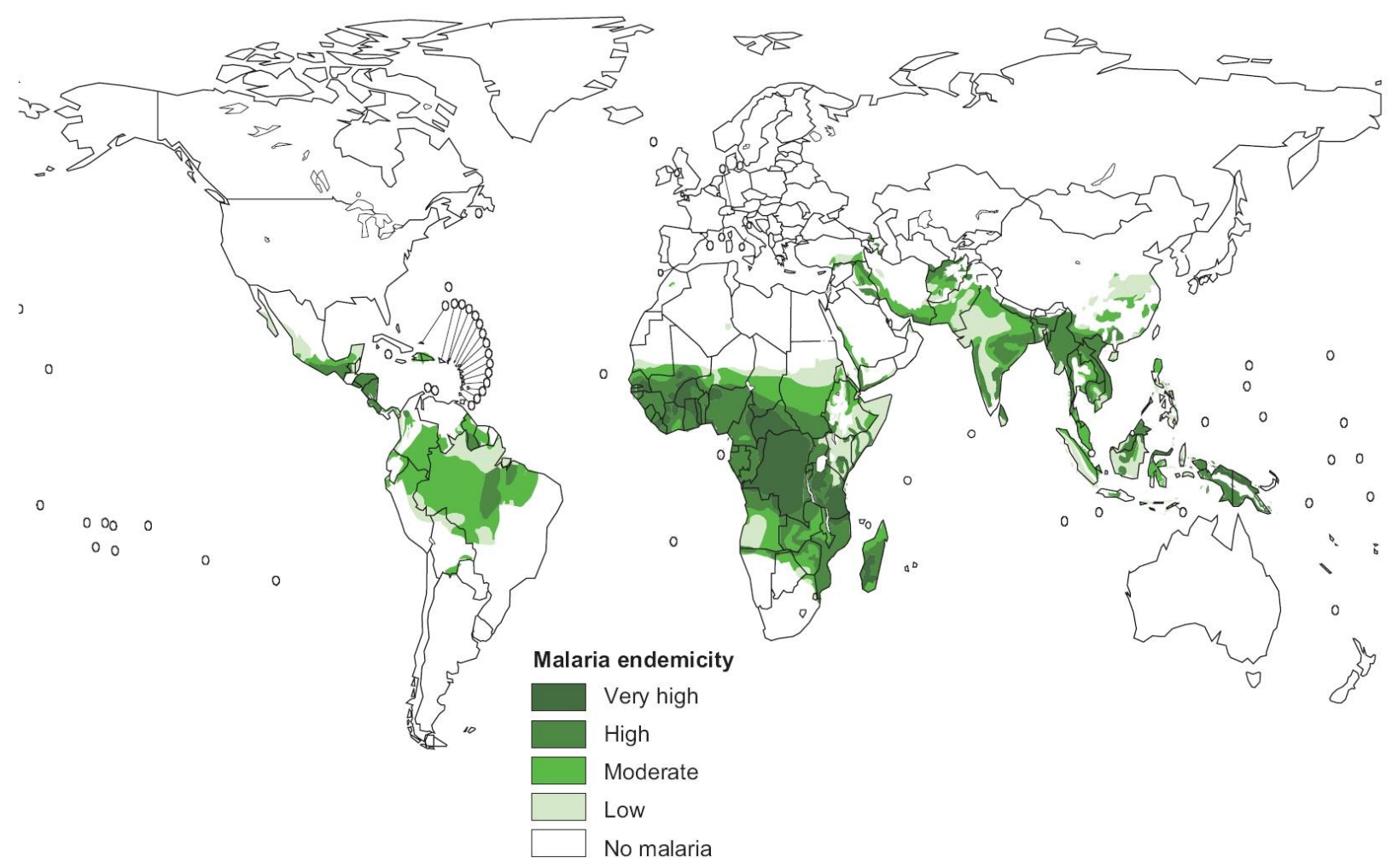

Figure 1: Global distribution of malaria transmission risk ${ }^{17}$

Even though it is impossible to precisely predict the malaria risk of the future, it is estimated that the effects of global warming and a growing population in malaria risk areas will result in an increased malaria incidence. Some studies expect a rise in the number of infections in the order of several hundred millions by the middle of the $21^{\text {st }}$ century. ${ }^{18}$

\subsubsection{Malaria in Sub-Saharan Africa}

Malaria exists in large parts of the tropics and subtropics (see figure 1), with sub-Saharan Africa and the Indian subcontinent forming the most affected regions (see figure 2 and table 2). Most malaria infections in Africa south of the Sahara are caused by Plasmodium falciparum, the most life-threatening of the malaria parasites. Moreover, this region is home to the most efficient species of the mosquitoes which transmit the disease. Moreover, many countries in this region lack both the (financial) resources and the infrastructure to effectively

17 WHO (2005), p. 281.

18 Martens, P. (1998), p. 53; OvergaArd, H. (2001), p. 7. 
combat malaria. One of the greatest challenges facing Africa in the fight against malaria is drug resistance: resistance to chloroquine, the cheapest and most widely used anti-malarial, is common throughout Africa. ${ }^{19}$

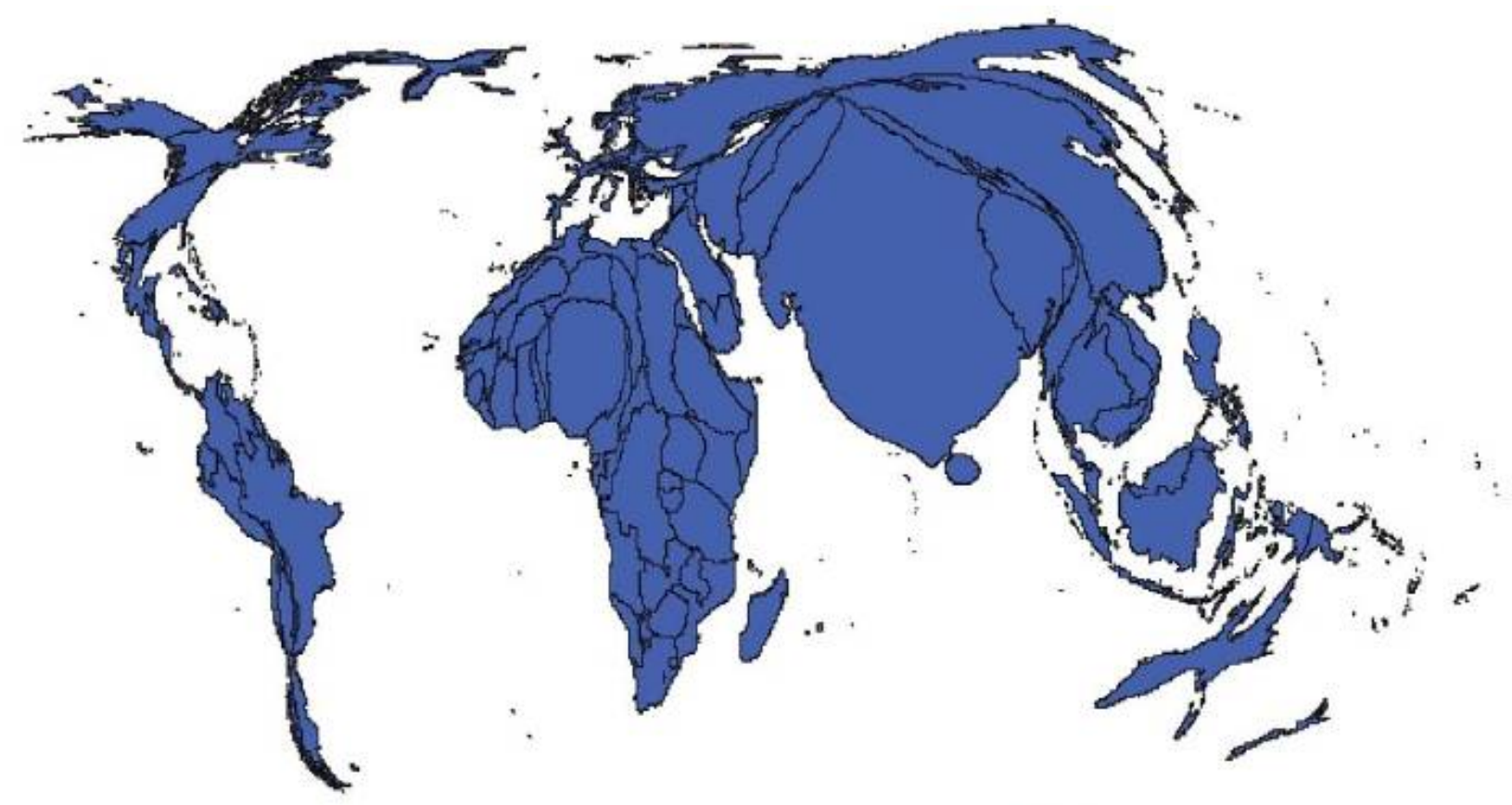

Figure 2: Cartogram of the population at risk of contracting Plasmodium falciparum malaria ${ }^{20}$

Even though the population living in regions of endemic Plasmodium falciparum malaria in Asia is slightly greater than in Africa (a fact that is certainly to a large part related to the much higher total population of Asia), $98 \%$ of the population living in areas of high transmission risk are Africans (see table 2).

19 http://www.rollbackmalaria.org/cmc upload/0/000/015/370/RBMInfosheet 3.htm, accessed 08/11/07.

20 Guerra, C.A; Snow, R.W. \& Hay, S.I. (2006), p. 356. 


\begin{tabular}{|c|c|c|c|c|c|}
\hline \multirow[t]{2}{*}{ Region } & \multirow{2}{*}{$\begin{array}{l}\text { Unstable } \\
\text { Risk }\end{array}$} & \multicolumn{3}{|c|}{ Stable Risk } & \multirow[t]{2}{*}{ Total } \\
\hline & & Low risk & $\begin{array}{l}\text { Moderate } \\
\text { risk }\end{array}$ & High Risk & \\
\hline Americas & 50,06 & 40,64 & 0 & 0 & 90,71 \\
\hline Africa+ & 21,88 & 114,5 & 196,83 & 345,28 & 678,49 \\
\hline Asia & 911,26 & 603,61 & 75,29 & 6,75 & 1596,91 \\
\hline \multicolumn{6}{|c|}{$\begin{array}{l}\text { Population figures in millions; Africa+ includes Yemen and Saudi-Arabia. } \\
\text { Risk stratification according to the prevalence rate of Plasmodium falciparum } \\
\left(\text { PfPR }_{2-10}\right) \text { in children between the ages of } 2 \text { and } 10 \text { years. } \\
\text { Low risk ............... PfPR } 2-10 \leq 5 \% \\
\text { Moderate risk ......... PfPR } 2-10>5 \% \text { to }<40 \% \\
\text { High Risk ............. PfPR } 2-10 \geq 40 \%\end{array}$} \\
\hline
\end{tabular}

Table 2: Population at risk of Plasmodium falciparum malaria in $2007^{21}$

Most of sub-Saharan Africa has stable endemic malaria because climatic conditions ideal for transmission coincide with the ranges of Anopheles gambiae, Anopheles arabiensis and Anopheles funestus, the most efficient vector mosquitoes in the world..$^{22} 60 \%$ of all malarial infections, $75 \%$ of severe infections caused by Plasmodium falciparum and $80 \%$ of all malaria deaths occur in sub-Saharan Africa. ${ }^{23}$ With a total population of nearly 300 million people, sub-Saharan West Africa represents the region with the largest population exposed to high levels of malaria transmission intensity in the world. ${ }^{24}$ Malaria is responsible for $18 \%$ of the childhood mortality in this region and accounts for $20 \%$ to $50 \%$ of hospital admissions and $15 \%$ to $35 \%$ of all hospital deaths in endemic countries. ${ }^{25}$ In West Africa, malaria infections are responsible for about 1 million deaths annually, mostly affecting children below the age of $5 .^{26}$

Moreover, malaria impedes economic progress and continues to be a severe challenge for local health care authorities. Malaria has been estimated to cost Africa more than US $\$ 12$ billion every year in lost GDP and accounts for $40 \%$ of Africa's public health expenditure. Malaria is both "a disease of poverty and a cause of poverty". ${ }^{27}$ The World Health Organization thus concludes that malaria control plays a "key role in poverty reduction in high burden countries". ${ }^{28}$

21 Hay, S.I.; Guerra, C.A. ; Gethin, P.W. et al. (2009), p. 295.

22 Killeen, G.; Seyoum, A. \& Knols, G.J. (2004), p. 87.

23 Hay, S.I.; Омumbo, J.A.; Craig, M.H. \& Snow R.W. (2000), p. 174; 194; WHO (2005), p. xvii.

24 Kleinschmidt, I.; Омumbo, J; Briët, O. et al. (2001), pp. 780.

25 WHO (2005), p. xvii; Hay, S.I.; Guerra, C.A.; TATem, A.J. et al. (2005), p. 81.

26 Hay, S.I.; Omumbo, J.A.; Craig, M.H. \& Snow R.W. (2000), p. 174.

27 http://www.rollbackmalaria.org/cmc upload/0/000/015/370/RBMInfosheet 3.htm, accessed 08/11/07.

28 World Health Organization (2005), p.1. 


\title{
1.1.3 Malaria - A Neglected Tropical Disease?
}

Infectious diseases are responsible for more than $25 \%$ of the global human disease toll. ${ }^{29}$ One group among these diseases are infections with a high prevalence in the developing world but little or no importance in industrialized nations: the neglected tropical diseases (NTDs).

\begin{abstract}
«The neglected diseases are burdens of forgotten populations diseases of the poorest of the poor- and generally do not affect developed countries, thus are largely ignored by medical science. $\gg^{30}$
\end{abstract}

For the 'neglected' tropical diseases, more than $99 \%$ of the global burden fall on low- and middle-income countries. ${ }^{31}$ However, since NTDs usually affect the poorest of the poor, there is no promising commercial market for drugs and vaccines against them ${ }^{32}$, and there is no indication that drug development for neglected diseases will significantly improve in the near future. ${ }^{33}$ Less than $10 \%$ of the global spending on health research is spent for those infectious diseases that cause $90 \%$ of the global disease burden. ${ }^{34}$ The discovery and development of most of the current pharmaceuticals against infectious tropical diseases was driven by colonial requirements during the first part of the $20^{\text {th }}$ century. As Western interests shifted away form these regions, tropical diseases have become progressively neglected, mainly because they do not offer sufficient financial returns for the pharmaceutical industry. ${ }^{35}$ Only $1 \%$ of the pharmaceutical drugs licensed in the recent past were for tropical diseases, many of them being merely byproducts from veterinary research.

In its program on Tropical Disease Research (TDR), the WHO distinguishes three groups of infectious tropical diseases, all of which are listed in table 3:

29 Harrus, S. \& Baneth, G. (2005), p. 1311.

30 Beyrer, C.; Villar, J.C.; SuWAnvanichiou, V. et al. (2007), p. 619.

31 MAY, R.M. (2007), pp. 498; 500.

32 Hotez P.J.; Molyneux, D.H.; Fenwick, A. et al. (2006), p. 577.

33 Trouiller, P.; Olliaro, P. \& Torreele, E. (2002), p. 2190.

34 Remme, J.H.F.; Blas, E.; Chitsulo, L. et al. (2002), p. 435.

35 Trouiller, P.; Olliaro, P. \& Torreele, E. (2002), p. 2188. 


\begin{tabular}{|l|l|l|}
\hline $\begin{array}{l}\text { TDR } \\
\text { Category }\end{array}$ & Features & Diseases \\
\hline Category 1 & $\begin{array}{l}\text { Emerging or re-emerging diseases for } \\
\text { which no effective control strategy } \\
\text { exists and for which better } \\
\text { intervention tools are needed }\end{array}$ & $\begin{array}{l}\text { African trypanosomiasis } \\
\text { Dengue fever } \\
\text { Leishmaniasis }\end{array}$ \\
\hline Category 2 & $\begin{array}{l}\text { Diseases for which a control strategy } \\
\text { exists, but for which a sustained } \\
\text { reduction in the disease burden has } \\
\text { not been achieved }\end{array}$ & $\begin{array}{l}\text { Malaria } \\
\text { Schistosomiasis } \\
\text { Tuberculosis }\end{array}$ \\
\hline Category 3 & $\begin{array}{l}\text { Diseases for which cost-effective } \\
\text { control strategies exist; falling } \\
\text { disease burden; targeted for } \\
\text { elimination }\end{array}$ & $\begin{array}{l}\text { Chagas' disease } \\
\text { Leprosy } \\
\text { Lymphatic filariasis } \\
\text { Onchocerciasis }\end{array}$ \\
\hline
\end{tabular}

Table 3: Diseases in the TDR portfolio ${ }^{36}$

Occasionally, malaria is excluded from the list in table 3 (and counted as one of the "big three", namely HIV/AIDS, tuberculosis and malaria) ${ }^{37}$. However, there are several reasons not to overlook malaria in the context of NTD research and intervention programs:

- Out of 10 NTDs, eight are vector-borne ("VBDs"), with malaria being the most prominent example. ${ }^{38}$

- Malaria causes $89 \%$ of the combined disease burden of these vectorborne NTDs. ${ }^{39}$

- Polyparasitism is frequent due to the geographic overlap of the regions affected by neglected tropical diseases. Coinfections may adversely affect the progression of each individual infection. Anemia, increased childhood mortality and impairments in physical growth, immune function and cognitive development are frequent results of malaria - NTD coinfections. ${ }^{40}$

NTDs are strongly connected with poverty which often goes hand in hand with low levels of education, literacy and understanding of disease processes and treatment options, thereby increasing the adverse consequences of infection. ${ }^{41}$ At the same time, poverty itself is potentiated by disease-related morbidity and mortality (figure 3).

36 Remme, J.H.F.; Blas, E.; Chitsulo, L. et al. (2002), p. 436.

37 Hotez P.J.; Molyneux, D.H.; Fenwick, A. et al. (2006), p. 576.

38 Remme, J.H.F.; Blas, E.; Chitsulo, L. et al. (2002), p. 436.

39 Remme, J.H.F.; Blas, E.; Chitsulo, L. et al. (2002), p. 438.

40 Hotez P.J.; Molyneux, D.H.; Fenwick, A. et al. (2006), pp. 577-579.

41 Alvar, J.; Yactayo, S. \& Bern, C. (2006), p. 552. 


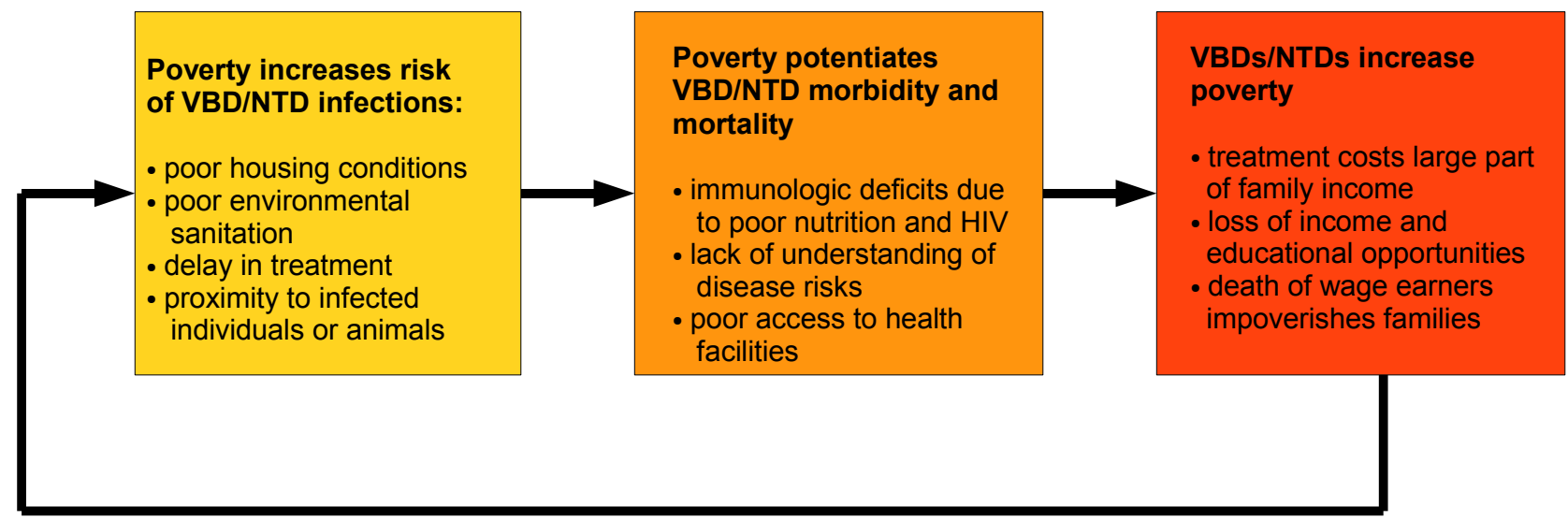

Figure 3: Links between poverty and VBDs/NTDs

Because of these links, the control of several vector-borne diseases and particularly malaria plays a vital for the achievement of the Millennium Development Goals (MDGs; see table 4). 


\begin{tabular}{|c|c|c|}
\hline Millennium & Development Goal & Relevance of Malaria \\
\hline & $\begin{array}{l}\text { MDG 1: } \\
\text { Eradicate extreme poverty } \\
\text { and hunger }\end{array}$ & $\begin{array}{l}\text { Malaria keeps people poor, costing } \\
\text { Africa US } \$ 12 \text { billion annually in lost } \\
\text { GDP and consuming up to } 25 \% \text { of } \\
\text { household incomes }\end{array}$ \\
\hline & $\begin{array}{l}\text { MDG 2: } \\
\text { Achieve universal primary } \\
\text { education }\end{array}$ & $\begin{array}{l}\text { Malaria is a leading cause of school } \\
\text { absenteeism in children and } \\
\text { teachers, impairs learning and can } \\
\text { cause neurological damages }\end{array}$ \\
\hline & $\begin{array}{l}\text { MDG 4: } \\
\text { Reduce childhood mortality }\end{array}$ & $\begin{array}{l}\text { Malaria is the leading cause of child } \\
\text { mortality in Africa }\end{array}$ \\
\hline & $\begin{array}{l}\text { MDG 5: } \\
\text { Improve maternal health }\end{array}$ & $\begin{array}{l}\text { Malaria in pregnant women has life- } \\
\text { threatening implications for mother } \\
\text { and child; pregnant women are } \\
\text { particularly susceptible to infections }\end{array}$ \\
\hline & $\begin{array}{l}\text { MDG 6: } \\
\text { Combat HIV/AIDS, malaria } \\
\text { and other diseases }\end{array}$ & $\begin{array}{l}\text { Malaria control programs directly } \\
\text { reduce morbidity and mortality } \\
\text { caused by malaria, including } \\
\text { coinfections }\end{array}$ \\
\hline & $\begin{array}{l}\text { MDG 8: } \\
\text { Develop a global } \\
\text { partnership for } \\
\text { development }\end{array}$ & $\begin{array}{l}\text { The cost of malaria research and } \\
\text { control is prohibitive for most } \\
\text { affected countries }\end{array}$ \\
\hline
\end{tabular}

Table 4: Relevance of malaria for MDG achievement ${ }^{42}$

The relevance of malaria with regard to six out of eight MDGs implies that overall MDG achievement is unlikely without progress in the field of malaria control.

42 Roll Back Malaria Partnership (2005), p. 42;

http://www.un.org/millenniumgoals/, accessed 18/06/09. 


\subsection{Geographic Contributions Towards Malaria Control}

Since colonial times, malaria distribution maps have been produced for many malarious regions. While early maps tended to show isolines. Such information is an important prerequisite for planning intervention programs. At the same time, geographic base data are needed -perhaps more than ever- as inputs for models and risk prediction systems, while new technologies such as GIS and remote sensing for the first time in history ensure the availability of spatiotemporal environmental data over large areas and the capacity for objective analyses.

\subsubsection{Malaria Mapping and Monitoring}

Good maps of malaria risk have long been recognized as an important tool for malaria control. ${ }^{43}$ Between the 1940 s and 1970 s, the WHO considered precise knowledge of the exact spatial extents of malaria transmission a prerequisite for their plan to eradicate malaria globally. Thus, huge investment was made to synthesize the available information on the distribution of malaria risk. ${ }^{44}$ In the 1950s, associations between climatic seasonality and malaria were discovered and crude risk maps for several African countries and the global distribution of malaria were prepared. ${ }^{45}$

Early maps identified (potentially) malarious areas based either on "expert opinion", past experience or simple climatic or geographical isolines, but this static information failed to reflect spatio-temporal variations in malaria transmission. ${ }^{46}$ When public health focus shifted away from malaria eradication in the 1970s, the interest in mapping global malaria risk therefore waned. ${ }^{47}$

Since the 1990s, there have been numerous efforts to map malaria on the regional, national, continental and global scale. One of the most comprehensive projects in the field of malaria mapping, the MARA/ARMA initiative ("Mapping Malaria Risk in Africa / Atlas du Risque de la Malaria en Afrique"), aims at the preparation of a malaria risk map of Africa by combining the results of published and unpublished malaria data on the one hand and spatial modeling of malaria distribution, seasonality and endemicity on the other. Despite some advances, there is still a lack of up-to-date maps providing more insight than global or continental overviews.

43 Kleinschmidt, I.; Bagayoko, M.; Clarke, G.P.Y. et al (2000), p. 355.

44 Guerra, C.A; Snow, R.W. \& Hay, S.I. (2006), p. 353.

45 Hay, S.I.; Omumbo, J.A.; Craig, M.H. \& Snow R.W. (2000), pp. 176; 191.

46 Hay, S.I.; Omumbo, J.A.; Craig, M.H. \& Snow R.W. (2000), pp. 176; 191.

47 Guerra, C.A; Snow, R.W. \& Hay, S.I. (2006), p. 353. 


\subsubsection{Malaria Modeling and Prediction}

Malaria transmission models try to explain variations in malaria transmission intensity by relating it to external factors. Even though there is no clear-cut distinction, malaria models are commonly classified as either statistical or causal. Statistical models identify statistical correlations between malaria transmission and factors that are believed to be determinants of disease transmission, whereas causal models identify determinants of disease transmission by observing biological links between the environment, the vector mosquito and the human host. This distinction is, however, fluent. A simplified, qualitative model of malaria transmission is presented in figure 4 and outlines the fundamental connections between malaria and its determinants.

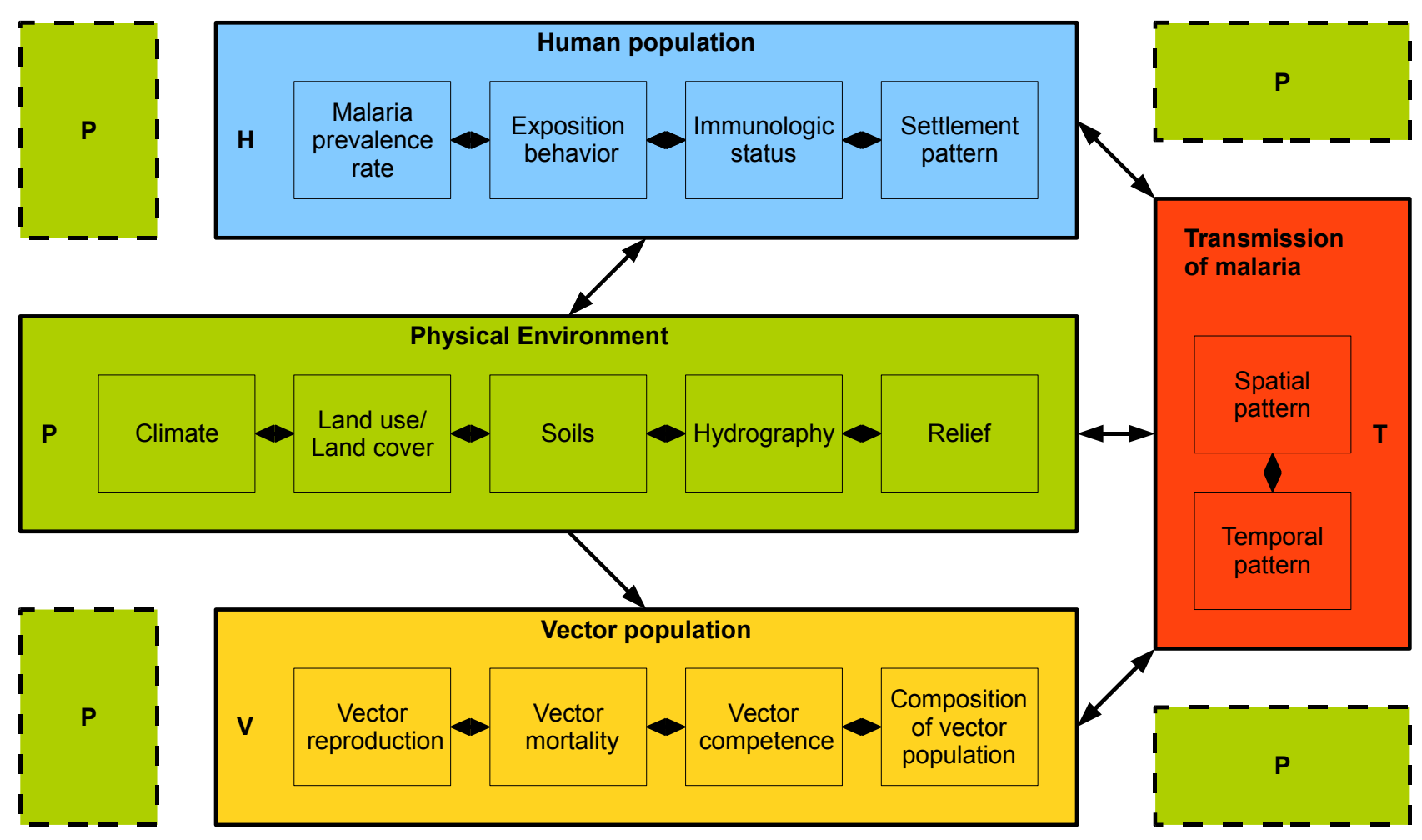

Figure 4: Levels of the malaria transmission process

The model distinguishes between three components that regulate the spatial and temporal pattern of malaria transmission intensity: the human and vector populations ( $\mathrm{H}$ and $\mathrm{V}$ ) interacting within the physical environment $(\mathrm{P})$.

The physical environment $(P)$ plays a key role in malaria epidemiology as it is linked to both the human population and to the mosquito vectors. The environment does not only determine the productivity of mosquito habitats but also has in influence on the malaria parasite's transmission cycle. 
Various studies have demonstrated statistical correlations between environmental parameters such as climate and land cover on the one hand and the life cycle of malarial vectors on the other hand. However, this link is not always "instantaneous": the highest correlation between malaria incidence and precipitation for example is found after a delay of approximately one month. ${ }^{48}$

The mosquito population ( $\mathrm{V}$ ) forms the link between the physical environment and the actual transmission process. Physical habitat characteristics play an important role for the reproduction and longevity/mortality of the mosquito vector. Reproduction and mortality rates govern the abundance of vector mosquitoes, which is a key determinant of the malaria transmission risk encountered in a certain region. However, the physical environment also influences the composition of the mosquito population and their biting behavior: different species/subspecies compositions of the vector population may lead to higher or lower transmission risks ${ }^{49}$, and even one and the same subspecies behaves differently depending on the environmental situation in its habitat. ${ }^{50}$

The actual transmission process $(T)$ depends not only on the abundance and transmission competence of the mosquito vectors found in a region, but also the human population $(\mathrm{H})$. First of all, different sociocultural or economic activities may bring higher or lower risks with them: fieldwork or other outdoor activities during the peak biting hours and close to mosquito breeding sites increase the risk of an infection, whereas personal prophylaxis (such as the use of insecticides or impregnated bednets in individual households) and other intervention strategies (such as the eradication of mosquito larvae or larval habitats) can reduce infection risks. Prompt and proper treatment of patients infected with malaria parasites also helps to prevent further transmission.

\subsubsection{GIS and Remote Sensing: New Tools For Malariology}

Since the 1980s and 1990s, rapid developments in the fields of remote sensing and geographic information systems (GIS) have provided unprecedented information and capacity for development of malaria risk maps. ${ }^{51}$ Remote sensing for the first time provided gapless environmental data for large regions, while the introduction of geographical information systems provided a quantitative basis for malaria mapping which used to be rather subjective in the past. ${ }^{52}$

48 Hay, S.I.; Omumbo, J.A.; Craig, M.H. \& Snow R.W. (2000), pp. $180 f$.

49 Service, M.W. (1993), p. $116 \mathrm{f}$.

50 Hoshen, M.B. \& Morse, A.P. (2004), doi:10.1186/1475-2875-3-32.

51 Gemperli, A.; Sogoba, N.; Eondjo, E. et al. (2006), p. 1033.

52 Hay, S.I.; Омumbo, J.A.; Craig, M.H. \& Snow R.W. (2000), p. 192. 
In the past few years, several studies dealt with the use of remote sensing data for predicting the transmission of malaria or other infectious diseases. Despite successful attempts of statistically correlating different environmental parameters with transmission pressure, the causal links between the geographical environment and vector density/transmission risk are not yet completely understood. ${ }^{53}$

Nevertheless, several types of remote sensing data can provide valuable background information for epidemiological studies. Aerial photographs and satellite images of high spatial resolutions can be used to identify small depressions which are potential mosquito breeding sites during rainy periods. They may also be used for the preparation of local-scale maps of human settlements and close by mosquito habitats. Digital elevation models based on radar measurements such as NASA's SRTM (Shuttle Radar Topography Mission) can be helpful in discerning malaria-free highland regions from high-risk lowland regions and can be a basis for modeling water flow directions.

Remotely sensed datasets with moderate spatial resolutions (typically a few hundred meters) can be equally useful if they offer high spectral or temporal resolutions. Typical examples are weather-related data such as thermal or rainfall indicators, and vegetation index data which may in fact be good proxies for mosquito population development and thus malaria risks.

Despite numerous studies on the application of remote sensing and geographic information systems in disease epidemiology in the recent past, real success stories have been more or less rare. Almost all projects cited in the framework of this study have focused on one single factor (and thus neglected the multitude of influences), and RS- and GIS-based prediction systems yet have to become operative in the world's most malarious regions in Sub-Saharan Africa.

\subsubsection{About this Study}

It may be an evident question to ask what contributions geographic research has to offer with regard to malaria. Malaria is at first sight a medical phenomenon and problem, and since the discovery of the transmission process by Alphonse Laveran in $1880^{54}$ malaria has often been the primary focus of tropical disease research. The protozoan parasites causing malaria are subject to microbiological investigations, and anopheline mosquitoes are studied by entomologists. Economists have produced studies on the financial burdens of malaria on both macro- and microeconomic levels, archaeologists and

53 Curran, P.J.; Atkinson, P.M.; Foody, G.M. \& Milton, E.J. (2000), p. 63.

54 Panjarathinam, R. (1990), p. 28. 
historians keep on discovering evidence about malaria in ancient civilizations. The many facets of malaria research are all part of malariology, a crossdisciplinary approach to the disease that is in theory curable but practically remains a major cause of mortality in the $21^{\text {st }}$ century.

The available malaria literature could fill libraries, but the death toll caused by a parasite too small to be visible but powerful enough to kill millions of people annually proves a solution in form of malaria eradication is not yet in sight. The Anopheles mosquito, which kills far more people than all terrestrial and aquatic predators combined, remains the clue to understanding the dynamics of malaria transmission. It is the most important of all disease vectors; but yet, the complex links between its reproduction and survival and its natural and anthropogenic environment are still not completely understood. These links, however, make malaria a subject of geographic research: it is a disease that has a clearly geographic dimension due to its uneven distribution and spatiotemporal variations. Malaria depends as much on physiogeographic determinants as it is influenced by sociogeographic variables. This makes malaria an ideal disease to be studied by medical geography. At the same time, the holistic view of geography coupled with advanced technologies in the field of spatial data acquisition and processing have the promise of providing much-needed baseline information for malaria control programs.

This study, carried out in the framework of a doctoral dissertation, first of all tries to provide a systematic overview of malaria epidemiology including all its geographic aspects. Both past efforts in the field of malaria mapping and advances in geographic information technology will be presented as prerequisites for prediction systems and intervention campaigns. This theoretic overview forms the basis for a case study of malaria transmission in Burkina Faso's Kossi Province, a rural dry savanna region close to the international border with Mali. This region seems ideal for such a project in many ways: high but seasonal transmission risks come together with a vulnerable population; different ecological settings ranging from undisturbed savanna to massive irrigation projects are found within a relatively small region; and last but not least, a network of rural health posts and a well-equipped health research center, the CRSN (Centre de Récherche en Santé de Nouna) record both health and demographic key data.

Malaria case data obtained from local health centers are presented against the backdrop of selected environmental and socio-geographic variables. Even though a relatively short observation period (2004 to 2008 for most parameters) and sometimes serious lacks in data availability mean this study is certainly not comprehensive enough to derive a numeric transmission model, it identifies important geographic variables that are linked to local-scale variations in malaria transmission dynamics. Methods for data acquisition are described and evaluated with regard to their feasibility for projects to be carried out at the regional level in a rural African dry savanna settings. Last but 
not least, both the geographic picture of Kossi Province and the methodology discussed have been prepared with regard to malaria transmission but may provide a basis for studies on other geomedical issues ranging from the many infectious diseases found in the region to other issues such as questions of water supply and food security.

Apart from analyzing the local situation in Kossi, the case study tries to assess the validity of four key hypotheses that are of a more general relevance to the application of GIS and RS in the field of malaria mapping:

1. a. Meteorological parameters, particularly rainfall, are reliable predictors of the temporal malaria pattern in dry savanna areas like Kossi Province.

b. In regions for which meteorological station data are scarce, remote the use of RS-based surrogates is a feasible alternative.

2. a. Other environmental and sociogeographic factors are important determinants of regional and local scale transmission pattern.

b. High spatial resolution RS imagery combined with ground truthing is a valuable resource for local-scale malaria risk mapping. 


\section{Malaria in West Africa: Transmission, Monitoring \& Control}

Malaria remains one of the most important vector-borne diseases in the world, with Sub-Saharan Africa being the most affected region. It causes not only an immense public health burden but is also linked to low levels of economic development in the region. Spatial pattern of malaria incidence are closely connected to the malaria transmission process and its dependence on environmental factors. The cyclical transmission process involves contacts between the human population, the mosquito (vector) population and the malaria parasite. This complex cycle is influenced by a set of both physical and anthropogenic factors.

This link between malaria and geography means that geographic techniques, ranging from the mapping of malaria cases and (potential) determinants of disease to advanced methods of data acquisition (remote sensing) and spatial and temporal analyses in geographic information systems, can be valuable tools for malariological research and the development of intervention strategies.

\subsection{Africa's Malaria Burden}

No other region in the world suffers from malaria to the degree that Africa does. It is commonplace in tropical Africa for more than half of the population to be infected with Plasmodium falciparum. ${ }^{55}$ The disease thrives particularly in the absence of effective health systems and in regions of social and environmental crisis. ${ }^{56}$ To this date, malaria remains among the most important causes of morbidity and mortality in most African nations south of the Sahara, quite frequently in fateful combination with other diseases. Malaria does not only affect the health sector but has implications for the general quality of life of local communities and has an impact on the economy and education sector of entire nations. The dimension of the malaria burden and its uneven distribution at both the continental and regional scales make malaria an important subject for geomedical research in Africa.

55 Killeen, G.; Seyoum, A. \& Knols, G.J. (2004), p. 87.

56 TANSER, F.C. \& le SUeur, D. (2002), doi: 10.1186/1476-072X-1-4. 


\subsubsection{Malaria Morbidity and Mortality in Africa}

Until recently, malaria was ranked as Africa's largest public health problem and is currently only surpassed by HIV/AIDS. Nevertheless, malaria accounts for $20 \%$ to $50 \%$ of all hospital admissions ${ }^{57}$ in Africa and is among the most important causes of death in large parts of the continent.

\subsubsection{Malaria-induced Morbidity}

In Sub-Saharan Africa, about 200 million people remain persistently infected with malaria. About $5 \%$ to $10 \%$ of the children suffering from cerebral malaria face long-term sequelae. ${ }^{58}$ Each year, malaria causes around 3000 cases of lifelong disability. ${ }^{59}$ This massive dimension of a disease that can theoretically be treated and prevented caused some scientists to speak of the "intolerable burden of malaria" (see figure 5 ).

Some degree of anemia is the rule in malarial infections. This is the consequence of several mechanisms:

- destruction of red blood cells by malaria parasites;

- reduced erythrocyte production in the bone marrow;

- formation of autoantibodies which

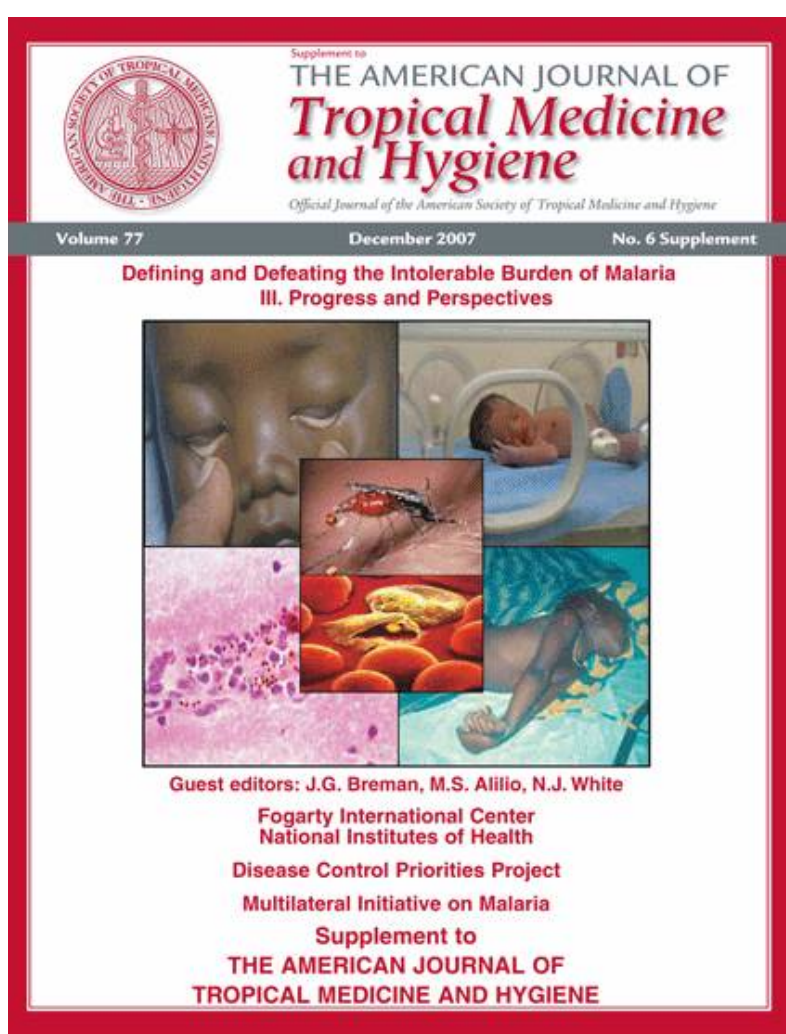

Figure 5: "Intolerable Burden of Malaria" destroy red blood cells. ${ }^{60}$

Malaria is often said to be immunosuppressive. When malaria is controlled, mortality from other causes usually falls, too. However, there are conflicting views on whether malaria predisposes to other acute infections such as pneumonia, or whether the illnesses observed are in fact a part of the clinical spectrum of malaria itself. ${ }^{61}$

57 Tanser, F.C. \& le Sueur, D. (2002), doi: 10.1186/1476-072X-1-4.

58 COULTER, J.B.S. (2002), p. 529.

59 HaY, S.I.; Omumbo, J.A.; Craig, M.H. \& Snow R.W. (2000), p. 194.

60 MARSH, K. (1993), p. 76.

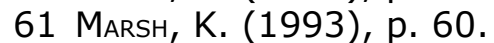


Plasmodium falciparum is the only one of the malaria parasites which has a direct impact on the central nervous system. Potential neurocognitive sequelae of Plasmodium falciparum infections include motor and coordination deficits, impairments of speech and perception and epilepsy. ${ }^{62}$ A Kenyan study showed subsequent neurological deficits in $10,9 \%$ of children who were hospitalized because of cerebral malaria. Another $17 \%$ died due to cerebral malaria. ${ }^{63}$

Malarial infections during pregnancy often have far-reaching consequences such as premature births, low birth weight and mental retardations ${ }^{64}$ and significantly increase the risk of death during the first month of life ${ }^{65}$ Malarial infections may directly and indirectly influence a child's development. The potential consequences of an infection depend on the child's age (i.e. on its neuronal state of development) and may become evident years after the original infection. ${ }^{66}$

Other complications in Plasmodium falciparum malaria include liver dysfunction, renal impairment and respiratory distress. ${ }^{67}$

\subsubsection{Malaria and Other Health Disorders}

In Africa, malaria often occurs in regions where other health problems are frequent, too. These problems may both increase the susceptibility to malaria and aggravate the outcomes of infections and typically include malnutrition and/or other infectious diseases.

Malnourished individuals, particularly children, have an increased susceptibility for severe malarial infections which is due to the limited function of the immune system. ${ }^{68}$ Data for West Africa suggest that more than half of all malaria-induced mortality is partly caused by malnutrition. ${ }^{69}$

Polyparasitism is frequent due to the geographic overlap of the regions affected by tropical diseases. Coinfections may adversely affect the progression of each individual infection. Perhaps the most important of the resulting comorbid conditions is anemia: many NTDs, including malaria, leishmaniasis, trypanosomiasis and schistosomiasis are major causes of anemia. Adverse health consequences are particularly relevant for three sub-populations: pregnant women, children and individuals infected with HIV/AIDS. In pregnancy, anemia is a leading contributor to maternal morbidity and mortality.

62 Mung'Ala-Odera, V.; Snow, R.W. \& Newton, C.R.J. (2004), p. 64.

63 Mung'Ala-Odera, V.; Snow, R.W. \& Newton, C.R.J. (2004), p. 65.

64 Holding, P.A. \& Kitsao-Wekulo, P.K. (2004), p. 74.

65 Roll Back Malaria Partnership (2005), p. 4.

66 Holding, P.A. \& Kitsao-Wekulo, P.K. (2004), p. 73.

67 BREMAN, J.G. (2009), pp. $13 f$.

68 Caulfield, L.E.; Richard, S. \& Black, R.E. (2004), p. 55.

69 Caulfield, L.E.; Richard, S. \& Black, R.E. (2004), p. 57. 
In young children, iron deficiency and anemia are associated with increased child mortality and impairments in physical growth, cognitive and motor development and immune function. Finally, among individuals with HIV/AIDS, anemia has been shown to be an independent risk factor for early death. ${ }^{70}$

Each year, about 19.000 people get infected with HIV since they receive contaminated blood for treating malaria-induced anemia. ${ }^{71}$ Because of the high prevalence of malaria and HIV/AIDS in Sub-Saharan Africa, co-infections are frequent. About $55 \%$ of Sub-Saharan Africa's HIV/AIDS victims are reproductive-age women. ${ }^{72}$ Coinfections of HIV and malaria are particularly risky during pregnancy and may cause maternal death, premature birth, low birth weights and high infant mortality rates ( 3 to 8 times higher than in infants infected solely with HIV!). ${ }^{73}$ TER KUILE et al. (2004) found the susceptibility to malaria of pregnant women to be correlated to HIV/AIDS prevalence. ${ }^{74}$

\subsubsection{Malaria-induced Mortality}

Malaria is an important cause of mortality among children in Africa, but the relation between malaria transmission intensity and childhood mortality remains controversial. In holoendemic areas, malaria-attributable mortality kills more than 3000 children below the age of five every day. ${ }^{75}$ There is evidence that the highest mortality may be at intermediate transmission intensities. $^{76}$

In case of Plasmodium falciparum, infected erythrocytes can obstruct small blood vessels. If this occurs in the brain, cerebral malaria results, often causing fatal complications. ${ }^{77}$ The majority of deaths occurs in infants and children under the age of 5 years. Mortality rates are of the order of $6 \%$ o in infants and $11 \%$ in children aged 1 to 4 years, representing between $10 \%$ and $30 \%$ of all deaths in these age groups. ${ }^{78}$ Mortality associated with cerebral malaria is around $20 \%$ among adults and $15 \%$ among children ${ }^{79}$ and has not improved in the past 30 years. ${ }^{80}$

70 Hotez P.J.; Molyneux, D.H.; Fenwick, A. (2006), pp. 577-579.

71 Hay, S.I.; Омumbo, J.A.; Craig, M.H. \& Snow R.W. (2000), p. 194.

72 Ter Kuile, F.O.; Parise, M.E.; Verhoeff F.H. et al. (2004), p. 41.

73 Ter Kuile, F.O.; Parise, M.E.; Verhoeff F.H. et al. (2004), pp. 43; 47.

74 Ter Kuile, F.O.; Parise, M.E.; Verhoeff F.H. et al. (2004), p. 44.

75 Yé, Y.; Sauerborn, R.; Seraphin, S. \& Hoshen, M. (2007), p. 375.

76 Gemperli, A.; Vounatsou, P.; Kleinschmidt, I. et al. (2004), p. 64.

77 TUTEJA, R. (2007), p. 4670.

78 Onori, E., Beales, P.F. \& Gilles, H.M. (1993), p. 274 f.

79 BREMAN, J.G. (2009), p. 11.

80 Tanser, F.C. \& le Sueur, D. (2002), doi: 10.1186/1476-072X-1-4. 
Appropriately and promptly treated, uncomplicated infections with Plasmodium falciparum result in a mortality rate of around $1 \%$. However, once dysfunctions of vital organs occur or parasites are found in more than $2 \%$ of the erythrocytes, mortality rises steeply. ${ }^{81}$

\subsubsection{Socio-Economic Implications of Malaria}

Malaria is essentially a disease of the poor. $58 \%$ of all malaria cases occur in the poorest $20 \%$ of the world population. ${ }^{82}$ The disease is both a consequence and a cause of poverty. The high incidence of malaria places a tremendous financial burden on affected households and poses a major problem for local health authorities. ${ }^{83}$ In areas where epidemic malaria occurs, sudden rises in the numbers of infected people frequently overburden public health services. ${ }^{84}$ In some African countries, families spend up to $25 \%$ of their income on malaria prevention and treatment. ${ }^{85}$ Moreover, malaria impairs the economic development of large parts of Africa:

«[...] the persistence of endemic malaria [...] particularly in Africa, is contributory to a perpetual state of depressed economic growth $[\ldots] \gg{ }^{86}$

Malaria is estimated to cost Africa more than $\$ 12$ billion annually ${ }^{87}$, and endemic malaria cripples the economies of sub-Saharan Africa and is thought to slow economic growth by about $1.3 \%$ per year. Malaria has been identified as a key contributor to weak economic growth and investment in Africa. ${ }^{88}$

For families in malaria-endemic regions, the disease often simultaneously causes income losses, declines in agricultural production (which may be needed for subsistence) and unaffordable treatment costs. Malaria epidemics frequently occur during sowing or harvest, and may thus have severe consequences for food security if field workers fall ill while their labor is needed

81 BREMAN, J.G. (2009), p. 10.

82 Roll Back Malaria Partnership (2005), p. 4.

83 Hay, S.I.; Omumbo, J.A.; Craig, M.H. \& Snow R.W. (2000), pp. 174, 194; SAMBA, E.M. (2004), p. ii.

84 Kiszewski, A.E. \& Teklehaimanot, A. (2004), p. 131.

85 Roll Back Malaria Partnership (2005), p. 4.

86 Hay, S.I.; Omumbo, J.A.; Craig, M.H. \& Snow R.W. (2000), p. 174, quoting SACHS, J.D. \& WERNER, A.M. (1997).

87 TutejA, R. (2007), p. 4670.

88 Killeen, G.; Seyoum, A. \& Knols, G.J. (2004), p. 87. 
most. Malaria epidemics occasionally cause complete crop failures. Many economic and social activities are brought to a standstill during epidemics, including classes at school, local markets and transports. ${ }^{89}$ Moreover, malarial infections in the family are also a frequent cause of absentism from schools. ${ }^{90}$

\subsubsection{Regional Pattern of Malaria Transmission}

About $80 \%$ to $90 \%$ of the world's malaria morbidity and $90 \%$ of malariarelated mortality occurs in sub-Saharan Africa. ${ }^{91}$ About 100 to 200 million people in sub-Saharan Africa contract malaria each year (2000: 213.6 million clinical cases ${ }^{92}$ ), of which around 1 million people die of Plasmodium falciparum malaria. More than $75 \%$ of the victims are children of preschool age. ${ }^{93}$ However, even within Sub-Saharan Africa this burden is very unevenly distributed: whereas malaria morbidity and mortality rates are low in southern Africa, they are extremely high in western and central Africa (see figure 6).

89 Kiszewski, A.E. \& TekLehaimanot, A. (2004), p. 131.

90 Holding, P.A. \& Kitsao-Wekulo, P.K. (2004), p. 74.

91 Gilles, H.M. $\left(1993^{2}\right)$, p. 125; Hay, S.I.; Omumbo, J.A.; Craig, M.H. \& Snow R.W. (2000), p. 174.

92 Mung'ala-Odera, V.; Snow, R.W. \& Newton, C.R.J. (2004), p. 65.

93 Onori, E., Beales, P.F. \& Gilles, H.M. (1993), p. 272. 


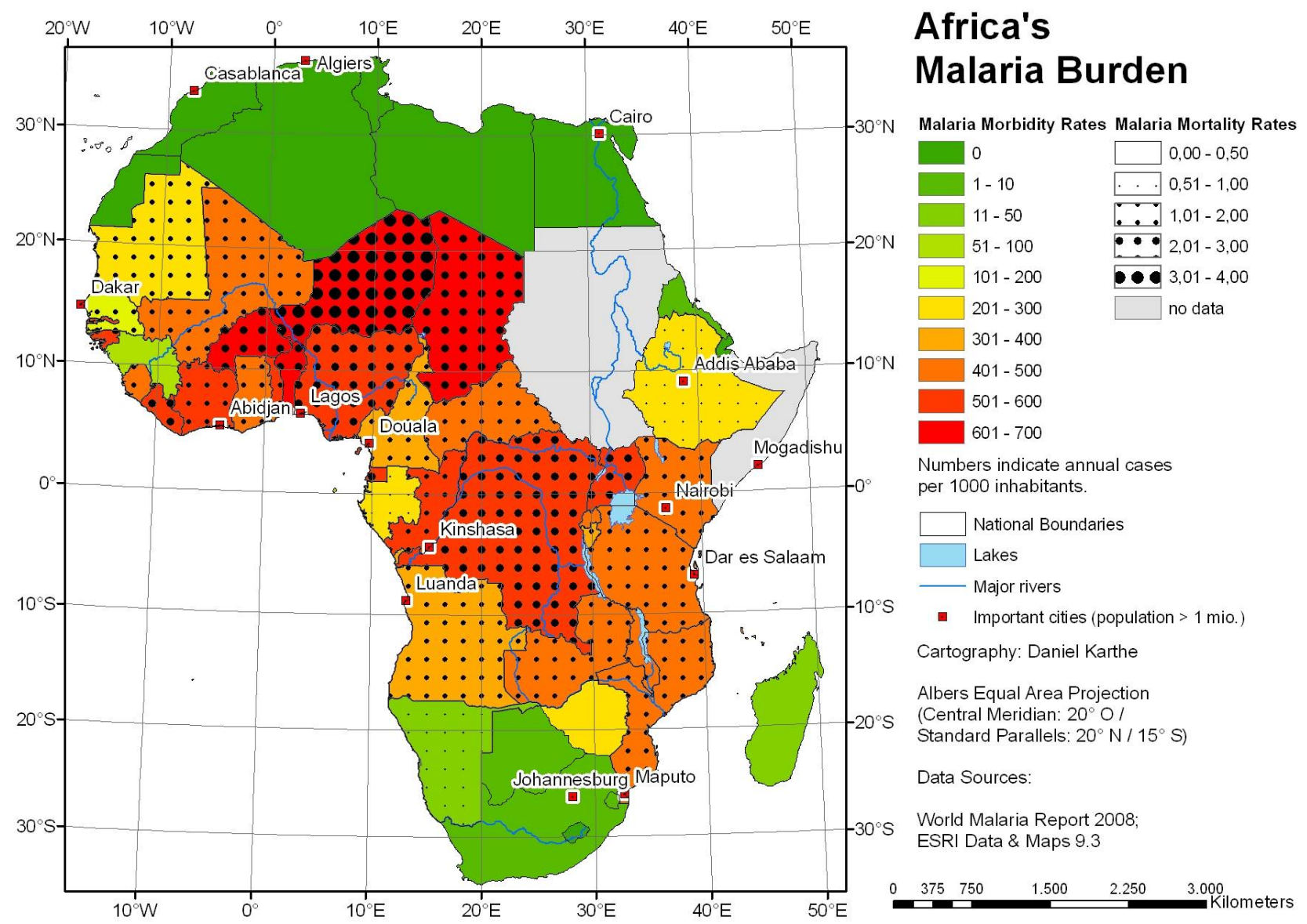

Figure 6: Malaria morbidity and mortality in Africa ${ }^{94}$

Throughout most of Africa south of the Sahara, malaria shows a high endemicity but has a low epidemic potential. In equatorial Africa, transmission is nearly perennial, and very high levels of prevalence are common in the continent's coastlands. ${ }^{95}$ In other endemic areas, the transmission risk varies seasonally and interannually - from singular cases of transmission over a period of several years to a constant risk. ${ }^{96}$

Most of sub-Saharan Africa can be classified as a high risk region (see table 5).

94 Based on World Health Organization (2008) and ESRI Data \& Maps 9.3.

95 Gilles, H.M. $\left(1993^{2}\right)$, p. 159.

96 HaY, S.I.; Омumbo, J.A.; Craig, M.H. \& Snow R.W. (2000), p. 176. 


\begin{tabular}{|c|c|c|c|c|}
\hline \multirow[b]{2}{*}{ Regions with ... } & \multicolumn{4}{|c|}{$\begin{array}{c}\text { Population at risk of contracting malaria in } \\
\text { sub-Saharan Africa (2000) }\end{array}$} \\
\hline & $\begin{array}{l}0 \text { to } 4 \\
\text { years }\end{array}$ & $\begin{array}{l}5 \text { to } 14 \\
\text { years }\end{array}$ & $\begin{array}{l}\text { above } 14 \\
\text { years }\end{array}$ & total \\
\hline ... low risk & 2,0 mio. & 3,7 mio. & 8,7 mio. & 14,4 mio. \\
\hline ... moderate risk ${ }^{97}$ & 22,0 mio. & 34,7 mio. & 69,1 mio. & 125,8 mio. \\
\hline$\ldots$ high risk ${ }^{98}$ & 73,4 mio. & 115,3 mio. & 228,1 mio. & 416,7 mio. \\
\hline
\end{tabular}

Table 5: Population at malaria risk in sub-Saharan Africa ${ }^{99}$

The malaria incidence in West Africa is one of the highest in the world and almost the entire region a zone of high malaria risk. As in other parts of subSaharan Africa, infections with Plasmodium falciparum constitute about 85 to $90 \%$ of all malaria cases. ${ }^{100}$ High transmission pressures and the dominance of the most dangerous malaria parasite are responsible for the tremendous malaria burden faced by this region.

\subsection{Epidemiology of Malaria}

Even though malaria is known to have existed in antiquity, the process of malaria transmission was only discovered at the end of the $19^{\text {th }}$ century. Two key processes, parasite development in human (or animal) hosts and anopheline mosquitoes, are linked by mosquito blood meals that are necessary for infections in both the vector and the host. The 'integrity' of this cycle is the key determinant of the transmission dynamics found in region.

\subsubsection{Discovery of the Transmission Process}

In his Rerum Rusticarum De Agri Cultura, the Roman general and encyclopedist Marcus Terentius VarRo reported that minute creatures living in swampy places may enter the body and cause serious diseases:

«[...] erunt loca palustria, $[\ldots]$ et quod crescunt animalia quaedam minuta, quae non possunt oculi consequi, et per aera intus in corpus per os ac nares perveniunt atque efficiunt difficilis morbos. ${ }^{101}$

97 low but constant risk or risk of epidemics

98 malaria-endemic areas

99 Mung'ala-Odera, V.; Snow, R.W. \& Newton, C.R.J. (2004), p. 65.

100 HaY, S.I.; Omumbo, J.A.; Craig, M.H. \& Snow R.W. (2000), p. 194.

101 Varro, M.T. (36 B.C.), Liber Primus, XII. 
This may in fact be the first written description of the malaria transmission process that preceded its "modern" discovery by more than 1900 years. ${ }^{102}$ Moreover, VARRO already linked disease to hydrological modifications related to agriculture. ${ }^{103}$

The word 'malaria' was introduced into the English language by the geologist JoHn MACCULOCH (1775-1835) in his 1827 book On Malaria: an essay on the production and localities of the places by which it is produced: with an enunciation of the diseases caused by it, and the means of preventing or diminishing them, both at home and in the naval and military service. The English word was derived from the Italian term mal'aria which referred to the bad air which was believed to be the prime cause of a variety of diseases. ${ }^{104}$ In early $19^{\text {th }}$ century Rome, scientists hoped that the identification of their distribution would reveal certain underlying facts about the nature of diseases such as malaria. Due to a shortage of scientific data, evidence was sought from local sayings and the experience of travelers. In popular opinion, location was seen as the key explanation of the nature of malaria. Based on the work of Giovanni Maria Lancisi (1654-1720), who had 'identified' airborne pathogenic matter as the cause of many diseases and related them to different soils, Roman scientists of the early $19^{\text {th }}$ century believed that malaria was caused by noxious emanations from soils. Depending on soil characteristics, locations in and around Rome were thus classified as "healthy", "unhealthy" and "very unhealthy" and maps produced accordingly. ${ }^{105}$ It was believed that archaeologists were most likely to be exposed to malaria since excavations removed the barriers that had existed between the modern atmosphere and ancient noxious 'miasmas'. However, two observations brought doubt to the theory that malaria was caused by bad air: Firstly, malaria was found to be localized unevenly, with individual houses in a street being affected and others not. Secondly, comparative studies of diseases in different places weakened the idea that they were particular in their locations - and that malaria was not just 'Roman fever'. ${ }^{106}$ In fact, the Italian parasitologist Ulisse AldRovandi had already hypothesized that there were links between bloodsucking insects and diseases in his De animalibus insectis which appeared in 1602. This opus on insects was largely ignored ${ }^{107}$, though, and it took nearly 300 years until the malaria transmission process was scientifically proven. Towards the end of the $19^{\text {th }}$ century, two important discoveries regarding the malaria transmission cycle were made: in 1880, the French army surgeon Charles Louis Alphonse Laveran

102 Aмici, R.R. (2001), p. 4.

103 VARRo, M.T. (36 B.C.), Liber Primus, XII.

104 WRIGHLEY, R. (2000), p. 207.

105 WRIGHLEY, R. (2000), pp. 209-211

106 WRIGHLEY, R. (2000), p. 215.

107 Амiсl, R.R. (2001), p. 4. 
observed the gametocyte form of the plasmodian parasite in the blood of an Algerian malaria patient and thus discovered the parasitic nature of malaria ${ }^{108}$, and in 1897, RonalD Ross, a British medical officer serving in India identified mosquitoes of the genus Anopheles as the sole vectors of malaria. ${ }^{109}$

\subsubsection{Outline of the Transmission Process}

Malaria is an infectious disease caused by protozoan organisms (Plasmodia) which are transmitted by vectors (mosquitoes of the genus Anopheles) from an infected to an uninfected (human) host. Specific developments of the parasites occur both inside the insect vector and the human host. Moreover, only certain (and different) developmental states of the malaria parasite may lead to an infection of either a mosquito or a human host. Therefore, malaria transmission can only occur if

- there is at least one infected host in a region;

- there is contact between parasite hosts and parasite vectors;

- the parasite vectors live long enough so that a form of the parasite develops which may infect another human host;

- environmental conditions are conducive to parasite development in mosquito vectors.

108 Doolan, D.L.; Dobaño, C. \& Baird, J.K. (2009), p. 13.

109 TutejA, R. (2007), p. 4670. 


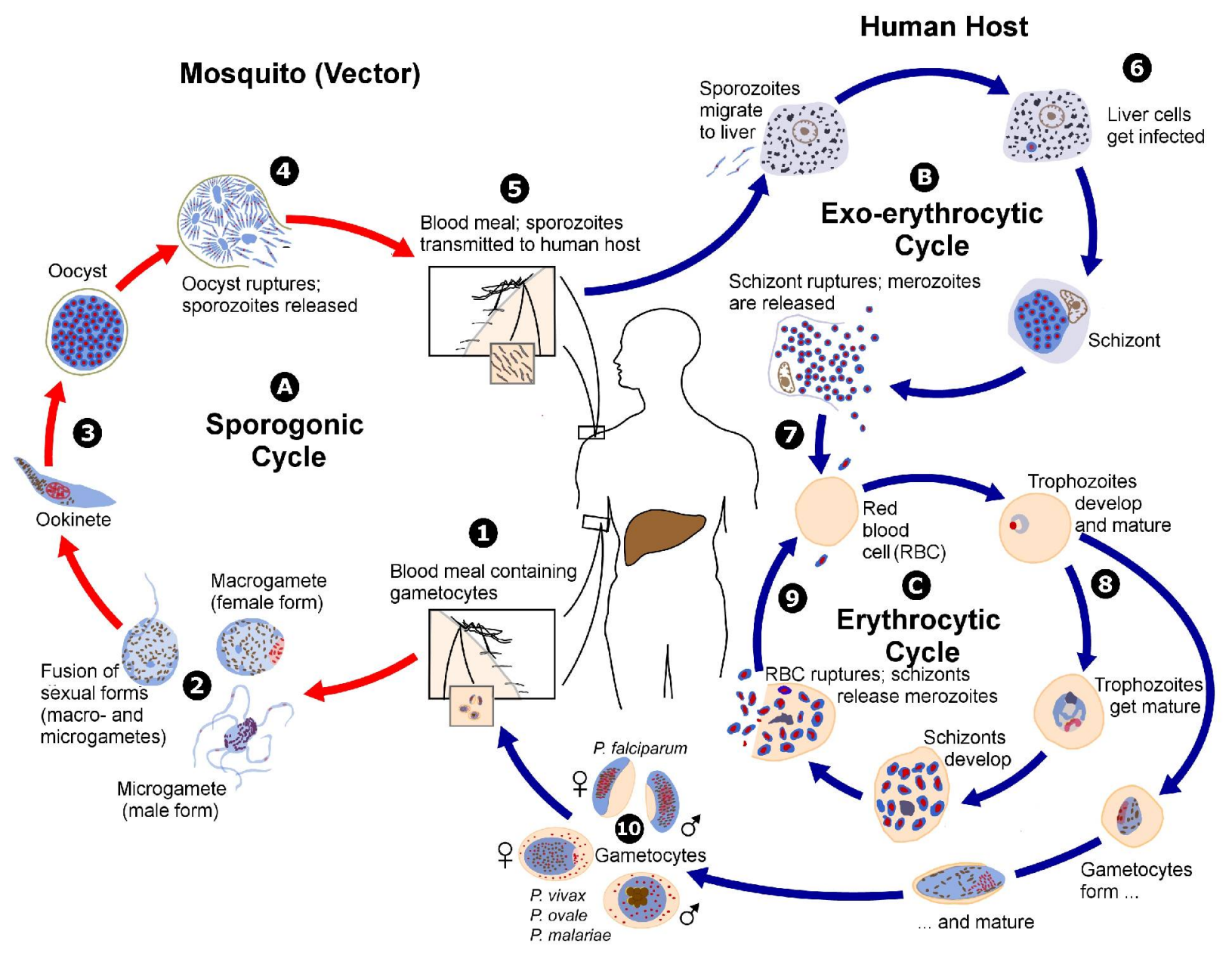

Figure 7: The malaria transmission cycle ${ }^{110}$

The transmission process (figure 8 ) begins when a female Anopheles mosquito takes a blood meal from an infected person (0). The mosquito may thereby ingest gametocytes, the gender-specific (i.e. male or female forms) of the plasmodian parasites which cause malaria. When the gametocytes reach the mosquito's intestinal tract, sexual reproduction of the parasites begins (2). The fusion of female macro- and male microgametes gives rise to ookinetes (3), which then develop into oocysts. Sporozoites are produced inside the oocysts. This whole process is also referred to as sporogony. The sporozoites which are released by ruptured oocysts (4) now migrate to the mosquito's salivary glands. When the female mosquito takes her next blood meal, these sporozoites may be injected into the victim's blood stream (5). The sporogonic cycle is now completed. ${ }^{111}$

110 Adapted from the US Center for Disease Control, http://www.cdc.gov/malaria. 111 Gilles, H.M. $\left(1993^{1}\right)$, p. 14 f. 
In the human host, the parasites first migrate to the liver (hepatic or exoerythrocytic stage) where they infect the liver cells and form schizonts (6). In the case of Plasmodium vivax and Plasmodium ovale, this stage may be prolonged and asymptomatic (dormant stage). In the liver, the schizonts develop and release merozoites which infect the red blood cells $(\boldsymbol{\theta})$; now, the erythrocytic stage begins. Most merozoites turn into trophozoites (8) and then into schizonts which produce new merozoites, giving rise to a repeated erythrocytic cycle $(\boldsymbol{\Theta})$. However, some merozoites ultimately differentiate into the gender-specific gametocytes which are infectious for the female

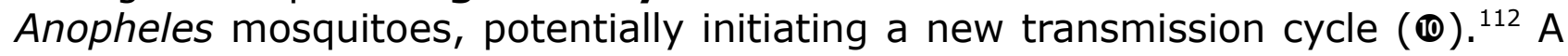
more detailed account of the mosquito vector's and malaria parasite's roles in the transmission process is presented in chapters 2.3 and 2.4.

\subsubsection{Classifications of Malaria Incidence and Transmission}

The source of human malaria is nearly always a human subject. This can be both a sick person or an asymptomatic carrier of the parasite. With the possible exception of chimpanzees in tropical Africa, which may carry an infection with Plasmodium malariae, no other animal reservoir of human malaria is known to exist. However, there have been a few cases of natural or accidental transmission of simian Plasmodia (malaria parasites normally infecting monkeys) to humans. ${ }^{113}$

Malaria cases can be categorized according to the localities of transmission and actual disease occurrence:

- autochthonous cases have been contracted locally;

- indigenous malaria occurs naturally in a certain area;

- imported cases have been contracted outside a certain area;

- introduced malaria refers to secondary infections which are contracted locally but derived from imported cases. ${ }^{114}$

Endemic malaria refers to a constant incidence of cases over a period of many successive years. Epidemic malaria, on the other hand, indicates a periodic or occasional sharp increase in the amount of malaria in a given indigenous community. ${ }^{115}$

An indicator of transmission stability is the reproduction rate of the disease: rates of less than one mean that malaria is unstable with a potential to die out, and rates higher than one imply that malaria is stable and likely to continue indefinitely. ${ }^{116}$

112 Gilles, H.M. $\left(1993^{1}\right)$, pp. 16-19.

113 Gilles, H.M. $\left(1993^{2}\right)$, p. 125.

114 Gilles, H.M. $\left(1993^{2}\right)$, p. 128.

115 Gilles, H.M. $\left(1993^{2}\right)$, p. 127.

116 Craig, M.H., Snow, R.W. \& le Sueur, D. (1999), p. 105. 


\subsubsection{Epidemic Malaria}

Malaria epidemics occur in areas where environmental conditions are marginal for vector survival and/or parasite development. ${ }^{117}$ The term may be applied to a sharp rise in of the incidence among a population in which the disease was formerly unknown or an unusual increase in an area of otherwise moderately endemic malaria. ${ }^{118}$

Malaria epidemics hit immunologically susceptible populations since long intervals between infections and spatial variability of transmission prevent the formation of immunity. This means that epidemics affect people of all age groups and not just children who have not yet developed immunity. The risk of dying from an untreated infection, which is around 2 to $3 \%$ in endemic areas, can be up to ten times higher during epidemics. ${ }^{119}$ This is not only due to a lack of immunity but also to the fact that the quality of medical treatment often deteriorates in the course of epidemics since hospital capacities or medical supplies may turn out to be insufficient. A study in southwest Uganda observed a sudden increase in the case fatality rate from $3 \%$ to $24,5 \%$ during the 1998 epidemic. ${ }^{120}$ Epidemics occurring after periods of drought are often particularly devastating. ${ }^{121}$

The genesis of malaria epidemics may be linked to several factors, including increases in susceptibility of the human population, increased parasite reservoirs and higher contact rates between vectors and hosts (see figure 10):

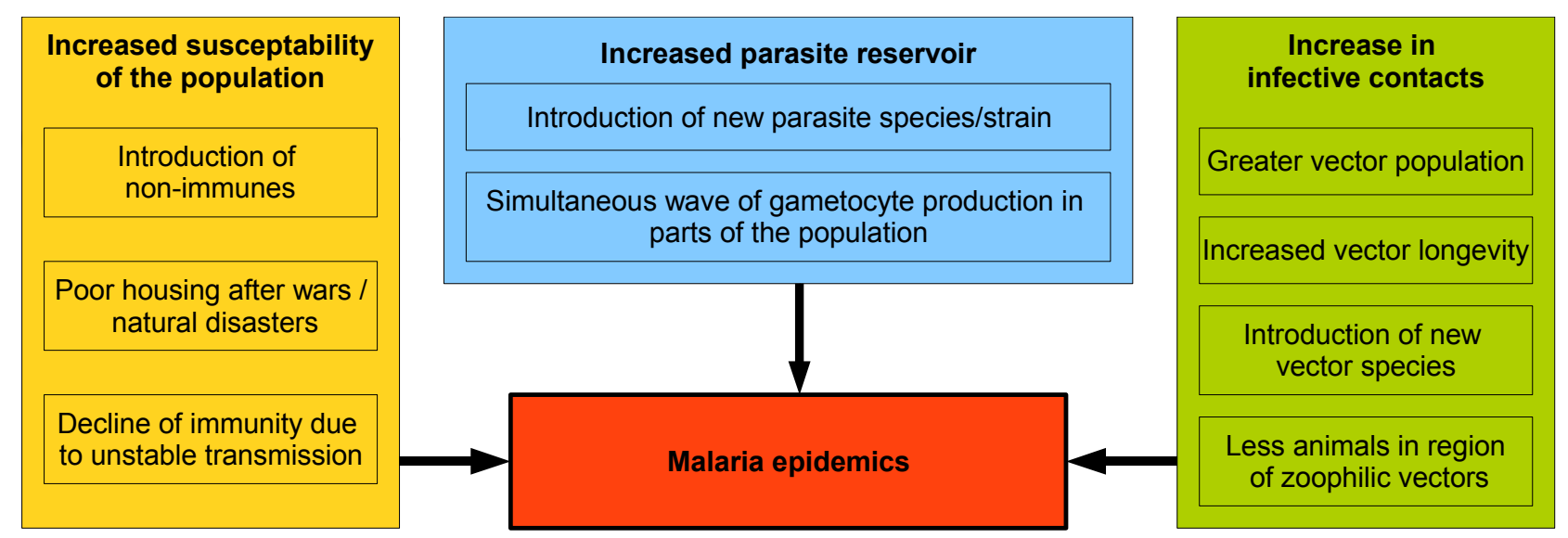

Figure 8: Causes of malaria epidemics ${ }^{122}$

117 Kiszewski, A.E. \& Teklehaimanot, A. (2004), p. 133.

118 Gilles, H.M. $\left(1993^{2}\right)$, p. 129.

119 Kiszewski, A.E. \& Teklehaimanot, A. (2004), p. 128.

120 Kiszewski, A.E. \& Teklehaimanot, A. (2004), p. 129.

121 Grover-Kopec, E.; Kawano, M.; Klaver, R.W. et al. (2005), doi:10.1186/1475-2875-4-6.

122 Based on Gilles, H.M. $\left(1993^{2}\right)$, p. 129. 


\subsubsection{Endemic Malaria}

Endemic malaria is found in areas where suitable conditions for transmission exist for several months each year. ${ }^{123}$ Endemic transmission may be characterized as either perennial (conditions always suitable for transmission) or seasonal (conditions suitable for some time each year). ${ }^{124}$

Endemic malaria may be present at various degrees:

- hypoendemicity denotes areas where there is little transmission;

- mesoendemicity is found in areas of varying intensity of transmission;

- hyperendemicity is seen in areas with intense but seasonal transmission where the immunity is insufficient to prevent the effects of malaria on all age groups;

- holoendemicity denotes a perennial transmission of high degree resulting in a considerable degree of immunity. ${ }^{125}$

In endemic areas, almost every individual experiences at least one mild clinical attack of malaria during his or her life-time. Approximately $0,25 \%$ of all malaria infections result in death. ${ }^{126}$ In endemic areas infants and young children often become victims of severe infections because they have not yet developed immunity. ${ }^{127}$ However, new-borns show a (partial) immunity for about three to six months after birth. In areas of high transmission, they may show considerable parasitemia but only mild fever and often no other symptoms of malaria. ${ }^{128}$ The rate of malaria morbidity is lower in the first year of life compared to the second year of life. ${ }^{129}$

Older children and adults are protected for a period of around 18 months after an infection, but lose their immunity when they are not re-infected within this time. ${ }^{130}$ Other reasons for a (partial) loss of immunity include pregnancies and migration. ${ }^{131}$

$123 \mathrm{M}_{\text {ARSH, }}$ K. (1993), p. 64.

124 Craig, M.H., Snow, R.W. \& le Sueur, D. (1999), p. 105.

125 Gilles, H.M. $\left(1993^{2}\right)$, p. 131.

126 SNOW, R.W. \& MARSH, K. (1998), p. 295.

127 Kiszewski, A.E. \& Teklehaimanot, A. (2004), p. 129.

128 MARSH, K. (1993), p. 64.

129 SNOW, R.W. \& MARSH, K. (1998), p. 299.

130 MaRTENS, P. (1998), p. 48.

$131 \mathrm{MARSH}$ K. (1993), p. 65. 


\subsection{The Mosquito Vector}

It is likely that mosquitoes appeared in the tropics around 200 million years ago, but their connection to pathogen transmission and importance as vectors of disease were only realized in the early 1900s. ${ }^{132}$ Vectors are (usually insect) species which "transport" microorganisms from one host organism to another. ${ }^{133}$ Malaria is a typical vector-borne disease since it is not directly transmitted from an infected individual to an uninfected person, but instead requiring anopheline mosquitoes as intermediate parasite carriers. In addition to transmitting malaria, anophelines may also transmit filariasis ${ }^{134}$ and viral diseases such as O'nyong-nyong fever ${ }^{135}$.

Mosquitoes (Culicidae) are insects of the order Diptera (two-winged flies) which develop from eggs to larvae, then pupae and finally to adults. There are around 3500 known mosquito species, but only some of those belonging the subfamily of the bloodfeeding Anophelinae are potential vectors of malaria. ${ }^{136}$

Out of more than 400 species of Anopheles mosquitoes, only around 60 are vectors of malaria under natural conditions ${ }^{137}$, and only 20 to 30 of them are of major importance for malaria transmission. ${ }^{138}$ Among the main factors determining whether a particular species of Anopheles is an important vector of malaria, the life span of the mosquitoes and their preference for feeding on humans are of particular importance ${ }^{139}$, as are an indoor resting behavior and the ability to quickly reach high population densities. ${ }^{140}$ The most common vector of Plasmodium falciparum malaria in Africa, Anopheles gambiae, fulfills all these "ideals." 141

\subsubsection{Anopheline Population Dynamics}

The key factors in insect population processes are the dynamics of abundance (population density) and dispersion. Anophelines are mosquitoes with a high reproductive potential, but nevertheless, mortality during immature stages is considerable and adult longevity and flight range limited.

132 KLOWDEN, M.J. (2007), p. 1.

133 Daly, H.V.; Doyen, J.T. \& Purcell, A.H. (1998), p. 265.

134 Otranto, D.; Stevens, J.R.; Cantacessi, C. \& Gasser, R.B. (2007), p. 117.

$135 \mathrm{TESH}$, R. B. (1982), p. 33.

136 KLOWDEN, M.J. (2007), p. 1.

137 Peters, W. (2002), p. 666.

138 TUTEJA, R. (2007), p. 4671.

139 SERVICE, M.W. (1993), p. 96.

140 Breman, J.G. (2009), p. 6.

141 Hoshen, M.B. \& Morse, A.P. (2004), doi:10.1186/1475-2875-3-32. 


\subsubsection{Population Biology of Insects}

Insect population growth is regulated both by density-dependent factors (e.g. increasing competition for food and prevalence of antagonists) and densityindependent factors (e.g. weather conditions). All these factors have a spatiotemporal dimension ${ }^{142}$, and their relative roles vary from one species to another. For most species, there is a certain maximum carrying capacity that is determined by the availability of food, space and other essential needs. This carrying capacity may be exceeded temporarily, ultimately causing (often rapid) declines in population densities. While so-called K-strategists tend to be adapted to stable habitats in which populations remain at relatively constant levels, r-strategists rapidly exploit new habitats by reproducing in an exponential manner. ${ }^{143}$ Anopheline mosquitoes are typical r-strategists ${ }^{144}$, i.e. insects with a high reproductive potential.

\subsubsection{Life Cycle of Anophelines}

Oviposition normally occurs during flight and eggs are laid by female anophelines on the type of water preferred by a particular species. Eggs are about $0.5 \mathrm{~mm}$ in length and normally provided with tiny air-filled floats which allow them to remain on the water surface. The number of eggs laid usually ranges between 100 and $150 .{ }^{145}$ Newly laid eggs require a period of two or three days for completion of embryonic development before they can hatch. Eggs of some Anopheles species can remain alive for 16 days or even longer on wet mud; when flooded, such eggs hatch within three to four minutes. However, anopheline eggs can usually not survive desiccation. ${ }^{146}$

Anopheline larvae hatch from their eggs about one to four days after oviposition. They now pass through different larval stages and float at the water surface and feed on particles contained in the water, particularly pollen, algae and bacteria at the water's surface. ${ }^{147}$ The larval stage typically lasts between eight and ten days; the larvae then turn into pupae. Two or three days later the adult mosquitoes hatch. This process lasts several minutes. The mosquitoes rest for a while so that their wings harden. ${ }^{148}$ Maturity is defined as the time of the first flight, soon after which the first blood meal is taken. ${ }^{149}$

142 ZWÖLFER, H. (2002), pp. $701 \mathrm{f}$.

143 Daly, H.V.; Doyen, J.T. \& PuRCell, A.H. (1998), p. 176.

144 Minakawa, N.; Munga, S.; Atielli, F. et al. (2005), p. 163.

145 Overgaard, H. (2001), p. 9; Service, M.W. (1993), p. 96.

146 Service, M.W. (1993), p. 111; Hoshen, M.B. \& Morse, A.P. (2004), doi:10.1186/1475-2875-3-32.

147 Ye-Ebiyo, Y.; Pollack, R.J.; Kiszewski, A. \& Spielmann, A. (2003), p. 748.

148 Ye-Ebiyo, Y.; Pollack, R.J.; Kiszewski, A. \& Spielmann, A. (2003), p. 748;

OvergaArd, H. (2001), p. 9.

149 Hoshen, M.B. \& Morse, A.P. (2004), doi:10.1186/1475-2875-3-32. 
The total duration of this development is species-specific and depends on meteorologic conditions. ${ }^{150}$ At a temperature around $30^{\circ} \mathrm{C}$, for example, the development from egg to adult takes around 10 days, a process which is slowed down considerably at lower temperatures (20 days at $20^{\circ} \mathrm{C}$ ). ${ }^{151}$ This dependence is partially responsible for the interannual variation of malaria incidence and implies that climate change may induce changes in malaria transmission pattern.

Vector mortality is particularly high at the larval stage and may be due to antagonists such as natural predators or parasitoids, diseases, flooding or drought. It is reported that only a small fraction (about $2 \%$ to $8 \%$ ) of the larvae that hatched eventually turns into adults. ${ }^{152}$ There is only very little data on adult vector longevity under natural conditions. It is believed that less than $10 \%$ of the mosquitoes live for more than 3 weeks. ${ }^{153}$ However, vector longevity is considered to be the most important factor governing the risk of malaria transmission. ${ }^{154}$

\subsubsection{Vector Competence and Transmission Risk}

The term vector competence refers to the capability of a certain mosquito species to transmit infectious diseases and in case of malaria varies considerably between different anophelines. Vector competence depends on the following factors:

- the species' physiological susceptibility to local strains of malaria ${ }^{155}$;

- their behavior, including both biting behavior and spatial behavior/flight range;

- the mosquitoes' population dynamics and average lifespan; ${ }^{156}$;

- the mosquito populations' ability to quickly recover even after prolonged periods of drought. ${ }^{157}$

Determination of the age of female mosquitoes is of importance for the full understanding of the epidemiology of malaria and for assessment of the efficacy of anti-anopheline measures, most of which aim at shortening the average lifespan of malaria vector populations. ${ }^{158}$

150 Overgaard, H. (2001), pp. 9 f.

151 BAYOH, M.N. \& LindSAY, S.W. (2003), pp. 377f; BAYOH, M.N. \& LindSAY, S.W. (2004), p. 174.

152 Paaijmans, K.P; Wandago, M.O.; Githeko, A.K. \& Takken, W. (2007), doi:10.1371/journal.pone.0001146.

153 Overgaard, H. (2001), p. 10.

154 Zavaleta, J.O. \& Rossignol, P.A. (2004), p. 611.

155 W HITE, G.B. (1982), p. 134.

156 Overgaard, H. (2001), p. 9.

157 Levine, R.S.; Peterson, T. \& Benedict, M.Q. (2004 ${ }^{1}$ ), p. 105

158 Service, M.W. (1993), p. $101 f$. 


\subsubsection{Host and Resting Preferences}

The adult female mosquito typically takes her first blood meal the night after she emerges from the pupal stage. Feeding occurs, almost without exception, between dusk and dawn, but anophelines may feed during the day-light hours in densely shaded woodland or dark interiors of shelters and houses. The times of biting can be epidemiologically important and are relatively constant among the members of one mosquito species. Some species have relatively early peaks of biting (e.g. Anopheles albimanus: $7 \mathrm{pm}$ until $9 \mathrm{pm}$ ) whereas others are late feeders (e.g. Anopheles gambiae: 0 am until 3 pm). ${ }^{159}$

The terms anthropophilic and zoophilic are used to indicate, respectively, a preference for feeding on humans or on animals, often domestic ones such as cattle. It must be understood, however, that such terms are relative, since many Anopheles species are ready to feed on alternate hosts when their favorite one is not available. ${ }^{160}$ The preference for human biting over other mammals is described by the human blood index (HBI; defined as the proportion of human blood ingested by a mosquito species as compared to blood of all sources), which is high (above 0.6) for anthropophilic Anopheles gambiae and much lower for zoophilic Anopheles arabiensis. ${ }^{161}$ The degree of variation within one species may be wide; for Anopheles culcifacies in India, for example, human bloodfeeding rates between $2 \%$ and $80 \%$ have been observed. This variation in feeding habit may have a great effect on the incidence of disease. ${ }^{162}$

Resting places are often inside houses, and particularly in the cooler lower portions where humidity tends to be higher. Depending on the resting and bloodfeeding habits, the following behavioral characteristics of mosquitoes can be distinguished:

- endophily: the habit of remaining within man-made shelters during much of the gonotrophic cycle;

- exophily: the habit of spending the greater part of the gonotrophic cycle outdoors;

- endophagy: the habit of taking blood meals within man-made structures;

- exophagy: the habit of taking blood meals outdoors. ${ }^{163}$

The nature of resting and bloodfeeding habits has important implications for the assessment of transmission risk and the implementation of intervention strategies.

159 Service, M.W. (1993), p. 114.

160 SerVice, M.W. (1993), p. 111.

161 Hoshen, M.B. \& Morse, A.P. (2004), doi:10.1186/1475-2875-3-32.

162 Gilles, H.M. $\left(1993^{2}\right)$, p. 140.

163 Service, M.W. (1993), p. 115. 


\subsubsection{Bloodfeeding and Reproduction}

The male Anopheles mosquito feeds exclusively on nectar and fruit juices while the female feeds primarily on blood. However, females usually also need a sugar meal before their host-seeking flight. ${ }^{164}$ Often within a day from hatching, anthropophilic anophelines such as Anopheles gambiae enter human dwellings to feed on blood. ${ }^{165}$ While one blood meal is often sufficient, some species, including Anopheles gambiae and Anopheles funestus, may require two blood meals before the first batch of eggs can develop. ${ }^{166}$ One study carried out in a moist savanna region near Bobo-Dioulasso in Burkina Faso reported that during the first gonotrophic cycle, $42 \%$ of female Anopheles gambiae and $63 \%$ of female Anopheles funestus required a secondary blood meal. ${ }^{167}$ Anophelines usually lay their first batch of eggs three to six days after emergence. In subsequent cycles a batch of eggs produced by the ovaries develops after each blood meal. ${ }^{168}$

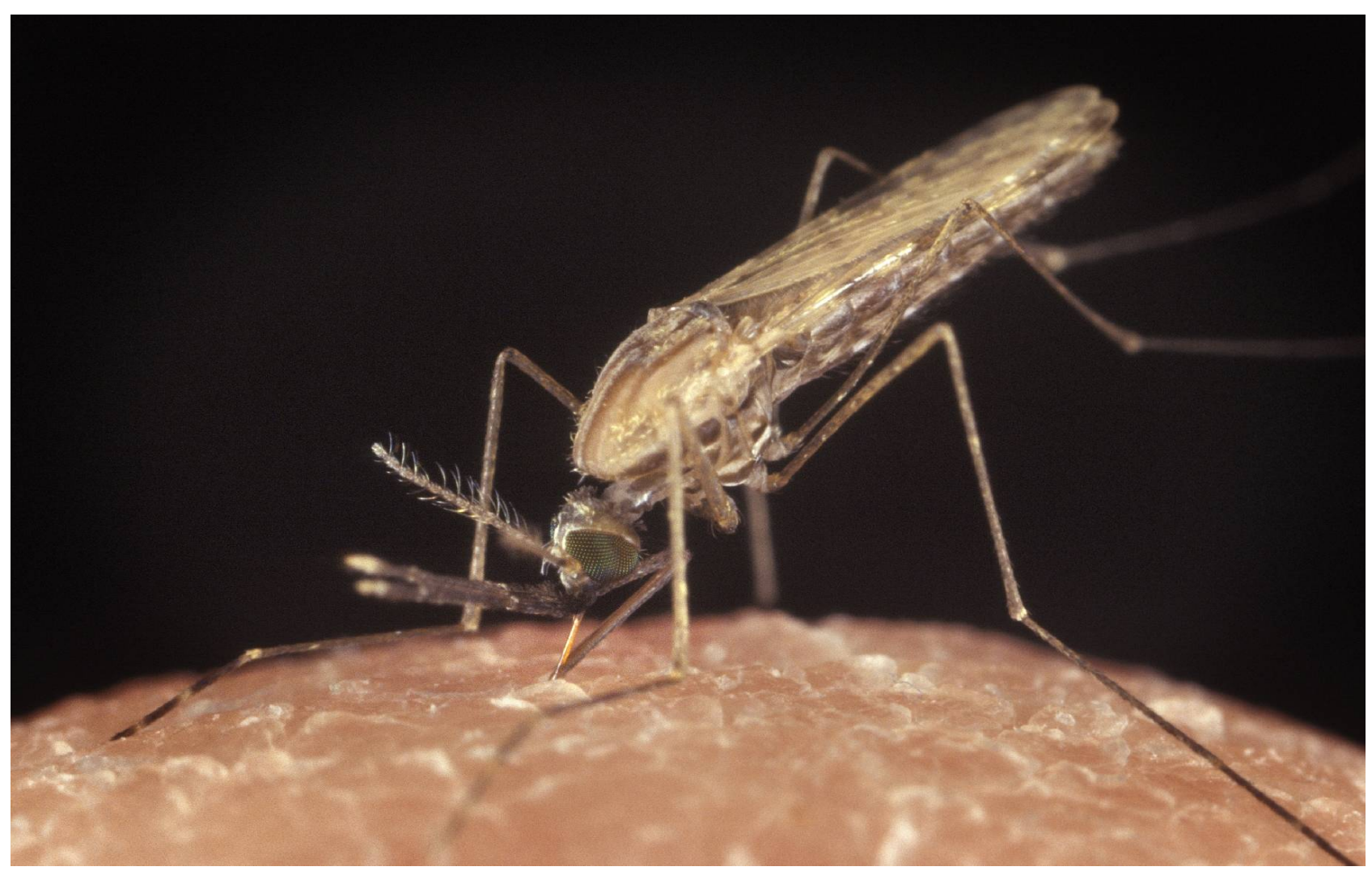

Figure 9: Female Anopheles gambiae during blood meal ${ }^{169}$

164 TAKKEN, W. \& KNOLS, B.G.J. (1999), p. 134.

165 KLOWDEN, M.J. (2007), p. 2.

166 Ramasamy, M.S.; Srikrishnaraj, K.A.; Hadjirin, N. et al. (2000), p. 1051.

167 Brengues, J. \& Coz, J. (1973), p. 107.

168 SERVICE, M.W. (1993), p. 110.

169 CDC Public Health Image Library (http://phil.cdc.gov/), image ID 1663, accessed 20/08/09. 
The mating of many species is preceded by the formation of male swarms which occur during twilight. It is probable that the females of most species receive enough sperm for all subsequent egg batches from a single mating. ${ }^{170}$ The fecundity or potential reproductive capacity of a mosquito is determined by several factors including body size, nutritional status and the quality of blood meals. ${ }^{171}$ Bloodfeeding, consisting of the approach for bite, the duration of the blood meal and the escape to a resting point, is the most dangerous stage in the life of an adult mosquito. ${ }^{172}$

The period between one oviposition and the next is called the gonotrophic cycle. The duration of the gonotrophic cycle is an important measure in malaria epidemiology as it determines the number of blood meals a female mosquito takes during her life. In the tropics one gonotrophic cycle typically lasts between two and four days. After oviposition, the gonotrophic cycle repeats. ${ }^{173}$ At temperatures above $23^{\circ} \mathrm{C}$, the gonotrophic cycle is completed within about 48 hours so that host-seeking for the next blood meal is repeated every two to three nights. ${ }^{174}$ However, adverse environmental conditions may lengthen the gonotrophic cycle considerably:

\begin{abstract}
«When the environmental temperature drops below a certain threshold, females of some species of Anopheles undergo a process of hibernation during which they develop fat bodies, and cease producing eggs. This process, known under the name of gonotrophic dissociation, may also occur in tropical Anopheles during the period of drought. The period of reproductive inactivity is termed diapause.» ${ }^{175}$
\end{abstract}

An estimated $40 \%$ and $60 \%$ of the mosquitoes survive the gonotrophic cycle. It is commonly assumed that survivorship is independent of the infective state, even though there are some reports that being infected is harmful to the mosquito. ${ }^{176}$ Charlwood et al. (2000) found 6,3\% of Anopheles gambiae, 4,0\% of Anopheles funestus and 2,0\% of Anopheles arabiensis to survive for four or more gonotrophic cycles under dry season and dry savanna conditions. ${ }^{177}$

170 Service, M.W. (1993), p. 110.

171 Ramasamy, M.S.; SRikrishnaraj, K.A.; Hadjirin, N. et al. (2000), p. 1052.

172 Hoshen, M.B. \& Morse, A.P. (2004), doi:10.1186/1475-2875-3-32.

173 Overgaard, H. (2001), p. 10.

174 Service, M.W. (1993), p. 110.

175 Service, M.W. (1993), p. 111.

176 Hoshen, M.B. \& Morse, A.P. (2004), doi:10.1186/1475-2875-3-32.

177 Charlwood, J.D.; Vij, R. \& Billingsley, P.F. (2000), p. 726. 
A mosquito species' bloodfeeding behavior is not only important because it regulates the number of potentially infective contacts but also because a second, non-infective blood meal following the infective one enhances the developmental potential of the parasite. ${ }^{178}$ Infectious Anopheles gambiae take larger and more multiple blood meals than uninfected mosquitoes. ${ }^{179}$

At the onset of the cold season, many mosquitoes are killed off. In some species, only the males die while the females seek shelter in relatively protected places and begin to hibernate. Prior to hibernation, a last blood meal is taken which, instead of resulting in the formation of eggs, is used to produce fat on which the females survive. Some mosquitoes take a prehibernation flight which is considerably longer than their normal flying range. ${ }^{180}$ A similar seasonal effect, aestivation, is seen in the hot dry seasons of some countries, where the females seek to avoid the dry atmosphere by remaining inactive in a cool damp place until more humid conditions return. ${ }^{181}$

\subsubsection{Vector Longevity}

The length of live of adult Anopheles mosquitoes varies somewhat between different species but much more due to environmental factors such as temperature, humidity and presence of natural enemies. The average duration of life of a female Anopheles under favorable climatic conditions is about 10 to 14 days, but occasionally much longer. Some females in a population live for up to 4 weeks; males tend to have a shorter lifespan than females. When the mean temperature is over $35^{\circ} \mathrm{C}$ or the humidity less than $50 \%$, the longevity of the mosquitoes is drastically reduced unless they find more favorable conditions in their microhabitat. The longevity of the local anopheline population is closely linked to the transmission risk. If the mean daily mortality of a population is $35 \%$, less than $1 \%$ of the mosquito population will survive the ten days necessary for the development of Plasmodium falciparum. ${ }^{182}$ An adult female mosquito has no prospects of transmitting malaria unless it lives through the time taken for sporogonic development of the malaria parasite; this is estimated to be between 8 and 25 days, depending on climatic conditions and parasite species. ${ }^{183}$

178 BEIER, J.C. (1998), p. 521.

179 TAKKEN, W. \& KNoLS, B.G.J. (1999), p. 146.

180 SeRVICE, M.W. (1993), pp. $113 f$.

181 SeRVICE, M.W. (1993), p. 114.

182 Service, M.W. (1993), p. 111.

183 Gilles, H.M. $\left(1993^{2}\right)$, p. 140. 
While physical inactivity in the form of hibernation and aestivation are survival strategies during periods of unsuitable environmental conditions, their effectivity is stage-dependent:

\begin{tabular}{|l|l|l|l|l|}
\hline $\begin{array}{l}\text { Aestivation } \\
\text { daily survival }\end{array}$ & 0.8 & Larvae & Pupae & Adults \\
\hline
\end{tabular}

Table 6: Stage-dependent daily survival rates during aestivation ${ }^{184}$

\subsubsection{Spatial Behavior}

The spatial behavior of mosquitoes is governed by physiological conditions and external stimuli ${ }^{185}$ and closely connected to their life cycle. There are five major reasons why mosquitoes fly to a particular place:

- Mosquitoes search for resting places, e.g. after a blood meal or before nesting.

- Mate seeking: Suitable mates are sought before the first blood meal, often during the first evening dawn after hatching. Swarms of female mosquitoes fly into swarms of male mosquitoes which are often found above large (vertical) objects such as trees, posts, huts or even large animals.

- Nectar seeking: Both male and -to a lesser extent- female mosquitoes look for sources of nectar.

- Host seeking: About one day after hatching, female mosquitoes require blood meals for the development of eggs. It is believed that body odors play an important role in the selection of human victims.

- Towards the end of the gonotrophic cycle, female mosquitoes look for breeding sites. ${ }^{186}$

Dispersal of anopheline adults is typically nocturnal when mating, oviposition and bloodfeeding occur. ${ }^{187}$ In most tropical regions, blood meals are taken between $6 \mathrm{pm}$ and 6 am. ${ }^{188}$

A whole range of external factors influences the mosquitoes' movement (see figure 12), among which the meteorologic conditions, the location of habitats and landscape features are the most important.

184 Depinay, J.M.O.; Mbogo, C.M.; Killeen, G. et al. (2004), doi:10.1186/1475-2875-3-29.

185 TAKKen, W. \& KNols, B.G.J. (1999), p. 134.

186 OvergaARD, H. (2001), pp. 10-12.

187 WHITE, G.B. (1982), pp. $134 f$.

188 Depinay, J.M.O.; Mbogo, C.M.; Killeen, G. et al. (2004), doi:10.1186/1475-2875-3-29. 


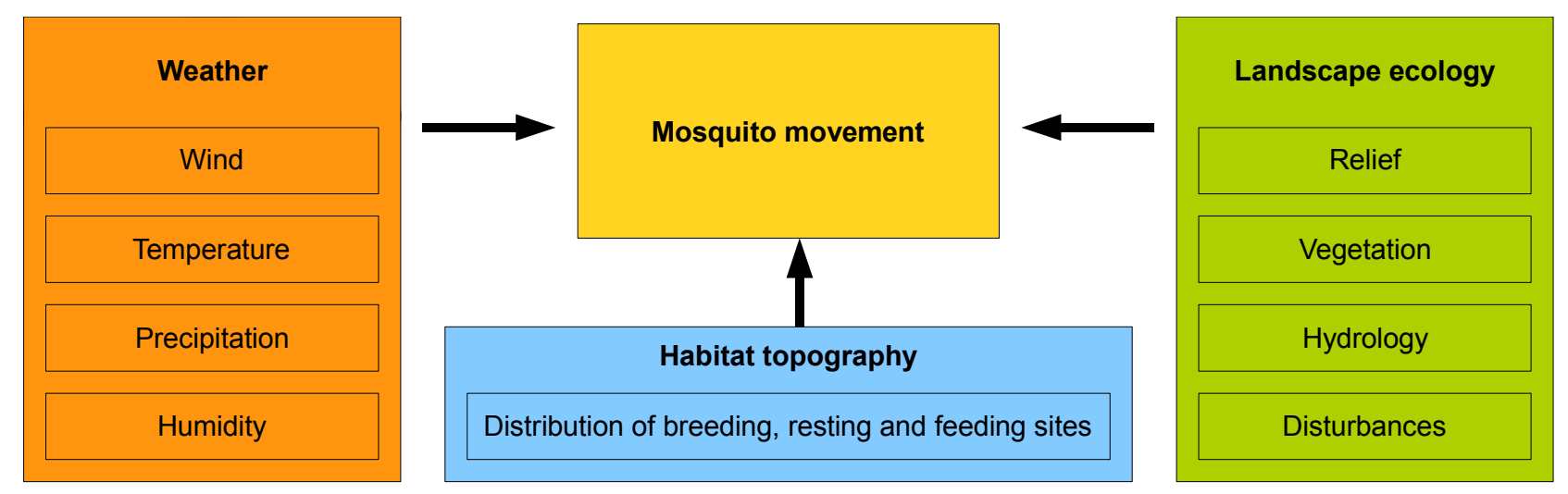

Figure 10: Macro-ecological factors influencing mosquito movement ${ }^{189}$

The immediate stimuli governing mosquito movement are often olfactory cues. While male mosquitoes respond mainly to plant odors, females are also attracted by breeding site volatiles, male mating pheromones and host odors. ${ }^{190}$ In fact, Anopheles gambiae odorant receptors are sex specific, and in females, the response to human odors is variable and down-regulated 12 hours after bloodfeeding. ${ }^{191}$

Some species readily breed in temporary rain pools and in small puddles of water, such as those formed by the imprints of animal hooves. Large expanses of open water, free from vegetation, are rarely chosen as breeding sites, although breeding may occur in isolated pockets of relatively still water along the grassy margins of lakes, streams and rivers. ${ }^{192}$ Irrigation without proper drainage frequently causes waterlogging and may result in "irrigation malaria". With crops like rice, breeding occurs in the water standing in the fields. ${ }^{193}$

The flight range of Anopheles mosquitoes is typically between 1 and $2 \mathrm{~km}^{194}$ and rarely goes beyond more than two or three kilometers from their breeding places. However, strong seasonal winds may carry anophelines up to $30 \mathrm{~km}$ or more from their main breeding place. Generally, tropical anophelines have a shorter flight range than mosquitoes present in temperate climates. ${ }^{195}$ Unfortunately, there is still very limited data on the flight range of Anopheles mosquitoes, including information on the influence of ecological settings, parasitation and other factors. An overview of the typical flight range of tropical mosquitoes is presented in table 7.

189 Based on OvergaARd, H. (2001), pp. $10 f$.

190 TAKKEN, W. \& KNols, B.G.J. (1999), p. 135.

191 Fox, A.N.; Pitts, R.G.; Robertson, H.M. et al. (2001), p. 14693.

192 Service, M.W. (1993), p. 112.

193 SerVice, M.W. (1993), p. $112 \mathrm{f}$.

194 Hoshen, M.B. \& Morse, A.P. (2004), doi:10.1186/1475-2875-3-32.

195 Service, M.W. (1993), p. 115. 


\begin{tabular}{|l|l|l|}
\hline Mosquito Species & $\begin{array}{l}\text { Information on ecological } \\
\text { setting and/or other potential } \\
\text { determinants }\end{array}$ & Flight range \\
\hline Anopheles minimus s.I. & Thailand & up to $2 \mathrm{~km}^{196}$ \\
\hline $\begin{array}{l}\text { Anopheles minimus } \\
\text { species E }\end{array}$ & Ryukyu Archipelago, Japan & up to $1 \mathrm{~km}^{197}$ \\
\hline Anopheles flavirostris & Philippines & about $2 \mathrm{~km}^{198}$ \\
\hline Anopheles funestus & Sub-Saharan Africa & up to $7 \mathrm{~km}^{199}$ \\
\hline Anopheles arabiensis & Marshland in Senegal & $\begin{array}{l}\text { typically less } \\
\text { than } 300 \mathrm{~m}^{200}\end{array}$ \\
\hline Anopheles gambiae & Côte d'Ivoire & $\begin{array}{l}\text { just above } \\
2 \mathrm{~km}\end{array}$ \\
\hline Anopheles gambiae & $\begin{array}{l}\text { Urban environments in Sub- } \\
\text { Saharan Africa }\end{array}$ & $\begin{array}{l}\text { typically less } \\
\text { than } 300 \mathrm{~m}^{202}\end{array}$ \\
\hline
\end{tabular}

Table 7: Flight ranges of tropical mosquitoes

Parasitation appears to play a role here: Anopheles stephensi mosquitoes infected with Plasmodium cynomolgi were found to show poorer flight performances than uninfected mosquitoes, which might be the result of carbohydrate consumption by the developing parasites. ${ }^{203}$

\subsubsection{Parasitation}

In entomological surveys, mosquitoes may be checked for parasites in their stomach and salivary glands. Since oocysts are usually not detectable until around seven days after bloodfeeding, the determination of the infection status of a mosquito is impossible for about a week after the ingestion of parasites. ${ }^{204}$ An Anopheles which shows oocysts on its stomach walls is infected; when it shows sporozoites in the salivary glands, it is infective. This is the more important parameter because it shows that the vector has lived long enough to be able to transmit malaria. The percentage of female Anopheles caught in nature showing sporozoites in the glands is the sporozoite rate. Sporozoite

196 Garros, C.; van Bortel, W.; Trung, H.D. et al. (2006), p. 105.

197 Garros, C.; van Bortel, W.; Trung, H.D. et al. (2006), p. 105.

198 Foley, D.H. \& TORRES, E.P. (2006), p. 400.

199 Michel, A.P.; Guelbeogo, W.M.; Grushro, O. et al. (2005), p. 379.

200 Robert, V.; Macintyre, K.; Keating, J. et al. (2003), p. 171.

201 Briët, O.J.T.; Dossou-Yovo, J.; Akodo, E. et al. (2003), p. 447.

202 Keiser, J.; Utzinger, J.; Caldas de Castro, M. et al. (2004), p. 120.

203 Bradley, C.A. \& Altizer, S. (2005), p. 297.

204 BeIer, J.C. (1998), p. 529. 
rates typically range from $1 \%$ to $20 \%{ }^{205}$, with rates of about $5 \%$ being common in African Anopheles gambiae. ${ }^{206}$ Rates of up to $30 \%$ have been observed seasonally in Anopheles gambiae and Anopheles funestus. ${ }^{207}$ Sporozoite rates tend to be lower in other species and outside Africa. ${ }^{208} \mathrm{~A}$ longstanding but potentially incorrect assumption is that mosquitoes carrying sporozoites in their salivary glands are infective. Until today, no reliable methods exist for assessing sporozoite infectivity. Moreover, predictions about the ability of a mosquito to eject sporozoites may not be feasible based on determinations of its sporozoite load. These are important limitations of current assessment techniques of vector competence. ${ }^{209}$

In the course of the extrinsic incubation period, malaria parasites can cause substantial damage to their vectors, and consequently, longevity of parasitized mosquitoes may be reduced. ${ }^{210}$ The consequences of plasmodian parasitation for malarial vectors are outlined in figure 11 .

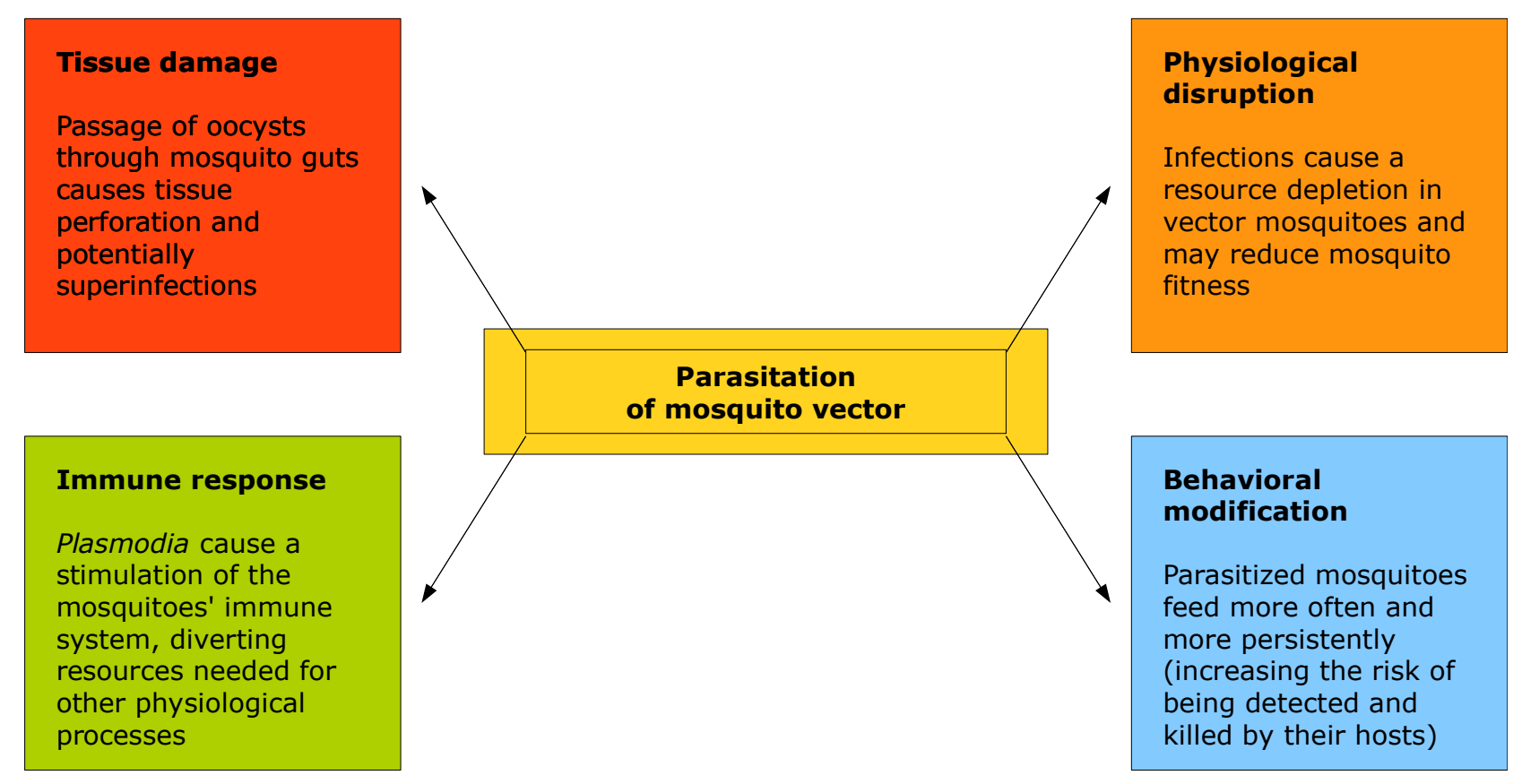

Figure 11: Consequences of mosquito parasitation ${ }^{211}$

205 Beier, J.C.; Killeen, G.F. \& Githure, J.I. (1999), p. 109.

206 SeRVICE, M.W. (1993), p. 122.

207 BEIER, J.C. (1998), p. 529.

208 SeRVICE, M.W. (1993), p. 122.

209 BEIER, J.C. (1998), pp. 532 f.

210 FERGUSON, H.M. \& READ, A.F. (2002), p. 256.

211 Based on Ferguson, H.M. \& Read, A.F. (2002), p. 257. 
While evidence that malaria parasites reduce the survival and fecundity of their mosquito vectors continues to accumulate, a hypothesized energy depletion could not be verified in a study on the impact of Plasmodia on the anopheline energy budget. Moreover, the magnitude of the impact of parasitation appears to be strain-specific. ${ }^{212}$

A single infective mosquito can inject sporozoites into several hosts who may all become infected. ${ }^{213}$

\subsubsection{Important Vectors in West Africa}

There are three main malaria vectors in sub-Saharan Africa: Anopheles gambiae and its sibling species Anopheles arabiensis, and Anopheles funestus. In large parts of Africa, all three vectors contribute significantly to malaria transmission, often in seasonal variation. ${ }^{214}$ Anopheles gambiae and Anopheles arabiensis are of particular importance in West Africa. Anopheles melas is an important local vector in some coastal areas of West Africa. ${ }^{215}$

\subsubsection{The Anopheles gambiae Complex}

The Anopheles gambiae sensu lato (s.l.) complex consists of seven morphologically similar species, which are responsible for about $80 \%$ of the worldwide malaria morbidity and mortality. ${ }^{216}$ The genetic structure of the complex is thought to have enabled its members to occupy different habitats. ${ }^{217}$ Anopheles gambiae is the most important malaria vector in Africa and until 1956, it was considered a single species with different varieties breeding in fresh and salt water. Today, the following sibling species are distinguished:

- Anopheles gambiae sensu stricto (s.s.) (formerly species A) is adapted to fresh-water breeding sites and predominates in humid areas. It is highly anthropophilic and is an important vector of malaria.

- Anopheles arabiensis (formerly species B) is also a fresh-water breeder but extends more into savanna areas. In many areas, it is more zoophilic and exophilic but nevertheless can be an efficient malaria vector.

- Anopheles quadriannulatus (formerly species C) is the third sibling species which is adapted to fresh-water habitats. It is highly zoophilic and therefore not a vector.

212 Rivero, A. \& Ferguson, H.M. (2003), p. 1365.

213 WHITE, G.B. (1982), p. 134.

214 TAKKEN, W. \& KNOLS, B.G.J. (1999), p. 133.

215 HaY, S.I.; Omumbo, J.A.; Craig, M.H. \& Snow R.W. (2000), pp. 175, 177.

216 Levine, R.S.; Peterson, T. \& Benedict, M.Q. (2004 ${ }^{1}$ ), p. 105

217 Edillo, F.E.; Tripét, F.; Touré, Y.T. et al. (2005), doi:10.1186/1475-2875-5-35. 
- The last fresh-water species, Anopheles bwambae (formerly species D), has a very restricted distribution in a small area of the Rift Valley, west of Ruwenzori, and breeds in geothermal waters. It is a minor and highly localized malaria vector.

- The two salt-water species, Anopheles melas of West Africa and Anopheles merus of East Africa, are local malaria vectors, but they are generally more exophagic and zoophilic and thus poorer vectors than Anopheles gambiae s.s. ${ }^{218}$

- The ancestral species of the complex, Anopheles quadriannulatus, is largely zoophilic and is not considered a vector of human malaria. ${ }^{219}$

\subsubsection{West African Vectors and Their Characteristics}

Anopheles gambiae and Anopheles arabiensis exist sympatrically in $70 \%$ of sub-Saharan Africa and are morphologically indistinguishable. ${ }^{220}$ Anopheles gambiae prefer more humid, vegetated areas; because of their relative longevity, they are important vectors. Anopheles arabiensis tend to predominate in drier savanna areas as they are able to reproduce rapidly after persisting periods of drought in small, remaining pools of water (e.g. in dry riverbeds). ${ }^{221}$ Anopheles gambiae s.s. is the most important vector in West Africa. It is widely distributed and is anthropophilic and rather endophilic and endophagic but may also feed and rest outdoors. Larvae of Anopheles gambiae and Anopheles arabiensis are commonly found in pools, usually exposed to the sun. Although they occur in all types of pools, they are more common in temporary ones than in those of long standing. Breeding in rice fields and swamps may occur. Owing to the nature of their breeding places, both species tend to be more numerous in the rainy season than in dry weather. ${ }^{222}$

Besides Anopheles gambiae and Anopheles arabiensis, Anopheles funestus is another important vector of malaria in sub-Saharan Africa. ${ }^{223}$ It is a very widely distributed carrier of Plasmodia and second in importance only to Anopheles gambiae. Anopheles funestus predominantly feeds on humans, is relatively long-lived and highly endophilic. Anopheles funestus prefers shadowy and vegetated areas for oviposition, and its larvae are found in rivers/streams

218 SeRVICE, M.W. (1993), p. $116 \mathrm{f}$.

219 TAKKEN, W. \& KNOLS, B.G.J. (1999), p. 133.

220 Gilles, H.M. $\left(1993^{2}\right)$, p. 160; HaY, S.I.;

Omumbo, J.A.; Craig, M.H. \& Snow R.W. (2000), p. 178.

221 Hay, S.I.; Omumbo, J.A.; Craig, M.H. \& Snow R.W. (2000), p. 179.

222 Gilles, H.M. $\left(1993^{2}\right)$, p. 160.

223 Hay, S.I.; Omumbo, J.A.; Craig, M.H. \& Snow R.W. (2000), p. 179. 
(particularly along grassy edges), swamps, seepages, rice fields. ${ }^{224}$ Owing to the nature of its breeding places, its season is often different from that of Anopheles gambiae and in many places, one species takes over from the other as chief vector when the season changes. ${ }^{225}$

Important characteristics of West Africa's most important vector species are summarized in table 8 :

\begin{tabular}{|l|l|l|l|}
\hline Anopheles & $\begin{array}{l}\text { Anopheles } \\
\text { gambiae } \\
\text { arabiensis }\end{array}$ & $\begin{array}{l}\text { Anopheles } \\
\text { funestus }\end{array}$ \\
\hline Host preference & $\begin{array}{l}\text { Highly } \\
\text { anthropophilic }\end{array}$ & Variable & Anthropophilic \\
\hline Feeding location & Endophagic & Variable & Endophagic \\
\hline Resting location & Endophilic & Exophilic ${ }^{226}$ & Endophilic \\
\hline $\begin{array}{l}\text { Susceptibility to } \\
\text { infection }\end{array}$ & $\begin{array}{l}\text { Highly susceptible } \\
\text { to Plasmodium } \\
\text { falciparum }\end{array}$ & $\begin{array}{l}\text { Moderately } \\
\text { susceptible to } \\
\text { Plasmodium } \\
\text { falciparum }\end{array}$ \\
\hline
\end{tabular}

Table 8: Characteristics of key African vectors ${ }^{227}$

Other vectors which are found in limited localities of West Africa include Anopheles moucheti (a secondary vector in riverine forest tracts) and Anopheles nili (important secondary vector breeding in streams) ${ }^{228}$. Anopheles melas, Anopheles coustani and Anopheles pharaoensis are reported to be secondary vectors of malaria in The Gambia. ${ }^{229}$

Different chromosomal forms of Anopheles gambiae are found in West Africa and can broadly be differentiated into savanna and forest forms. ${ }^{230}$ They apparently have both macro- and microgeographic differences in their habitats. Anopheles gambiae Mopti seems able to exploit man-made habitats in dry areas normally occupied by Anopheles arabiensis. In Burkina Faso, Anopheles gambiae Mopti is found at higher relative frequency in irrigated areas whereas Anopheles gambiae Savanna appears to be more prevalent in natural sites

224 HaY, S.I.; Omumbo, J.A.; CRAig, M.H. \& Snow R.W. (2000), p. 179; Gilles, H.M. $\left(1993^{2}\right)$, p. 160.

225 Gilles, H.M. $\left(1993^{2}\right)$, p. 160.

226 SerVice, M.W. (1993), p. $116 f$.

227 TAKKEN, W. \& KNOLS, B.G.J. (1999), p. 133.

228 Gilles, H.M. $\left(1993^{2}\right)$, p. 160.

229 Fillinger, U.; Sombroek, H.; Majambere, S. et al. (2009), doi:10.1186/1475-2875-8-62.

230 Bayoh, M.N.; Thomas, C.J. \& Lindsay, S.W. (2001), p. 268. 
except for swamps. ${ }^{231}$ Anopheles gambiae Bissau is found most frequently in the western parts of Burkina Faso. In regions where they are sympatric, the chromosomal differentially fluctuate seasonally ${ }^{232}$ and seem to be reproductively more or less isolated ${ }^{233}$.

\subsection{The Malaria Parasite}

Malaria is caused by Plasmodia, a group of unicellular organisms (protozoa), which are transmitted from an infected to an uninfected person by anopheline vectors. More than one hundred species of Plasmodia can infect numerous animal species such as reptiles, birds and mammals, but only five of them infect humans. These species differ morphologically, immunologically, in their geographic distribution, clinical picture of infection and treatment. ${ }^{234}$

- Plasmodium vivax is the most widespread malaria parasite. It typically causes a gradual health deterioration ${ }^{235}$ but infections with this species are rarely fatal. ${ }^{236}$ It occurs in parts of the temperate and subtropical zones and throughout the tropics. However, it is not very common in West Africa. ${ }^{237}$

- Plasmodium ovale is the least common malaria parasite and largely restricted to tropical West Africa. ${ }^{238}$ It usually causes less serious infections than Plasmodium vivax. ${ }^{239}$

- Plasmodium malariae occurs in the tropics and subtropics, especially West and East Africa. Plasmodium malariae also affects chimpanzees who are the natural hosts of the parasite. Infections with Plasmodium malariae may persist in a human host for several years - perhaps even a lifetime. ${ }^{240}$

- Plasmodium falciparum leads to severe infections, which may terminate fatally in non-immune persons if proper treatment is not promptly given. In the malaria-endemic regions of Africa, Plasmodium falciparum occurs more frequently than the other parasites. Plasmodium falciparum causes very high levels of parasitemia ( $>300.000$ parasites $/ \mu$ l blood). The parasite is confined to tropical and subtropical areas, because the parasites' development in the vector is greatly retarded at temperatures of $20^{\circ} \mathrm{C}$ and below; at $20^{\circ} \mathrm{C}$, the maturation of sporozoites takes around 3 weeks ${ }^{241}$ which is longer than the average lifespan of anopheline mosquitoes.

231 Edillo, F.E.; Tripét, F.; Touré, Y.T. et al. (2005), doi:10.1186/1475-2875-5-35.

232 BAYOH, M.N.; THOMAS, C.J. \& LindSAY, S.W. (2001), p. 268.

233 TAKKEN, W. \& KNOLS, B.G.J. (1999), p. 137.

234 TUteJA, R. (2007), p. 4671.

235 Ruan, S.; XiaO, D. \& Beier, J.C. (2008), p. 1098.

236 TUtEJA, R. (2007), p. 4671.

237 Gilles, H.M. $\left(1993^{1}\right)$, p. 20.

238 Tuteja, R. (2007), p. 4671.

239 Panjarathinam, R. (1990), p. 39.

240 Gilles, H.M. $\left(1993^{1}\right)$, pp. $24 f$.

241 Gilles, H.M. $\left(1993^{1}\right)$, pp. $25 f$. 
- Plasmodium knowlesi is a newly confirmed human species that is morphologically similar to Plasmodium malariae and has recently been demonstrated in patients in Malaysia, the Philippines, Thailand and Myanmar. ${ }^{242}$ The parasite has its main reservoir in macaques but routinely infects humans living in proximity to the monkeys. ${ }^{243}$

Important characteristics of human infections with these parasites are presented in table 9.

\begin{tabular}{|c|c|c|c|c|}
\hline Species & $\begin{array}{c}\text { Plasmodium } \\
\text { vivax }\end{array}$ & $\begin{array}{c}\text { Plasmodium } \\
\text { malariae }\end{array}$ & $\begin{array}{c}\text { Plasmodium } \\
\text { ovale }\end{array}$ & $\begin{array}{l}\text { Plasmodium } \\
\text { falciparum }\end{array}$ \\
\hline $\begin{array}{l}\text { Parasitemia } \\
\text { (parasites } / \mu \mathrm{l} \\
\text { blood) }\end{array}$ & $\approx 20.000$ & $\approx 6000$ & $\approx 9000$ & $\begin{array}{l}\approx 20.000 \\
\text { to } 500.000\end{array}$ \\
\hline Risk of recursion & very high & low & very high & low \\
\hline $\begin{array}{l}\text { Duration of an } \\
\text { untreated } \\
\text { infection }\end{array}$ & $1 \frac{1}{2}$ to 5 years & 3 to 50 years & $1 \frac{1}{2}$ to 5 years & 1 to 2 years \\
\hline
\end{tabular}

Table 9: Characteristics of different malarial infections ${ }^{244}$

Sympatric combinations of the four key main species affecting humans occur within human populations and within infected individuals. ${ }^{245}$ Mixed infections involving Plasmodium falciparum and Plasmodium malariae or Plasmodium falciparum and Plasmodium ovale are very common in the malaria-endemic parts of the tropics. ${ }^{246}$ In Madagascar and New Guinea, the co-occurrence of all four species has been observed. ${ }^{247}$

\subsubsection{Life Cycle of the Malaria Parasites}

The life cycle of the four malaria parasites affecting man is largely identical. It comprises

- an exogenous stage (sporogony), during which the parasite multiplies sexually in the mosquito's gut;

- an endogenous stage (schizogony), during which the parasite multiplies asexually in the human host. ${ }^{248}$

242 BREMAN, J.G. (2009), p. 6.

243 Doolan, D.L.; Dobaño, C. \& Baird, J.K. (2009), p. 14.

244 Gilles, H.M. $\left(1993^{1}\right)$, p. 27.

245 McKenzie, F.E. \& Bossert, W.H. (1997), p. 593.

246 Gilles, H.M. $\left(1993^{1}\right)$, p. 27.

247 McKenzie, F.E. \& Bossert, W.H. (1997), p. 596.

248 Gilles, H.M. $\left(1993^{1}\right)$, p. 13. 
Chapter 2 - Malaria in West Africa: Transmission, Monitoring \& Control

The hematophagous adult female Anopheles mosquito needs blood before it can produce eggs. Soon after oviposition, it requires another blood meal to produce a new batch of eggs. Only this constant need of blood meals makes the transmission of parasites possible. ${ }^{249}$

The infection of a human host begins when an infected mosquito injects a sporozoite during a blood meal. It takes between half an hour and four hours until the parasites infect the host's liver cells (hepatic stage). The parasites multiply in the liver cells and release up to 30.000 merozoites which now infect the red blood cells (erythrocytes). This asymptomatic period lasts for about one week in the tropics. ${ }^{250}$

The subsequent intracellular and asexual reproduction of the parasites (erythrocytic stage) results in the destruction of erythrocytes and the release of toxins. Bouts of fever, which recur every 48 hours in the case of Plasmodium falciparum, Plasmodium vivax and Plasmodium ovale and 72 hours in infections with Plasmodium malariae are related to these toxin releases. In the course of asexual reproduction and the invasion of fresh erythrocytes a sexual differentiation of some parasites into macrogametocytes (female) and microgametocytes (male) takes place. These forms of the parasite are infectious for mosquitoes. ${ }^{251}$

The sexual cycle of the parasite begins, when gametocytes are ingested by the vector during a blood meal from an infected host. Their fertilization occurs in the mosquito's midgut, where oocysts are formed on the stomach walls. Sporozoites develop inside the oocysts and migrate to the mosquito's salivary glands after the oocysts burst. Several aspects of this complex cycle of development, which is also called the extrinsic incubation period, are influenced by climatic conditions ${ }^{252}$ (see section 2.5.1).

\subsubsection{The Exogenous Stage}

When a mosquito takes a blood meal, there is a certain risk that it ingests the sexual or asexual forms of the malaria parasite. The asexual forms of the parasite are digested along with the red blood cells, while the sexual forms (gametocytes) may undergo further development. The abrupt environmental change caused by the blood meal triggers gametocytes to emerge from the erythrocytes within minutes. ${ }^{253}$ Parasites now encounter the insect's immune

249 Hay, S.I.; Omumbo, J.A.; Craig, M.H. \& Snow R.W. (2000), p. 178. 250 Hay, S.I.; Omumbo, J.A.; Craig, M.H. \& Snow R.W. (2000), p. 178. 251 HaY, S.I.; Omumbo, J.A.; Craig, M.H. \& Snow R.W. (2000), p. 178. 252 Hay, S.I.; Omumbo, J.A.; Craig, M.H. \& Snow R.W. (2000), p. 178; Overgaard, H. (2001), p. 8. 253 Alano, P. (2007), p. 291. 
system. ${ }^{254}$ Surviving macrogametocytes turn into macrogametes while the process of exflagellation releases eight mobile microgametes from each microgametocyte. 255 This transformation occurs in the mosquito's midgut quickly after a blood meal. ${ }^{256}$ Fertilized zygotes are formed after the fusion of male and female gametocytes). When the zygotes become mobile, they are called ookinetes; these ookinetes migrate towards the stomach wall where they turn into immobile oocysts. ${ }^{257}$ This transformation must occur before ookinetes are digested along with the blood meal. ${ }^{258}$ The oocysts gradually enlarge as their nucleus divides and sporozoites are formed. This process is temperature-dependent. The mobile sporozoites break through the oocyst's wall and migrate towards the mosquito's salivary glands, from where they can be transmitted to a human host during the next blood meal. It is estimated that one oocyst can release up to 1000 sporozoites. ${ }^{259}$ However, the process of sporozoite invasion of the salivary glands is rather ineffective with less than $25 \%$ of the sporozoites produced reaching their destination. ${ }^{260}$ The typical duration of sporogony in the tropics is presented in table 10.

\begin{tabular}{|l|l|c|c|c|}
\hline Species & $\begin{array}{c}\text { Plasmodium } \\
\text { vivax }\end{array}$ & $\begin{array}{c}\text { Plasmodium } \\
\text { malariae }\end{array}$ & $\begin{array}{c}\text { Plasmodium } \\
\text { ovale }\end{array}$ & $\begin{array}{c}\text { Plasmodium } \\
\text { falciparum }\end{array}$ \\
\hline $\begin{array}{l}\text { Duration of } \\
\text { sporogony }\end{array}$ & 8 to 10 days & 14 to 16 days & 12 to 14 days & $\begin{array}{l}9 \text { to } 10 \\
\text { days }\end{array}$ \\
\hline
\end{tabular}

Table 10: Duration of sporogony at a temperature of $28^{\circ} \mathrm{C}$

Figure 13 illustrates the stages involved in the exogenous cycle of the malaria parasite.

254 Dimopoulos, G. (2003), p. 3.

255 TUTEJA, R. (2007), p. 4673.

256 BEIER, J.C. (1998), p. 524.

257 Gilles, H.M. $\left(1993^{1}\right)$, pp. 14 f.

258 BEIER, J.C. (1998), p. 525.

259 Gilles, H.M. $\left(1993^{1}\right)$, p. 15.

260 BeIER, J.C. (1998), p. 526.

261 Gilles, H.M. $\left(1993^{1}\right.$ ), p. 17. 


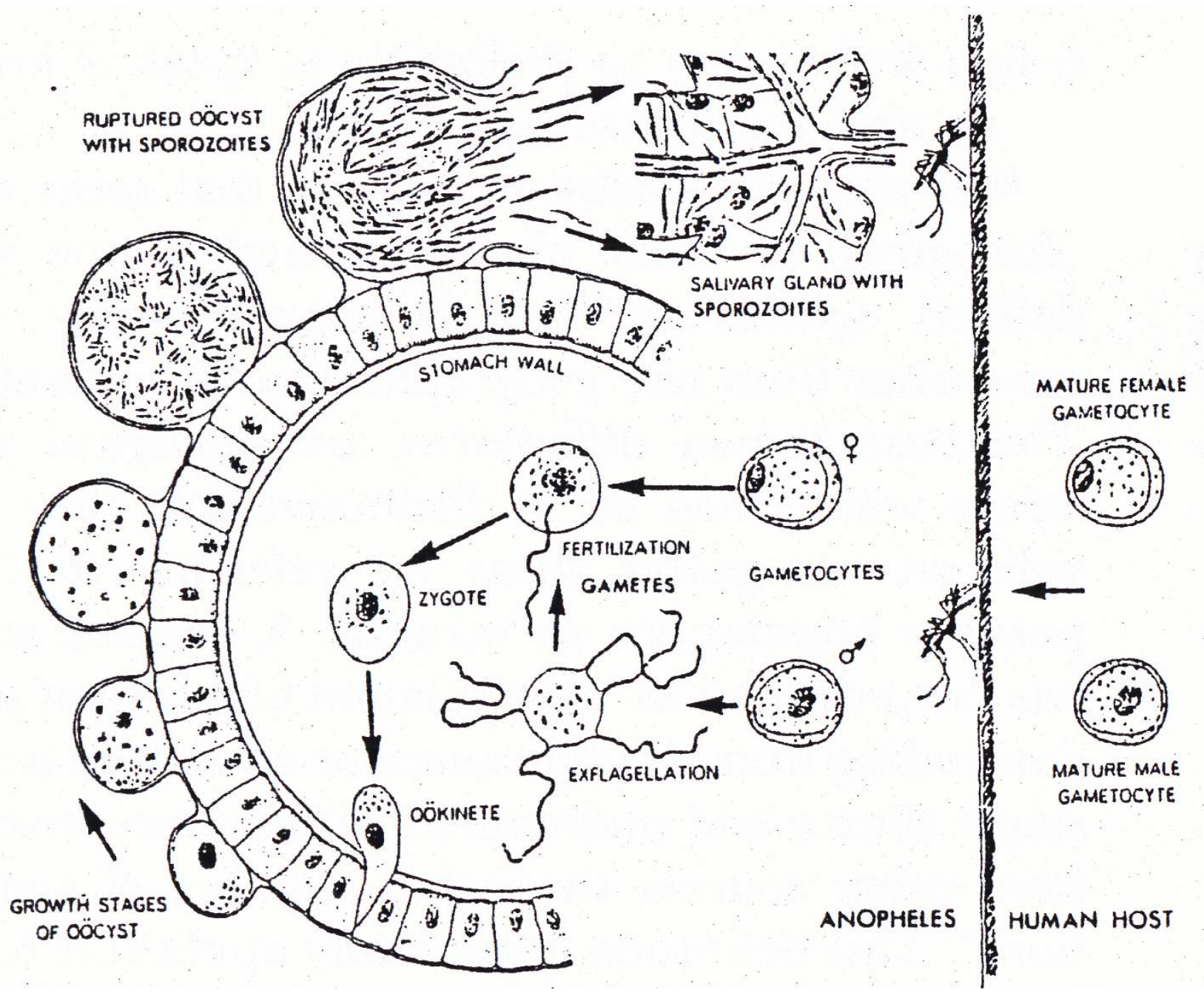

Figure 12: The exogenous cycle of the malaria parasite ${ }^{262}$

Once sporozoites have migrated to a mosquito's salivary glands, it remains infective for one or two months. ${ }^{263}$

The malaria parasite suffers large losses during its sporogonic development, and only a small fraction of the ingested gametocytes eventually reach the mosquito's salivary glands. ${ }^{264}$ In the course of the exogenous cycle, there are three main stages where parasite development may be disrupted. These are the developmental transitions between gametocyte and ookinete, between ookinete and mature oocyst and between oocysts and salivary gland sporozoites. The magnitude of the stage-specific parasite losses are an important determinant of a mosquito species' vector competence. ${ }^{265}$

262 Gilles, H.M. $\left(1993^{1}\right)$, p. 14.

263 TUTEJA, R. (2007), p. 4673.

264 Dimopoulos, G. (2003), p. 8.

265 BeIER, J.C. (1998), pp. $523 f$. 


\subsubsection{The Endogenous Stage}

The endogenous stage comprises the parasite's development cycle in the parenchyma cells of the liver (exo-erythrocytic schizogony) and its development in the red blood cells (erythrocytic schizogony). ${ }^{266}$

Infective sporozoites from the salivary gland of the Anopheles mosquito are injected into a human host along with anticoagulant-containing saliva and begin to circulate in the bloodstream. ${ }^{267}$ They remain at the site of the bite for at least five but not more than 15 minutes after blood-feeding. ${ }^{268}$ However, some sporozoites may be injected into the skin and move on to regional lymph nodes within 6 hours. ${ }^{269}$ After the injection of sporozoites into the human blood in the course of a mosquito bite, the blood remains infected for roughly half an hour. Most of the sporozoites are destroyed by the human immune system; the remaining sporozoites infect the liver. Now the process of pre-erythrocytic schizogony begins: In the cases of Plasmodium falciparum and Plasmodium malariae schizonts are directly formed; in the case of Plasmodium vivax and Plasmodium ovale some sporozoites develop into hypnozoites which may remain dormant in the liver for several years. ${ }^{270}$ The mechanism of targeting and invading the liver cells is not yet well understood. ${ }^{271}$ At the end of the preerythrocytic stage, about 6 to 16 days after the original infection, several thousand merozoites are released. Some of them are destroyed by phagocytes while others invade the red blood cells. ${ }^{272}$ This invasion marks the beginning of the erythrocytic stage. The interval between the date of the infection and the time when malaria parasites are detectable in the peripheral blood is known as the pre-patent period. ${ }^{273}$ Table 11 presents the duration of the endogenous parasite cycles of human malaria parasites.

266 Gilles, H.M. $\left(1993^{1}\right)$, p. 13.

267 TUTEJA, R. (2007), p. 4671.

268 BeIER, J.C. (1998), p. 533.

269 Doolan, D.L.; Dobaño, C. \& Baird, J.K. (2009), p. 14.

270 TUTEJA, R. (2007), p. 4671.

271 TUTEJA, R. (2007), p. 4671.

272 Gilles, H.M. $\left(1993^{1}\right)$, p. 16.

273 Gilles, H.M. $\left(1993^{1}\right)$, p. 18. 
Chapter 2 - Malaria in West Africa: Transmission, Monitoring \& Control

\begin{tabular}{|l|l|l|l|l|l|}
\hline Species & $\begin{array}{c}\text { Plasmodium } \\
\text { vivax }\end{array}$ & $\begin{array}{c}\text { Plasmodium } \\
\text { malariae }\end{array}$ & $\begin{array}{c}\text { Plasmodium } \\
\text { ovale }\end{array}$ & $\begin{array}{l}\text { Plasmodium } \\
\text { falciparum }\end{array}$ \\
\hline $\begin{array}{l}\text { Duration of pre- } \\
\text { erythrocytic } \\
\text { schizogony }\end{array}$ & 6 to 8 days & 14 to 16 days & 9 days & $\begin{array}{l}51 / 2 \text { to } 7 \\
\text { days }\end{array}$ \\
\hline Pre-patent period & 8 to 27 days & 15 to 30 days & 9 to 17 days & $\begin{array}{l}8 \text { to } 25 \\
\text { days }\end{array}$ \\
\hline Erythrocytic cycle & $48 \mathrm{~h}$ & $72 \mathrm{~h}$ & $50 \mathrm{~h}$ & $48 \mathrm{~h}^{276}$ \\
\hline
\end{tabular}

Table 11: Duration of pre-erythrocytic schizogony, pre-patent period and erythrocytic cycle (depending on parasite)

In immune persons antibodies prevent the invasion of erythrocytes by merozoites ${ }^{277}$; in non-immunes, asexual parasite division starts inside the erythrocyte. The early stages of parasites which have invaded the red blood cells are called trophozoites. The trophozoites absorb the erythrocytes' hemoglobin and grow until asexual reproduction, i.e. erythrocytic schizogony, begins. The end of this stage is marked by the formation of schizonts, each of which contain around 20 merozoites. ${ }^{278}$ Infected red blood cells burst and release merozoites which now invade fresh erythrocytes. This process continues and leads to increasing levels of parasitemia until it is stopped by the host's immune response. ${ }^{279}$ The rupture of red blood cells and release of merozoites coincides with a rise in body temperature. ${ }^{280}$

The process of erythrocytic schizogony repeats, taking around 48 hours except for Plasmodium malariae, where it takes around 72 hours. The cycle occurs quite synchronously and merozoites are released at approximately the same time of day. ${ }^{281}$

While merozoites originating from pre-erythrocytic schizogony may also give rise to sexually differentiated gametocytes, it is usually only after several erythrocytic cycles that these forms are produced in greater numbers. The gametocytes invade red blood cells where they mature. Such infected red blood cells may be ingested during a mosquito's blood meal. ${ }^{282}$ The onset of sexual differentiation instead of continued erythrocytic schizogony is a real

274 Gilles, H.M. $\left(1993^{1}\right)$, p. 17.

275 TUtEJA, R. (2007), p. 4673.

276 Gilles, H.M. $\left(1993^{1}\right)$, p. $17 ; 27$.

277 Gilles, H.M. $\left(1993^{1}\right)$, p. 18.

278 TutejA, R. (2007), p. 4672.

279 Gilles, H.M. $\left(1993^{1}\right)$, p. 19.

280 TUTEJA, R. (2007), p. 4672.

281 TUTEJA, R. (2007), p. 4673.

282 Gilles, H.M. $\left(1993^{1}\right)$, p. 19. 
branch point in the otherwise rigidly deterministic Plasmodium life cycle. Gametocyte production generally increases in response to conditions negatively affecting asexual multiplication, including drug treatment. ${ }^{283}$ Figure 14 illustrates the stages of the endogenous cycle of human Plasmodia.

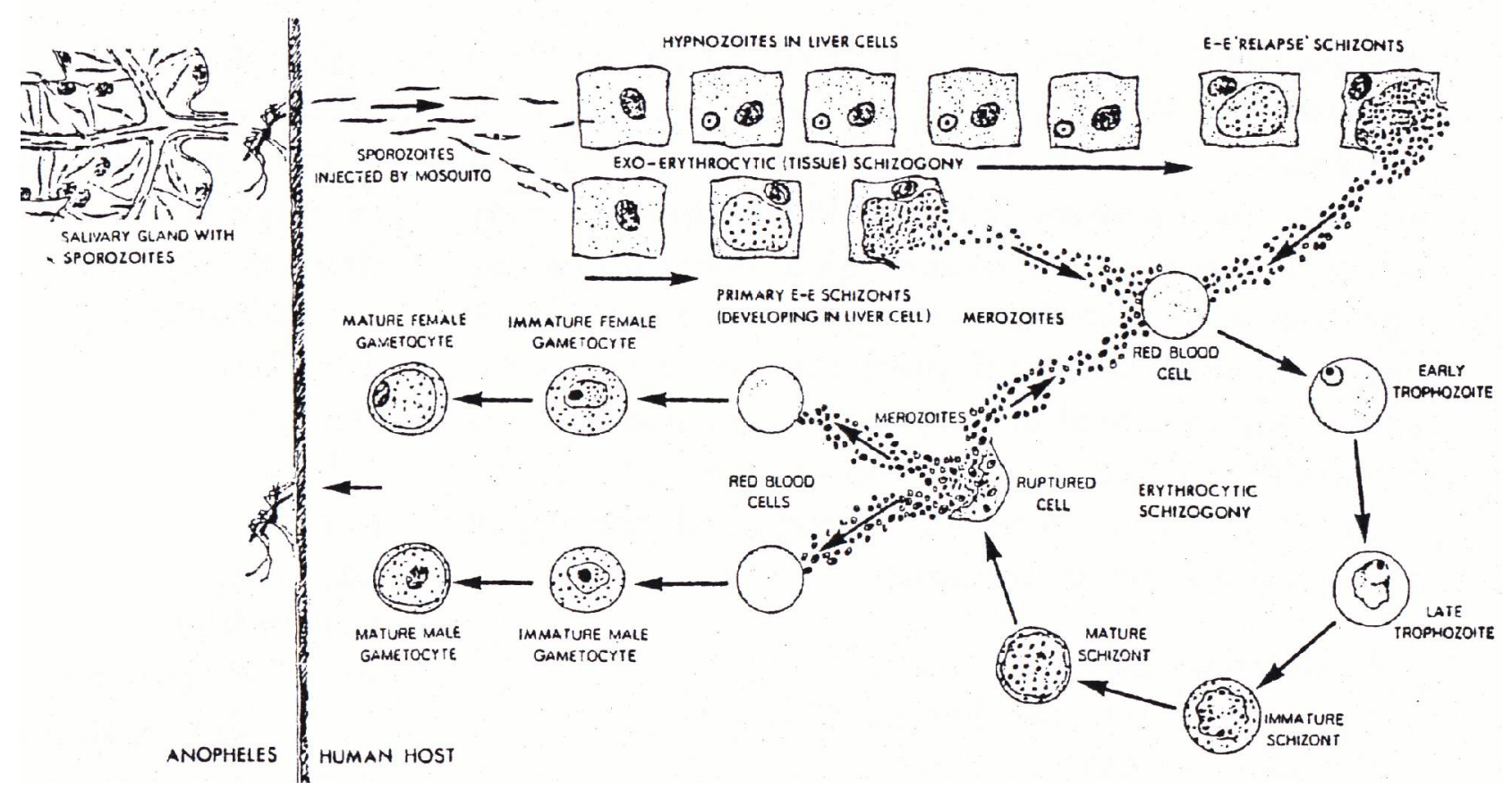

Figure 13: Endogenous cycle of the parasite 284

The entire process is independent of temperature and the first gametocytes are typically produced about two weeks after the initial bite. ${ }^{285}$

\subsubsection{Immunity Against Malaria}

The malaria parasite has to face a succession of challenges within the host; it has to attach to, enter and thrive in hepatocytes and erythrocytes and then leave the host to carry on the next part of its cycle in the mosquito. ${ }^{286}$ The symptomatic stage of a malaria infection begins when parasite densities of about $50 / \mu \mathrm{l}$ are reached in the blood. ${ }^{287}$ Then immune system begins to control this acute infection, leading to a chronic infection with intermittent febrile

283 Alano, P. (2007), p. 292.

284 Gilles, H.M. $\left(1993^{1}\right)$, p. 14.

285 Hoshen, M.B. \& MoRSE, A.P. (2004), doi:10.1186/1475-2875-3-32.

$286 \mathrm{M}_{\mathrm{ARSH}}, \mathrm{K} .(1993)$, p. 60.

287 BREMAN, J.G. (2009), p. 4. 
episodes marking the peaks of higher parasitemia. These peaks are progressively lower until after many months, the infection may be eliminated. ${ }^{288}$

Immunity against malaria can be either innate or acquired. However, this differentiation is not clear-cut as innate immunity may play a role in the development of acquired immunity. ${ }^{289}$

\subsubsection{Innate Resistance to Malaria}

Innate resistance to malaria is often linked with abnormalities of red or white blood cells even though the exact mechanisms are not yet understood. In many forms of innate resistance against malaria, it is unclear whether this resistance is an adaptation of populations to malaria or not. ${ }^{290}$

Modifications of red blood cells which appear to provide resistance against malaria include the following:

- Hemoglobin S: In some parts of Africa more than $20 \%$ of the population have a particular amino acid substitution in their hemoglobin molecules (hemoglobin S). This trait, which leads to sickle cell anemia, seems to give a strong protection against the clinical effects of Plasmodium falciparum malaria since schizonts appear to have problems utilizing this abnormal hemoglobin. Therefore, the process of schizogony is normally not completed in patients with sickle cell anemia. ${ }^{291}$ Because of the selectional advantage offered, the percentage of the population with the hemoglobin $S$ trait has increased over thousands of generations. ${ }^{292}$

- Hemoglobin C: In localized parts of West Africa, around Burkina Faso and Ghana, hemoglobin $C$ is present at high frequencies. Red blood cells may inhibit parasite multiplication due to their resistance to bursting and releasing merozoites. ${ }^{293}$

- Thalassemia hemoglobin and hemoglobin E provide some protection against Plasmodium vivax malaria. ${ }^{294}$

- As the parasites have to gain entry into red blood cells to survive, modifications of the structure and function of the red blood cell membrane may affect the parasite's ability to invade red blood cells.

288 Langhorne, J.; Noungu, F.M.; Sponaas, A.-M. \& Marsh, K. (2008), p. 725.

289 MARSH, K. (1993), p. 60.

290 MARSH, K. (1993), pp. $60 \& 62$.

291 Panjarathinam, R. (1990), p. 35.

292 DiesfelD, H.J. (1995), p. 47.

293 MARSH, K. (1993), p. 62.

294 Panjarathinam, R. (1990), p. 35. 
Human leucocyte antigens (HLAs) also appear to play a role in innate resistance against malaria. Currently, evidence is emerging for associations between a few HLA antigens common in African populations and protection from severe disease. ${ }^{295}$

\subsubsection{Acquired Immunity}

Acquired resistance against malaria may be due to nutrition and previous experiences of malaria. Whereas some dietary components appear to increase the risk of severe malaria (particularly iron), it appears that a diet which is low in riboflavin or para-amino benzoic acid tends to limit the risk of severe infections. African children suffering from marasmus (a general loss of strength and emaciation caused by energy and protein deficiency) or kwashiorkor (a state of protein deficiency in populations subsisting mostly on cereals) rarely suffer from severe malaria. ${ }^{296}$ Acquired immunity can have different forms, ranging from anti-disease immunity (the prevention of clinical symptoms) to antiparasitic immunity (protection against parasitemia). ${ }^{297}$

In stable endemic areas, a heavy toll of morbidity and mortality falls on young children but malaria tends to be rather mild in adults. This is due to the acquisition of specific immunity. Children remain relatively protected for a period of between three and six months following birth. If transmission is heavy, the child may become parasitemic during this time, but rarely manifests any severe features of malaria. It appears that a substantial degree of protection can be transferred from mother to child. ${ }^{298}$

Following the period of relative protection, children become increasingly susceptible to the more severe clinical manifestations of malaria. ${ }^{299}$ The level of immunity then rises again, until at the age of 5, most children have achieved immunity. However, this immunity initially appears to be antitoxic rather than antiparasitic: children may not show a severe reaction towards toxic parasite products despite high levels of parasitemia. Nevertheless, they may soon after asymptomatically (!) die of cerebral malaria as too many red blood cells were destroyed by the high parasite load. ${ }^{300}$

295 MARSH, K. (1993), p. 64.

$296 \mathrm{M}_{\text {ARSH, K. }}$ (1993), p. 64.

297 Doolan, D.L.; Dobaño, C. \& Baird, J.K. (2009), p. 14.

$298 \mathrm{M}_{\mathrm{ARSH}}, \mathrm{K} .(1993)$, pp. 64f.

299 Doolan, D.L.; Dobaño, C. \& Baird, J.K. (2009), p. 14.

300 MARSH, K. (1993), pp. 64f. 
«Recent field studies have provided direct evidence that the frequency of parasite exposure from birth determines the speed with which effective clinical immunity is acquired among the host population, the clinical spectrum of life-threatening disease in a community and the extent of active immunization early in life during an innate period of clinical protection.»301

In malaria-endemic regions, repeated exposures to malaria first lead to the induction of immunity against severe disease, then to mild disease (i.e. febrile episodes) and finally to asymptomatic infection, even though complete immunity (i.e. absolute sterility) is probably never achieved ${ }^{302}$. Moreover, malaria immunity may not be stable and it is often reported that immune subjects who spend time outside an endemic area are prone to malaria on reexposure:

\begin{abstract}
«The $[\ldots]$ most striking conclusion is that immunity to malaria seems to need a long period of exposure to the parasite for induction, and probably continuing exposure for maintenance. The most plausible hypothesis is that immunity to malaria is, at least during the early years, largely strain-specific.» ${ }^{303}$
\end{abstract}

The strain-specific theory suggests that malaria may exist as a composite of several independently transmitted antigenic types. Although immunity to a particular strain could be life-long, development of immunity to malaria is not complete without experiencing all constituent strains. ${ }^{304}$ Both strain-specific and cross-reactive (i.e. strain-transcending) immunity have been observed in the laboratory and field. ${ }^{305}$

It appears that malaria-immunity is stage-specific and that the following forms of immunity can be distinguished:

- Pre-erythrocytic immunity: Humans in endemic areas produce antibodies against sporozoites. Levels of immunity are low in childhood and rise with age.

- Immunity to erythrocytic stages: Immune reactions towards the erythrocytic stages of Plasmodia are not yet well-understood.

301 Oмumbo, J.A.; Ouma, J.; Repuoda, B. et al. (1998), p. 8.

302 Langhorne, J.; Ndungu, F.M.; Sponaas, A.-M. \& Marsh, K. (2008), p. 725.

$303 \mathrm{M}_{\mathrm{ARSH}}, \mathrm{K}$. (1993), p. 65.

304 Gu, W.; Killeen, G.F.; Mbogo, C.M. et al. (2003), p. 45.

305 Doolan, D.L.; Dobaño, C. \& Baird, J.K. (2009), p. 24. 
- Immunity to sexual stages: Antibodies against gametocytes may continue to be effective after blood has been ingested by mosquitoes. However, in the case of Plasmodium falciparum, antigametocyte responses appear to be more frequent in non-immune persons than in individuals living under constant transmission. ${ }^{306}$

The development of various forms of antimalarial immunity in children and their protection against malaria-associated mortality are illustrated in figure 14.

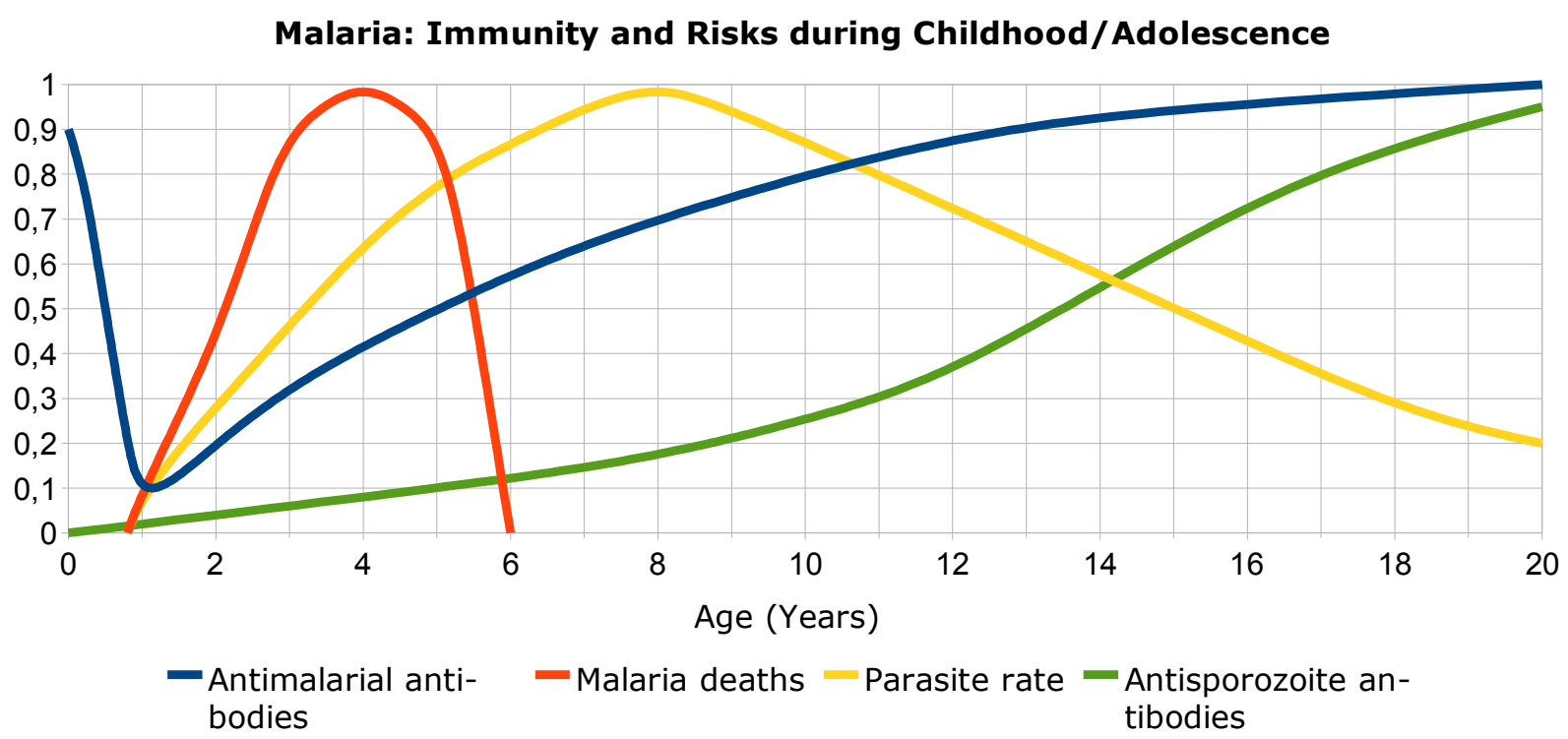

Figure 14: Development of antimalarial immunity during childhood ${ }^{307}$

To date, it is not really known what to measure as a correlate for immunity against malaria, and what mechanisms regulate the immunological responses in semi-immune people. Such knowledge would, however, be an important step towards a malaria vaccine. ${ }^{308}$

$306 \mathrm{M}_{\mathrm{ARSH}}$ K. (1993), pp. 69-71.

307 Adapted from MARSH, K. (1993), p. 72.

308 Langhorne, J.; Noungu, F.M.; Sponaas, A.-M. \& Marsh, K. (2008), p. 730. 


\title{
2.4.2.3 Loss of Immunity
}

Different factors may interfere with acquired immunity against malaria, including pregnancies, exposure to a different strain of malaria and the prevention of exposure through malaria control measures.

Even if complete immunity is lost, immunity to life-threatening disease persists. Nevertheless, fully immune individuals may be susceptible to malaria when moving into another malarious area, but the course of the infection will then usually be limited to fever and minor symptoms. ${ }^{309}$

Pregnancy is often involved with a certain loss of immunity:

\begin{abstract}
«This is manifest as an increased prevalence and increased density of parasitemia which may lead to severe anemia. At delivery, the placenta is often parasitized to a degree far beyond that expected from the peripheral parasitemia and one of the most important health effects is the associated reduction in birthweight. ${ }^{310}$
\end{abstract}

Many malariologists believe that in areas with holoendemic (stable) malaria, man should not interfere with the established exposure to malaria since that would increase the quantity and severity of clinical infections. Even the treatment of young children who experience their first attack of malaria is sometimes disadvised to allow the development of immunity. ${ }^{311}$

\subsection{Determinants of Malaria Transmission}

Any attempt to map, model or predict the malaria risk for a defined area and period requires an exact knowledge of the factors which determine the disease's transmission dynamics. The life cycle of the malaria vectors and parasites, the vectors' behavior and vector-to-host contact are influenced by an often closely interwoven set of environmental parameters. In addition, malaria models and prediction systems also have to take into account characteristics of the host population such as parasitation, immunological status and population density and distribution: 
«It is the combined effect of varying rainfall, temperature and humidity as well as vector-host-contact over the annual cycle that will determine malaria transmission risk. [...] Furthermore, behavioral traits and genetic variation in the human population may be important in determining both malaria exposure and outcome in individuals, and may account for variation in disease patterns among different communities. $\gg^{312}$

\subsubsection{Malaria and the Environment}

Several environmental parameters have a more or less direct impact on the quality of vector habitats and some of them even on the parasite development. Whereas climatic seasonality and variation is often seen as one key driver of transmission dynamics, terrain characteristics such as relief, soils, hydrography and land cover also play an important role for the spatio-temporal pattern of vector population dynamics and thus potential malaria risks.

Both the development of mosquito larvae and the survival and behavior of adult mosquitoes depend on various environmental factors, but the demands of the two stages are different. ${ }^{313}$ Despite a general agreement that such factors affect malaria transmission, conflicting opinions persist about their relative importance. ${ }^{314}$ This may partly be due to differences between controlled labbased studies and investigations under field conditions with considerable variations between different localities.

Whereas some environmental factors, e.g. climate, are important predictors of malaria transmission pattern at continental level, a great number of other parameters becomes important within smaller areas. ${ }^{315}$ One important difficulty involved in such studies is the indirect link between environmental determinants and malaria transmission: Environmental conditions may be favorable for mosquito reproduction and survival, but for actual transmission several other conditions have to be met, including a parasite reservoir in the human population and sufficient vector-to-host contact.

\subsubsection{Malaria and Temperature}

Malaria transmission takes place at temperatures between $18^{\circ} \mathrm{C}$ and $32^{\circ} \mathrm{C}$ to $33^{\circ} \mathrm{C}$ in the case of Plasmodium falciparum and between $16^{\circ} \mathrm{C}$ and $32^{\circ} \mathrm{C}$ in case of Plasmodium vivax, Plasmodium malariae and Plasmodium ovale. ${ }^{316}$

312 Thomson, M.C.; Connor, S.J.; Milligan, P. \& Flasse, S.P. (1997), p. 314.

313 HoSHEN, M.B. \& MoRse, A.P. (2004), doi:10.1186/1475-2875-3-32.

314 Yé, Y.; Louis, V.R.; Simboro, S. \& Sauerborn, R. (2007), doi:10.1186/1471-2458-7-101.

315 Martin, C.; Curtis, B.; Fraser, C. \& Sharp, B. (2002), p. 227.

316 Hay, S.I.; Omumbo, J.A.; Craig, M.H. \& Snow R.W. (2000), p. 195; Martens, P. (1998), p. 50. 
When a mosquito becomes infected with malaria parasites (gametocytes), it cannot immediately pass on the disease. Only after the sporogonic cycle is completed, the mosquito becomes infective (infectious) and carries sporozoites in its salivary glands, which may be injected into the bloodstream of a human host during a blood meal.

The time needed for the completion of the sporogonic cycle is known as the extrinsic incubation period. The duration of this period is temperaturedependent and optimum conditions are found between $25^{\circ} \mathrm{C}$ and $30^{\circ} \mathrm{C}$. Parasite development is retarded or suppressed at temperatures below $16^{\circ} \mathrm{C}$ and above $40^{\circ} \mathrm{C}$, which often means that the sporogonic cycle is not completed during the mosquito's lifetime. ${ }^{317}$ The effect of temperature on the duration of the sporogonic cycle can be described as a more or less linear negative correlation at temperatures between $20^{\circ} \mathrm{C}$ and $30^{\circ} \mathrm{C}$ : increasing temperatures are connected with proportionally shorter durations of the cycle. At lower temperatures, the extrinsic incubation period lasts considerably longer, while higher temperatures seem to be connected to a further shortening to less than 5 days at $40^{\circ} \mathrm{C}$ (see figure 16 )..$^{318}$

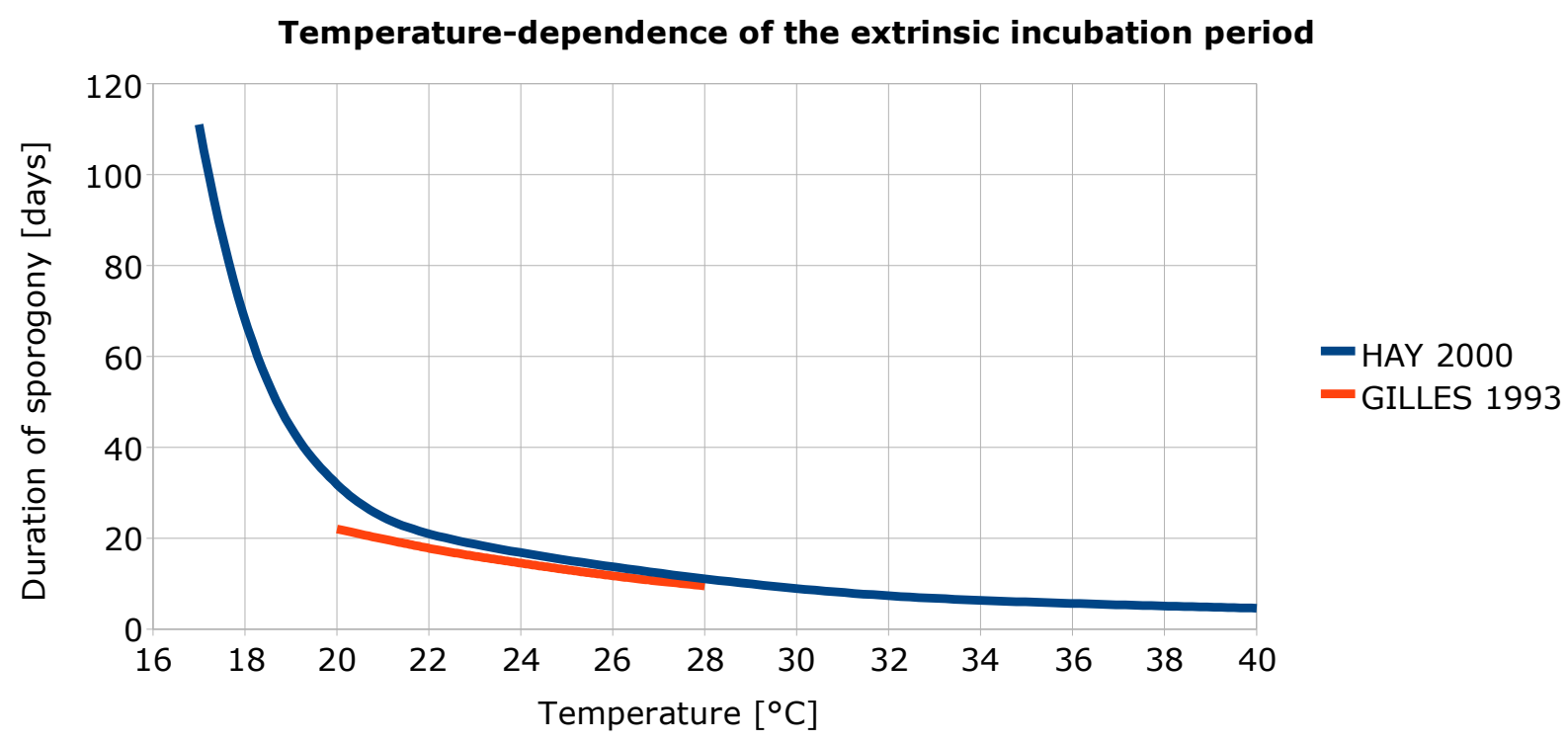

Figure 15: Duration of the extrinsic incubation period and its relation to temperature ${ }^{319}$

317 Hay, S.I.; Omumbo, J.A.; Craig, M.H. \& Snow R.W. (2000), p. 179.

318 Craig, M.H., Snow, R.W. \& le Sueur, D. (1999), p. 106.

319 Adapted from Hay, S.I.; Oмumbo, J.A.; Craig, M.H. \& Snow R.W. (2000), p. 180 and Gilles, H.M. $\left(1993^{1}\right)$, p. 27. 
At a temperature of $28^{\circ} \mathrm{C}$ the sporogonic cycle is completed within less than 12 days, a period that is survived by about $5 \%$ of the initial mosquito population which may now pass on the infection. At $19^{\circ} \mathrm{C}$ and less, the sporogonic cycle lasts for months and the survival probability of mosquitoes becomes extremely small. 320

Since warmer temperatures mean shorter extrinsic incubation periods, mosquitoes who ingested parasites during a blood meal become infective more rapidly the warmer it becomes. However, adult mosquito longevity is also temperature-dependent, and the effect of rising temperatures is very different here: In the range of $18^{\circ} \mathrm{C}$ to $26^{\circ} \mathrm{C}$, an increase in temperature of only $1 \mathrm{~K}$ can extend mosquito lifespan by more than a week. ${ }^{321}$ At temperatures of more than $30^{\circ} \mathrm{C}$, the likelihood of mosquitoes surviving the process of sporogony decreases. In a laboratory based test, only $50 \%$ of the Anopheles gambiae mosquitoes survived a temperature of $40^{\circ} \mathrm{C}$ for 67 minutes..$^{322}$ In contrast to the timing of sporogony, little is known the impact of temperature at stagespecific parasite densities and development in the vector. ${ }^{323}$

An increase in feeding frequencies at warmer temperatures typically results increased proportions of infective mosquitoes. ${ }^{324}$ Moreover, temperatures do not only have an influence on adult mosquitoes, but also on the development of eggs and the larval and pupal stages, a factor that is closely related to the malaria risk encountered in a certain area a short while later:

\footnotetext{
«The longevity of the aquatic stages of mosquitoes (Diptera: Culicidae) dictates the rate of production of adults and hence the intensity of disease transmission. ${ }^{325}$
}

Even though it is well known that insect embryogenesis and egg hatching are influenced by temperature, relatively little is known about this link in case of anophelines. HuANG et al. (2006) found prolonged temperatures beyond $40^{\circ} \mathrm{C}$ to be harmful to anopheline eggs, with about $45^{\circ} \mathrm{C}$ being the limit of tolerance even for brief periods (see figure 17). They concluded that anopheline eggs are thermally adapted to residing on water or moist mud where evaporation has a local cooling effect but that sun-exposed dry soil readily reaches lethal temperatures at which protein degeneration and desiccation takes place. ${ }^{326}$

320 Hoshen, M.B. \& Morse, A.P. (2004), doi:10.1186/1475-2875-3-32.

321 Depinay, J.M.O.; Mbogo, C.M.; Killeen, G. et al. (2004), doi:10.1186/1475-2875-3-29.

322 LINDSAY, S.W. \& \& KIRBY, M.J. (2004), p. 441.

323 BEIER, J.C. (1998), p. 532.

324 Teklehaimanot, H.D.; Lipsitch, M.; Teklehaimanot, A. \& Schwartz, J. (2004), doi:10.1186/1475-2875-3-41.

325 BAYOH, M.N. \& LINDSAY, S.W. (2004), p. 174.

326 Huang, J.; Walker, E.D.; Vulule, J. \& Miller, J.R. (2006), doi:10.1186/1475-2875-5-87. 


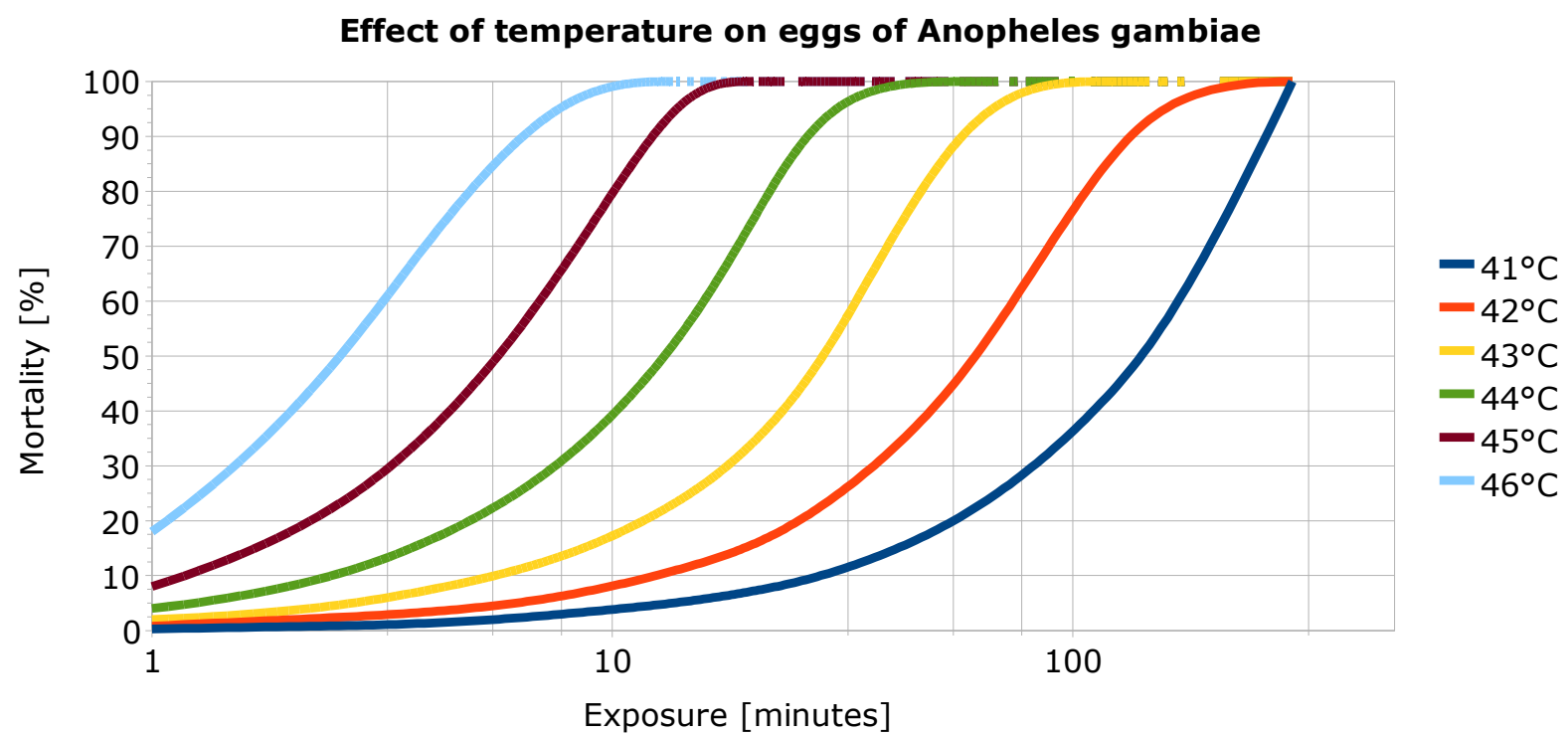

Figure 16: Effect of temperature on eggs of Anopheles gambiae $e^{327}$

The influence of temperature on the aquatic stages of mosquitoes has been studied for some species, including the most important vector mosquito in Africa (see table 12). Findings of these studies demonstrate that the immature stages of most species are extremely sensitive to temperatures above $40^{\circ} \mathrm{C} .{ }^{328}$ Temperatures exceeding $40^{\circ} \mathrm{C}$ are often only experienced for limited periods, so it is important for mosquito larvae to be able to survive brief exposure to extremely high temperatures. Alternatively, high temperatures may be avoided by mosquito larvae moving into shade or diving to the bottom of the water column. ${ }^{329}$

\begin{tabular}{|l|c|c|c|c|c|c|}
\hline \multirow{2}{*}{ Stage } & Eggs & \multicolumn{4}{|c|}{ Larval stages } & Pupae \\
\cline { 3 - 6 } & & L1 & L2 & L3 & L4 & \\
\hline $\begin{array}{l}\text { Maximum } \\
\text { temperature }\end{array}$ & $40^{\circ} \mathrm{C}$ & $40^{\circ} \mathrm{C}$ & $38^{\circ} \mathrm{C}$ & $36^{\circ} \mathrm{C}$ & $34^{\circ} \mathrm{C}$ & $34^{\circ} \mathrm{C}$ \\
\hline
\end{tabular}

Table 12: Impact of temperature on immature stages of Anopheles gambiae ${ }^{330}$

Larval mortality increases significantly at temperatures above $30^{\circ} \mathrm{C}$, with death $(>70 \%)$ rather than metamorphosis into adult mosquitoes being the rule. ${ }^{331}$ DePinAY et al. (2004) observed that temperatures exceeding the thermal death point of anophelines by $1 \mathrm{~K}, 2 \mathrm{~K}$ or $3 \mathrm{~K}$ resulted in daily mortalities of $10 \%, 50 \%$

327 Adapted from Huang, J.; Walker, E.D.; Vulule, J. \& Miller, J.R. (2006), doi:10.1186/1475-2875-5-87.

328 BAYOH, M.N. \& LINDSAY, S.W. (2004), p. 174.

329 BAYOH, M.N. \& LINDSAY, S.W. (2004), p. 175.

330 Huang, J.; Walker, E.D.; Vulule, J. \& Miller, J.R. (2006), doi:10.1186/1475-2875-5-87.

331 BAYOH, M.N. \& LINDSAY, S.W. (2004), p. 174. 
and $100 \%$ respectively. ${ }^{332}$ However, there is a severe shortage of empirical data on the effects of water temperature on larval survival. ${ }^{333}$ Moreover, virtually all available data is based on laboratory-based studies and may not be representative for field conditions.

Larvae of Anopheles gambiae develop into adult mosquitoes at temperatures between $16^{\circ} \mathrm{C}$ to $18^{\circ} \mathrm{C}$ and $34^{\circ} \mathrm{C} . .^{334}$ At temperatures below $12^{\circ} \mathrm{C}$ and above $38^{\circ} \mathrm{C}$, larval survival is less than 7 days; the metamorphosis into adult mosquitoes is not completed. Between 14 and $20^{\circ} \mathrm{C}$, the larval stage lasts for more than 30 days $^{335}$ which results in a high risk of predation. ${ }^{336}$ The speed of adult development is greatest at temperatures between $28^{\circ} \mathrm{C}$ and $32^{\circ} \mathrm{C}$, whereas adult emergence is highest between 22 and $26^{\circ} \mathrm{C}$. ${ }^{337}$ This demonstrates that the optimum temperatures for survival are lower than the temperatures at which mosquito development is quickest (see figure 18).

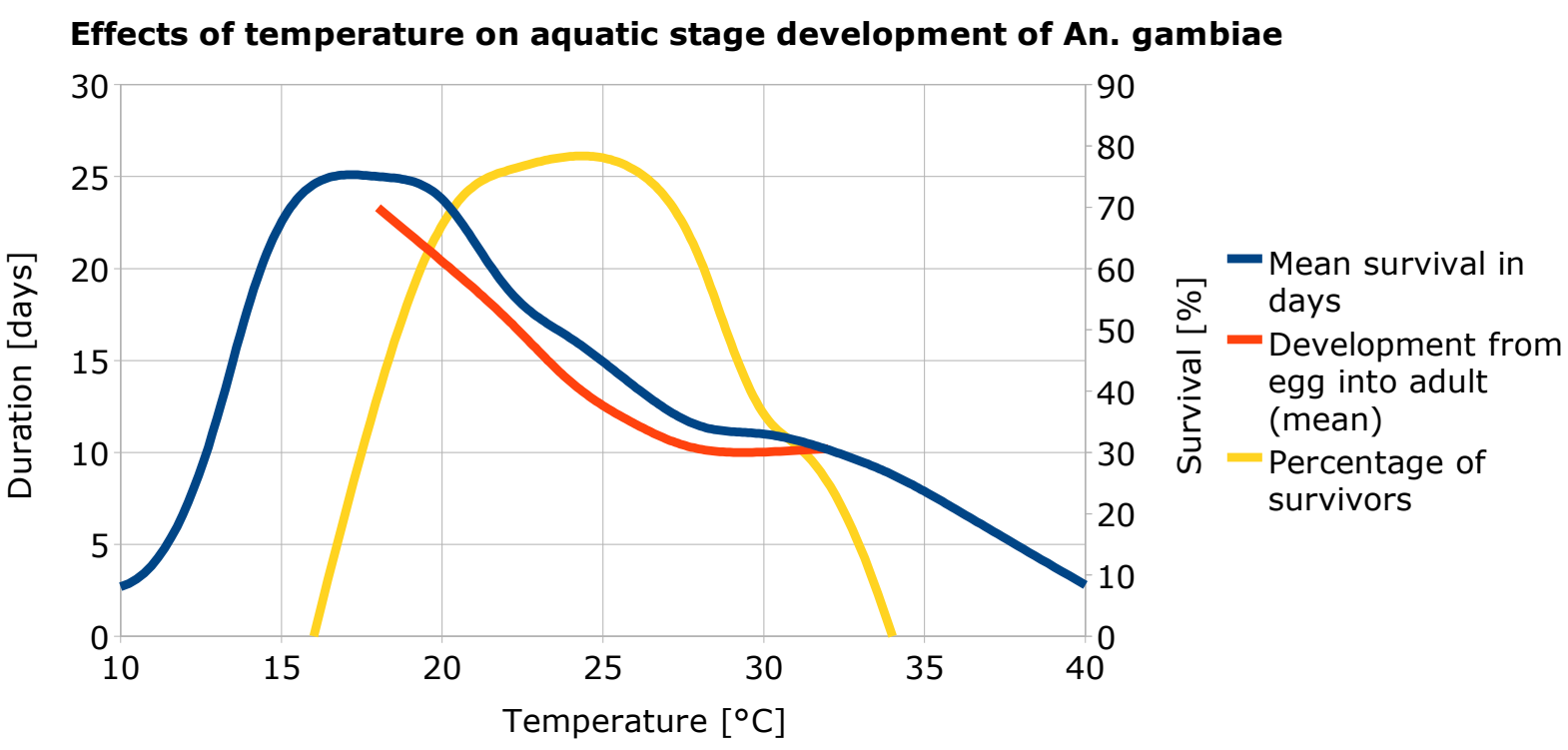

Figure 17: Effect of temperature on the aquatic stage development of Anopheles gambiae ${ }^{338}$

332 Depinay, J.M.O.; Mbogo, C.M.; Killeen, G. et al. (2004), doi:10.1186/1475-2875-3-29.

333 Hoshen, M.B. \& Morse, A.P. (2004), doi:10.1186/1475-2875-3-32.

334 BAYOH, M.N. \& LindSAY, S.W.(2004), p. 174; BAYOH, M.N. \& LiNDSAY, S.W. (2003), p. 377.

335 BAYOH, M.N. \& LiNDSAY, S.W. (2004), p. 174.

336 ZWÖLFER, H. (2002), p. 706.

337 BAYOH, M.N. \& LINDSAY, S.W. (2003), p. 378.

338 Adapted from BAYOH, M.N. \& LiNDSAY, S.W.(2004), p. 174-179 and

BAYOH, M.N. \& LiNDSAY, S.W. (2003), p. 377. 
Data on water temperature under natural conditions are often unavailable. This is particularly problematic since the temperature in shallow pools is sometimes significantly higher than the ambient temperature. ${ }^{339}$ In a study on mosquito larvae in market-garden wells in Senegal, RoBert et al. (1998) found temperatures of $28^{\circ} \mathrm{C}$ to $30^{\circ} \mathrm{C}$ to be ideal for Anopheles arabiensis. ${ }^{340}$

Even though higher temperatures mean faster parasite and vector development, there is a certain threshold above which a reduced lifespan of adult mosquitoes or high larval death rate becomes counterproductive to malaria transmission. Figure 19 illustrates the combined impact of temperature on several parameters related to malaria epidemiology.

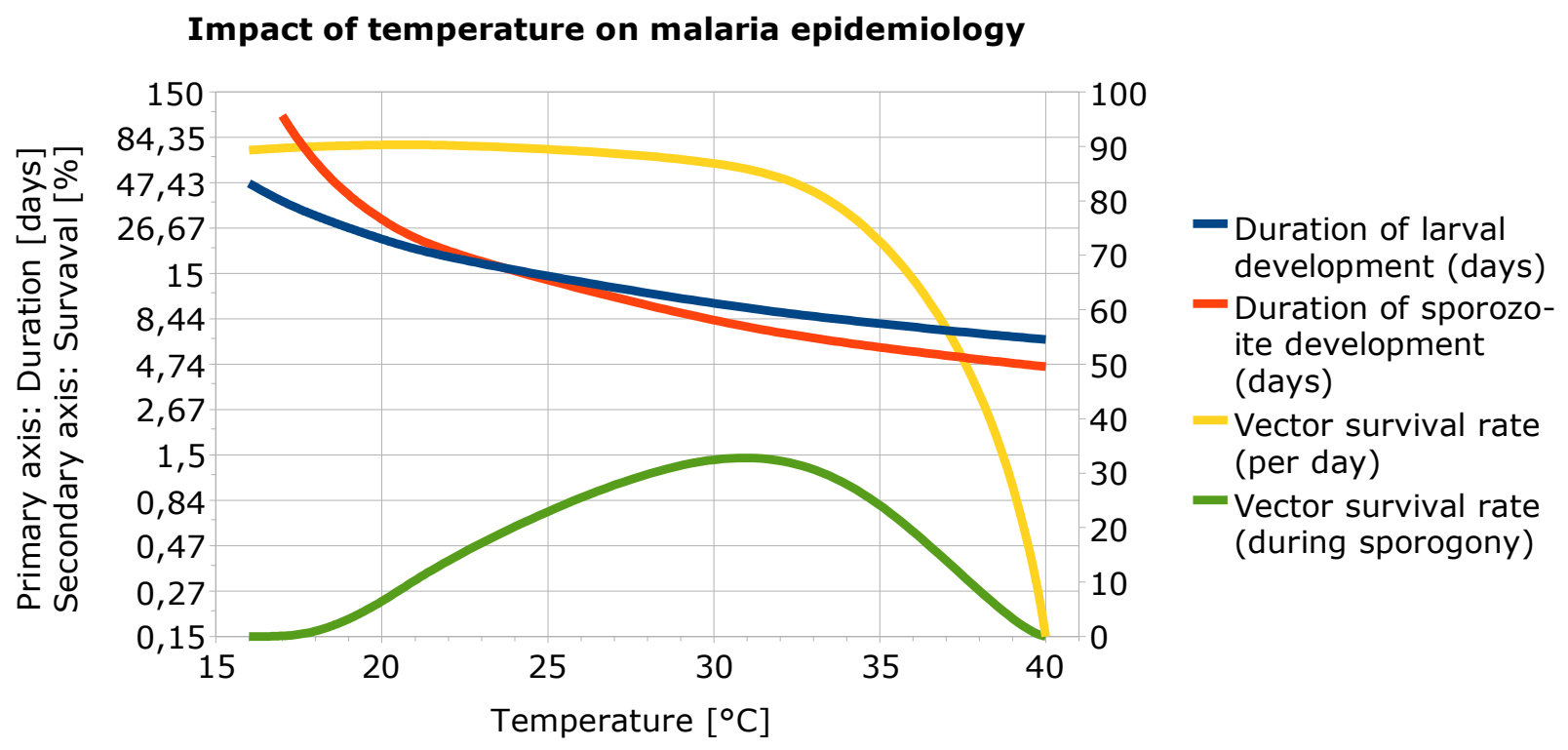

Figure 18: Combined impact of temperature on important epidemiological factors ${ }^{341}$

Despite the difficulties in predicting the combined epidemiological relevance of temperature, the epidemiological potential of malaria appears to be highest at temperatures between 29 and $33^{\circ} \mathrm{C}$, since a faster mosquito and parasite development (and a higher biting rate) cannot compensate for the decreasing vector longevity at higher temperatures. ${ }^{342}$

339 Hoshen, M.B. \& Morse, A.P. (2004), doi:10.1186/1475-2875-3-32.

340 Robert, V.; Awono-Ambene, H.P. \& Thioulouse, J. (1998), p. 948.

341 Gilles, H.M. (1993²), p. 126 and Hay, S.I.; Omumbo, J.A.; Craig, M.H. \& Snow R.W. (2000), p. 180.

342 Martens, P. (1998), p. 50. 
Using the Garki model, GemperLi et al. (2006) estimated the dependence of the prevalence measure $E$ (see section 2.5.4.2) on maximum monthly temperature (in ${ }^{\circ} \mathrm{C}$ ) as illustrated by figure 21 ). In the graph, long season corresponds to areas of perennial malaria transmission, whereas short season refers to areas where malaria is only just endemic at a transmission season of 2 months. The highest malaria prevalence is found at temperatures of around $31^{\circ} \mathrm{C}$ to $32^{\circ} \mathrm{C} .{ }^{343}$

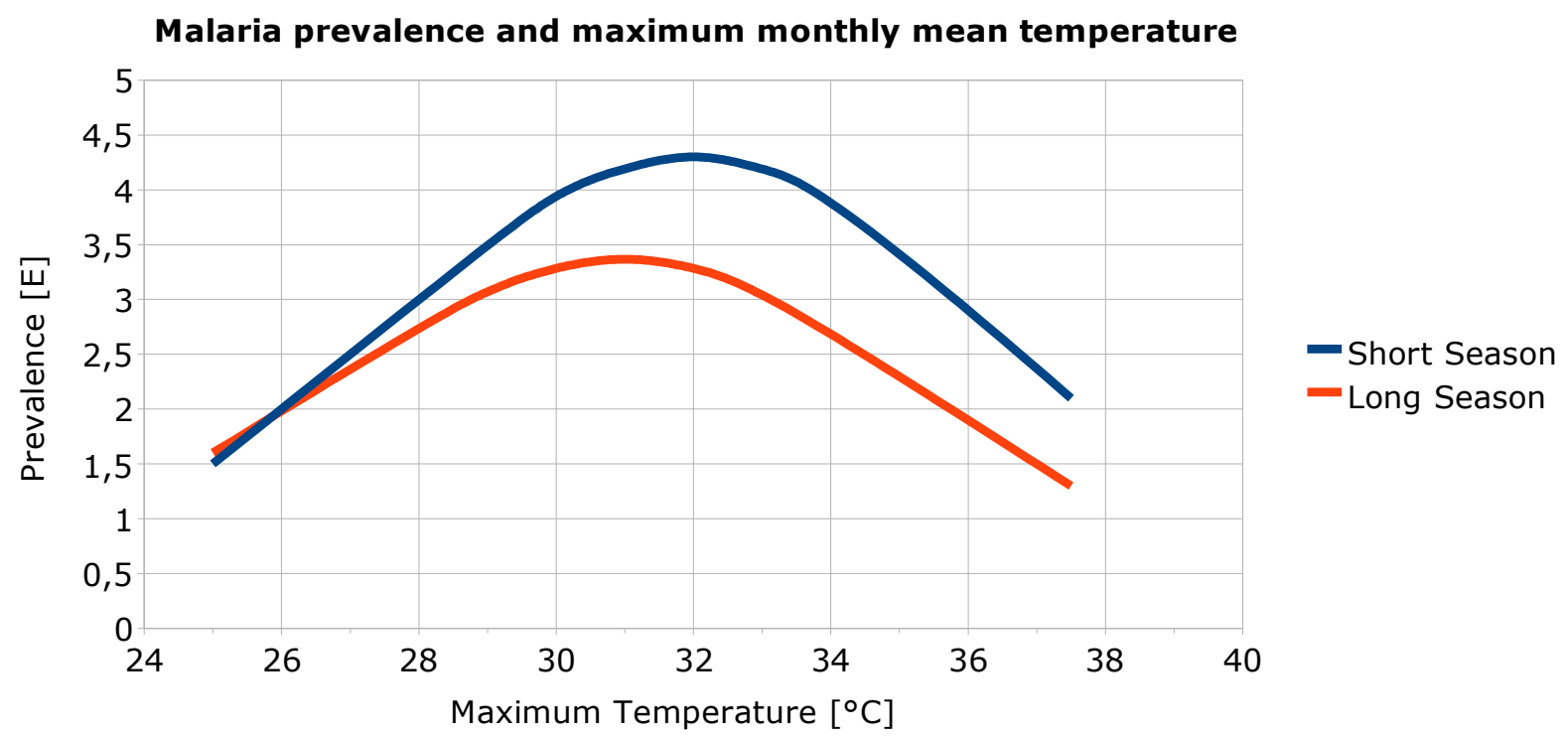

Figure 19: Malaria prevalence and maximum monthly temperature ${ }^{344}$

Various studies see temperature as one of the key determinants of malaria transmission but make different assumptions regarding spatio-temporal temperature pattern and temperature thresholds. The following examples have been chosen to illustrate how temperature can be used to map or model malaria transmission under environmental conditions such as those found in West Africa.

In summary, Plasmodium falciparum malaria transmission is likely to take place at temperatures between $18^{\circ} \mathrm{C}$ and $33^{\circ} \mathrm{C}$ and the following conditions:

- Winter temperatures should not fall below $3^{\circ} \mathrm{C}$ for mosquito populations to survive.

- Monthly mean temperatures must exceed $18^{\circ} \mathrm{C}$ and should exceed $22^{\circ} \mathrm{C}$ for transmission to take place.

- Stable transmission takes place when monthly rainfall is at least $80 \mathrm{~mm}$.

- Favorable conditions should persist for at least 3 months. ${ }^{345}$

343 Gemperli, A.; Sogoba, N.; Fondjo, E. et al. (2006), p. 1038.

344 Gemperli, A.; Sogoba, N.; Fondjo, E. et al. (2006), p. 1038.

345 HaY, S.I.; Omumbo, J.A.; Craig, M.H. \& Snow R.W. (2000), p. 193. 
Figure 20 illustrates the importance of threshold temperatures for processes of malariological relevance.

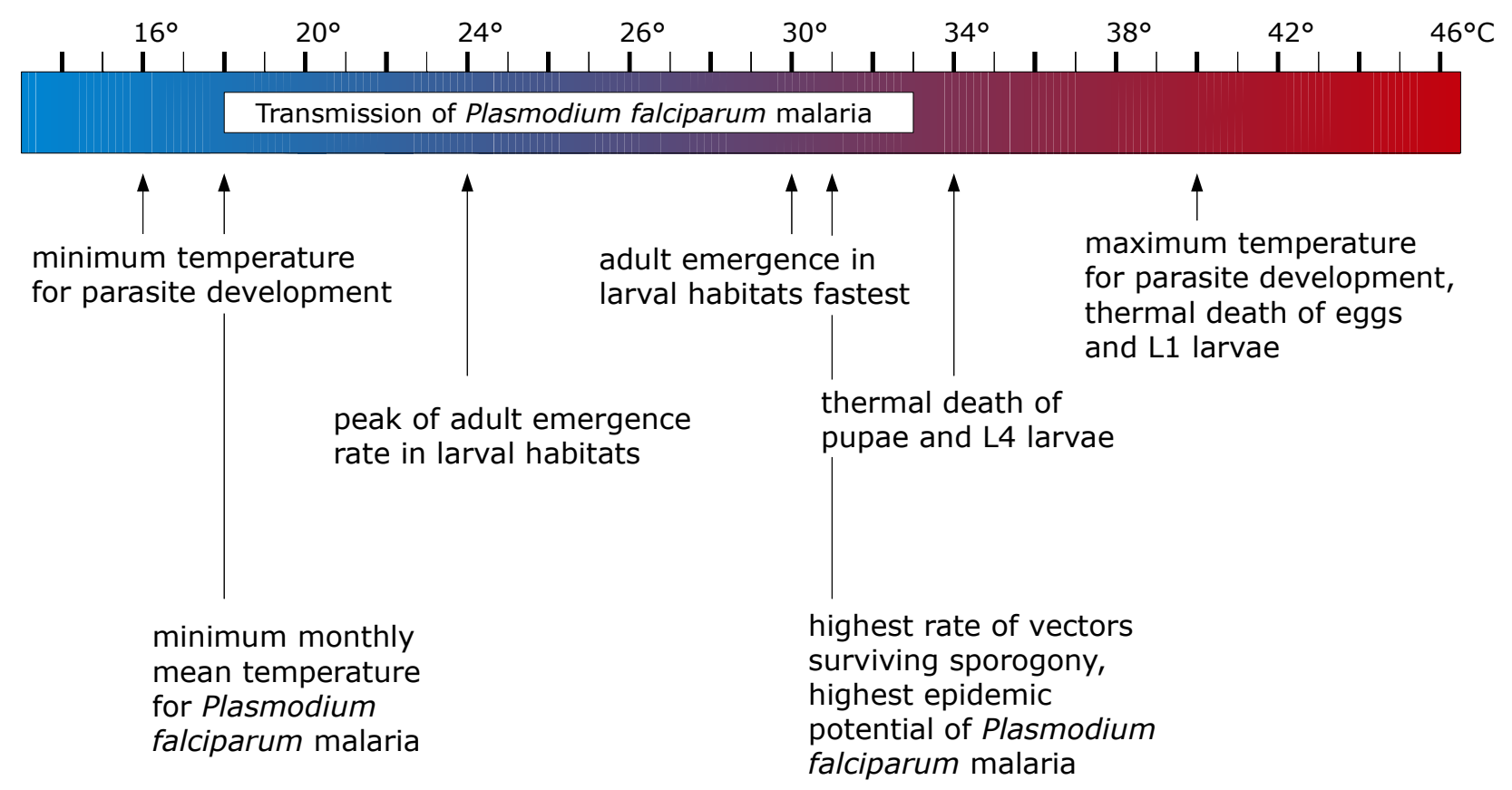

Figure 20: Temperature and Plasmodium falciparum malaria (summary)

While the temperature range between $16^{\circ} \mathrm{C}$ and $40^{\circ} \mathrm{C}$ marks the absolute limits of potential malaria transmission, the is a much narrower optimum range of just a few degrees above and below $31^{\circ} \mathrm{C}$.

\subsubsection{Malaria and Precipitation}

Precipitation has a major impact on the malaria transmission potential since

- female Anopheles mosquitoes seek surface water for oviposition and

- the longevity of the mosquitoes depends on humidity. ${ }^{346}$

The immature stages of Anopheles gambiae require an aquatic environment and are often found in transient pools created by precipitation. The frequency, duration and intensity of rainfalls determine the creation, density and persistence of breeding sites. ${ }^{347}$

346 Hay, S.I.; Omumbo, J.A.; Craig, M.H. \& Snow R.W. (2000), pp. $180 f$.

347 Paaijmans, K.P; Wandago, M.O.; Githeko, A.K. \& Takken, W. (2007), doi:10.1371/journal.pone.0001146. 
In (semi)arid regions where temperatures are suitable, malaria transmission sets in shortly after rainfalls provide temporary breeding sites for vectors. Outside the rainy season, malaria transmission in arid regions only takes place along rivers, in oases and close man-made water reservoirs. Most existing studies have identified a maximum correlation between rainfall and malaria after one month. ${ }^{348}$ Fillinger et al. (2009) found mosquito larval densities to be highest around two months after the onset of the rainy season in The Gambia. ${ }^{349} \mathrm{H}_{\mathrm{AY}}$ et al. (1998) successfully used cold cloud duration (CCD) imagery to predict malaria prevalence two weeks later. ${ }^{350}$ Despite this time lag, precipitation may be the most important predictor of malaria transmission in arid regions:

«The sources of instability in malaria transmission on the fringes of the Sahel from the west coast of Africa to Sudan appear to be determined more by rainfall than temperature. $\gg^{351}$

Since warmer temperatures accelerate several steps in the process of mosquito and parasite development, the time lag between rainfall and new malaria cases shortens as temperatures rise:

\begin{tabular}{|l|c|c|}
\hline Temperature & $20^{\circ} \mathbf{C}$ & $30{ }^{\circ} \mathbf{C}$ \\
\hline Aquatic Stage & 28 days & 12 days \\
\hline Sporogonic Cycle & 28 days & 8 days \\
\hline Incubation & \multicolumn{2}{|c|}{10 to 16 days } \\
\hline $\begin{array}{l}\text { Total time } \\
\text { (from oviposition to first malaria cases) }\end{array}$ & 9 to 10 weeks & 4 to 5 weeks \\
\hline
\end{tabular}

Table 13: Influence of temperature on the lag between rainfall and malaria incidence ${ }^{352}$

Even though the two most common vectors of malaria in West Africa, Anopheles gambiae and Anopheles arabiensis, occur at nearly the same temperatures, the amount of precipitation in their habitats differs considerably: $\mathrm{H}_{\mathrm{AY}}$ et al.(2000) observed that Anopheles gambiae was present in areas with an annual precipitation between $330 \mathrm{~mm}$ and $3224 \mathrm{~mm}$, whereas Anopheles arabiensis was found in drier regions with an annual precipitation between $237 \mathrm{~mm}$ and $415 \mathrm{~mm}$. Recognizing that there are no sharp boundaries of vector presence defined by precipitation alone, the authors suggested the use of a moisture index:

\footnotetext{
348 Hay, S.I.; Omumbo, J.A.; Craig, M.H. \& Snow R.W. (2000), pp. $180 f$.

349 Fillinger, U.; Sombroek, H.; Majambere, S. et al. (2009), doi:10.1186/1475-2875-8-62.

350 Curran, P.J.; Atkinson, P.M.; Foody, G.M. \& Milton, E.J. (2000), p. 64.

351 Kiszewski, A.E. \& Teklehaimanot, A. (2004), p. 130.

352 Teklehaimanot, H.D.; Lipsitch, M.; Teklehaimanot, A. \& Schwartz, J. (2004), doi: 10.1186/1475-2875-3-41.
} 


$$
\text { Moisture index }=\frac{\text { precipitation }}{\text { evapotranspiration }_{p o t}} 353
$$

For human malaria, a moisture index of 0,7 defines the lower limit of transmission. ${ }^{354}$ Rainfall does not only directly have a direct impact on mosquito reproduction by providing breeding sites but may also alter their character by reducing the temperature of pools or washing out predators..$^{355}$ Relative humidities below $60 \%$ shorten the lifespan of mosquitoes. ${ }^{356}$ HoSHEN and MoRSE (2004) estimate that the oviposition rate is roughly proportionate to both the ovipositing mosquito number and the rainfall during the previous ten days. ${ }^{357}$

The most important weather-related cause of larval death is probably desiccation, even though eggs may survive for weeks without water. ${ }^{358}$ Since eggs of Anopheles gambiae remain viable for 12 to 16 days under dry conditions, egg dormancy is seen as a short term survival mechanism of the species. Moreover, larvae emerging on damp soil have the capability of reaching nearby breeding sites. ${ }^{359}$

Prolonged periods of drought have a major impact on mosquito populations. Relative humidities below $60 \%$ shorten the lifespan of adult mosquitoes ${ }^{360}$, and the desiccation of larval habitats prevents reproduction. Nevertheless, both Anopheles arabiensis and Anopheles gambiae can survive in sufficient numbers during the dry season in Africa's dry savanna zone. ${ }^{361}$ The exact manner of this survival is unknown, but it is believed that local populations can be sustained either by few dispersed survivors or larger populations surviving in isolated localities. Populations may also become locally extinct and become re-colonized at the beginning of the rainy season by few migrants from adjacent areas where permanent breeding is allowed or mass migration or expansion of populations inhabiting stable areas. ${ }^{362}$ Since drought conditions also suppress predators of anophelines, they may increase malaria risks at the time of habitat recolonization. ${ }^{363}$

353 Hay, S.I.; Omumbo, J.A.; CRAig, M.H. \& Snow R.W. (2000), p. 195. 354 HaY, S.I.; Omumbo, J.A.; Craig, M.H. \& Snow R.W. (2000), p. 195.

355 Paaijmans, K.P; Wandago, M.O.; Githeko, A.K. \& Takken, W. (2007), doi:10.1371/journal.pone.0001146.

356 Yé, Y.; Louis, V.R.; Simboro, S. \& SAUerborn, R. (2007), doi:10.1186/1471-2458-7-101.

357 Hoshen, M.B. \& MoRse, A.P. (2004), doi:10.1186/1475-2875-3-32.

358 Hoshen, M.B. \& Morse, A.P. (2004), doi:10.1186/1475-2875-3-32.

359 Koenraadt, C.J.M.; Paaijmans, K.P.; Githeko, A.K. et al. (2003), doi:10.1186/1475-2875-2-20.

360 Yé, Y.; Louis, V.R.; Simboro, S. \& Sauerborn, R. (2007), doi:10.1186/1471-2458-7-101.

361 Charlwood, J.D.; ViJ, R. \& Billingsley, P.F. (2000), p. 726.

362 Simard, F.; Lehmann, T.; Lemasson, J.-J. et al. (2000), p. 467.

363 Anyamba, A.; Chretien, J-P.; Small, J. et al. (2006), doi: 10.1186./1476-072X-5-60. 
Strong rainfall may cause runoff from larval habitats that results in flushing of larvae. However, this does not necessarily mean the death of mosquito larvae as they are able to move actively over moist soil or may be washed into an adjacent habitat. However, larvae that are swept out of their natural habitat are likely to suffer higher mortalities. ${ }^{364}$

\subsubsection{Malaria and Surface Water}

Mosquito habitats are usually close to water bodies, particularly during the breeding phase. The physical processes which govern the links between vector densities and land cover are much more complex, however, and include population-internal processes, interdependencies between mosquito and host populations and other environmental influences (e.g. microclimate) which are sometimes difficult to capture. ${ }^{365}$

A mosquito's probability to oviposit, and for the larvae to survive, is proportional to the amount of water it finds. ${ }^{366}$ In a field study in Kenya during the dry season, Mushinzimana et al. (2006) found more than three fourths of all anopheline-positive habitats to be within 50 meters of streams; this percentage decreased only slightly during the rainy season. ${ }^{367}$ Studies in Mozambique showed that the prevalence of Plasmodium falciparum decreased from $40 \%$ to $60 \%$ in the direct vicinity of swampy breeding sites to $5 \%$ to $11 \%$ within $500 \mathrm{~m}$. People living within a $200 \mathrm{~m}$ radius of the breeding sites had a 6.2 times greater malaria risk than those living between $200 \mathrm{~m}$ and $500 \mathrm{~m}$ away. ${ }^{368}$ LACAux et al. (2007) pointed out that a few relatively small breeding sites may cause a relatively large zone of transmission. ${ }^{369}$

Larvae of Anopheles gambiae are typically found in small, temporary, sunlit and turbid puddles over bare soils, often created by human or animal activities (e.g., footprints, pits left after house-building, drainage ditches). ${ }^{370}$ Soil particles are often abundant in such transient water bodies, particularly towards the end of the rainy season when these puddles contract. Few other

364 Paaijmans, K.P; Wandago, M.O.; Githeko, A.K. \& Takken, W. (2007), doi:10.1371/journal.pone.0001146.

365 Curran, P.J.; Atkinson, P.M.; Foody, G.M. \& Milton, E.J. (2000), p. 64.

366 Hoshen, M.B. \& Morse, A.P. (2004), doi:10.1186/1475-2875-3-32.

367 Mushinzimana, E.; Munga, S.; Minakawa, N. et al. (2006), doi:10.1186/1475-2875-5-13.

368 HaY, S.I.; Omumbo, J.A.; Craig, M.H. \& Snow R.W. (2000), p. 201.

369 Lacaux, J.P.; Tourre, Y.M.; Vignolles, C. et al. (2007), p. 73.

370 Huang, J.; Walker, E.D.; Giroux, P.Y. et al. (2005), p. 443; Mutuku, F.M.; Bayoh, M.N.; Gimnig, J.E. et al. (2006), p. 54. 
multicellular organisms share this peculiar ecological niche. ${ }^{371}$ However, Anopheles gambiae females are quite flexible in their choice of oviposition sites; pools in streambeds appear to present refuge habitats when other habitats are unavailable. ${ }^{372}$

Not only do Anopheles gambiae s.s. females preferentially select small, open habitats for oviposition but also is larval predation less prevalent in temporary habitats than in large, permanent habitats. ${ }^{373}$ In fact, ovipositing mosquitoes are known to avoid habitats colonized by predators. ${ }^{374}$ Moreover, Anopheles gambiae s.s. exploit the increased production of algae in warm, open habitats. Warmer temperatures encountered in small and open habitats have the additional benefit of shortening larval-to-pupae development. ${ }^{375}$ Even under optimal conditions, however, the development of larvae into pupae takes at least six days. The limited stability of small habitats may therefore critically affect their otherwise high productivity. Under tropical conditions, pools containing less than $1 \mathrm{~m}^{3}$ of water are prone to desiccation before emergence of adult mosquitoes can take place. ${ }^{376}$

The quality of larval habitats influences the quality of resulting adults, including their vector competence. ${ }^{377}$ Food availability is an important determinant of larval development success, rapidity of development and the size of resulting adults. Large adults live longer, produce more eggs and bite more frequently than their smaller counterparts, all factors that are highly relevant for malaria transmission. ${ }^{378}$ RoBeRT et al. (1998) found warm temperatures $\left(28^{\circ} \mathrm{C}\right.$ to $30^{\circ} \mathrm{C}$ ), shallow water $(\leq 40 \mathrm{~cm})$, high carbonate concentrations, high $\mathrm{pH}$ and presence of water lettuce to be ideal for Anopheles arabiensis larvae. ${ }^{379}$ At the same time, conductivities above $2000 \mu \mathrm{S} / \mathrm{cm}$ (indicating high mineral contents) were recognized as limiting factors for Anopheles gambiae s.s. and Anopheles arabiensis breeding. ${ }^{380}$

A study carried out in natural and artificial larval habitats in the Kenyan Highlands demonstrated that Anopheles gambiae s.s. primarily breeds in small, sun-exposed habitats. Typical larval habitats include swamp margins, roadside ditches, and animal footprints devoid of large vegetation. Small pools were found to be about 10 times more likely to be colonized by larvae than moving streams and large water bodies. ${ }^{381}$ In a study on breeding site productivity in

371 Ye-Ebiyo, Y.; Pollack, R.J.; Kiszewski, A. \& Spielmann, A. (2003), p. 748.

372 Mutuku, F.M.; Bayoh, M.N.; Gimnig, J.E. et al. (2006), p. 60.

373 Minakawa, N. \& Sonye, G. (2004), p. 304.

374 Fillinger, U.; Sombroek, H.; Majambere, S. et al. (2009), doi:10.1186/1475-2875-8-62.

375 Minakawa, N. \& Sonye, G. (2004), p. 304.

376 Minakawa, N.; Sonye, G. \& Yan, G. (2005), pp. 295 \& 297.

377 OKeCH, B.A.; Gouagna, L.C.; YAN, G. et al. (2007), doi:10.1186/1475-2875-6-50.

378 Ye-Ebiyo, Y.; Pollack, R.J.; Kiszewski, A. \& Spielmann, A. (2003), p. 750.

379 Robert, V.; Awono-Ambene, H.P. \& Thioulouse, J. (1998), p. 952.

380 Fillinger, U.; Sombroek, H.; Majambere, S. et al. (2009), doi:10.1186/1475-2875-8-62.

381 Minakawa, N. \& Sonye, G. (2004), p. 303. 
The Gambia, Fillinger et al. (2009) observed a reduction of larval density with increasing size of water bodies. ${ }^{382}$ However, a characterization of aquatic habitat quality based on observed larval densities can be misleading for two reasons: adult mosquitoes emerging from habitats with high larval densities tend to be smaller, equipped with lower energy reserves and produce less eggs than their larger counterparts originating from habitats with lower larval densities. ${ }^{383}$ Moreover, the emergence rate of adult mosquitoes -and not larval density- is the malariologically relevant factor.

The pupal habitat productivity of Anopheles gambiae is one of the key determinants of vector density. ${ }^{384}$ In a study carried out to assess the productivity of different types of pupal habitats, burrow pits were found to be the most effective, when productivity was expressed as either total pupae per habitat or the number of pupae $/ \mathrm{m}^{2}$ of habitat (see figure 22 ). ${ }^{385}$

Pupal Productivity of Different Aquatic Habitats

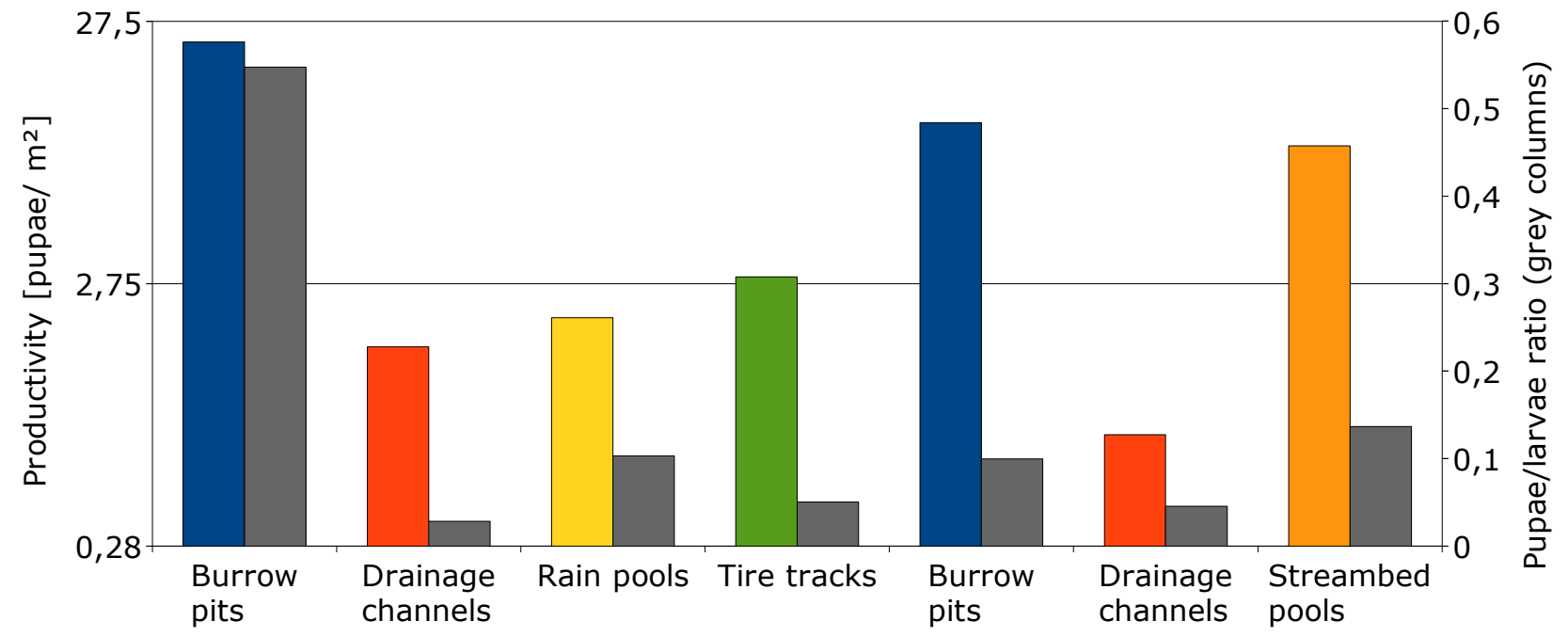

Figure 21: Pupal habitats and their productivity ${ }^{386}$

As the pupal stage represents the final step in metamorphosis, and moreover, the transition from the aquatic larval to the terrestrial adult mosquito, estimates of pupal density are a much better proxy measure for habitat productivity and adult emergence than larval density. Small pools often appear to contain a large number of anopheline larvae and are thus usually considered to be very productive, but this may not be true; larval density and pupal productivity are rather decoupled in many habitats. ${ }^{387}$ However, low

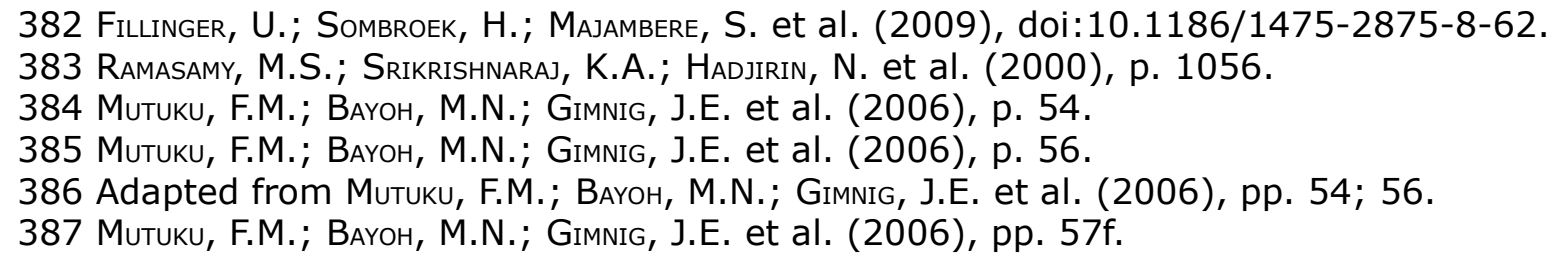


productivity is not necessarily a question of habitat stability: whereas unstable habitats are an obvious cause of larval mortality, predators and parasites often inhabit stable habitats and may be very effective in suppressing pupal production. ${ }^{388}$

There is still very limited data on habitat productivity under natural conditions. One study reports weekly adult emergences ranging from around 0.13 to 1.8 individuals $/ \mathrm{m}^{2}{ }^{389}$, but these numbers are based on very limited observations and may not be representative.

There are conflicting views regarding the role of water turbidity. Particles causing turbidity include clay and silt, finely divided organic matter and microorganisms. Turbidity is promoted by rainfall, biological activity (e.g. algal growth) and disturbances by humans or animals. ${ }^{390}$ Such conditions are shortlived, but well-mixed pools may remain turbid for several days. ${ }^{391}$ Since these factors all vary in time, turbidity itself is also dynamic. ${ }^{392}$ Robert et al. (1998) found turbid water to be unfavorable for larvae of Anopheles arabiensis. ${ }^{393}$ By contrast, Muturi et al. (2007) found Culex quinquefasciatus to prefer habitats with turbid water caused by organic matter. ${ }^{394}$ YE-EBIYo et. al. (2003) noted a correlation between water turbidity and the presence of Anopheles gambiae larval stages, as long as food is abundant at the water's surface. ${ }^{395}$ It appears that the nature of the particles causing turbidity plays an important role: water turbid from food particles may represent a suitable habitat while particles not edible for larvae could disfavor their production. ${ }^{396}$ Water turbidity can also have an effect on water temperature that can be in the order of $\pm 3 \mathrm{~K}$ in the upper water layer (where mosquito larvae are usually found). ${ }^{397}$ However, this effect varies with the time of day and is greatest around midday on clear days. This rise in temperatures can have two effects. At temperatures of up to $37^{\circ} \mathrm{C}$, increases are likely to enhance larval development. However, in small, very turbid water bodies, temperatures may rise beyond the thermal death point of anopheline larvae. Depending on the pools' depth, mosquito larvae may avoid these unfavorable conditions by diving and remaining submerged. ${ }^{398}$

\footnotetext{
388 Mutuku, F.M.; Bayoh, M.N.; Gimnig, J.E. et al. (2006), pp. $58 f$.

389 Fillinger, U.; Sombroek, H.; Majambere, S. et al. (2009), doi:10.1186/1475-2875-8-62.

390 Paaijmans, K.P.; Takken, W.; Githeko, A.K. \& Jacobs, A.F.G. (2008), pp. $747 f$.

391 Lacaux, J.P.; Tourre, Y.M.; Vignolles, C. et al. (2007), p. 67.

392 PaAjumans, K.P.; Takken, W.; Githeko, A.K. \& Jacobs, A.F.G. (2008), p. 752.

393 Robert, V.; Awono-Ambene, H.P. \& Thioulouse, J. (1998), p. 952.

394 Muturi, E.J.; Shililu, J.I.; Gu, W. et al. (2007), p. 101.

395 Ye-Ebiyo, Y.; Pollack, R.J.; Kiszewski, A. \& Spielmann, A. (2003), p. 748.

396 Jacob, B.G.; Muturi, E.J.; Mwangani, J.M. et al. (2007), doi:10.1186/1476-072X-6-21.

397 PaAijmans, K.P.; Takken, W.; Githeko, A.K. \& Jacobs, A.F.G. (2008), p. 747.

398 PaAijmans, K.P.; Takken, W.; Githeko, A.K. \& Jacobs, A.F.G. (2008), pp. $751 \mathrm{f}$.
} 
In 2000, two independent studies in India and Kenya revealed that the use of nitrogenous fertilizers coincided with an increase in anopheline larval populations. A control experiment conducted in Kenya showed that the total numbers of anopheline and culicine larvae were higher in ponds to which fertilizer was added than in controls. One hypothetical explanation was an observed reduction in water turbidity after the application of fertilizer, which may influence mosquito oviposition behavior. ${ }^{399}$

\subsubsection{Malaria and (Soil) Surface Characteristics}

Surface characteristics that play a role for the spatial distribution of mosquito breeding sites and habitats include the relief and the infiltration characteristics of the soil. ${ }^{400}$ Not only surface runoff and the formation of water bodies, but also soil moisture and color have an impact on mosquito oviposition. ${ }^{401}$ The effect of soil color is still poorly understood, but ovipositing Anopheles gambiae s.l. generally seem to prefer darker substrates. ${ }^{402}$

Laboratory and field based studies showed a strong correlation between soil moisture and egg-laying behavior of Anopheles gambiae; the number of eggs laid generally increases towards the stage of saturation and free-standing water over soil and sand. ${ }^{403}$ Larvae hatching on wet substrates may crawl to adjacent aquatic habitats or be washed there by rain. ${ }^{404}$ Figure 23 illustrates the results of a Kenyan study on anopheline oviposition and soil moisture even though it only vaguely differentiated between sandy and "mixed" soil.

399 Mutero, C.M.; NG'Ang'A, P.N.; Wekoyela, P. et al. (2004), pp. 188-191.

400 Hay, S.I.; Omumbo, J.A.; Craig, M.H. \& Snow R.W. (2000), p. 181.

401 Huang, J.; Walker, E.D.; Giroux, P.Y. et al. (2005), p. 443.

402 Paajumans, K.P.; Takken, W.; Githeko, A.K. \& Jacobs, A.F.G. (2008), p. 748.

403 Huang, J.; Walker, E.D.; Giroux, P.Y. et al. (2005), p. 448.

404 Huang, J.; Walker, E.D.; Vulule, J. \& Miller, J.R. (2006), doi:10.1186/1475-2875-5-87. 
Egg Laying Behavior and Soil Moisture

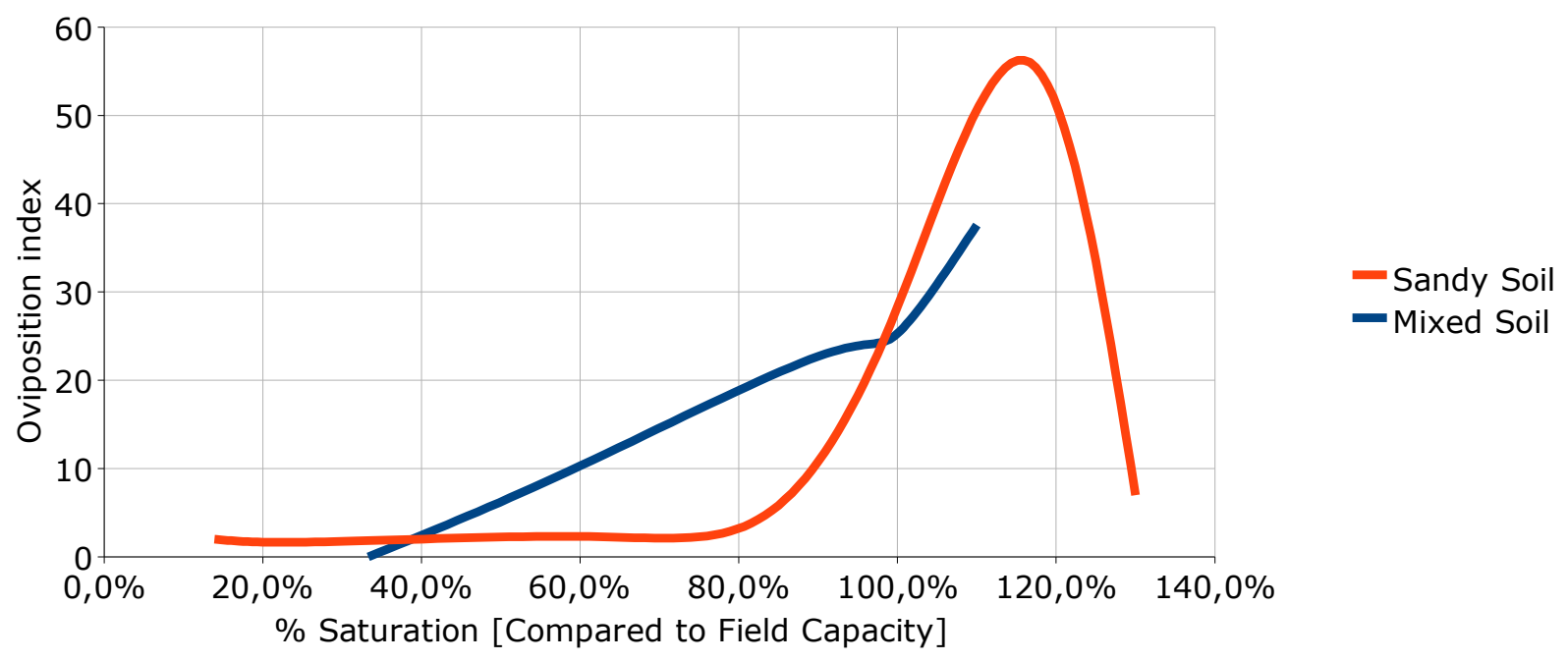

Figure 22: Egg-laying behavior of anophelines depending on soil moisture ${ }^{405}$

However, the experiments showed that the mosquitoes laid about $10 \%$ of their eggs on relatively dry substrates.

«This finding suggests that a range of hydrated habitats in nature, including mud without free-standing water, may be acceptable for oviposition. Perhaps such sites have a high probability of being flooded during the rainy season. Such an hypothesis would further suggest that $A n$. gambiae females might spread their bets by distributing eggs in sites ranging from fully saturated to only slightly hydrated. $\gg^{406}$

In fact, laying eggs on damp soils may pay off when the site are flooded later on since they may not yet be colonized by predators and pathogens. While small breeding sites dry up quickly even during the wet season, rain showers usually follow within the 12 to 16 day period eggs remain viable. Nevertheless, this is only a short-term strategy for population survival that does not work throughout the dry season ${ }^{407}$; anopheline eggs cannot survive for more than 15 days on completely dry soil. ${ }^{408}$

405 Adapted from Huang, J.; Walker, E.D.; Giroux, P.Y. et al. (2005), p. 446.

406 Huang, J.; Walker, E.D.; Giroux, P.Y. et al. (2005), p. 448.

407 Koenraadt, C.J.M.; Paajumans, K.P.; Githeko, A.K. et al. (2003), doi:10.1186/1475-2875-2-20.

408 Depinay, J.M.O.; Mbogo, C.M.; Killeen, G. et al. (2004), doi:10.1186/1475-2875-3-29. 
Soil moisture (lagged by 2 to 4 weeks) has been shown to explain between one third and half of the Anopheles gambiae / Anopheles funestus biting rates. ${ }^{409}$ Moreover, a study carried out in Kisian, Kenya, demonstrated that soil moisture is a better predictor of mosquito biting variability than precipitation, yielding similar results as vegetation indices (see table 14 ):

\begin{tabular}{|l|l|}
\hline Environmental Parameter & $\begin{array}{l}\text { Coefficients of determination }\left(\mathbf{r}^{\mathbf{2}}\right) \\
\text { for Anopheles gambiae human } \\
\text { biting rate }\end{array}$ \\
\hline Soil moisture (after 2 weeks) & $0.31(0.45)$ \\
\hline Precipitation (after 4 weeks) & $0.03(0.13)$ \\
\hline NDVI & 0.42 \\
\hline
\end{tabular}

Table 14: Soil moisture, precipitation, NDVI as determinants malaria transmission ${ }^{410}$

\section{2,5.1.5 Malaria and Land Cover}

Land cover is one landscape feature that plays a central role in the epidemiology of malaria. ${ }^{411}$

Anopheles gambiae s.l. is generally thought to utilize puddles over bare soil as its prime larval habitat while avoiding standing water populated with vegetation. However, it is unclear whether presence of larvae in grassy habitats results from hatches of eggs placed on and around wet grasses, or whether larvae were carried there by flowing water. ${ }^{412}$

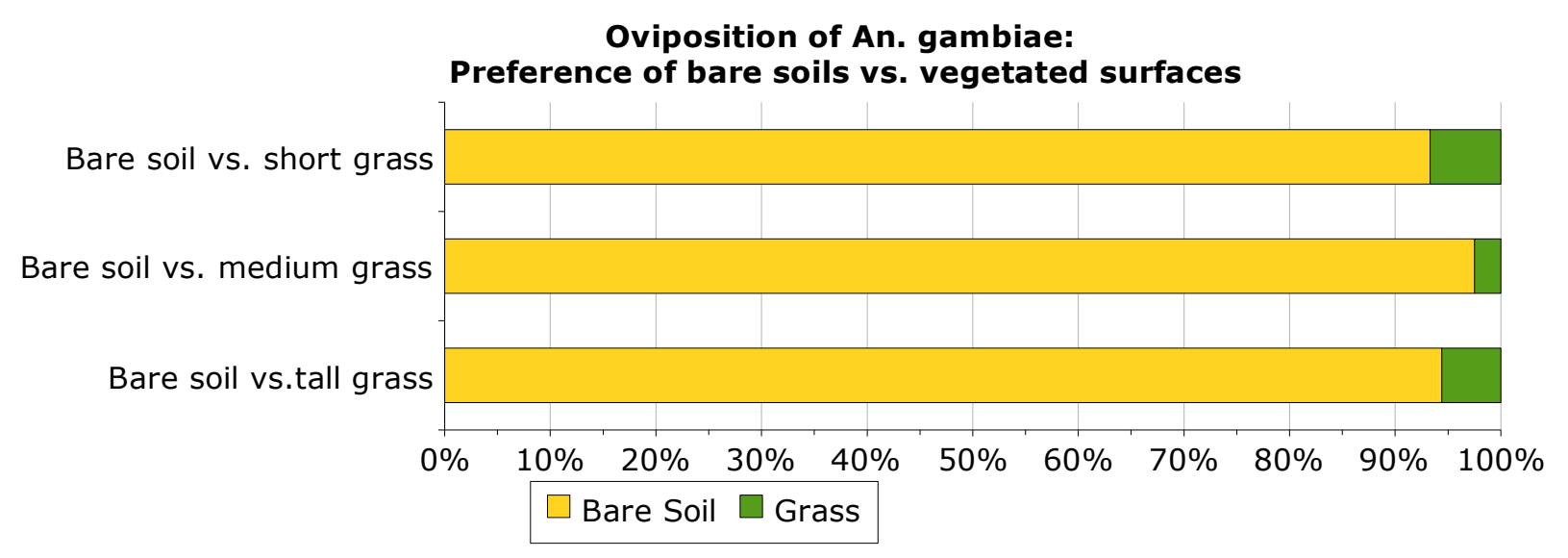

Figure 23: Grass cover and mosquito oviposition ${ }^{413}$

409 Patz, J.A.; Strzepek K.; Lele, S. et al. (1998), p. 818.

410 Patz, J.A.; Strzepek K.; Lele, S. et al. (1998), pp. 818; 822.

411 Mutuku, F.M.; Bayoh, M.N.; Hightower, A.W. et al. (2009), doi:10.1186/1476-072X-8-19.

412 Huang, J.; Walker, E.D.; Otienoburu, P.E. et al. (2006), doi: 10.1186/1475-2875-5-88. 
However, this preference for bare soils does not seem to be universal among anophelines, as studies on the oviposition behavior of Anopheles minimus show a preference in the order small-leaved plants $>$ large-leaved plants $>$ grasses $>$ soil. ${ }^{414} \mathrm{~A}$ study carried out in the Kenyan Highlands also showed that water bodies with short vegetation are more often inhabited by anopheline larvae than those having tall plants growing in or around them. Pools with floating plants were always found to be devoid of larvae. ${ }^{415}$

\section{Acquatic Vegetation and Anopheles gambiae Breeding}

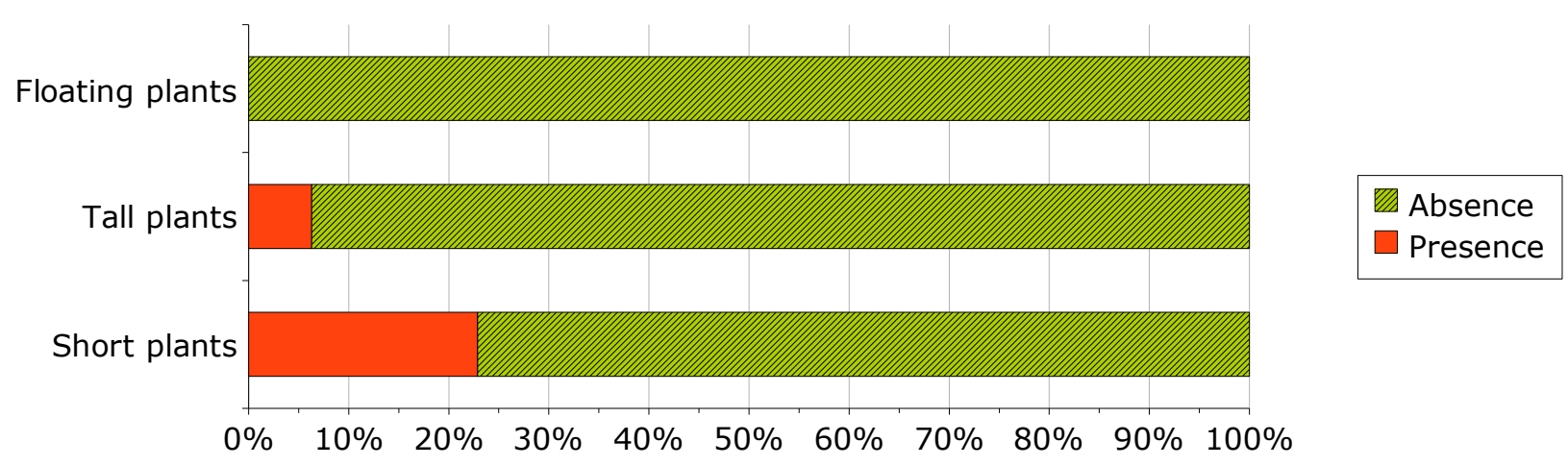

Figure 24: Aquatic vegetation and mosquito larval habitats ${ }^{416}$

\subsubsection{Anthropogenic Determinants of Malaria Transmission}

Human activities are linked to malaria transmission in several ways. On the one hand, human settlement pattern and population densities may decide over the fact that host-to-vector contact occurs frequently enough for malaria transmission to take place. Man-made changes in land cover, and in particular the creation of (permanent) vector breeding sites due to irrigation projects, may alter the presence, composition, density and longevity of a vector population.

\subsubsection{Malaria and Settlement Pattern}

Malaria is only found in areas where the population density is high enough to sustain transmission; population densities of less than one person per square kilometer are considered unsuitable. ${ }^{417}$

\footnotetext{
413 Adapted from Huang, J.; Walker, E.D.; Otienoburu, P.E. et al. (2006), doi: $10.1186 / 1475-2875-5-88$.

414 OvergaARD, H.J. (2007), p. 193.

415 Minakawa, N. \& Sonye, G. (2004), pp. $303 \mathrm{f}$.

416 Adapted from Minakawa, N. \& Sonye, G. (2004), p. 303.

417 Guerra, C.A; Snow, R.W. \& Hay, S.I. (2006), p. 355.
} 
As a general rule, the health status of urban populations in Africa is better than in rural areas, and cities normally present unfavorable conditions for malaria. ${ }^{418}$ Reasons include pollution of mosquito habitats, mosquito avoidance behavior of urban populations (e.g. better housing) and a higher ratio of humans to mosquitoes. ${ }^{419}$ Nevertheless, there is evidence for malaria transmission in most urban areas in Africa. ${ }^{420}$ One reason is that urban environments in Africa often show great spatial variations in their development level, with well-developed centers often being surrounded by underdeveloped and inadequately serviced settlements. ${ }^{421}$ Poor housing and lack of sanitation and drainage on the one side and a rapid adaptation of malaria vectors to urban areas on the other side pose great challenges for malaria control. ${ }^{422}$ Malaria transmission risks differ greatly between and within Africa's urban areas, reflecting their heterogeneity and the focal nature of malaria transmission. ${ }^{423}$

Only three anopheline species are responsible for urban malaria in Africa, most notably Anopheles gambiae and Anopheles arabiensis and in rare cases Anopheles funestus. ${ }^{424}$ In urban environments, the dispersion of Anopheles gambiae appears to be more or less restricted to a zone within $200 \mathrm{~m}$ to $300 \mathrm{~m}$ around their main breeding sites. ${ }^{425}$

Urban areas in Africa often have a "pseudo-urban" character, with rural elements dominating at least parts of them. This is exemplified by an investigation into the role of agriculture in urban areas was carried out by Afrane et al. in the city of Kumasi, Mali (see table 15):

418 HAY, S.I.; Guerra, C.A.; TATEM, A.J. et al. (2005), pp. $81 \mathrm{f}$.

419 Robert, V.; Macintyre, K.; Keating, J. et al. (2003), p. 169.

420 Robert, V.; Macintyre, K.; Keating, J. et al. (2003), p. 170.

421 Keiser, J.; Utzinger, J.; Caldas de Castro, M. et al. (2004), p. 119.

422 Keiser, J.; Utzinger, J.; Caldas de Castro, M. et al. (2004), p. 118.

423 Siri, J.G.; Lindblade, K.A.; Rosen, D.H. (2008); doi:10.1186/1475-2875-7-34.

424 Robert, V.; Macintyre, K.; Keating, J. et al. (2003), p. 170.

425 Keiser, J.; Utzinger, J.; Caldas de Castro, M. et al. (2004), p. 120. 


\begin{tabular}{|l|l|}
\hline City district & Typical breeding sites of anopheline larvae \\
\hline $\begin{array}{l}\text { Urban areas } \\
\text { without } \\
\text { agriculture }\end{array}$ & $\begin{array}{l}\text { - mostly in temporary pools and puddles created after } \\
\text { rains in the unpaved streets and in between houses, } \\
\text { exposed directly to the sun and without vegetation; } \\
\text { - common in the rainy and rare during the dry season }\end{array}$ \\
\hline $\begin{array}{l}\text { Urban areas with } \\
\text { agriculture }\end{array}$ & $\begin{array}{l}\text { - } \begin{array}{l}\text { shallow wells dug for irrigation } \\
\text { irrigated fields }\end{array} \\
\text { - similar breeding sites as in the areas without } \\
\text { agriculture were found. }\end{array}$ \\
\hline Periurban areas & $\begin{array}{l}\text { - edges of slow-moving streams } \\
\text { isolated pools in drying riverbeds }\end{array}$ \\
\hline
\end{tabular}

Table 15: Mosquito breeding sites and urban agriculture ${ }^{426}$

Consequently, the man biting rate and the entomological inoculation rate (EIR) were significantly higher in the periurban areas and the urban areas with agriculture than in those without (see figure 25):

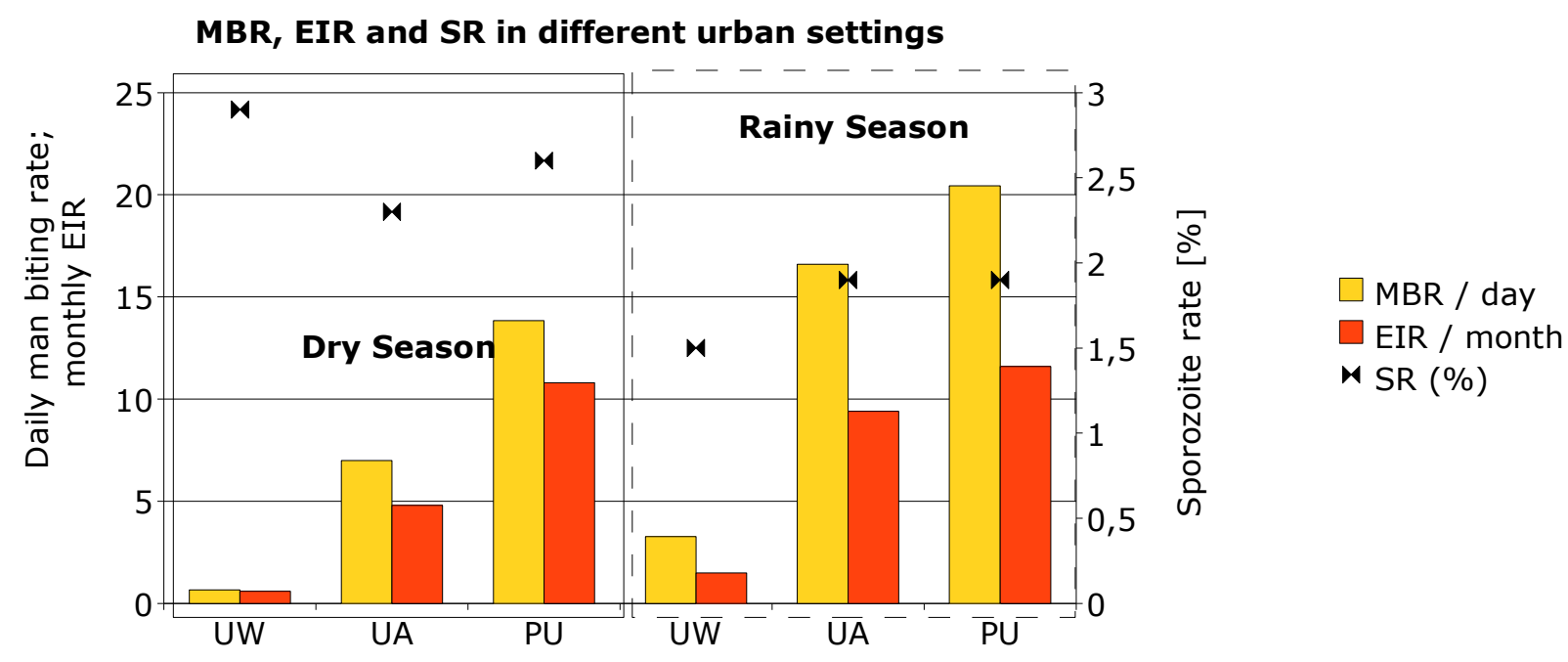

Settings: $U W=$ urban, without agriculture; $U A=$ urban, with agriculture; $P U=$ periurban

Figure 25: Mosquito biting rate / EIR and urban agriculture ${ }^{427}$

426 Afrane, Y.A.; Klinkenberg, E.; Drechsel, P. et al. (2004), pp. $128 \mathrm{f}$.

427 Adapted from Afrane, Y.A.; Klinkenberg, E.; Drechsel, P. et al. (2004), p. 131. 
Given the heterogeneity of population distribution in many African countries, there is still relatively little information on links between malaria and settlement pattern. Despite the increasing role of malaria in urban areas, the disease is still a largely rural phenomenon, with changes in agricultural practices frequently being paralleled by alterations in malaria incidence.

\subsubsection{Malaria and Land Cover Change}

The drastic land-use modifications currently occurring in many African countries can promote vector-borne disease transmission in several ways.

Deforestation, which is often linked to an expansion of agricultural activities, may also be a cause of increased malaria transmission. A case study in Loreto district, Peru found that malaria incidence rose from 2.1 cases per 1000 population in the early 1990 s to 343 cases per 1000 population in the late 1990s, nearly half of which were caused by Plasmodium falciparum. During that time, the construction of a major road between Iquitos and Nauta had led to massive deforestation, an enormous population growth rate and a continued expansion of agriculture into the forest fringes. ${ }^{428}$ Anopheles darlingi, the most efficient vector of malaria in the Amazon basin, had never been found in areas predominantly covered by forest (forest cover $>60 \%$ ). Average human biting rates rose considerably in areas with less forest cover (see table 16).

\begin{tabular}{|l|l|l|l|}
\hline Forest Cover & $<20 \%$ & $20 \ldots 60 \%$ & $>60 \%$ \\
\hline $\begin{array}{l}\text { Average human biting rate of } \\
\text { Anopheles darlingi per } 6 \text { hour } \\
\text { interval }\end{array}$ & 6.5 & 1.7 & 0.0429 \\
\hline
\end{tabular}

Table 16: Forest cover and mosquito biting rates (in Loreto District, Peru)

Case studies in Kenya have come to similar conclusions and found increased incidence of malaria in areas where forests were cleared. ${ }^{430}$

In swamp areas where tall grasses form the natural vegetation, the introduction of agriculture may cause a reduction of inundation but at the same time lead to more sunlit pools and thus more favorable habitats for Anopheles gambiae. ${ }^{431}$

428 Vittor, A.Y.; Gilman, R.H.; Tielsch, J. et al. (2006), p. 3.

429 Vittor, A.Y.; Gilman, R.H.; Tielsch, J. et al. (2006), p. 6.

430 Minakawa, N.; Munga, S.; Atielli, F. et al. (2005), p. 163.

431 Minakawa, N.; Munga, S.; Atielli, F. et al. (2005), p. 157. 
A study conducted by KEBEDE et al. (2005) in Bure district of Ethiopia revealed that an expansion of malaria transmission coincided with the replacement of traditional crops with maize (Zea mays) ${ }^{432}$; the malaria incidence rate differed according to the intensity of maize cultivation (see table 17):

\begin{tabular}{|l|l|l|}
$\begin{array}{l}\text { Intensity of maize } \\
\text { cultivation }\end{array}$ & $\begin{array}{l}\text { Incidence rate / } \\
\mathbf{1 0 . 0 0 0} \text { person years }\end{array}$ & Incidence density ratio \\
\hline Low & 25,1 & 1,00 \\
\hline Medium & 92,4 & 3,68 \\
\hline High & 239,8 & 9,54 \\
\hline
\end{tabular}

Table 17: Maize cultivation and malaria transmission intensity in Ethiopia ${ }^{433}$

An increase in animal densities may cause both the creation of open pastures and temporary mosquito habitats in form of animal footprints. ${ }^{434}$ However, depending on the degree of anthropophily/zoophily of vector populations, higher animal densities may also result in protective effects (zooprophylaxis). ${ }^{435}$ 


\subsubsection{Malaria and Irrigated Agriculture}

The high population growth rate on the African continent has driven many governments to improve food production by initiating large-scale irrigation projects, involving reclamation of arid and semi-arid land for the cultivation of crops. Although crop irrigation promises one solution to alleviating hunger and poverty, irrigation has often been blamed for aggravating disease in local communities. ${ }^{436}$ Depending on local conditions, this is in particular true for malaria: The overwhelming impact of malaria mortality and morbidity, especially in an African context, led the Consultative Group on International Agricultural Research (CGIAR) to launch the "System-wide Initiative on Malaria and Agriculture" (SIMA) in 2001. ${ }^{437}$

Nearly half of all arable land in Africa is too dry for rain-fed agriculture and large areas experience rainfall which is sparse and highly variable. Since only $4 \%$ of Africa's land is currently irrigated, a great potential exists for increasing food and cash crop production through irrigation development. ${ }^{438}$

Rice is rapidly replacing other cereals as the staple diet in many developing countries, including those in (West) Africa, where the total area under rice cultivation is expected to increase. ${ }^{439}$ At the same time, it is by far the most common crop grown under irrigation, comprising about one third of all irrigated crops grown in Africa. Whereas upland rice does not require flooding for its growth, lowland rice is maintained in 10 to $15 \mathrm{~cm}$ of water. ${ }^{440}$

Increased numbers of vectors following irrigation can lead to increased malaria in areas of unstable transmission, whereas it appears to have little impact in areas of stable transmission ${ }^{441}$ :

«Irrigation might push malaria transmission over a threshold in areas where transmission would otherwise be very low or nonexistent, such as desert fringes or highlands. [... In areas of stable but seasonal transmission], irrigation, especially during the dry season, might alter the transmission pattern from seasonal to annual. $\gg^{442}$

436 IJUMBA, J.N. \& LindSAY, S.W. (2001), p. 1.

437 van der Hoek, W. (2004), p. 95.

438 IJUMBA, J.N. \& LiNDSAY, S.W. (2001), p. 2.

439 Mutero, C.M.; Blank, H.; Konradsen, F.; vaqn der Hoek, W. (2000), p. 254.

440 IJUMBA, J.N. \& LiNDSAY, S.W. (2001), p. 2.

441 IJumba, J.N. \& Lindsay, S.W. (2001), p. 1.

442 Sissoko, M. S; Dicko, A.; Briët, O.J.T. et al. (2004), p. 162. 
In the semi-arid savanna zone of Africa, irrigated rice cultivation can alter malaria transmission pattern from seasonal to perennial. Rice is considered to pose the greatest threat among irrigated crops since it is grown under flooded conditions, and studies in various parts of Africa have demonstrated the presence of up to 35 mosquito species in irrigated rice agro-ecosystems. ${ }^{443}$ Flooded paddy fields provide ideal breeding sites for the principal vectors of malaria in West Africa, namely members of the Anopheles gambiae complex (and Anopheles arabiensis in particular). These vectors are pioneer species which rapidly colonize recently flooded fields, although they decline in numbers as the rice grows and begins to cover the water surface. Irrigated rice cultivation, depending on the number of cropping cycles, may also extend their breeding season and hence increase the annual duration of transmission. Moreover, in dry regions, irrigation will elevate relative humidity that aids survival of these vectors. ${ }^{44}$

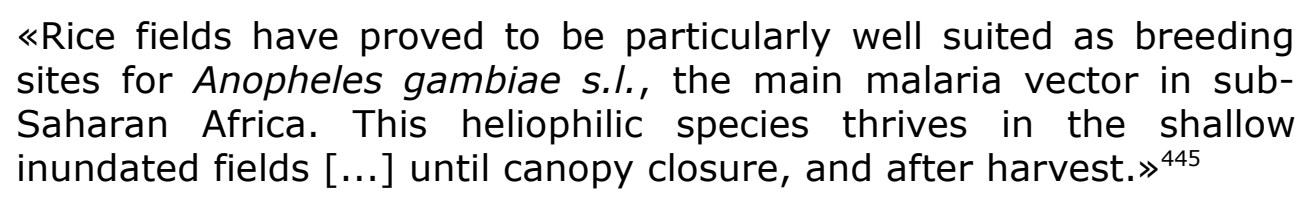

In general, the predominant vector is the one found in surrounding areas, although there is at least one notable exception: The Mopti form of Anopheles gambiae s.s. thrives in rice fields located in the northern fringes of the Sahel in Burkina Faso, where it outnumbers the savanna form found elsewhere. ${ }^{446}$

During the dry season, irrigated agriculture often leads to anophelism without malaria: While enormous numbers of vectors are produced by rice fields during the dry season, extremely high temperatures (often rising to above $40^{\circ} \mathrm{C}$ ) during the day reduce survival of adult mosquitoes and, perhaps more importantly, kill the developing parasites within the vector. ${ }^{447}$

Not only the introduction of irrigated rice, but also of irrigated wheat, cotton and sugarcane has led to an increase of malaria in some parts of Africa. Even though these crops require less water than rice, an increase in humidity may lead to an increase in malaria. Moreover, the construction of dams and artificial lakes (e.g. for aquaculture) has been blamed for increased prevalence of endemic diseases including malaria. ${ }^{448}$ LAUTZE et al. (2007) found malaria case

443 Muturi, E.J.; Shilitu, J.I.; Gu, W. et al. (2007), p. 95.

444 Ijumba, J.N. \& Lindsay, S.W. (2001), p.4; Marrama, L.; Jambou, R.; Rakotorivony, I. et al. (2004), p. 200.

445 Dolo, G.; Briët, O.J.T.; Dao, A. et al. (2004), p. 147.

446 IJUMBA, J.N. \& LiNDSAY, S.W. (2001), p. 4.

447 IJumba, J.N. \& LiNDSAY, S.W. (2001), p. 4.

448 IJumba, J.N. \& Lindsay, S.W. (2001), pp. $7 f$. 
rates within $3 \mathrm{~km}$ of the Koka Reservoir in Ethiopia to be around 2.31 times higher than those between $6 \mathrm{~km}$ and $9 \mathrm{~km}$ away. This effect was observed despite the much more widely practiced application of indoor residual spraying in houses around the reservoir. ${ }^{449}$

Studies in Zimbabwe showed that the nature of irrigation schemes plays an important role. In areas of sprinkler irrigation, anopheline larval densities were found to be higher than in surface irrigated zones. The distance of water reservoirs to houses and their construction (i.e. suitability as breeding sites) also determine the impact irrigation has on malaria transmission. ${ }^{450}$

In regions where malaria transmission is connected to irrigated rice cultivation, intermittent irrigation with sufficiently long periods of dry soils has been proposed as a larval control strategy, but at least five "dry" days are required to kill most of the larvae. ${ }^{451}$ Intermittent irrigation was introduced as a vector control strategy in the first half of the $20^{\text {th }}$ century but fell into disuse with the arrival of modern insecticides such as DDT. Recently, however, intermittent irrigation has become popular among farmers in China and India, mainly as a strategy to save water in water-scarce areas. ${ }^{452}$ In Szechuan Province, China, a campaign primarily directed at water conservation led to the introduction of a system of "wet/dry crop rotation", reducing the area of flooded paddy fields by about $90 \%$ :

The gradual reduction of breeding sites has apparently reduced the reproductive rate of vector populations to such a degree, that even without adult control, mosquito populations do not return to previous levels. ${ }^{453}$

Intermittent irrigation has not been widely evaluated or used in Africa. One case study carried out in the Mwea rice irrigation scheme in Kenya did not show clear results, possibly because seepage from plots under water and drained plots prevented sufficient desiccation to kill anopheline larvae. ${ }^{454}$

In some areas, malaria morbidity and mortality was reduced considerably after the introduction of irrigated agriculture. One plausible explanation is the creation of wealth in local communities, which allows farmers to make improvements to their homes (so that they become inhospitable to mosquitoes), improve their living standards and increase their disposable income (which can be used to pay for health services and drugs). ${ }^{455}$ Another

449 Lautze, J.; McCartney, M.; Kirshen, P. et al. (2007), pp. 985-987.

450 Chimbari, M.J.; Chirebvu, E. \& Ndlela, B. (2004), p. 209.

451 Koenraadt, C.J.M.; Paaijmans, K.P.; Githeko, A.K. et al. (2003), doi:10.1186/1475-2875-2-20.

452 Mutero, C.M.; Blank, H.; Konradsen, F.; vaqn der Hoek, W. (2000), p. 254.

453 Qunhua, L.; Xin, K.; Chnagzhi, C. et al. (2004), p. 246.

454 Mutero, C.M.; Blank, H.; Konradsen, F.; vaqn der Hoek, W. (2000), pp. 254 \& 261.

455 IJUmBA, J.N. \& LindSAY, S.W. (2001), p. 6. 
hypothesized explanation is that "[...] high anopheline densities lead to smaller adults, who do not live so long and hence are less efficient for transmitting the disease." ${ }^{456}$ The nuisance caused by high numbers of mosquitoes may also induce people to use their bednets more often. ${ }^{457} \mathrm{~A}$ case-study in the southern Sahel of Mali (Niono District, Ségou region; annual rainfall: $400 \mathrm{~mm}$ ) revealed a decline of malaria cases in irrigated parts of the region to roughly half the levels found in non-irrigated areas. ${ }^{458}$

One major limitation of most studies on the impact of irrigated agriculture is the small number of villages used for the comparison for regions with and without irrigation. Villages with irrigated agriculture often differ from other villages in numerous ways even before the onset of irrigation. The presence of vector habitats may for example be wrongly attributed to irrigation in regions where flooding also occurs naturally. ${ }^{459}$

\subsubsection{Exposure and Preventive Measures}

The degree of an individual's exposure to potentially infectious mosquito bites is an important determinant of his or her risk to contract malaria.

Since in many regions of the world, malaria is transmitted by endophagic mosquitoes, the quality of housing structures plays an important role for the exposure risk of their inhabitants. In a field trial in rural Gambia, the fitting of local houses with insect-screen ceilings reduced the number of Anopheles gambiae entering the huts by up to $80 \% .{ }^{460}$ In Burkina Faso, malaria vectors were found in greater numbers in simple mud-roofed structures than in more elaborate buildings with iron roofs. ${ }^{461}$

456 Diuk-Wasser, M.A.; Toure, M.B.; Dolo, G. et al. (2005), p. 725.

457 Diuk-Wasser, M.A.; Toure, M.B.; Dolo, G. et al. (2005), p. 725.

458 Sissoko, M. S; Dicko, A.; Briët, O.J.T. et al. (2004), p. 162.

459 Briett, O.J.T.; Dossou-Yovo, J.; Akodo, E. et al. (2003), pp. 439 \& 446.

460 Lindsay, S.W.; JaWARa, M.; Paine, K. et al. (2003), p. 512-514.

461 Yé, Y.; Hoshen, M.; Louis, V. et al. (2006), doi:10.1186/1475-2875-5-8. 


\subsection{Monitoring, Mapping and Modeling Malaria Transmission}

There are numerous measures of malaria transmission intensity, ranging from epidemiological indicators (transmission risk) to impact indicators (such as malaria mortality), which can be used as the basis for malaria maps. Due to a scarcity of data, maps are often based on ecological risk indicators. However, models linking environmental parameters and malaria transmission dynamics tend to be complex, and a multitude of different models, often based on different assumptions and input parameters, make comparisons difficult. Nevertheless, malaria models are an important prerequisite for the development of early warning systems.

\subsubsection{Malaria Surveys}

Malaria surveys have the goal of objectively assessing the risk and/or burden of malaria in a given area. Such surveys may either be based on diagnoses of malaria cases, or indices characterizing the transmission risk.

Passive case detection is based on reporting of malaria cases from static medical units such as dispensaries and hospitals. Active case detection is carried out by house-to-house visits at fortnightly intervals. During the final or maintenance phase, any occurrence of imported or indigenous cases of malaria is watched out for and appropriate measures are taken. ${ }^{462}$

\subsubsection{Diagnostic Methods}

Malaria is diagnosed using a combination of clinical observations, case history and diagnostic tests (usually a microscopic examination of blood).

Malaria symptoms can appear as soon as 6 to 8 days or as late as several months or even years after being bitten by an infected mosquito. Typical signs of an infection are fever, shivering, respiratory distress, general pain, diarrhea, vomiting and convulsions. ${ }^{463}$

462 Onori, E., Beales, P.F. \& Gilles, H.M. (1993), pp. $268 \mathrm{f}$.

463 TutejA, R. (2007), p. 4674. 
Since many of the symptoms of malaria are relatively unspecific, it is relatively difficult to diagnose malaria confidently without laboratory confirmation. ${ }^{464}$ Blood tests should ideally be taken when the patient's temperature is rising since this is the time when parasites are most likely to be detected in the blood. ${ }^{465}$ A lack of suitable laboratory equipment in many malarious areas often results in symptomatic diagnoses without confirmation of malaria and/or definite exclusion of other infections:

\begin{abstract}
«Misclassification might result from the tendency of physicians working in areas of high malaria transmission intensity to attribute most fevers to malaria, [... accompanied by a] lower sensitivity for other causes of deaths in such settings. ${ }^{466}$
\end{abstract}

In most countries in Sub-Saharan Africa, causes of death are assessed using the verbal autopsy method: postmortem interviews are conducted with family members about the circumstances leading to death and the symptoms observed before death. ${ }^{467}$

\begin{abstract}
«Despite obvious limitations, verbal autopsies are at present the best possible method to obtain reasonably precise information on cause-specific deaths in poor countries.»468
\end{abstract}

The uncertainties involved in such relatively subjective methods mean that malaria incidence and mortality data should be treated with care, and that other sources of information, such as entomological quantifiers of transmission intensity, may be valuable additional indicators for local malaria burdens.

\title{
2.6.1.2 Measures of Malaria Incidence
}

Accurate measurements of malaria morbidity and mortality are prerequisites for malaria mapping, public health planning and the assessment of control activities.

Incidence describes the frequency of illnesses commencing during a certain period of time whereas prevalence refers to the number of cases of disease or infection existing in a population at a given point of time. The term morbidity is usually used synonymously with "incidence" and normally expressed with regard to 1000 or 10.000 population. Information regarding malaria morbidity is often based on recorded hospital admissions or medical consultations. In

464 Becher, H.; Kynast-Wolf, G.; Sié, A. et al. (2008), p. 112.

465 TUTEJA, R. (2007), p. 4673.

466 Hammer, G.P.; Somé, F.; Müller, O. et al. (2006), doi:10.1186/1475-2875-5-47.

467 Becher, H.; Kynast-Wolf, G.; Sié, A. et al. (2008), p. 106.

468 Becher, H.; Kynast-Wolf, G.; SiÉ, A. et al. (2008), p. 106. 
areas of high endemicity with a large proportion of asymptomatic carriers the morbidity may severely underrepresent the incidence (of parasitation) as it only refers to clinical cases. ${ }^{469}$ Moreover, passive case detection data "almost always vastly underrepresent the true case incidences". ${ }^{470}$

One of the earliest methods used for estimation of the amount of malaria in a given locality is that of determining the proportion of persons with an enlargement of the spleen. Even though it is only a crude measure, it is still widely used. The proportion of enlarged spleens in a sample of the population is known as the spleen rate. ${ }^{471}$ The other important measure of the prevalence of malaria in an area is the evaluation of the proportion of persons in a given community who harbor malaria parasites in their blood. Blood examinations are used to calculate the parasite rate, which is normally done for different age groups. The infant parasite rate is of special importance as it is a good indicator of recent transmission of malaria. ${ }^{472}$

Malaria mortality represents the number of deaths from malaria, usually per 100.000 of the population and should be distinguished from the fatality rate, which is the number of deaths in relation to all cases of malaria (or all cases of infections with Plasmodium falciparum) ${ }^{473}$ In many cases, such mortality data only include deaths which occurred in health facilities or that are reported to them. ${ }^{474}$

\subsubsection{Measures of Transmission Intensity}

For most malaria-endemic areas, there is no reliable data on the actual number of infections. When comprehensive information is missing, it may be feasible to conduct malaria surveys in young children to derive statements on malaria transmission risks. ${ }^{475}$ In most endemic countries, children represent the most significant reservoir of infection as they tend to have the highest prevalence and density of parasites. ${ }^{476}$ Because of the scarcity of reliable clinical malaria case data in sub-Saharan Africa, many studies have used other malariological indices but it should always be kept in mind that they do not measure actual malaria incidence. ${ }^{477}$

469 Gilles, H.M. (19932), p. 129.

470 Carter, R. \& Mendis, K. (2006), p. 187.

471 Panjarathinam, R. (1990), p. 40.

472 Gilles, H.M. $\left(1993^{2}\right)$, pp. $134 f$.

473 Gilles, H.M. $\left(1993^{2}\right)$, p. 129.

474 SNow, R.W. \& HAY, S.I. (2006), p. 189.

475 Martens, P. (1998), p. 62.

476 BeIER, J.C. (1998), p. 530.

477 Mabaso, M.L.H.; Craig, N.; Ross, A. \& Smith, T. (2007), p. 33. 
While morbidity and mortality rates measure the actual burden of malaria, other indicators focus on the transmission process. Approaches for evaluating the dynamics of malaria parasite transmission include the determination of vectorial capacity or EIR. ${ }^{478}$ The entomological inoculation rate (EIR) is the number of infective mosquito bites per human per unit time. ${ }^{479}$ This measure is routinely used to estimate the intensity of transmission under field conditions and is calculated as the product of the mosquitoes' biting rate and the proportion of mosquitoes carrying sporozoites in their salivary glands (sporozoite rate). In malaria-endemic areas of Africa, EIRs usually range from less than 1 to more than 1000 infective bites per year. ${ }^{480}$ However, some factors may lead to lower infection rates than predicted by the EIR, including (acquired or innate) immunity and small host size ${ }^{481}$. The entomologic inoculation rate (EIR), which is routinely determined by observing human landings on adult males, may have the inherent flaw of overestimating the risk to large parts of the population since the biting rate of malaria vectors per host depends on the person's body mass. Moreover, the actual infection rate is not proportional to EIR since the probability that an inoculation is effective decreases as the EIR increases. ${ }^{482}$

RoBert et al. (2003) criticized that the infectivity success rate is rarely taken into account for estimations of transmission pressure. In endemic areas, it typically ranges from $5 \%$ to $26 \%$, with lower success rates in regions of higher transmission (mainly due to protective immunity and multiple infections). ${ }^{483}$

Entomological surveys are necessary to determine the characteristics of vector populations. Such surveys can give important insights into the composition of local mosquito populations, including information on the (relative) abundance of malaria vectors, their vectorial competence and actual parasitation rate. Three techniques are commonly used: The Human landing catch (HLC) is the most accurate method to determine vector-to-host contact, but it is also the most risky one since it deliberately exposes test persons to potentially infectious mosquito bites. Moreover, its results tend to be subjective, depending on both the motivation and skill of mosquito collectors. ${ }^{484}$ Pyrethrum spray capture (PSC) is an alternative method for sampling endophagic mosquitoes since it relies on an indoor insecticide application followed by a collection of mosquitoes falling down onto white sheets. ${ }^{485}$ Light trap capture (LTC) is perhaps the most widely used method and attracts mosquitoes to an electric light bulb located inside a wire net. However, this

478 BEIER, J.C. (1998), p. 528.

479 Mabaso, M.L.H.; Craig, M.; Ross, A. \& SMith, T. (2007), p. 33.

480 Beier, J.C.; Killeen, G.F. \& Githure, J.I. (1999), p. 109.

481 Smith, T.; Maire, N.; Dietz, K. et al. (2006), p. 15.

482 Smith, T.; Killeen, G.; Lengeler, C. \& Tanner, M. (2004), p. 80.

483 Robert, V.; Macintyre, K.; Keating, J. et al. (2003), p. 170.

484 Mathenge, E.M.; Killeen, G.F.; Oulo, D.O. (2002), p. 68.

485 Govella, N.J.; Chaki, P.P.; Geissbuhler, Y. et al. (2009), doi:10.1186/1475-2875-8-157. 
method attracts a large variety of insects ${ }^{486}$, and has been found to underestimate the relative abundance of some anthropophilic species. Light traps hung besides occupied bednets can help to overcome this limitation. ${ }^{487}$ Different collection methods often yield incomparable results since attract mosquitoes by different stimuli. ${ }^{488}$

\subsubsection{Levels of Endemicity}

The spleen rate or parasite rate can be used to estimate the degree of endemicity in a given area:

- hypoendemicity: spleen rate (or parasite rate) in children (two to nine years) < $10 \%$;

- mesoendemicity: spleen rate (or parasite rate) in children (two to nine years) between $11 \%$ and $50 \%$;

- hyperendemicity: spleen rate (or parasite rate) in children (two to nine years) constantly $>50 \%$; adult rates also high ( $>25 \%$ );

- holoendemicity: spleen rate (or parasite rate) in children (two to nine years) constantly $>50 \%$ but adult rates low. ${ }^{489}$

In their studies in Kenya, Омимво et al. (1998) defined three levels of stable endemicity:

- high endemicity: more than $60 \%$ of the population are infected;

- moderate endemicity: $20 \%$ to $59 \%$ of the population are infected;

- low endemicity: less than $20 \%$ of the population are infected. ${ }^{490}$

The authors justified the distinction between three levels of endemicity by pointing out their relevance for the development of immunity and the clinical outcomes of infections:

«These definitions assume that where less than one in five of the
childhood population is found to have evidence of infection on cross-
sectional survey, parasite exposure in childhood will be so low that
disease incidence may simply be a function of parasite encounters
and not greatly modified by acquired immune responses. In these
areas, disease risk in childhood has been shown to be low and
spread evenly across all age-groups. Conversely, communities which
experience a high frequency of infection ( $60 \%$ or greater) will
represent a high rate of parasite exposure from birth, early
acquisition of immunity, a concentration of disease risk within the

486 Serfling, R.E. (1952), p. 1020.

487 Mathenge, E.M.; Killeen, G.F.; Oulo, D.O. (2002), p. 68.

488 Serfling, R.E. (1952), p. 1020.

489 Gilles, H.M. $\left(1993^{2}\right)$, p. 136.

490 Омиmвo, J.A.; Ouma, J.; Repuoda, B. et al. (1998), p. 12. 
first 2 years of life and a paradoxically lower risk of disease throughout childhood compared with settings with moderate transmission (i.e. with parasite ratios of 20\%-59\%). Among the latter, disease risk is spread over the first 5 years of life and more commonly involves pathologies with cerebral involvement. $\gg^{491}$

Knowledge about the level of malaria endemicity in a region are not only important for risk predictions but also for planning intervention measures and assessing their outcomes.

\subsubsection{Limitations of Malaria Surveys and Statistics}

Malaria surveys and statistics in many malarious regions of the world suffer from several restrictions:

- The place for which malaria is reported is often not identical with the place of transmission. Patients may have traveling (thus importing a malaria case), and even when staying at home, they may not visit the nearest clinic.

- Diagnostic methods, particularly in the developing world, differ enormously, even within countries and parts thereof. In many malarious regions, malaria diagnosis is not verified with blood tests.

- People suffering from malaria may not visit a clinic, for example because treatment costs may seem prohibitive to them. ${ }^{492}$

Since these uncertainties tend to add up, all limitations should be kept in mind when interpreting or analyzing data, particularly when addressing spatiotemporal transmission pattern by geographic information systems.

\subsubsection{Malaria Mapping}

The interest in malaria mapping arose largely during the colonial age. Since then, there have been both periods of waning and increasing interest. In the past two decades, the arrival of geographic information systems (GIS) and remote sensing have coincided with a renewal of efforts, with dynamic and interactive maps being seen as promising tools for public health planning.

491 Omumbo, J.A.; Ouma, J.; Repuoda, B. et al. (1998), p. 12.

492 Sipe, N.G. \& Dale, P. (2003), doi:10.1186/1475-2875-2-36. 
Reasons for malaria mapping and monitoring include the determination of optimal intervention strategies (e.g. magnitude, timing, spatial focus of control program) and the assessment of control interventions. ${ }^{493}$ Moreover, in resource-constrained environments, dynamic malaria risk maps could be used for optimizing the distribution of antimalarials. ${ }^{494}$

\subsubsection{History of Malaria Mapping}

Before the introduction of geographical information systems, there were only a few maps which depicted the worldwide or regional pattern of malaria transmission. These early maps were commonly based on simple climatic or geographical isolines and on expert opinion. Empirical studies were only carried out in a few cases. Consequently, the high degree of spatial and temporal heterogeneity of transmission was not properly taken into account. ${ }^{495}$ Since these maps are not based on clear and reproducible numerical definitions, their comparative value is limited. ${ }^{496}$

The map depicting important tropical diseases (figure Fehler: Referenz nicht gefunden) was prepared in 1942 by the German Ministry for Colonial Affairs. Based on the expertise of two doctors serving in the German military's medical corps, it identified risk areas for yellow fever, malaria, the plague, trypanosomiasis (sleeping sickness) and hookworm infections. Regarding malaria, the map distinguished between areas of permanent and seasonal transmission, thus offering a very coarse categorization of malaria risk.

493 Tatem, A.J.; Goetz, S.J. \& Hay, S.I. (2004), p. 34.

494 HAY, S.I.; SNow, R.W. \& Rogers, D.J. (1998), p. 311.

495 HaY, S.I.; Omumbo, J.A.; Craig, M.H. \& Snow R.W. (2000), p. 191.

496 Craig, M.H., Snow, R.W. \& le Sueur, D. (1999), p. 105. 


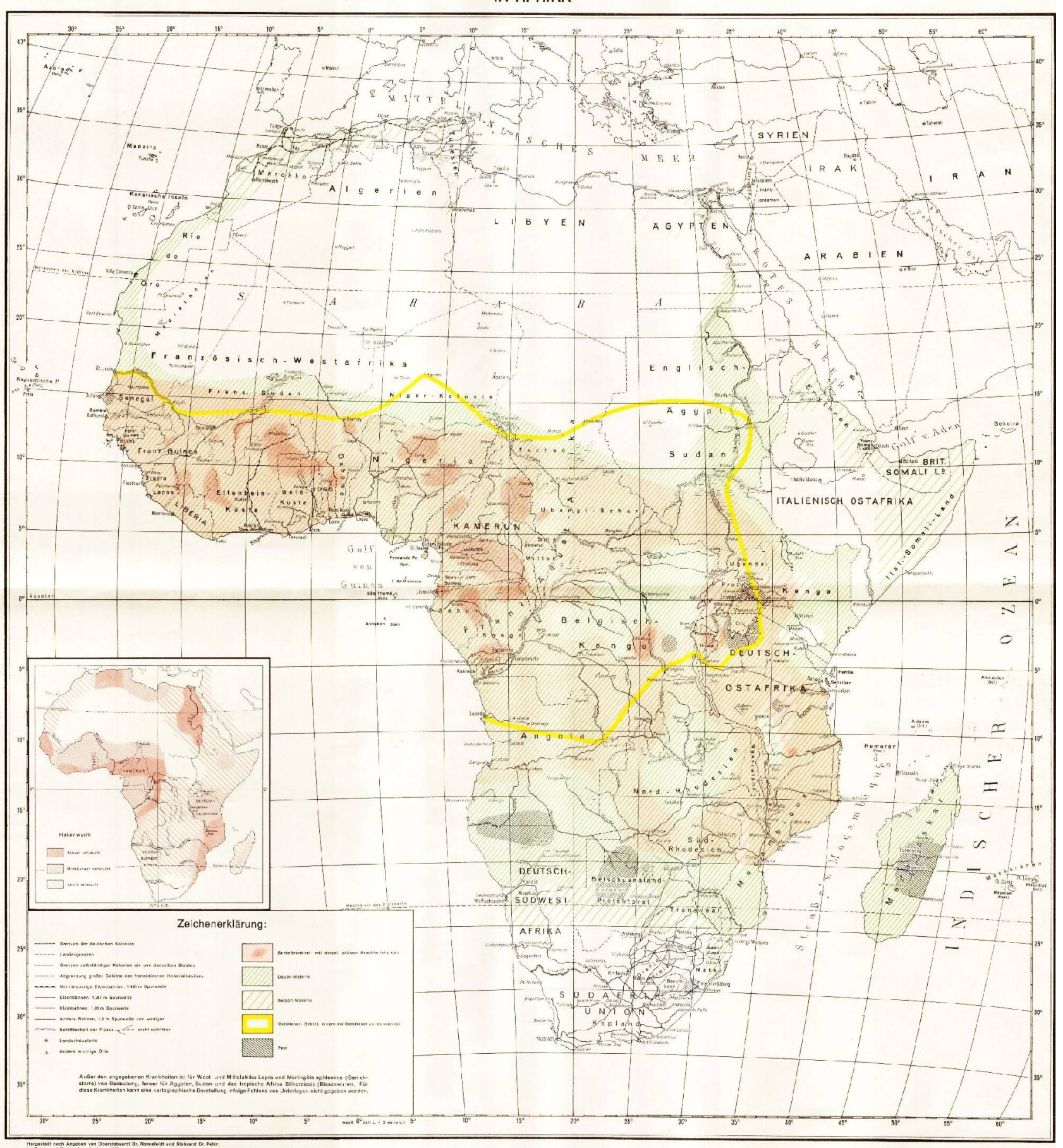

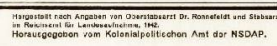

Mafistab 1: 15090000

Figure 26: Colonial map depicting tropical diseases in Africa ${ }^{497}$

497 Kolonialpolitisches Amt der NSDAP (Ed.) (1942). 


\subsubsection{Malaria Mapping Today}

While most current maps of malaria transmission are still based on relatively rough risk estimates, there have also been attempts to empirically validate such maps by using either malaria incidence or transmission intensity data. Such attempts have been relatively rare, however. For example, no empirical map of the global distribution of endemic malaria was produced between 1968 and the very recent publication of the "World Malaria Map" in 2009.498

The MARA/ARMA project (Mapping Malaria Risk in Africa / Atlas du Risque de la Malaria en Afrique), a collaborative network of African scientists and institutions, aims at a comprehensive, empirical and standardized set of maps of malaria transmission in Africa based on all data on malaria in Africa available in formal and informal literature. The first continental model was based on the assumption that malaria transmission is mainly driven by climate. ${ }^{499}$ One of the first objectives of MARA was to find the limits of stable malaria transmission. ${ }^{500}$ MARA scientists also collect parasite prevalence data to validate and improve theoretical, climate-based models. ${ }^{501}$

498 Hay, S.I.; Guerra, C.A. ; Gethin, P.W. et al. (2009), p. 286.

499 Hay, S.I.; Omumbo, J.A.; Craig, M.H. \& Snow R.W. (2000), p. 192; Gemperli, A.; Sogoba, N.; Fondjo, E. et al. (2006), p. 1032.

500 Craig, M.H., Snow, R.W. \& le Sueur, D. (1999), p. 105.

501 Gemperli, A.; Sogoba, N.; Fondjo, E. et al. (2006), p. 1032. 


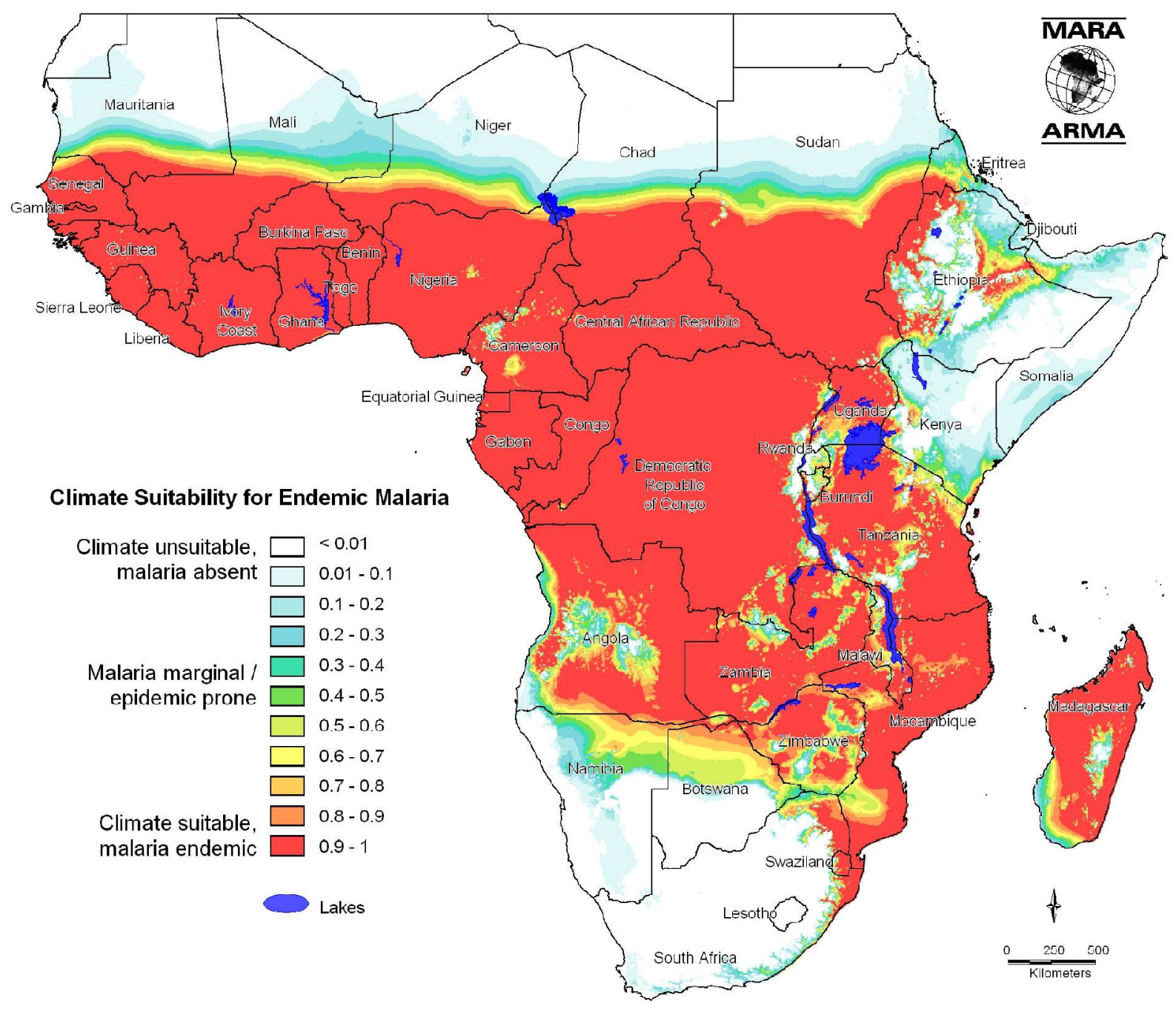

Figure 27: Climate suitability for endemic malaria in sub-Saharan Africa ${ }^{502}$

Two major drawbacks of the MARA data are that compilations of prevalence data comprise survey results from different seasons and that these surveys were based on non-standardized age groups of the population. This makes it difficult to account for the seasonality and age dependence of the malaria prevalence. ${ }^{503}$

KLEINSCHMIDT et al. (2001) produced a malaria distribution map for West Africa (figure 28) that is based on both actual malaria prevalence and a model driven by environmental parameters and vector-to-host contact: For 450 data points scattered throughout large parts of West Africa, a total of about 250.000 children between the ages of 2 and 10 were surveyed for malaria parasites, and four classes of malaria prevalence were distinguished, namely low

502 MARA/ARMA, http://www.mara.org.za/pdfmaps/AfDistributionGrad.pdf.

503 Gemperli, A.; Sogoba, N.; Fondjo, E. et al. (2006), p. 1033. 
incidence (less than $10 \%$ of the children infected with Plasmodium falciparum), intermediate incidence (10 to $30 \%$ of the children infected), high incidence (30 to $70 \%$ of the children infected) and very high incidence (more than $70 \%$ of the children infected with Plasmodium falciparum). In addition to ecological factors (temperature, rainfall, drainage density), the population density was used as a (very rough) proxy for vector-host-contact: areas with population densities of less than one person per square kilometer were regarded uninhabited, areas with densities of more than 386 persons $/ \mathrm{km}^{2}$ were considered urban. Based on the model outlined above, the authors produced a malaria distribution map (see figure 28) for West Africa which coincided with $77.6 \%$ of the empirical observations. However, the authors expected relatively large errors for urban areas, and that it is not possible to estimate the error for parts of the map not covered by malariometric survey locations. ${ }^{504}$ The uneven distribution of survey locations is in fact a limitation of most existing studies.

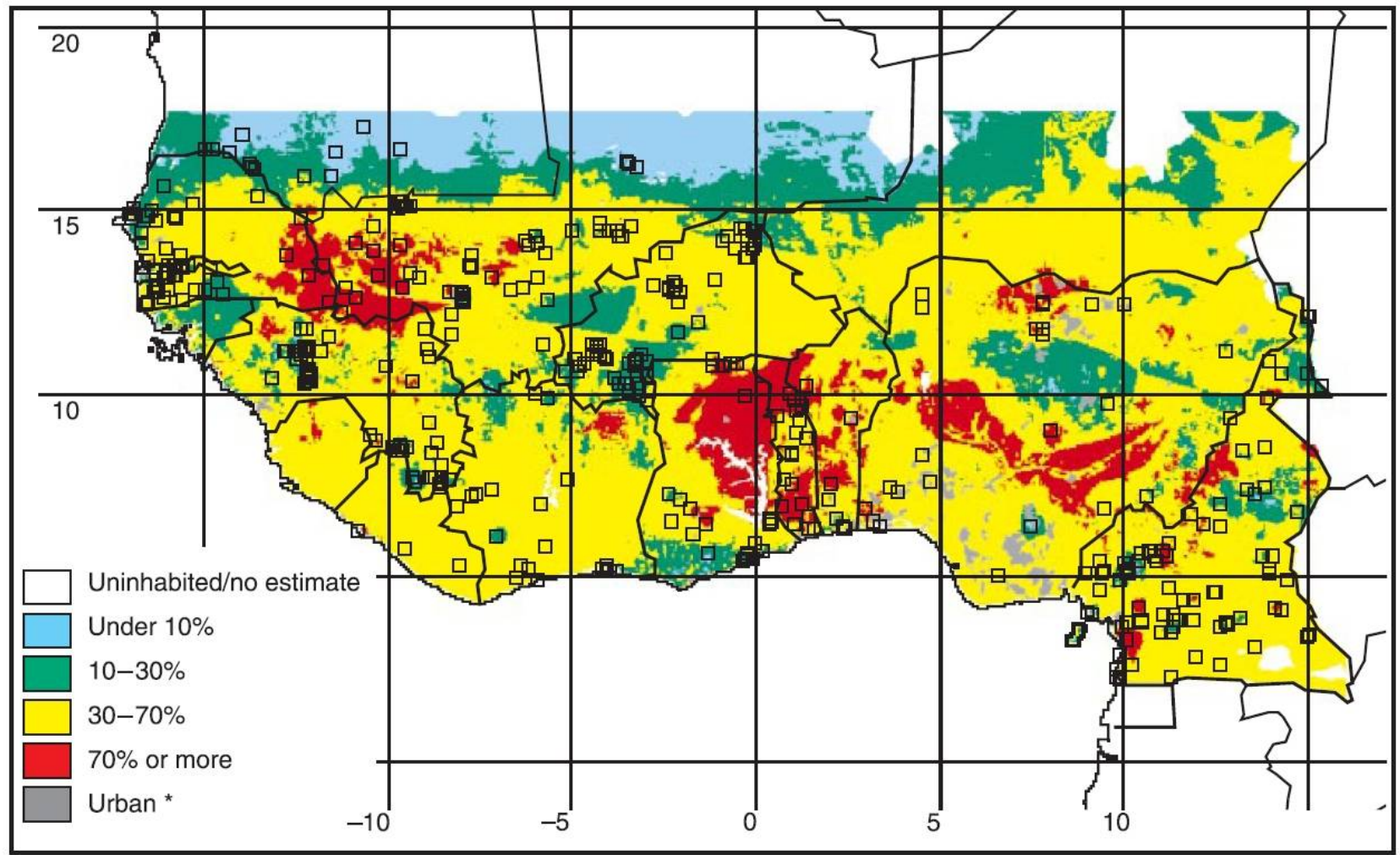

Figure 28: Malaria distribution in West Africa: incidence rates for children ${ }^{505}$

GemperLi et al. (2006) cautioned that many malaria transmission maps may be inaccurate due to a neglect of temporal transmission pattern. Moreover, seasonal effects as well as different measures used for malaria transmission intensity or infection rates make it very difficult to compare malaria transmission maps of different regions:

504 Kleinschmidt, I.; Omumbo, J; Briët, O. et al. (2001), pp. 780-784.

505 Kleinschmidt, I.; Oмumbo, J; Briët, O. et al. (2001), p. 783. 


\begin{abstract}
«Seasonality in transmission maps is an important, but neglected, consideration in malaria mapping, both because the season at which the data were collected may be important, and because the malaria maps themselves may be season-specific. [...] Many surveys are deliberately carried out during the peak transmission season, and this introduces a bias in the maps unless it is allowed for. Seasonality also affects the relationship between prevalence and inoculation rates, because when many inoculations occur over a short period of time the proportion resulting in erythrocytic infections is reduced. $\gg^{506}$
\end{abstract}

At least by name, two dynamic malaria risk maps for the African continent have been developed recently ${ }^{507}$, but both of them are in fact little more than rainfall maps which still require interpretation (see chapter 2.7.6).

\title{
2.6.3 Malaria Modeling and Prediction
}

The production of malaria risk maps often relies on modeling to predict the risk for most of the map, with actual observations of malaria prevalence usually only known at a limited number of specific locations. Estimation is complicated by the fact that there is often local variation of risk that cannot be accounted for because data points of measured malaria prevalence are not evenly or randomly spread across the area to be mapped. ${ }^{508}$ Mathematical malaria transmission models can help to overcome this obstacle, since they use parameters that tend to be more widely available than malariologic data, allowing the conversion of "a set of heterogeneous malariologic indices onto a common scale for risk mapping purposes"509. Moreover, such malaria models can be tools for integrating information from different disciplines ${ }^{510}$ such as ecology, biology and entomology.

The dynamics of malaria transmission depend on both extrinsic (e.g. meteorological) and intrinsic (e.g. immunological) effects. The balance of these factors depends upon the levels of malaria transmission and changes over time. Malaria early warning systems therefore require malaria models that incorporate the temporal dynamics of these factors. ${ }^{511}$

506 Gemperli, A.; Sogoba, N.; Fondjo, E. et al. (2006), p. 1040.

507 Grover-Kopec, E.; Kawano, M.; Klaver, R.W. et al. (2005), doi:10.1186/1475-2875-4-6.

508 Kleinschmidt, I.; Bagayoko, M.; Clarke, G.P.y. et al. (2000), p. 355.

509 Gemperli, A.; Sogoba, N.; Eondjo, E. et al. (2006), p. 1033.

510 McKenzie, F.E. (2000), p. 515.

511 Rogers, D.J.; Randolph, S.E.; Snow, R.W. \& HaY, S.I. (2002), p. 710. 
The history of (mathematical) malaria modeling is nearly as old as the discovery of the transmission process. In the early 1900s, the malariologist RonaLD Ross tried to capture the quantitative links between several determinants of malaria transmission and the disease's transmission dynamics. ${ }^{512}$ Several malaria models have been developed since then, but due to the complexity of the malaria transmission process, these models tend to concentrate on a certain component of the transmission cycle, e.g. the role of human hosts or mosquito vectors. Obviously, such models can never completely predict the dynamics of malaria transmission. Nevertheless, the enormous malaria burden on the one hand and scarce resources available for malaria control mean that there is an urgent need for early warning systems ${ }^{513}$ which need to rely on some sort of malaria transmission model.

\subsubsection{1 'Classic Models' by Ross and Macdonald}

In 1908, RonalD Ross developed the first biomathematical model characterizing the transmission of vector-borne diseases. ${ }^{514}$ Ross divided the population into two fractions, one of them malaria-positive (infected; $Y$ ) and the other malarianegative (not infected). Two factors, the contact rate between malaria vectors and human hosts (C), and the recovery rate of infected individuals ( $r$ ), govern the transmission risk: ${ }^{515}$

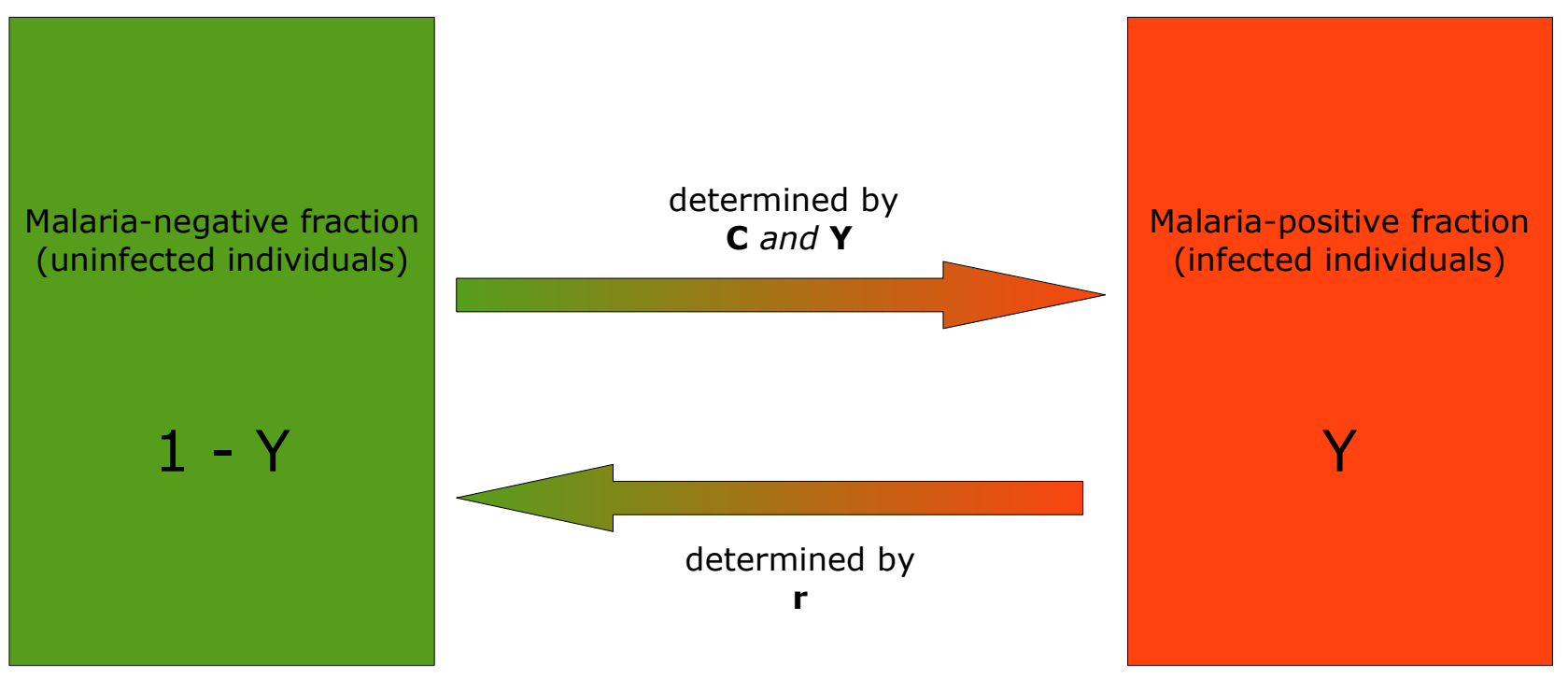

Figure 29: Ross' model of malaria transmission ${ }^{516}$

512 MaRTENS, P. (1998), p. 47.

513 Teklehaimanot, H.D.; Lipsitch, M.; Teklehaimanot, A. \& Schwartz, J. (2004), doi:10.1186/1475-2875-3-41.

514 Zavaleta, J.O. \& Rossignol, P.A. (2004), p. 611.

515 Molineaux, L. (1985), p. 743.

516 Adapted from Molineaux, L. (1985), p. 743. 
According to Ross' model, the number of future infections can be estimated if the present number of infections, the host-to-vector contact rate and the recovery rate are known:

$$
Y_{t+1}=Y_{t}+Y_{t} * C *\left(1-Y_{t}\right)-r Y_{t} 517
$$

Moreover, an equilibrium prevalence can be assumed when the recovery rate compensates for the number of new infections:

$$
Y=1-\frac{r}{C} 518
$$

\begin{tabular}{|ll|}
\hline Symbol & Definition \\
\hline$Y$ & Infected fraction of the population \\
$Y_{t}$ & Infected fraction of the population at time $t$ \\
\hline$C$ & Contact rate between human population and vectors \\
\hline$r$ & Recovery rate of infected individuals \\
\hline
\end{tabular}

Table 18: Variables used in the Ross model

Ross' model provided several important insights into malaria epidemiology. First of all, there is a (non-zero) critical level of the vectorial capacity below which malaria cannot maintain itself; second, above the critical level, the relationship is highly non-linear: close to the critical level, small changes in the vectorial capacity produce large changes in the prevalence of malaria; at higher levels, even large changes in the vectorial capacity produce little or no change in the prevalence of malaria (figure 32). ${ }^{519}$ The vectorial capacity is an index which is proportional to the basic reproduction rate, the number of secondary infections resulting from one primary case. It is defined as the average maximum number of infective contacts between a vector and a host population. ${ }^{520}$ Diseases caused by vector-borne pathogens vary in magnitude through space and time much more than directly transmitted pathogens: while basic reproduction rates for vector-borne diseases reach hundreds or even thousands, they are typically less than ten for directly transmitted pathogens. ${ }^{521}$ 


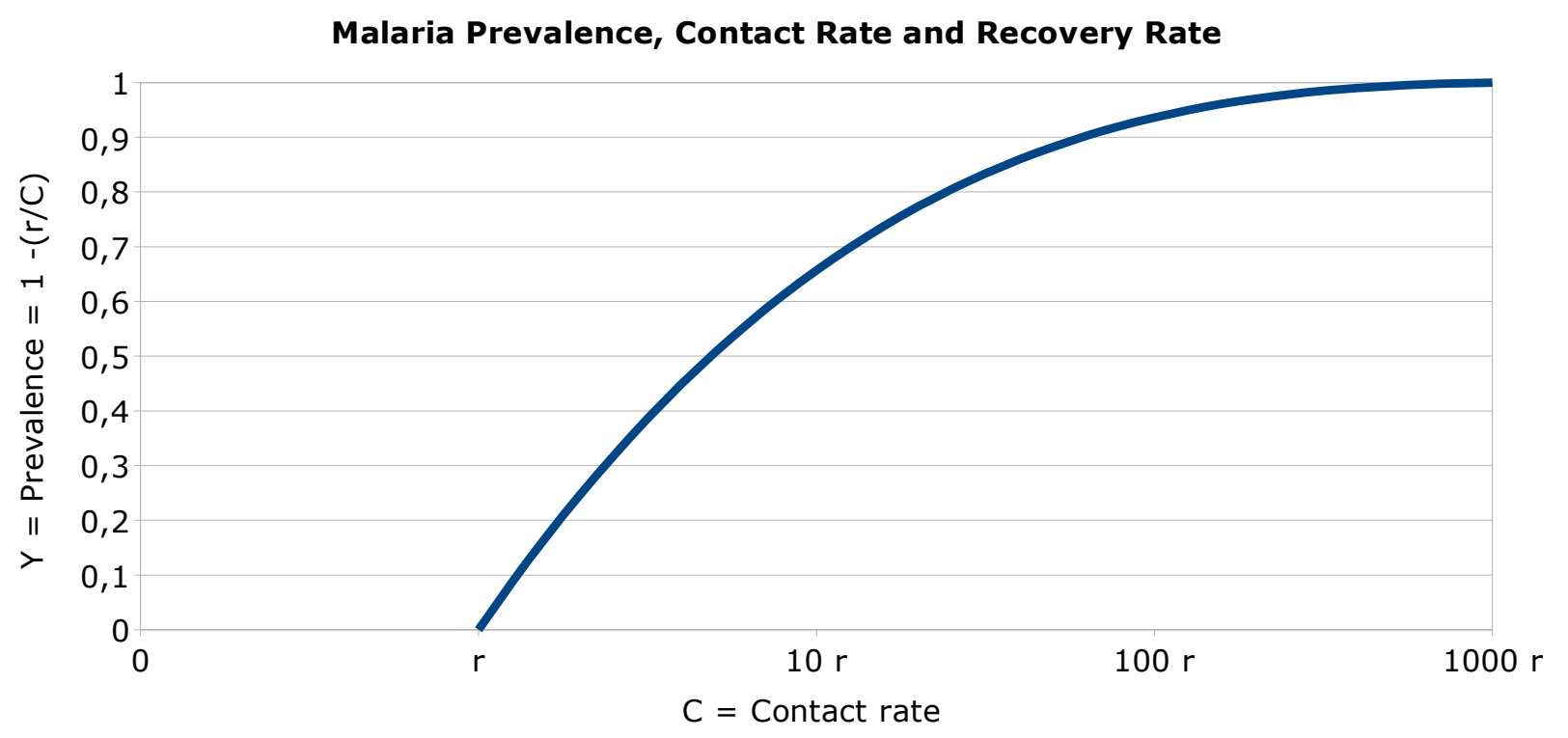

Figure 30: Relevance of the vector-to-host contact rate t22 $^{522}$

As illustrated by figure 31 , a drastic reduction in the prevalence of malaria (the parasite reservoir), without alteration of the vectorial capacity is followed by a return to the previous status quo; the impact of a reduction in the vectorial capacity (short of the critical level) seems to wear off with time until a new equilibrium is reached. A final insight is that the critical vectorial capacity is lower for longer-lasting infections (it is lower for Plasmodium vivax than for Plasmodium falciparum). ${ }^{523}$ 


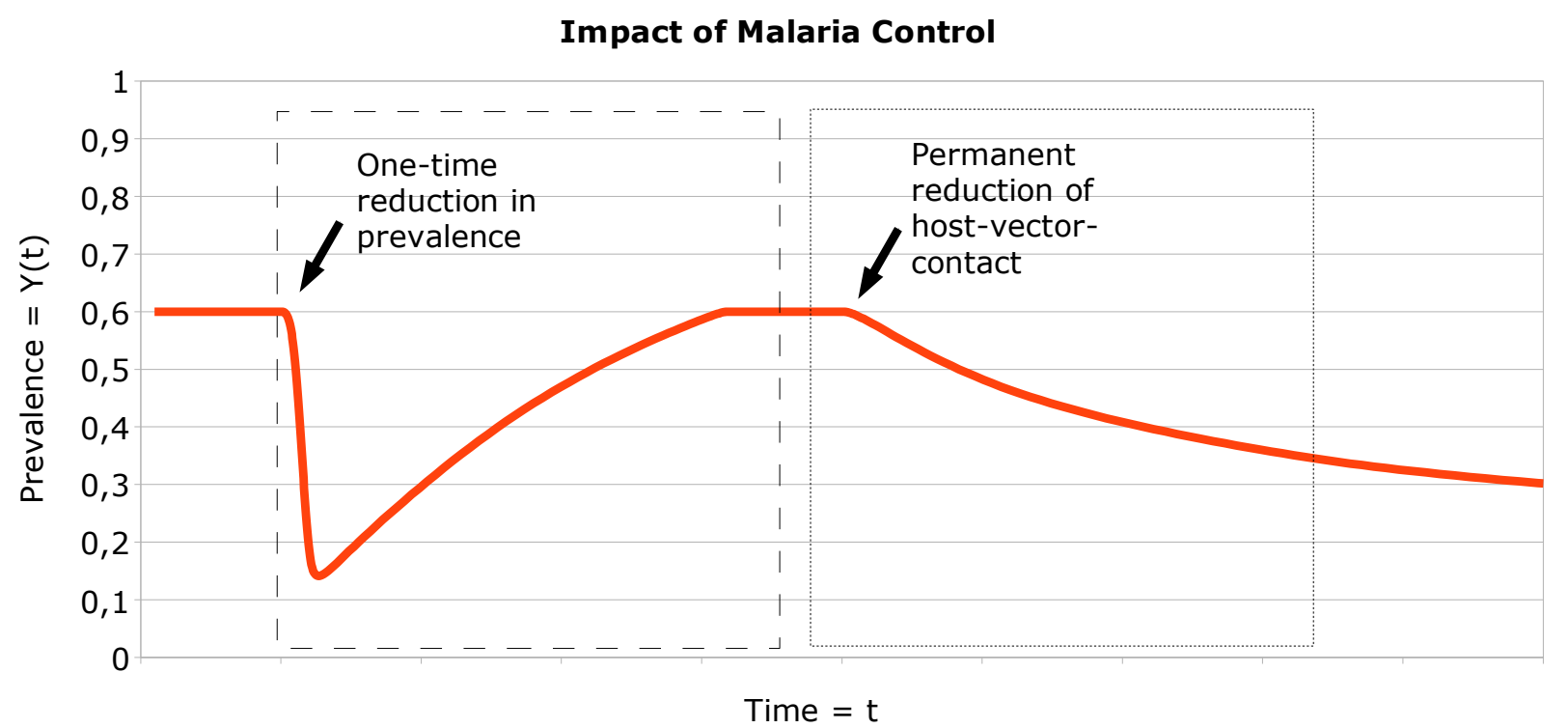

Figure 31: Impacts of reductions in malaria prevalence and host-to-vector contact ${ }^{524}$

Ross' model was popularized for malaria in 1952 by George MaCdonald (figure 33) and focused on the basic reproduction rate, i.e. the number of secondary infections that can arise from a single primary case. ${ }^{525}$ MACDONALD identified the longevity of mosquito vectors as the single most important variable that determines the transmission pressure. ${ }^{526}$ Both Ross and MACDONALD assumed that there are certain thresholds of mosquito density and longevity below which transmission is not maintained and that mosquito longevity is a greater determinant of risk than abundance. ${ }^{527}$

524 Adapted from Molineaux, L. (1985), p. 744.

525 Zavaleta, J.O. \& Rossignol, P.A. (2004), p. 611.

526 Hoshen, M.B. \& Morse, A.P. (2004), doi:10.1186/1475-2875-3-32.

527 Zavaleta, J.O. \& Rossignol, P.A. (2004), p. 611;

Diuk-Wasser, M.A.; Toure, M.B.; Dolo, G. et al. (2005), p. 726. 


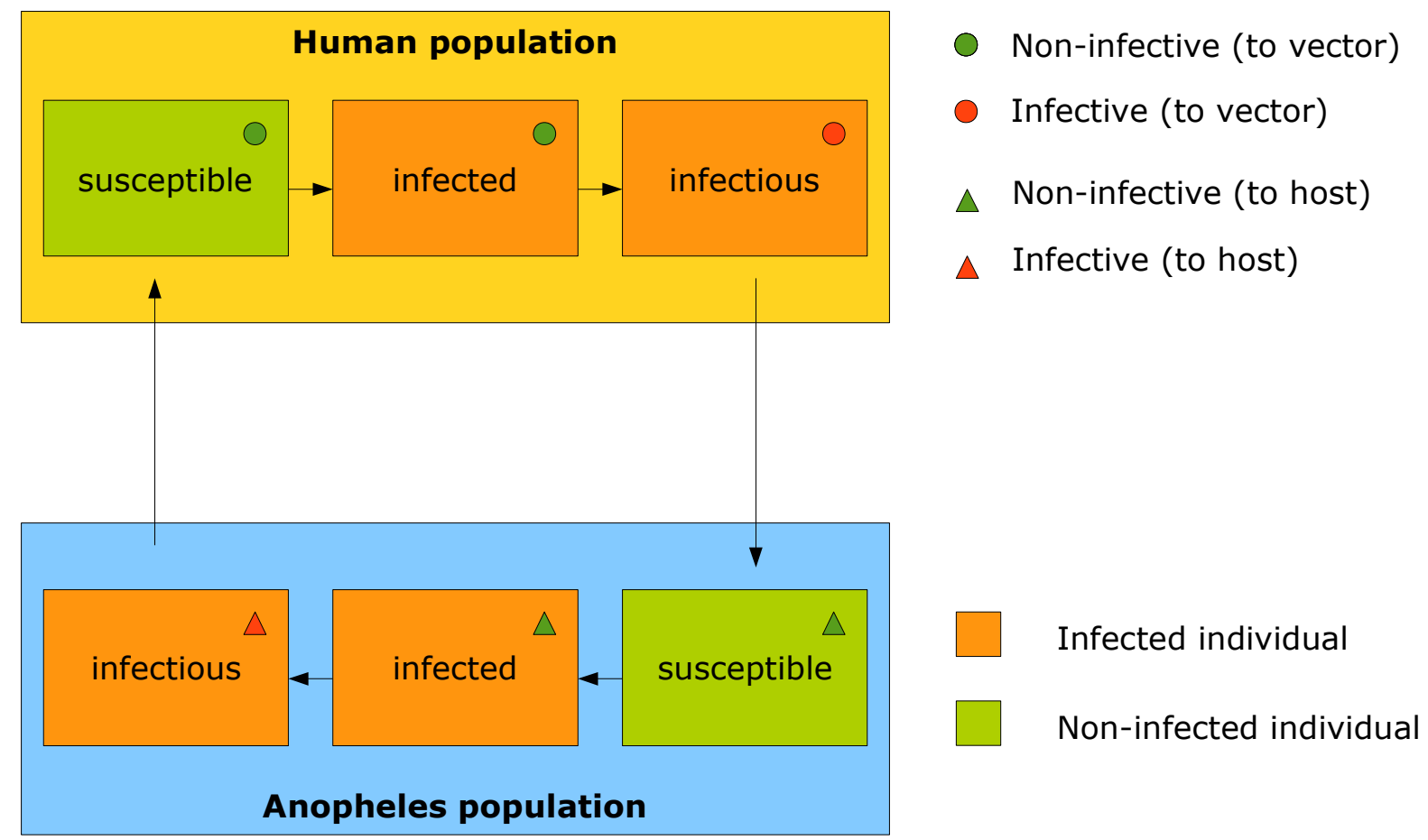

Figure 32: States of the human and anopheles population in the MACDONALD mode/528

According to MACDONALD, the level of endemicity is most sensitive to changes in vector survival and the duration of sporogony, less sensitive to changes in biting behavior and least sensitive to vector density and the recovery rate. ${ }^{529}$ Even though MACDONALD was aware that such a sensitivity analysis which identifies the weakest point in the transmission process does not automatically identify the factors to focus on in malaria control, he stated that

«The worst conditions in Africa could [...] be overcome by an increase in the daily mortality of the vector from about 5 per cent to about 45 per cent. ${ }^{530}$

Even though the malaria models developed by Ross and Macdonald were the first systematic attempts to quantify the impact of different factors on malaria transmission, they are nevertheless simplifications of the truth. The models did neither account for the incubation periods of the parasite in mosquitoes nor did they look at individual behavior, mosquito habitat distribution and the resulting spatial heterogeneity of transmission. ${ }^{531}$

528 Adapted from McKenzie, F.E. \& Samba, E.M. (2004), p. 95.

529 Molineaux, L. (1985), p. 745.

530 Molineaux, L. (1985), p. 745.

531 RuAn, S.; XIAO, D. \& BeIER, J.C. (2008), p. 1100. 


\subsubsection{The Garki Model}

From 1969 to 1976, the WHO and the government of Nigeria carried out a large field study on the epidemiology and control of malaria in the Garki region of Nigeria ("Garki Study"). The development of a mathematical model of transmission and its testing against hard data were part of this effort. ${ }^{532}$

The Garki model is a dynamic compartment model which considers basic characteristics of immunity to malaria and the dynamics of the interactions among humans, mosquitoes and malaria. Given entomological measures of transmission intensity as input, the model predicts age-specific prevalence and vice versa. ${ }^{533}$

The model was developed for field data from northern Nigeria and describes transitions among seven categories of hosts distinguished by their infection and immunological status. ${ }^{534}$ It has been optimized for use under the conditions found in the Sudan savanna, where Anopheles gambiae, Anopheles arabiensis and Anopheles funestus are the main vectors. ${ }^{535}$ The model attempts to represent the natural course of infections and their transmission: Man is born into the non-immune non-infected ("negative") status $x_{1}$ (passive immunity and the transfer of infections from mother to child are ignored). Non-immune negatives are inoculated at a rate $h$ and transferred to the incubating class $x_{2}$ in which they remain for a fixed incubation period of $N$ days. After that they become infected ("positive") and are now infective to mosquitoes (class $y_{1}$ ). Infectivity is lost at a constant rate $\alpha_{1}$, at which persons move to the state $y_{2}$ in which they are non-infective but still positive. A person may now either recover and return to the non-immune negative state, or progress to the immune positive $\left(y_{3}\right)$ and immune negative states $\left(x_{3}\right) .{ }^{536}$

Most patients go through several cycles $x_{1} \rightarrow x_{2} \rightarrow y_{1} \rightarrow y_{2} \rightarrow x_{1}$ before moving to $y_{3}$. As the inoculation rate increases, the actual recovery rate decreases and superinfections may therefore prevent recovery. Thus an increasing proportion of persons travel the route $x_{1} \rightarrow x_{2} \rightarrow y_{1} \rightarrow y_{2} \rightarrow y_{3}$ without returning to $x_{1}$. Once in $y_{3}$, they may either remain there or become reinfected after recovery and return to $y_{3}$ through $x_{4}{ }^{537}$

532 Molineaux, L. \& Gramiccia, G. (1980), pp. 11; 15.

533 Gemperli, A.; Sogoba, N.; Fondjo, E. et al. (2006), p. 1033.

534 Gemperli, A.; Sogoba, N.; Fondjo, E. et al. (2006), p. 1043.

535 Molineaux, L. \& Gramiccia, G. (1980), p. 289.

536 Molineaux, L. \& Gramiccia, G. (1980), pp. $262 f$.

537 Molineaux, L. \& Gramiccia, G. (1980), p. 265. 


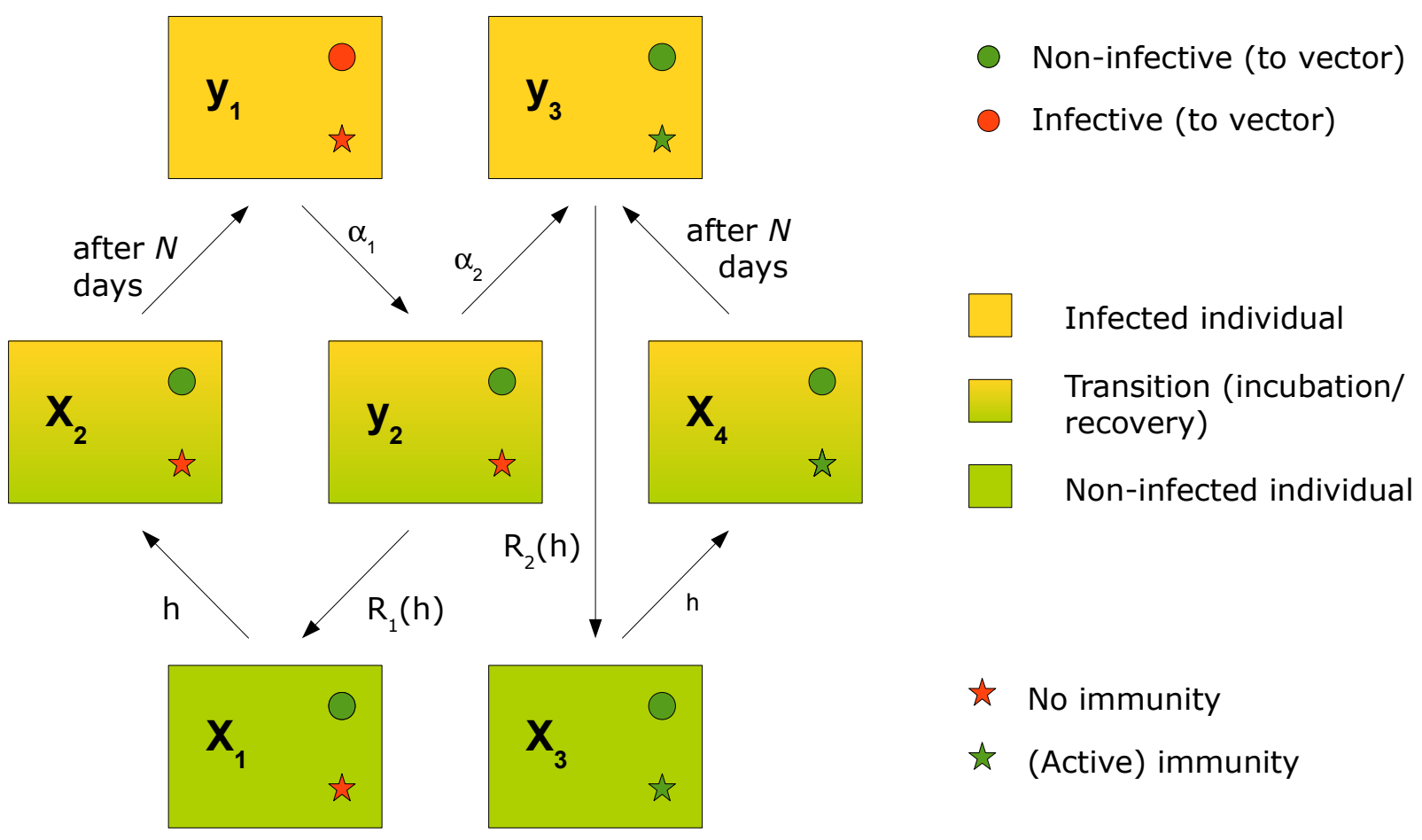

Figure 33: States and transitions in the Garki mode/538

The entire information on the vector-population is incorporated into one timedependent variable, the vectorial capacity $C(t)$. It is defined as the number of bites on man that the vectors distribute on day $t$ after the completion of the sporogonic cycle during the rest of their life. It is thus the number of potential infective contacts an individual makes, through the vector population. ${ }^{539}$

A vector population can be characterized by the vector density $m$ (number of vectors per human individual), their man-biting habit $a$ and the daily survival probability $p$. An individual is thus bitten by $m * a$ vectors in one day; a fraction of $p^{n}$ vectors survive the sporogonic cycle. If there are several vector populations with different characteristics, the total vectorial capacity can be calculated as the sum of the vectorial capacities of the individual populations:

$$
C(t)=\sum_{j=1}^{J} \frac{m_{j}(t) * a_{j}{ }^{2} * p_{j}^{n}}{-\ln p_{j}} 540
$$


The biting behavior is contained twice in this equation - a primary bite leads to the infection of the vector, and a secondary bite to the infection of a new host. The vector life expectancy is also of special importance as it contributes exponentially. ${ }^{541}$

Tables 19 and 20 explain the symbols used in the Garki model and the calculation of the vectorial capacity. The input parameters fall into two categories: constants which govern the interaction between parasites and man (e.g. the rates describing loss of infectivity, development of immunity and the speed of recovery; these constants are presented in table 20), and variables which distinguish one epidemiological situation from another (e.g. vectorial capacity).${ }^{542}$

\begin{tabular}{|ll|}
\hline Symbol & Definition \\
\hline$x_{1}$ & non-immune negatives (uninfected persons without immunity) \\
$x_{2}$ & non-immunes in the incubation stage \\
$x_{3}$ & immune negatives (uninfected persons with immunity) \\
$x_{4}$ & immunes in the incubation stage \\
$y_{1}$ & infectious positives (infected persons which may pass on \\
$y_{2}$ & parasites to vectors) \\
$y_{3}$ & non-immunes, slowly recovering from malaria \\
\hline
\end{tabular}

Table 19: States of human individuals in the Garki model

\begin{tabular}{|c|c|c|}
\hline Symbol & Definition & Estimates \\
\hline h & rate of infection & \\
\hline$\alpha_{1}$ & loss of infectivity rate for infectious positives & 0.002 / day \\
\hline$\alpha_{2}$ & $\begin{array}{l}\text { development of immunity rate for non- } \\
\text { immune positives }\end{array}$ & \\
\hline$R_{1}(h)$ & recovery rate of non-immunes & 0.05 / day \\
\hline $\mathrm{R}_{2}(\mathrm{~h})$ & recovery rate of immunes & $10 \mathrm{R}_{1}(\mathrm{~h})$ \\
\hline$C(t)$ & vectorial capacity & \\
\hline ] & $\begin{array}{l}\text { number of vector populations found in a } \\
\text { region }\end{array}$ & \\
\hline j & index for an individual vector population & \\
\hline $\mathrm{m}$ & vector density (vector number per potential & \\
\hline
\end{tabular}

541 Zavaleta, J.O. \& Rossignol, P.A. (2004), p. 612.

542 Molineaux, L. \& Gramiccia, G. (1980), pp. $273 \mathrm{f}$. 


\begin{tabular}{|lll|}
\hline Symbol & Definition & Estimates \\
\hline a & $\begin{array}{l}\text { human host) } \\
\text { man-biting rate, an index for the } \\
\text { anthropophily/zoophily of a vector population }\end{array}$ \\
\hline $\mathrm{p}$ & daily survival probability of vectors & 0.95 \\
$\mathrm{n}$ & duration of the sporogonic cycle & 10 days \\
$\mathrm{N}$ & duration of the incubation period in man & 15 days \\
\hline
\end{tabular}

Table 20: Parameters used in the Garki model

Even though the Garki model ignores several epidemiologically relevant parameters, including the use of antimalarial drugs, maternal immunity, the delayed appearance of gametocytes and the loss of immunity, it incorporated more about the biology of malaria than previous models, for instance by considering the acquisition of a partial immunity ${ }^{543}$ :

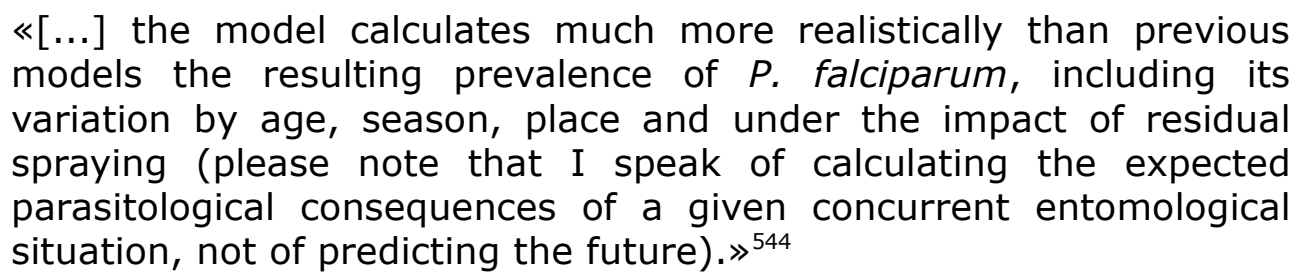

Based on the Garki model, the relationship between different measures of "epidemiological intensity" has been investigated. Figure 35 illustrates that

- (entomological) measurements of the vectorial capacity are difficult at low transmission levels;

- the prevalence or incidence of infection and the proportion of seropositives are sensitive to the vectorial capacity at low levels and may be used for an indirect estimation;

- at high transmission levels, incidence, prevalence and seropositivity are insensitive but the entomological inoculation rate is sensitive to the vectorial capacity and may be used for an indirect estimation. ${ }^{545}$ 


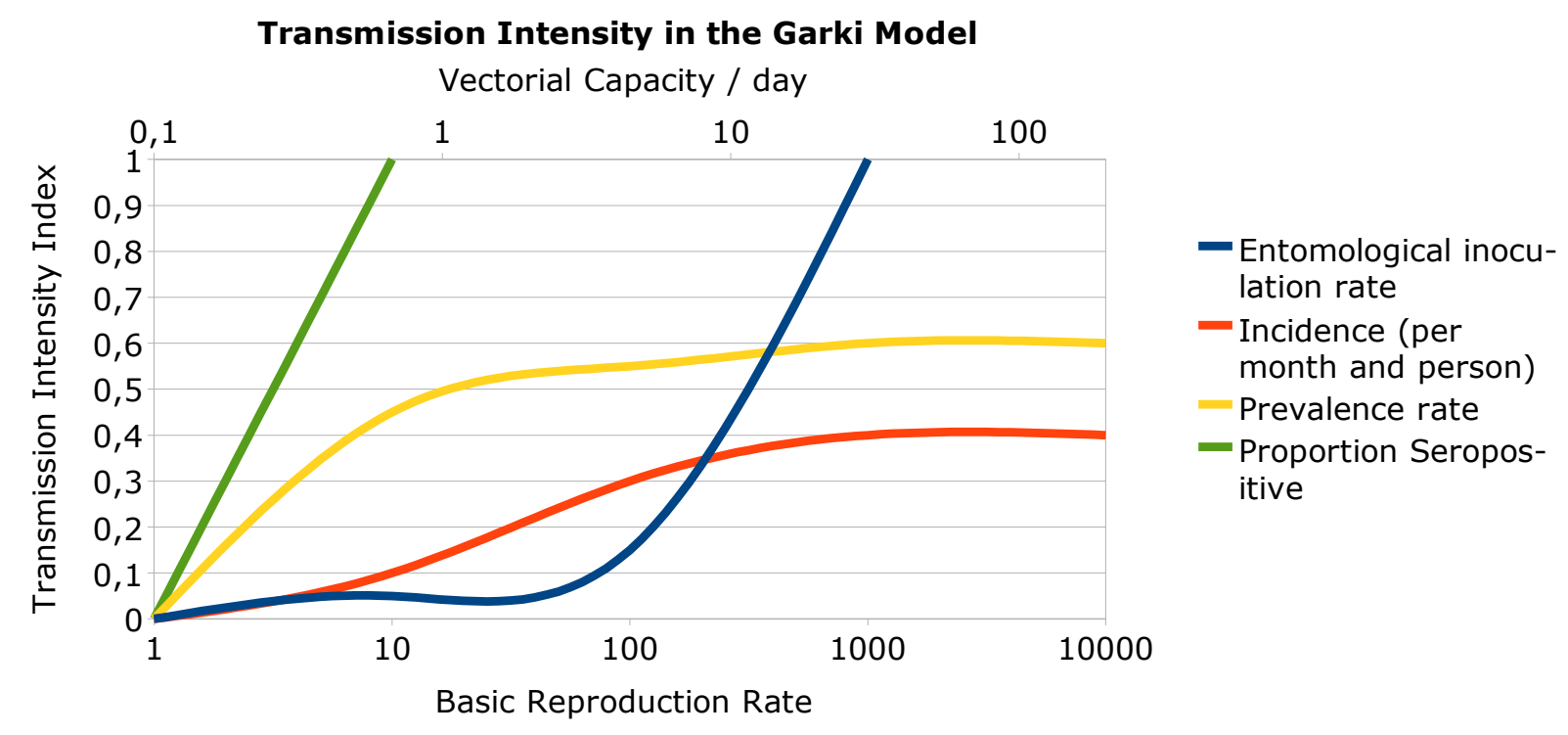

Figure 34: Measures of transmission intensity in the Garki mode/ ${ }^{546}$

The model uses a measure for the entomological inoculation rate $(E)$ that is not always equivalent to the EIR calculated from entomological data because $E$ has an upper limit, and for prevalences above this limit $E$ is below the EIR. ${ }^{547}$

The Garki model and its variants remain to date the most accepted integrated models of malaria transmission dynamics and immunity. ${ }^{548}$ During their global campaign to eradicate malaria (see chapter 2.8.2), the WHO also used transmission models based on vectorial capacity. ${ }^{549}$

Theoretically, 0.01 is the critical vectorial capacity for malaria multiplication (assuming that the mean period of human recovery is 100 days), but field observations showed that in practice vectorial capacities of at least 0.02 to 0.03 are necessary for malaria transmission to be maintained. Seasonal vectorial capacities of 10 to 100 are not rare, implying an epidemic potential of 1000 to 10000 -fold multiplication of malaria cases in non-immune population. ${ }^{550}$

546 Adapted from Molineaux, L. (1985), p. 746.

547 Gemperli, A.; Sogoba, N.; Fondjo, E. et al. (2006), p. 1035.

548 Smith, T.; Maire, N.; Dietz, K. et al. (2006), p. 11.

549 Zavaleta, J.O. \& Rossignol, P.A. (2004), p. 612.

550 WHITE, G.B. (1982), p. 211. 


\subsubsection{Individual-based Models}

In the decades following the Garki study, several malaria transmission models focusing on the human host were developed.

MaRTens (1998) proposed to distinguish three groups: infected, susceptible and immune individuals, which are then divided into different age groups. According to the model, all newborns initially enter the group of susceptible individuals. However, as these individuals grow older, their susceptibility status changes. At the same time, the number of susceptible individuals in a certain age group changes as some become infected and thus leave the group whereas others enter the group as recovered non-immunes. Infected individuals may attain immunity, which typically lasts for 18 months, but lose their immunity in the long run. Finally, a "natural", malaria-independent mortality rate also means that all three subgroups constantly lose a certain percentage of their members. ${ }^{551}$

The rate of infections now depend on the malaria transmission potential found in a region (expressed as the vectorial capacity by the model) and the proportion of infected individuals within the population.

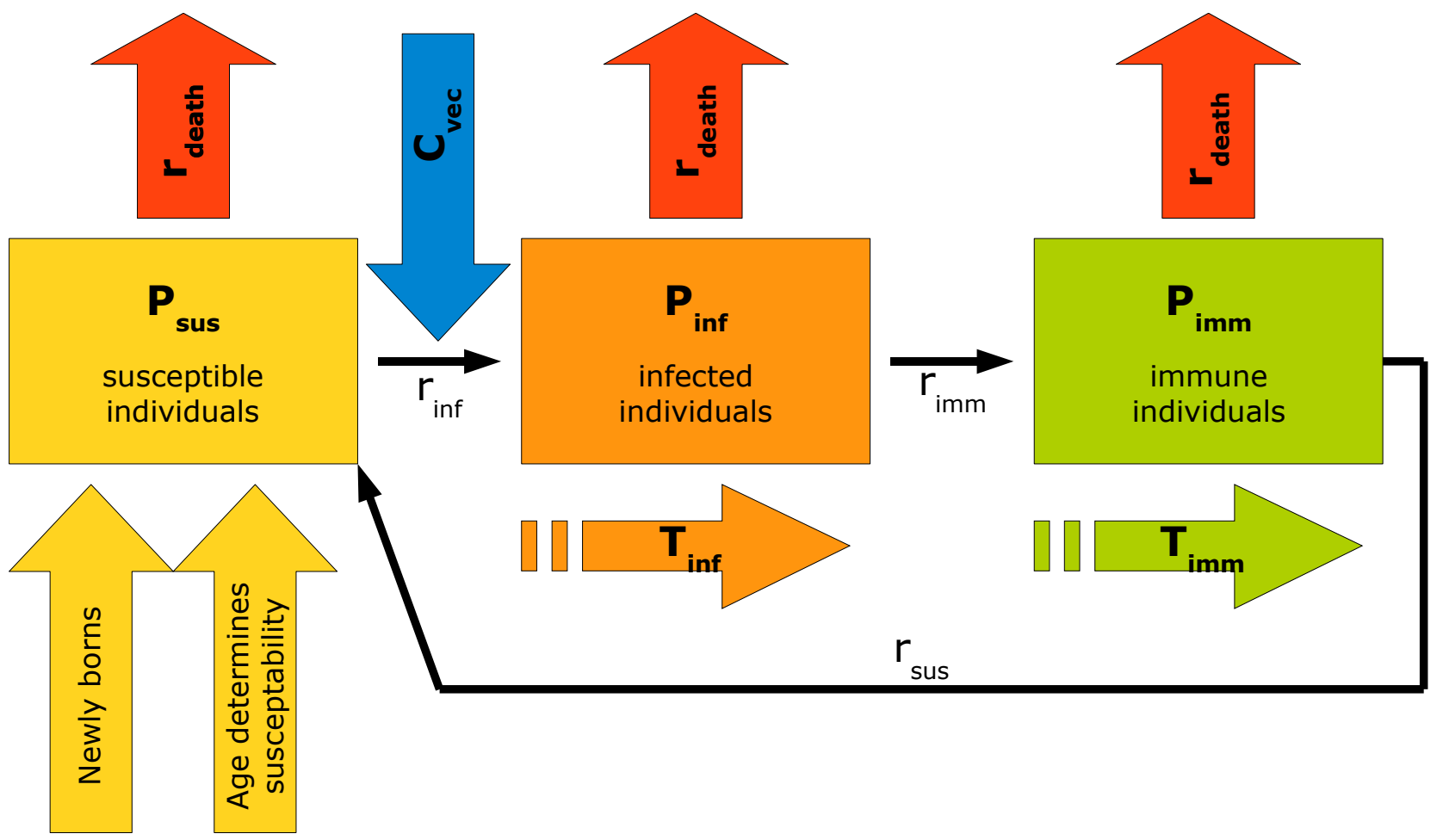

Figure 35: Symbolic presentation of MARTENS' individual-based model ${ }^{552}$

551 Martens, P. (1998), p. 48.

552 Adapted from MARTENS, P. (1998), p. 48. 
Since both infections and immunity last for a certain period of time, the following equations can be derived ${ }^{553}$ :

(1) $\quad r_{\text {inf }}(t)=C_{v e c}(t) * P_{\text {inf }}(t)$

(2) $\quad r_{\text {sus }}(t)=\frac{r_{\text {inf }}(t)}{-e^{r_{\text {inf }}(t) * T_{\text {inf }}-1}}$

(3) $\quad r_{\text {imm }}(t)=\frac{r_{\text {inf }}(t)}{-e^{r_{\text {inf }}(t) * T_{\text {imm }}-1}}$

The number of susceptible, infected and immune persons can now calculated using the following equations:

(4)

$$
\frac{\delta \mathrm{P}_{\text {sus }}}{-\delta \mathrm{t}}+\frac{\delta \mathrm{P}_{\text {sus }}}{\delta a}=\mathrm{r}_{\text {sus }}(\mathrm{t}) * \mathrm{P}_{\text {imm }}-\left[\mathrm{r}_{\text {inf }}(\mathrm{t})+\mathrm{r}_{\text {death }}\right] * \mathrm{P}_{\text {sus }}(\mathrm{t}, \mathrm{a})
$$

(5) $\frac{\delta P_{\text {inf }}}{--\bar{y}}+\frac{\delta P_{\text {inf }}}{\delta a}=r_{\text {inf }}(t) * P_{\text {sus }}-\left[r_{\text {imm }}(t)+r_{\text {death }}\right] * P_{\text {inf }}(t, a)$

(6) $\frac{\delta P_{\text {imm }}}{-----}+\frac{\delta P_{\text {imm }}}{\delta t}+\frac{----}{\delta a}=r_{\text {imm }}(t) * P_{\text {inf }}-\left[r_{\text {sus }}(t)+r_{\text {death }}\right] * P_{\text {imm }}(t, a)$ 


\begin{tabular}{|ll|}
\hline Symbol & Definition \\
\hline$P_{\text {inf }}$ & infected individuals \\
$\mathrm{P}_{\text {sus }}$ & susceptible individuals \\
$\mathrm{P}_{\text {imm }}$ & immune individuals \\
\hline$a$ & age group \\
$\mathrm{r}_{\text {inf }}(\mathrm{t})$ & infection rate of susceptible individuals at time $t$ \\
$\mathrm{r}_{\text {imm }}(\mathrm{t})$ & immunity acquisition rate of infected individuals at time $t$ \\
$\mathrm{r}_{\text {sus }}(\mathrm{t})$ & loss of immunity rate of immune individuals at time $t$ \\
$\mathrm{r}_{\text {death }}$ & death rate \\
$\mathrm{C}_{\text {vec }}(\mathrm{t})$ & Vectorial capacity (during period $t)$ \\
$\mathrm{T}_{\text {inf }}$ & Period of an infection \\
$\mathrm{T}_{\text {imm }}$ & Period of immunity \\
\hline
\end{tabular}

Table 21: Parameters used in MARTENS' individual-based mode ${ }^{554}$

According to Gu et al. (2003), a susceptible individual can be infected according to the individual's susceptibility which is dependent on his or her exposure history. An infected person recovers when infection lasts beyond the individualspecific infection period. Superinfections may result in prolonged recovery. ${ }^{555}$

Repeated exposure leads to a reduced susceptibility, but the effect of immunity on the recovery rate has a ceiling after about 20 infections, when a $\approx 50 \%$ reduction of the duration of the innate infection period is achieved (typically, from 270 days to 135 days without treatment). For regions of low transmission ( 0.5 mosquito bites per person and day), the reductions in susceptibility and the duration of the innate infection period can be calculated as follows:
(1) $\Delta S=\frac{1}{\left(1+N^{2} / 200\right)}$
(2) $\Delta I P=\frac{1}{\left(1+N^{2} / 400\right)}$ for $\mathrm{N} \leq 20 ; \Delta I P=0.5$ for $\mathrm{N}>20$

554 Martens, P. (1998), p. 48.

555 Gu, W.; Killeen, G.F.; Mbogo, C.M. et al. (2003), p. 44. 


\begin{tabular}{|ll|}
\hline Symbol & Definition \\
\hline$\Delta S$ & proportion by which the individual susceptibility is reduced \\
$\Delta \mathrm{IP}$ & $\begin{array}{l}\text { proportion by which the individual infection period is } \\
\text { reduced }\end{array}$ \\
$\mathrm{N}$ & number of infections experienced \\
\hline
\end{tabular}

Table 22: Parameters used in the individual-based model (Gu et al.) $)^{556}$

\subsubsection{Ecological Models}

Experts regard the development of ecological models an essential prerequisite for planning and evaluating vector control interventions. ${ }^{557}$ Ecological niche models (ENM) such as the GARP ("Genetic Algorithm for Rule-set Prediction") and the integrated ecological model developed by Killeen et al. are two examples of models that attempt to take into account the ecologic complexity of mosquito habitats.

Predictive modeling of species' distributions now represents and important tool in biogeography. ${ }^{558}$ Ecological niche models (ENMs) can take two forms: process-based models based on detailed physiological information, and empirical reconstructions based on the known geographic occurrences of a species and the ecological characteristics of the associated landscapes. While process-based models have the theoretical advantage of not being limited by sampling inaccuracies, they require full knowledge of the factors influencing a species' distribution. ${ }^{559}$

The Genetic Algorithm for Rule-set Prediction (GARP) was proposed by STOCKWELL (1991) to improve on existing ecological models by relating species' ecologic niches to heterogeneous rule sets ${ }^{560}$, incorporating both categorical and continuous ecological variables. ${ }^{561}$ With the introduction of geographic information systems, it has become increasingly feasible to model a species' distribution pattern based on its ecological niche ${ }^{562}$ :

«GARP is a superset of other approaches, and should always have greater predictive ability than any one of them. $\gg^{563}$

556 Gu, W.; Killeen, G.F.; Mbogo, C.M. et al. (2003), p. 45.

557 Killeen, G.; Seyoum, A. \& Knols, G.J. (2004), p. 87.

558 Anderson, R.P.; Lew, D. \& Peterson, A.T. (2003), p. 212.

559 Peterson, A.T. (2007), p. 395.

560 Peterson, A.T. \& Cohoon, K.P. (1999), p. 160.

561 Stockwell, D.R.B. \& Peterson, A.T. (2002), p. 10.

562 Peterson, A.T. (2001), pp. 599 f.

563 Peterson, A.T. (2001), p. 600. 
A species habitat is only part of its ecological niche which includes the range of conditions that permit its survival and reproduction. ${ }^{564}$ Ecological niches are areas in which one species can maintain its population without immigration of individuals from other areas. ${ }^{565}$ Realized ecological niches and fundamental ecological niches (potential habitats of a species) need to be distinguished in this context: While a realized ecological niche describes the ecological conditions in a single habitat, the identification of many habitats of a species allows the derivation of potential ranges of its distribution. ${ }^{566}$ The GARP algorithm is used to model fundamental ecological niches by an iterative process of rule selection, evaluation, testing and incorporation or rejection. ${ }^{567}$ Both biotic (e.g. vegetation) and abiotic factors (e.g. climate) are used in this process, but it nevertheless remains difficult to include all of the possible ecological niche dimensions. ${ }^{568}$

Based on this concept, Levine et al. (2004) developed models of the fundamental ecological niches of Anopheles gambiae s.s., Anopheles arabiensis and Anopheles quadriannulatus in order to predict their distribution. Individual occurrences of the species were marked on electronic maps underlaid by relevant ecological information. On this basis, a heterogeneous set of rules was derived that modeled the potential occurrence of the species, and continentalscale maps produced for each species at a resolution of $0.1^{\circ}$. According to the authors, the model proved to be both accurate and relatively simple:

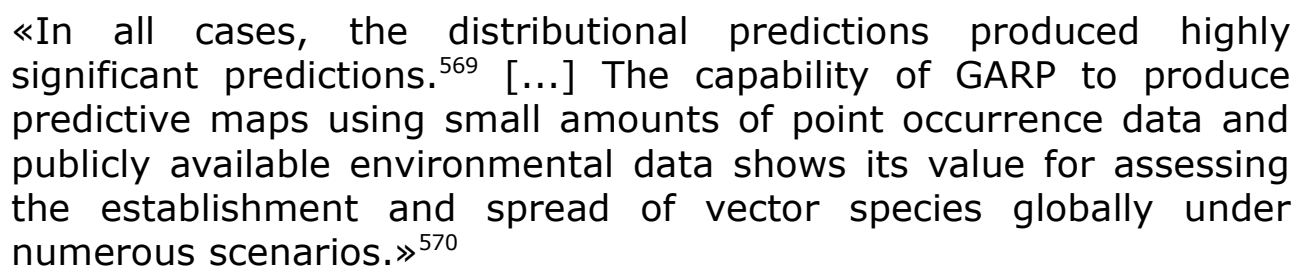

While the predictive power of the GARP algorithm is currently around $70 \%{ }^{571}$, present implementations only utilize species' presence data rather than both presence and absence. ${ }^{572}$ This causes notable discrepancies between reality and model predictions at local scales:

564 Daly, H.V.; Doyen, J.T. \& Purcell, A.H. (1998), p. 182.

565 Peterson, A.T. (2006), p. 1822.

566 Peterson, A.T. (2001), p. 600.

567 Peterson, A.T. \& Cohoon, K.P. (1999), p. 160.

568 Anderson, R.P.; Lew, D. \& Peterson, A.T. (2003), p. 212.

569 Levine, R.S.; Peterson, T. \& Benedict, M.Q. $\left(2004^{1}\right)$, p. 105.

570 Levine, R.S.; Peterson, T. \& Benedict, M.Q. (2004²), p. 607.

571 Stockwell, D.R.B. \& Peterson, A.T. (2002), p. 10.

572 Anderson, R.P.; Lew, D. \& Peterson, A.T. (2003), p. 211. 
«It [the GARP] illustrates the limitation of computer modeling for species distribution, particularly where breeding sites in arid areas are highly localized at permanent springs, river edges, or irrigational projects, and are not affected by local rainfall. $\gg^{573}$

The efforts and costs involved in surveys of species' distribution practically mean that sampling sizes have to be limited, while at the same time the number of occurrence points used for a species greatly affects the quality of GARP-based models. ${ }^{574}$ In a study on the distribution of 109 bird species in Mexico, Stockwell et al. (2002) found the accuracy of the GARP algorithm to be $64 \%$ with 10 sampling points and $69 \%$ with 50 sampling points, with widespread species being less accurately modeled than those with more restricted habitats. ${ }^{575}$

The integrated ecological model proposed by KiLLeEN et al. (2004) considers mosquito host-seeking and biting behavior as two key determinants of the transmission process that are related to the environmental setting. The length of the gonotrophic cycle $f$, which determines the interval between two blood meals, is the sum of the gestation period $g$ (over which eggs develop) and the seeking intervals required by female mosquitoes to obtain the resources $n_{r}$ required for egg fertilization and development. These include sperm, carbohydrate meals, aquatic habitats and blood from vertebrate hosts. ${ }^{576}$ The latter two are often the limiting factors, as expressed by the following equation (variables are explained in table 23 ):

$$
f=g+\sum_{r}^{N r} n_{r} \approx g+n_{v}+n_{a}
$$

The proportion of mosquitoes surviving the gonotrophic cycle can thus be estimated as a function of the total feeding cycle length:

$$
P_{f}=P^{g+n_{v}+n_{a}} 577
$$

573 Coetzee, M. (2004), p. 103.

574 Stockwell, D.R.B. \& Peterson, A.T. (2002), pp. 1 f.

575 Stockwell, D.R.B. \& Peterson, A.T. (2002), pp. 5-9.

576 Killeen, G.; Seyoum, A. \& Knols, G.J. (2004), p. 88.

577 Killeen, G.; Seyoum, A. \& Knols, G.J. (2004), p. 88. 


\begin{tabular}{|c|c|}
\hline Symbol & Definition \\
\hline$f$ & duration of the gonotrophic cycle (feeding cycle) \\
\hline g & $\begin{array}{l}\text { gestation period, i.e. duration of the period required for egg } \\
\text { maturation prior to oviposition }\end{array}$ \\
\hline $\mathrm{r}$ & a resource required by the mosquito population \\
\hline $\mathrm{n}_{\mathrm{r}}$ & the period required to obtain the resource $r$ \\
\hline$n_{v}$ & the time required to find a vertebrate host \\
\hline $\mathrm{n}_{\mathrm{a}}$ & the time required to find aquatic habitats \\
\hline$P_{f}$ & probability that a mosquito survives the gonotrophic cycle \\
\hline
\end{tabular}

Table 23: Parameters used in the integrated ecological model proposed by KILLEEN et al. ${ }^{578}$

For the conditions prevalent southern Tanzania, the mean daily survival rate was estimated to be around 0.9 , and a total of 2.93 bites, including 0.044 infectious bites, were observed per vector lifetime. 60 bites per human per day resulted in an EIR of 327 infectious bites per person per year. ${ }^{579}$

Even though the exact characterization of vector habitats is a complex task, ecological models have the advantage of relying on parameters which can be measured and monitored relatively easily. In regions where data on entomological processes and transmission itself is missing, they may therefore be the only way to quantify and predict disease risks.

\subsubsection{Feasibility and Limitations of Malaria Models}

Ideally, timely predictions of mortality and morbidity should be the final outputs of malaria models. TekLehaimanot et al. (2004) found weather-based models incorporating incidence data to be well suited for case prediction of of Plasmodium falciparum malaria about four weeks ahead. ${ }^{580}$

Even though the available evidence indicates that malaria prevalence, incidence, morbidity and mortality increase with transmission intensity, the relationship between transmission intensity and disease burden is still poorly understood. ${ }^{581}$

578 Killeen, G.; Seyoum, A. \& Knols, G.J. (2004), p. 88.

579 Killeen, G.; Seyoum, A. \& Knols, G.J. (2004), p. 88.

580 Teklehaimanot, H.D.; Schwartz, J.; Teklehaimanot, A. \& Lipsitch, M. (2004), doi:10.1186/1475-2875-3-44.

581 Killeen, G.F.; McKenzie, F.E.; Foy, B.D. et al. (2000), p. 535. 
Many contemporary malaria models focus on the connection between malaria prevalence and meteorological parameters that partly determine vector population dynamics. Such models are typically statistical but may involve fuzzy logic and be rule-based or process-based. ${ }^{582}$ However, the observed correlations may not directly represent physical, i.e. causal links ${ }^{583}$ :

\begin{abstract}
«The qualitative predictions of simple models may be more biologically meaningful than the precise quantitative predictions of complex models involving many parameters. $>^{584}$
\end{abstract}

Even though there is general agreement on the role climatic variables play for malaria transmission, there is still no consensus about the relative importance and predictive value of different factors. ${ }^{585}$ Moreover, the complexity of modern malaria models may turn into a disadvantage since they require numerous input parameters which may not be available at the desired accuracy or spatiotemporal resolution:

\begin{abstract}
«A major hindrance to compiling a clear picture of the relationship between transmission intensity and malaria disease patterns throughout Africa has always been, and is likely to remain, the paucity of detailed accurate epidemiological data (especially relating to severe disease and death) collected in space and time in a manner that accurately reflects what is happening in the communities. ${ }^{586}$
\end{abstract}

The absence or imprecision of field estimates, wrong fundamental assumptions and the necessity to simplify the complex life cycle of the malaria parasite limit the accuracy and precision of all malaria models. ${ }^{587}$ Consequently, the purely statistical models have only limited ability to predict the impact of changing environmental conditions, human interventions or resistance patterns. ${ }^{588}$

582 Yé, Y.; Sauerborn, R.; Seraphin, S. \& Hoshen, M. (2007), p. 376.

583 Curran, P.J.; Atkinson, P.M.; Foody, G.M. \& Milton, E.J. (2000), p. 68.

584 Killeen, G.F.; McKenzie, F.E.; Foy, B.D. et al. (2000), p. 535.

585 Teklehaimanot, H.D.; Lipsitch, M.; Teklehaimanot, A. \& Schwartz, J. (2004), doi: 10.1186/1475-2875-3-41.

586Thomson, M.C.; Connor, S.J.; Milligan, P. \& Flasse, S.P. (1997), p. 314.

587 Killeen, G.F.; McKenzie, F.E.; Foy, B.D. et al. (2000), p. 541.

588 Yé, Y.; Sauerborn, R.; Seraphin, S. \& Hoshen, M. (2007), p. 376. 
Most existing malaria transmission models unrealistically assume single, enclosed populations of hosts, vectors ${ }^{589}$ and parasites and do not consider emigration or immigration. ${ }^{590}$ Moreover, while the process of parasite transmission from vector to host has been studied quite thoroughly, relatively few studies have evaluated the role of the human infectious reservoir in malaria-endemic regions. ${ }^{591}$

\subsubsection{Synopsis: Determinants of Malaria Transmission}

The input variables used for most malaria maps, monitoring systems and models can be broadly classified into three categories: environmental parameters, entomological and parasitological indicators, and characteristics of the host population. The following tables provide a systematic overview of parameters used by studies in the recent past.

Among the environmental parameters, climate has been studied most extensively. Moreover, several studies have looked at land cover and its dynamics and other land surface characteristics (see table 24).

589 Gu, W. ; Killeen, G.F.; Mbogo, C.M. et al. (2003), p. 46.

590 Ruan, S.; XIAO, D. \& BeIER, J.C. (2008), p. 1109.

591 Bonnet, S.; Gouagna, L.C.; Paul, R.E. et al. (2003), p. 53. 


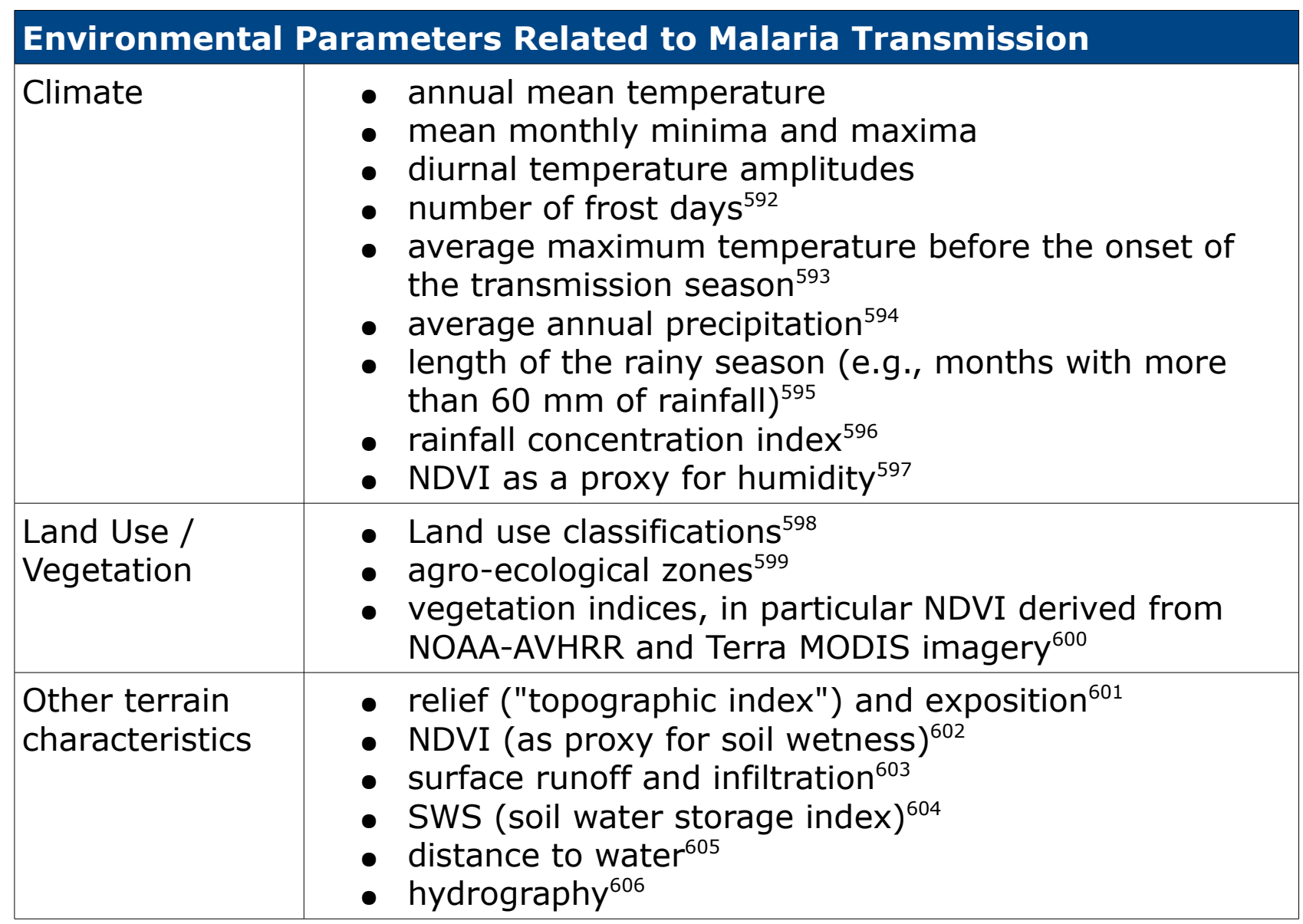

Table 24: Environmental parameters related to malaria transmission

592 Levine, R.S.; Peterson, T. \& Benedict, M.Q. (2004 ${ }^{1}$ ), p. 105.

593 Kleinschmidt, I.; Bagayoko, M.; Clarke, G.P.Y. et al (2000), p. 357.

594 Levine, R.S.; Peterson, T. \& Benedict, M.Q. (2004 ${ }^{1}$ ), p. 105.

595 Kleinschmidt, I.; Bagayoko, M.; Clarke, G.P.Y. et al (2000), p. 357.

596 Mabaso, M.L.; Craig, M.; Ross, A. \& Smith, T. (2007), p. 33.

597 Kleinschmidt, I.; Bagayoko, M.; Clarke, G.P.Y. et al (2000), p. 357.

598 Gemperli, A.; Sogoba, N.; Fondjo, E. et al. (2006), p. 1034.

599 Gemperli, A.; Sogoba, N.; Fondjo, E. et al. (2006), p. 1034.

600 Gemperli, A.; Sogoba, N.; Fondjo, E. et al. (2006), p. 1033.

601 Levine, R.S.; Peterson, T. \& Benedict, M.Q. $\left(2004^{1}\right)$, p. 105.

602 Gemperli, A.; Sogoba, N.; Fondjo, E. et al. (2006), p. 1033.

603 Levine, R.S.; Peterson, T. \& Benedict, M.Q. (2004 ${ }^{1}$ ) p. 105.

604 Gemperli, A.; Sogoba, N.; Fondjo, E. et al. (2006), p. 1034.

605 Gemperli, A.; Sogoba, N.; Fondjo, E. et al. (2006), p. 1034;

Kleinschmidt, I.; Bagayoko, M.; Clarke, G.P.Y. et al (2000), p. 357.

606 Gemperli, A.; Sogoba, N.; Fondjo, E. et al. (2006), p. 1034. 
Even though there are clear links between mosquito vectors, malaria parasites and the environment, some studies have concentrated on the characteristics of the vector and parasite population excluding their environmental determinants. Parameters observed in such studies are presented in table 25 . While environmental parameters tend to be easily measurable, entomological and parasitological quantifiers have the advantage of being more direct predictors of the malaria transmission pressure.

\section{Entomological and Parasitological Parameters}

\begin{tabular}{|c|c|}
\hline $\begin{array}{l}\text { General } \\
\text { characteristics of } \\
\text { mosquito } \\
\text { population }\end{array}$ & $\begin{array}{l}\text { - vector density } \\
\text { - vectorial capacity of the mosquito population }{ }^{607} \\
\text { - longevity / daily survival probability } \\
\text { - vectors per host rate } \\
\text { - length of gonotrophic cycle }\end{array}$ \\
\hline $\begin{array}{l}\text { Mosquito } \\
\text { behavior }\end{array}$ & - man-biting rate 611 \\
\hline $\begin{array}{l}\text { Parasitological } \\
\text { indicators }\end{array}$ & $\begin{array}{l}\text { - infective state of mosquitoes } \\
\text { - } \text { sporozoite rate }^{613} \\
\text { - duration of sporogonic cycle } \\
614\end{array}$ \\
\hline
\end{tabular}

Table 25: Parameters related to the malaria vector

Finally, the host population (see table 26) plays a major role for malaria modeling and has been the focus of various studies. Important aspects include general demographic and socioeconomic characteristics, infectiological states and processes and host-to-vector contact as the link between man, mosquito and malaria parasite.

607 Molineaux, L. \& Gramiccia, G. (1980), p. 271.

608 Molineaux, L. \& Gramiccia, G. (1980), p. 271; Zavaleta, J.O. \& Rossignol, P.A. (2004), p. 612.

609 ZaVAleta, J.O. \& Rossignol, P.A. (2004), p. 612.

610 Killeen, G.; Seyoum, A. \& Knols, G.J. (2004), p. 88.

611 Molineaux, L. \& Gramiccia, G. (1980), p. 271.

612 McKenzie, F.E. \& Samba, E.M. (2004), p. 95.

613 Beier, J.C.; Killeen, G.F. \& Githure, J.I. (1999), p. 109.

614 Molineaux, L. \& Gramiccia, G. (1980), p. 271; Zavaleta, J.O. \& Rossignol, P.A. (2004), p. 612. 


\begin{tabular}{|c|c|}
\hline $\begin{array}{l}\text { Demographic } \\
\text { and } \\
\text { socioeconomic } \\
\text { indicators }\end{array}$ & $\begin{array}{l}\text { - Population density }{ }^{615} \\
\text { - birth order and birth intervals between children } \\
\text { - women's (mothers') education and mother's age at } \\
\text { infant birth }{ }^{617} \\
\text { - mortality rate }{ }^{618}\end{array}$ \\
\hline $\begin{array}{l}\text { Vector-to-host } \\
\text { contact }\end{array}$ & $\begin{array}{l}\text { - } \text { contact rate }^{619} \\
\text { - (entomological) inoculation rate }\end{array}$ \\
\hline $\begin{array}{l}\text { Infectiological } \\
\text { characteristics of } \\
\text { host population }\end{array}$ & $\begin{array}{l}\text { - infective state }{ }^{621}, \text { prevalence of infections }^{622} \\
\text { - loss of infectivity rate } \\
\text { - recovery rate upon infection } \\
\text { - development of immunity rate } \\
\text { - loss of immunity rate }{ }^{626} \\
\text { - basic reproduction rate }\end{array}$ \\
\hline
\end{tabular}

Table 26: Role of the human hosts in malaria transmission

Even though malaria models have been increasingly refined in the recent past, they cannot provide accurate numerical predictions of outcomes since the biologic and social systems involved are sufficiently complex that "it may not be possible to even define all of the variables, much less get precise predictions about their interactions and overall results in specific real-world situations." 628

615 Gemperli, A.; Sogoba, N.; Fondjo, E. et al. (2006), p. 1034; Gemperli, A.; Vounatsou, P.;

KLEINSCHMIDT, I. et al. (2004), p. 65.

616 Gemperli, A.; Vounatsou, P.; Kleinschmidt, I. el al. (2004), p. 64.

617 Gemperli, A.; Vounatsou, P.; Kleinschmidt, I. et al. (2004), p. 64.

618 Gu, W.; Killeen, G.F.; Mbogo, C.M. et al. (2003), p. 45.

619 Molineaux, L. (1985), p. 743.

620 Molineaux, L. \& Gramiccia, G. (1980), pp. 262f; Gu, W.; Killeen, G.F.; Mbogo, C.M. et al.

(2003), p. 46; Gemperli, A.; Sogoba, N.; Fondjo, E. et al. (2006), p. 1035.

621 McKenzie, F.E. \& Samba, E.M. (2004), p. 95.

622 Molineaux, L. (1985), p. 747.

623 Molineaux, L. \& Gramiccia, G. (1980), pp. $262 f$.

624 Molineaux, L. \& Gramiccia, G. (1980), p. 265; Molineaux, L. (1985), p. 743.

625 Molineaux, L. \& Gramiccia, G. (1980), p. 264;

Gu, W.; Killeen, G.F.; Mbogo, C.M. et al. (2003), p. 45.

626 Gu, W.; Killeen, G.F.; Mbogo, C.M. et al. (2003), p. 45.

627 Zavaleta, J.O. \& Rossignol, P.A. (2004), p. 611.

628 McKenzie, F.E. \& Samba, E.M. (2004), p. 95. 


\title{
2.7 GIS and Remote Sensing: New Tools For Malariology
}

Mosquito habitats are defined by a complex set of ecological factors such as topography, hydrography, climate and land use pattern. Periodic changes of the spatial pattern of those factors show co-incidence with changes of habitat and density of disease-transmitting vectors and prevalence of infectious agents:

\begin{abstract}
«The presence or absence of a [vector] species in any area is often distinguished not only by the absolute levels of climate or vegetation values, but also by subtle differences in the seasonality of these variables, which can only be captured by repeated measurements over time. ${ }^{629}$
\end{abstract}

In the early 1970s, NASA scientists first investigated the use of color and infrared aerial photography to identify mosquito larval habitats. ${ }^{630}$ Since then, numerous studies have demonstrated that remote sensing data are a suitable tool for surveying spatio-temporal changes in (vector) habitats. The provision of remotely-sensed real-time information allows the monitoring of spatiotemporal changes of climate, vegetation and land use - all parameters which affect the transmission process of infectious diseases such as malaria.

\subsubsection{Basics of Remote Sensing}

Remote sensing is the process of acquiring information about an object from a distance. While active remote sensing systems generate their own energy, passive systems rely on ambient energy, usually the sun. ${ }^{631}$ Earth observing satellites provide data on numerous processes in the geosphere and atmosphere which have several advantages over other data sources: remotely sensed images are available at wavelengths invisible to the human eye, and they provide near real-time synoptic overviews over large areas. ${ }^{632}$

629 Scharlemann, J.P.; Benz, D.; Hay, S.I. et al. (2008), p. 1.

630 HaY, S.I.; SNOW, R.W. \& Rogers, D.J. (1998), p. 306.

$631 \mathrm{HAY}_{\mathrm{AY}}$, S.I. (2000), p. 2.

632 De Lange, N. (2006), p. 366. 


\subsubsection{Physical Basics of Remote Sensing}

Depending on its state, any object absorbs and/or reflects the incoming radiation. A part of the absorbed energy is emitted in form of thermal radiation. ${ }^{633}$

Several spectral ranges are of particular importance for remote sensing (see table 27), but it should be noted that transitions are not clearly defined:

\begin{tabular}{|l|l|}
\hline Wavelength & Spectral range \\
\hline $100 \mathrm{~nm}-280 \mathrm{~nm}$ & Short wavelength ultraviolet (UV-C) \\
\hline $280 \mathrm{~nm}-320 \mathrm{~nm}$ & Medium wavelength ultraviolet (UV-B) \\
\hline $320 \mathrm{~nm}-400 \mathrm{~nm}$ & Long wavelength ultraviolet (UV-A) \\
\hline $400 \mathrm{~nm}-700 \mathrm{~nm}$ & Visual spectrum \\
\hline $700 \mathrm{~nm}-1,1 \mu \mathrm{m}$ & Near infrared (NIR) \\
\hline $1,1 \mu \mathrm{m}-3 \mu \mathrm{m}$ & Short wavelength infrared (SWIR) \\
\hline $3 \mu \mathrm{m}-7 \mu \mathrm{m}$ & Medium wavelength infrared (MWIR) \\
\hline $7 \mu \mathrm{m}-15 \mu \mathrm{m}$ & Long wavelength infrared (LWIR) \\
\hline $15 \mu \mathrm{m}-30 \mu \mathrm{m}$ & Far infrared (FIR) \\
\hline $1 \mathrm{~mm}-1 \mathrm{~m}$ & Microwave \\
\hline
\end{tabular}

Table 27: Spectral regions with relevance to remote sensing ${ }^{634}$

NIR and SWIR are sometimes called "reflected infrared" while MWIR and LWIR are sometimes referred to as "thermal infrared." 635

Passive remote sensing is based on the fact that all objects which have temperature of more than $0 \mathrm{~K}\left(-273^{\circ} \mathrm{C}\right)$ emit electromagnetic radiation. According to the Stefan-Boltzmann law, the amount of energy emitted increases rapidly with temperature:

$$
\begin{aligned}
& M=\sigma * T^{4} \\
& M=\text { emitted energy per area }\left[\mathrm{W} / \mathrm{m}^{2}\right] \\
& \sigma=\text { Stefan-Boltzmann constant }\left[5.6 * 10^{-8} \mathrm{~W} / \mathrm{m}^{2} \mathrm{~K}^{4}\right] \\
& T=\text { absolute temperature of an object }[\mathrm{K}]
\end{aligned}
$$


The radiometers or "sensors" of satellites measure the intensity of the emitted or reflected radiation within a narrow range of wavelengths, often called "bands". Processed band signals are usually referred to as "channels". 636

The amount of electromagnetic radiation reflected by an object depends on two factors: its physical properties, and the wavelength of the radiation. The combined reflexion characteristics of an object are called its spectral signature, but since such a signature is often ambiguous, multitemporal data or terrestrial examinations may be required to identify ground objects. ${ }^{637}$

One of the main disadvantages of passive optical systems is their weatherdependence. By contrast, radar remote sensing usually relies on active sensors which operate at wavelengths between $1 \mathrm{~mm}$ and $1 \mathrm{~m}$. Such a system can operate independently from both solar illumination and atmospheric conditions. ${ }^{638}$ For the purpose of mosquito habitat detection, SAR sensors have the added advantage of water discernability. ${ }^{639}$

There are two main types of radar systems: real aperture radar which use an antenna of fixed size, while synthetic aperture radar (SAR) systems electronically "synthesize" longer antennas than they actually have. ${ }^{640}$ Interferometric radar systems (InSAR) use two or more synthetic aperture radars and make use of the phase differences of the waves returning to the sensor. Such InSAR systems can provide high precision three-dimensional topographic information. ${ }^{641}$

Radar remote sensing uses microwave radiation of wavelengths between $2,4 \mathrm{~cm}$ and $30 \mathrm{~cm}$. Contrary to optical sensors, radar sensors are active, and since they were first used for military applications, radar bands are denoted by NATO codes. For the production of digital elevation models, the $X$-band ( $\lambda=$ $2,4 \mathrm{~cm}$ to $\lambda=3,75 \mathrm{~cm})$ and the C-band $(\lambda=3,75 \mathrm{~cm}$ to $\lambda=7,5 \mathrm{~cm})$ are of particular interest. Whereas $X$-band radiation is (due to its short wavelength) largely reflected by the surface, C-band penetrates into the upper layer of the surface. ${ }^{642}$

$636 \mathrm{H}_{\mathrm{AY}}$, S.I. (2000), p. 3.

$637 \mathrm{H}_{\mathrm{AY}}$, S.I. (2000), p. 4.

638 De Lange, N. (2006), p. 389.

$639 \mathrm{HAY}_{\text {AY }}$ S.I. \& TATEM, A.J. (2005), p. 655.

640 JENSEN, J.R. (2000), p. 286.

641 JenSEN, J.R. (2000), p. 323.

642 Kessels, O. (2006), p. 24. 


\subsubsection{Sensor Resolution Characteristics}

While the sensor of polar-orbiting satellites constantly moves in relation to the earth's surface, constructing images ("scenes") line by line, the radiometers of geostationary satellites remain in a constant position in relation to the earth. Since both the storage capacities aboard satellites and the communication links between satellites and ground stations are limited, remote sensing data tend to have either high temporal, high spatial or high spectral resolutions, but not a combination of all three. ${ }^{643}$

Spatial resolution refers to the smallest separation between two objects that can be resolved by a sensor. Ideally, the resolution of a sensor system should be less than half of the dimension of the smallest object to be identified. ${ }^{644}$ Due to the curvature of the earth, the spatial resolution of satellites decreases towards the margins of images. ${ }^{645}$

The limited spatial resolution of satellites means that one pixel often represents more than one ground object (mixed pixels). This is particularly a problem when remote sensing data are used as inputs for land cover classifications which normally assume that each pixel belongs to a certain, discrete class. On the one hand, land cover classes may be continuous and not have clear class boundaries, and on the other hand, a single pixel may represent a heterogeneous area composed of several land cover classes. ${ }^{646}$ There are two approaches to overcome this problem: spectral unmixing and soft classifications. While spectral unmixing tries to separate a pixel into different classes, soft or "fuzzy" classifications allow a pixel to have partial and multiple class memberships. ${ }^{647}$

The temporal resolution refers to how often a remote sensing system records imagery of a certain area and is of particular importance when the parameter to be monitored is highly variable. ${ }^{648}$

The spectral resolution refers to the number and ranges of specific wavelength intervals monitored by a remote sensing instrument. While some systems were developed to collect data in just a single band, multispectral systems acquire data in several and hyperspectral instruments in hundreds of spectral bands. ${ }^{649}$ The radiometric resolution determines the capability of a sensor to differentiate the signals within a certain spectral band. ${ }^{650}$

643 HAY, S.I. (2000), pp. $6 f$.

644 JENSEN, J.R. (2000), p. 16.

$645 \mathrm{HAY}_{\text {AY }}$ S.I. (2000), pp. $6 \mathrm{f}$.

646 Curran, P.J.; Atkinson, P.M.; Foody, G.M. \& Milton, E.J. (2000), p. 58.

647 Curran, P.J.; Atkinson, P.M.; Foody, G.M. \& Milton, E.J. (2000), pp. $59 f$.

648 JENSEN, J.R. (2000). p. 16.

649 JENSEN, J.R. (2000). p. 12.

650 De Lange, N. (2006), p. 378. 


\subsubsection{Image Preprocessing}

The raw data provided by satellite sensors have to be preprocessed before image analysis and interpretation. This includes rectification and georeferencing on the one hand, and the removal of disturbances such as atmospheric attenuation on the other.

Raw images delivered by satellites need to be rectified to remove geometric distortion so that individual pixels are in their proper planimetric map locations. Georegistering of images acquired by geostationary satellites is normally unproblematic, while polar-orbiting satellites produce series of strips which need to be co-registered and geometrically corrected before being merged into images. Georeferencing can cause a certain loss of spatial resolution. ${ }^{651}$

Since electromagnetic radiation is partly absorbed and partly scattered in the earth's atmosphere (and in particular in the lower troposphere), a satellite sensor cannot directly measure the radiation emitted by an object. However, this effect is wavelength-dependent and there are certain "atmospheric windows" in which there is comparatively little atmospheric attenuation. ${ }^{652}$

Even though images are affected by atmospheric attenuation irrespective of their spatial resolution, high temporal resolutions provide the potentials for synthetic images such as MVCs (maximum value composites). Moreover, scattering by aerosols and absorption by gases such as water vapor, carbon dioxide and ozone can be compensated for if their concentration in the local atmosphere is known. ${ }^{653}$

651 JENSEN, J.R. (2005), p. 228; HAY, S.I. (2000), pp. $15 f$.

$652 \mathrm{HAY}_{\mathrm{A}}$, S.I. $(2000)$, p. 3.

$653 \mathrm{HAY}_{\text {AY }}$ S.I. (2000), pp. $16 \mathrm{f}$. 


\subsubsection{Digital Elevation Models}

Digital elevation models (DEMs) can be used in several ways for geomedical research. First of all, altitude may directly be the focus of interest, for example when delimiting high-altitude malaria-free from low-altitude malarious areas. Secondly, digital elevation data may be used for modeling slopes, which are of interest when surface water movements and accumulation are to be investigated (e.g. identification of major vector breeding sites). Thirdly, digital elevation data may be used in combination with other remotely sensed data, e.g. for improving land cover classification accuracy.

While digital elevation models may be created without remote sensing data, e.g. by interpolating information from point altitude information or isolines on maps, remote sensing has greatly enhanced the quality and large-scale availability of such data.

In February 2000, the Shuttle Radar Topography Mission (SRTM) recorded interferometric synthetic aperture radar data (InSAR) of the entire land mass of the earth between $60^{\circ} \mathrm{N}$ and $57^{\circ} \mathrm{S} .{ }^{654}$ During the SRTM mission, data capable of producing a 3D image of $80 \%$ of the earth's land surface were acquired ${ }^{655}$. Global digital elevation models had existed before the mission, but since they were based on data acquired with a variety of sensors, their local quality was not uniform and their spatial resolution limited to $1 \mathrm{~km}$. ${ }^{656}$ The GTOPO30 dataset, which had been completed in 1996 by USGS, was based on various sources of elevation information that largely ranged from $\pm 30 \mathrm{~m}$ to $\pm 650 \mathrm{~m}$ vertical accuracy (outside the US). ${ }^{657}$ In comparison, the SRTM dataset has a horizontal resolution of $90 \mathrm{~m}$ and a typical vertical accuracy of $\pm 16 \mathrm{~m}$ or better ${ }^{658}$ and is thus "about 100 times more detailed than other existing freely available global elevation data". 659

The simultaneous acquisition of data with single-pass interferometry guaranteed that any object on ground was imaged under virtually the same atmospheric condition by two radar antennas about $60 \mathrm{~m}$ apart from each other. ${ }^{660}$ Terrain heights were deduced by trigonometry using the phase difference between the signals of the two antennas, their distance and the space shuttle's location. ${ }^{661}$ Two single pass radar interferometers, a C-band system $(\lambda=5,6 \mathrm{~cm})$ and an $X$-band system $(\lambda=3,1 \mathrm{~cm})$ were used during the

654 Rabus, B.; Eineider, M.; Roth, A. \& Bamler, R. (2003), p. 241. 655 VAN ZYL, J.J. (2001), p. 559.

656 Rabus, B.; Eineider, M.; Roth, A. \& Bamler, R. (2003), p. 241.

657 Miliaresis, G.C. \& Argialas, D.P. (1999), p. 715.

658 SANDERS, B.F. (2007), p. 1832; van ZYL, J.J. (2001), p. 562.

659 Reuter, H.I.; Nelson, A. \& Jarvis, A. (2007), p. 984.

660 Rabus, B.; Eineider, M.; Roth, A. \& Bamler, R. (2003), p. 243.

661 SANDERS, B.F. (2007), p. 1832. 
SRTM mission. ${ }^{662}$ The C-Band SRTM system, which covered the entire region between $60^{\circ} \mathrm{N}$ and $57^{\circ} \mathrm{S}$ was designed to achieve a vertical accuracy of better than $\pm 16 \mathrm{~m}^{663}$, while a relative vertical accuracy of $\pm 6 \mathrm{~m}$ was realized at a local $200 \mathrm{~km}$ scale. ${ }^{664}$ The X-band system covered a much smaller area but was designed for a higher vertical accuracy $( \pm 10 \mathrm{~m}$ absolute and $\pm 2.8 \mathrm{~m}$ relative accuracy). ${ }^{665}$

Several factors may limit the accuracy of radar-based DEMs. These include high relief, vegetation cover and "random noise" which appears to be strongest on floodplains. ${ }^{666}$ Terrain slopes between $30^{\circ}$ and $60^{\circ}$ are a particular problem for the SRTM-based DEM. ${ }^{667}$ Investigations into the accuracy of SRTM data revealed that vertical accuracy errors are close to zero in flat terrain but increase to more than $\pm 30 \mathrm{~m}$ at slopes of $50^{\circ}$ and more. ${ }^{668}$ The SRTM-based DEMs are technically digital surface models since they include buildings, trees and other objects as part of the elevation value. This may be a problem for hydrologic modeling, one key application of DEMs. ${ }^{669}$ Moreover, no DEM can resolve channels smaller in width than twice the DEM resolution. ${ }^{670}$ Due to these problems, automated approaches using SRTM DEM data for hydrologic modeling often seriously over- or underestimate stream densities in a region. ${ }^{671}$

In addition to these limitations, the C-band product of the SRTM mission has significant areas of missing data. For such regions, either auxiliary sources of elevation data were used (for example, ASTER DEMs, GTOPO30, topographic maps) or voids were filled using interpolation algorithms. Both gap filling methods led to local accuracy degradations. ${ }^{672}$

The incorporation of DEMs into land cover classifications has been shown to improve the classification accuracy by reducing topographic effects which can produce large errors in image classifications. This is due to the variation of brightness values between plain surfaces and slopes of the same land cover type. ${ }^{673}$

662 VAN ZYL, J.J. (2001), p. 561; LUdWig, R. \& Schneider, P. (2006), p. 340.

663 van ZYL, J.J. (2001), p. 562.

664 Rabus, B.; Eineider, M.; Roth, A. \& Bamler, R. (2003), p. 256.

665 Rabus, B.; Eineider, M.; Roth, A. \& Bamler, R. (2003), p. 246.

666 SANDERS, B.F. (2007), pp. 1832-1834.

667 Reuter, H.I.; Nelson, A. \& Jarvis, A. (2007), p. 985.

668 Ludwig, R. \& SCHneider, P. (2006), pp. 346f. ; Kessels, O. (2006), p. $105 f$.

669 Ludwig, R. \& SCHNEIDER, P. (2006), p. 342.

670 SANDERS, B.F. (2007), p. 1842.

671 Islam, M.A.; Thenkabail, P.S.; Kulawardhana, R.W. et al. (2008), p. 7077.

672 Reuter, H.I.; Nelson, A. \& Jarvis, A. (2007), p. 987.

673 Fahsi, A.; Tsegaye, T.; Tadesse, W. \& Coleman, T. (2000), p. 57. 


\subsubsection{Land Cover Mapping}

Both land cover types (e.g., woodland, agricultural land, urban land) and quantitative identifiers of biomass may be of concern in epidemiological studies. ${ }^{674}$ In case of vector-borne diseases, land cover has a significant influence on vector density, which may in turn be used as a predictor of disease risk. ${ }^{675}$ Several study groups investigated the feasibility of utilizing land surface data for monitoring mosquito population dynamics in the framework of the Global Monitoring and Disease Prediction Program (GMDPP). In the 1980s, remote sensing-based vegetation indices were for the first time successfully used to model mosquito population dynamics in rice-growing regions. ${ }^{676}$ The Terra MODIS working group identified public health issues, including the detection of mosquito breeding sites, as one of the key applications of MODIS vegetation index data. ${ }^{677}$

One major advantage of remote sensing imagery is that it allows the identification of land cover changes. ${ }^{678}$ Change detection techniques can be classified as pre- and post-classification methods, and the choice regarding data types and methods depends on the ecosystems to be monitored. ${ }^{679}$ However, for change to be identified with confidence, atmospheric differences between different sampling dates have to be considered. ${ }^{680}$

\subsubsection{Land Use Classifications}

Computerized classification techniques that use remotely sensed data of the spectral properties of surface materials are an objective way of mapping land cover types. ${ }^{681}$ In case of unsupervised classifications, a software automatically creates groups of pixels based on similar multispectral response pattern. However, these automatically generated classes may not coincide with actual land use classes. Supervised classifications try to overcome this limitation by generating a classification algorithm based on user-defined examples. ${ }^{682}$ However, most existing classification algorithms only use individual pixel values for classification, thus neglecting texture and other contextual information. Such data could greatly enhance class separability, as could additional data such as altitude information or a soil typology particularly when vegetation units that are known to be located in different

674 Curran, P.J.; Atkinson, P.M.; Foody, G.M. \& Milton, E.J. (2000), p. 52.

675 Curran, P.J.; Atkinson, P.M.; Foody, G.M. \& Milton, E.J. (2000), p. 63.

676 HaY, S.I.; Omumbo, J.A.; Craig, M.H. \& Snow R.W. (2000), p. 185.

677 Huete, A.; Justice, C. \& van Leeuwen, W. (1999), p. 2.

678 Treitz, P. \& Rogan, J. (2004), p. 271.

679 LunettA, R.S.; KNIGHT, J.F.; Ediriwickrema, J. et al. (2006), p. 144.

680 Treitz, P. \& Rogan, J. (2004), p. 271.

681 TReitz, P. \& Rogan, J. (2004), p. 271.

682 Curran, P.J.; Atkinson, P.M.; Foody, G.M. \& Milton, E.J. (2000), pp. $53 f$. 
Geographic Determinants of Malaria Transmission

environments have a similar appearance on satellite images. ${ }^{683}$

Land cover mapping of large areas became possible with the introduction of the first Landsat satellite in 1972, but for a long time, a low temporal resolution, cloud cover in some regions and limited computer capacities prevented the production of continental-scale maps. ${ }^{684}$ In the 1980s, the use of remote sensing for the monitoring land use and cover change (LUCC) became a key topic in global change research ${ }^{685}$.

In recent years, several global land cover datasets were produced at spatial resolutions of $1 \mathrm{~km}$ or better. The GeoCover LC dataset is the only $30 \mathrm{~m}$ land cover dataset, but for the year 2000, its coverage is somewhat less than $50 \%$ of the world's land surface (data for another nearly $50 \%$ are available for 1990). datasets with $1 \mathrm{~km}$ resolution are more widely available and were derived from NOAA Advanced Very High Resolution Radiometer (AVHRR) data in the early 1990s and MODIS imagery for the year 2000. The perhaps most widely used dataset (the GLC 2000) is based mostly on SPOT-4 images of the year $2000 .{ }^{686}$

The Global Land Cover 2000 (GLC 2000) dataset was developed by the European Commission's Joint Research Center in collaboration with research teams from around the world. ${ }^{687}$ It includes a land cover map of Africa which was produced at a spatial resolution of $1 \mathrm{~km}$ using data from four different satellites: While SPOT Vegetation imagery was used for the identification of grasslands, shrub- and woodlands and bare soils, SAR radar data of the Japanese JERS-1 satellite and ESA's ERS-1 and ERS-2 satellites were used for moist tropical forest regions. Additionally, images of the US Defense Meteorological Satellite Program (DMSP) were used for the detection of human settlements due to their good nocturnal light imaging capability. ${ }^{688}$ One outstanding feature of the GLC 2000 approach is that is was based both on the FAO Land Cover Classification System (LCCS) and the expertise of individual working groups who created land use legends specific to their needs. ${ }^{689}$

However, the GLC 2000 map shows several general limitations. In semi-arid regions such as Burkina Faso, the GLC 2000 map tends to overestimate croplands. Many regions classified as "agricultural" are indeed characterized by a significant (or even dominant) proportion of non-agricultural land cover. ${ }^{690}$ In

683 Curran, P.J.; Atkinson, P.M.; Foody, G.M. \& Milton, E.J. (2000), p. 58.

684 Mayaux, P.; Bartholomé, E.; Fritz, S. \& Belward, A. (2004), p. 862.

685 Erasmi, S.; Kappas, M.; Twele, A. \& Ardiansyah, M. (2007), p. 438.

686 Nelson, G.C. \& Robertson, R.D. (2007), pp. 4244; 4246.

687 Nelson, G.C. \& Robertson, R.D. (2007), p. 4245.

688 Mayaux, P.; Bartholomé, E.; Fritz, S. \& Belward, A. (2004), pp. 863-865.

689 Nelson, G.C. \& Robertson, R.D. (2007), p. 4256.

690 Mayaux, P.; Bartholomé, E.; Fritz, S. \& Belward, A. (2004), p. 867. 
fact, two land cover categories are explicitly identified as mosaics. ${ }^{691}$ The use of several sensors and regional teams for map production reduced the internal consistency of the product. ${ }^{692}$ The GLOBCOVER dataset follows a similar concept as the GLC2000 but is based on Envisat MERIS imagery with a spatial resolution of $300 \mathrm{~m} .{ }^{693}$

Three alternative datasets used different variations of the International Geosphere Biosphere Project (IGBP) classification system. The first dataset was produced within the framework of the IGBP project itself and was based solely on AVHRR NDVI data, while a subsequent global dataset produced by the University of Maryland used the same AVHRR data, but this time included information from all five spectral bands. The latest global landcover dataset was made available by the MODIS working group and used an extended version of the IGBP classification. ${ }^{694}$ However, the methodological differences mean that the existing global datasets are difficult to compare (and indeed often disagree with regard to their classification):

\begin{abstract}
«All land cover datasets were created using different classification methods, but with the same purpose of providing accurate land cover information for environmental modelers and policy makers. [...However, to date] there is no internationally accepted land cover classification system. ${ }^{695}$
\end{abstract}

Both the relatively general classification system and the low spatial resolution of global land cover datasets mean that they are not well suited for local scale mapping. Several African countries have therefore been mapped under the FAO's Africover project, but data for many regions, including West Africa, are still entirely missing. ${ }^{696}$

Several satellite systems provide multispectral data suitable for land cover mapping, with Landsat being the oldest land-surface observation satellite system. ${ }^{697}$ Landsat 7 (table 28), the most recent satellite in its family, was launched in 1999 and brought two major improvements over the previous Landsat generations: a $15 \mathrm{~m}$ resolution panchromatic band and an increased resolution in the thermal infrared band $(60 \mathrm{~m}$ instead of $120 \mathrm{~m}) \cdot{ }^{698}$

691 Nelson, G.C. \& Robertson, R.D. (2007), p. 4246.

692 Mayaux, P.; Bartholomé, E.; Fritz, S. \& Belward, A. (2004), p. 873.

693 Erasmi, S.; Kappas, M.; Twele, A. \& Ardiansyah, M. (2007), p. 458;

http://ionia1.esrin.esa.int/; accessed 11/08/09.

694 McCallum, I.; Obersteiner, M.; Nilsson, S. \& Shvidenko, A. (2006), pp. $247 \mathrm{f}$.

695 McCallum, I.; Obersteiner, M.; Nilsson, S. \& Shvidenko, A. (2006), pp. $253 \mathrm{f}$.

$696 \mathrm{http}: / /$ www.africover.org/system/africover data.php (accessed 17/04/09).

697 JENSEN, J.R. (2000). p. 185.

698 JENSEN, J.R. (2000). p. 199. 


\begin{tabular}{|l|l|}
\hline Characteristics of Landsat 7 ETM+ \\
\hline Spatial Resolution & $\begin{array}{l}15 \mathrm{~m} \text { (panchromatic) } \\
30 \mathrm{~m} \text { (Bands 1-5) } \\
60 \mathrm{~m} \text { (Band 6) }\end{array}$ \\
\hline Spectral Resolution & $\begin{array}{l}7 \text { spectral bands (between } 450 \mathrm{~nm} \text { and } 2.35 \mu \mathrm{m}) \\
\text { panchromatic band }(520-900 \mathrm{~nm})\end{array}$ \\
\hline Temporal Resolution & 16 days \\
\hline
\end{tabular}

Table 28: Characteristics of Landsat 7 ETM+

In May 2003, Landsat 7's scan-line corrector (SLC), a small mirror meant to compensate for the forward motion of the satellite during data acquisition, failed. This results in image overlaps and large physical gaps (on average, about $22 \%$ of a scene are missing data). ${ }^{699}$ Due to high demand for Landsat 7 ETM+ datasets, techniques have been developed to fill in the data gaps in the SLC-off imagery, e.g. by geostatistical interpolation methods such as ordinary kriging. ${ }^{700}$

\subsubsection{Vegetation Indices}

Vegetation indices are dimensionless, radiation-based measurements that are used to infer land cover properties by isolating vegetation characteristics from other materials (e.g. soil, water). They are well correlated with different vegetation parameters, including green biomass, leaf area index (LAI) and chlorophyll concentration. ${ }^{701}$ Most spectral vegetation indices (SVIs) are based on the fact that chlorophyll and carotinoid pigments in plant tissues absorb light in the red part of the visible spectrum while mesophyll reflects light in the near infrared. A healthy, actively photosynthesizing plant therefore appears darker in the visible red channel and lighter in the near infrared channel than an unhealthy or wilting plant. Denser vegetation also increases absorption at red and reflexion at infrared wavelengths. ${ }^{702}$

Some vegetation indices such as the simple ratio index (SRI) are problematic at low vegetation densities since soils may "shine through", particularly if they are reddish or dark. The Normalized Difference Vegetation Index (NDVI) is less prone to such effects. ${ }^{703}$ The NDVI has proven to be very useful in measuring and mapping the density of green vegetation because it partially compensates for changing illumination conditions, surface slope and viewing

699 Zhang, C; Li, W. \& Travis, D. (2007), p. 5103.

700 Zhang, C; LI, W. \& Travis, D. (2007), p. 5119.

701 JaCoB, B.G.; Muturi, E.J.; Mwangani, J.M. et al. (2007), doi:10.1186/1476-072X-6-21.

702 HAY, S.I. (2000), p. 18.

703 HAY, S.I. (2000), pp. $19 f$. 
aspect, all of which are factors that strongly affect observed radiances. Therefore, the NDVI has become by far the most often used spectral vegetation index but is still affected by atmospheric effects, sparse vegetation and dark soils. ${ }^{704}$

Several attempts have been made to make vegetation indices more robust against external influences. The soil-adjusted vegetation index (SAVI) introduced a soil adjustment factor to minimize the influence of soil on vegetation spectra. ${ }^{705}$ The MSAVI is a modified version of the SAVI which uses a soil adjustment factor that depends on vegetation density (i.e. higher correction factor at low vegetation densities). ${ }^{706}$ The atmospherically resistant vegetation index (ARVI) utilizes differences between the blue and red channel to correct radiance in the red channel. Atmospheric and canopy background corrections were combined for the soil and atmospherically resistant vegetation index (SARVI and SARVI2). ${ }^{707}$ An overview of important vegetation indices in presented in table 29.

\begin{tabular}{|c|c|c|}
\hline $\begin{array}{l}\text { Vegetation } \\
\text { Index }\end{array}$ & Calculation & Characteristics \\
\hline $\begin{array}{l}\text { Simple Ratio } \\
\text { Index } \\
\text { (SRI) }\end{array}$ & $S R I=\frac{N I R}{V R E D}$ & $\begin{array}{l}\text { First index used; } \\
\text { problematic with } \\
\text { sparse/dense vegetation }\end{array}$ \\
\hline $\begin{array}{l}\text { Normalized } \\
\text { Difference } \\
\text { Vegetation } \\
\text { Index (NDVI) }\end{array}$ & $N D V I=\frac{N I R-V R E D}{N I R+V R E D}$ & $\begin{array}{l}\text { Standard; atmospheric } \\
\text { and soil effects smaller } \\
\text { than for SRI but still } \\
\text { present }\end{array}$ \\
\hline $\begin{array}{l}\text { Enhanced } \\
\text { Vegetation } \\
\text { Index (EVI) }\end{array}$ & $E V I=2 \frac{N I R-V R E D}{L+N I R+C_{1} V R E D+C_{2} V B L U E}$ & $\begin{array}{l}\text { Developed for MODIS } \\
\text { imagery; robust against } \\
\text { soil and atmospheric } \\
\text { effects }\end{array}$ \\
\hline \multicolumn{3}{|c|}{$\begin{array}{l}\text { NIR : near infrared radiance } \\
\text { VRED : visible red radiance } \\
\text { L : background adjustment term } \\
\text { C1 and C2: weighting factors of red/blue channels for aerosol corrections }\end{array}$} \\
\hline
\end{tabular}

Table 29: Important vegetation indices

704 Geerken, R. \& Ilaiwi, M. (2004), p. 491.

705 Qi, J.; Chehbouni, A.; Huete, A.R. et al. (1994), p. 119.

706 Qi, J.; Chehbouni, A.; Huete, A.R. et al. (1994), p. 126.

707 Huete, A.R.; Liv, H.W.; Batchily, K. \& van Leeuwen, W. (1997), pp. 441 f. 
In semi-arid regions, a strong relation exists between NDVI and rainfall. Therefore, it may be used to detect both drought conditions and humid periods ${ }^{708}$. The NDVI has been successfully used for drought monitoring and famine early warning systems in regions with sparse pluviometric networks ${ }^{709}$ as well as for the assessment of interannual changes of green biomass. ${ }^{710}$ Moreover, NDVI has been shown to be a good predictor of malaria incidence in Kenya. ${ }^{711}$

However, human activities resulting in land degradation may interfere with this more or less direct link. ${ }^{712}$ Urban expansion and vegetation degradation around urban areas often causes a decline in NDVIs, as does overgrazing in regions of intensive agro-pastoralism ${ }^{713}$. In dryland areas, rainfed cultivation is another frequent cause of human-induced land degradation. ${ }^{714}$ Moreover, the erratic nature of rainfall in arid regions make direct comparisons of meteorological station data and vegetation indices difficult. ${ }^{715}$

The Moderate Resolution Imaging Spectrometers (MODIS) are among the key instruments on board the EOS Terra and Aqua satellites which were launched in 1999 and 2002. ${ }^{716}$ At the time of their introduction, 36 spectral bands and a 12 bit radiometric resolution were unmatched for moderate resolution global coverages. In comparison to older systems (such as Landsat), capabilities in the shortwave and longwave infrared were added. ${ }^{717}$ The Terra satellite is already beyond its original scheduled operational lifespan but is currently expected to remain in operation until 2013. ${ }^{718}$ However, the moderate spatial resolution of MODIS data means that changes at fine scales, e.g. in urban areas, are likely to remain undetected. ${ }^{719}$

Two vegetation index algorithms are produced from MODIS data. The NDVI was chosen as a "continuity index" since global NDVI datasets have been produced from 1981 onwards. ${ }^{720}$ The Enhanced Vegetation Index (EVI) was introduced with the MODIS sensor and offered improvements over the NDVI both at high and low vegetation covers (reduction of saturation and soil background effects). ${ }^{721}$

708 Li, J.; LeWis, J.; RowLAND, J. et al. (2004), pp. $464 f$.

709 Anyamba, A. \& Tucker, C.J. (2005), p. 598f.

710 Geerken, R. \& Ilaiwi, M. (2004), p. 492.

711 HaY, S.I.; SNow, R.W. \& Rogers, D.J. (1998), p. 310.

712 Li, J.; LEWIS, J.; RowLAND, J. et al. (2004), p. 477.

713 Budde, M.E.; Tappan, G.; Rowland, J. et al. (2004), pp. $490 f$.

714 Geerken, R. \& Ilaiwi, M. (2004), p. 492.

715 OLSSON, L.; EKLUNDH, L. \& ARdö, J. (2005), p. 560.

716 Justice, C.O.; Townshend, J.R.G.; Vermote, E.F. et al. (2002), p. 3.

717 Justice, C.O.; Townshend, J.R.G.; Vermote, E.F. et al. (2002), p. 4.

718 Scharlemann, J.P.; Benz, D.; Hay, S.I. et al. (2008), p. 1.

719 LUNeTtA, R.S.; KNIGHT, J.F.; EdiriwiCKREMA, J. et al. (2006), p. 152.

720 Huete, A.; Justice, C. \& van Leeuwen, W. (1999), p. 1.

721 Justice, C.O.; Townshend, J.R.G.; Vermote, E.F. et al. (2002), p. 6. 
Chapter 2 - Malaria in West Africa: Transmission, Monitoring \& Control

\begin{tabular}{|l|l|l|l|}
\hline MODIS Band & RED (Band 1) & NIR (Band 2) & BLUE (Band 3) \\
Wavelength & $620-670 \mathrm{~nm}$ & $841-876 \mathrm{~nm}$ & $459-479 \mathrm{~nm}$ \\
\hline
\end{tabular}

Table 30: Spectral bands used for MODIS vegetation indices ${ }^{722}$

Imagery of both indices is available at resolutions of $250 \mathrm{~m}$ or $1 \mathrm{~km}$ as 16 day or monthly composites. ${ }^{723}$

\subsubsection{High Resolution Imagery}

Modern very high spatial resolution imagery such as provided by the IKONOS or QuickBird satellites are much better suited for larval habitat identification than lower resolution imagery available in the past. ${ }^{724}$ The key characteristics of the two satellites are presented in table 31 .

\begin{tabular}{|l|l|l|}
\hline Sensor Characteristics & IKONOS $^{725}$ & QuickBird $^{726}$ \\
\hline Spatial resolution & $\begin{array}{l}\text { Panchromatic: 0,82 m } \\
\text { Multispectral: 3,2 m }\end{array}$ & $\begin{array}{l}\text { Panchromatic: 0,6 m } \\
\text { Multispectral: 2,4 m }\end{array}$ \\
\hline Spectral resolution & Blue: $445-516 \mathrm{~nm}$ & Blue: $450-520 \mathrm{~nm}$ \\
& $\begin{array}{l}\text { Green: } 506-595 \mathrm{~nm} \\
\text { Red: } 632-698 \mathrm{~nm} \\
\text { Near-IR: 757 }-853 \mathrm{~nm}\end{array}$ & $\begin{array}{l}\text { Green: } 520-600 \mathrm{~nm} \\
\text { Red: } 630-690 \mathrm{~nm} \\
\text { Near-IR: } 760-900 \mathrm{~nm}\end{array}$ \\
\hline Revisit time & about 3 days & 2 to 3 days \\
\hline Radiometric resolution & 11 bit & 11 bit \\
\hline
\end{tabular}

Table 31: Characteristics of the IKONOS and QuickBird satellites

Mushinzimana et al. (2006) found IKONOS imagery to be superior to both panchromatic aerial photographs and Landsat 7 ETM+ imagery for the detection of mosquito habitats of more than $100 \mathrm{~m}^{2}$, while all techniques performed poorly for smaller sizes. ${ }^{727} \mathrm{~J}_{\mathrm{ACOB}}$ et al. (2006) utilized QuickBird imagery for the distinction between rice fields of high and low larval productivities by observing the amount of surface cover by the plants. Mosquito larvae typically increase when rice fields are flooded but their population begins to decline drastically when rice plants cover much of the surface. ${ }^{728}$

722 Huete, A.; Justice, C. \& van Leeuwen, W. (1999), p. 16.

723 TATEm, A.J.; Goetz, S.J. \& Hay, S.I. (2004), p. 39.

724 Mutuku, F.M.; Bayoh, M.N.; Hightower, A.W. et al. (2009), doi:10.1186/1476-072X-8-19.

725 http://www.geoeye.com/CorpSite/products/imagery-sources/Default.aspx\#ikonos, accessed 20/06/09.

726 http://www.digitalglobe.com/index.php/85/QuickBird, accessed 20/06/09.

727 Mushinzimana, E.; Munga, S.; Minakawa, N. et al. (2006), doi:10.1186/1475-2875-5-13.

728 JACOB, B.G.; Muturi, E.; Funes, J.E. et al. (2006), doi: 10.1186/1475-2875-5-91. 
Mutuku et al. (2009) proposed the use of "pan-sharpened" IKONOS images for larval habitat detection. ${ }^{729}$ This image construction technique which may be used for most sensor systems builds on the fact that many satellites simultaneously acquire higher resolution panchromatic and lower resolution multispectral images. Ideally, the combined pan-sharpened image exhibits the spectral properties of the multispectral and the resolution of the panchromatic image. ${ }^{730}$

Using SPOT-5 imagery for a feasibility study in Senegal, LACAUX et al. (2007) proposed two indices to detect and characterize aquatic habitats: a Normalized Difference Pond Index (NDPI) to determine the presence of ponds, and a Normalized Difference Turbidity Index (NDTI) to assess their degree of turbidity. The latter index is based on the assumption that pure water has a specific radiometric response that changes with increasing levels of turbidity so that it resembles bare soils in the visible range. ${ }^{731}$

\begin{tabular}{|l|l|l|}
\hline Index & Calculation & Characteristics \\
\hline $\begin{array}{l}\text { Normalized } \\
\text { Difference } \\
\begin{array}{l}\text { Pond Index } \\
\text { (NDPI) }\end{array}\end{array}$ & $N D P I=\frac{M I R}{V G R E E N}$ & $\begin{array}{l}\text { Helps in automatic } \\
\text { detection of ponds; } \\
\text { thresholds for NDPI vary } \\
\text { temporally }\end{array}$ \\
\hline $\begin{array}{l}\text { Normalized } \\
\text { Difference } \\
\text { Turbidity } \\
\text { Index (NDTI) }\end{array}$ & $N D T I=\frac{V R E D}{V G R E E N}$ & $\begin{array}{l}\text { Characterizes turbidity of } \\
\text { ponds without vegetation }\end{array}$ \\
\hline
\end{tabular}

Table 32: Indices for detection and characterization of ponds

The detectability of small habitats by remote sensing is limited not only by their size but also by their temporal brevity. ${ }^{732}$ The low frequency of image capture of high resolution sensors, which are often cloud-contaminated, only adds to the problem, limiting the use of high resolution sensors in epidemiology. ${ }^{733}$

729 Mutuku, F.M.; Bayoh, M.N.; Hightower, A.W. et al. (2009), doi:10.1186/1476-072X-8-19.

730 Mateos, J.; Vega, M.; Molina, R. \& Katsaggelos, A.K. (2008), doi:10.1088/1742$6596 / 139 / 1 / 012022$.

731 Lacaux, J.P.; Tourre, Y.M.; Vignolles, C. et al. (2007), pp. 70-71.

732 JACOB, B.G.; Muturi, E.; Funes, J.E. et al. (2006), doi: 10.1186/1475-2875-5-91.

733 Tatem, A.J.; Goetz, S.J. \& Hay, S.I. (2004), p. 35. 


\subsubsection{Surface Temperature Products}

Temperature and precipitation are two of the key factors ruling Anopheles population dynamics, mosquito biting behavior and parasite development. Both meteorological station data and remote sensing data can be used to survey temporal changes in these parameters. Whereas meteorological station data may be more accurate, the use of remote sensing data has the advantage of providing continuous data over larger areas:

«Remotely sensed observations by satellite sensors are the only
feasible means of obtaining regional- and continental-scale
measurements of climate at regular intervals for real-time
epidemiological applications, such as disease early warning
systems.» 734

Satellite-based land surface temperature (LST) measurements have a number of advantages, including a spatial data density that is higher than for any meteorological network and the availability of data precisely for the points for which it is needed. On the other hand, however, sensor data need to be processed, and overpass times and frequencies may be limiting factors. ${ }^{735}$

\subsubsection{Thermal Infrared Remote Sensing}

All objects that have a temperature of more than $0 \mathrm{~K}$ emit thermal electromagnetic radiation at wavelengths between $3 \mu \mathrm{m}$ and $14 \mu \mathrm{m}$. This is due to the collision of molecular particles, a process which creates kinetic heat (temperature). The electromagnetic radiation exiting an object is responsible for its "radiant temperature", which usually correlates highly with kinetic temperature. ${ }^{736}$ Thermal scanners on a variety of satellite platforms can be used to measure these thermal infrared (TIR) emissions which vary as a function of surface temperature and emissivity. ${ }^{737}$

A part of the solar radiation reaches the earth's surface and is partly reflected, and partly absorbed and re-emitted as terrestrial infrared radiation after heating up the surface. ${ }^{738}$ The terrestrial emission is maximal at a wavelength of about $9,7 \mu \mathrm{m}$; satellite sensors receive maximum input at this wavelength. At around $3,78 \mu \mathrm{m}$ in the midinfrared (MIR or MWIR), the reflection of the solar irradiance and terrestrial emission are of the same order of magnitude. ${ }^{739}$

734 GREen, R.M. \& HAY, S.I. (2002), p. 166.

735 De Wit, A.J.W.; Boogaard, H.L. \& van Diepen, C.A. (2004), pp. $188 \mathrm{f}$.

736 Jensen, J.R. (2000). p. 246.

737 PRIHODKo, L. \& GoWARD, S. (1997), p. 336.

738 DASH, P. (2005), p. 7.

739 DASH, P. (2005), p. 9. 
Therefore, IR sensors for LST determination usually operate at wavelengths of around $3,5 \mu \mathrm{m}$ and $11 \mu \mathrm{m} .{ }^{740}$ Land surface temperature (LST) products provide an estimate of the earth's surface temperature up to a depth of about $12 \mu \mathrm{m} .^{.71}$

In order to derive surface temperatures from thermal infrared RS imagery, the absorption of radiation by atmospheric gases and aerosols, the emissivity of the land surface and the role of topographic effects must be known. ${ }^{742}$ In regions of relatively flat terrain, atmospheric and emissivity corrections are of particular concern.

An object's emissivity $(\varepsilon)$ is defined as the radiant flux exiting at a certain temperature $\left(M_{r}\right)$ as compared to the radiant flux that would be emitted from a blackbody (a theoretical construct that radiates energy at the maximum possible rate) of the same temperature $\left(M_{b}\right)$ :

$$
\varepsilon=\frac{M_{r}}{M_{b}}
$$

The emissivity of an object is influenced by a number of factors, including color, surface roughness and moisture content: dark colors, greater surface areas due to roughness and high water contents increase emissivities. ${ }^{73}$ Emissivities of important natural materials are presented in table 33.

\begin{tabular}{|l|l|}
\hline Material & Emissivity $\boldsymbol{\varepsilon}$ \\
\hline Water & $0.92-0.98$ \\
\hline Rocks: granite / basalt & $0.86 / 0.95$ \\
\hline Soil: sand / dry loam / wet loam & $0.90 / 0.92 / 0.95$ \\
\hline Vegetation: grass / forest & $0.97 / 0.97^{744}$ \\
\hline
\end{tabular}

Table 33: Emissivity of selected materials

In order to retrieve kinetic temperatures, radiometric temperatures must be corrected for emissivity effects. ${ }^{745}$ However, it is almost impossible to exactly determine emissivity at pixel level due to large spatial variations and difficulties related to remote-sensing based measurements of emissivity. ${ }^{76}$ Most satellite thermal infrared radiometers have been calibrated over water bodies, in order

740 DASH, P. (2005), p. 10.

741 Pinheiro, A.C.T.; Mahoney, R.; Privette, J.L. \& Tucker, C.J. (2006), p. 153.

742 TANG, B.; BI, Y.; LI, Z.-L. \& XIA, J. (2008), p. 934.

743 JENSEN, J.R. (2000). p. 249.

744 JeNSEN, J.R. (2000). p. 251.

745 Coll, C.; Caselles, V.; Galve, J.M. et al. (2005), p. 293.

746 Pinheiro, A.C.T.; Mahoney, R.; Privette, J.L. \& Tucker, C.J. (2006), p. 157. 
to minimize the variability of surface temperature and emissivity. ${ }^{747} \mathrm{~A}$ proper LST validation over land surfaces is more difficult because the derived LST is representative for the whole pixel, while point temperature measurements can vary over short distances, particularly during daytime. Therefore, a field validation is only possible for homogeneous areas such as dense vegetation or desert. ${ }^{78}$ Satellite-derived LST data are well-suited for relative assessments rather than for absolute values. ${ }^{749}$

The temperature-vegetation index method (TVX) additionally takes the negative correlation between vegetation indices and daytime surface temperatures into consideration: plants have a thermal capacity that greatly differs from soil backgrounds. While the surface temperature of soil can exceed air temperatures by as much as $30 \mathrm{~K}$ under dry conditions, the low thermal capacity of leaves and evapotranspiration prevent their temperature from heating up considerably. ${ }^{750}$ Soil moisture also increases the thermal inertia of soils. ${ }^{751}$

In the 8 to $13 \mu \mathrm{m}$ infrared window, water vapor is mainly responsible for atmospheric effects; aerosol absorption and scattering are negligible, except for exceptional conditions such as a severely dusty atmosphere. Atmospheric gases such as $\mathrm{CO}_{2}$ also influence atmospheric transmission but are rather evenly distributed. Water vapor is more problematic as it is unevenly distributed and as the water vapor content of the atmosphere may vary within a short period of time. ${ }^{752}$ Therefore, cloud contamination is a severe problem in thermal remote sensing, particularly for low resolution sensors ${ }^{753}$, and complicates the production of continuous time-series. ${ }^{754}$

\subsubsection{MODIS Surface Temperature Products}

Whereas sea surface temperature (SST) retrieval accuracy from MODIS data is about $\pm 0.26 \mathrm{~K}$, LST estimation is much more complicated because of the much more variable land surface emissivity (LSE). For the sea surface, which is rather homogeneous, a constant emissivity can be assumed; for land surfaces, emissivities have a wide range and may vary considerably over short distances. ${ }^{755}$

747 Coll, C.; Caselles, V.; Galve, J.M. et al. (2005), p. 289.

748 WAN, Z. \& LI, Z.-L. (2008), p. 5374.

749 Pinheiro, A.C.T.; Mahoney, R.; Privette, J.L. \& Tucker, C.J. (2006), p. 161.

750 Stisen, S.; Sandholt, I.; Nørgaard, A. et al. (2007), p. 263.

751 Stisen, S.; Sandholt, I.; Nørgaard, A. et al. (2007), p. 264.

752 DASH, P. (2005), p. 10.

753 Stisen, S.; Sandholt, I.; Nørgaard, A. et al. (2007), p. 272.

754 De Wit, A.J.W.; Boogaard, H.L. \& van Diepen, C.A. (2004), p. 18.

755 DASH, P. (2005), p. 2. 
Several procedures of deriving LST from remote sensing data are commonly used:

- LST can be retrieved from a single infrared channel through an accurate radiative transfer model if surface emissivity is known and temperature / water vapor profile is given by either satellite soundings or conventional radiosonde data.

- Split-window LST methods require known surface emissivities to make corrections for the atmospheric and surface emissivity effects based on differential atmospheric absorption in the 10 to $13 \mu \mathrm{m}$ split window. ${ }^{756}$

Because the accuracy of LST retrieved by single channel and split-window methods depends on the accuracy of surface emissivity, these methods do not work well in semi-arid and arid regions, where surface emissivity may vary significantly with location and time. ${ }^{757}$

\begin{abstract}
«Recent efforts to develop continental scale emissivity maps based on the MODIS emissivity product remain unvalidated to date. However, advances by both the MODIS and ASTER teams suggest that accurate and dynamic emissivity maps may be possible in the coming years. $>^{758}$
\end{abstract}

Errors in LST tend to be larger over bare soils and in highly heterogeneous sites because of uncertainties regarding surface emissivity 759 which varies with vegetation coverage and surface moisture content. However, it does not significantly change in several days unless rain (or snow) occurs during the period of time for which the surface of the ground is normally dry. ${ }^{760}$

The MODIS LST data products are arranged in scenes of 2030 (or 2040) by 1354 pixels and have a spatial resolution of about $1 \mathrm{~km}$ or $5 \mathrm{~km}$. The first product, MOD11_L2, is an LST product which is generated using a splitwindow algorithm together with the MODIS sensor radiance data product (MOD021KM), the geolocation product (MOD03), the cloud mask product (MOD35_L2), the atmospheric temperature and water vapor product (MOD07_L2), the quarterly land cover (MOD12Q1) and snow product (MOD10_L2). It contains nine scientific datasets, among them LST and QC (quality control). The quality control dataset uses 16 bits. Bits 00 and 01 are used for mandatory quality assurance information. Values of 00 represent pixels for which LST is produced in good quality (and for which more detailed

756 WAN, Z. (1999), p. 3.

757 WAN, Z.; ZHANG, Y.; ZHANG, Q. et al. (2002), p. 163.

758 Pinheiro, A.C.T.; Mahoney, R.; Privette, J.L. \& Tucker, C.J. (2006), p. 161.

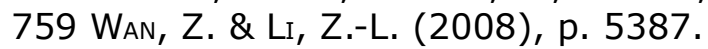

760 WAN, Z.; ZHANG, Y.; ZHANG, Q. et al. (2002), p. 166. 
quality assurance bits can be ignored); values of 01 mean that LST was produced in unreliable or unquantifiable quality (which means that an examination of further QC data is recommended); values of 10 stand for pixels not produced due to cloud effects (LST is only produced for pixels in clear-sky conditions at $99 \%$ confidence); values of 11 represent pixels not produced primarily due to reasons other than cloud. ${ }^{761}$

MOD11A1, is a daily LST product at about $1 \mathrm{~km}$ spatial resolution $(0.927 \mathrm{~km}$ or $0.5^{\prime}$ at the equator) which is generated by mapping the pixels from the MOD11_L2 products for a day to the earth locations on the integerized sinusoidal projection. MOD11A2 is the 8-day LST produced by averaging the MOD11A1 product in a series of 8 days. ${ }^{762}$

The MODIS Rapid Response System has been designed to provide MODIS land products as quickly as possible. This is in part achieved by a simplification in data processing, including modified atmospheric corrections based on past observations. ${ }^{763}$ The rapid response product tends to underestimate the standard LST product at daytime and to overestimate nighttime values. These differences are greatest (up to $2 \mathrm{~K}$ ) at low latitudes $\left(20^{\circ} \mathrm{N}\right.$ to $\left.20^{\circ} \mathrm{S}\right) .{ }^{764}$

Although state-of-the-art techniques based on multiple MODIS bands are used in the MODIS cloud mask products and LSTs are produced only for clear-sky pixels at the highest confidence $(99 \%)$, there are still some small possibilities that MODIS LSTs are contaminated with cloud effects (for example in the case of subpixel clouds). ${ }^{765}$

Numerous attempts have been made to validate MODIS LST data and to assess its value for prediction of air temperatures. WAN \& LI (2008) observed a typical accuracy of $\pm 1 \mathrm{~K}$ for current version (V5) MODIS LST data under clear sky conditions, with "slightly" larger inaccuracies found over bare soil and highly heterogeneous sites. ${ }^{.66}$ However, a true validation of remote-sensing based LST is difficult since both surface temperatures and emissivities vary considerably. ${ }^{767}$

One particular advantage of the Terra and Aqua satellites with respect to LST retrieval is that a second sensor, ASTER, allows to "zoom into" areas of special interest due to its five thermal infrared bands offering a spatial resolution of $90 \mathrm{~m}^{768}$

761 WAN, Z.; ZHANG, Y.; ZHANG, Q. et al. (2002), p. 168.

762 WAN, Z.; ZHANG, Y.; ZHANG, Q. et al. (2002), p. 168.

763 Pinheiro, A.C.T.; Descloitres, J.; Privette, J.L. et al. (2007), p. 328.

764 Pinheiro, A.C.T.; Descloitres, J.; Privette, J.L. et al. (2007), p. 330.

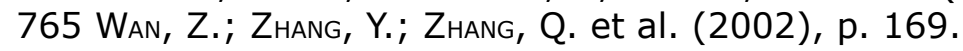

766 WAN, Z. \& LI, Z.-L. (2008), p. 5393.

767 Stisen, S.; Sandholt, I.; NørgaArd, A. et al. (2007), p. 271.

768 TATem, A.J.; Goetz, S.J. \& Hay, S.I. (2004), p. 39. 


\subsubsection{Surface vs. Air Temperature}

There are several reasons for using remotely-sensed temperature information in addition to or in place of terrestrial measurements: Meteorological stations have often been located "more for convenience than for representative sampling", and the network density is low in many parts of the world. ${ }^{769}$ In Africa, for example, data are available for only around 250 WMO member stations, resulting in a density of approximately one station per $12.000 \mathrm{~km}^{2} .{ }^{770}$

Surface and air temperatures are two different physical quantities that are related to each other through the exchange of energy fluxes near the earth's surface. ${ }^{771}$ The estimation of air temperature from satellite surface temperatures is a complex issue since there is no direct correlation between the two. Several factors have an influence on the surface energy balance, including wind speed, soil moisture, surface roughness ${ }^{772}$, vegetation cover and micrometeorological conditions. ${ }^{773}$

«Remotely sensed observations of the surface reflectance and radiant emissions may have the potential for estimating nearsurface environmental conditions at spatial scales appropriate for regional to global bioclimatic research. $>^{774}$

PRinodko \& Goward (1997) found the precision of the TVX method for estimation of near-surface air temperatures to be in the order of $3 \mathrm{~K} .{ }^{775}$ GREEN AND HAY observed "significant correlations" between terrestrial measurements of air temperature and remotely sensed land surface temperature. Even though seasonal variability in the strength of correlations was observed, variability pattern appeared to be consistent between years. ${ }^{776}$

It is common practice to use maximum values composites for the estimation of surface temperatures to reduce effects of cloud contamination. This is certainly one reason why LSTs typically exceed air temperatures. ${ }^{777}$ In Kossi Province in the dry savanna zone of Burkina Faso, an average difference of $9.7 \mathrm{~K}$ between average air temperatures and composited daytime LSTs was observed, with

769 PRIHOdKo, L. \& GoWARD, S. (1997), p. 335.

770 HAY, S.I. \& LENNON, J.J. (1999), p. 59.

771 De Wit, A.J.W.; Boogaard, H.L. \& van Diepen, C.A. (2004), p. 191.

772 Stisen, S.; Sandholt, I.; Nørgaard, A. et al. (2007), p. 263.

773 De Wit, A.J.W.; Boogaard, H.L. \& van Diepen, C.A. (2004), p. 191.

774 PRIHOdKo, L. \& GoWARD, S. (1997), p. 336.

775 PRIHodko, L. \& GoWARD, S. (1997), p. 343.

776 GREen, R.M. \& HAY, S.I. (2002), p. 166.

777 HAY, S.I. \& LeNNON, J.J. (1999), pp. $61 \& 64$. 
differences growing larger during the dry season (up to almost $15 \mathrm{~K}$ ) and smaller during the rainy season (around $3 \mathrm{~K}$ ). This constant overestimation is likely to be related to both midday LST measurements and immense heating of dry soils and rocks. ${ }^{778}$

\subsubsection{Rainfall Estimates}

Satellite data have been used for rainfall estimates since the 1970s, and several techniques, based on visible, infrared and microwave data, have been proposed since then. ${ }^{779}$ Most precipitation indices are based on the observation of the temperatures prevailing on the top of clouds (cold cloud duration, CCD). ${ }^{780}$ This technique is well-suited in tropical latitudes where weather systems are dominated by convection processes resulting in clouds with high and cold tops. ${ }^{781}$ Cold cloud tops indicate vertically developed clouds that typically produce rainfall. ${ }^{782}$ CCD lagged by around two months has been demonstrated to be a good predictor of malaria incidence in areas with unimodal rainfall pattern. ${ }^{783}$

The use of cloud-top temperatures may, however, be misleading since rain falls from the bases of clouds. ${ }^{784}$ More sophisticated estimation techniques also include information on cloud formation and decay. ${ }^{785}$

In recent years, infrared imagery has increasingly replaced visible imagery since only the former provides information during day and night-time. However, rainfall indices derived from both visible and infrared images are physically indirect since they detect cloud characteristics but not rain. Microwave systems give physically more direct evidence of atmospheric water, but have difficulties in identifying rainfall under some conditions, including areas with frozen or bare soils. ${ }^{786}$ Several commonly-used rainfall indices combine different types of data, exploiting both the better temporal resolution of IR / visible information and the closer physical link provided by microwave imagery. ${ }^{787}$

778 KaRThE, D. \& KaPPAS, M. (2007), p. 14.

779 BaRRET, E.C. (1993), p. 119.

$780 \mathrm{HAY}_{\text {AY }}$ S.I. (2000), p. 23.

$781 \mathrm{H}_{\text {AY, }}$ S.I. \& LENNON, J.J. (1999), p. 62.

782 Nicholson, S. (2005), p. 623.

783 HAY, S.I.; SNow, R.W. \& Rogers, D.J. (1998), pp. $311 \mathrm{f}$.

784 BarretT, E.C. (1993), p. 122.

785 HAY, S.I. (2000), p. 23.

786 Barrett, E.C. (1993), pp. 122-125.

787 NiCHOLSON, S. (2005), p. 624. 
In the framework of the Tropical Rainfall Measuring Mission (TRMM), highresolution radar data, passive microwave and visible/infrared radiometry was used for assessing precipitation. ${ }^{788}$ The precipitation radar on board the Tropical Rainfall Measuring Mission (TRMM) satellite was the first spaceborne radar used for this purpose and was designed to detect rain rates of $0.7 \mathrm{~mm} / \mathrm{h}$ and more at a spatial resolution of $250 \mathrm{~m} .{ }^{789}$ Even though the TRMM algorithms were originally developed for maritime regions, it provides reasonable estimates for the Sahel. ${ }^{790}$

Two near-global datasets based on remote sensing data are currently available. The GPCP (Global Precipitation Climatology Project) dataset provides data at a temporal resolution of 1 day and a spatial resolution of $1^{\circ}$. The PERSIANN (Precipitation Estimation from Remotely Sensed Information using Artificial Neural Networks) datasets have a temporal resolution of $6 \mathrm{~h}$, a spatial resolution of $0.25^{\circ}$ and cover regions between $50^{\circ} \mathrm{N}$ and $50^{\circ} \mathrm{S} .{ }^{791}$

Dinku et al. (2008) compared several satellite-based rainfall products with rain gauge data and concluded that all perform reasonably well for the detection of rainfall but rather poor in quantifying precipitation. ${ }^{792}$ For two test sites located in Zimbabwe and Ethiopia, correlations between predicted and observed rainfall ranged between 0.26 (using the NOAA Climate Prediction Center's revised RFE2 [revised rainfall prediction estimate] algorithm for Ethiopia) and 0.64 (using the same algorithm for Zimbabwe), while detection probabilities were $69 \%$ and $63 \%$ respectively. Results were found to be particularly poor in regions where orographic rainfall dominates, which is probably due to cloud-top temperatures exceeding the normal thresholds used for IR imagery. ${ }^{793}$ Similar problems were observed for PERSIANN data which seriously underestimate rainfall in mountainous areas. ${ }^{794}$

An important issue noted by most recent studies is that local calibration is essential. Even though the relationship between cloud temperatures and the probability of rainfall has been well established, spatial and temporal differences of the threshold temperatures and associated amounts of rainfall mean that empirical observations are needed for calibration. ${ }^{795}$ Moreover, precipitation is extremely variable in both the spatial and temporal domains.

$788 \mathrm{HAY}_{\text {AY }}$ S.I. (2000), p. 23.

789 Kawanishia, T.; Kuroiwa, H.; Kojima, M. et al. (2000), p. 969.

790 Nicholson, S. (2005), pp. $626 \mathrm{f}$.

791 Hughes, D.A. (2006), p. 400.

792 Dinku, T.; Chidzambwa, S.; Ceccato, P. et al. (2008), p. 4097.

793 Dinku, T.; Chidzambwa, S.; Ceccato, P. et al. (2008), pp. 4104 - 4109.

794 Hughes, D.A. (2006), p. 408.

$795 \mathrm{HAY}_{\text {AY }}$ S.I. (2000), p. 23. 
This is of particular significance in the tropics, where geostationary satellites are best when short period rainfall estimates are required. ${ }^{796}$ Despite these problems, Hughes (2006) concluded that "results are encouraging enough to suggest that further detailed investigations are justified". ${ }^{797}$

A lack of reliable rain gauge data not only over thinly populated areas but also from some countries which in the past provided relatively good surface data means that estimates based on remote sensing may for some regions be the only available source of information. ${ }^{798}$

\begin{abstract}
«Networks of ground-based hydro-meteorological observations are frequently sparse in developing countries and the situation is not improving. [...] However, these are also the very same countries where improved estimates of water resource availability are required. $\gg^{799}$
\end{abstract}

The difficult access to or complete absence of meteorological station data mean that satellite data have become increasingly important for routine monitoring of precipitation over West Africa. Unfortunately, most datasets have neither been developed nor validated for this region. ${ }^{800}$

\title{
2.7.6 Geographic Information Systems (GIS)
}

Both environmental factors in the physical sense and social, economic and cultural factors play a role in the epidemiology of many diseases. Their pattern usually cause a non-uniformity of disease distribution ${ }^{801}$ and thus make them the topic of investigation for medical geography. One of the key developments in medical geography in the past two decades has been the increase in the availability and power of computing systems and the development of software to deal with the relations of spatially explicit data ${ }^{802}$, a fact that is particularly relevant for many of the diseases found on the African continent:

«GIS is a tool of great inherent potential for health in Africa as health is largely determined by environmental factors (including the sociocultural and physical environment). $\gg^{803}$

796 BaRRET, E.C. (1993), p. 120.

797 Hughes, D.A. (2006), p. 409.

798 BARRET, E.C. (1993), p. 120.

799 Hughes, D.A. (2006), p. 399.

800 NiCHOLSON, S. (2005), p. 622.

801 Moore, D.A. \& CARPenter, T.E. (1999), p. 143.

802 GLASS, G.E. (2000), p. 138.

803 Tanser, F.C. \& le Sueur, D. (2002), doi: 10.1186/1476-072X-1-4. 
The dynamic malaria situation requires ongoing research and intervention efforts to control it. ${ }^{804}$ Geographic information systems (GIS) are ideal tools for monitoring temporal variations and changes in malaria transmission pattern and its determinants.

GIS systems are used in malaria research for a variety of purposes. The most basic application involves mapping of malaria case data (e.g. incidence, burden of disease, mortality, ...) to see if obvious spatial and/or temporal pattern exist. Such maps may be overlaid by information on environmental variables such as temperature, rainfall, land cover / land use, terrain, hydrography and population distribution. GIS systems may then be used to assess statistical relationships between malaria incidence and these variables. Since availability of terrestrial data is often one of the key factors limiting such analyses, GIS systems may also be used for integrating and interpreting remotely sensed information. Once relationships between malaria occurrence and its (statistical) determinants are established, GIS systems may be used for modeling and prediction of malaria risks. ${ }^{805}$

A conceptually simple but meaningful application of GIS and Remote Sensing for risk mapping of mosquito-borne diseases was proposed by LACAUx et al. (2007). The authors suggested the derivation of zones potentially occupied by mosquitoes (ZPOM) based on the RS-based detection of breeding sites and vector flight ranges. ${ }^{806}$

Several GIS packages are currently used in the public health sector. These include ArcGIS, EpiInfo/EpiMap (developed by the US Centers for Disease Control) and Health Mapper (developed by the WHO and UNICEF). ${ }^{807}$

Recently, two web GIS systems indicating current/near-future malaria risks have become operational for the African continent. Both provide raster maps for online display and export to geographic information systems. The US Agency for International Development provides an estimation of malaria risks in the framework of the Famine Early Warning System (FEWS). The Malaria Early Warning System (MEWS) developed by the International Research Institute for Climate Prediction also provides GIS-compatible data. ${ }^{808}$

804 Martin, C.; Curtis, B.; Fraser, C. \& Sharp, B. (2002), p. 227.

805 SiPE, N.G. \& DALE, P. (2003), doi:10.1186/1475-2875-2-36.

806 Lacaux, J.P.; Tourre, Y.M.; Vignolles, C. et al. (2007), p. 73.

807 Sipe, N.G. \& Dale, P. (2003), doi:10.1186/1475-2875-2-36.

808 Grover-Kopec, E.; Kawano, M.; Klaver, R.W. et al. (2005), doi:10.1186/1475-2875-4-6. 


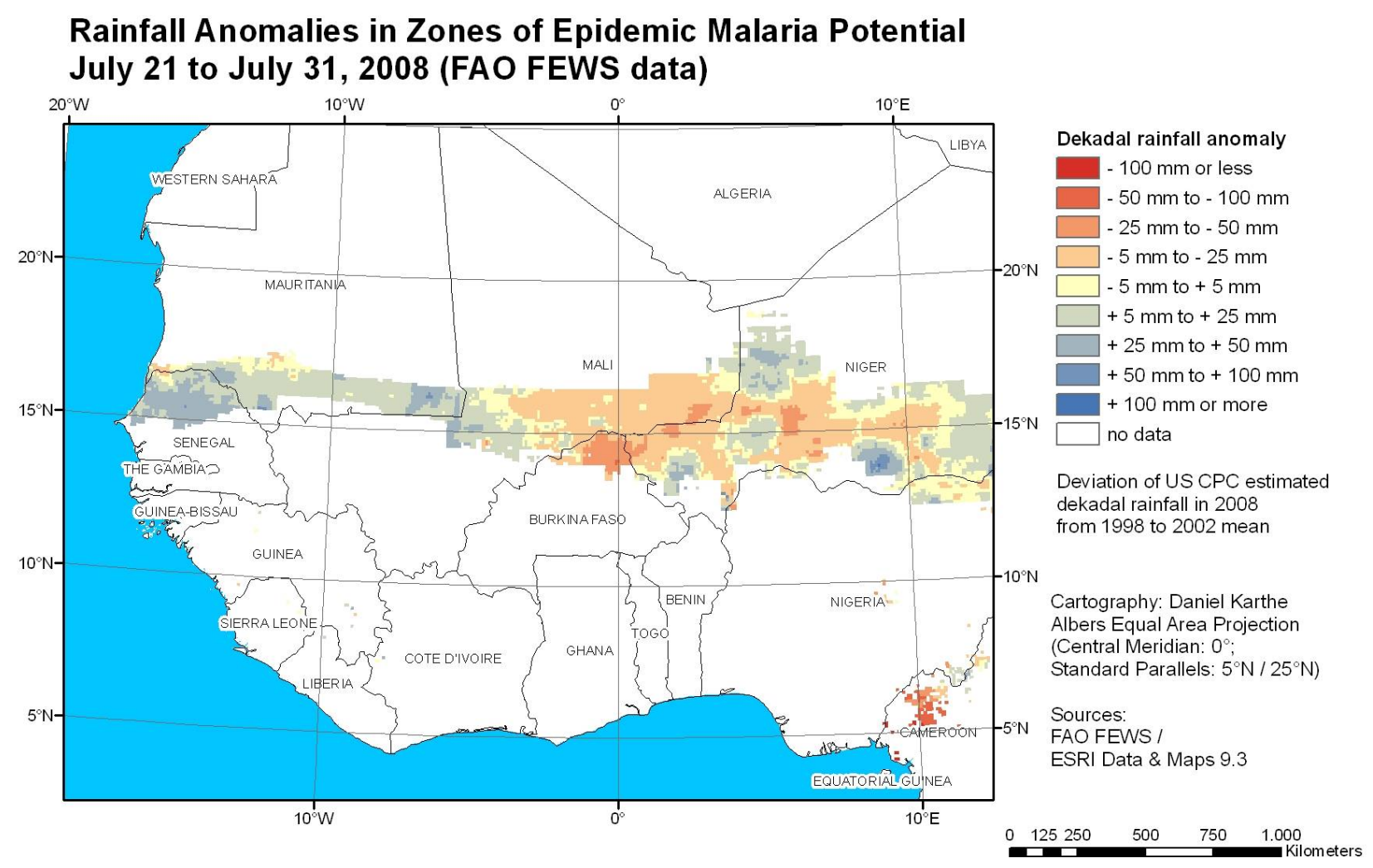

Figure 36: Rainfall anomalies in zones of epidemic malaria in West Africa (21 to 31 July 2008)

In both cases, the term "malaria early warning system" is a misnomer since both actually provide information on rainfall: while the continental scale maps provided by FEWS provide information on dekadal (10-day) rainfall anomalies in areas prone to malaria epidemics (see figure 36), the MEWS maps (see page 335) are in fact rainfall maps based on the CPCs rainfall estimate RFE 2.0. For regions of endemic malaria within Africa, only the latter dataset is of value since the former does not provide data for these. However, both datasets have in common that they only look at a single determinant of malaria transmission. In fact, this may be the reason why they do not directly indicate malaria risks, instead leaving this interpretation up to their users.

\subsubsection{Limitations of RS and GIS in Malariology}

Even though numerous studies have demonstrated the feasibility of using remotely sensed data for malaria mapping, not all projects have produced successful results. JACOB et al. (2007) attempted to predict Anopheles arabiensis habitats using several vegetation indices (NDVI, SAVI, ARVI) but found these indices unsuitable for determining local-scale ecological conditions suitable for vector breeding. ${ }^{809}$ Mushinzimana et al. (2006) found Landsat 7 ETM+

809 Jacob, B.G.; Muturi, E.J.; Mwangani, J.M. et al. (2007), doi:10.1186/1476-072X-6-21. 
imagery to be completely useless for anopheline breeding site detection in the Kenyan Highlands. ${ }^{810}$ Even high-resolution imagery often fails in the detection of small larval habitats. On a study on the feasibility of pan-sharpened IKONOS imagery for aquatic habitat detection in Kenya, Mutuku et al. (2009) found "the majority of streams and still water bodies (such as man-made ponds) [to be] undetectable by satellite imagery, either because of a closed vegetation canopy (streams) or because the spectral characteristics were similar to bare soil because of turbid water". Moreover, small but highly productive larval habitats remained undetected due to limited sensor resolution. ${ }^{811}$

One particular problem is that ecological variables which can be monitored with the help of satellites are not the only determinants of transmission. In regions of high anopheline abundance, sociological factors such as the usage of insecticide-treated bednets may create transmission situations which cannot be predicted solely with RS data. ${ }^{812}$

Even though the potentials of geographic information technology and remote sensing in malariology have been highlighted in case studies from around the world, they are still rarely used in typical field situations. Reasons include a lack of qualified staff in many malarious regions, financial implications (e.g. high cost of some RS imagery and geographic data) and data limitations (e.g. limited data availability, imprecise identification of the transmission localities, ...). ${ }^{813}$ Health data from official or government sources are often subject to underreporting and lack accurate spatial information. Moreover, data from different sources are often inconsistent with regard to the diagnostic methods used. ${ }^{814}$ In the absence of uniform diagnostic standards, they often range between "educated guesses and wild speculation". ${ }^{815}$

Besides all limitations regarding data availability and quality and some controversies about the links between malaria and various environmental factors, the use of RS and GIS in malariology is still in the experimental phase: So far GIS applications regarding health in Africa are pilot studies linked to initiatives funded or supported by international donors rather than large-scale operational systems. ${ }^{816}$

810 Mushinzimana, E.; Munga, S.; Minakawa, N. et al. (2006), doi:10.1186/1475-2875-5-13.

811 Mutuku, F.M.; BaYoh, M.N.; Hightower, A.W. et al. (2009), doi:10.1186/1476-072X-8-19.

812 HAY, S.I.; SNOW, R.W. \& Rogers, D.J. (1998), p. 310.

813 Sipe, N.G. \& Dale, P. (2003), doi:10.1186/1475-2875-2-36.

814 Moore, D.A. \& Carpenter, T.E. (1999), p. 155.

815 TANSER, F.C. \& LE SUeUr, D. (2002), doi: 10.1186/1476-072X-1-4.

816 Tanser, F.C. \& le Sueur, D. (2002), doi: 10.1186/1476-072X-1-4. 


\subsection{Malaria Control and Eradication}

Successfully controlling malaria would yield multiple benefits, including the prevention of illness and the reduction of productivity losses and household expenditures for malaria treatment. Therefore, effective malaria control is essential for progress towards the Millennium Development Goals in many affected countries ${ }^{817}$ and thus supported by numerous international organizations, including more formal bodies such as the WHO and collaborations at the NGO level such as the Afro-European "Stop Malaria Now!" initiative (see figure 37).

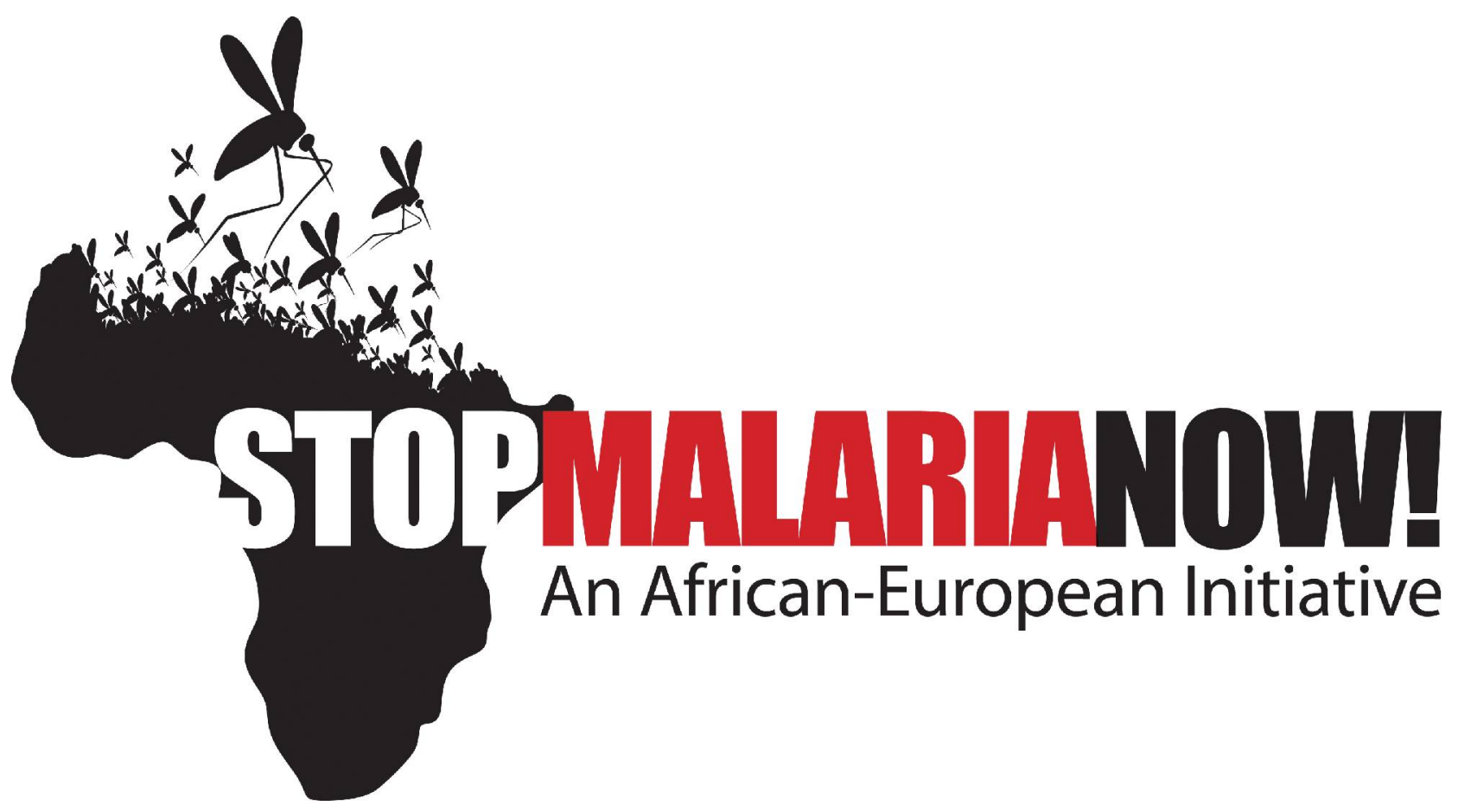

Figure 37: Logo of the Stop Malaria Now initiative ${ }^{818}$

The vector-borne nature of malaria means that eradication and control programs frequently target the mosquito population (vector control) or vectorto-host contact (exposition control), but may also focus on the prophylaxis and treatment of infections in order to reduce or suppress the transmission pressure and malaria prevalence in a region. Malaria control programs have been carried out around the world - with mixed results: whereas eradication was attained in some regions, others (particularly on the African continent) still face an almost unchanged malaria burden.

817 Roll Back Malaria Partnership (2005), p. 4.

818 http://www.stopmalarianow.org/, accessed 20/08/09. 


\subsubsection{Principles of Malaria Control and Eradication}

The very general term malaria control comprises a varied set of measures aiming at a reduction in transmission intensity. Typical control activities include

- exposure prophylaxis, such as the use of (impregnated) bednets ${ }^{819}$ and indoor application of pesticides ${ }^{820}$,

- vector control, i.e. measures to decrease vector abundance and reduce the vector survival rate (e.g. use of larvicides/insecticides, genetic manipulation or replacement of the vector population) $)^{821}$,

- environmental modifications which avoid the negative side effects of insecticides (e.g. eradication of mosquito habitats close to human dwellings ${ }^{822}$ and use of biological agents agains mosquitoes and their larvae $^{823}$ ),

- health education (in particular of women, who play a leading role in the household and in childcare $)^{824}$,

- provision of malaria-related health services (e.g. stocking of antimalarial drugs, development and administration of vaccines).

Antivector measures in malaria control normally aim for a cost-effective reduction of the transmission potential to just below the critical level for sustained transmission. ${ }^{825}$ RonaLD Ross concluded from his model that to counteract malaria, it is not necessary to entirely eradicate malaria vectors but that a reduction of their number below a certain limit is sufficient. ${ }^{826}$ However, experience from malaria eradication programs showed that malaria can rapidly resurge in epidemic form even after an almost total interruption of transmission. ${ }^{827}$

When designing malaria control programs, it is essential to know the distribution and abundance of the disease to target intervention strategies. In many African countries, the paucity of epidemiological data hinders the quantification of disease for basic planning. ${ }^{828}$ Reliable empirical maps of the geographical distribution of malaria are urgently needed for accurate estimation of disease burden, to identify areas which should be prioritized in terms of resource allocations and for assessing the progress of intervention programs. ${ }^{829}$ Therefore, the eco-epidemiological stratification of malaria risk

819 Hougard, J.M.; Fontenille, D.; Chandre, F. et al. (2002), p. 283.

820 Organisation Mondiale de la Santé (Ed.) (1995), p. 20.

821 Hougard, J.M.; Fontenille, D.; Chandre, F. et al. (2002), p. 283; Coluzzi, M. (1992), p. 113.

822 GarCia, R. \& HufFAKER, C.B. (1979), p. 295.

823 Organisation Mondiale de la Santé (Ed.) (1995), p. 20.

824 A'Rahman, S.H.; Mohamedani, A.A.; Mirgani, E.M. \& Ibrahim, A.M. (1996), p. 1433.

825 Coluzzi, M. (1992), p. 113.

826 McKenzie, F.E. \& Samba, E.M. (2004), p. 94.

827 BREMAN, J.G. (2001), p. 6.

828 BROOKer, S.; HAY, S.I. \& Bundy, D.A.P. (2002), p. 70.

829 Gemperli, A.; Sogoba, N.; Eondjo, E. et al. (2006), p. 1032. 
zones is the first prerequisite for planning intervention strategies. ${ }^{830}$ This usually begins with the determination of important vector species and their distribution. ${ }^{831}$ At the local level, the proper identification of all potential aquatic mosquito habitats is an absolute prerequisite for successful control operations. ${ }^{832}$ Control efforts should concentrate on the source population whether these are local survivors or insects migrating in from other areas. ${ }^{833}$

In areas of high transmission pressure, the effect of vector control programs is often only transitory. Some scientists even argue that efficient vector control programs may be counter-productive since they prevent the natural development of immunity to the infection ${ }^{834}$ :

Routine exposure to hyper- and holoendemic malaria protects a majority of individuals while killing a minority. Aggressive interventions that consider only that vulnerable minority risk compromising or eliminating the solid protection against severe malaria in the majority. ${ }^{835}$

Malaria eradication aims at the cessation of transmission and elimination of the reservoir of infected cases. A malaria eradication program requires a "total coverage" - malaria needs to be completely eliminated in order to prevent a resumption of transmission later on. ${ }^{836}$ Local elimination of malaria is feasible in low transmission areas. However, a small amount of immigration by infected people can prevent local eradication. ${ }^{837}$ In malaria intervention programs, people or communities in the proximity of important larval breeding sites should receive priority. ${ }^{838}$ Because of the size and cost of the undertaking, a malaria eradication program is normally organized on a national scale. It does not only involve the fight against vectors and/or parasites but also a surveillance program based on passive or active case detection. ${ }^{839}$

The experiences of malaria eradication and control programs have shown that strategies to effectively control malaria in one ecological setting may not be appropriate in the other. ${ }^{840}$ The following sections provide an overview of the different intervention strategies and discuss the scopes and limitations of malaria control.

830 Organisation Mondiale de la Santé (Ed.) (1995), p. 19.

831 TOURÉ, Y.T. (1989), p. 39.

832 Muturi, E.J.; Shilitu, J.I.; Gu, W. et al. (2007), p. 96.

833 Simard, F.; Lehmann, T.; Lemasson, J.-J. et al. (2000), p. 468.

834 Greenwood, B.M. (1997), p. 90.

835 Doolan, D.L.; Dobaño, C. \& Baird, J.K. (2009), p. 14.

836 Onori, E., Beales, P.F. \& Gilles, H.M. (1993), p. 267.

837 Gu, W.; Killeen, G.F.; Mbogo, C.M. et al. (2003), p. 43.

838 Organisation Mondiale de la Santé (Ed.) (1995), p. 20.

839 Onori, E., Beales, P.F. \& Gilles, H.M. (1993), pp. $268 \mathrm{f}$.

840 BEIER, J.C. (1998), p. 521. 


\subsubsection{Vector Control through Insecticides}

Vector control through insecticides may take place either at the larval breeding sites, or through the indoor application of residual insecticides.

Regular application of oil or insecticides to potential breeding sites does not reduce their availability to ovipositing females but suppresses their mean productivity. ${ }^{841}$ During the First World War, petroleum oil and "Paris Green" (copper acetoarsenite) were used as larvicides in mosquito breeding sites. ${ }^{842}$ To treat the breeding sites it is necessary to find them, which is not always easy. ${ }^{843}$ Larval control may be more effective if implemented during the dry season when the distribution of larval habitats is more strictly confined. ${ }^{844}$

Since the home is the setting where many vector-borne diseases, including malaria, are transmitted, strategies for their control have to involve the active participation of the residents living there. ${ }^{845}$ The indoor application of residual insecticides can be an appropriate constituent of malaria intervention programs under the following conditions:

- the vector population is predominantly endophile;

- a high percentage of buildings is treated, and surfaces are suitable for residual insecticide application;

- the vector population is susceptible to the insecticides used. ${ }^{846}$

Reduction or break-down of the malaria transmission by house-spraying with residual insecticides has been the basis of many malaria control programs since the 1950 s. ${ }^{847}$

The first insecticide that experienced mass application against malaria was DDT (dichloro-diphenyl-trichloroethane). Any female mosquito carrying malarial parasites was likely to pick up a lethal dose of the insecticide during the two weeks required for the extrinsic incubation period ${ }^{848}$, since Anopheles mosquitoes typically take a rest indoors after bloodfeeding. Spraying all surfaces of dwellings with long-lasting insecticides thus kills a substantial portion of the mosquitoes before they can transmit malaria. ${ }^{849}$ The use of DDT

841 Killeen, G.; Seyoum, A. \& Knols, G.J. (2004), p. 88.

842 RIECKMANN, K.H. (2006), p. 648.

843 Carnevale, P. \& Mouchet, J. (1987), p. 184.

844 Minakawa, N.; Munga, S.; Atieli, F. et al. (2005), p. 164.

845 Winch, P.J.; Lloyd, L.S.; Hoemeke, L. \& Leontsini, E. (1993), p. 327.

846 Organisation Mondiale de la Santé (Ed.) (1995), pp. 23.

847 Carnevale, P. \& Mouchet, J. (1987), p. 183.

848 RiECKMANN, K.H. (2006), p. 648.

849 Onori, E., Beales, P.F. \& Gilles, H.M. (1993), p. 267. 
was the key method for malaria eradication in the temperate zone, and in India its application helped to reduce the malaria incidence by $99.8 \%$ between the $1930 \mathrm{~s}$ and $1960 \mathrm{~s}$ but also induced physiological resistance among anophelines. ${ }^{850}$

A study on the effects of residual house-spraying with the insecticide dieldrin in Pare Taveta, Tanzania resulted in an infant mortality reduction from 165 per 1000 live births to 78 per 1000 life birth. One year after completion of the program, a rebound in infant mortality to 132 per 1000 life births was observed and after seven to eight years, the mortality among children aged 1 to 4 years returned to pre-intervention levels. Unfortunately, for other large malaria control projects such as the Garki study, the situation after the suspension of control measures was not monitored. ${ }^{851}$

Experience from major insecticide-based antimalarial programs demonstrated that the continued large-scale application of insecticides is not a viable option because of high costs, increasing insecticide-resistance of vector populations and adverse environmental effects such as those experienced with DDT. ${ }^{852}$ Nevertheless, some countries (including South Africa, Mozambique and Swaziland) once again promote the usage of DDT. ${ }^{853}$

Indian scientists have proposed the use of azadirachtin, a natural substance produced by neem trees (Azadirachta indica; see figure 60, page 211), to combat Anopheles mosquitoes. At a concentration of $1 \mathrm{ppm}$, the substance showed almost $100 \%$ larval mortality. ${ }^{854}$

At the household level, the use of mosquito coils and fumigation mats are popular methods to avoid mosquito nuisance and reduce the risk of indoor transmission. ${ }^{855}$

\subsubsection{Vector Control through Environmental Management}

Environmental management, even though scarcely used in Africa during the last 50 years or so, is one promising approach to control malaria, particularly since other control strategies have been found to be unaffordable, unreliable or detrimental to the environment.

850 Pates, H. \& CuRtis, C. (2005), p. 53.

851 SNOW, R.W. \& MARSH, K. (1998), p. 305.

852 Organisation Mondiale de la Santé (Ed.) (1995), pp. $4 ; 21$.

853 Roll Back Malaria Partnership (2005), p. 12.

854 Nathan, S.; Kalaivani, K. \& Murugan, K. (2005), p. 47.

855 Carnevale, P. \& Mouchet, J. (1987), p. 185. 
«The renewed interest in environmental-management-based approaches for the control of malaria vectors follows the rapid development of resistance by mosquitoes to the widely used insecticides, the increasing cost of developing new chemicals, logistical constraints involved in the implementation of residualspraying programs and the environmental concerns linked to the use of persistent organic pollutants. ${ }^{856}$

The most common environmental control strategy aims at a reduction of larval habitat productivity and is most promising in relatively small areas where the targeted habitats are clustered. ${ }^{857}$ Mosquito larval control through hydrologic environmental modification does not only reduce biting rates, but also mosquito survival and sporozoite prevalence because of the increased length of time spent seeking for oviposition sites:

\begin{abstract}
«The increased length of time gravid female mosquitoes would spend foraging for a reduced number of suitable oviposition sites was predicted to substantially extend the mean length of the gonotrophic cycle and increase the mortality associated with each feeding cycle.» ${ }^{858}$
\end{abstract}

An entirely different environmental management approach is the use of biological agents. The diversity of mosquito species and the wide range of aquatic habitats used by them dictates that the use of organisms for control must also be broad and wide ranging. ${ }^{859}$ The use of plants to interfere with mosquito development was recognized in the early 1920s. Shade has successfully been used as a control measure against vector anophelines that prefer sunlit oviposition sites such as Anopheles maculatus ${ }^{860}$; the strategy is certainly promising for other important vector species such as Anopheles gambiae.

Fish are the only biological control predators used widely for mosquito control. The most commonly used species is the mosquito fish Gambusia affinis which is adaptable to many different mosquito habitats due to its small size and its relative tolerance for temperature extremes, salinity and pollution. However, its adaptability and aggressive behavior have resulted in the displacement or extermination of several indigenous species. ${ }^{861}$ A summary of fish species that have been found useful for mosquito control are covered in table 34 .

856 Konradsen, F.; van der Hoek, W.; Amerasinghe, F.P. et al. (2004), p. 99.

857 Minakawa, N.; Sonye, G. \& Yan, G. (2005), p. 295.

858 Killeen, G.; Seyoum, A. \& Knols, G.J. (2004), p. 89.

859 GARCIA, R. (1983), p. 73.

860 GARCIA, R. (1983), p. 75.

861 GARCIA, R. (1983), p. 73. 


\begin{tabular}{|l|l|}
\hline Fish species & Experiences \\
\hline $\begin{array}{l}\text { Oreochromis spilurus } \\
\text { (tilapia) }\end{array}$ & $\begin{array}{l}\text { Very effective for larval reduction in large water } \\
\text { storage tanks; of little help for areas where temporary } \\
\text { rainpools are dominant for breeding sites }\end{array}$ \\
\hline $\begin{array}{l}\text { Gambusia affinis } \\
\text { (mosquito fish) }\end{array}$ & $\begin{array}{l}\text { Well suited for very different aquatic habitats; may } \\
\text { disturb aquatic ecosystems }\end{array}$ \\
\hline $\begin{array}{l}\text { Poecilia reticulata } \\
\text { (common guppy) }\end{array}$ & Well-suited for warm polluted waterbodies \\
\hline $\begin{array}{l}\text { Cyprinodon macularius } \\
\text { (desert pupfish) }\end{array}$ & $\begin{array}{l}\text { Desert regions; saline water at temperatures around } \\
45^{\circ} \mathrm{C}\end{array}$ \\
\hline $\begin{array}{l}\text { Gasterosteus } \\
\text { aculeatus (stickleback) }\end{array}$ & \begin{tabular}{l} 
Estuarine waterways \\
\hline 63
\end{tabular} \\
\hline
\end{tabular}

Table 34: Fish species used for mosquito control

One important limitation of biological control using larvivorous fish is their ineffectiveness in controlling very large mosquito broods resulting from the synchronous hatching of large numbers of mosquito eggs. ${ }^{864}$ The large-scale use of fish for temporary habitats such as rice fields is a particular problem and depends on the availability of large quantities of fish available for release at a certain time. ${ }^{865}$

862 Carnevale, P. \& Mouchet, J. (1987), p. 184.

863 GARCIA, R. (1983), p. 74.

864 Pates, H. \& Curtis, C. (2005), pp. $61 \mathrm{f}$.

865 GARCIA, R. (1983), p. 73. 


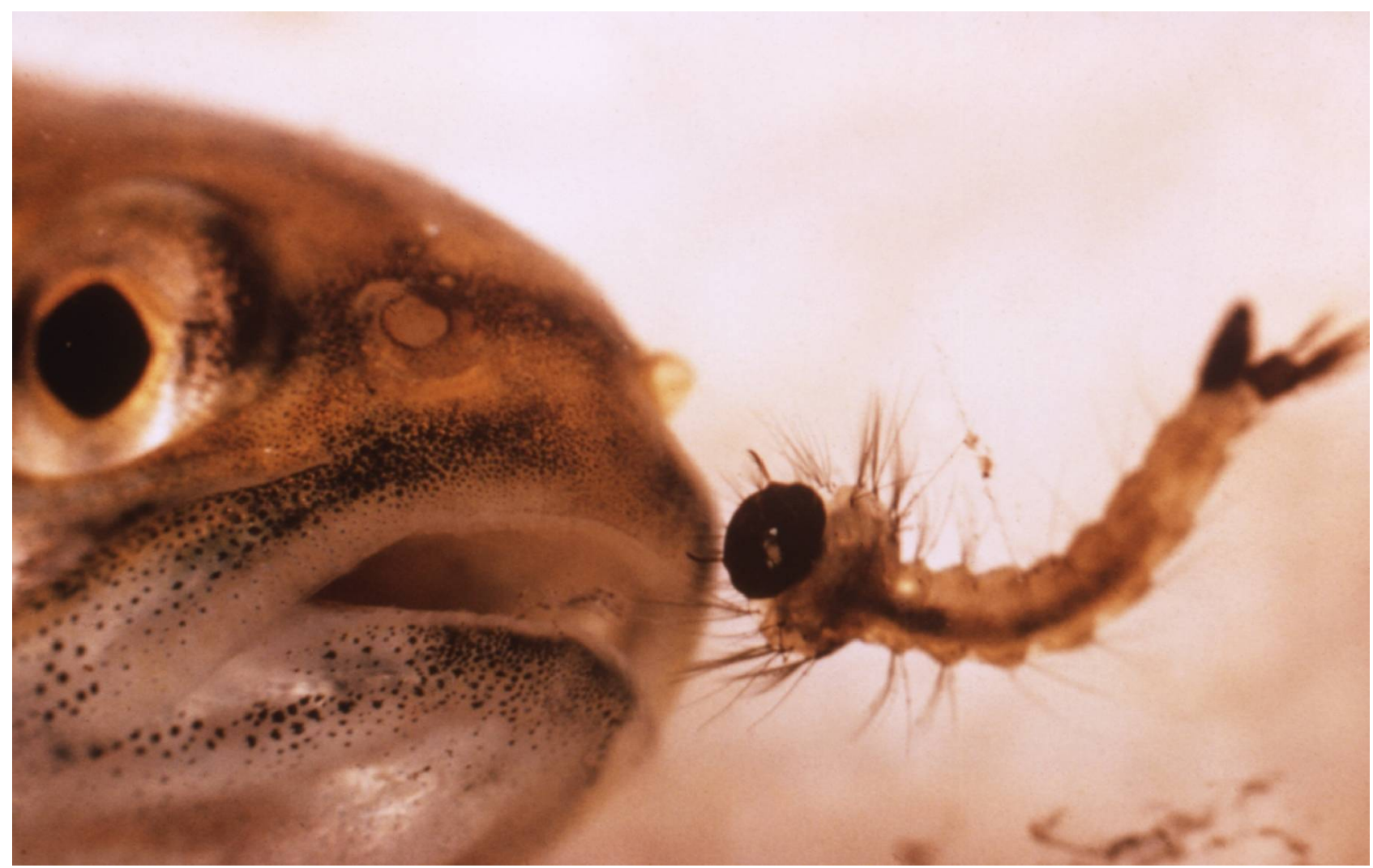

Figure 38: The mosquito fish Gambusia affinis and larval-stage Anopheles gambiae ${ }^{866}$

One predatory group of arthropods that has shown promise for mass rearing and release are predatory mosquitoes of the genus Toxorynchites. However, low egg production and a lack of synchrony in predator-prey life cycles may lead to poor vector control. Inoculative release of flatworms of the genus Mesostoma has been shown to be useful for mosquito control in irrigated rice fields. ${ }^{867}$

The Bacillus thuringiensis $\mathrm{H} 14$ is very toxic to anophelines and is considered safe for the environment. However, the residual effect of the available formulations is quite limited, so that a weekly application is necessary to keep pools free of pupae. ${ }^{868}$ Another spore-forming pathogen, Bacillus sphaericus, has been under investigation for use as a microbial insecticide. Investigations with this organism in roadside ditches have revealed evidence of long-term suppression of mosquito larvae. Coelomomyces fungi have been found to cause high mortality among natural populations of mosquitoes. This pathogen seems to persist in mosquito habitats for long periods and may reduce mosquito populations by more than $90 \% .{ }^{869}$

866 CDC Public Health Image Library (http://phil.cdc.gov/), image ID 4958, accessed 20/08/09.

867 GARCIA, R. (1983), p. 74.

868 Carnevale, P. \& Mouchet, J. (1987), p. 184.

869 GARCIA, R. (1983), p. 75. 
Chapter 2 - Malaria in West Africa: Transmission, Monitoring \& Control

\subsubsection{Prevention of Vector-Host Contact}

Several strategies can be pursued to prevent or reduce exposition to mosquito bites. Since Anopheles mosquitoes are particularly active during the night (especially dusk and dawn), bednets can offer a substantial degree of protection against malaria. A very different concept is zooprophylaxis: potential mammal hosts may divert mosquitoes from humans, thereby reducing the risk of malaria transmission.

The earliest recorded use of bednets goes back as far as the $6^{\text {th }}$ century BC, probably used as a barrier against blood-sucking insects for uninterrupted sleep. ${ }^{870}$ Today, the use of insecticide-treated bednets (ITNs) is recommended by the $\mathrm{WHO}$ and one of the key strategies for malaria control. ${ }^{871}$

A study carried out in The Gambia showed that malaria incidence may be inversely related to mosquito density and activity, since high biting rates may cause people to use (permethrine-impregnated) bednets. Conversely, in regions of low mosquito density, exposition prophylaxis is often regarded less important and may lead to a higher incidence of malaria. ${ }^{872}$

Very often bednets are wrongly placed especially when people sleep on mats. Moreover, the maintenance of nets is often poor and when they are perforated they do not work properly. ${ }^{873}$ Low retreatment rates are another major cause of concern in areas where insecticide-treated bednets are used. Whereas free re-treatment of bednets typically results in retreatment rates of more than $60 \%$, only $5 \%$ to $30 \%$ of the nets are re-treated in most operational projects with a cost recovery element. ${ }^{874}$ The cost of retreatment for a double net is around US\$ 0.50 to US\$ 1.00 for permethrine, the most commonly used insecticide which should be applied every six months. Using cheaper insecticides with longer life expectancies can reduce re-treatment costs: deltamethrine requires only one treatment per year and typically costs US\$ 0.10 to US $\$ 0.60$ (all costs based on a bulk re-treatment; costs for individual packages may be higher). ${ }^{875}$

Even though regular re-treatment of bednets is viewed as critical for malaria control, many control programs in Africa report low retreatment rates. A study carried out in The Gambia showed, however, that the use of untreated nets which are otherwise in good condition is associated with a significantly lower

870 GUYAT, H.L. \& SnOW, R.W. (2002), p. 12.

871 Noor, A.M.; Mutheu, J.J.; TAtem, A.J. et al. (2009), p. 58.

872 Thomson, M.C.; D'Alessandro, U.; Bennet, S. et al. (1994), p. 641.

873 Carnevale, P. \& Mouchet, J. (1987), p. $184 \mathrm{f}$.

874 GuYAT, H.L. \& SNOW, R.W. (2002), p. 12.

875 GuYat, H.L. \& Snow, R.W. (2002), p. 14. 
prevalence of malaria. ${ }^{876}$ Other studies comparing insecticide-treated and untreated bednets suggest that untreated nets are at least half as effective against malaria transmission as treated ones. ${ }^{877}$ Despite a general increase in bednet use, coverage rates in Africa are sill low. In Burkina Faso, for example, the proportion of children sleeping under ITNs increased from $2 \%$ in 2003 to $10 \%$ in $2006 .{ }^{878}$ Despite great progress in recent years, millions of African children do still not have access ITNs.

While most studies have found at least some degree of malaria reduction in areas of high bednet use, some scientists believe that bednet use may be counterproductive in the long run:

«[...] such systems [...] induce changes in [mosquitoes'] biting cycle and indoor/outdoor biting behavior and may therefore render bednets useless $[\ldots] .{ }^{879}$

From their mathematical transmission model, ChIYAKA et al. (2008) deduced that personal protective measures such as bednet use may contribute to overall disease control only if their efficacy and compliance are very high. ${ }^{880}$

The abundance of cattle as alternative blood meal hosts and their proximity to humans have been implicated as important determinants of transmission by Anopheles arabiensis as they may divert mosquitoes from feeding on humans:

«If effective tsetse fly control and improved water management were to enable cattle rearing as an alternative agricultural practice, previous analyses have suggested that stocking densities of one animal per person could confer substantial zooprophylactic protection against this particular vector. ${ }^{881}$

Since cattle are no Plasmodia hosts, blood meals taken from cattle prevent potential infections of human hosts and vector mosquitoes; infectious mosquitoes cannot pass on the parasite to cattle, and mosquitoes taking most blood meals from cattle do not become infected themselves. ${ }^{882}$

876 Clarke, S.E.; Bøgh, C.; Brown, R. et al. (2001), p. 457-462.

877 GuYat, H.L. \& SnOW, R.W. (2002), p. 14.

878 Noor, A.M.; Mutheu, J.J.; TAtem, A.J. et al. (2009), p. 60.

879 TAKKEN, W. \& KNols, B.G.J. (1999), p. 132.

880 Chiyaka, C.; TChuenche, J.M.; Garira, W. \& Dube, S. (2008), p. 641.

881 Killeen, G.; Seyoum, A. \& Knols, G.J. (2004), p. 89.

882 Hoshen, M.B. \& Morse, A.P. (2004), doi:10.1186/1475-2875-3-32. 


\title{
2.8.1.4 Treatment and Chemoprophylaxis
}

Malaria is a curable disease if treated adequately and promptly. ${ }^{883}$ Since the advent of the first synthetic antimalarials in the early 20th century, only a small number of compounds has proved suitable for human use. ${ }^{884}$

Quinine had been the mainstay for malaria treatments for centuries until, in the 1920s, three substitutes were developed. One of them, chloroquine, became the standard treatment in the 1940s, and in the 1950s a combination therapy of chloroquine and primaquine was introduced to prevent relapses in Plasmodium vivax malaria. At the same time, proguanil and pyrimethamine and sulfadoxine were introduced for malaria chemoprophylaxis. ${ }^{885}$

Drug resistance of Plasmodium falciparum is expanding rapidly and is a serious threat for chemotherapy and chemoprophylaxis. It has been known since 1954 that resistance to pyrimethamine was developing quickly in areas where this drug was used mainly for chemoprophylaxis. ${ }^{886}$ Other reasons for resistance of malaria parasites include inadequate or incomplete treatments of infections, high parasite adaptability and a massive reproduction rate that allows selected parasite populations to emerge relatively rapidly. ${ }^{887}$ Chloroquine-resistant malaria first emerged in Thailand in $1957^{888}$ and reached Africa in $1979 .{ }^{889}$ But the late 1980 s, resistance had spread to virtually all of sub-Saharan Africa ${ }^{890}$ :

\begin{abstract}
«The rapid spread of resistance against chloroquine, for decades the most important safe, effective and affordable antimalarial drug worldwide, was considered a public health disaster for SSA by 1998. ${ }^{891}$
\end{abstract}

Since 1984, mefloquine has been used to cure multidrug-resistant malaria and is still widely recommended as a prophylactic for visitors to endemic areas. However, resistance to mefloquine has been observed since 1989. In the 1990s, malarone, a combination of proguanil and atovaquone, was developed as an alternative to mefloquine. Since malarone is relatively expensive to produce, it is unaffordable for most people living in malarious areas. ${ }^{892}$ Pyrethamine-sulfadoxine, a more affordable combined formulation, has been

883 TUTEJA, R. (2007), p. 4674.

884 HYDE, J.E. (2007), p. 4688.

885 Rieckmann, K.H. (2006), p. 650f; Hyde, J.E. (2007), p. 4689.

886 Carnevale, P. \& Mouchet, J. (1987), p. 182.

887 HYDE, J.E. (2007), p. 4689.

888 HYDE, J.E. (2007), p. 4689.

889 RieCKMANN, K.H. (2006), p. 651.

890 HYDE, J.E. (2007), p. 4689.

891 Kouyaté, B.; Sié, A.; Yé, M. et al. (2007), p. 997.

892 RieCKMAnN, K.H. (2006), p. 653. 
extensively used to combat chloroquine-resistant parasites in Africa since the 1990s, but its efficacy is already decreasing. ${ }^{893}$ Several Sub-Saharan African countries now officially recommend artemisinin-based combination therapies (ACTs), but again, due to high costs and short supply, this is more a matter of policy than practice. ${ }^{894}$ Moreover, first indications of resistance against some ACTs have been reported from East Africa. ${ }^{895}$ Because of the high cost of 'modern' drugs and resistances to them, traditional forms of treatment are a potential source of new antimalarial compounds. ${ }^{896}$

In developing countries, antimalarials are sometimes a target of criminal action. Fake drugs are often of substandard quality and may increase malariarelated mortality. ${ }^{897}$ Moreover, in Africa many people prefer to use traditional plant extracts for the curative treatment of malaria. ${ }^{898}$

An important consideration in the framework of malaria control projects is that the treatment of existing infections alone is insufficient:

\begin{abstract}
«Antimalarial drugs have little impact at the intensity of transmission at the community level because most drugs do not reduce the production of Plasmodium gametocytes, the parasite stage responsible for initiation of infection in mosquitoes.» ${ }^{899}$
\end{abstract}

Therefore, treatment campaigns have to be accompanied by other control strategies such as antivectorial measures.

To date, chemoprophylaxis remains the best way to protect non-immune people who stay for a limited period in endemic areas. With regard to local populations, chemoprophylaxis of pregnant women is often advised but prophylaxis of infants and children below the age of five seen more critically. For children, there is a higher risk of side effects, and the mass administration of antimalarials often leads to the development of resistant strains of parasites and a lower antibody rate of the local population. ${ }^{900}$

893 HYDE, J.E. (2007), p. 4692.

894 KouYaté, B.; Sié, A.; Yé, M. et al. (2007), p. 997.

895 HYDE, J.E. (2007), p. 4693.

896 Ouattara, Y.; Sanon, S.; Traoré, Y. et al. (2006), p. 75.

897 Tipke, M.; Diallo, S.; Coulibaly, B. et al. (2008), doi:10.1186/1475-2875-7-95.

898 Ouattara, Y.; Sanon, S.; Traoré, Y. et al. (2006), p. 75.

899 Mushinzimana, E.; Munga, S.; Minakawa, N. et al. (2006), doi:10.1186/1475-2875-5-13.

900 Carnevale, P. \& Mouchet, J. (1987), p. 183. 


\subsubsection{Development of Malaria Vaccines}

In the past, the development of vaccines usually relied on the inactivation of pathogens or their toxins, while more recently focus centered on an induction of quasi-natural immunity ${ }^{901}$. The first human malaria vaccine trials were conducted shortly after the Global Malaria Eradication Program was abandoned in $1969 .{ }^{902}$

Malaria vaccines may be directed either at interrupting transmission or at preventing clinical malaria. ${ }^{903}$ The observation of that a transfer of immunoglobulins from semi-immune adults can help to cure the clinical complications of malaria led to the development of a malaria vaccine based on Plasmodium antigens. ${ }^{904}$

So far, vaccine candidates were found to have an efficacy of up to $30 \% .{ }^{905}$ Such partially effective vaccines would need to be a part of an integrated control program because otherwise transmission would continue in a setting where lower levels of natural immunity might result in higher fatality rates. ${ }^{906}$ Combining vaccines that target multiple stages of the parasite's life cycle may help to avoid such problems. ${ }^{907}$

So far, all expectations that a malaria vaccine was "just around the corner" proved to be false and the deployment of an effective vaccine is probably still years away. ${ }^{908}$

\subsubsection{The History of Malaria Control and Eradication}

In 1911 Ronald Ross wrote that malaria could be completely eradicated in a locality if three preventive measures were adopted: personal protection, mosquito reduction and treatment. ${ }^{909}$ Management of mosquitoes through the manipulation of the environment had been practiced since the initiation of organized mosquito control programs and formed their backbone until the advent of modern synthetic insecticides in the 1940 s. ${ }^{910}$

901 TODRYK, S. \& BEJON, P. (2009), p. 2007.

902 Greenwood, B. \& TARget, G. (2009), p. 582.

903 Greenwood, B. \& TARgett, G. (2009), p. 584.

904 Matuschewski, K. \& Mueller, A.K. (2007), p. 4681.

905 Greenwood, B. \& TARGett, G. (2009), p. 584.

906 TODRYK, S. \& BEJON, P. (2009), p. 2009.

907 TODRYK, S. \& BEJON, P. (2009), p. 2009.

908 Greenwood, B. \& TARgett, G. (2009), p. 582.

909 RiECKMANN, K.H. (2006), p. 647.

910 GARCIA, R. (1983), p. 73. 
Paul Müller's discovery in 1939 of the insecticidal properties of DDT (dichlorodiphenyl-trichloroethane) introduced a new concept for the control of malaria. For many months after walls and other surfaces of a house were sprayed with this long-acting insecticide, the mosquitoes landing on a sprayed surface were killed. By the late 1940s, wide-spread spraying campaigns had reduced the longevity of mosquitoes sufficiently to interrupt the transmission of malaria parasites in several areas. ${ }^{911}$

Soon after World War II, the World Health Organization recognized that malaria killed more people than any other disease and that it severely impaired the economic development of the affected regions. ${ }^{912}$ Malaria control was actively undertaken in the post-war years and brought impressive success in some countries. ${ }^{913}$ In 1955, the concept of malaria eradication was adopted by the Eighth World Health Assembly and a global strategy for malaria eradication was launched in the following year. ${ }^{914}$ By the 1950s and 1960s, DDT sprayings had become the backbone of most intervention programs. ${ }^{915}$ In most tropical countries (except for Sub-Saharan Africa, where eradication was never attempted), a moderate to marked drop in the prevalence of malarial infection in infants was observed. ${ }^{916}$ Because of the the presumed intensity of transmission and the lack of health infrastructure, Africa was initially not included in the global malaria eradication program. The excellent results obtained elsewhere with DDT house-spraying in interrupting malaria transmission encouraged the initiation of more than 20 pilot projects in various African countries during the mid 1950s and early 1960s. ${ }^{917}$

Although technical problems, such as insecticide-resistance in the mosquitoes and drug-resistance in the parasites, hampered eradication in some places, the reluctance of local populations became a major problem and a lack of information about changing local epidemiological and social conditions made control efforts fruitless. ${ }^{918}$ In 1968, it was recognized that eradication could not be attained everywhere. By 1970, eradication programs had freed more than 700 million people of the risk of malaria - more than half of the population living in originally malarious areas. ${ }^{919}$ As experience showed that malaria eradication could not be achieved in some countries, a conversion of many eradication programs to malaria control programs took place. In the following years, the malaria situation greatly deteriorated and malaria resurged in

911 Rieckmann, K.H. (2006), p. 648.

912 Onori, E., Beales, P.F. \& Gilles, H.M. (1993), p. 267.

913 Carnevale, P. \& Mouchet, J. (1987), p. 181.

914 RieckMANn, K.H. (2006), p. 649.

915 Organisation Mondiale de la Santé (Ed.) (1995), p. 21.

916 RieCKMANN, K.H. (2006), p. 649.

917 Molineaux, L. \& Gramiccia, G. (1980), p. 11.

918 RiECKMANN, K.H. (2006), p. 649.

919 Onori, E., Beales, P.F. \& Gilles, H.M. (1993), p. 270. 
several South Asian and Latin American countries. The number of reported cases of malaria more than doubled by $1977 .{ }^{920}$ In that year, the World Health Organization reported 10 million cases annually, but Africa was not included in these statistics ${ }^{921}$ :

\begin{abstract}
«In Asia, vector control measures (based on integrated vector control strategies and insecticide spraying) have been used with relative success to reduce anopheline populations and control the spread of the disease. However, over the same period of time it seems that, in sub-Saharan Africa, the malaria situation has either remained constant or continued to worsen. $\gg^{922}$
\end{abstract}

It is difficult to assess the success of malaria control on a global scale as case detection activities in many countries have been greatly reduced because of their high cost. Moreover, in tropical Africa most countries report only cases of malaria that attend health institutions, which cover not more than 10 to $20 \%$ of the population. ${ }^{923}$

In 1975, the UNDP, the World Bank and the WHO created a program for Tropical Disease Research (TDR) to address the need for research into neglected tropical diseases that represent major public health problems in developing countries. Since then, four of these diseases - Chagas' disease (American trypanosomiasis), lymphatic filariasis, onchocerciasis and leprosy (not a vector-borne disease) have been targeted for elimination as public health problems. ${ }^{924}$ The main areas of research envisaged by the TDR program are

- the acquisition of basic knowledge about the biological, socioeconomic and behavioral determinants of "neglected" tropical diseases (NTDs) and

- the development of improved methods, strategies and tools for use in infectious disease prevention and control. ${ }^{925}$

Unfortunately, there is no universal agreement on which diseases are regarded NTDs. Therefore, malaria is not always included in programs on NTD research and control. ${ }^{926}$

920 Onori, E., Beales, P.F. \& Gilles, H.M. (1993), pp. $270 f$.

921 Carnevale, P. \& Mouchet, J. (1987), p. 181.

922 De Plaen, R.; Seka, M.-L. \& Koutoua, A. (2004), p. 136.

923 Onori, E., Beales, P.F. \& Gilles, H.M. (1993), p. 271.

924 Remme, J.H.F.; Blas, E.; Chitsulo, L. et al. (2002), p. 435.

925 Remme, J.H.F.; Blas, E.; Chitsulo, L. et al. (2002), p. 438.

926 Hotez P.J.; Molyneux, D.H.; Fenwick, A. (2006), p. 576. 


\title{
2.8.3 Major Campaigns in Operation
}

Even though the global malaria eradication drives appear to be a thing of the past, several major international campaigns against malaria have been launched in the past few decades.

\begin{abstract}
«After a 25-year period of apathy, the international health community has once again taken up the challenge of malaria control and moved this higher up the agenda.» ${ }^{927}$
\end{abstract}

In the late 1990s, the "Roll Back Malaria" (RBM) campaign was initiated to counter the enormous malarial burden in Africa. ${ }^{928}$ In 2000, the Abuja Summit on Malaria was held in Nigeria, where African heads of state established control objectives for the next decade. ${ }^{929}$ RBM's current efforts are outlined in its 2005 to 2015 strategic plan (see table 35):

\section{Targets}

- $80 \%$ of people at risk from malaria are protected

- $80 \%$ of malaria patients are treated with effective antimalarials within one day of the onset of illness

- $80 \%$ of pregnant women living in areas of stable transmission receive preventive treatment

- malaria burden is reduced by $50 \%$ as compared to the situation in 2000

\section{Targets}

- malaria morbidity and mortality are reduced by $75 \%$ in comparison to the situation in 2005

- malaria related MDGs are achieved across all affected countries

- the poorest groups in all affected countries profit particularly from effective interventions

Table 35: Goals of the Roll Back Malaria initiative ${ }^{930}$

927 Greenwood, B. \& TARGet, G. (2009), p. 583.

928 Hay, S.I.; Omumbo, J.A.; Craig, M.H. \& Snow R.W. (2000), p. 174.

929 BREMAN, J.G. (2009), p. 17.

930 Roll Back Malaria Partnership (2005), p. 2. 
The RBM initiative intends to achieve these goals by initiating malaria monitoring systems and supporting countries to implement effective malaria control interventions but recognizes the necessity of more research on the development of locally appropriate and effective intervention practices. ${ }^{931}$ Beyond 2015, the RBM partnership envisions the complete eradication of malaria. ${ }^{932}$

The overwhelming impact of malaria mortality and morbidity, especially in the African context, led the Consultative Group on International Agricultural Research (CGIAR) to launch a System-wide Initiative on Malaria and Agriculture (SIMA) in 2001. ${ }^{933}$ The goals of SIMA are to promote research and capacity building needed to increase the understanding of the links between malaria and agriculture, which is the only economic activity of many rural communities in malaria risk zones. ${ }^{934}$

The creation of the Global Fund to Fight AIDS, Tuberculosis and Malaria in 2002 renewed the focus on the diseases which are the leading causes of death in much of the developing world and particularly Africa. The Global Fund pursues two key strategies: to raise financial resources needed for the control of AIDS, tuberculosis and malaria, and to provide grants to projects at the national level. ${ }^{935}$

\subsubsection{Current Limitations of Malaria Control}

So far, malaria eradication programs have been a complete failure in some regions, and many control programs have only had limited success. In the early $21^{\text {st }}$ century, the global malaria burden was higher than ever before. ${ }^{936}$

The reasons for the reverses of the global malaria eradication program are complex. In some cases, vector control programs were simply stopped. ${ }^{937}$ Technical obstacles such as the exophilic habits of some anopheline species, resistance of malaria vectors to insecticides, resistance of Plasmodia to antimalarial drugs, inaccessibility of some outlying houses, inadequate basic health services and the primitive structure of houses have contributed to the

931 Roll Back Malaria Partnership (2005), p. 3.

932 Roll Back Malaria Partnership (2008), p. 26.

933 VAN der Hoek, W. (2004), p. 95.

934 Mutero, C.M.; Amerasinghe, F.; Boelee, E. et al. (2005), p. 12.

935 The Global Fund to Fight Aids, Tuberculosis and Malaria (2007), p. 6.

936 Hay, S.I.; Guerra, C.A.; TATem, A.J. et al. (2005), p. 81.

937 Doolan, D.L.; Dobaño, C. \& Baird, J.K. (2009), p. 13. 
failure of eradication programs. Extensive agricultural development projects have aggravated the situation. ${ }^{938}$ Because of market constraints, few new insecticides have been developed against common disease vectors in developing countries over the past three decades. ${ }^{939}$

Many malaria control programs have failed for the simple reason that they did not take into account suitable epidemiological data:

\begin{abstract}
«Despite the established tradition of defining malaria transmission in communities by stability, intensity and seasonality, there are few examples of how these descriptions have been used to guide and select interventions. ${ }^{940}$
\end{abstract}

Due to a lack of comprehensive health information systems and national registrations, there is only little information about the epidemiological pattern of malaria in many parts of sub-Saharan Africa. Since the rough maps of seasonal malaria risk produced in the 1950s, there has only been a limited number of mapping efforts. ${ }^{941}$ One important consequence is that rises in malaria incidence still often come surprisingly, resulting in the unpreparedness of local health authorities:

\begin{abstract}
«Currently, epidemic responses in many nations are reactive. Awareness of epidemics arises anecdotally or in the media and if the indications seem serious enough, investigators are sent to confirm the presence of an epidemic. [...] If these responses occur quickly enough, then supplies may be augmented before an epidemic peaks. Too often, however, such augmentation takes place too late to have any substantial effect on the course of an epidemic. ${ }^{942}$
\end{abstract}

Any campaign directed against malaria transmission may have unwanted results unless it is carried out properly. In areas of low-transmission, malaria prevalence readily reverts to previous levels when vector and parasite populations survive the perturbations caused by intervention. ${ }^{943}$ In highly endemic areas, a campaign which is not stringent enough in fighting vectors and parasites may ultimately cause a rise in malaria transmission. This may be the case when mosquitoes or parasites adapt and develop resistance against the insecticides or antiprotozoals used. ${ }^{944}$ Campaigns using both strategies at low dosages have often proven to be particularly counterproductive..$^{945}$

938 Onori, E., Beales, P.F. \& Gilles, H.M. (1993), pp. $276 \mathrm{f}$.

939 Hemingway, J.; Beaty, B.J.; Rowland, M. et al. (2006), p. 308.

940 Oмumbo, J.A.; Ouma, J.; Repuoda, B. et al. (1998), p. 7.

941 HaY, S.I.; Oмumbo, J.A.; CRAIG, M.H. \& Snow R.W. (2000), p. 176.

942 Kiszewski, A.E. \& Teklehaimanot, A. (2004), p. 132.

943 Gu, W.; Killeen, G.F.; Mbogo, C.M. et al. (2003), p. 44.

944 Martens, P. (1998), p. 99.

945 Martens, P. (1998), p. 101. 
One major deficit of malaria control initiatives in the past was their short lifespan and a neglect of long-term effects: In most cases, organizations promoting vector control either through residual spraying or bednets did not set up long term model control zones in which "the fundamental question of delayed acquisition of effective clinical immunity could be studied". ${ }^{946}$

\title{
2.8.5 Prospects for Malaria Control
}

Future intervention campaigns may build on both the experiences of control efforts in the past and new developments in malariology. In the recent past, progress has been made in several fields including malaria modeling, environmental monitoring, vector control, drug and vaccine development.

Malaria models have given important insights into vector control. In the 1950s, MACDONALD already concluded from his malaria model that antilarval measures are one of the most ineffective means of malaria control. Based on sensitivity analyses of the model parameters, he found that reductions in biting rate and mosquito longevity have a greater impact on malaria transmission than the application of larvicides. ${ }^{947}$ The Garki model revealed that there is a critical vectorial capacity $C^{*}$ below which malaria transmission ceases. This happens on the condition that the basic reproduction rate is less than 1 , i.e. if less than one secondary case can be associated with each primary infection. A rate of 0.022 infectious contacts per person per day is seen as the critical vectorial capacity by the developers of the Garki model; above this critical level, the level of endemicity quickly reaches $100 \% .{ }^{948}$

While models can help to predict the outcomes of interventions, malaria risk maps are essential tools for their effective implementation.

\begin{abstract}
«That there are so few examples of the use of epidemiological maps in malaria control may be explained by the lack of suitable, spatially defined data and of an understanding of how epidemiological variables relate to disease outcome. However, recent evidence suggests that the clinical outcomes of infection are determined by the intensity of parasite exposure, and developments in geographical information systems (GIS) provide new ways to represent epidemiological data spatially. ${ }^{949}{ }^{\text {pro }}$
\end{abstract}

946 SNOW, R.W. \& MARSH, K. (1998), p. 306.

947 RuAN, S.; XIAO, D. \& BEIER, J.C. (2008), p. 1098.

948 Molineaux, L. \& Gramiccia, G. (1980), pp. $281 \mathrm{f}$.

949 Oмumbo, J.A.; Ouma, J.; Repuoda, B. et al. (1998), p. 7. 
Such advances may, in the future, contribute to more effective intervention campaigns, as may new developments in genetic engineering and drug development. Research into the genome sequence of the Anopheles gambiae mosquito, one of the main vectors of human malaria, has begun to provide opportunities to improve on existing and develop new vector control tools. ${ }^{950}$ There have been promising advances in the field of genetic control, which is in fact not a single strategy but a range of techniques that may be subdivided into two broad categories: population suppression and population replacement. While the sterile insect technique (SIT) has already been used successfully for the control of various insect species ${ }^{951}$, the development of transgenic mosquitoes which are incapable of spreading malaria parasites have recently appeared as an option for malaria control. Despite recent successes in genetically modifying mosquito vector competence in the laboratory, a lot of research on their release into the wild is still needed. ${ }^{952}$ Both techniques still have some limitations, with the mass rearing and release of modified mosquitoes being one of the key problems to be solved. ${ }^{953}$ The sterile insect technique in many cases yielded low success rates because of the low mating capacities of released insects. ${ }^{954}$

950 Touré, Y.; Oduola, A.M.J \& Morel, C.M. (2004), p. 142.

951 Coleman, C. \& Alphey, L. (2004), p. 433.

952 TUteja, R. (2007), pp. $4674 f$.

953 Coleman, C. \& Alphey, L. (2004), pp. $434 f$.

954 ChRistophides, G.K. (2005), p. 327. 


\section{Case Study: Malaria in Kossi Province}

Kossi Province is a malaria-endemic region in western Burkina Faso with a seasonal transmission pattern typical for the transition zone between the Sahel to the north and the Sudanian zone to the south. Despite this general pattern, transmission is far from uniform in the spatial and interannual perspective.

In Kossi Province, a vulnerable population is confronted with a high risk of malaria transmission. Residents often live in close proximity to vector breeding sites, and many villagers suffer from a poor state of nutrition and the cooccurrence of several infectious diseases. Inadequate protective measures (sometimes caused by misapprehensions about malaria transmission) on the one hand and limited access to adequate health care on the other hand mean that malaria continues to be an important cause of death in the region, particularly in young children.

The high frequency of malaria cases, large spatio-temporal variations in malaria transmission intensity, the routine recording of malaria cases at a dense network of rural health centers and the presence of one of Burkina Faso's most important health (and particularly malaria) research centers, the CRSN (Centre de Recherche en Santé de Nouna) in Nouna mean that Kossi Province is quite ideal for geomedical studies of malaria transmission. Moreover, the low level of mobility of a population living largely from subsistence farming and animal herding mean that recorded malaria cases at a certain health center are likely to be locally contracted rather than imported. Despite all limitations, data availability on demographic trends and malaria incidence are better than in many other parts of West Africa.

The following case study provides an overview of the physical and socioeconomic environment in Kossi Province before addressing the spatiotemporal distribution of malaria in the region. These variations and their determinants -environmental and anthropogenic- are then analyzed in order to assess their relative importance. Based on the results, the feasibility of a malaria monitoring and prediction system for the region are discussed. 


\subsection{Physical Environment}

Burkina Faso is a land-locked country located in West Africa. It shares borders with Ivory Coast, Mali, Niger, Benin, Togo and Ghana. Kossi Province is located in the western part of Burkina Faso, close to the border with Mali, around $13^{\circ} \mathrm{N}$ and $4^{\circ} \mathrm{W}$.

The relief is characterized by flatness and relatively low elevations (see figure 39); the climate is semi-arid to semi-humid with annual mean temperatures around $28^{\circ} \mathrm{C}$. There is a positive north-south moisture gradient which is paralleled by the vegetation which ranges from semi-desert in the north to tropical forests in the south.

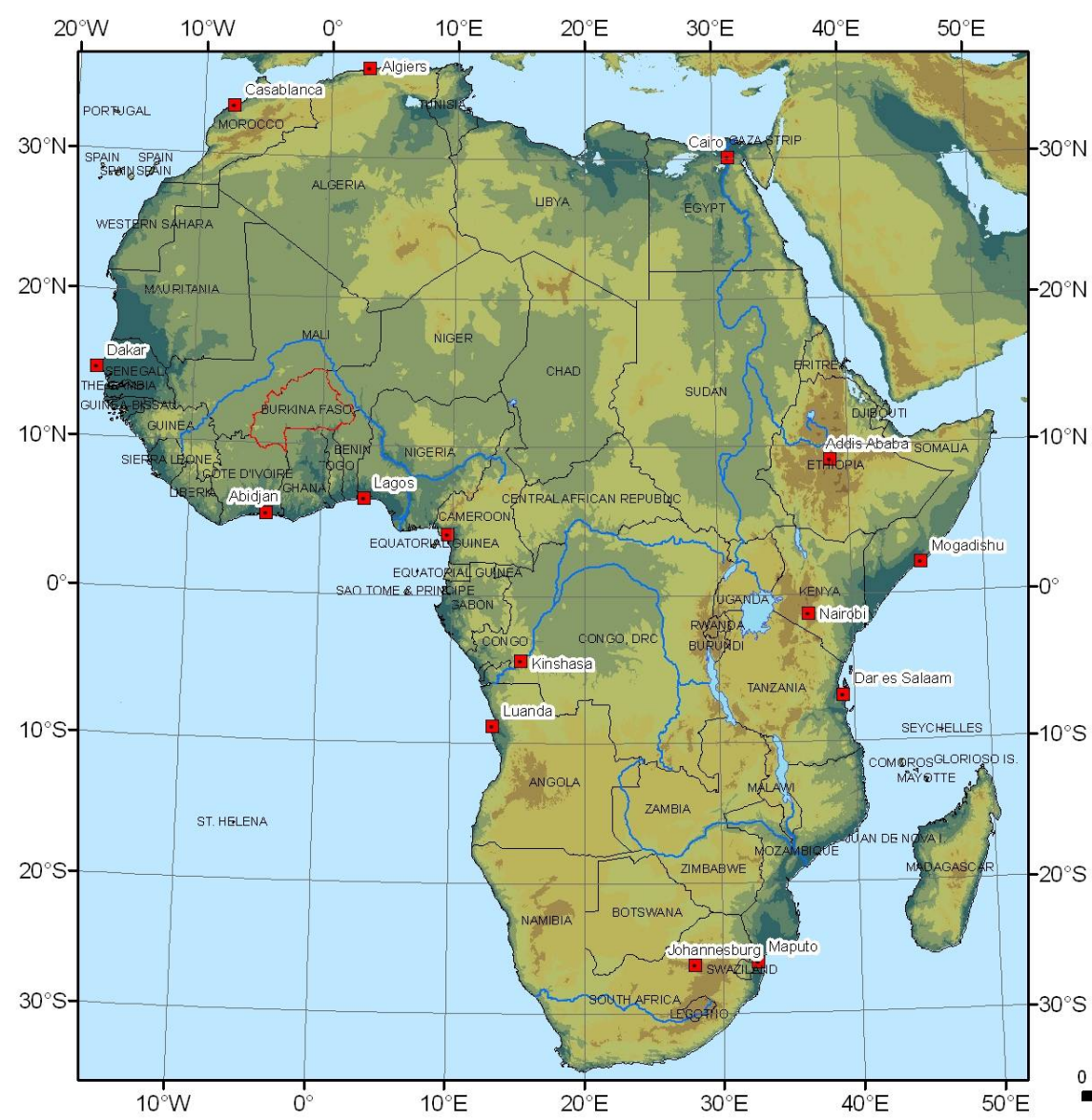

\section{Physical Map of Africa}

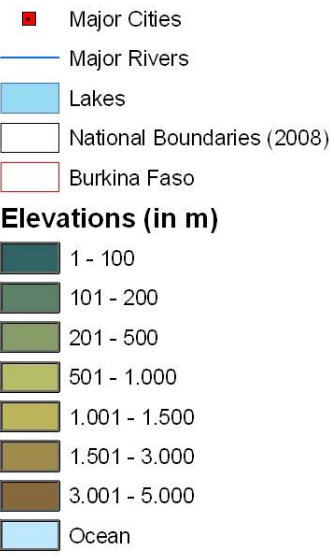

Cartography: Daniel Karthe

Albers Equal Area Projection (Central Meridian: $20^{\circ} \mathrm{OI}$

Standard Parallels: $20^{\circ} \mathrm{N} / 15^{\circ} \mathrm{S}$ )

Data Sources:

USGS GTOPO30;

ESRI Data \& Maps 9.3

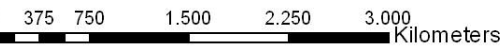

Figure 39: Physical map of Africa ${ }^{955}$

955 Based on USGS GTOPO 30 dataset, ESRI Data \& Maps 9.3 and KAPPAS, M. (2009), p. 118. 


\subsubsection{Land Surface Characteristics}

The geology of Burkina Faso reflects the general geological pattern found in West Africa and is characterized by a basement of old crystalline rocks that was formed in precambrian times. This basement is covered by a massive layer of sediments, mainly sandstones and calcites. Minimal tectonic activities have resulted in a relatively flat relief, with elevations typically ranging between 250 and $350 \mathrm{~m} .{ }^{956}$

Both the hydrography and the soil pattern of Burkina Faso are largely determined by the country's geological structures and climatic conditions. Hydrologically, Burkina Faso makes up parts of the Volta Basin (in the center and west of the country, including Kossi Province), the Comoé Basin (southern Burkina Faso) and the Niger Basin (north and east of Burkina Faso). ${ }^{957}$ Soils rich in sand and/or clay dominate much of the country, with hydromorphic soils found around major water bodies. Clayey soils in general and ferralitic soils in particular are prone to surface crusting. ${ }^{958}$

Despite some differences to international nomenclature, the geological and soil classifications introduced by the French colonial administration are used in the following sections as they are still widely used in francophone West Africa.

\subsubsection{Geology}

Burkina Faso can be divided into 3 major geological zones: the precambrian shield covering around three fourths of the country; and two zones dominated by sedimentary covers in the west/northwest and southeast of the country (see figure 40).

The precambrian shield consists of metamorphic and volcanic rocks ${ }^{959}$; these rocks are locally folded. In addition to granites, the bedrock contains gneiss and mica schists. It is covered by a massive layer of clayey sandstones and calcites which are sometimes heavily eroded. This layer of sediments is most profound in the region around Bobo-Dioulasso and around the Burkina Faso Benin boarder. ${ }^{960}$ Tectonic movements have been negligible since precambrian times; therefore, the rock formations are consolidated and abraded. This explains the general flatness of the relief in Burkina Faso. ${ }^{961}$

956 Kappas, M. (2006), pp. 12f.; Yahmed, D.B. (2005), p. 58.

957 YAHMED, D.B. (2005), p. 58.

958 HaMmeR, T. (2005), pp. 24f; YAHMED, D.B. (2005), p. 64.

959 YAHMED, D.B. (2005), p. 62.

960 KaPpAS, M. (2006), p. 12.

961 Yahmed, D.B. (2005), p. 62. 


\section{Burkina Faso: Geology}

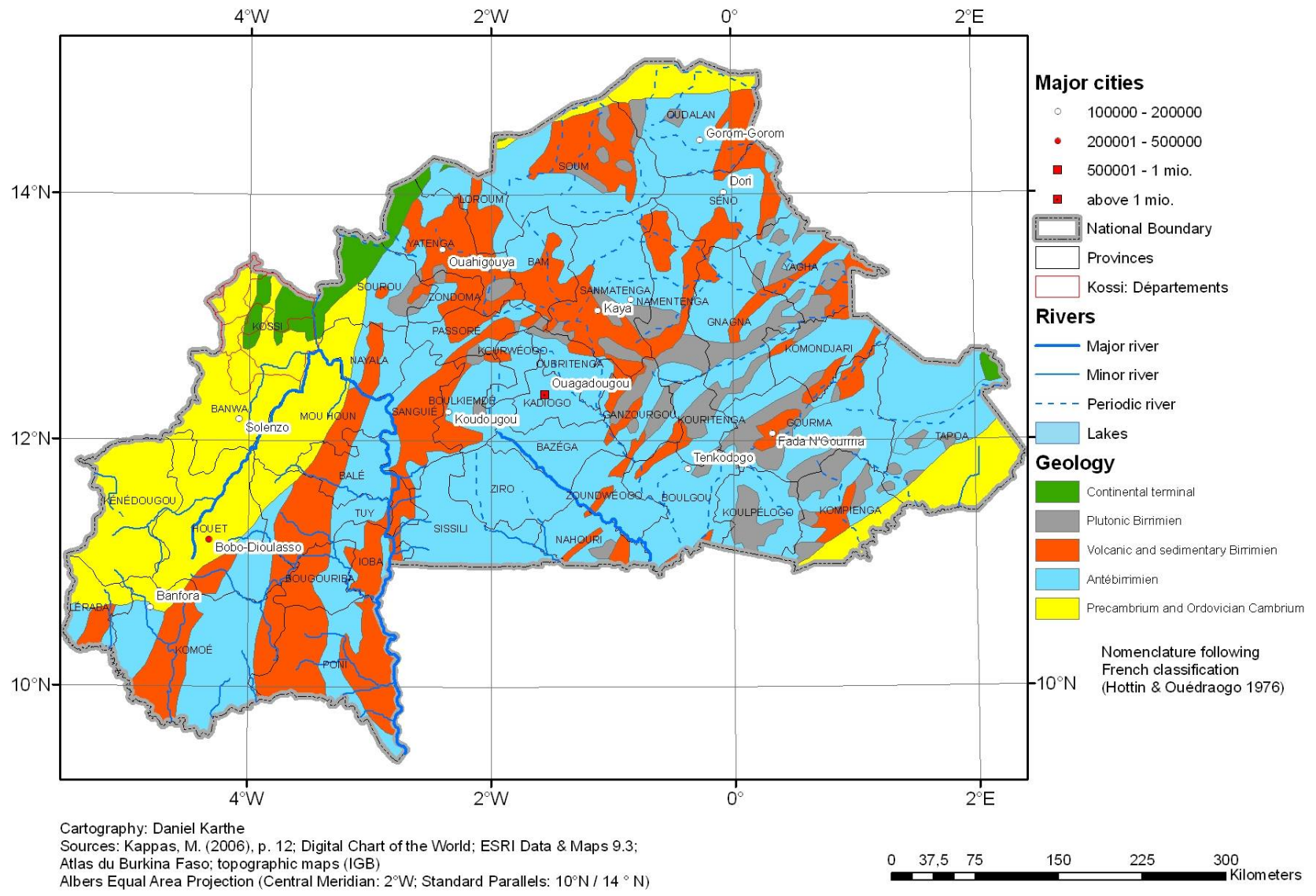

Figure 40: Geological map of Burkina Faso ${ }^{962}$

Laterite crusts containing iron and aluminum are well developed in most parts of Burkina Faso. The thickness of these crusts is quite variable and ranges from $50 \mathrm{~cm}$ to more than $10 \mathrm{~m} .{ }^{963}$

The sedimentary cover in western Burkina Faso consists of paleozoic and tertiary formations (see figure 41). The Continental terminal, a sedimentary layer consisting of sand / sandstone and clay, was deposited in the tertiary and is only found in the Gondo Plain in western Burkina Faso. The region around the Burkina Faso-Mali border is characterized by paleozoic sandstone, the Grès de Bandiagra and the Grès de Koutiala, which occasionally form hills. Towards the border between Kossi and Sourou provinces, at a longitude of about $3^{\circ} 30^{\prime}$ $\mathrm{W}$, the sandstone alternates with slate (Schistes de Toun) or slate and dolomite

962 Map based on KaPpAS, M. (2006), p. 12; Digital Chart of the World; YAHMED, D.B. (2005);

ESRI Data \& Maps 9.3 and topographic maps (IGB).

963 YAHMED, D.B. (2005), p. 63. 
(Etage schistogrèso-dolomitique). With this exception, the remainder of western Burkina Faso is largely covered by a sedimentary layer of sandstones, most notably the Grès de Sotuba, which are occasionally intermingled with larger pebbles of quartz (Grès à galets de quartz). ${ }^{964}$

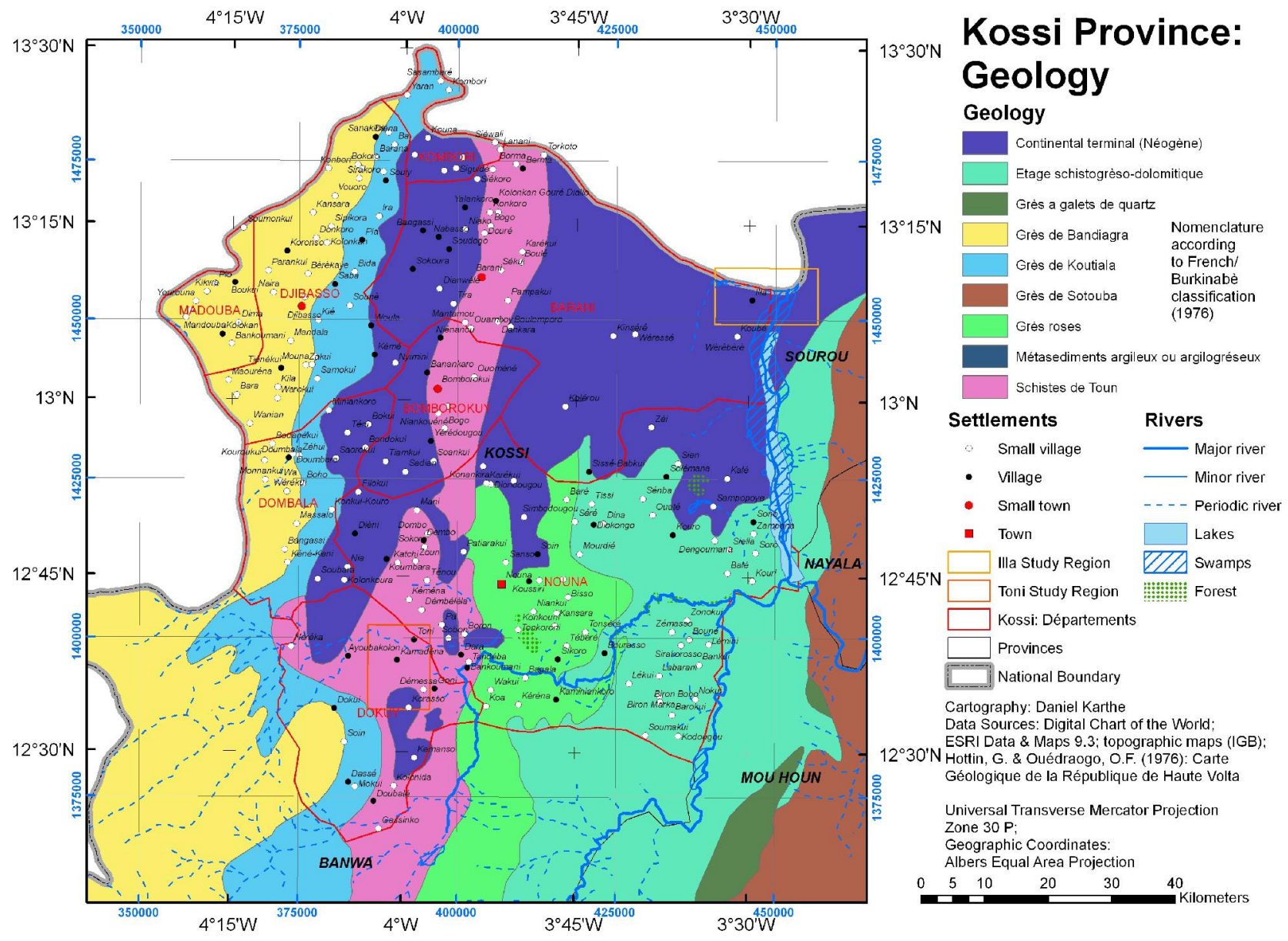

Figure 41: Geological map of western Burkina Faso ${ }^{965}$

964 Yahmed, D.B. (2005), pp. 62f; Hottin, G. \& Ouedraogo, O.F. (1976): Carte Géologique de la République de Haute-Volta.

965 Based on Hottin, G. \& Ouedraogo, O.F. (1976); Digital Chart of the World; YAHMED, D.B. (2005); ESRI Data \& Maps 9.3 and topographic maps (IGB). 


\subsubsection{Relief}

Burkina Faso has a flat relief with around half of the country lying between $250 \mathrm{~m}$ and $350 \mathrm{~m}$ above sea level. At an elevation of $749 \mathrm{~m}$, the Ténakourou is the highest mountain in the country, whereas altitudes fall to below $200 \mathrm{~m}$ along the borders with Togo and Benin in the southeast of Burkina Faso (see figure 42). ${ }^{966}$

\section{Burkina Faso: Physical Map}

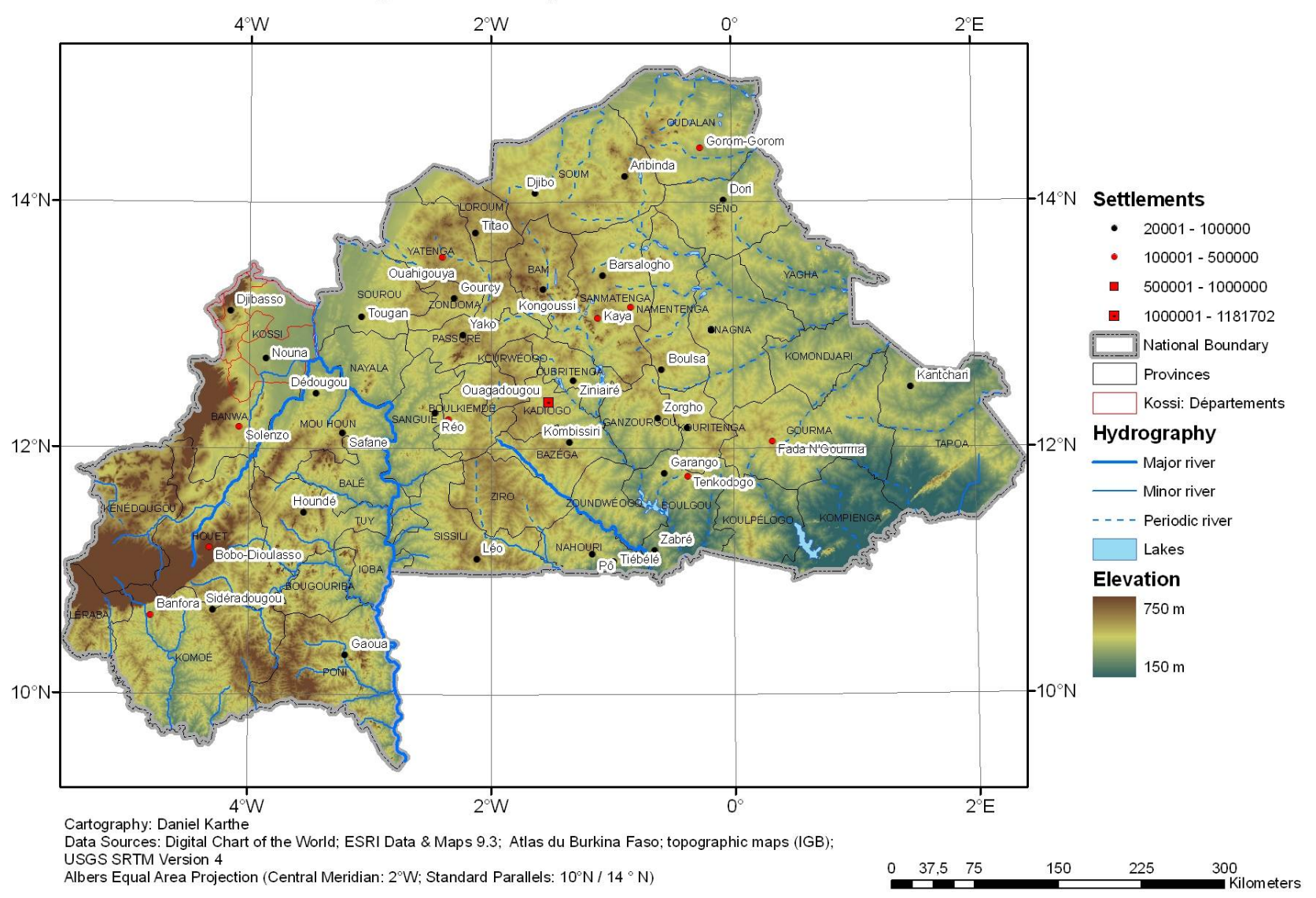

Figure 42: Physical map of Burkina Faso ${ }^{967}$

In Kossi Province, there is an altitudinal gradient ranging from more than $450 \mathrm{~m}$ in the west to around $250 \mathrm{~m}$ in the Sourou Valley. The relief in the western part of Kossi has a relatively high energy compared to minor variations in altitude in the eastern half (see figures 42 and 43 ).

966 YAHMED, D.B. (2005), p. 58.

967 Based on USGS SRTM version 4; Digital Chart of the World; YAHMED, D.B. (2005);

ESRI Data \& Maps 9.3 and topographic maps (IGB). 


\subsubsection{Hydrography}

Burkina Faso has limited renewable water resources, which are currently estimated at $906 \mathrm{~m}^{3}$ per inhabitant ${ }^{968}$. According to UNEP, a situation with per capita freshwater resources of less than $1700 \mathrm{~m}^{3}$ is considered water stress and less than $1000 \mathrm{~m}^{3}$ as water scarcity. ${ }^{969}$ Within Burkina Faso, the availability of water differs greatly between the Sahelian provinces in the north and moister regions in the South (see figures 42 and 50).

Burkina Faso does not have access to the large rivers of the Sahel like the Niger or Senegal. The Volta Basin is Burkina Faso's most important river basin and covers an area of around $120.000 \mathrm{~km}^{2}$ in the center and west of the country. It consists of three subbasins related to the Mouhoun (formerly Black Volta), Nakambé (formerly White Volta) and Pendjari. ${ }^{970}$ The basin is characterized by a generally flat terrain, and all rivers of the basin drain into Lake Volta, one of the largest artificial lakes in the world. ${ }^{971}$ The two other important river basins of the country are the Niger basin, covering around $72.000 \mathrm{~km}^{2}$ in the northeast of the country, and the Comoé basin, covering $18.000 \mathrm{~km}^{2}$ in southwestern Burkina Faso (see figure 42). ${ }^{972}$

The major rivers which flow through the Sahelo-Sudanian zone of Burkina Faso are mostly fed in regions receiving higher rainfall in the South. Few of these rivers have large enough reservoirs or sufficient flows to support large-scale irrigation. ${ }^{973}$ Moreover, runoff is highly sensitive to rainfall, with small changes in annual rainfall causing large changes in river flow. ${ }^{974}$

Two rivers are of major importance for Kossi Province: the Mouhoun and the Sourou (see figure 43), the only perennial rivers of the region.

968 OeCD \& African Development Bank (2007), p. 157.

969 United Nations Environment Programme (2008), p. 20.

970 YAHMED, D.B. (2005), p. 58.

971 JUNG, G. (2006), p. 12.

972 YAHMED, D.B. (2005), p. 58.

973 Ingram, K.T.; Roncoli, M.C. \& Kirshen, P.H. (2002), p. 333.

974 Jung, G. (2006), p. 13. 


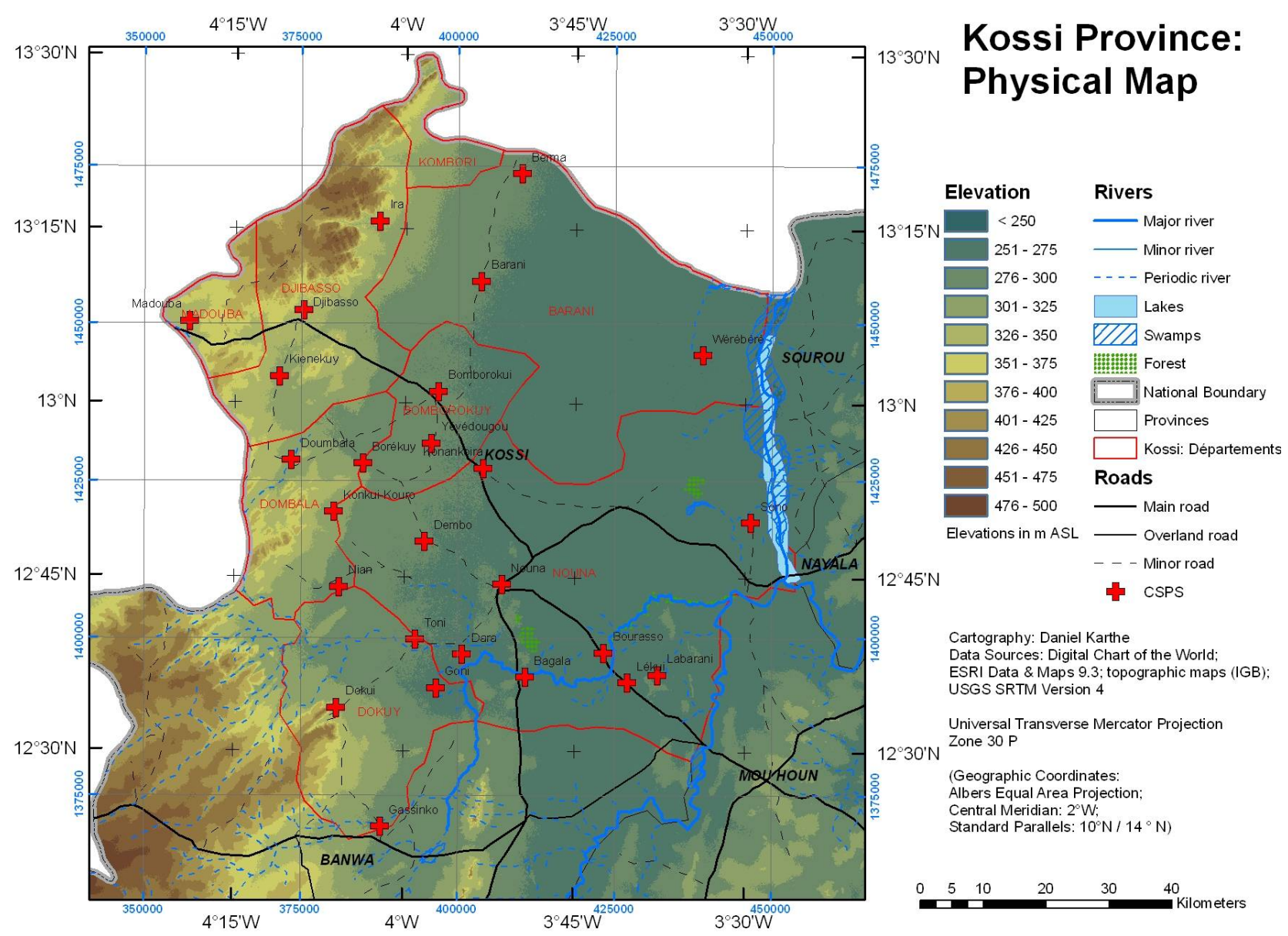

Figure 43: Physical map of Kossi Province ${ }^{975}$

The Mouhoun enters Kossi Province from the south in Dokuy département, then bends eastward towards the confluence of Mouhoun and Sourou and finally makes a sharp southward turn. Barrages along the Mouhoun are used for both the generation of hydroelectric energy and irrigation purposes. ${ }^{976}$ Most of the rain falling in the Mouhoun subbasin evaporates, leaving only around $4 \%$ for surface runoff (see table 36 ):

\begin{tabular}{|l|l|l|l|}
\hline Rainfall & $\begin{array}{l}\text { Evapo- } \\
\text { Transpiration }\end{array}$ & Infiltration & Surface runoff \\
\hline $74.5 \mathrm{~km}^{3}$ & $59.16 \mathrm{~km}^{3}$ & $12.4 \mathrm{~km}^{3}$ & $2.94 \mathrm{~km}^{3}$ \\
\hline
\end{tabular}

Table 36: Hydrological balance in the Mouhoun subbasin (annual data) ${ }^{977}$

975 Based on USGS SRTM version 4; CRSN Nouna; Digital Chart of the World; YAHMED, D.B. (2005); ESRI Data \& Maps 9.3 and topographic maps (IGB).

976 Barbier, B.; Dembelé, Y. \& Comparoré, L. (2006), p. 21.

977 Barbier, B.; Dembelé, Y. \& Comparoré, L. (2006), p. 21. 
The Sourou, sometimes referred to as "West Africa's miracle river", is at first sight a relatively minor river that stretches around $120 \mathrm{~km}$ from Toroli (Mali) to its confluence with the Mouhoun near Léri (Burkina Faso). The Sourou flows through a nearly meridional trough, the so-called Sourou Depression. ${ }^{978}$ Its tributaries only carry water during the rainy season ${ }^{979}$, but hydrological modifications have transformed the Mouhoun into a reservoir receiving up to 200 million cubic meters of water during the rainy season (mostly from the Mouhoun). Around December, the Mouhoun inverts its direction of flow, restituting some of its water stocks into the Mouhoun. ${ }^{980}$ While this is essentially a natural process, a regulation mechanism was installed in 1984 to control the flow of both rivers. ${ }^{981}$ Between $40 \%$ and $50 \%$ of the water in the reservoir eventually evaporates, a fact that is related not only to the hot, dry climate but also the flat relief and thus the limited depth of the reservoir. ${ }^{982}$

Burkina Faso has a network of roughly 2100 small dams built mostly in rural areas to harvest rain water runoff ${ }^{983}$, but many of the ephemeral streams in Burkina Faso's northern half are too undependable for irrigation. ${ }^{984}$ In Kossi Province, the construction of a dam is currently planned near Toni. ${ }^{985}$

Given the countries rapidly growing population, Burkina Faso experiences a fast growing demand for water for domestic consumption, generation of electricity and irrigation. ${ }^{986}$ Since Burkina Faso currently only irrigates around $1 \%$ of its agricultural land (compared to $4 \%$ in Sub-Saharan Africa and $20 \%$ worldwide), irrigation is perceived as one of the key strategies for improving food security. ${ }^{987}$ Moreover, hydraulic resources currently account for $25 \%$ of Burkina Faso's energy production, but since the remaining $75 \%$ depend on imported fossil fuels, hydro-energy is promoted by the government ${ }^{988}$, even more so because the cost of electrical energy in Burkina Faso is among the world's highest. ${ }^{989}$ Despite natural limitations, the construction of new dams and reservoirs can be expected in the future.

978 JUnG, G. (2006), p. 13.

979 Bethemont, J.; Faggi, P.; Zoungrana, T.P. (2003), pp. 9-11.

980 Bethemont, J.; Faggi, P.; Zoungrana, T.P. (2003), p. 19.

981 JUNG, G. (2006), p. 13.

982 Barbier, B.; Dembelé, Y. \& Comparoré, L. (2006), p. 21.

983 United Nations Environment Programme (2008), p. 99.

984 Ingram, K.T.; Roncoli, M.C. \& Kirshen, P.H. (2002), p. 333.

985 Personal communication with Issouf Traoré.

986 Barbier, B.; Dembelé, Y. \& Comparoré, L. (2006), p. 20.

987 Bharati, L.; Rodgers, C.; ERdenberger, T. et al. (2008), p. 925.

988 OeCD \& African Development Bank (2008), p. 174.

989 Barbier, B.; Dembelé, Y. \& Comparoré, L. (2006), p. 23. 


\subsubsection{Soils}

The distribution of major soil types in Africa is linked to the geologic and climatic situation on the continent. Whereas ferrasols, acrisols and nitisols are the predominant soils in large parts of the humid equatorial regions, towards the tropics of cancer and capricorn there are soil types which reflect the semihumid, semi-arid and arid nature of the climate. Whereas lixisols are frequently found in the semi-humid to semi-arid regions, arenosols dominate in the driest regions of the continent. ${ }^{990}$ An overview of important soil types according to the FAO classification is presented in figure 44 and table 37.

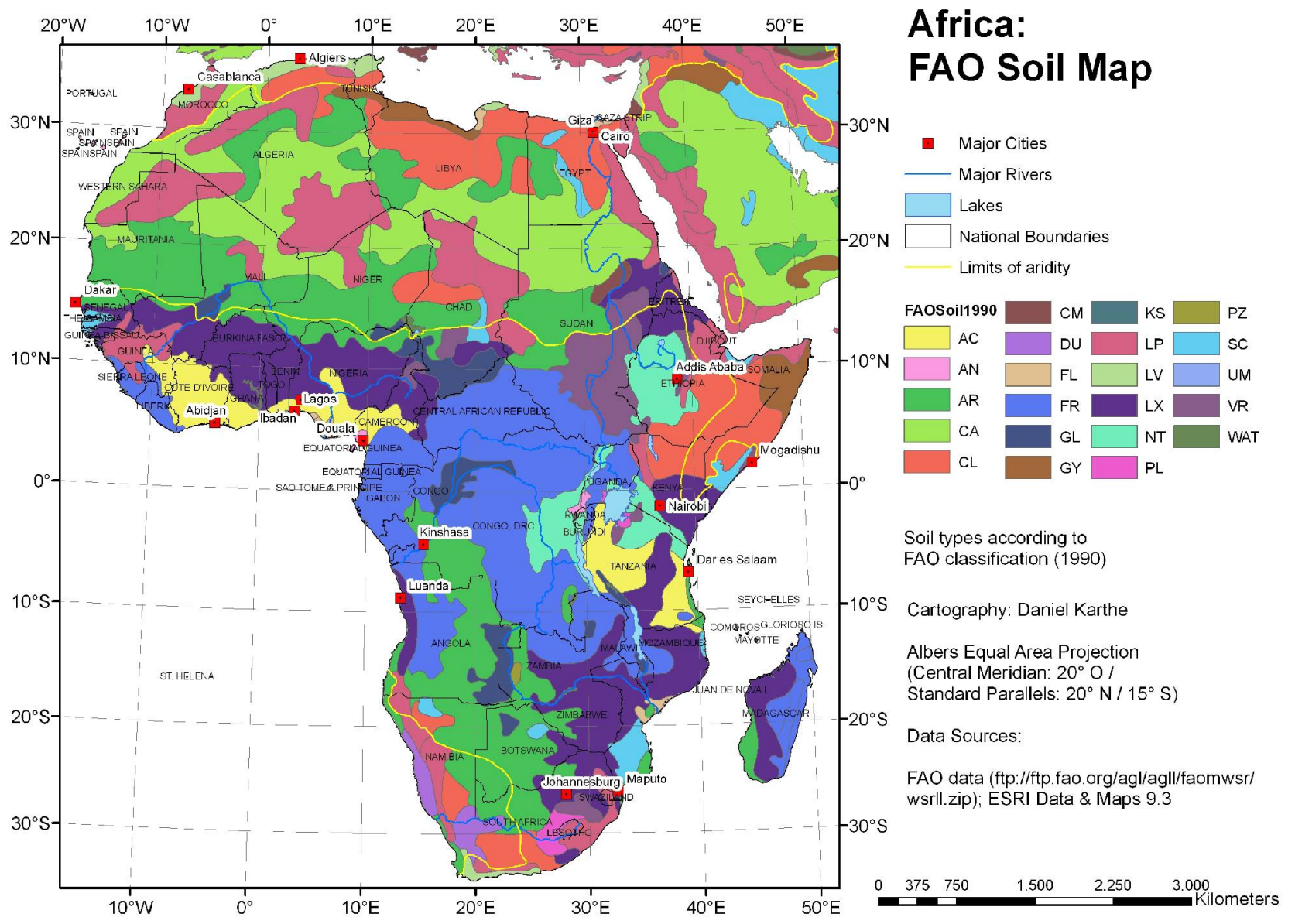

Figure 44: FAO soil resources map of Africa ${ }^{991}$

$990 \mathrm{ftp}: / / \mathrm{ftp}$.fao.org/agl/agll/faomwsr/wsrll.zip; accessed 09/09/08.

991 Based on FAO Soil map (ftp://ftp.fao.org/agl/agll/faomwsr/wsrll.zip) and ESRI Data \& Maps 9.3. 


\begin{tabular}{|l|l|l|l|}
$\begin{array}{l}\text { FAO } \\
\text { ID }\end{array}$ & Soil Types & $\begin{array}{l}\text { FAO } \\
\text { ID }\end{array}$ & Soil Types \\
\hline AC & Acrisols, Alisols, Plinthosols & KS & Kastanozems, Solonetz \\
\hline AN & Andosols & LP & Leptosols, Regosols \\
\hline AR & Arenosols & LV & Luvisols, Cambisols \\
\hline CA & Calcisols, Regosols, Arenosols & LX & Lixisols \\
\hline CL & Calcisols, Cambisols, Luvisols & NT & Nitisols \\
\hline CM & Cambisols & PL & Planosols \\
\hline DU & Durisols & PZ & Podsols, Histosols \\
\hline FL & Fluvisols, Gleysols, Cambisols & SC & Solonchaks, Solonetz \\
\hline FR & Ferrasols, Acrisols, Nitisols & UM & Umbrisols \\
\hline GL & Gleysols, Histosols, Fluvisols & VR & Vertisols \\
\hline GY & Gypsisols, Calcisols & WAT & water \\
\hline
\end{tabular}

Table 37: Legend to the soil map of Africa (figure 44) ${ }^{992}$

According to the FAO map of world soil resources, Burkina Faso falls into a zone characterized by lixisols in the south and arenosols in the north. The massive potential evaporation causes a quick desiccation of most soils during the dry season..$^{993}$ Therefore, the USDA soil taxonomy categorizes virtually all soils of the Sahel as Aridisols, based only on their water balance ${ }^{994}$. However, even the FAO map is an oversimplification, and several other important soil types are found in the semiarid belts of Central and Western Africa (see table 38).

$992 \mathrm{ftp}: / / \mathrm{ftp}$. fao.org/agl/agll/faomwsr/wsavcl.jpg; accessed 09/09/08.

993 HAMMER, T. (2005), p. 25.

994 HaMmer, T. (2005), p. 24. 


\begin{tabular}{|c|c|c|}
\hline $\begin{array}{l}\text { FAO } \\
\text { ID }\end{array}$ & Soil Types & Characteristics \\
\hline LX & Lixisols & $\begin{array}{l}\text { Soils with subsurface accumulation of low activity clays } \\
\text { and high base saturation }\end{array}$ \\
\hline \multirow[t]{2}{*}{ LP } & Leptosols & $\begin{array}{l}\text { Very shallow soils over hard rock or in unconsolidated } \\
\text { very gravelly material }\end{array}$ \\
\hline & Regosols & Soils with very limited soil development \\
\hline AR & Arenosols & $\begin{array}{l}\text { Sandy soils featuring very weak or no soil } \\
\text { development }\end{array}$ \\
\hline \multirow[t]{3}{*}{ GL } & Gleysols & $\begin{array}{l}\text { Soils with permanent or temporary wetness } \\
\text { near the surface }\end{array}$ \\
\hline & Histosols & Soils which are composed of organic materials \\
\hline & Fluvisols & Young soils in alluvial deposits \\
\hline VR & Vertisols & $\begin{array}{l}\text { Soils with dark-colored cracking and swelling clays (see } \\
\text { figure 46) }\end{array}$ \\
\hline
\end{tabular}

Table 38: Important soil types in West Africa (according to FAO classification) ${ }^{995}$

Lixisols are the predominant soils in the semiarid belt of West Africa and are found in large parts of Burkina Faso. They have a higher clay content in the subsoil than in the topsoil as a result of pedogenetic processes (especially clay migration). Lixisols have a high base saturation and low-activity clays at certain depths. ${ }^{996}$

Arenosols are found in the northern parts of Burkina Faso. They comprise sandy soils, including both soils developed in residual sands after in situ weathering of usually quartz-rich sediments or rock, and soils developed in recently deposited sands such as dunes. In the French classification system, the sols minéraux bruts and sols peu évolués are the corresponding soils. ${ }^{997}$

The most common soil classification used in francophone West Africa was developed by the French Office de la Recherche Scientifique et Technique d'Outre-Mer (ORSTOM) from the 1950s onwards. ${ }^{998}$

As figure 45 illustrates, the soils found in and around Kossi Province vary considerably.

\footnotetext{
995 http://www.fao.org/ag/agl/agll/wrb/soilres.stm and

ftp://ftp.fao.org/agl/agll/faomwsr/wsrll.zip;accessed 09/09/08.

996 IUSS Working GRoup WRB (2006), p. 85.

997 IUSS Working Group WRB (2006), p. 72.

998 KAPPAS, M. (2006), p. 34.
} 


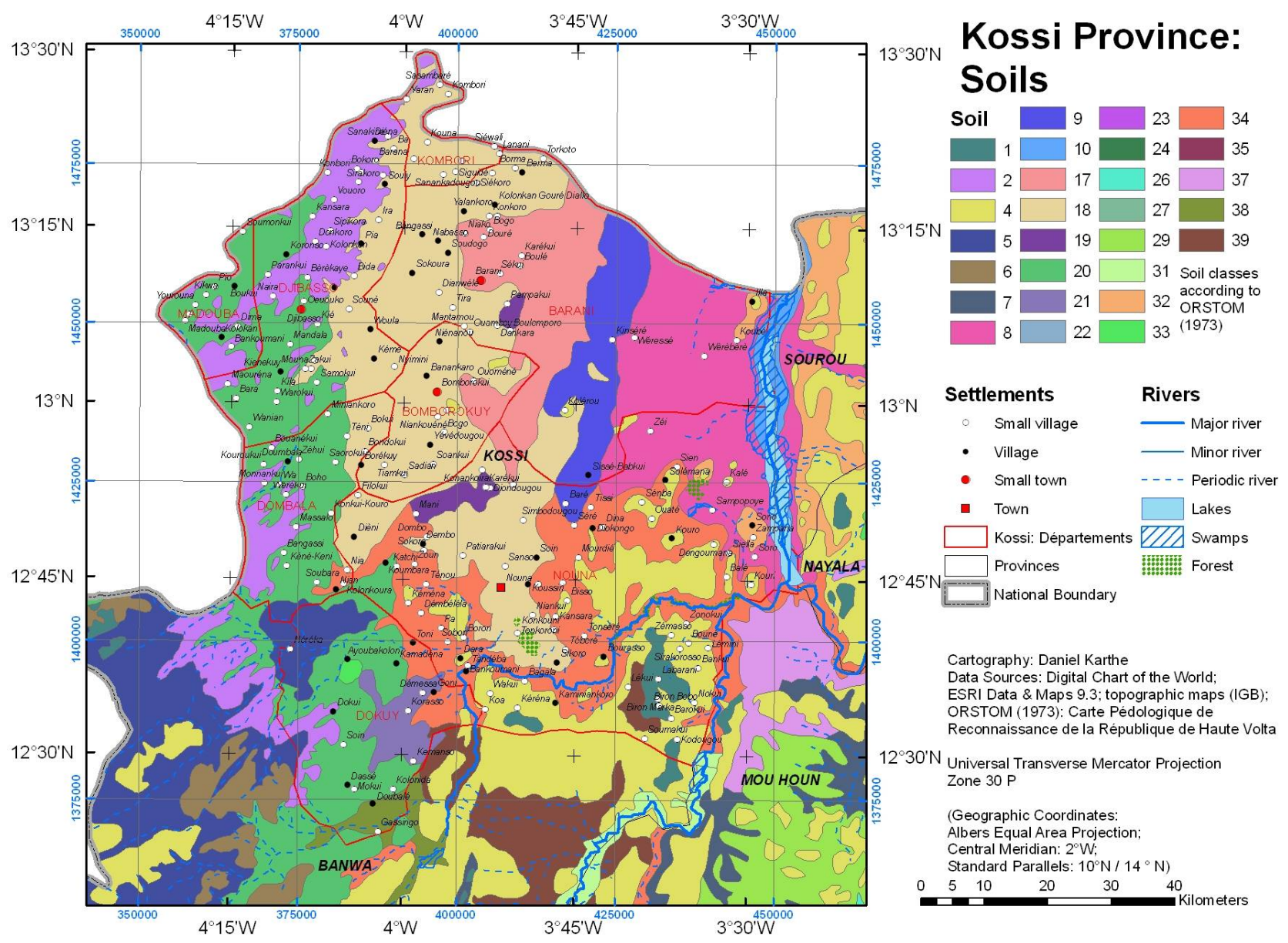

Figure 45: Soil map of Kossi Province ${ }^{999}$

\begin{tabular}{|l|l|}
\hline No. & $\begin{array}{l}\text { Soil Classification } \\
\text { (according to ORSTOM) }\end{array}$ \\
\hline Raw & Mineral Soils (Sols minéraux bruts) \\
\hline 1 & Lithosol on ferralitic crust \\
\hline 2 & Lithosol on sandstone \\
\hline 3 & Lithosol on ferralitic crust \\
\hline Poorly Developed Soils (Sols peu evolués) \\
\hline 4 & Lithosol on ferralitic crust \\
\hline 5 & Lithosol and leached ferralitic soil \\
\hline 6 & Lithosol on ferralitic crusts and sandstone \\
\hline 7 & Lithosol on sandstone \\
\hline
\end{tabular}

999 Based on ORSTOM (1973); Digital Chart of the World; YAHMED, D.B. (2005); ESRI Data \& Maps 9.3 and topographic maps (IGB). 
No. Soil Classification (according to ORSTOM)

Vertisols (Vertisols)

8 Vertisol on clayey alluvium

9 Vertisol on clayey alluvium, with clayey/sandy superstratum

10 Vertisol with hydromorphic character on clayey alluvium

Soils rich in sesquioxides and with rapidly mineralizing organic matter (Sols à sesquioxides et à matière organique rapidement minéralisée)

17 Ferralitic tropical soil on eolian sand

18 Ferralitic tropical soil on eolian sand (with limited drainage)

19 Ferralitic tropical soil on gravel (poorly developed)

20 Leached or impoverished hydromorphic soil on sand or clay

21 Leached or impoverished poorly developed soils on gravel and ferralitic crusts

22 Leached or impoverished soil on sand or clay (with concretions)

23 Poorly developed eroded soils on gravel and crusted lithosols (with concretions)

24 Moderately desaturated ferralitic soils

26 Impoverished tilled soil on gravel, crusts or slate

27 Impoverished tilled soil on gravel and hard crusts

29 Impoverished tilled soil on gravel (ferralitic)

Hydromorphic Soils (Sols hydromorphes)

31 Pseudogley on alluvium (often clayey)

32 Pseudogley on limnic sand or sandy/clayey alluvium or colluvium

33 Pseudogley on clay or sandy/clayey alluvium or colluvium

34 Hydromorphic pseudogley on clayey to sandy alluvium

35 Pseudogley on gravel

37 Leached ferralitic hydromorphic soils with concretions

38 Pseudogley on limnic clay

39 Poorly developed pseudogley on gravel

Table 39: Key to the ORSTOM soil classification 
Vertisols dominate in the eastern parts of Kossi, particularly in the Sourou Depression and develop massive cracks during the dry season (see figure 46). Hydromorphic soils (particularly pseudogleys) are found around the Mouhoun. Ferralitic tropical soils cover much of the central part of Kossi, while lithosols and leached/impoverished soils dominate in western Burkina Faso, corresponding to higher elevations and sandstone formations (Grès de Bandiagra / Grès de Koutiala).

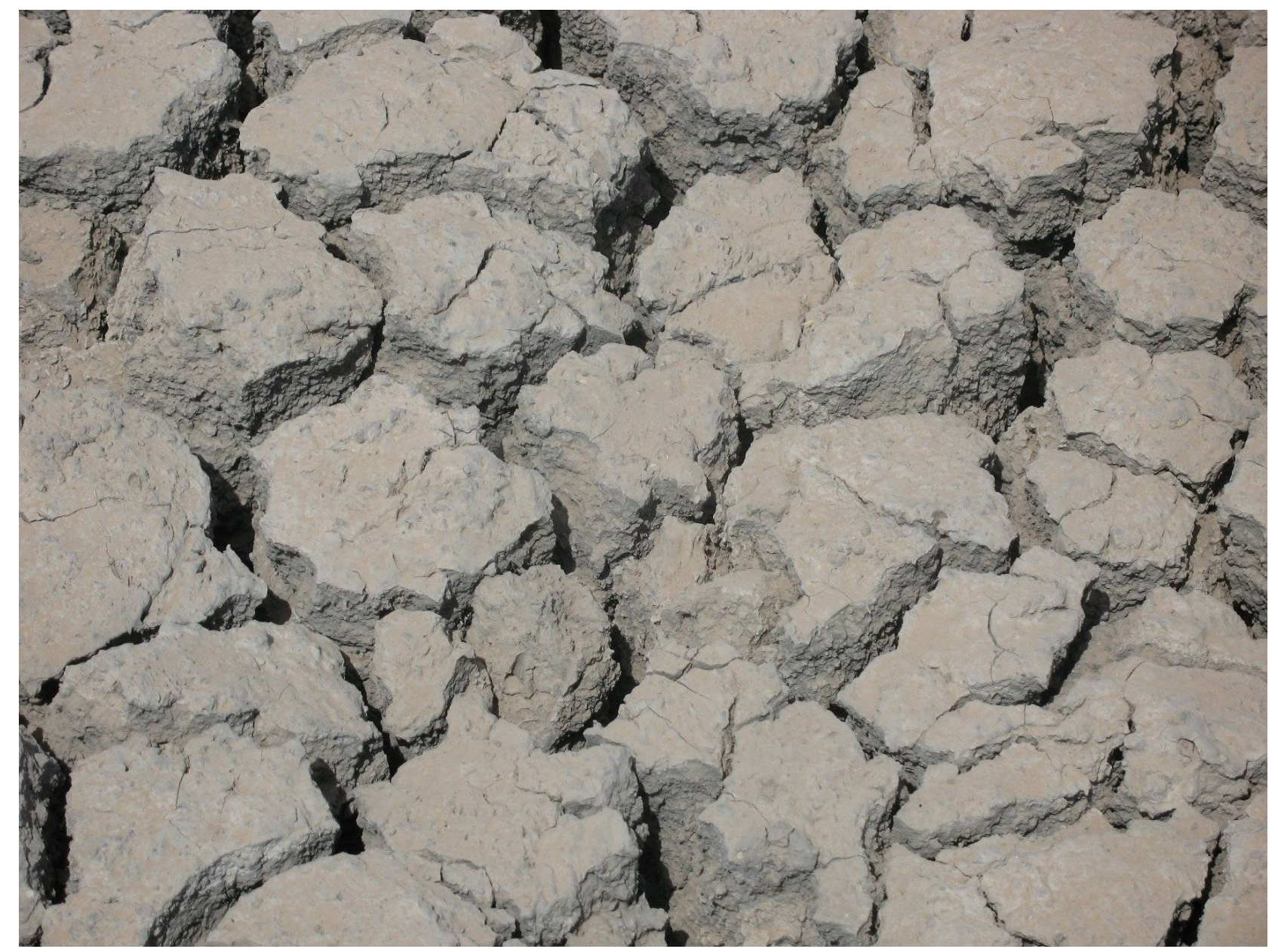

Figure 46: Vertisol at the height of the dry season (Toni)

Table 40 outlines the characteristics of the dominating soil types in Kossi Province. 


\section{English and French denomination}

\begin{tabular}{|c|c|}
\hline $\begin{array}{l}\text { Vertisols } \\
\text { Vertisols sur alluvions ou } \\
\text { matériau argileux }{ }^{1000}\end{array}$ & $\begin{array}{l}\text { Vertisols (see figure } 46 \text { ) are mainly found on } \\
\text { alluvium in the Sourou Valley and are the } \\
\text { dominant soil in northeastern Kossi province. } \\
\text { They are characterized by high contents of } \\
\text { expanding clays which increase their volume due } \\
\text { to water retention but shrink under desiccation. } \\
\text { They have a relatively high mineral content, a } \\
\text { weak porosity and surface crusts. }{ }^{1001}\end{array}$ \\
\hline $\begin{array}{l}\text { Hydromorphic soils and } \\
\text { pseudogleys } \\
\text { Sols hydromophes } \\
\text { minéraux à pseudogley }\end{array}$ & $\begin{array}{l}\text { Hydromorphic soils, mainly pseudogleys, are } \\
\text { found along the major rivers such as the } \\
\text { Mouhoun and Sourou and at the bottom of large } \\
\text { depressions. These soils are characterized by a } \\
\text { temporary excess of water and are traditionally } \\
\text { used for sorghum planting. }{ }^{1002}\end{array}$ \\
\hline $\begin{array}{l}\text { Ferruginous tropical soils } \\
\text { Sols ferrugineux tropicaux } \\
\text { peu lessivés et lessivés }\end{array}$ & $\begin{array}{l}\text { Ferruginous and ferralitic tropical soils cover } \\
\text { about half of the surface of Burkina Faso and are } \\
\text { found in central Burkina Faso. They cover nearly } \\
\text { one third of Kossi province and are typically } \\
\text { found on gravel or sandy material that is rich in } \\
\text { iron and aluminum oxides and hydroxides. }{ }^{1003} \\
\text { Ferralitic soils are typical for rocky plateaus and } \\
\text { hilly landscapes. Their low content of nutrients } \\
\text { and organic matter and their high acidity mean } \\
\text { that they are only loosely covered with } \\
\text { vegetation. Ferralitic soils are highly susceptible } \\
\text { to crust formation, lateritization and erosion. }{ }^{1004}\end{array}$ \\
\hline $\begin{array}{l}\text { Lithosols } \\
\text { Sols minéraux bruts: } \\
\text { lithosols sur roches diverses } \\
\text { et cuirasses }\end{array}$ & $\begin{array}{l}\text { Lithosols are defined by the FAO as soils limited } \\
\text { in depth by continuous hard rock within } 10 \mathrm{~cm} \text { of } \\
\text { the surface. }{ }^{1005} \text { They develop on slightly or non- } \\
\text { weathered rocks, often on sandstone or ferrous } \\
\text { crusts. }{ }^{1006} \text { They tend to be concentrated in the } \\
\text { southern part of Kossi. }\end{array}$ \\
\hline
\end{tabular}

\section{Characteristics and distribution}

Vertisols (see figure 46) are mainly found on . Valley and are the They are characterized by high contents of They are characterized by high contents of expanding clays which increase their volume due to water retention but shrink under desiccation. content, a

Hydromorphic soils, mainly pseudogleys, are found along the major rivers such as the Mouhoun and Sourou and at the bottom of large depressions. These soils are characterized by a exporary excess of water and are traditionally Ferruginous and ferralitic tropical soils cover about half of the surface of Burkina Faso and are one third of Kossi province and are typically ound on gravel or sandy material that is rich in Ferralitic soils are typical for rocky plateaus and hilly landscapes. Their low content of nutrients d organic matter and their high acidity mean vegetation. Ferralitic soils are highly susceptible Lithosols are defined by the FAO as soils limited in depth by continuous hard rock within $10 \mathrm{~cm}$ of in the

1000 YAHMED, D.B. (2005), p. 64.

1001 YAHMED, D.B. (2005), p. 64.

1002 YaHMED, D.B. (2005), pp. 64f.

1003 IUSS Working GROUP WRB (2006), p. 18.

1004 HAMMER, T. (2005), p. 19.

1005 http://www.fao.org/Ag/AGL/agll/key2soil.stm (accessed 20/05/08).

1006 YAHMED, D.B. (2005), p. 64. 


\section{English and French denomination}

Poorly developed soils on gravel

Sols peu evolués d'érosion sur matériau gravillonnaire

\section{Characteristics and distribution}

These poorly developed soils have a thicker surface horizon than lithosols and may develop after breakup of ferrous crusts. They are characterized by poor water retention and low mineral content and therefore rarely used for agricultural purposes. These soils are only found in parts of southern Kossi. ${ }^{1007}$

Table 40: Important soil types in Kossi province

In general, the soils in West Africa are characterized by low organic matter content, nutrients deficiency and low water holding capacity. Field capacities are typically between $15 \%$ and $20 \%(\mathrm{v} / \mathrm{v})$ and the wilting point between $7 \%$ and $9 \%(\mathrm{v} / \mathrm{v}) .{ }^{1008}$ Sandy soils dominate in the Sahel and are primarily the result of eolian sand deposition which occurred as early as the Pleistocene ("fossil dunes"). They have a low water retention capacity and are highly erodible by wind and water. Clayey and loamy soils are typical for depressions and alluvial areas and are -if moisture permits- more productive than sandy soils. ${ }^{1009}$ However, some studies have come to contrasting results. According to KUmAR et al. (2002), higher vegetation indices are measured over sandy soils than over loamy and clayey soils in semiarid Burkina Faso - particularly during the early part of the dry season. ${ }^{1010}$ Rain falling on sandy soil largely infiltrates and becomes available for plant growth, whereas rain falling on clayey soil is largely lost as runoff and evaporation. The difference in infiltration rates is greatly increased in the Sahel due to intensive crusting on clayey soils. ${ }^{1011}$

The main malariologic importance of soils is related to their infiltration characteristics. Depending on the relief, surface crusts and poorly permeable horizons may either cause runoff or lead to water stagnation. By contrast, highly permeable substrates such as sandy soils absorb rainfall quickly enough to avoid inundation.

1007 YAHMED, D.B. (2005), p. 64.

1008 Korodjouma, O.; Badiori, O.; Ayemou, A. \& Michel, S.P. (2006), p. 218.

1009 HaMmer, T. (2005), pp. $24 f$.

1010 Kumar, L.; Rietkerk, M.; v. Langevelde, F. et al. (2002), p. 147.

1011 Kumar, L.; Rietkerk, M.; v. Langevelde, F. et al. (2002), p. 149. 


\subsubsection{Climate}

The semiarid climate of Burkina Faso's Sahelo-Sudanian zone is characterized by a rainy season in the summer, a dry winter and an annual average temperature of about $28^{\circ} \mathrm{C}$. The temperature difference between the warmest and coldest month is less than $10 \mathrm{~K}$ in monthly means. While temperatures increase towards the north between April and October, this trend is reversed during the winter months (November to March). The highest temperatures occur just before the onset of the summer monsoon and may exceed $45^{\circ} \mathrm{C}$. ${ }^{1012}$ Rains fall during a single wet season consisting of intense storms over a threeto five-months period, with about $90 \%$ of the rains falling during July, August and September. ${ }^{1013}$ Annual rainfall may differ significantly between neighboring villages. ${ }^{1014}$ Figure 47 shows a comparison of climate data for 2008 (a fairly typical but slightly dry year) with the 1961 to 1990 mean. Dédougou, located just outside Kossi in Mouhoun Province and around $50 \mathrm{~km}$ southeast of Nouna, is the closest station to the study region for which a more or less uninterrupted time series is available.

Climate in Dédougou

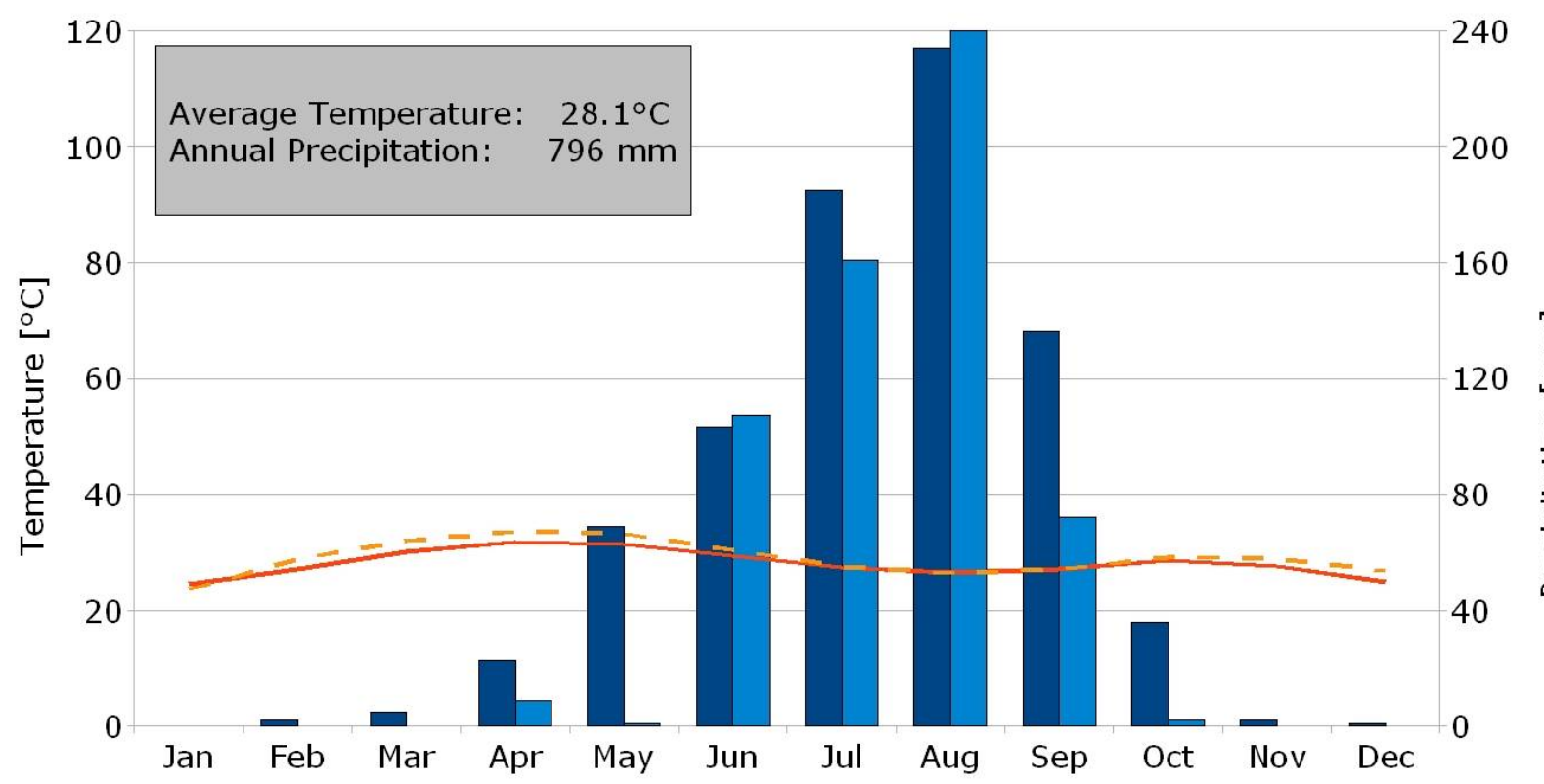

Precip 1961-1990 — Precip 2008 - Temp 1961-1990 - Temp 2008

Figure 47: Climate in Dédougou (2008 vs. 1961-1990 mean) $)^{1015}$

1012 GRifFiths, J.F. (1972), pp. 195 f.

1013 Ingram, K.T.; Roncoli, M.C. \& KirShen, P.H. (2002), p. 333.

1014 Ingram, K.T.; Roncoli, M.C. \& Kirshen, P.H. (2002), p. 333.

1015 Data source: FAO ClimNET, http://geonetwork3.fao.org/climpag/agroclimdb en.php, accessed 15 March 2009. 
Because of more complete and longer-term data series and the fulfillment of WMO criteria, this study used meteorological data from Dédougou unless intra-province differences, microclimatic issues or high temporal resolution are a concern.

\subsubsection{Temperature}

In the Sahelo-Sudanian zone of Burkina Faso, insolation is highest in April, just before the onset of rain, and reaches a minimum during the months of July and August when cloud cover is typically between $6 / 8$ and $7 / 8 .{ }^{1016}$ This is directly reflected by air and surface temperatures.

The annual mean temperature in Kossi Province is between 28 and $29^{\circ} \mathrm{C}$, with April being the hottest month (around $34^{\circ} \mathrm{C}$ ) and December the coolest (around $26^{\circ} \mathrm{C}$ ). The variation in diurnal temperature is greatest in the cool period (December and January). ${ }^{1017}$

Even though monthly mean values suggest that temperatures are quite uniform throughout the year, there is a considerable intradiurnal variation. This is illustrated at the example of data from Dédougou (figure 48).

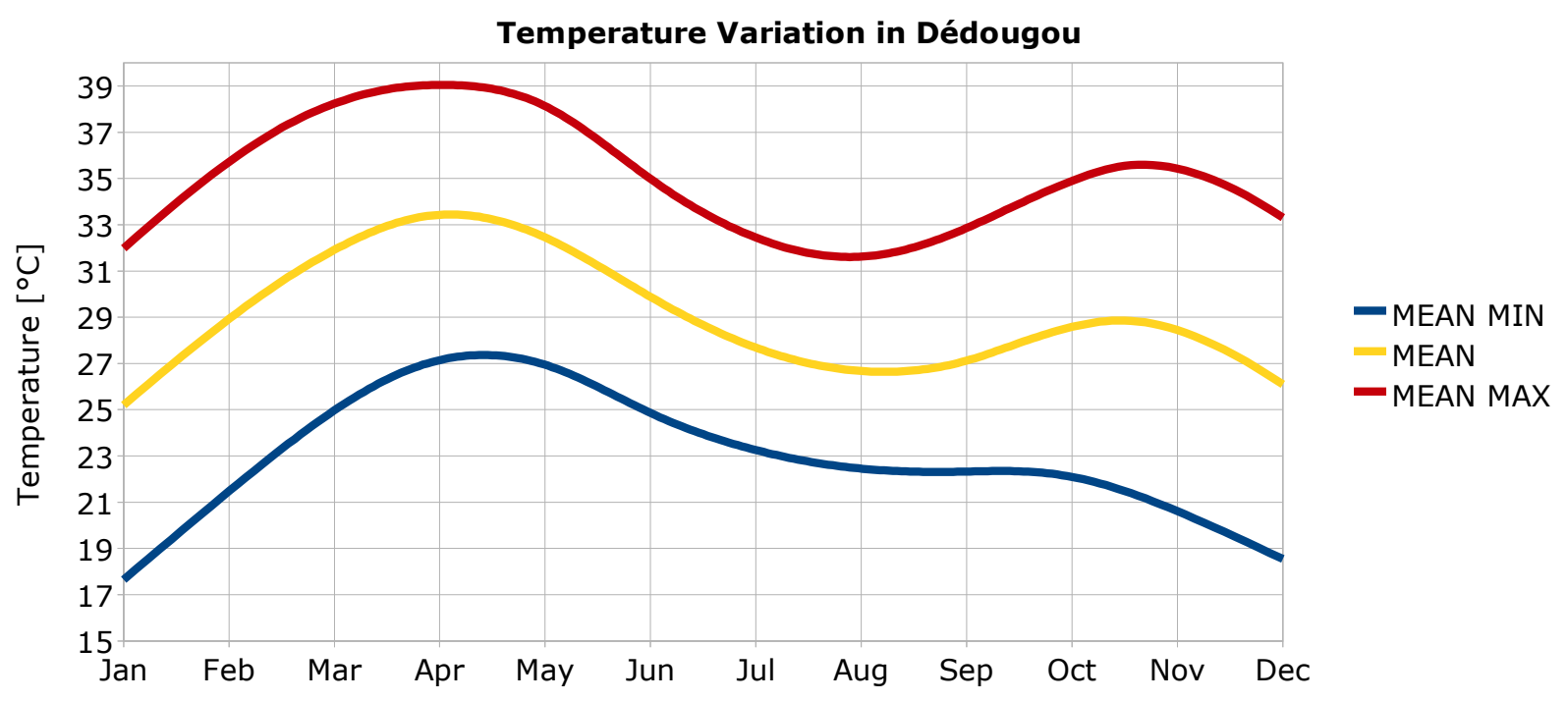

Figure 48: Seasonal and diurnal temperature variation in Dédougou (1983 to 2008 mean) $)^{1018}$

1016 GrifFiths, J.F. (1972), pp. 195f.

1017 Yé, Y.; Sauerborn, R.; Seraphin, S. \& Hoshen, M. (2007), p. 376.

1018 Data source: FAO ClimNET, http://geonetwork3.fao.org/climpag/agroclimdb en.php, accessed 15 March 2009. 
Between 1961 and 1990, the annual mean temperature in Dédougou was $28.1^{\circ} \mathrm{C}$, with January being the coolest month $\left(24.6^{\circ} \mathrm{C}\right)$ and April the warmest $\left(31.8^{\circ} \mathrm{C}\right)$. A certain warming trend as compared to this normal period could already be observed: for the 1983 to 2008 period, the annual mean was $29.0^{\circ} \mathrm{C}$ with $25.2^{\circ} \mathrm{C}$ in January and $33.1^{\circ} \mathrm{C}$ in April. This indicates not only a general warming but also an increase in temperature amplitudes. Albeit small, such changes are malariologically relevant (in this case causing an increase in the epidemiological potential which is maximal around $31^{\circ} \mathrm{C}$ ).

Temperatures in August, at the height of the wet season, are similar to those in January, making it the second-coolest month of the year. The annual temperature amplitude of $9.6 \mathrm{~K}$ is considerably exceeded by diurnal temperature variations, which are typically in the order of $15 \mathrm{~K}$ (dropping to around $8 \mathrm{~K}$ to $9 \mathrm{~K}$ during the wet season). However, the mean minimum and maximum temperatures somewhat mask the large total variation between absolute minima and maxima (see table 41 ).

\begin{tabular}{|c|c|c|c|c|c|c|}
\hline Month & T avg & $\begin{array}{l}T \text { min } \\
\text { (mean) }\end{array}$ & $\begin{array}{l}T \text { max } \\
\text { (mean) }\end{array}$ & $\begin{array}{l}\Delta T \\
\text { (typical) }\end{array}$ & $\begin{array}{l}T \min \\
\text { (abs) }\end{array}$ & $\begin{array}{l}T \text { max } \\
\text { (abs) }\end{array}$ \\
\hline $\begin{array}{l}\text { January } \\
\text { (dry \& cool) }\end{array}$ & $25,4^{\circ} \mathrm{C}$ & $17,8^{\circ} \mathrm{C}$ & $32,1^{\circ} \mathrm{C}$ & $14,3 \mathrm{~K}$ & $12,4^{\circ} \mathrm{C}$ & $35,1^{\circ} \mathrm{C}$ \\
\hline $\begin{array}{l}\text { April } \\
\text { (dry \& hot) }\end{array}$ & $34,0^{\circ} \mathrm{C}$ & $25,4^{\circ} \mathrm{C}$ & $38,6^{\circ} \mathrm{C}$ & $13,3 \mathrm{~K}$ & $22,0^{\circ} \mathrm{C}$ & $43,0^{\circ} \mathrm{C}$ \\
\hline \multirow[t]{2}{*}{$\begin{array}{l}\text { August } \\
\text { (wet) }\end{array}$} & $26,4^{\circ} \mathrm{C}$ & $22,4^{\circ} \mathrm{C}$ & $31,3^{\circ} \mathrm{C}$ & $8,9 \mathrm{~K}$ & $24,1^{\circ} \mathrm{C}$ & $34,4^{\circ} \mathrm{C}$ \\
\hline & \multicolumn{4}{|c|}{$\begin{array}{l}\text { Long-term averages (1983 to } 2008 \text {, due to partial } \\
\text { non-availability of data for the } 1961 \text { to } 1990 \text { normal } \\
\text { period) })^{1019}\end{array}$} & \multicolumn{2}{|c|}{2008 data $^{1020}$} \\
\hline
\end{tabular}

Table 41: Temperature extrema and variation in Dédougou

These large variations are important since both the minimum and maximum thresholds for several epidemiologically relevant processes are exceeded at some time of the year.

The coolest temperatures are typically recorded towards the end of the night, just before sunrise (see figure 49). During the dry period, the daytime rise is both faster and more intensive than during the wet season. Temperatures remain relatively between noon and late afternoon; during the wet season, the temperature usually peaks during the afternoon, but this pattern also depends on the timing of rains which often result in a slight cool-off.

1019 Data source: FAO ClimNET, http://geonetwork3.fao.org/climpag/agroclimdb en.php, accessed 15 March 2009.

1020 http://www.tutiempo.net/clima/Dedougou/2008/655050.htm, accessed 21/08/08. 


\section{Diurnal variation of temperature}

Nouna, 15 March \& 15 July 2004

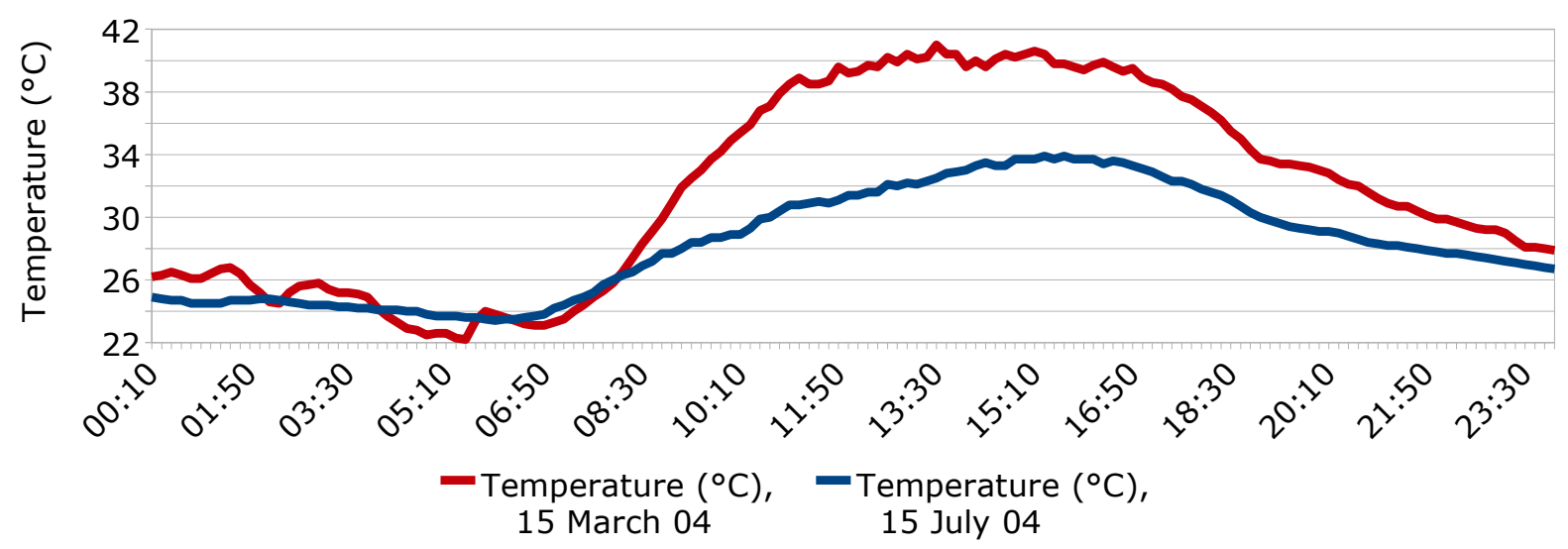

Figure 49: Diurnal variation of temperature in Nouna ${ }^{1021}$

These diurnal temperature variations are malariologically as relevant as daily or monthly mean temperatures: during the rainy season, temperatures remain relatively stable and do not exceed critical thresholds affecting vectors and parasites. During the dry season, high daytime temperatures frequently exceed the maxima tolerated by vectors and malaria parasites, indicating that at this time, refugia with a cooler microclimate play a vital role for sustained malaria transmission.

\subsubsection{Precipitation and Humidity}

Rainfall is the most important single factor for many ecological and environmental processes in the tropics. ${ }^{1022}$ In Burkina Faso, latitudinal differences in precipitation are much more marked than temperature gradients. From south to north, the amount of annual rainfall decreases substantially (see figure 50$){ }^{1023}$ 


\section{Burkina Faso: Rainfall}

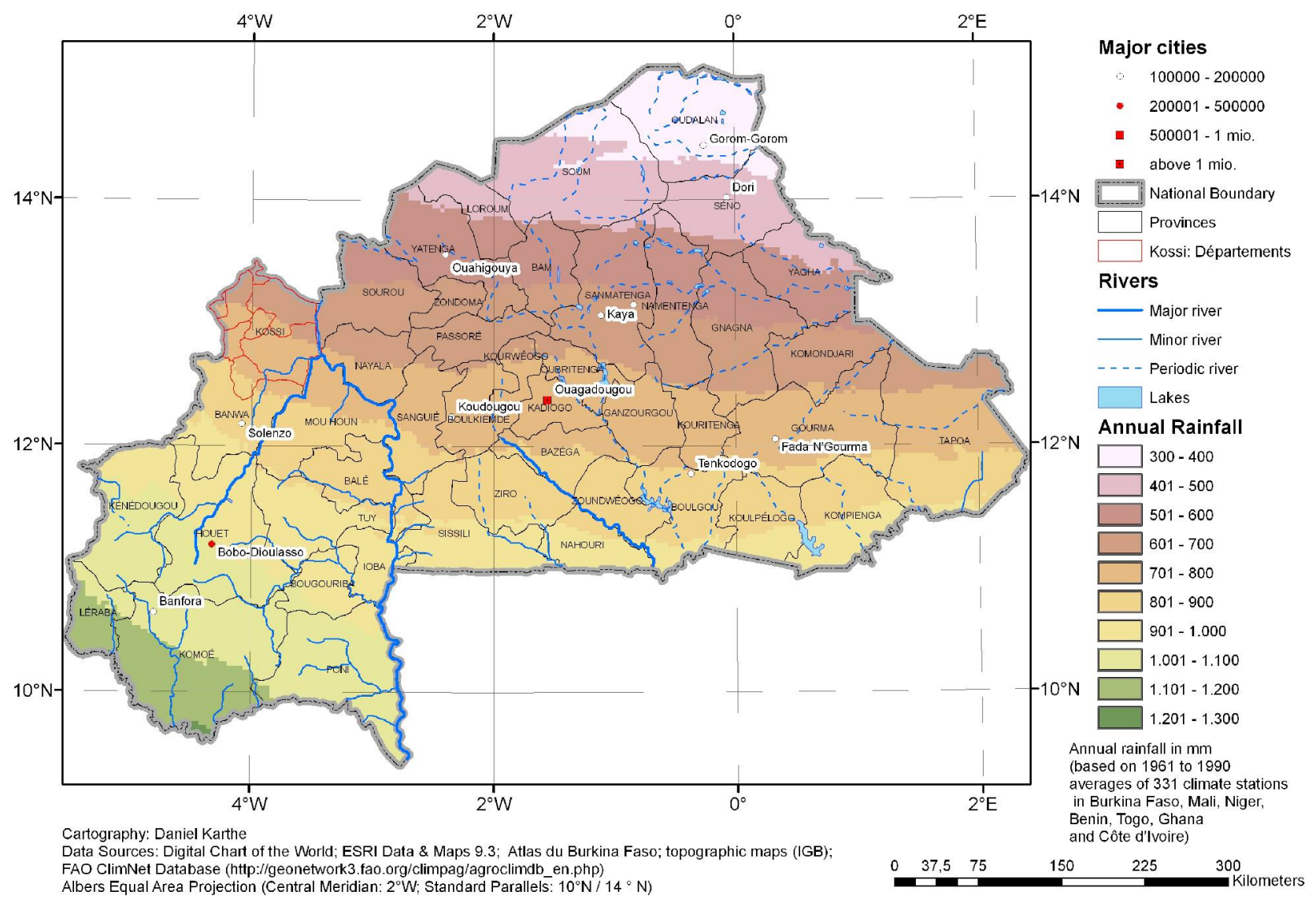

Figure 50: Spatial distribution of precipitation ${ }^{1024}$

Based on the amounts of annual rainfall, Burkina Faso can be divided into three major regions (see figure 57).

- In the Sahel (zone sahélienne) in the north of the country, the annual amounts of rainfall do not exceed $600 \mathrm{~mm}$. There are not more than two humid months with a precipitation of less than $150 \mathrm{~mm}$ in dry years. ${ }^{1025}$ The semi-arid region of the Sahel largely falls beyond the boundary of rain-fed agriculture (which requires a rainfall of about 450 to $500 \mathrm{~mm} /$ year). ${ }^{1026}$

- The Sahelo-Sudanian zone (northern Sudan region; zone soudanosahélienne) occupies the center of the country, where most of the $600 \mathrm{~mm}$ to $900 \mathrm{~mm}$ of annual rain fall during the four to five months of the rainy season.

1024 Based on FAO ClimNet Database (331 stations falling within and $50 \mathrm{~km}$ around Burkina Faso); Digital Chart of the World; YAHMED, D.B. (2005); ESRI Data \& Maps 9.3 and topographic maps (IGB).

1025 Laclavère, G. (1996), p. 15.

1026 Weischet, W. \& Endlicher, W. (2000), p. 274. 
- The Sudanian zone (southern Sudan region; zone soudanienne) is located in the south of the country, where the annual precipitation ranges between $900 \mathrm{~mm}$ and $1300 \mathrm{~mm}$ and the rainy season lasts for around 6 months. ${ }^{1027}$

In this terminology, Kossi Province falls into the Sahelo-Sudanian zone. Even within the limited region of the province, a clear precipitation gradient of about $25 \mathrm{~mm}$ per 10 to $20 \mathrm{~km}$ can be observed (see figure 51). Such immense differences over short distances are certainly of malariologic relevance and result in differences of about $150 \mathrm{~mm}$ between northern and southern Kossi.

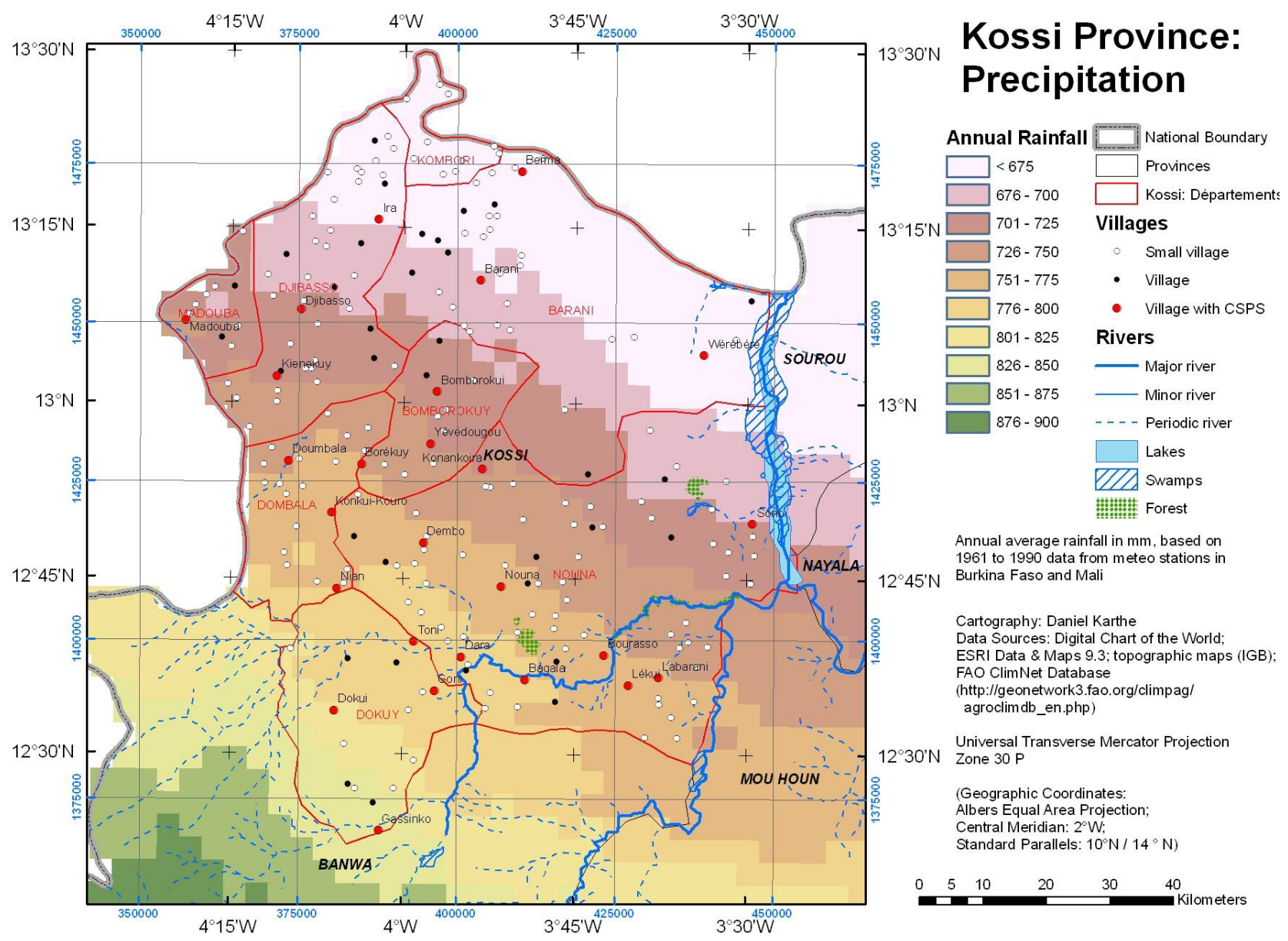

Figure 51: Precipitation gradient in Kossi Province ${ }^{1028}$

1027 LACLAVÈre, G. (1996), p. 15.

1028 Based on FAO ClimNet Database (331 stations falling within and $50 \mathrm{~km}$ around

Burkina Faso); Digital Chart of the World; YAHMED, D.B. (2005); ESRI Data \& Maps 9.3 and topographic maps (IGB). 
In the northern half of Burkina Faso, the amount of precipitation very frequently deviates from the long-term mean and typically ranges between $50 \%$ and $150 \%$ of the average. ${ }^{1029}$ There is a considerable climatic variability, and both prolonged dry spells of several months and downpours of $150 \mathrm{~mm}$ of rainfall on a single day are common. ${ }^{1030}$

«Fluctuations between "wet" and "dry" in the Sahel / Soudan zone are extreme even on decadal and multi-decadal time scales.»1031

Nicholson (2005) observed that rainfall variability in the region is strongly related to August rainfall which -as the wettest month- contributes more to annual variability than any other month. ${ }^{1032}$ This is not only a major concern for agriculture but also for vector-borne disease epidemiology.

\section{Precipitation in Dédougou}

Dry (1996) and wet (1994) years as compared to long-term means

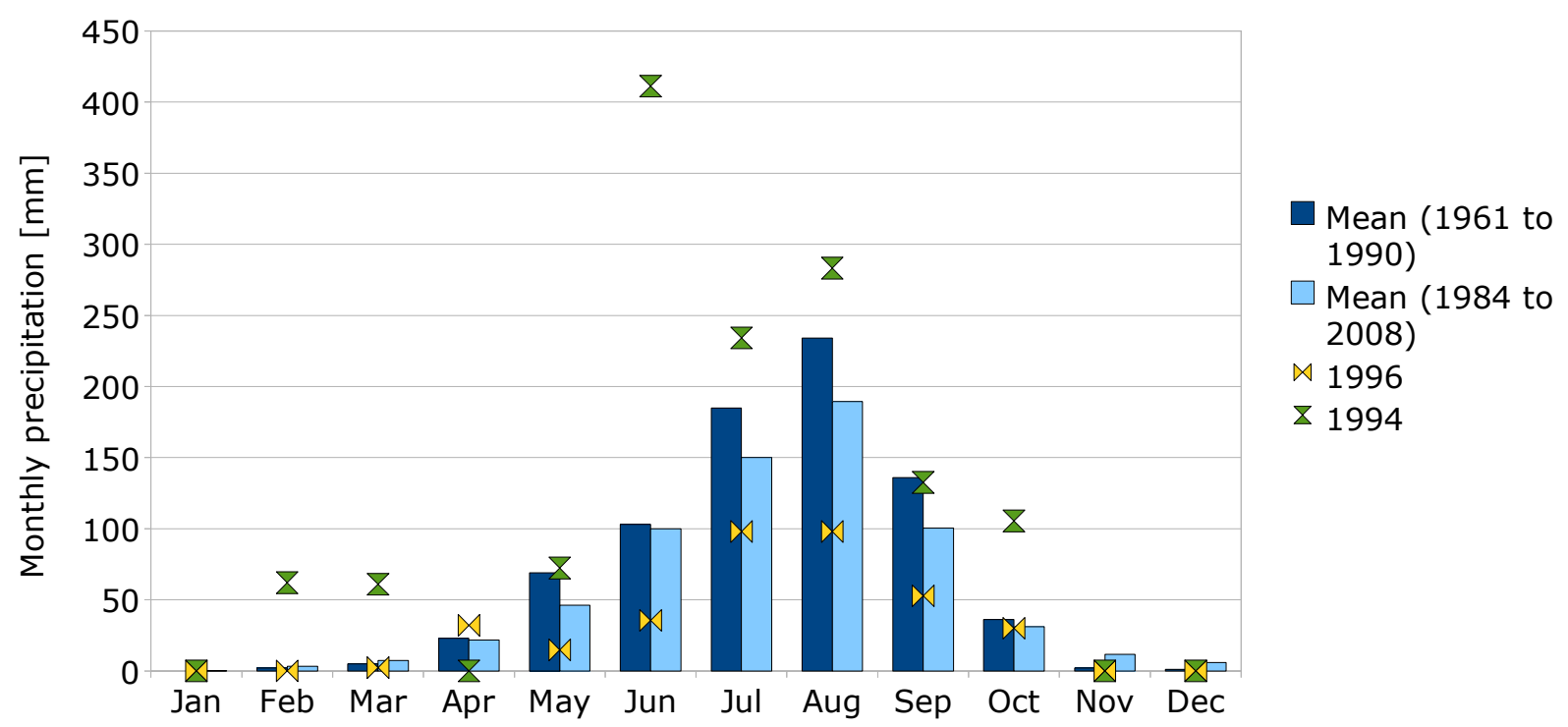

Figure 52: Precipitation in Dédougou: average (1984-2008), 1994, $1996^{1033}$

1029 GRIFFITHS, J.F. (1972), p. 197.

1030 GRIFFITHS, J.F. (1972), p. 198.

1031 Nicholson, S. (2005), p. 631.

1032 Nicholson, S. (2005), p. 616.

1033 http://www.tutiempo.net/clima/Dedougou/655050.htm, accessed 28/07/09.

1984 is the first year for which monthly data from Dédougou meteo station are available; data for 1999 and 2000 have been omitted for the calculation of the means due to poor data quality. 
In Dédougou, the current average annual precipitation is around $667 \mathrm{~mm}$, around $84 \%$ of the 1961 to 1991 normal period mean of $796 \mathrm{~mm}$. For the period between 1984 and 2007, the lowest annual rainfall was $364 \mathrm{~mm}$ (1996; $55 \% / 46 \%$ of the respective means) and the highest annual rainfall was $1362 \mathrm{~mm}(1994 ; 204 \% / 171 \%)$. Both the length of the rainy season and the intensity of the summer rains fluctuate enormously: whereas in 1996, the monthly precipitation never exceeded $100 \mathrm{~mm}$, the June rainfalls in 1994 amounted to $411 \mathrm{~mm}$. At the same time, the rainy season (here defined as the number of months with a precipitation exceeding $60 \mathrm{~mm}$ ) may be as short 2 months (1996) or as long as 8 months (1994). ${ }^{1034}$ The fact that Dédougou is the only WMO registered meteorological station in the study region is particularly problematic in the light of large spatial variations in precipitation which may be of the factor two over distances of less then ten kilometers in West Africa. ${ }^{1035}$ Even though other stations do exist, they were found to be highly unreliable (see chapter 3.1.2.5.).

Humidity in Kossi Province typically ranges between $10 \%$ and $90 \%$, with only minor differences observed between different meteorological stations regarding these extrema. ${ }^{1036}$ Figure 53 shows the annual course of humidity and precipitation in Nouna for 2004, which was moderately (around 25\%) drier than a "normal" year.

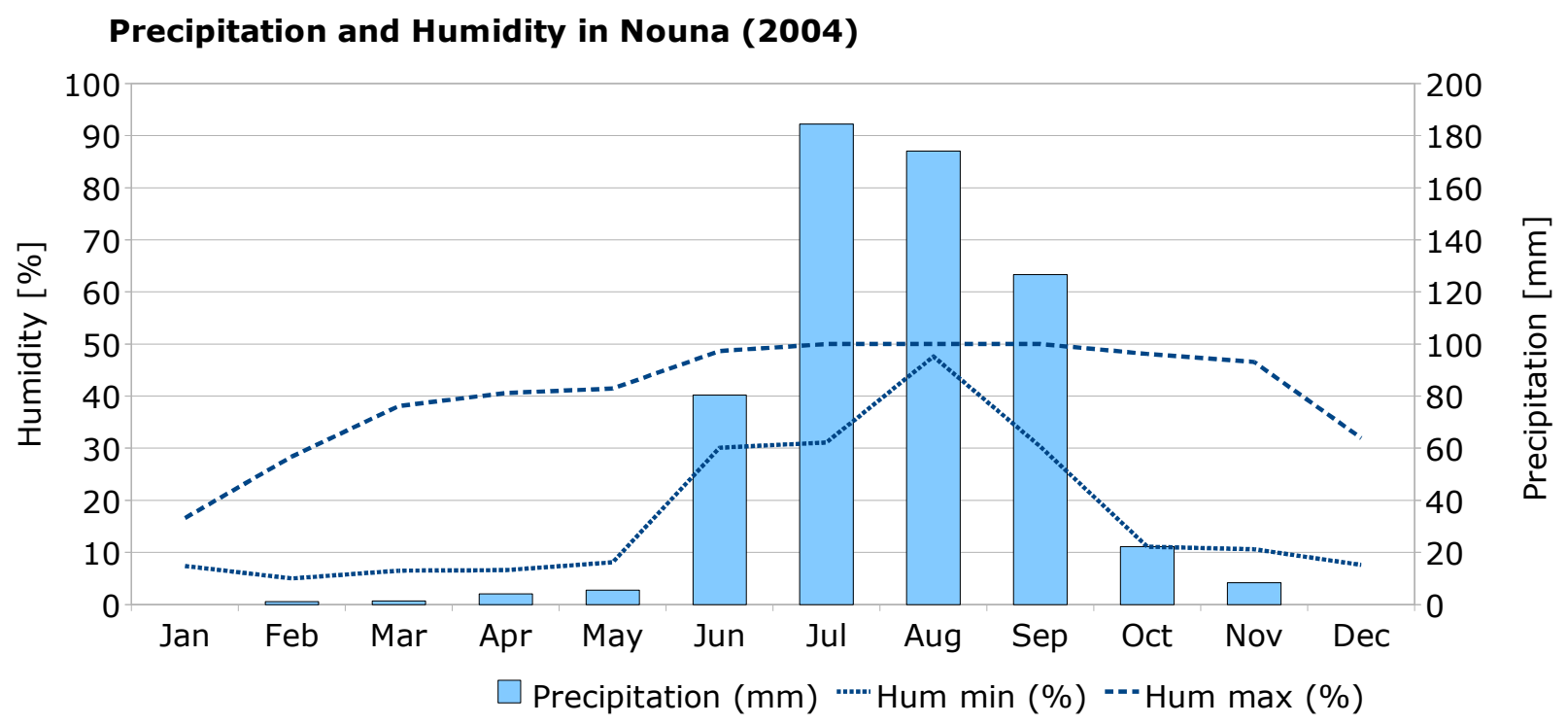

Figure 53: Precipitation and humidity in Nouna (based on meteo station data, 2004) ${ }^{1037}$

1034 http://www.tutiempo.net/clima/Dedougou/655050.htm, accessed 28/07/09.

1035 HAY, S.I. \& LenNon, J.J. (1999), p. 68.

1036 Yé, Y.; Louis, V.R.; Simboro, S. \& SaUerborn, R. (2007), doi:10.1186/1471-2458-7-101.

1037 Data from Nouna meteo station, operated by CRSN Nouna. 
In Kossi, the minimum and maximum aerial humidities are closely linked with the rainfall pattern (see figures 53 and 54): during the rainy summer months, the maximum humidity regularly peaks at $100 \%$ and the minimum humidity rarely falls below $30 \%$, conditions that are favorable to mosquito survival. During the dry period, on the other hand, the minimum humidity is typically around $10 \%$. High relative humidities are rare during hot daytime hours but may occur due to nighttime cool-off.

Precipitation and Humidity in Nouna, July 2004

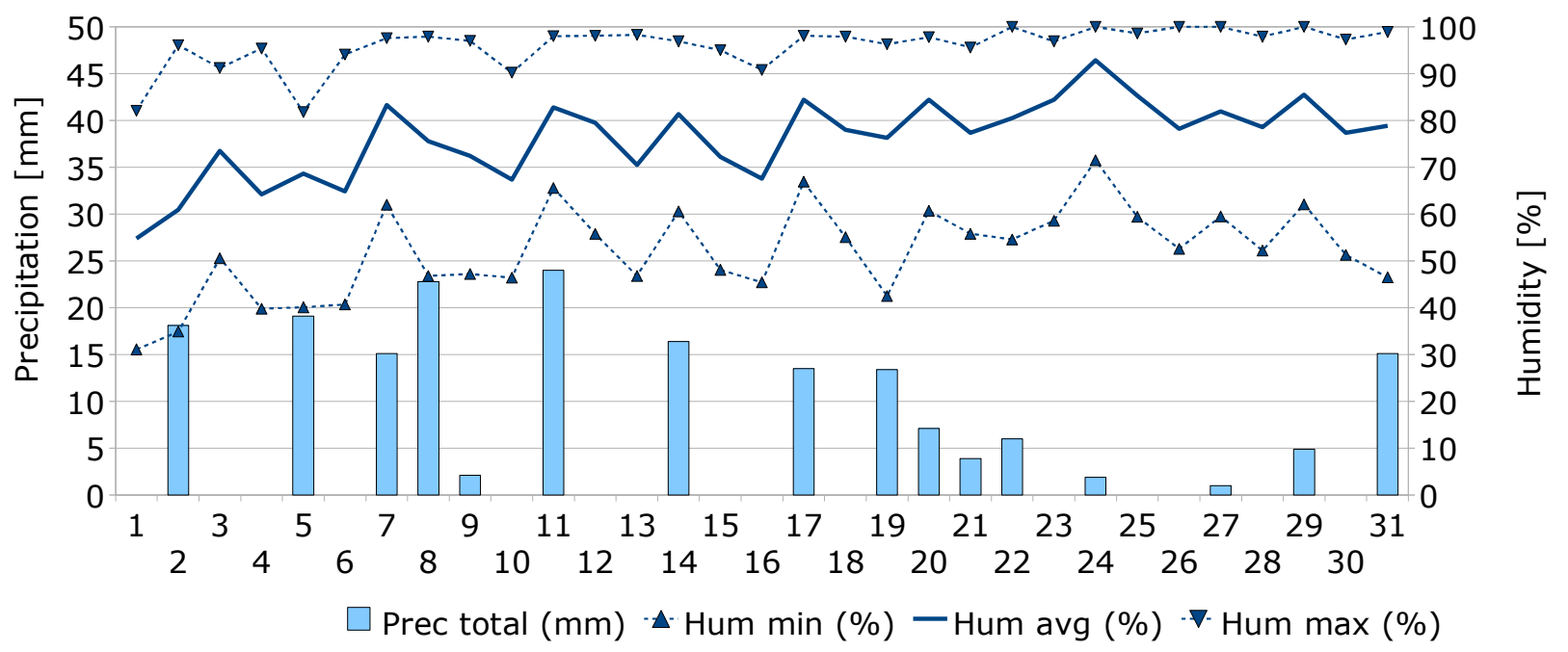

Figure 54: Precipitation and humidity in Nouna, July $2004^{1038}$

During the rainy season, the humidity closely reflects rainfall pattern. The average humidity ranges around $80 \%$, with the daily maxima frequently above $90 \%$ and the minima often above $50 \%$ (figure 54). Such conditions are favorable for prolonged mosquito survival.

\subsubsection{Climatological Dynamics}

Within the global circulation, the Hadley circulation (the mean meridional circulation of the lower latitudes that is also referred to as the trade wind circulation) and the Walker circulation (the mean zonal circulation along the meteorological equator) govern the dynamics and variability of the West African climate. Moreover, most flow characteristics in West Africa are related to the location of the Intertropical Convergence Zone (ITCZ) which separates dry, continental air masses from moist monsoonal air from the Gulf of Guinea. ${ }^{1039}$ Rainfall over West Africa is primarily controlled by the advection of moist air from the Gulf of Guinea in the low levels of the atmosphere. This

1038 Data from Nouna meteo station, operated by CRSN Nouna.

1039 Jung, G. (2006), pp. 9f. 
"West African monsoon" develops during the spring and summer of the northern hemisphere and brings the ITCZ and associated rainfall maxima to their northernmost position in August. ${ }^{1040}$ The annual shift of the ITCZ over West Africa is shown in figure 55.

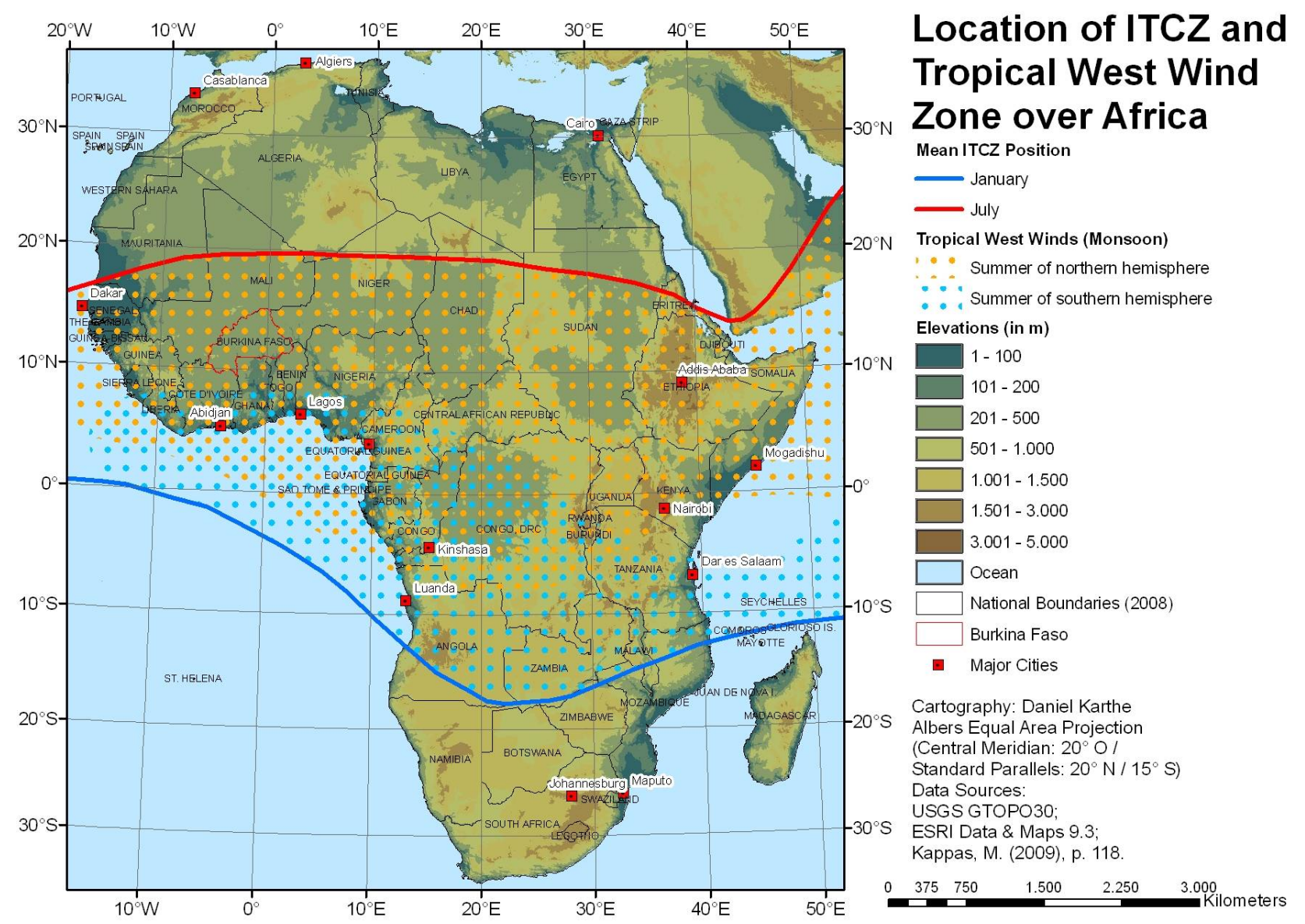

Figure 55: Location of the ITCZ and tropical west wind zone over Africa ${ }^{1041}$

Burkina Faso - and the Nouna region in particular - are far north of the ITCZ during the winter months. At this time, the weather is determined by northeasterly trade winds which bring in dry air from the Sahara ("harmattan"). Under these conditions, the sky is usually cloudless except for cirrus clouds. Despite a massive radiation-induced convection, no clouds are formed due to the aridity of the air, thus preventing any rainfall. After the vegetation withers, the atmosphere is blurred by dust which is either raised locally or brought in by harmattan winds. Around this time, air temperatures reach very high daytime maxima, while there is a considerable drop in temperatures during the night due to outgoing radiation. ${ }^{1042}$

1040 Sultan, B. \& JANICOT, S. (2003), pp. $3407 f$.

1041 Based on USGS GTOPO 30 dataset, ESRI Data \& Maps 9.3 and KAPPAS, M. (2009), p. 118.

1042 Weischet, W. \& EndLICHER, W. (2000), p. 262. 
During the transitional seasons between the summerly wet season and the winterly dry season (usually in November and March/April), Burkina Faso lies at the northern fringe of the ITCZ, which at ground marks the boundary between the harmattan-induced dry air from the north and moist monsoonal air from the south. In the flat layer of monsoonal air that is delineated by the trade wind inversion convectional clouds attain only a limited vertical extension. In rare cases, moist air penetrates the trade wind inversion; this can result in the formation of isolated thunderstorm cells. However, the precipitation which falls from these cumulonimbus clouds often evaporates before reaching the ground (this phenomenon is also referred to as "virga" clouds). Intensive convection can cause the raising of sand and dust from dry soils devoid of vegetation. ${ }^{1043}$

During the humid summer months Burkina Faso comes under the influence of monsoonal air of a vertical expansion of $1500 \mathrm{~m}$ to $2000 \mathrm{~m} \cdot{ }^{1044}$ The monsoon winds are controlled by the pressure gradient between the heat low along the ITCZ and high pressure over the southern Atlantic near St. Helena. ${ }^{1045}$ At that time, convective cloud formation is much more intensive. ${ }^{1046}$ However, most convectional clouds do not result in any precipitation and the onset of the monsoon is typically preceded by isolated showers that are often misinterpreted as the start of the rainy season. In fact, the West African monsoon arrives on the continent in two phases. The arrival of rains on the West African coast typically occurs in February. Clouds then move northward and reach central Burkina Faso in May ("pre-onset"). At this time, the intertropical convergence zone establishes itself around $5^{\circ} \mathrm{N}^{1047}$ while the intertropical front (ITF), i.e. the confluence line between moist southwesterly monsoonal air and the dry air masses of the northeasterly harmattan, is located around $15^{\circ} \mathrm{N} .{ }^{1048}$ With the northward shift of the ITCZ to about $10^{\circ} \mathrm{N}$ and the ITF reaching around $20^{\circ} \mathrm{N}$ in July/August, the monsoon sets in fully between $9^{\circ} \mathrm{N}$ and $13^{\circ} \mathrm{N} .{ }^{1049}$ The fact that this shift occurs abruptly only in the western part of West Africa appears to be linked to strong advection of moist oceanic air. ${ }^{1050}$

The circulation system over West Africa is not explainable solely through the Hadley circulation. The strength and location of both the African Easterly Jet (AEJ) and the Tropical Easterly Jet (TEJ) as well as instabilities of the AEJ, the so called African Wave Disturbances (AWDs), play an important role for the formation of rain-bearing clouds. ${ }^{1051}$ Rainfall events are usually connected with one of two weather phenomena: simple thunderstorms and so-called

1043 Weischet, W. \& Enditcher, W. (2000), pp. $263 f$.

1044 Weischet, W. \& EndLICHER, W. (2000), p. 264.

1045 Sultan, B. \& Janicot, S. (2003), p. 3409.

1046 Weischet, W. \& Endlicher, W. (2000), p. 264.

1047 Laux, P.; Kunstmann, H. \& Bárdossy, A. (2008), p. 330.

1048 Sultan, B. \& Janicot, S. (2003), p. 3407.

1049 Eineder, F. (2009), p. 20; Sultan, B. \& JANicot, S. (2003), p. 3409.

1050 Sultan, B. \& JaNiCOT, S. (2003), p. 3424.

1051 Jung, G. (2006), p. 10. 
lignes de grains (squall lines; "West African tornadoes").

Lignes de grains are belts of intensive thunder storms which are driven by the TEJ and which traverse Burkina Faso in a westerly direction. The passage of such a storm belt begins when the southwest monsoon ceases at ground level due to an intensive vertical movement of air. Stormy winds fall in from the east and frequently cause dust storms, particularly at the beginning of the rainy season. Intensive stormy downpours are connected with sudden falls in temperature of around $10 \mathrm{~K}$. Intensive rains falling from cumulonimbus clouds are gradually replaced by a steady drizzle. Such lignes de grains are of fundamental importance for the water supply of the Sahel. ${ }^{1052}$ Up to 50 such events per year occur in the southern Sahel, with a maximum incidence of around 10 in the month of July (at $12^{\circ} \mathrm{N}$ ). From the ecological viewpoint, it is important that most of the rain falls in short, intensive showers so that most of the water does not infiltrate the soil but is lost due to run-off. ${ }^{1053}$

The highly localized nature of convection events mean that both precipitation rates and onset dates of the rainy season vary considerably at the regional scale. ${ }^{1054}$ Daytime surface heating, topography and local moisture supply are all determinants of convective rain. ${ }^{1055}$

Nicholson (2005) observed that during wet years, the African Easterly Jet is displaced several degrees northward, bringing rains into the Sahel. ${ }^{1056}$ Such circulation changes may be a consequence of changes in sea surface temperatures in the nearby Atlantic. Warm temperatures in the Atlantic and Indian Ocean seem to promote dry conditions, particularly if the subtropical Atlantic is anomalously cold. ${ }^{1057}$

\subsubsection{Climatic Variability and Trends}

Several studies have shown that the climate in large parts of Africa has become drier during the $20^{\text {th }}$ century. The Sahel received relatively good rainfall during the period from 1931 to $1960^{1058}$, and the unusually moist conditions that prevailed in the 1950 s tend to receive much less attention than the drought years that followed. ${ }^{1059}$ Since the mid-1960s, the Sahel experienced a decrease in rainfall ${ }^{1060}$ and nearly three decades of abnormally

1052 Weischet, W. \& EndLicher, W. (2000), pp. $264 f$.

1053 Weischet, W. \& EndLicher, W. (2000), p. 268.

1054 Laux, P.; Kunstmann, H. \& Bárdossy, A. (2008), p. 330.

1055 JUnG, G. (2006), p. 11.

1056 Nicholson, S. (2005), p. 617.

1057 NiCHOLSON, S. (2005), p. 620.

1058 NiCHOLSON, S. (2005), p. 617.

1059 EINEDER, F. (2009), p. 16.

1060 Oguntunde, P.G.; Friesen, J.; van de Giesen, N. \& Savenije, H.H.g. (2006), p. 1180. 
dry conditions followed the drought years between 1968 and $1973 .{ }^{1061}$ Nevertheless, parts of the region experienced above normal rains during this period. ${ }^{1062}$ Wide-spread drought again occurred during the 1982 to 1985 period ${ }^{1063}$ and relatively low rainfall continued until $1997 .{ }^{1064}$ Speculations about the climatology of these droughts are still largely unresolved ${ }^{1065}$, and even though they provide "the most dramatic example of multi-decadal climate variability that has been quantitatively and directly measured"1066 and the magnitude and duration of the drought events were unprecedented in the $20^{\text {th }}$ century, it is unclear whether they were unique in the Holocene. ${ }^{1067}$ Whenever precipitation data from the Sahel are compared to the 1961 to 1990 normal period, it must be kept in mind that both the relatively humid 1960s and the extremely arid 1970 s and 1980 s fell into this period ${ }^{1068}$ and that there are marked differences in the long-term means of different normal periods (see table 42).

\begin{tabular}{|l|l|l|l|l|l|}
\hline Period & $\begin{array}{l}1931 \text { to } \\
1960\end{array}$ & $\begin{array}{l}1941 \\
1970\end{array}$ & $\begin{array}{l}1951 \text { to } \\
1980\end{array}$ & $\begin{array}{l}1961 \text { to } \\
1990\end{array}$ & $\begin{array}{l}1971 \text { to } \\
2000\end{array}$ \\
\hline Rainfall & $520 \mathrm{~mm}$ & $512 \mathrm{~mm}$ & $488 \mathrm{~mm}$ & $428 \mathrm{~mm}$ & $410 \mathrm{~mm}$ \\
\hline
\end{tabular}

Table 42: Sahelian rainfall trends according to WMO normal periods ${ }^{1069}$

In the Volta Basin, the last three decades of the $20^{\text {th }}$ century were drier than any other comparable period for which data exist. ${ }^{1070}$ Nevertheless, it is still debated whether this drought was a natural low probability event or an indicator of long-lasting climatic changes. ${ }^{1071}$ While potential evaporation remained relatively stable, ranging between $1541 \mathrm{~mm} /$ year and $1679 \mathrm{~mm} /$ year (mean: $1601 \mathrm{~mm} /$ year), the annual amount of precipitation was much more variable, ranging between $730 \mathrm{~mm} /$ year and $1314 \mathrm{~mm} /$ year (mean: $1067 \mathrm{~mm}$ /year). ${ }^{1072}$ This variability is illustrated in figure 56 .

1061 NiCHOLSON, S. (2005), p. 616.

1062 Paturel, J.E.; Boubacar, I, \& L'Aour, A. (2004), p. 41.

1063 Oguntunde, P.G.; Friesen, J.; van de Giesen, N. \& Savenije, H.H.G. (2006), p. 1180.

1064 Nicholson, S. (2005), p. 617.

1065 OlsSON, L.; EKLUNDH, L. \& ARdö, J. (2005), p. 556.

1066 HuLme, M. (2001), p. 19.

1067 HerRmann, S.M. \& HutChinson, C.F. (2005), p. 542.

1068 Eineder, F. (2009), p. 18.

1069 HuLme, M. (2001), p. 24.

1070 Oguntunde, P.G.; Friesen, J.; van de Giesen, N. \& Savenije, H.H.G. (2006), p. 1180.

1071 Jung, G. (2006), p. 15.

1072 Oguntunde, P.G.; Friesen, J.; van de Giesen, N. \& Savenije, H.H.G. (2006), p. 1183. 


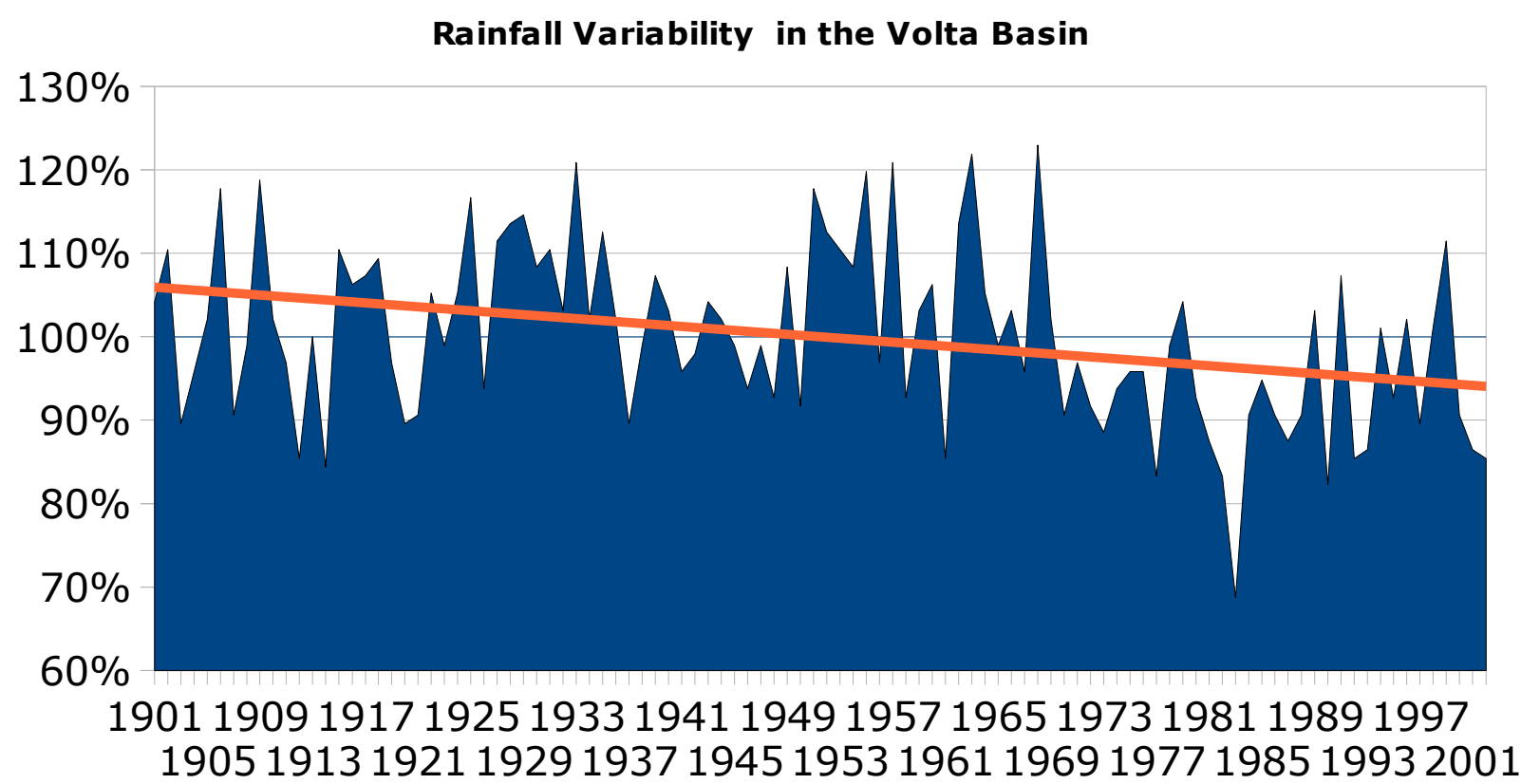

Figure 56: Precipitation variability and trend in the Volta Basin (1901-2001) ${ }^{1073}$

Burkina Faso's central plateau region was hit particularly hard by the drought: while rainfall averaged around $700 \mathrm{~mm}$ in the 1960s, this declined to $550 \mathrm{~mm}$ in the $1970 \mathrm{~s}$ and $80 \mathrm{~s}$. Average rainfall at the peak of the drought (1982 to 1985) was only $381 \mathrm{~mm} \cdot{ }^{1074}$

In Burkina Faso, one consequence of the large-scale drought was the migration of many farm families to regions of higher rainfall in the South or in Ivory Coast. The consequences of the drought led to initiatives like the formation of a national council for environmental management (CONAGESE, Conseil National pour la Gestion de l'Environnement $)^{1075}$ and an international collaboration of the Sahel countries to fight drought and desertification (CILSS, Comité permanent Inter-États de Lutte contre la Sécheresse dans le Sahel).

Some studies have demonstrated an increase in extreme precipitation events ${ }^{1076}$ as well as a general increase in rainfall in arid and semiarid parts of West Africa since the 1990s. For the Sahel, Nicholson (2005) observed some recovery as compared to the $1970 \mathrm{~s}$ and 1980s. This trend has been most marked in the central Sahel $\left(12^{\circ} \mathrm{N}\right.$ to $\left.14^{\circ} \mathrm{N}\right)$ where conditions since 1997 have been comparable to the wet decade of the 1950s. In the northern Sahel $\left(14^{\circ} \mathrm{N}\right.$ to $18^{\circ} \mathrm{N}$ ) annual totals have exceeded the long term mean but not reached the levels of the 1950s. Around the Saharan margin, no increases have been

1073 Calculated from Oguntunde, P.G.; Friesen, J.; van de Giesen, N. \& Savenije, H.H.G. (2006), p. 1184.

1074 Reij, C.; Tappan, G. \& Belemvire, A. (2005), p. 646.

1075 Reij, C.; Tappan, G. \& Belemvire, A. (2005), p. 643.

1076 Hountondu, Y.-C.; Sokpon, N. \& Ozer, P. (2006), pp. $875 f$. 
observed. ${ }^{1077}$ Hountondj et al. (2006) noted an increase in rainfall in Burkina Faso during the 1990s that was paralleled by a strong increase in NDVI, which the authors interpreted as a vegetation recovery that partly reversed the previous period of land degradation. ${ }^{1078}$ In Burkina Faso's Oudalan province, even a revitalization of fossil dunes has been observed, including a recovery of both herbaceous and woody vegetation. ${ }^{1079}$ By contrast, PAtURel et. al. (2004) noted a net decline of rainfall in West Africa in the $20^{\text {th }}$ century. ${ }^{1080}$ EINEDER (2009) compared the precipitation totals recorded in Burkina Faso between 1978 and 2007 to the 1961 to 1990 normal period and found declines in the order of $4 \%{ }^{1081}$

Ecologically, the annual rainfall totals are not the only factor of relevance. The onset, length and end of the rainy season also play a major role for many environmental processes ${ }^{1082}$, including vector population dynamics. Over the past three decades, a considerable delay of the rainy season has been observed, particularly in the Sahel of Burkina Faso. ${ }^{1083}$ The onset of the rainy season is malariologically relevant since it marks the imminent increase in vector habitat availability and thus malaria transmission risks. However, various definitions for this onset exist in the West African context:

\begin{tabular}{|l|l|}
\hline Author & Definition \\
\hline WaLteR (1967) & $\begin{array}{l}\text { The day when accumulated rainfall has reached } 2 \text { inches } \\
\text { (about } 51 \mathrm{~mm} \text { ) }\end{array}$ \\
\hline DAVEY (1976) & $\begin{array}{l}\text { First } 10 \text { day period of the year to receive } 20 \mathrm{~mm} \text { of rainfall } \\
\text { or more }\end{array}$ \\
\hline VIRMANI (1975) & $\begin{array}{l}\text { The week with more than } 20 \mathrm{~mm} \text { of rains provided that } \\
\text { more than } 10 \mathrm{~mm} \text { are likely in the subsequent week }\end{array}$ \\
\hline BeNOIT (1977) & $\begin{array}{l}\text { The day when cumulative rainfall exceeds } 50 \% \text { of the } \\
\text { cumulative potential evapotranspiration }\end{array}$ \\
\hline
\end{tabular}

Table 43: Definitions for rainy season onset ${ }^{1084}$

1077 Nicholson, S. (2005), p. 628.

1078 Hountondji, Y.-C.; SokPon, N. \& Ozer, P. (2008), pp. $875 f$.

1079 Rasmussen, K.; Fog, B. \& Madsen, J.E. (2001), p. 281.

1080 Paturel, J.E.; Boubacar, I, \& L'Aour, A. (2004), p. 44.

1081 EINEDER, F. (2009), p. 34.

1082 Laux, P.; Kunstmann, H. \& Bárdossy, A. (2008), p. 329.

1083 EINEDER, F. (2009), p. 20.

1084 Stern, R.D.; Dennett, M.D. \& Garbutt, D.J.(1981), p. 59. 
In the future, warming in Africa is likely to be larger than the global annual mean warming, with arid regions warming more than the moist tropics. The factors that determine the southern boundary of the Sahara and rainfall in the Sahel have attracted special interest because of the extended drought experienced in this region in the 1970s and 1980s. ${ }^{1085}$ However, it is unclear how rainfall in the Southern Sahara and Sahel will evolve, since there is doubt on the reliability of the models for this region. ${ }^{1086}$

It is now widely accepted that sea surface temperatures (SSTs) in the tropical Atlantic have an influence on West African rainfall ${ }^{1087}$, and explain around $25 \%$ to $35 \%$ of interannual variations. ${ }^{1088}$

\begin{abstract}
«The dominant SST anomaly configuration associated with the Sahelian desiccation has been an inter-hemispheric temperature contrast. The pattern whereby southern oceans are warmer and northern oceans are cooler than average has tended to persist during multi-year periods of Sahelian desiccation. ${ }^{1089}$
\end{abstract}

The seasonal response to SST variations is strongest in summer when the region comes under monsoonal influence and decreases from the Guinea Coast towards the northwest. ${ }^{1090}$ Variations of SSTs in the Atlantic affect both the African Easterly Jet and the Tropical Easterly Jet. ${ }^{1091}$ Dry years in West Africa's Sahelo-Sudanian zone are typically connected to warm SST anomalies in the Gulf of Guinea ${ }^{1092}$ and a weaker TEJ but stronger AEJ, while the opposite was observed during wet years. ${ }^{1093}$ In West Africa, rainfall north of $10^{\circ} \mathrm{N}$ also appears to be linked "to Indian Ocean SST via large-scale atmospheric circulation". ${ }^{1094}$ Moreover, there is a teleconnection to the tropical Pacific basin via the Walker circulation. After El Niño events, the Sahelo-Sudanian region tends to be unusually dry whereas La Niña events precede positive rainfall anomalies. ${ }^{1095}$ The oscillation between El Niño and La Niña events appears to be the primary cause of high-frequency variability ${ }^{1096}$ and has been found to be linked to variations in vector-borne disease transmission in various parts of the world. ${ }^{1097}$

1085 Anyamba, A. \& Tucker, C.J. (2005), p. 597.

1086 IPCC $\left(2007^{1}\right)$, p. 866.

1087 HerRmann, S.M. \& HutChinson, C.F. (2005), p. 542.

1088 OlsSON, L.; EKLUNDH, L. \& ARdö, J. (2005), p. 557.

1089 HuLme, M. (2001), p. 25.

1090 Paeth, H. \& Hense, A. (2004), pp. 179 \& 203.

1091 HerRmann, S.M. \& HutChinson, C.F. (2005), p. 542.

1092 Paeth, H. \& Hense, A. (2004), p. 180.

1093 Jung, G. (2006), p. 11.

1094 Jung, G. (2006), p. 11.

1095 Paeth, H. \& Hense, A. (2004), pp. $180 f$.

1096 Hulme, M. (2001), p. 26.

1097 Anyamba, A.; Chretien, J-P.; Small, J. et al. (2006), doi: 10.1186./1476-072X-5-60. 
However, varying SSTs and large-scale circulations are not the only factor influencing Sahelian rainfall. It is believed that the Sahelian desiccation was also the result of self-reinforcing regional feedback processes through changes in land cover and surface albedo. ${ }^{1098}$

\subsubsection{Data Availability}

Over time, meteorological station networks have changed considerably in West Africa. A dense network of stations had been established by the French colonial government, but many stations have ceased to operate and many African meteorological services have discontinued to make data freely available. ${ }^{1099}$

\section{«[Since the] mid-1990s it has become exceedingly difficult to acquire African precipitation data.» 1100}

Moreover, for those stations that still operate data quality may be a serious problem. Even for important meteorological stations, time series are very frequently incomplete. EINEDER (2009) observed that in the past 20 years, more than $15 \%$ of the rainy season data from Ouagadougou and Bobo-Dioulasso were not registered, a figure that more than doubles for remote regions. ${ }^{1101}$

The reliability for the only WMO-registered station in the study region (Dédougou) has deteriorated in recent years (see table 44), as exemplified by data availability situation in 2008 as compared to 1984 (the first year for which daily data have been made available):

\begin{tabular}{|l|l|l|l|l|l|l|l|l|l|l|l|l|l|l|}
\hline & Jan & Feb & Mar & Apr & May & Jun & Jul & Aug Sep & Oct & Nov & Dec \\
\hline 2008 & 7 & 5 & 5 & 7 & 11 & 2 & 3 & 3 & 0 & 6 & 5 & 1 & 55 \\
\hline 1984 & 2 & 1 & 2 & 2 & 0 & 1 & 1 & 0 & 0 & 0 & 0 & 2 & 11 \\
\hline
\end{tabular}

Table 44: Data availability for Dédougou meteo station (2008 vs. 1984)

The period for which data are not available has increased five-fold, from around $3 \%$ to $15 \%$. This situation is particularly problematic for the estimation of rainfall: for the half year period in which malariologically relevant amounts of rain can be expected (May to October), data gaps increased from two days to 25 days. Such gaps complicate the dating of the onset of the rainy season as well as the derivation of moisture-driven malaria risk indicators.

Even though a total of 10 meteo stations have been set up by the CRSN in

1098 Hulme, M. (2001), pp. $22 \& 26$.

1099 NiCHOLSON, S. (2005), p. 622.

1100 NiCHOLSON, S. (2005), p. 616.

1101 Eineder, F. (2009), p. 20. 
Kossi province, lack of staff and financial resources mean that data availability is even much more restricted, as exemplified by the meteo stations representing the three study locations at Illa, Toni and Kodougou (see tables 45 and ).

\begin{tabular}{|c|l|l|l|l|l|l|l|l|l|l|l|l|l|l|}
\hline & Jan & Feb & Mar & Apr & May Jun & Jul & Aug Sep & Oct & Nov & Dec $\Sigma$ \\
\hline Illa & 31 & 12 & 6 & 1 & 18 & 30 & 31 & 27 & 0 & 7 & 6 & 21 & 190 \\
\hline Toni & 29 & 0 & 0 & 0 & 0 & 0 & 30 & 2 & 0 & 17 & 21 & 19 & 118 \\
\hline Kod. $^{*}$ & 26 & 16 & 0 & 0 & 0 & 1 & 31 & 31 & 30 & 31 & 23 & 0 & 189 \\
\hline
\end{tabular}

Numbers indicate days for which data are not available; Kod. = Kodougou * no rainfall data available for July to October

Table 45: Data availability for the meteo stations operated by CRSN Nouna (2004)

In 2004 , data non-availability was a problem for $32,2 \%$ to $51,9 \%$ of all days, with extremely severe limitations during the peak of the rainy season (average data non-availability for July and August: $81,7 \%$ ).

\begin{tabular}{|c|c|c|c|c|c|c|c|c|c|c|c|c|c|}
\hline & Jan & Feb & Mar & Apr & May & Jun & Jul & Aug & Sep & Oct & Nov & Dec & $\Sigma$ \\
\hline Illa & 0 & 0 & 10 & 0 & 22 & 30 & 31 & 31 & 30 & 31 & 30 & 31 & 246 \\
\hline Toni* $^{*}$ & 31 & 29 & 31 & 30 & 31 & 30 & 31 & 31 & 30 & 31 & 30 & 31 & 366 \\
\hline Kod. & 0 & 0 & 0 & 0 & 0 & 23 & 13 & 0 & 0 & 0 & 7 & 18 & 61 \\
\hline
\end{tabular}

Table 46: Data availability for the meteo stations operated by CRSN Nouna (2008)

By 2008 , non-availability of data had increased to an average level of $61,5 \%$, again with serious gaps $(73,7 \%$ of the days) during the height of the rainy season in July/August. Even though the meteorological stations had originally been erected to improve the availability of weather data for epidemiological studies, the poor reliability of the stations mean that they are currently of little value for geomedical investigations and that data from alternate sources (RS data, meteo data from Dédougou) have to be used. In fact, the poor continuity of the time series mean that they are not even suited for the truthing and calibration of external data. 


\subsubsection{Vegetation}

Burkina Faso's vegetation is dominated by savannas that reflect the gradient from the moist tropics in the south to the arid tropics in the north of the country. Kossi Province is a typical dry savanna region. While large parts of the savanna have come under anthropogenic influence, particularly in form of agricultural land use, trees and shrubs (some actually introduced into the region by man) have often been left standing, acting as indicators for certain eco-epidemiological conditions (see table 51). However, both the wide ecological amplitudes and the anthropogenic selection of species mean that single specimens may not be precise ecological proxies.

\subsubsection{Ecological Regions of West Africa}

West Africa's natural vegetation ranges from the desert and semi-desert regions of the Sahara and north Sahel to rainforests towards the south coast. Even though there are several different classifications, particularly with respect to the deliminations of the belts (which are almost universally based on rainfall), the following north-to-south sequence can be generally observed: 
Chapter 3 - Case Study: Malaria in Kossi Province

\begin{tabular}{|l|l|l|l|}
\hline Belt & Precipitation & Vegetation & $\begin{array}{l}\text { Traditional } \\
\text { forms of } \\
\text { agriculture }\end{array}$ \\
\hline Desert & almost none & almost none & almost none \\
\hline
\end{tabular}




\begin{tabular}{|c|c|c|c|}
\hline Belt & Precipitation & Vegetation & $\begin{array}{l}\text { Traditional } \\
\text { forms of } \\
\text { agriculture }\end{array}$ \\
\hline
\end{tabular}

Transition between Sahara and Sahel $(\approx 50 \mathrm{~mm})$ Limit of pastoralism $(\approx 100 \mathrm{~mm})$ 
Chapter 3 - Case Study: Malaria in Kossi Province

\begin{tabular}{|l|l|l|l|}
\hline Belt & Precipitation & Vegetation & \multicolumn{1}{|l|}{$\begin{array}{l}\text { Traditional } \\
\text { forms of } \\
\text { agriculture }\end{array}$} \\
\hline Semi-desert & $\begin{array}{l}<250 \mathrm{~mm}, 9 \text { to } \\
11 \text { arid months }\end{array}$ & $\begin{array}{l}\text { succulents, low } \\
\text { shrubs and } \\
\text { grasses }\end{array}$ & $\begin{array}{l}\text { irrigated } \\
\text { agriculture in } \\
\text { oases; nomadic } \\
\text { rearing of animals }\end{array}$ \\
\hline $\begin{array}{l}\text { Grass and thorn } \\
\text { savanna }\end{array}$ & $\begin{array}{l}250 \text { to } 500 \mathrm{~mm}, 8 \\
\text { to } 10 \text { arid months }\end{array}$ & thorny bushes, \\
grasses & $\begin{array}{l}\text { irrigated } \\
\text { agriculture in } \\
\text { oases; nomadic } \\
\text { rearing of animals }\end{array}$ \\
\hline Dry savanna & $\begin{array}{l}500 \text { to } 1100 \mathrm{~mm}, \\
4 \text { to } 8 \text { arid months }\end{array}$ & $\begin{array}{l}\text { high grass with } \\
\text { individual trees; } \\
\text { dry forests }\end{array}$ & $\begin{array}{l}\text { rainfed agriculture } \\
\text { during summer } \\
\text { (but frequent crop } \\
\text { failures) }\end{array}$ \\
\hline \multicolumn{3}{|c|}{ Penck's limit of aridity (evaporation $>$ precipitation) } \\
\hline Moist savanna & $\begin{array}{l}1100 \text { to } 1600 \mathrm{~mm}, \\
2 \text { to } 4 \text { arid months }\end{array}$ & $\begin{array}{l}\text { monsoonal forests } \\
\text { and high grasses }\end{array}$ & $\begin{array}{l}\text { rainfed agriculture } \\
\text { with two annual } \\
\text { harvests }\end{array}$ \\
\hline
\end{tabular}




\begin{tabular}{|l|l|l|l|}
\hline Belt & Precipitation & Vegetation & $\begin{array}{l}\text { Traditional } \\
\text { forms of } \\
\text { agriculture }\end{array}$ \\
\hline Tropical rainforest & $\begin{array}{l}>1500 \mathrm{~mm} \text {, less } \\
\text { than two arid } \\
\text { months }\end{array}$ & $\begin{array}{l}\text { high evergreen } \\
\text { trees }\end{array}$ & $\begin{array}{l}\text { permanent } \\
\text { agriculture }\end{array}$ \\
\hline
\end{tabular}

Table 47: Vegetation belts of West Africa ${ }^{1102}$

Burkina Faso almost completely falls into the savanna zone, with the grass and thorn savannas of the Sahel forming the northern third of the country, the dry savannas of the Sahelo-Sudanian zone (by some authors referred to as the zone soudanienne ${ }^{1103}$ ) the center and the wet savannas of the Sudanian zone (sometimes referred to as the zone guinéenne ${ }^{1104}$ ) occupying the south. Kossi province is situated in the very north of the Sahelo-Sudanian zone, very close to the Sahel sensu stricto (see figure 57).

1102 Nicholson, S. (2005), p. 621; Anhuf, D. \& Frankenberg, P. (1991), p. 245.

1103 AnhuF, D. \& Frankenberg, P. (1991), p. 245.

1104 AnhuF, D. \& Frankenberg, P. (1991), p. 245. 


\section{Burkina Faso: Ecozones}

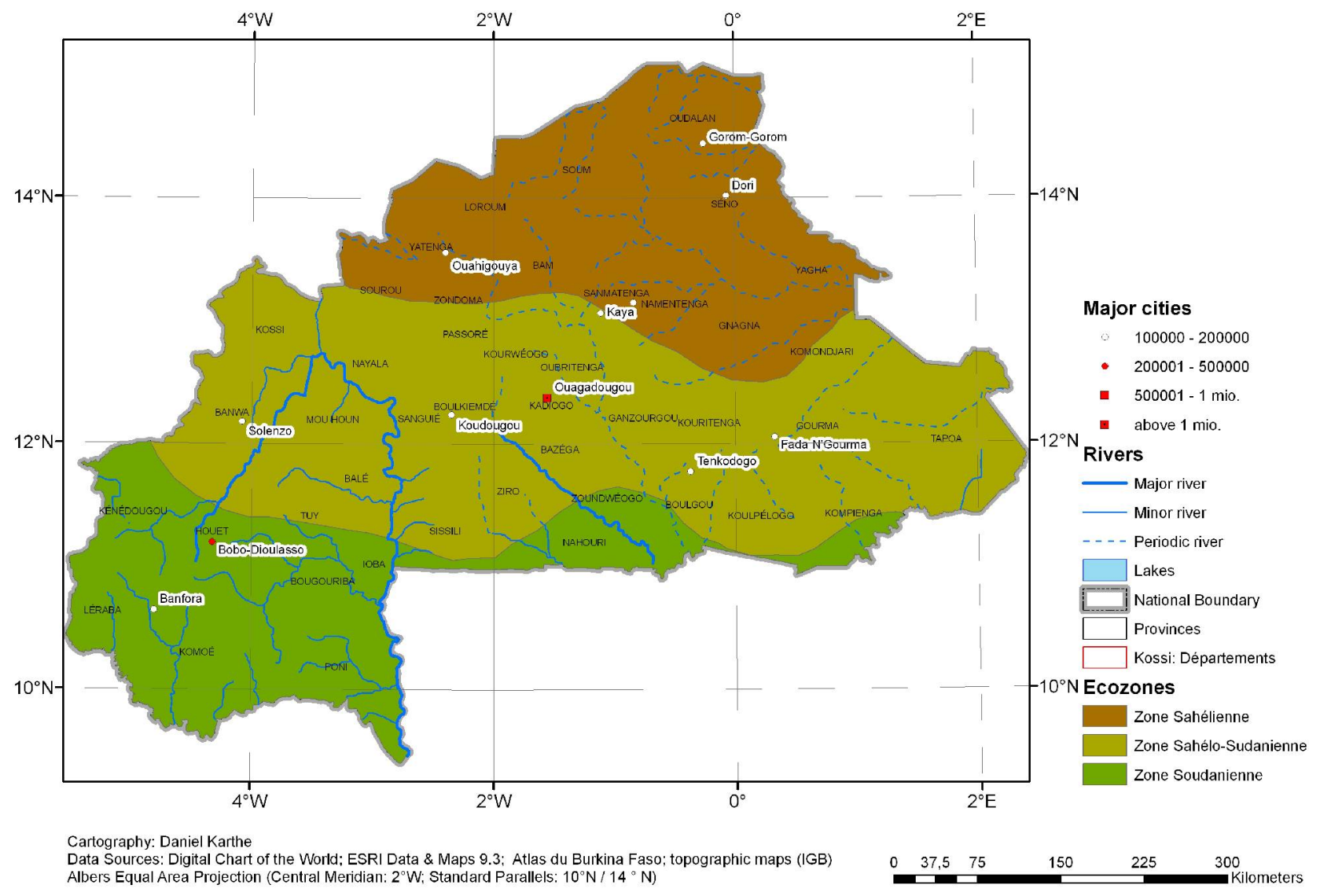

Figure 57: Agro-ecological zones of Burkina Faso ${ }^{1105}$

Some authors consider the dry savanna region in and around Kossi province to be a part of the Sahel, which they define as the region with an annual precipitation ranging between $100 \mathrm{~mm}$ and $1000 \mathrm{~mm} .{ }^{1106}$ HeLD et al. (2005) even denote the Sahel as "the transition zone between the Saharan desert and the rainforests of Central Africa and the Guinean coast"1107, a view that is far from being universally accepted. By contrast, Anhuf and Frankenberg (1991) use the term 'Sahel' only for regions with a precipitation between at least $200 \mathrm{~mm}$ and at the most $600 \mathrm{~mm} .{ }^{1108}$ As the rainfall decreases from south to north in the Sudanian and Sahel savanna, the woody trees and shrubs decrease in height rather than in their amount of ground cover, but towards the Sahara the

1105 Based on YAHMED, D.B. (2005), p. 67; Digital Chart of the World ; ESRI Data \& Maps 9.3 and topographic maps (IGB).

1106 HAMMER, T. (2005), p. 19.

1107 HeLd, I.M.; DeLworth, T.L.; Lu, J. et al. (2005), pp. 17891.

1108 AnhuF, D. \& Frankenberg, P. (1991), p. 260. 
cover also decreases. ${ }^{1109}$ The dry savannas of the Sahel are dominated by grass in the north, with individual bushes and small tress being present. To the south follows a belt dominated by thorny shrubs, which further to the south gives way to tree-dominated savannas and dry forests. ${ }^{110}$

Endemism is not an outstanding feature of the West African savanna, and many species occurring there are also found in other parts of Africa or the world. None of the 370 or so species of trees and bushes found in the West African savanna belongs to a family endemic to West Africa, and most of them belong to pantropical or cosmopolitan families. ${ }^{1111}$

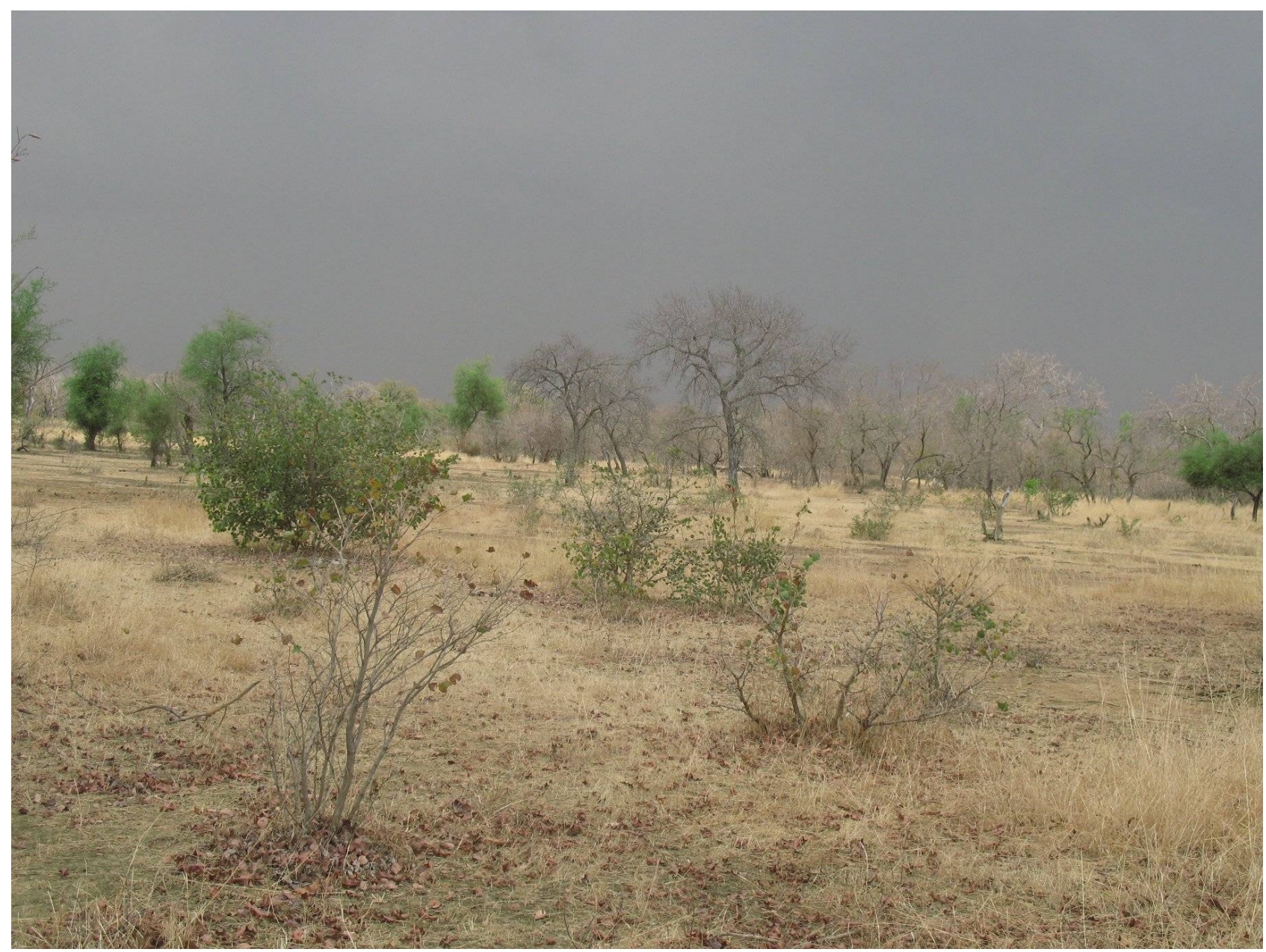

Figure 58: Dry savanna near Bomborokuy (before a 'winter' shower)

According to the GLC 2000 land cover map, most of the land in Burkina Faso is -at least to some extent- used agriculturally (see table 48 and figure 59).

1109 Geerling, C. (1985), p. 247.

1110 HAMMER, T. (2005), p. 19.

1111 Geerling, C. (1985), p. 247. 


\begin{tabular}{|l|l|l|l|l|l|}
\hline & Woodland & Shrubland & Grassland & $\begin{array}{l}\text { Cultivated } \\
\text { land }\end{array}$ & Bare soil \\
\hline Area $\left[\mathrm{km}^{2}\right]$ & 11570 & 49410 & 4538 & 173860 & 50 \\
\hline
\end{tabular}

Table 48: Land cover in Burkina Faso (according to GLC 2000) 1112

Most of Kossi Province is categorized as 'cropland' or 'cropland with woody vegetation', indicating an intense anthropogenic modification of the natural savanna landscape. The GLC's tendency to overestimate cropland should be kept in mind, however. ${ }^{1113}$ The Sourou Valley, located in the east of Kossi Province, is the site of Burkina Faso's northernmost large-scale irrigation project and the only region in the country that is designated as irrigated cropland by the GLC 2000 land cover map. Open grassland is found in the very north of Kossi Province (see figure 59).

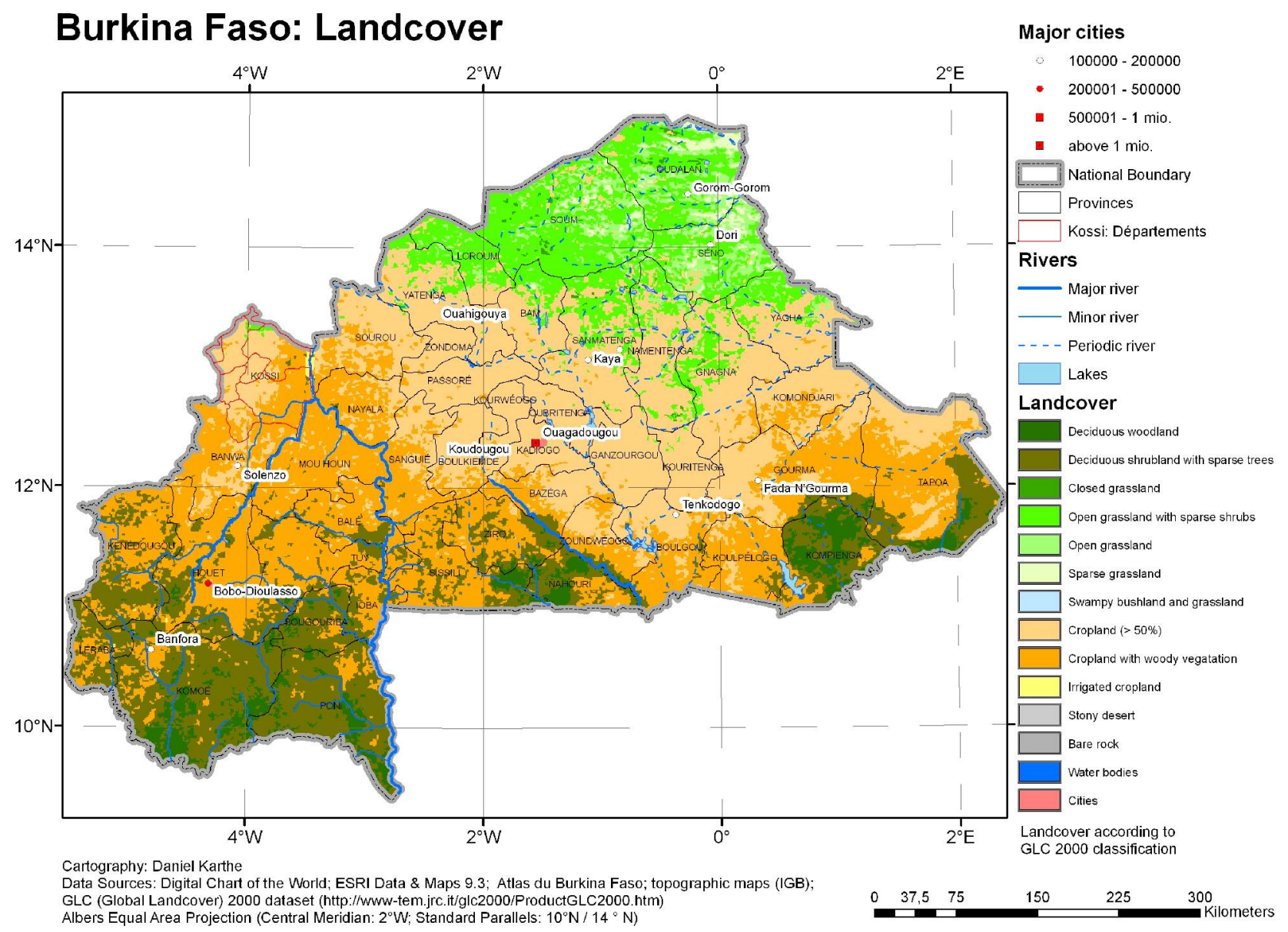

Figure 59: Landcover and landuse in Burkina Faso ${ }^{1114}$

1112 Mayaux, P.; Bartholomé, E.; Fritz, S. \& Belward, A. (2004), p. 868.

1113 Mayaux, P.; Bartholomé, E.; Fritz, S. \& Belward, A. (2004), p. 873.

1114 Based on GLC 2000 dataset; YAHMED, D.B. (2005); Digital Chart of the World;

ESRI Data \& Maps 9.3 and topographic maps (IGB). 


\subsubsection{Important Species}

Even though a considerable part of the savanna has been transformed into agricultural land, the presence of individual bushes and trees is not uncommon in cultivated fields. Nevertheless, the tree layer of the dry savanna is sometimes limited to species selected by man; in fact, some species have been artificially introduced into the region. ${ }^{1115}$ OuadBa (1991) found that soil types determined the species richness on protected land in Burkina Faso's Central Plateau, ranging from 12 woody species per hectare on vertisols to 27 species per hectare on hydromorphic soils, not counting minor plants. ${ }^{116}$ Except for a few exceptions like Acacia albida ${ }^{1117}$, most trees are totally devoid of foliage for at least a part of the dry season. ${ }^{1118}$ 
Chapter 3 - Case Study: Malaria in Kossi Province

\begin{tabular}{|c|c|c|c|c|}
\hline \multirow[t]{2}{*}{ Common name } & \multirow[t]{2}{*}{ Botanic name } & \multicolumn{3}{|c|}{ Local names } \\
\hline & & Bambara & Moré & Peulh \\
\hline Neem tree & Azadirachta indica & & neem & \\
\hline \multirow[t]{2}{*}{ Wild grape tree } & Lannea microcarpa & $\begin{array}{l}\text { pegu, } \\
\text { m-peku }\end{array}$ & sabgha & falfahi \\
\hline & $\begin{array}{l}\text { Combretum } \\
\text { glutinosum }\end{array}$ & tyangara & $\begin{array}{l}\text { dandegha, } \\
\text { koagenga }\end{array}$ & $\begin{array}{l}\text { buski, } \\
\text { dooki, ookai }\end{array}$ \\
\hline Kinkeliba & $\begin{array}{l}\text { Combretum } \\
\text { micranthum }\end{array}$ & singolobe & dandegha & \\
\hline Seyal acacia & Acacia seyal & $\begin{array}{l}\text { sadee, } \\
\text { zayee }\end{array}$ & $\begin{array}{l}\text { gomiga, } \\
\text { gimpelaga }\end{array}$ & $\begin{array}{l}\text { bidehi, } \\
\text { boulbi, } \\
\text { komanahi }\end{array}$ \\
\hline Desert date & $\begin{array}{l}\text { Balanites } \\
\text { aegyptiaca }\end{array}$ & $\begin{array}{l}\text { seguene, } \\
\text { zegene }\end{array}$ & $\begin{array}{l}\text { kieghaligha, } \\
\text { kielega, } \\
\text { tjaralra }\end{array}$ & $\begin{array}{l}\text { goleteki, } \\
\text { mourotouki, } \\
\text { tane }\end{array}$ \\
\hline $\begin{array}{l}\text { African locust } \\
\text { bean tree }\end{array}$ & Parkia biglobosa & nere & $\begin{array}{l}\text { doaaga, } \\
\text { roanga, } \\
\text { ghoaga }\end{array}$ & narehi, neré \\
\hline Jujube tree & Ziziphus mauritania & $\begin{array}{l}\text { domo, } \\
\text { tomonou }\end{array}$ & $\begin{array}{l}\text { bagandre, } \\
\text { magunuga }\end{array}$ & $\begin{array}{l}\text { barkewi, } \\
\text { djabe }\end{array}$ \\
\hline $\begin{array}{l}\text { Butter tree, } \\
\text { karité }\end{array}$ & Vitellaria paradoxa & si & taga, taanga & $\begin{array}{l}\text { karedie, } \\
\text { kolo }\end{array}$ \\
\hline Tamarind tree & Tamarindus indica & domi, ntomi & $\begin{array}{l}\text { bupugubu, } \\
\text { puaga }\end{array}$ & $\begin{array}{l}\text { damé, } \\
\text { ngatabbi }\end{array}$ \\
\hline Mango tree & Mangifera indica & mankuru & & \\
\hline $\begin{array}{l}\text { Baobab, } \\
\text { monkey bread } \\
\text { tree }\end{array}$ & Adansonia digitata & sira & toega & \\
\hline
\end{tabular}

Table 49: Botanic, English and local names of important woody species found in Kossi ${ }^{1119}$

Table 49 provides an overview of the English, botanic and local names of the tree and bush species that are found in the dry savanna landscape of Kossi province. These trees (some of which also occur in form of small shrubs under less favorable conditions) are covered here for two principal reasons: the species composition of the tree 'layer' is an indicator for certain environmental conditions that may be of malariologic relevance, and certain parts of some of these trees are used as ingredients for traditional medicine and natural insecticides.

1119 Based on von Maydell, H.J. (1990) \& Schütt, P.; Weisgerber, H.; Schuck, H.J. et al. (2006). 
Neem trees (Azadirachta indica) have been planted in the environs of several villages in Kossi province and are considered an integral component of agroforestry projects in the Sahel. The species was introduced from the dry forest regions of India and several parts of the plant are considered to be of pharmaceutical and insecticidal value. ${ }^{1120}$ Azadirachta indica occurs in regions where monthly mean temperatures range between $10^{\circ} \mathrm{C}$ and $36^{\circ} \mathrm{C}$ but extremes of up to $49^{\circ} \mathrm{C}$ are tolerated..$^{1121}$ The trees develop best at $450 \mathrm{~mm}$ to $750 \mathrm{~mm}$ annual precipitation but are very drought-resistant. They may subsist on $150 \mathrm{~mm}$ of rain per year and improve degraded soils ${ }^{1122}$ but also tolerate extremely high amounts of rainfall (up to $4000 \mathrm{~mm}$ per year). Azadirachta indica is not found on hydromorphic soils. ${ }^{1123}$

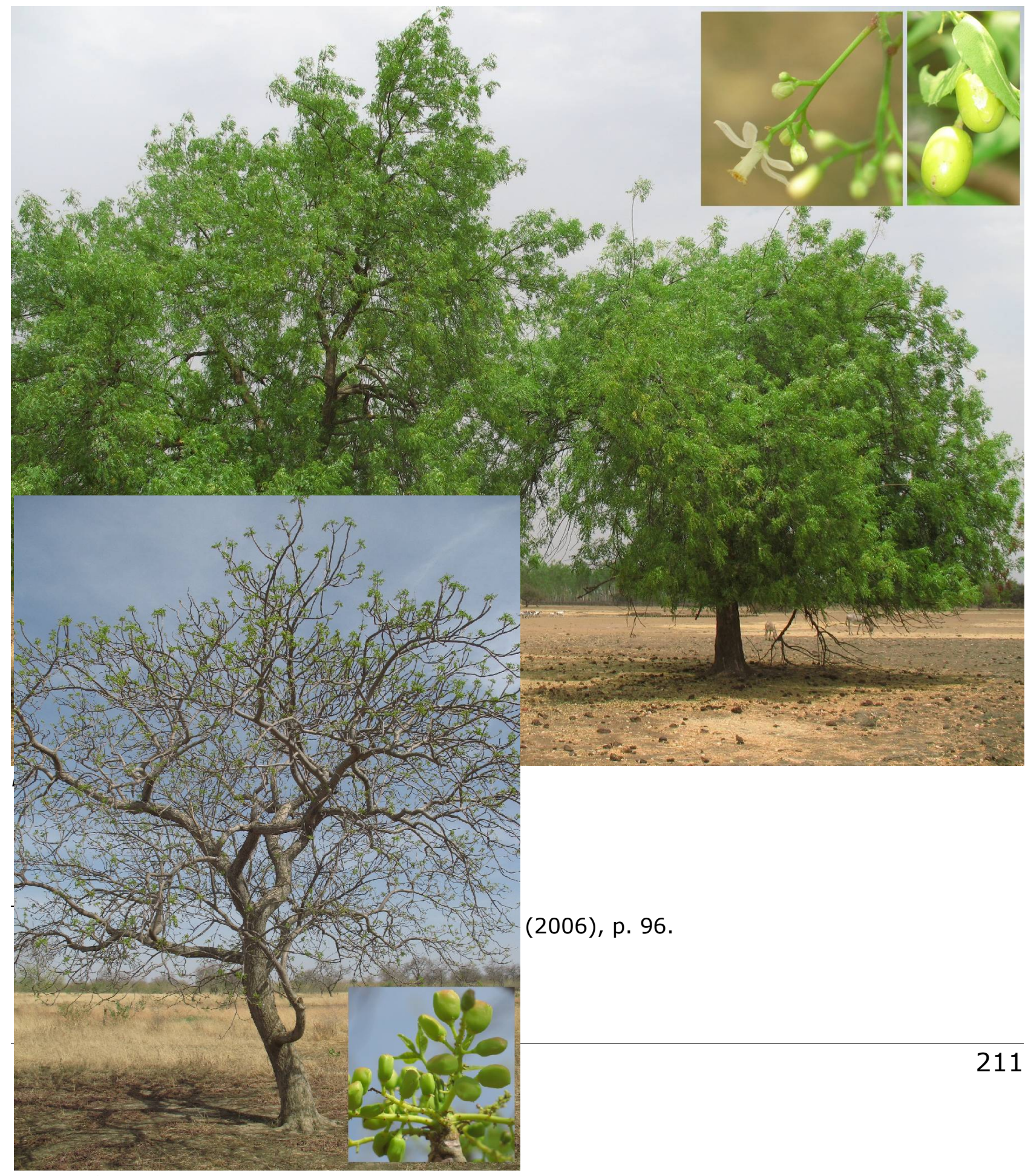


The wild grape tree (Lannea microcarpa) actually comprises two taxa, one that is restricted to eastern Nigeria and Cameroon, and one that is quite commonly found in West Africa's dry savannas. ${ }^{1124} 900$ to $1100 \mathrm{~mm}$ of rain are ideal, but the plant may subsist on as little as $500 \mathrm{~mm}$ per year ${ }^{1125}$ and is occasionally found in the north of Burkina Faso's Sahelo-Sudanian zone. The tree is often found on lateritic rocks. ${ }^{1126}$ Its fruits may be dried and eaten like raisins or used for making a sweet beverage. The leaves, which remain green until the onset of the rainy season are used for feeding goats. ${ }^{1127}$

Several species of the Combretaceae family occur in the Sahel and SaheloSudanian zone of West Africa, including Combretum aculateum, a small bush which is frequently found in dry locations ${ }^{1128}$ near termite mounds ${ }^{1129}$, Combretum glutinosum, a small tree or bush which is very common between the western Sahel and Cameroon ${ }^{1130}$, Combretum micranthum, an indicator of very poor soils which grows primarily in the Sahel ${ }^{1131}$, Combretum nigricans, a small tree which is frequent in dry locations ${ }^{1132}$ in the dry and wet savannas ${ }^{1133}$, particularly on sandy soils ${ }^{1134}$, and Combretum paniculatum, a tree which has red flowers during the dry season and occurs in savanna regions and gallery forests south of the Sahel. ${ }^{1135}$ Combretum glutinosum, one of the most frequent species in the Kossi savanna, may be found on surface crusts and hard concretions ${ }^{1136}$ but grows on many soil types, preferentially well-drained sandy soils. The species is drought-resistant and present in areas where monthly mean temperatures range between $21^{\circ} \mathrm{C}$ and $36^{\circ} \mathrm{C}^{1137}$ and where the mean annual rainfall is between 300 and $700 \mathrm{~mm}$, but minima of $200 \mathrm{~mm}$ (where it may be found in inundated locations) ${ }^{1138}$ and maxima of $2000 \mathrm{~mm}$ are tolerated. ${ }^{1139}$ The species' distribution therefore extends deep into the Sahel in the north and the rainforest zone in the south. ${ }^{1140}$ Extracts of young leaves of

1124 Geerling, C. (1982), p. 55.

1125 http://en.sl.life.ku.dk/upload/123net.pdf; accessed 16/09/08.

1126 Boudet, G. \& Lebrun, J.P. (1986), p. 205.

1127 von MAYDelL, H.J. (1990), p. 289.

1128 BOUdet, G. \& LeBRUn, J.P. (1986), p. 81

1129 von MAYdelL, H.J. (1990), p. 211.

1130 von MAYDELL, H.J. (1990), p. 213.

1131 von Maydell, H.J. (1990), p. 215.

1132 Boudet, G. \& LeBrun, J.P. (1986), p. 81

1133 Von MAYDELL, H.J. (1990), p. 217.

1134 Geerling, C. (1982), p. 113.

1135 von MaYdelL, H.J. (1990), p. 219.

1136 Boudet, G. \& LeBRun, J.P. (1986), p. 81.

1137 THIES, E. (1995), p. 182.

1138 http://en.sl.life.ku.dk/upload/combretum glutinosum 128.pdf; accessed 29/06/09.

1139 THIES, E. (1995), p. 182.

1140 Geerling, C. (1982), p. 110. 
Combretum glutinosum are used against fever, malaria and jaundice in Burkina Faso, and laboratory tests have confirmed some antiplasmodial activity of methanolic leaf extracts. ${ }^{1141}$ Combretum micranthum, another very frequent species in the Kossi savanna, is a relatively ubiquitous species that is distributed between Mauritania and Senegal and from Nigeria to Niger. ${ }^{1142}$ The savanna plant is native to western Africa, found on dry sites and usually an indicator of poor, low nutrient soils ${ }^{1143}$, including skeletal soils and soils with surface crusts. ${ }^{1144}$ It grows where monthly mean temperatures range between $20^{\circ} \mathrm{C}$ and $37^{\circ} \mathrm{C}^{1145}$ and where annual rainfall is between 300 and at least 1500 $\mathrm{mm}$. The tree may be locally abundant, and is often found in pure, dense stands ${ }^{1146}$ or in association with Combretum nigricans and Acacia macrostachya. ${ }^{1147}$

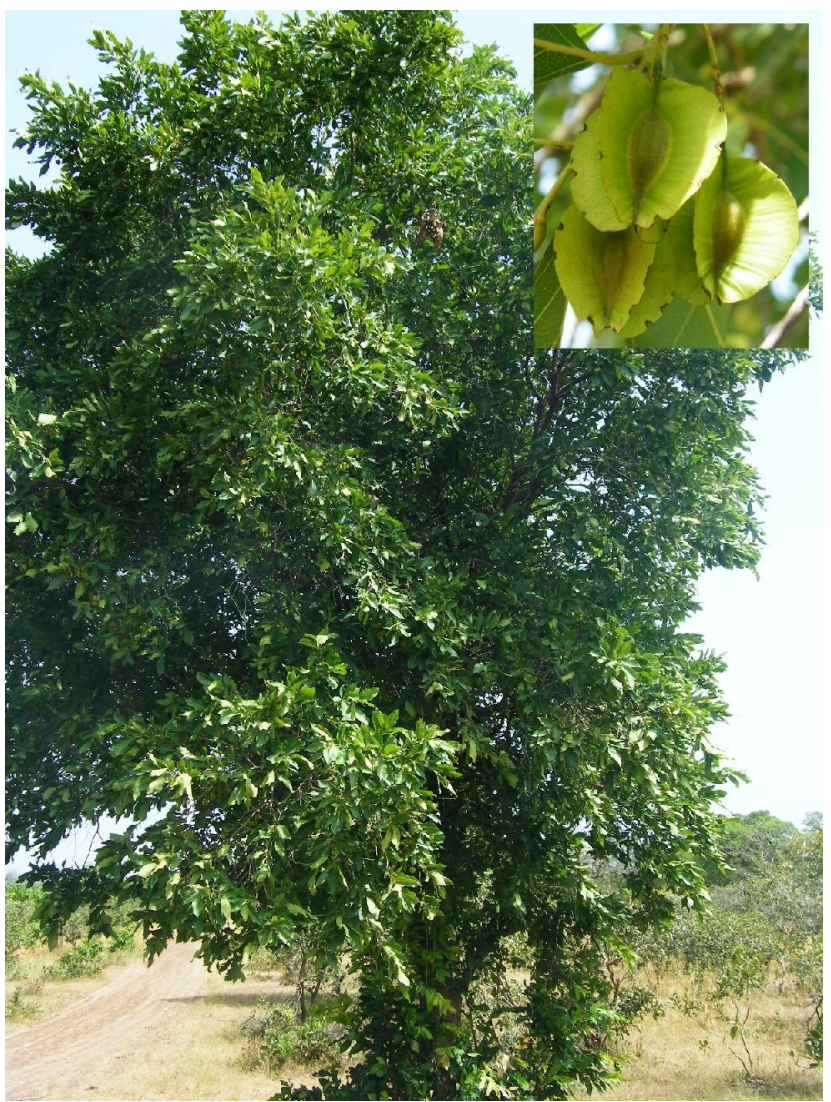

Figure 62: Combretum micranthum

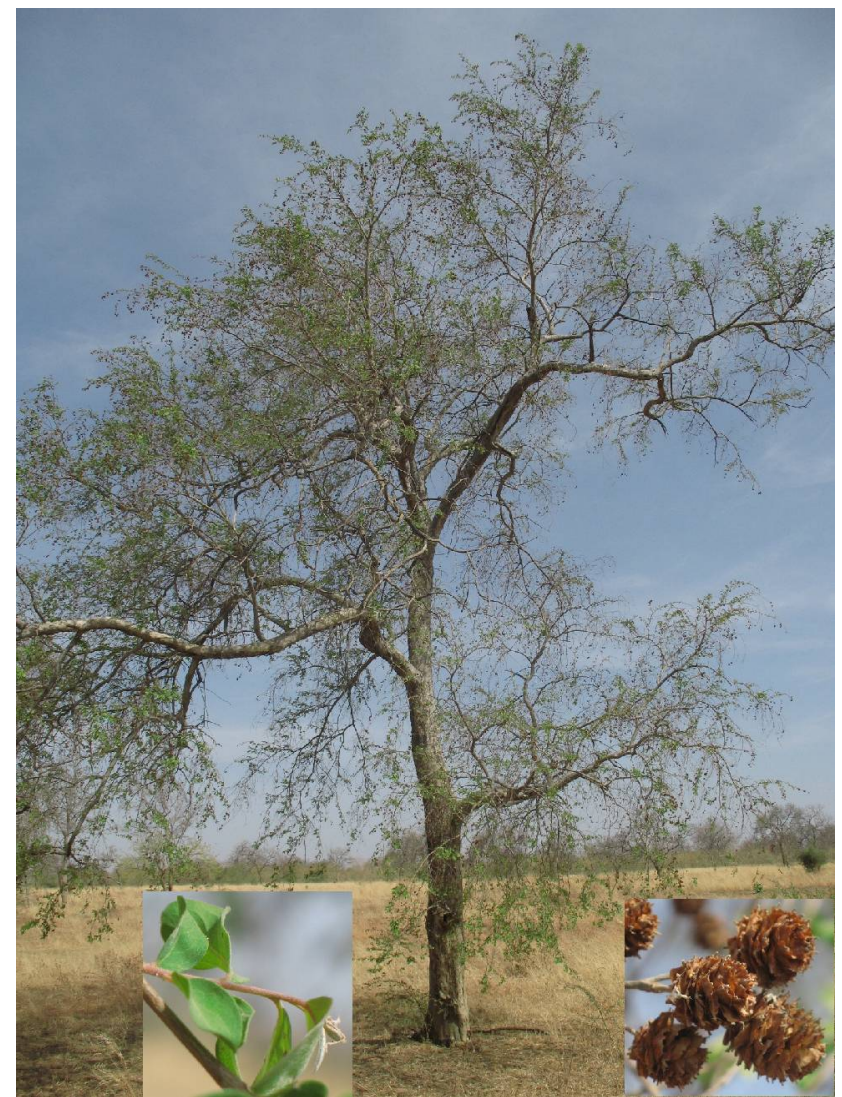

Figure 63: Combretum glutinosum

1141 Ouattara, Y.; Sanon, S.; Traoré, Y. et al. (2006), p. 80.

1142 Boudet, G. \& LEBRun, J.P. (1986), p. 80 and

http://en.sl.life.ku.dk/upload/c micranthum.pdf; accessed 29/06/09.

1143 THIES, E. (1995), p. 184.

1144 Geerling, C. (1982), p. 111.

1145 THIES, E. (1995), p. 184.

1146 http://en.sl.life.ku.dk/upload/c micranthum.pdf; accessed 29/06/09.

1147 THIES, E. (1995), p. 185. 
In Burkina Faso, traditional healers use leaf extracts of Combretum micranthum for the treatment of malaria. ${ }^{1148}$

Several Acacia species are present in Burkina Faso's dry savannas. These include Acacia albida (also referred to as Faidherbia albida), which is found in large parts of the African continent where the annual precipitation is between 300 and more than $1800 \mathrm{~mm}$. It is, however, most common in semiarid regions with 400 to $900 \mathrm{~mm}$ of rain. The trees can subsist even during prolonged drought periods (which may last several years), low temperatures in winter (a monthly mean of $6^{\circ} \mathrm{C}$ is sufficient) and daytime maxima of more than $40^{\circ} \mathrm{C}$ in the summer. Due to their deep roots, the trees do not depend on fertile upper soils. ${ }^{149}$ Acacia albida is sometimes called the "miracle tree" of the Sahel, since it is the most important source of fodder, provides good-quality wood and improves the quality of the soil. The leaves and fruits are used for a multitude of purposes, including the fight of several infectious diseases, and the production of soap. ${ }^{1150}$ Its is therefore protected by local populations, but irregular in its distribution. ${ }^{1151}$ In contrast to most other tree species found in the region, Acacia albida sheds its leaves during the rainy season. ${ }^{1152}$ Acacia seyal also belongs to the Acacia species that are almost ubiquitously found in the dry and thornbush savannas, preferentially on clayey soils. ${ }^{1153}$ The trees

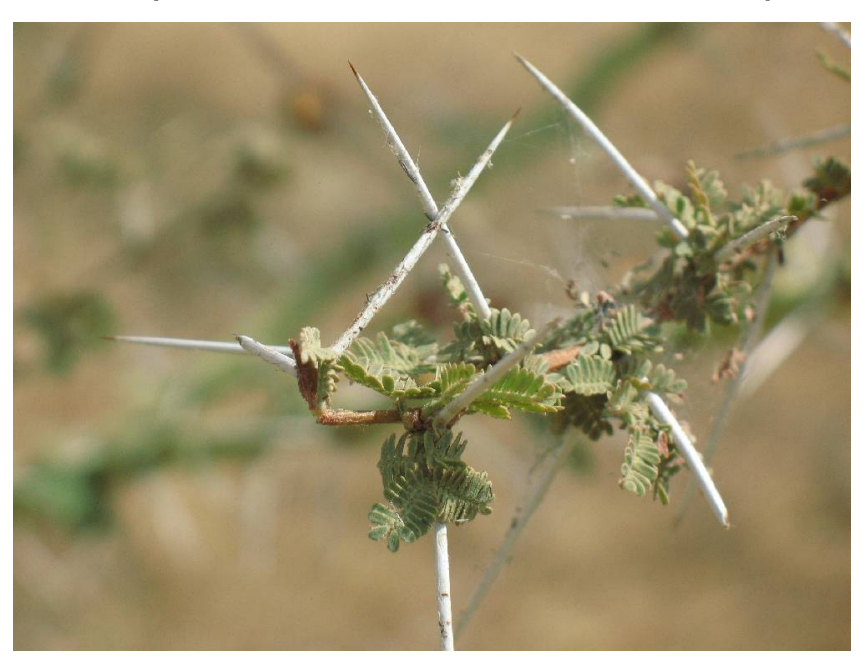

Figure 64: Acacia cf. seyal have -sometimes only very fewpinnate leaves and typically attain a height of 9 to $17 \mathrm{~m}$; most of the roots are just below the ground but some reach a depth of up to $8 \mathrm{~m}$. This, together with a root system of up to $9 \mathrm{~m}$ diameter, allows the plant to thrive in semiarid regions. Acacia seyal is typically found in regions where the annual precipitation is between 500 and $1200 \mathrm{~mm}$ and the annual average temperature between $25^{\circ} \mathrm{C}$ and $30^{\circ} \mathrm{C}$. However, the trees can bear temperatures between $2^{\circ} \mathrm{C}$ and $50^{\circ} \mathrm{C}$ but do not tolerate frost. They can tolerate inundation ${ }^{1154}$, are well-adapted to vertisols and tend to grow in bas fonds and other depressions ${ }^{1155}$. Acacia ataxacantha is found between the Sahel and the rain forest zone ${ }^{1156}$, and a common species in

1148 http://en.sl.life.ku.dk/upload/c micranthum.pdf; accessed 29/06/09.

1149 von MaYdelL, H.J. (1990), p. 91.

1150 von MaYdeLL, H.J. (1990), pp. $92 f$.

1151 Geerling, C. (1982), p. 177.

1152 Boudet, G. \& Lebrun, J.P. (1986), p. 134.

1153 Boudet, G. \& Lebrun, J.P. (1986), p. 139.

1154 Geerling, C. (1982), p. 186.

1155 Schüt, P.; Weisgerber, H.; Schuck, H.J. et al. (2006), pp. 10-13.

1156 Geerling, C. (1982), p. 178. 
the Sahelo-Sudanian zone, particularly in association with Combretum micranthum, in gallery forests ${ }^{1157}$ and at the periphery of dry forests. The heliophilic species is well-adapted to ferralitic crusts and found in regions of $250 \mathrm{~mm}$ to $1700 \mathrm{~mm}$ of rainfall where monthly mean temperatures range between $21^{\circ} \mathrm{C}$ and $36^{\circ} \mathrm{C} .{ }^{1158}$ Acacia dudgeoni is a small bush or tree that is requires at least $800 \mathrm{~mm}$ of rain ${ }^{1159}$ and moderately fertile soils ${ }^{1160}$, whereas the Acacia ehrenbergiana bush is a Sahelo-Saharan species ${ }^{1161}$ that may subsist on as little as $50 \mathrm{~mm}$ on sandy and $150 \mathrm{~mm}$ on clayey soils ${ }^{1162}$ and is typically found between Senegal and Ethiopia. ${ }^{163}$ Other members of the genus Acacia that are found in the Sahelo-Sudanian zone include Acacia gourmaensis (600 to $1250 \mathrm{~mm}$ of rain, preference for humous soils), ${ }^{1164}$ Acacia laeta (Sahelian species $^{1165}, 250 \mathrm{~mm}$ to $750 \mathrm{~mm}$ of rain, drought-resistant ${ }^{1166}$ ), Acacia macrostachya (a relatively common species most commonly found in the Sudanian savanna ${ }^{1167}$ ) Acacia nilotica (a Sahelo-Sudanian species that is usually found on poorly drained soils ${ }^{1168}$ such as inundation zones close to mares and major rivers ${ }^{1169}$ ) and Acacia pennata (found throughout the Sahel and SaheloSudanian zone). Acacia raddiana and Acacia senegal are among the most characteristic trees of the Sahel. They are highly drought-resistant (50 to 100 $\mathrm{mm}$ of rain are sufficient), require well-drained soils and are rarely found in areas with an annual precipitation of more than 800 to $1000 \mathrm{~mm} .{ }^{1170}$ Acacia senegal is the most important source of natural gum in West Africa. ${ }^{1171}$

Balanites aegyptiaca is a small to medium-sized tree which is found in the entire Sahel and adjoining Sahelo-Sudanian zone. ${ }^{1172}$ It is found on very different soils, including sand, clay, rocky and alluvial material. Its oil-rich fruits, often referred to as desert dates (dattier sauvages), are edible and are used to produce soap and insecticide effective against Schistosoma parasites and the vectors of dracunculiasis. ${ }^{1173}$ Balanites aegyptiaca typically grows in areas with an annual precipitation between 400 and $800 \mathrm{~mm}$, but $250 \mathrm{~mm}$ are usually sufficient to produce a bush of $2 \mathrm{~m}$ height. The species tolerates bushfires and degraded soils and is often found on vertisols and sandy alluvial

1157 von MaYdelL, H.J. (1990), p. 95.

1158 THIES, E. (1995), p. 71.

1159 von MAYDELL, H.J. (1990), p. 97.

1160 Geerling, C. (1982), p. 178.

1161 Geerling, C. (1982), p. 179.

1162 von MaYdelL, H.J. (1990), p. 99.

1163 Boudet, G. \& Lebrun, J.P. (1986), p. 136.

1164 von MaYdeLL, H.J. (1990), p. 101.

1165 Geerling, C. (1982), p. 177.

1166 von MAYDELL, H.J. (1990), p. 103.

1167 Geerling, C. (1982), p. 183.

1168 Geerling, C. (1982), p. 186.

1169 Boudet, G. \& Lebrun, J.P. (1986), p. 138.

1170 von MaYdeLL, H.J. (1990), pp. $121 \& 125$.

1171 Boudet, G. \& Lebrun, J.P. (1986), p. 138.

1172 Geerling, C. (1982), p. 77.

1173 von Maydell, H.J. (1990), p. 165. 
soils, not seldom tens of meters apart from the next bush or tree. ${ }^{1174}$ In the dry regions of tropical Africa, it is commonly found on relatively moist soils. ${ }^{1175}$ The natural distribution of the plant is obscured by cultivation. After the seedling stage, Balanites aegyptiaca is intolerant to shade and prefers open woodland or savanna for natural regeneration. ${ }^{1176}$

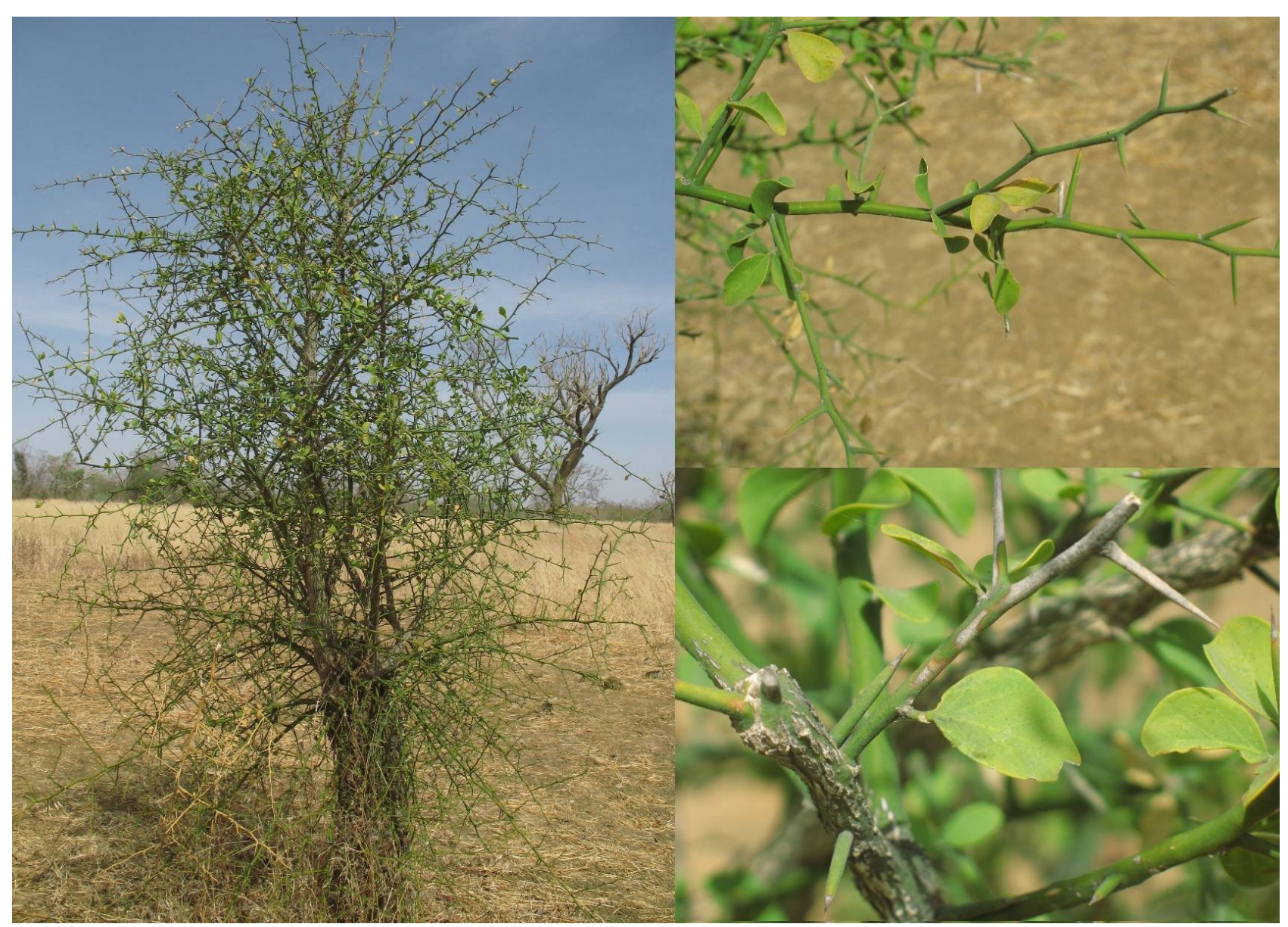

Figure 65: Balanites aegyptiaca

The African locust bean tree Parkia biglobosa, a large tree that typically grows to a height of 15 to $20 \mathrm{~m}$, is frequently found dry forests of the SaheloSudanian zone but also occurs in the transition zone towards the Sahel as $500 \mathrm{~mm}$ of annual rainfall are sufficient for its growth. ${ }^{1177}$ The species is most common in the Sudanian and Guinean savannas ${ }^{1178}$ and grows under different edaphic conditions ${ }^{1179}$ but in the Sahelo-Sudanian zone, it is usually an indicator for deep sandy ${ }^{1180}$ or loamy soils. The tree occurs in regions where

1174 Schütr, P.; Weisgerber, H.; Schuck, H.J. et al. (2006), p. 115.

1175 BOUdET, G. \& LeBrun, J.P. (1986), p. 103.

1176 http://en.sl.life.ku.dk/upload/124net.pdf ; accessed 29/06/09.

1177 von MaydelL, H.J. (1990), p. 313.

1178 Geerling, C. (1982), p. 198.

1179 THIES, E. (1995), p. 288.

1180 Boudet, G. \& LeBrun, J.P. (1986), p. 131. 
monthly mean temperatures range between $17^{\circ} \mathrm{C}$ and $36^{\circ} \mathrm{C}^{1181}$ and is well adapted to strongly seasonal climates where the dry season lasts 4 to 8 months and the annual precipitation is no more than $1400 \mathrm{~mm}$. The trees may be severely damaged by bush fires. ${ }^{1182}$ Its fruits are edible and when fermented form the base for a local beverage and a vegetable cheese ("soumbara"). Due to the high protein content and their value in traditional medicine, the fruits of the tree are locally traded. ${ }^{1183}$ Therefore, the species is protected by local communities. ${ }^{1184}$

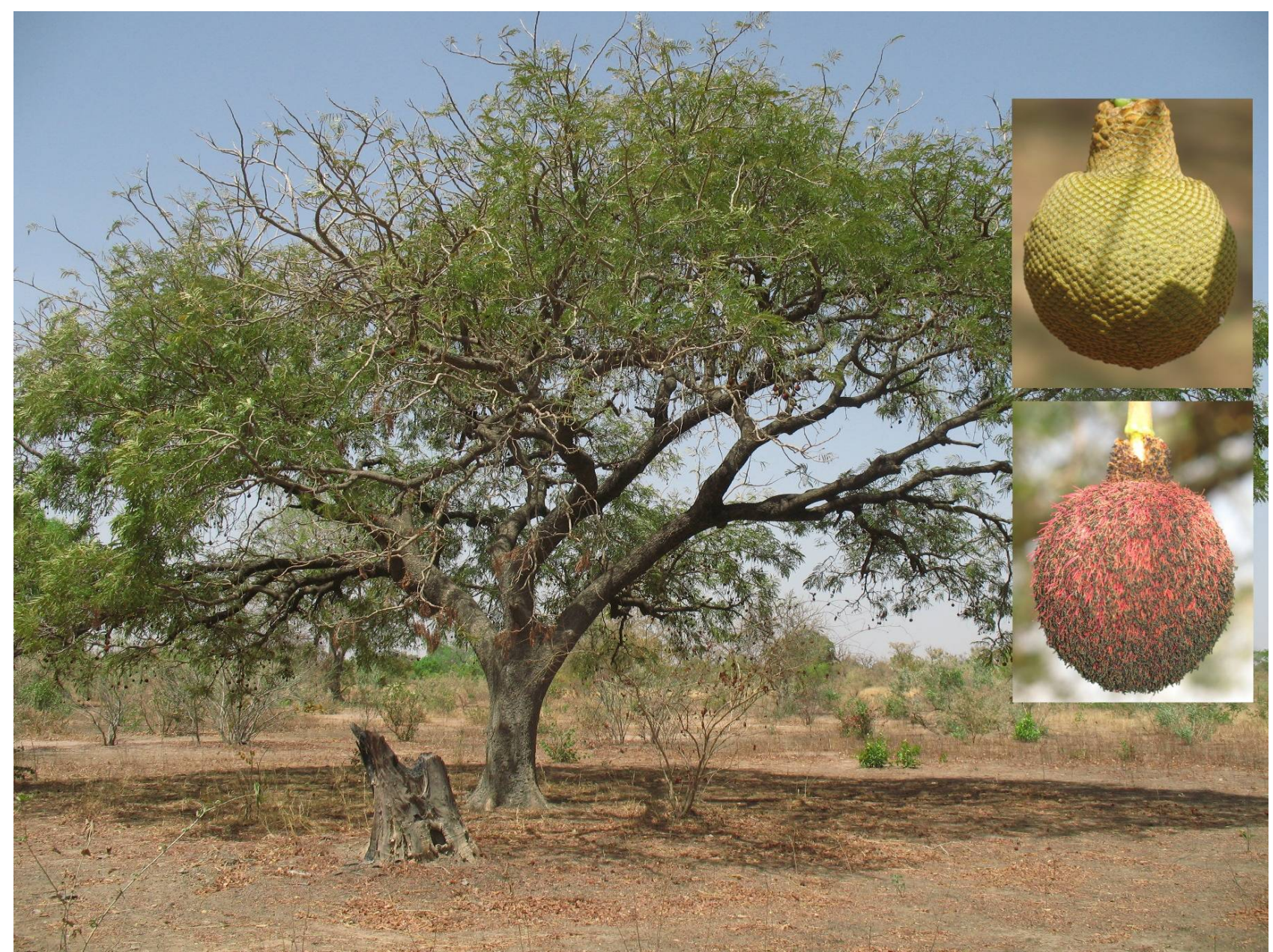

Figure 66: Parkia biglobosa

1181 Thies, E. (1995), p. 288.

1182 http://en.sl.life.ku.dk/upload/124net.pdf; accessed 29/06/09.

1183 von MaYdelL, H.J. (1990), p. 313.

1184 Geerling, C. (1982), p. 198. 


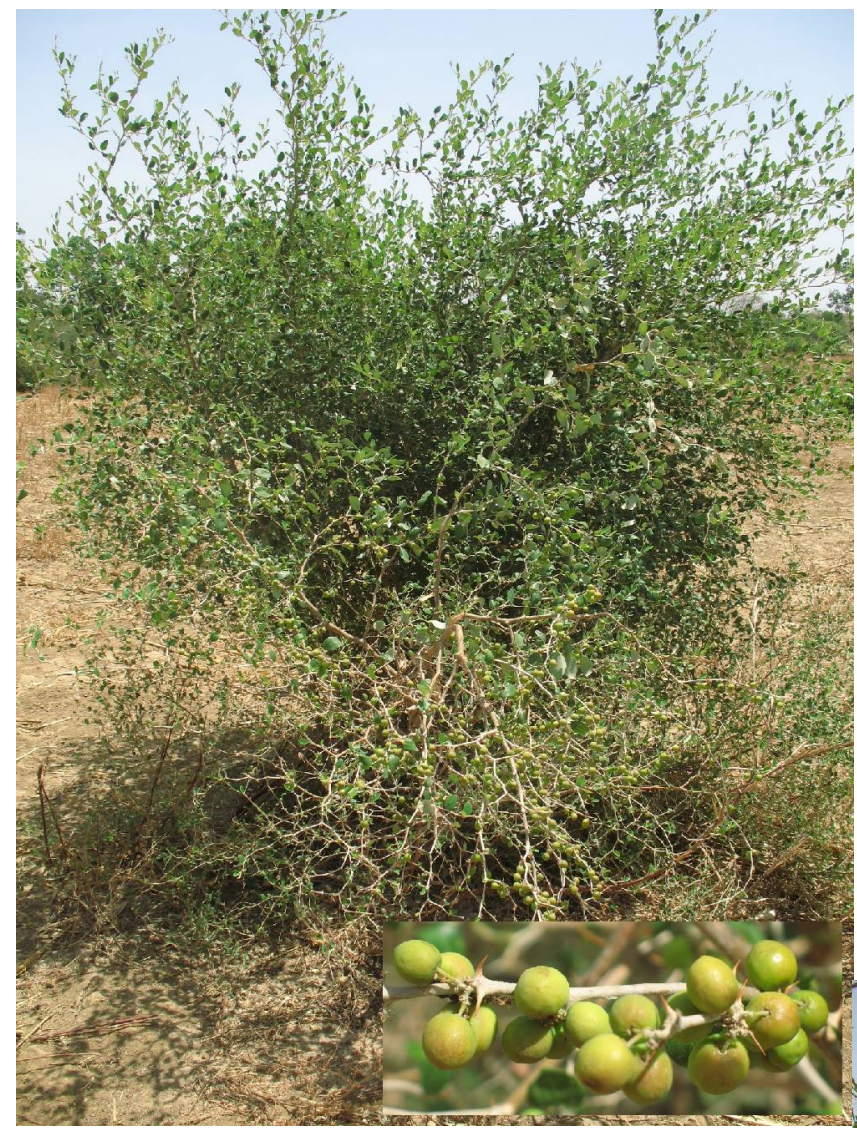

Figure 67: Ziziphus mauritania

Vitellaria paradoxa, also named Butyrospermum parkii, and locally often referred to by its French name, "karité", is a tree of 10 to $15 \mathrm{~m}$ (and sometimes up to $25 \mathrm{~m}$ ) height that is common in the savannas between the southern Sahel and the Sudanian zone, where rainfall is between 600 $\mathrm{mm}$ and $1500 \mathrm{~mm}$. It prefers sandy and dry clayey soils with a wellestablished layer of humus but is also found on gravel and lateritic subsoil. As the trees cannot endure prolonged inundation, they are not found in swampy regions and close to water bodies. The plants both occur as isolated specimens and as
Ziziphus mauritania is, with its well-developed root system, found all over the semiarid regions of Africa. The plant survives intensive heat (more than $50^{\circ} \mathrm{C}$ ), and despite a preference for well-drained soils ${ }^{1185}$, it tolerates both drought and inundation, but no frost and humid air. It is typically found in regions with an annual precipitation between 150 and $500 \mathrm{~mm}$. The fruits of Ziziphus mauritania are eaten fresh or after drying and the leaves are used as vegetables in couscous. The multipurpose trees also provide fodder, fuel and timber. ${ }^{1186}$

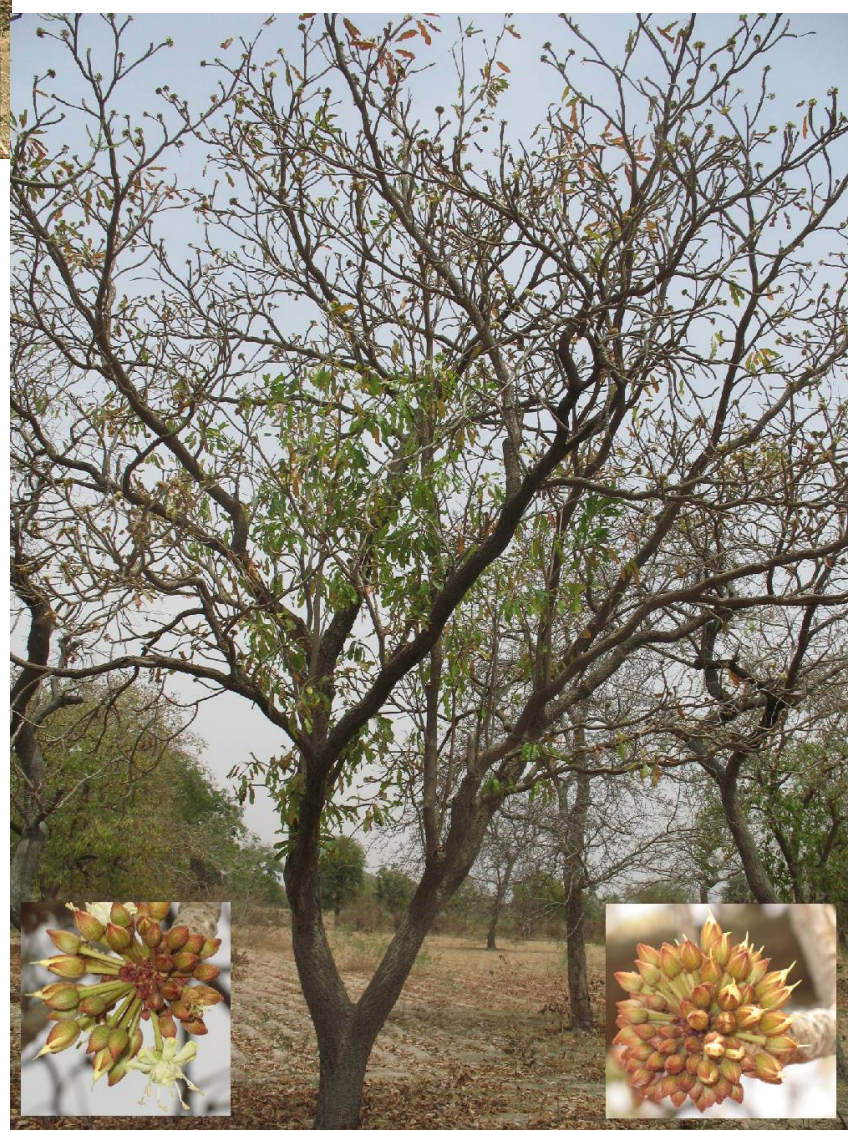

Figure 68: Vitellaria paradoxa 
large, closed populations. ${ }^{1187}$ Vitellaria paradoxa tolerates bush fires which may kill of competing species. ${ }^{1188}$ The most important product of Vitellaria paradoxa is shea butter (karité), which plays an economically important role over much of the region where the species is distributed. ${ }^{1189}$ Shea butter is one of the most affordable and widely used vegetable fats in the Sahel and particularly appreciated in regions with less than $1000 \mathrm{~mm}$ of annual rain where oil palms cannot be grown. Since the trees also play an important role in soil conservation and water storage, they are traditionally favored and protected by local populations ${ }^{1190}$ and may even be cultivated in large numbers. ${ }^{1191}$ Because of anthropogenic selection, Vitellaria paradoxa makes up more than $80 \%$ of the woody vegetation in some parts of Burkina Faso. ${ }^{192}$

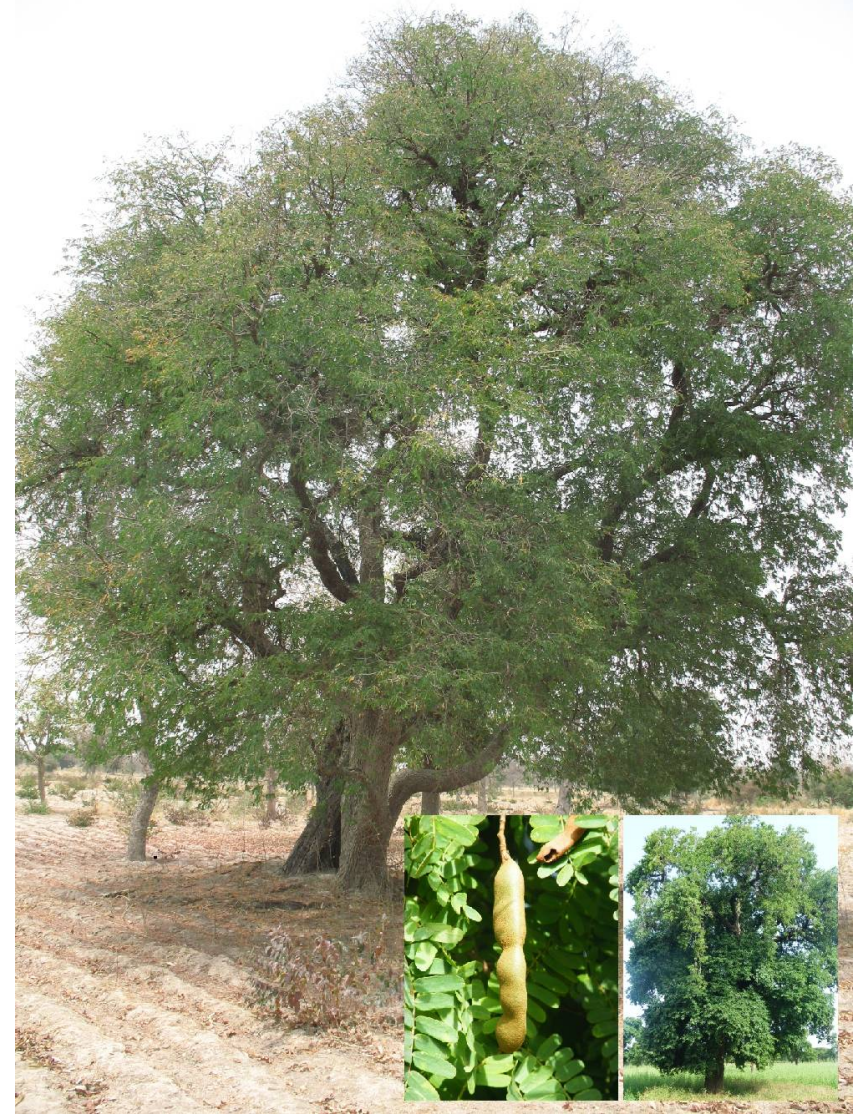

Figure 69: Tamarindus indica
Despite its botanic name, the large tamarind tree (Tamarindus indica) which often attains heights of $30 \mathrm{~m}$ and more originates from tropical Africa. ${ }^{193}$ The species has become pantropical because of anthropogenic dissemination ${ }^{1194}$ and performs well in both semi-arid and humid monsoon climates and are found between the semiarid belts of tropical Africa in regions with an annual precipitation of at least 400 $\mathrm{mm}$. A marked dry season is vital for the development of its fruits. The species neither occurs in swampy areas nor in regions with rocky subsoil which prevents a deep penetration by the plant's roots but prefers profound and permeable soils. ${ }^{1195}$ On well-drained soils, up to $1900 \mathrm{~mm}$ of annual precipitation are tolerated, as are temperatures between $2^{\circ} \mathrm{C}$ and $47^{\circ} \mathrm{C}$. However, the

1187 von Maydell, H.J. (1990), p. 184.

1188 THIES, E. (1995), p. 364.

1189 von MAYdeLL, H.J. (1990), p. 185.

1190 http://en.sl.life.ku.dk/upload/vitellaria paradoxa int.pdf; accessed 29/06/09.

1191 Geerling, C. (1982), p. 310.

1192 Maranz, S. \& Wiesmann, Z. (2003), p. 1506.

1193 Schüt, P.; Weisgerber, H.; SChuck, H.J. et al. (2006), p. 624.

1194 GeERLing, C. (1982), p. 177.

1195 von MaYdeLL, H.J. (1990), p. 185 and

http://en.sl.life.ku.dk/upload/tamarindus indica int.pdf; accessed 29/06/09. 
plant is highly sensitive to frost. Tamarindus indica frequently occurs around settlements and on agricultural fallows ${ }^{1196}$ where it is cultivated by villagers ${ }^{1197}$ but is also found in open savannas (sometimes co-occurring with Adansonia digitata) and gallery forests. ${ }^{1198}$

The mango tree Mangifera indica originates from tropical eastern Asia, but its many varieties have been introduced to tropical regions all over the world. ${ }^{1199}$ Mangifera indica is the most widely distributed fruit tree in the Sahel. The tree only requires modestly fertile soils but does not cope well with frequent or prolonged inundation. It prefers deep and well-drained soils ${ }^{1200}$, and soils rich in humus, clay and iron produce particularly large trees. Mangifera indica does not tolerate frost and maximum temperatures that go far beyond $40^{\circ} \mathrm{C}$. An annual precipitation of 700 to $2000 \mathrm{~mm}$ is required for a good growth of fruits, of which several thousands can be produced by a single tree each year. ${ }^{1201}$

1196 SchütT, P.; Weisgerber, H.; Schuck, H.J. et al. (2006), p. 626 and http://en.sl.life.ku.dk/upload/tamarindus indica int.pdf; accessed 29/06/09.

1197 BOUdET, G. \& LeBRUn, J.P. (1986), p. 129.

1198 THIES, E. (1995), p. 336.

1199 BOUDET, G. \& LeBRUn, J.P. (1986), p. 205.

1200 von MaYdell, H.J. (1990), p. 185.

1201 Schütr, P.; Weisgerber, H.; Schuck, H.J. et al. (2006), p. 424. 


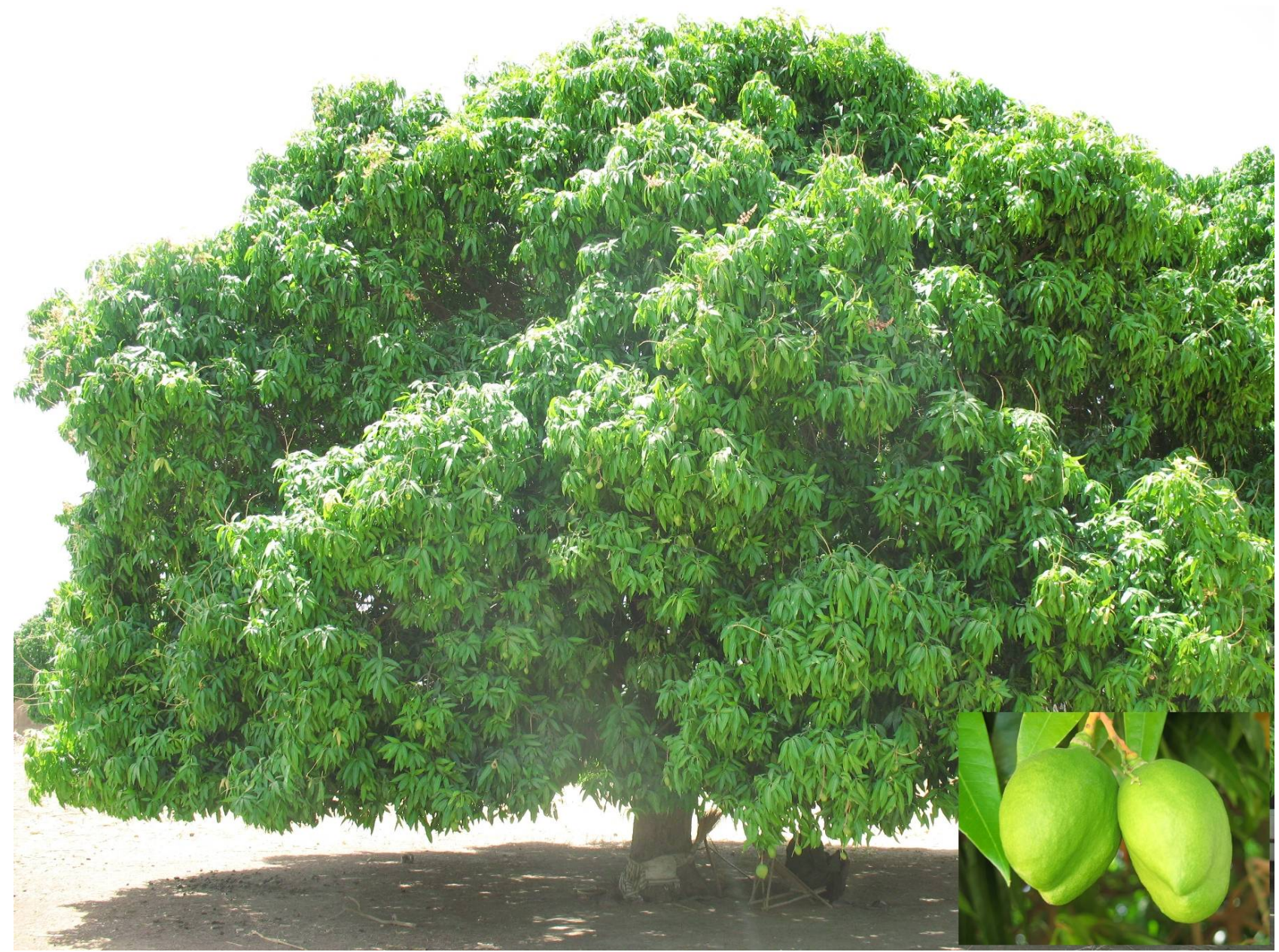

Figure 70: Mangifera indica 
The baobab (Adansonia digitata) is most common in the Sudanian savanna zone ${ }^{1202}$ but also one of the most characteristic trees of the dry savannas south of the Sahara. The tree is most common at altitudes between $450 \mathrm{~m}$ and 600 $\mathrm{m}$, but it is present at elevations between sea level and $1500 \mathrm{~m}$. The species occurs between the Sahel in the north to the Transvaal in the south, but is completely absent in the tropical rain forests. In West Africa, Adansonia digitata is often found in association with Tamarindus indica, Balanites aegyptiaca and Vitellaria paradoxa. ${ }^{1203}$ Since local populations often protect baobab trees, they are particularly frequent close to recent human habitats ${ }^{1204}$ or previously settled land. ${ }^{1205}$ The trees are often left standing when land is cleared for cultivation ${ }^{1206}$ (see figure 71 ).

The baobab can be best recognized by the shape of its enormous trunk and its branches. It reaches a height of up to $30 \mathrm{~m}$, and the trunks typically have a diameter of $3 \mathrm{~m}$ to $6 \mathrm{~m} .{ }^{1207}$ The water stored in its trunk is not only used by local populations but also by animals. ${ }^{1208}$ Both the leaves and fruits of the trees are eaten locally, sometimes as a natural medication against fever, malaria and filariasis. ${ }^{1209}$

Adansonia digitata requires only $90 \mathrm{~mm}$ of precipitation a year and grows on poor soils. It prefers welldeveloped calcareous and sandy soils but does not tolerate inundation on clayey soils. The tree is most common in the semi-arid regions south of the Sahara, at an annual precipitation ranging between 300 $\mathrm{mm}$ and $500 \mathrm{~mm}$, but tolerates up to $1400 \mathrm{~mm}$ on well-drained soils. In

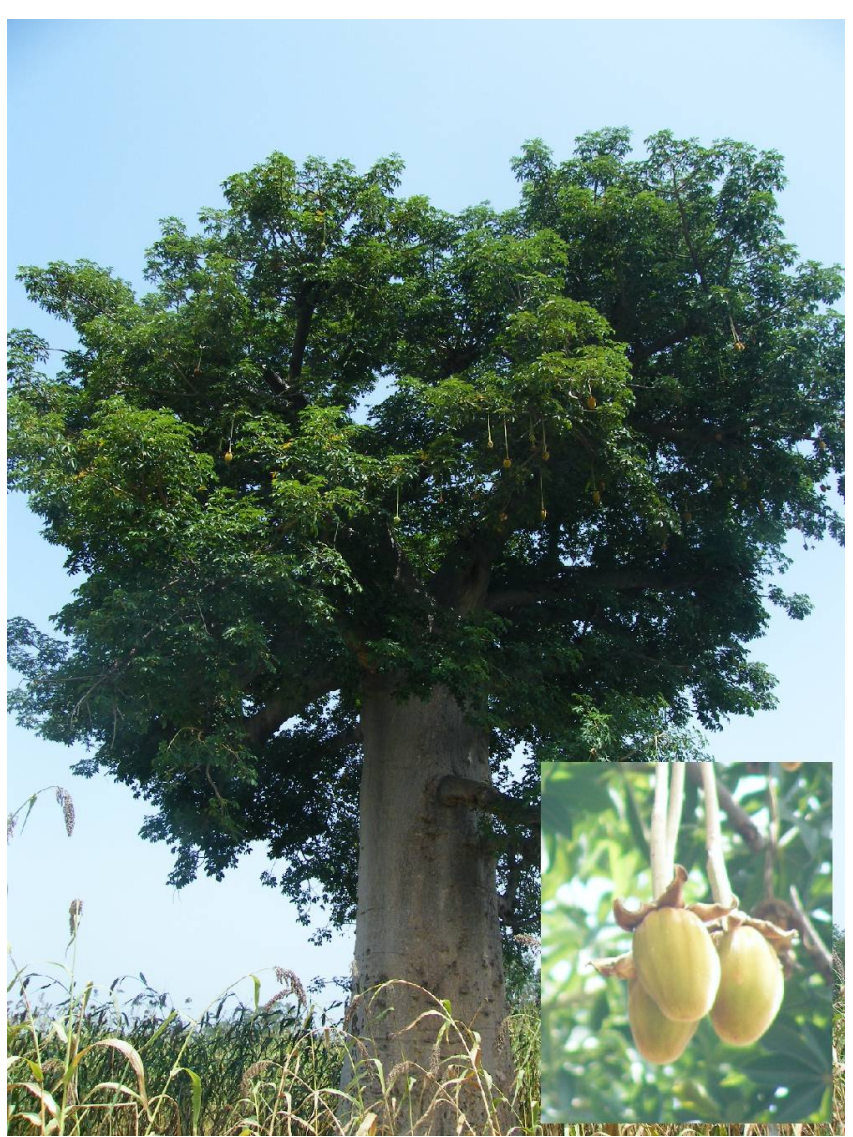

Figure 71: Adansonia digitata the areas of its distribution, the rainy season ranges from 6 weeks to 5 months and temperatures are typically 1202 Geerling, C. (1982), p. 81.

1203 Schütr, P.; Weisgerber, H.; Schuck, H.J. et al. (2006), p. 40.

1204 Boudet, G. \& LeBRun, J.P. (1986), p. 91.

1205 THIEs, E. (1995), p. 82.

1206 http://en.sl.life.ku.dk/upload/adansonia_109.pdf; accessed 29/06/09.

1207 von MaydelL, H.J. (1990), p. 135.

1208 Schüt, P.; Weisgerber, H.; Schuck, H.J. et al. (2006), p. 40.

1209 von Maydell, H.J. (1990), p. 137. 
between $18^{\circ} \mathrm{C}$ and $40^{\circ} \mathrm{C}$ to $46^{\circ} \mathrm{C}$. The plant does not survive frost. ${ }^{1210}$ Adansonia digitata flowers just before the onset of the rainy season. ${ }^{1211}$ Bush burning in the dry season, grazing and seed diseases limit the number of trees. The baobab population is declining and there is very low regeneration in its natural environment probably because of poor seed germination in some places and livestock, which readily eats the young trees. ${ }^{1212}$

Trees and bushes that are used for the production of natural insecticides and used as natural remedies against malaria are summarized in table 50 . While the pharmaceutical value of some species has been recognized by Western medicine, not much is known about many others.

\begin{tabular}{|l|l|l|}
\hline Species & Parts used & Use \\
\hline $\begin{array}{l}\text { Combretum glutinosum } \\
\text { Combretum micranthum }\end{array}$ & Young leaves & $\begin{array}{l}\text { Traditional antimalarial } \\
\text { and antipyretic }\end{array}$ \\
\hline Adansonia digitata & Leaves, fruits & Traditional antimalarial \\
\hline Azadirachta indica & Leaves, bark, fruits & Natural insecticide \\
\hline Acacia albida & Leaves, bark, fruits & Traditional antipyretic \\
\hline
\end{tabular}

Table 50: Tree and bush species of antimalarial relevance

An overview of the ecological conditions indicated by important tree and shrub species found in Kossi Province is presented in table 51. While the presence of one individual species may not be a reliable indicator, combined presence/absence pattern can be good clues for eco-epidemiological conditions.

1210 Schütr, P.; Weisgerber, H.; Schuck, H.J. et al. (2006), p. 44;

BOUdeT, G. \& Lebrun, J.P. (1986), p. 91.

1211 von MAYdelL, H.J. (1990), p. 135.

1212 http://en.sl.life.ku.dk/upload/adansonia 109.pdf; accessed 29/06/09. 


\begin{tabular}{|l|l|l|}
$\begin{array}{l}\text { Eco- } \\
\text { epidemiological } \\
\text { situation }\end{array}$ & $\begin{array}{l}\text { Conditions favorable for } \\
\text { malaria transmission }\end{array}$ & $\begin{array}{l}\text { Conditions unfavorable } \\
\text { for malaria transmission }\end{array}$ \\
\hline Microclimate & $\begin{array}{l}\text { Species requiring } \geq 750 \mathrm{~mm} \text { of } \\
\text { rainfall }(\dagger) \text { and/or intolerant of } \\
\text { massive heat }\left(>45^{\circ} \mathrm{C} ; \neq\right) \\
\text { Lannea microcarpa }(\dagger), \\
\text { Vitellaria paradoxa }(\dagger), \\
\text { Mangifera indica }(\dagger, \neq), \\
\text { Combretum micranthum }(\dagger)\end{array}$ & $\begin{array}{l}\text { Species requiring } \leq 500 \mathrm{~mm} \text { of } \\
\text { rainfall }(*) \text { and/or tolerating } \\
\text { massive heat }\left(>45^{\circ} \mathrm{C}, \#\right)\end{array}$ \\
$\begin{array}{l}\text { Azadirachta indica }(*), \\
\text { Balanites aegyptiaca }(*), \\
\text { Ziziphus mauritania }(*, \#), \\
\text { Combretum glutinosum }(*)\end{array}$ \\
conditions & $\begin{array}{l}\text { Species tolerating indundation } \\
\text { and preferring moist soils } \\
\text { Ziziphus mauritania }\end{array}$ & $\begin{array}{l}\text { Species found on sandy or well- } \\
\text { drained soils } \\
\text { Tamarindus indica, } \\
\text { Mangifera indica, } \\
\text { Combretum glutinosum } \\
\text { Parkia biglobosa, } \\
\text { Vitellaria paradoxa, } \\
\text { Combretum glutinosum }\end{array}$ \\
\hline
\end{tabular}

Table 51: Trees as eco-epidemiological indicators ${ }^{1213}$

1213 Species indicating partly favorable and partly unfavorable conditions have been omitted. 
Other tree species found in the Kossi savanna include Sclerocarya birrea which is often found on well-drained sandy soils and even on lateritic crusts ${ }^{1214}$ and rocky hills. The species occurs at low to medium altitudes in areas with 200 to $1600 \mathrm{~mm}$ rain per year. ${ }^{1215}$ Guiera senegalensis and Piliostigma thonningii are frequently found on cultivated fields. ${ }^{1216}$ Guiera senegalensis is a bush achieving a height of up to $3 \mathrm{~m}$ that often occurs on dry, sandy soils and is often an indicator of overgrazing. ${ }^{1217}$ Piliostigma thonningii occurs between the southern Sahel and the humid savannas. It does not tolerate drought and normally requires at least $700 \mathrm{~mm}$ of rainfall. ${ }^{1218}$

In the dry savannas of Burkina Faso, gramineae (also referred to as poaceae or 'true grasses') cover a greater part of the ground than any other group of plants. KAPPAS (2006) reported that gramineae make up $62 \%$ of the leaf area index in the country's savannas ${ }^{1219}$, but this proportion certainly varies widely. The grass layer is dominated by fine-leaved annual grasses, including Schoenefeldia gracilis, Dactyloctenium aegypticum, Aristida mutabilis, Cenchrus biflorus ${ }^{1220}$ and Panicum laetum. ${ }^{1221}$

\subsubsection{Vegetation Dynamics}

Fire is a major ecological factor in the Sahelo-Sudanian zone of West Africa. It eliminates the fire-sensitive species from the savanna and favors the herb layer - usually a continuous and non-sclerophylous layer of grass. ${ }^{1222}$ Savanna vegetation is for the greater part a fire climax. Fire destroys young woody plants and the litter which suffocates the grasses. The grass fires are today practically all lit by man; natural fires are rare. ${ }^{1223}$ AnhuF and FrankenberG consider West Africa's moist and dry savanna regions as secondary formations which have evolved under human influence, with natural savannas occurring only north of the $500 \mathrm{~mm}$ isohhyet. ${ }^{1224}$ Fire-sensitive species are limited to moist habitats such as swamps and gallery forests. ${ }^{1225}$

1214 von Maydell, H.J. (1990), p. 345.

1215 http://en.sl.life.ku.dk/upload/sclerocarya_birrea_int.pdf; accessed 10/07/09.

1216 Reij, C.; Tappan, G. \& Belemvire, A. (2005), p. 653.

1217 von MAYDELL, H.J. (1990), p. 279.

1218 von MAYDeLL, H.J. (1990), p. 327.

1219 KaPPAS, M. (2006), p. 112.

1220 Stisen, S.; Sandholt, I.; Nørgaard, A. et al. (2007), p. 264.

1221 Bethemont, J.; Faggi, P.; Zoungrana, T.P. (2003), p. 17.

1222 Geerling, C. (1985), pp. $247 \mathrm{f}$.

1223 Geerling, C. (1985), p. 249.

1224 AnhuF, D. \& Frankenberg, P. (1991), p. 244.

1225 Geerling, C. (1985), p. 247. 


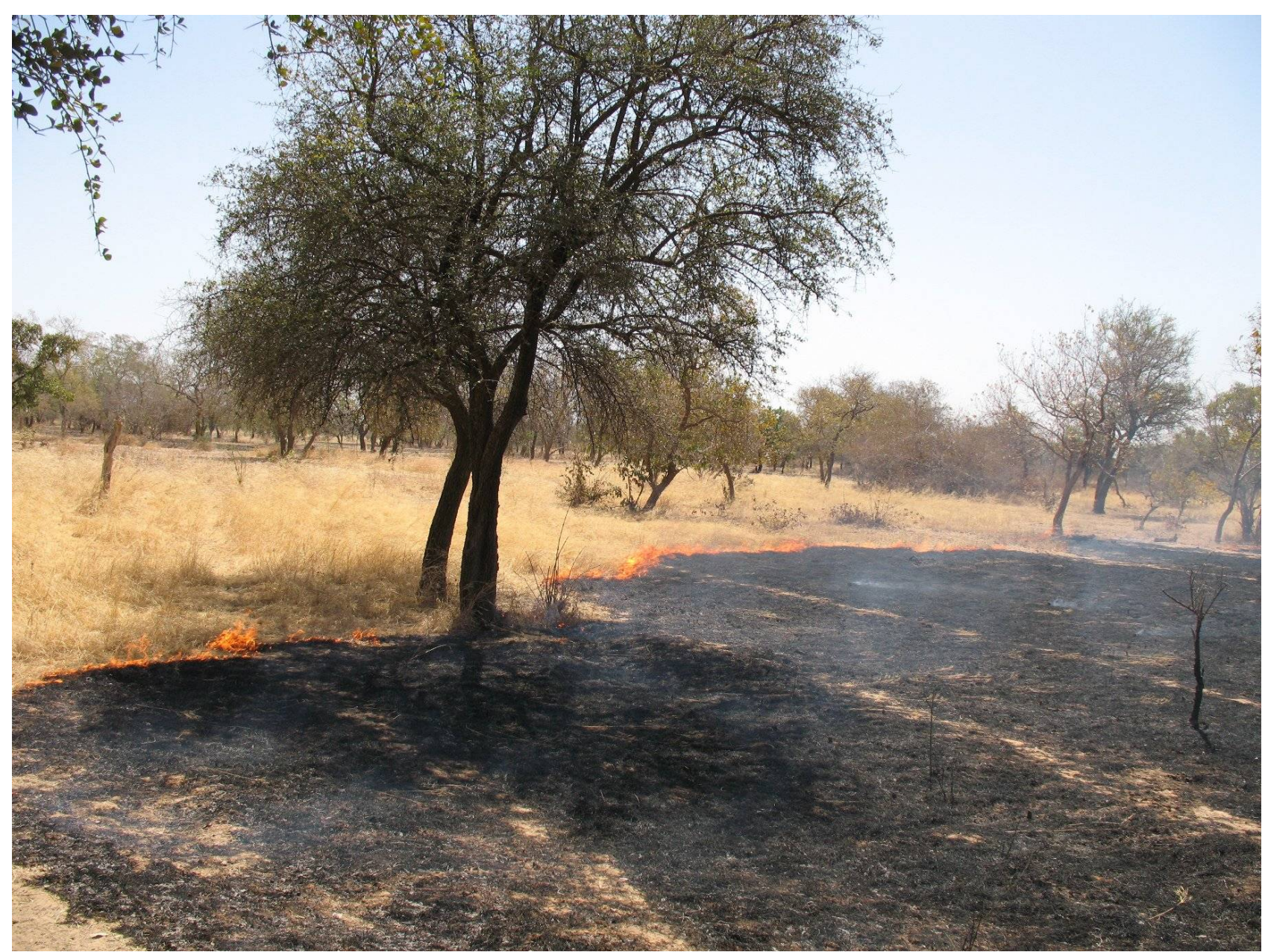

Figure 72: Bush fire in Kossi

In the past, climatic variability caused several shifts in vegetation. During the Sahelian drought years, the northern limits of distribution of species like Acacia senegal have moved south for about $200 \mathrm{~km}$, returning to the limits where they were found in the 1930s. During the more humid period between the 1930 s and the 1960s, these species had become established further north. ${ }^{1226}$ During the extended drought from 1969 to 1985, many pseudo-equatorial species died off in the dry forests (forêts sèches), often being replaced by Combretaceae. ${ }^{1227}$ Moreover, there has been an influx of Sahelian species into the moister regions south of the Sahel in recent decades. ${ }^{1228}$

Overgrazing and cutting for firewood have led to the disappearance of natural vegetation over large areas ${ }^{1229}$ : 
«The present exploitation of the Sudan and Sahel zones is in no way stable or sustainable. [... This] has led to a situation where few complete and intact ecosystems still exist, let alone have a future. $\gg^{1230}$

Due to population growth, firewood harvesting has increased by around 30 per cent since 1990, resulting in the depletion of forest resources near population centers. ${ }^{1231}$

Once the natural savanna vegetation has been destroyed by human impact, the cycles of regeneration are very long since a destruction of the vegetation often negatively impacts soil moisture and fertility; the risk of soil erosion increases and the ground water table may be lowered. ${ }^{1232}$

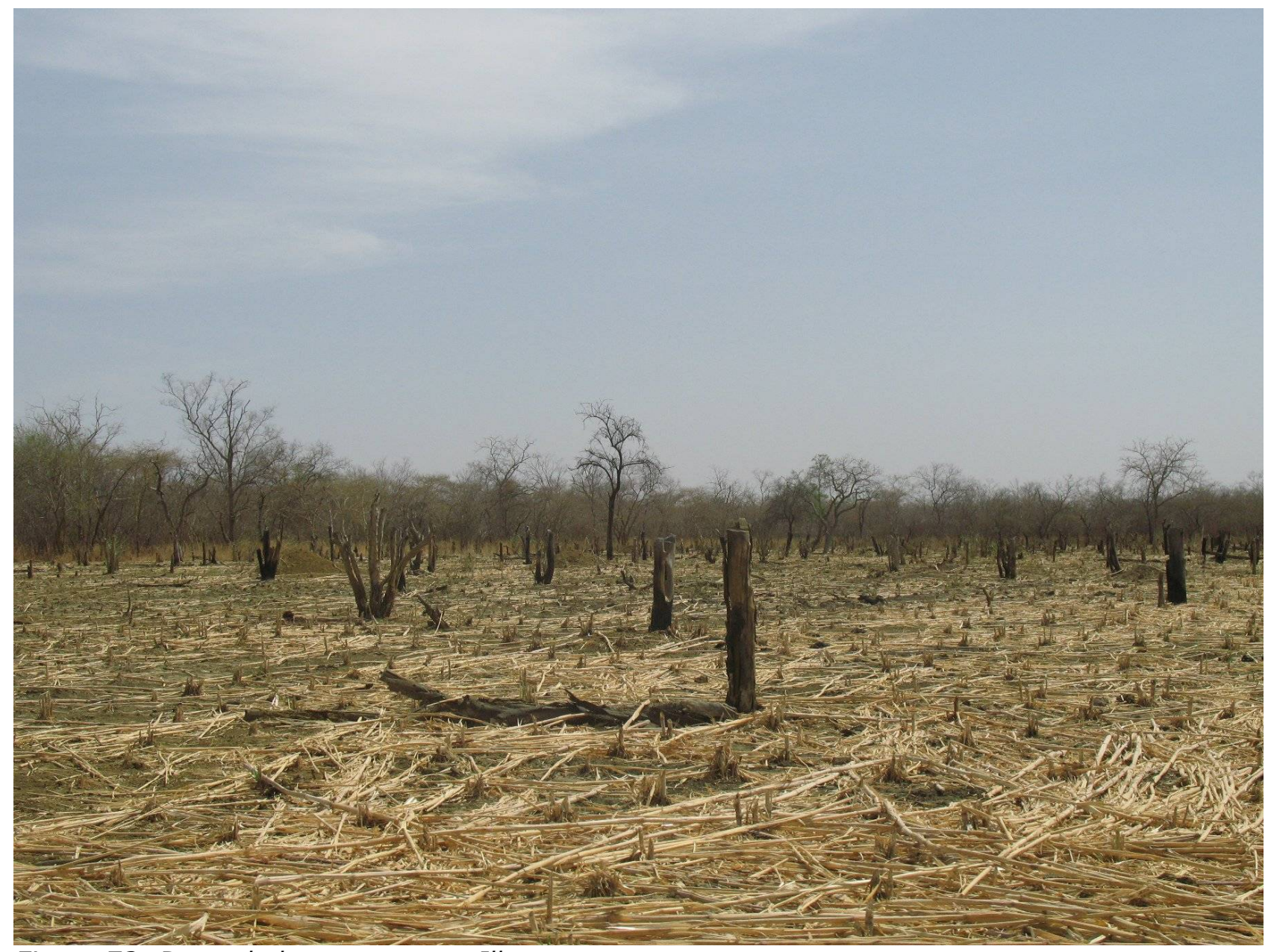

Figure 73: Degraded savanna near Illa

1230 Geerling, C. (1985), p. 254.

1231 United Nations Environment Programme (2008), p. 99.

1232 HaMmer, T. (2005), p. 26. 
In the recent past, a strong trend of increasing vegetation cover in the Sahel has been observed, but appears to be only partly related to increased rainfall. ${ }^{1233}$

\subsection{Sociogeographic Environment and Public Health}

Burkina Faso is a developing country that currently ranks among the world's least developed nations. A weak economy goes hand in hand with poor records in the field of public health.

While Burkina Faso's population has just exceeded 15 millions, the country's population growth rate is high even by West African standards. Burkina Faso's population is a largely agrarian society, and despite a relatively constant economic growth in recent years, Burkina Faso remains one of the poorest nations in West Africa. The nation's state of development is also characterized by low levels of literacy and education and relatively poor but improving health records. Consequently, Burkina Faso ranks in the $176^{\text {th }}$ position out of 177 countries in the UNDP's 2007/2008 Human Development Index (HDI). ${ }^{1234}$

\subsubsection{Population}

Burkina Faso's largely rural population is multi-ethnic and one of the fastest growing in the world. Kossi Province is home to around 300.000 people or $2 \%$ of Burkina Faso's national population and one of the more sparsely settled region's of the country.

\subsubsection{Settlement History and Ethnic Groups}

The drying of the Sahara about 3000 to 4000 years ago led to a migration into riverine valleys and lake districts of Sub-Saharan Africa. This caused a concentration of the population on the fringes of the expanding desert and semi-desert. ${ }^{1235}$ In the past 1000 years, the Sahel saw the rise and decline of several great empires, including Mossi kingdoms of present-day Burkina Faso. The fate of these empires was closely linked to geo-ecological changes: drought periods and large-scale epidemics often left their mark in local population dynamics and political power. ${ }^{1236}$

1233 OLSSON, L.; EKLUNDH, L. \& ARdö, J. (2005), p. 559.

1234 OeCD \& African Development Bank (2008), p. 177.

1235 HAMMER, T. (2005), p. 11.

1236 HAMMER, T. (2005), p. 15. 
Historically, the terms Sahel and Sudan had an entirely different meaning from their present (exogenous) perception, even though they are still embedded in endogenous views: locally, the Sahel region is usually not seen as a hostile environment. ${ }^{1237}$ Historically, Sudan (Arabic: bilad-es-sudan, referred to the "Land of the Blacks" and encompassed in the view of Arabian geographers the region we now call Sahel. The term Sahel (arabic: essahil, سـاحل), on the other hand, referred to the border or "shore" of the Sahara and originates from the era of trans-Saharan trade when caravans of merchants and nomads were relieved to cross the southern limit of the desert. ${ }^{1238}$

Burkina Faso's population is made up of roughly 60 different ethnic groups, speaking several different regional languages. ${ }^{1239}$ The most important groups include the Mossi, the Peulh, the Bobo and the Lobi:

\begin{tabular}{|l|l|l|}
\hline Ethnic group & $\begin{array}{l}\text { Percentage } \\
\text { of national } \\
\text { population }\end{array}$ & Regional concentration \\
\hline Mossi & $48 \%$ & Central Burkina Faso \\
\hline Peulh & $10 \%$ & Sahel, western Burkina Faso \\
\hline Bobo & $7 \%$ & Western Burkina Faso \\
\hline Lobi & $7 \%$ & Southern Burkina Faso \\
\hline
\end{tabular}

Table 52: Important ethnic groups in Burkina Faso

The population of Kossi province is essentially a mixture of Dafing/Marka (who dominate in the eastern part), Bobo (dominating the western part) and Peulh (dominating the northern part of the province). In the Nouna area, the biggest groups are the Dafing with a population share of about $49 \%$, followed by the Bwaba (20\%), the Mossi (13\%), the Peulh (9\%) and the Samo (8\%). ${ }^{1240}$ However, most villages are multi-ethnic, as illustrated by the following examples:

\begin{tabular}{|l|l|l|}
\hline Village & Majority group & Minority groups \\
\hline Toni & Mossi & $\begin{array}{l}\text { Samo, Peulh, Bobo, } \\
\text { Bwaba }\end{array}$ \\
\hline Illa & Dafing & Samo \\
\hline
\end{tabular}

Table 53: Ethnic groups in the study villages ${ }^{1241}$

1237 HaMmer, T. (2005), p. 17.

1238 HaMmer, T. (2005), pp. $11 ; 18$.

1239 Berié, E. \& Kobert, H. (2005), p. 96.

1240 WürthWEIN, R. (2002), p. 140.

1241 Survey in February 2008, carried out together with Issouf Traoré. 
The Mossi are believed to have originated in the area around Lake Chad. In Burkina Faso, they founded important kingdoms like Oubritenga (present-day Ouagadougou) and Yatenga (Ouahigouya) and still have their own head, the "Moro Naba", who resides in Ouagadougou and today only has a representative function. Many Mossi have resisted the conversion to Islam, and traditionally live as cultivators. ${ }^{1242}$ The Peulh (also referred to as Fulani or Fulbe), who are traditionally cattle herders, probably originated from East Africa and Arabia and settled in West Africa more than one thousand years ago. Some groups became sedentary and converted to Islam, while others remained nomadic and preserved their traditional philosophy. ${ }^{1243}$ The Bobo and Bwaba dwelled in present-day Burkina Faso since at least the $16^{\text {th }}$ century (there are no reliable sources for the period before), and were joined by Lobi who came from the west of the Mouhoun. The Samo and Dafing (or Marka) are subgroups of the Mandé who originated in the upper Niger valley and colonized the margins of Mossi-dominated areas. ${ }^{1244}$ 


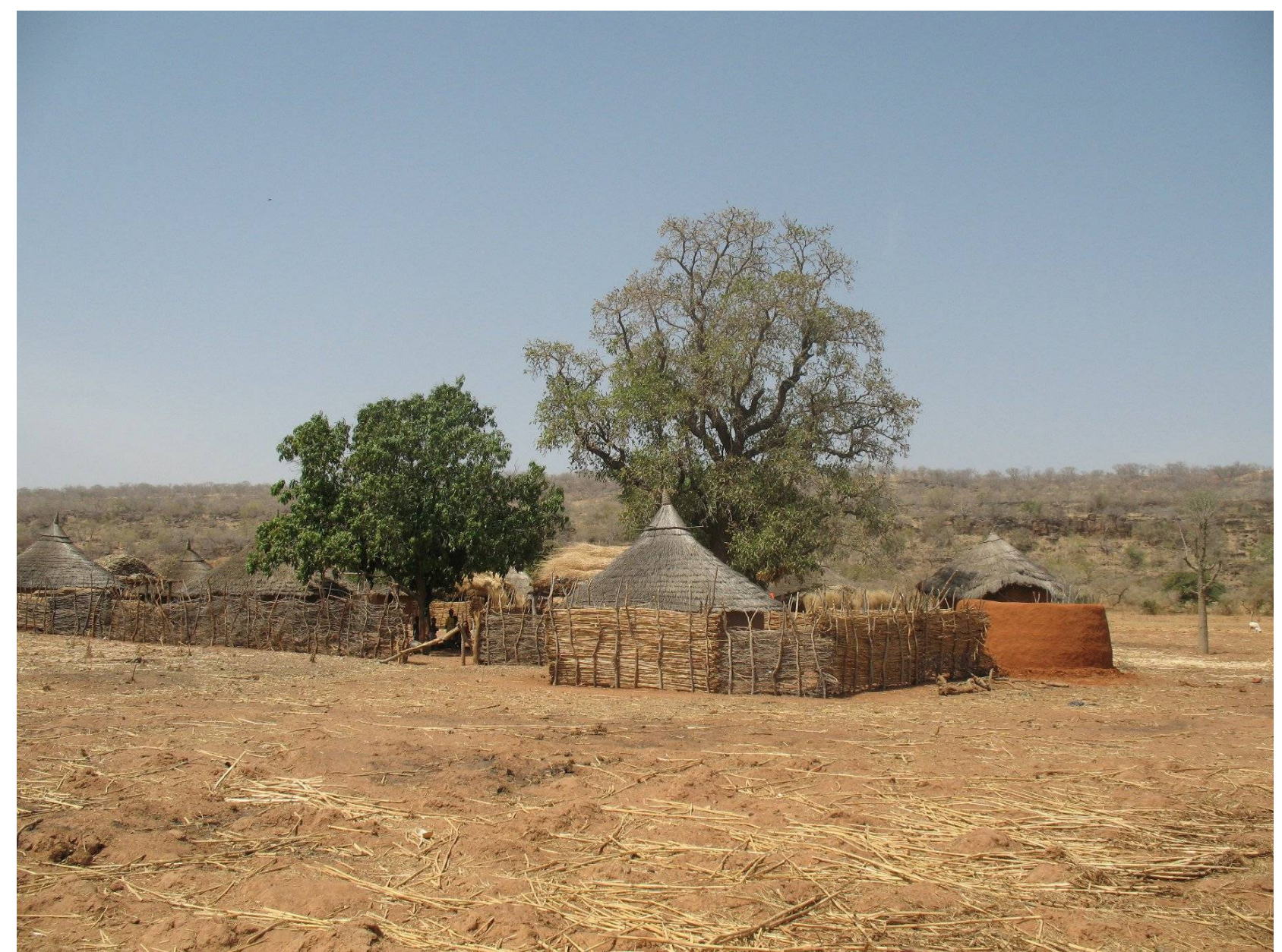

Figure 74: Peulh settlement outside Djibasso

A study carried out in Kossi Province found that the Peulh have significantly higher risks of contracting malaria than Mossi and Samo. However, studies in other regions have come to the opposite result, and it thus remains unclear whether other (i.e. environmental) factors were the actual causes of observed differences. ${ }^{1245}$

\subsubsection{Population Distribution}

Burkina Faso currently has an average population density of around 55 inhabitants $/ \mathrm{km}^{2}$ which is quite unevenly distributed (see figure 75 ). Within the next four decades, this density is expected to rise to between 132 and 167 inhabitants $/ \mathrm{km}^{2}$ (see figure 76 ). About $82 \%$ of the population live in rural

1245 Yé, Y.; Kyobutingi, C.; Louis, V.R. \& SAUerborn, R. (2007), doi:10.1186/1475-2875-6-46. 
regions, making Burkina Faso one of the least urbanized countries in the world. ${ }^{1246}$ Nevertheless, Burkina Faso's urban population grew by 200 per cent between 1975 and 2000 and is projected to continue expanding at a similar pace over the next quarter century. ${ }^{1247}$

Peripheral areas in the north, east and southwest are very sparsely settled, with population densities typically ranging between unsettled and less than 25 inhabitants $/ \mathrm{km}^{2}$. The highest densities are recorded in and around the country's major cities, most notably Ouagadougou, where the population density exceeds 1000 inhabitants $/ \mathrm{km}^{2}$. Kossi province is fairly typical for the country's western region, but even within the province there are marked differences. The département of Nouna is close to the national average of 55 persons $/ \mathrm{km}^{2}$, but the départements of Bourasso in the south and Barani in the north of the province are much less populated at around 10 persons $/ \mathrm{km}^{2}$.

\section{Burkina Faso: Population Distribution}

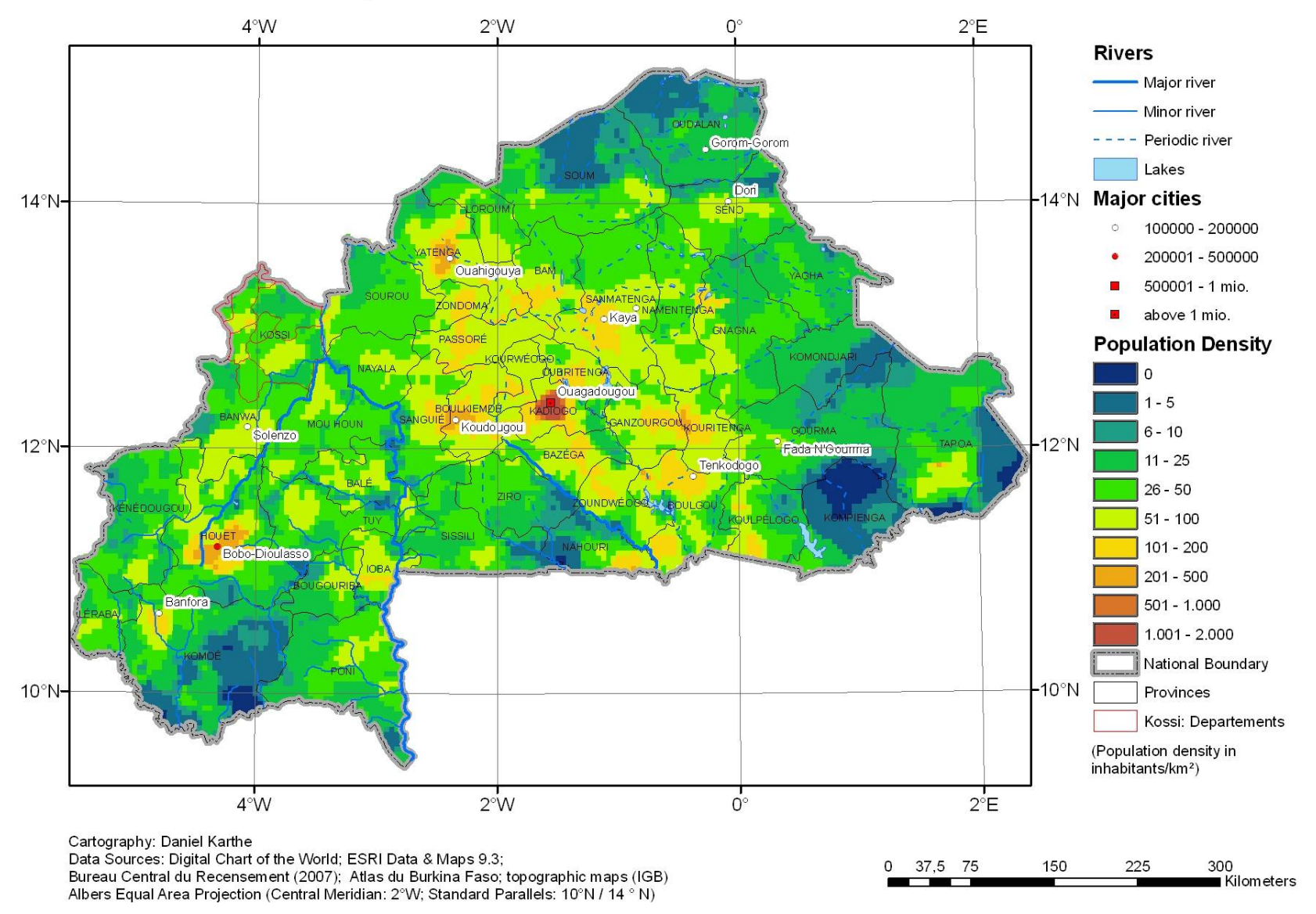

Figure 75: Population density in Burkina Faso ${ }^{1248}$

1246 WoRLd BANK (2007), p. 321.

1247 United Nations Environment Programme (2008), p. 100.

1248 Based on Digital Chart of the World; Bureau Central du Recensement (2007);

YAHMED, D.B. (2005); ESRI Data \& Maps 9.3 and topographic maps (IGB). 


\subsubsection{Demographic Structure and Trends}

Today, the countries in the Sahel and Sahelo-Sudanian zone are characterized by high birthrates and thus population growth. Burkina Faso currently has a population of 15.2 millions, which is projected to reach 37.2 millions by 2050 (see figure 76). This is due to the high total fertility rate (TFR) of 6.2, which leads to an annual population growth rate of $3.0 \%$, but this is projected to decrease in the future. ${ }^{1249}$ According to the medium to high scenarios of the United Nations Population Division, Burkina Faso's population could even exceed 45 millions by 2050; if the TFR remained at the present level, this figure would even be in the order of 67 millions. ${ }^{1250}$

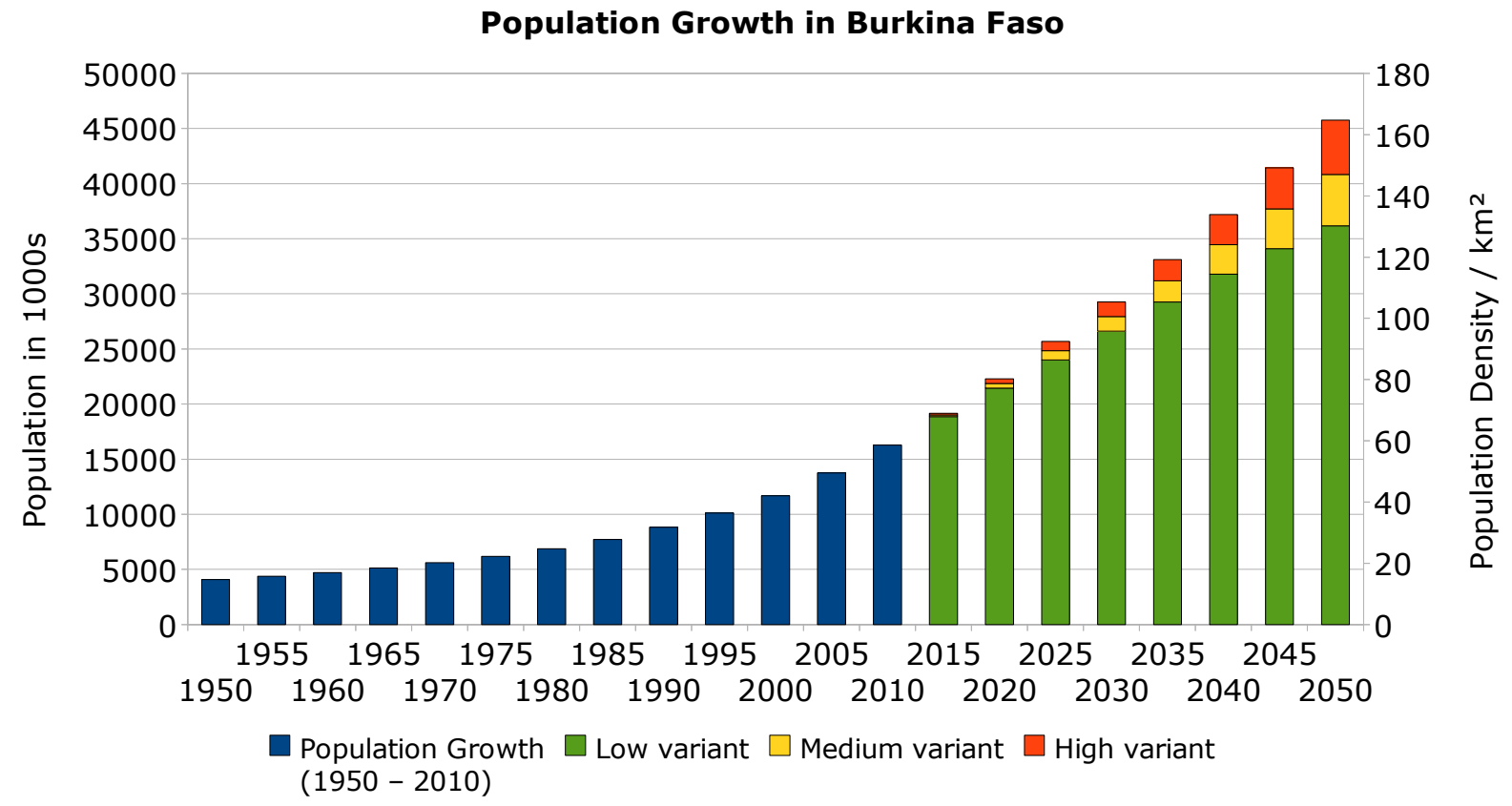

Figure 76: Population growth in Burkina Faso (1950-2050) ${ }^{1251}$

As illustrated by table 54 , this situation is fairly typical for other countries in sub-Saharan West Africa, and slightly above the region's average.

1249 Population Reference Bureau (2008), p. 7.

1250 UN World Population Database, http://esa.un.org/unpp/index.asp, accessed 29/05/2009. 1251 UN World Population Database, http://esa.un.org/unpp/index.asp, accessed 29/05/2009. 


\begin{tabular}{|l|l|l|l|l|} 
& TFR & $\begin{array}{l}\text { Annual } \\
\text { growth }\end{array}$ & Birth rate & Death rate \\
\hline Burkina Faso & 6.2 & $3.0 \%$ & $45 \%$ o & $15 \%$ o \\
\hline Niger & 7.1 & $3.1 \%$ & $46 \%$ o & $15 \%$ o \\
\hline Ghana & 4.3 & $2.2 \%$ & $32 \%$ o & $10 \%$ o \\
\hline West Africa & 5.7 & $2.6 \%$ & $42 \%$ o & $15 \%$ o \\
\hline
\end{tabular}

Table 54: Key demographic indicators for selected West African countries ${ }^{1252}$

Burkina Faso exhibits the typical demographic pattern of a developing country (see figure 77): more than half of the population are less than 18 years old, while only a very small fraction are senior citizens.

Population Pyramid of Burkina Faso (2009)

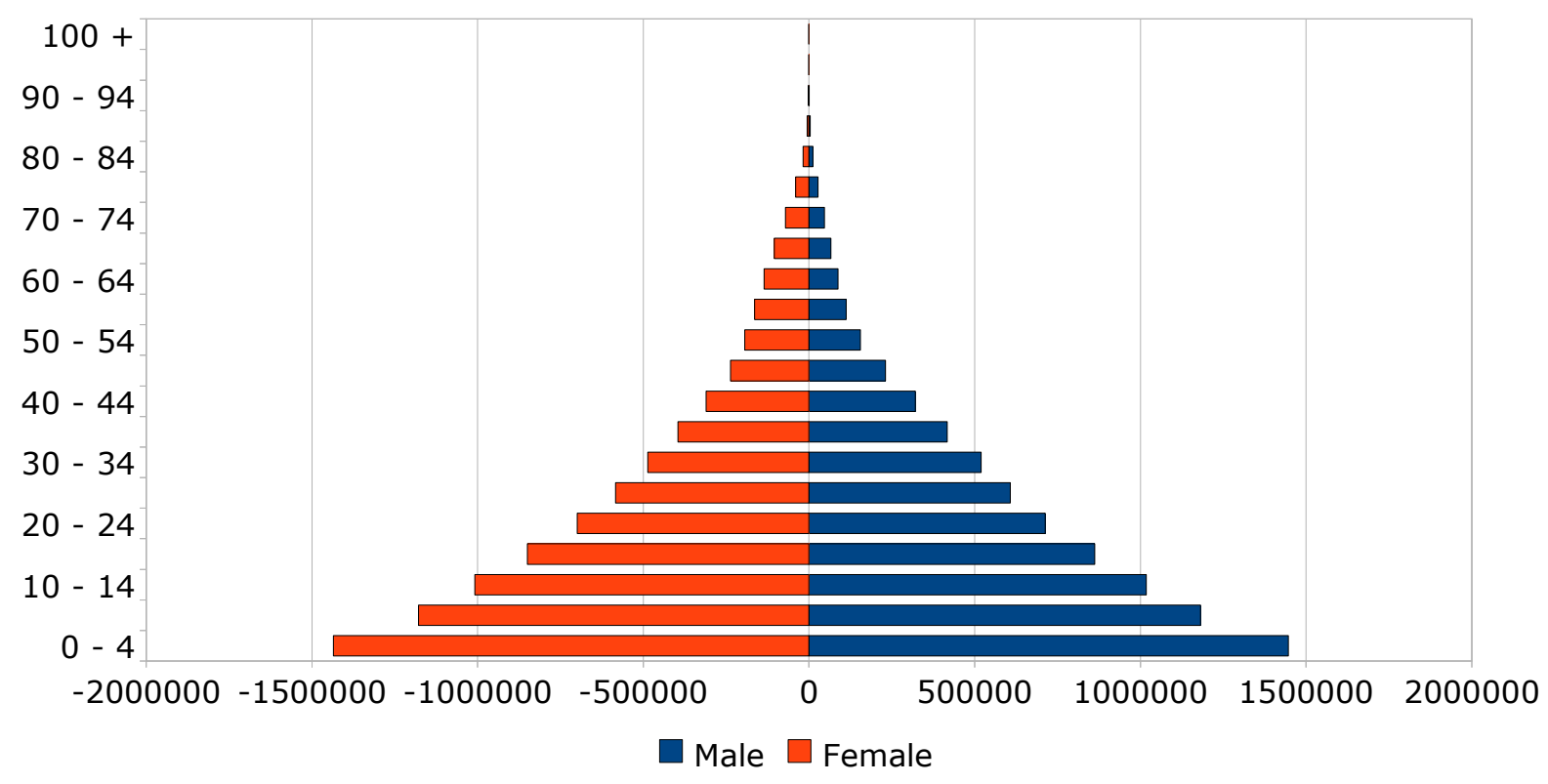

Figure 77: Population pyramid of Burkina Faso, midyear $2009^{1253}$

In Kossi Province, about $17 \%$ of the population are below 5 years of age, and almost half the population are below 15 years of age ${ }^{1254}$.

1252 Population Reference Bureau (2008), p. 7.

1253 US Census Bureau International Database,

http://www.census.gov/ipc/www/idb/country.php, accessed 02/07/2009.

1254 WürthWEIN, R. (2002), pp. $139 f$. 


\subsubsection{Economy and Development}

Burkina Faso ranges among the countries with the lowest per capita income in the world. The national economy still depends greatly on agriculture and employs the absolute majority of the population. Albeit at a low level, Burkina Faso's GDP has grown more or less consistently in recent years. Within the country, there are considerable regional disparities and Kossi Province is one of the least developed regions. 


\subsubsection{General Indicators and Structures}

Burkina Faso's per capita GDP is around $\$ 345$ per year, or $\$ 1300$ in purchasing power parities. Unemployment and underemployment are frequent in Burkina Faso, and almost half of the population live below the poverty line ${ }^{1255}$ which is currently defined as an annual per capita income of 82.672 FCFA ( $€ 126$ or US\$179). ${ }^{1256}$ The national poverty rate of $46.4 \%{ }^{1257}$ is unevenly distributed between rural areas and urban areas $(48 \%$ vs. $18 \%$ respectively). ${ }^{1258}$

Agriculture contributes $29.7 \%$ to the national income but employs around $85 \%$ to $90 \%$ of the national labor force. ${ }^{1259}$ It is estimated that more than $90 \%$ of all Burkinabès live on subsistence farming. ${ }^{1260}$ These are some of the highest proportions found anywhere in Africa. ${ }^{1261}$ The remaining $10 \%$ are occupied in the industrial and service sectors, which yield $19.4 \%$ and $50.9 \%$ of the national income, respectively. A large part of the male labor force migrates annually to neighboring countries for seasonal employment. ${ }^{1262}$

\section{Economic Structure (Percentage of GDP)}

$\square$ Service $\square$ Industry $\square$ Agriculture

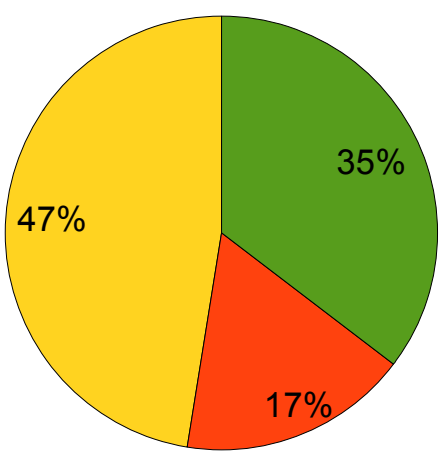

Economic Structure (Employment)

$\square$ Informal $\square$ Formal $\square$ Agriculture

Figure 78: Burkina Faso's economic structure ${ }^{1263}$

1255 CIA World Fact Book 2008, https://www.cia.gov/library/publications/the-worldfactbook/geos/uv.html, accessed 01/10/08.

1256 Direction Générale de l'Institut National de la Statistique et de la Démographie (2005), p. 11.

1257 Direction Générale de l'Information et des Statistiques Sanitaires $\left(2009^{1}\right)$, p. 4.

1258 OeCD \& African Development Bank (2006), p. 163.

1259 CIA World Fact Book 2008, https://www.cia.gov/library/publications/the-worldfactbook/geos/uv.html, accessed 01/10/08.

1260 Berié, E. \& Kobert, H. (2005), p. 96.

1261 United Nations Environment Programme (2008), p. 99.

1262 CIA World Fact Book 2008, https://www.cia.gov/library/publications/the-worldfactbook/geos/uv.html, accessed 01/10/08.

1263 OeCD \& African Development Bank (2008), p. 179. 
Geographic Determinants of Malaria Transmission

\begin{tabular}{|l|l|l|l|l|l|}
\hline Primary Sector & \multicolumn{3}{|l|}{ Secondary Sector } & Tertiary Sector \\
\hline Agriculture & $18 \%$ & $\begin{array}{l}\text { Mining \& } \\
\text { manufacturing }\end{array}$ & $16 \%$ & Services & $23 \%$ \\
\hline Livestock & $13 \%$ & Construction & $6 \%$ & $\begin{array}{l}\text { Government } \\
\text { services }\end{array}$ & $22 \%$ \\
\hline $\begin{array}{l}\text { Forestry \& } \\
\text { Fisheries }\end{array}$ & $2 \%$ & & & & \\
\hline
\end{tabular}

Table 55: Burkina Faso's GDP by economic sectors (2005) ${ }^{1264}$

Burkina Faso's economy is very vulnerable because of its dependence on agriculture. Climatic hazards such as droughts or floods or locust invasions are enough to jeopardize substantial parts of the harvest. ${ }^{1265}$ In addition, the largely disorganized agricultural sector results in bad management of stocks and low farmer incomes. ${ }^{1266}$ Cotton alone brings in about half of Burkina Faso's foreign earnings, and its trade balance depends heavily on the state of the cotton sector. ${ }^{1267}$ The provinces of the Boucle du Mouhoun region are among the country's principal cotton producers; changes in cotton prices therefore have a direct impact on living conditions in the region. ${ }^{1268}$

Burkina Faso's dependence on foreign aid is illustrated by the fact that nearly one third of the national income is received in the form of international donations, mainly from France and the European Union and debt reliefs by the world bank. ${ }^{1269}$

The Kossi economy has a very strong subsistence character, and the per capita cash income is to the order of only $\$ 50$ per year. The subsistence income (cash income plus the value of subsistence farming products) amounts to about $\$ 250$ per capita and year. ${ }^{1270}$ There is no notable industry within Kossi province. The closest centers of small-scale industry are Dédougou (cotton ginning) and Koudougou (cotton ginning and textile industry). ${ }^{1271}$ Around $60 \%$ of the population live below the poverty line. ${ }^{1272}$ Kossi is thus one of the least developed regions within Burkina Faso.

1264 OeCD \& African Development Bank (2007), p. 150; in case of Burkina Faso, the OeCD does not publish separate figures for mining, which is strictly speaking not a primary sector activity.

1265 OeCD \& African Development Bank (2007), p. 149.

1266 OeCD \& African Development Bank (2002), p. 61.

1267 OeCD \& African Development Bank (2002), p. 66.

1268 Direction Générale de l'Institut National de la Statistique et de la Démographie (2005), p. 6.

1269 YAHMED, D.B. (2005), p. 92.

1270 WÜRTHWEIN, R. (2002), p. 135.

1271 YAHMED, D.B. (2005), p. 103.

1272 Direction Générale de l'Institut National de la Statistique et de la Démographie (2005), p. 6. 


\subsubsection{Economic Development}

Burkina Faso's economy has been growing at an average of $5,9 \%$ between 1997 and 2006 but slowed down to 4,3\% in 2007 due to a fall in cotton production. Despite this constant growth, Burkina Faso remains one of the poorest countries in the world, with $44,8 \%$ of its population living on less than US- $\$ 1$ per day. ${ }^{1273}$ Moreover, the global economic crisis may invert the positive economic trend of the past decade of years. ${ }^{1274}$

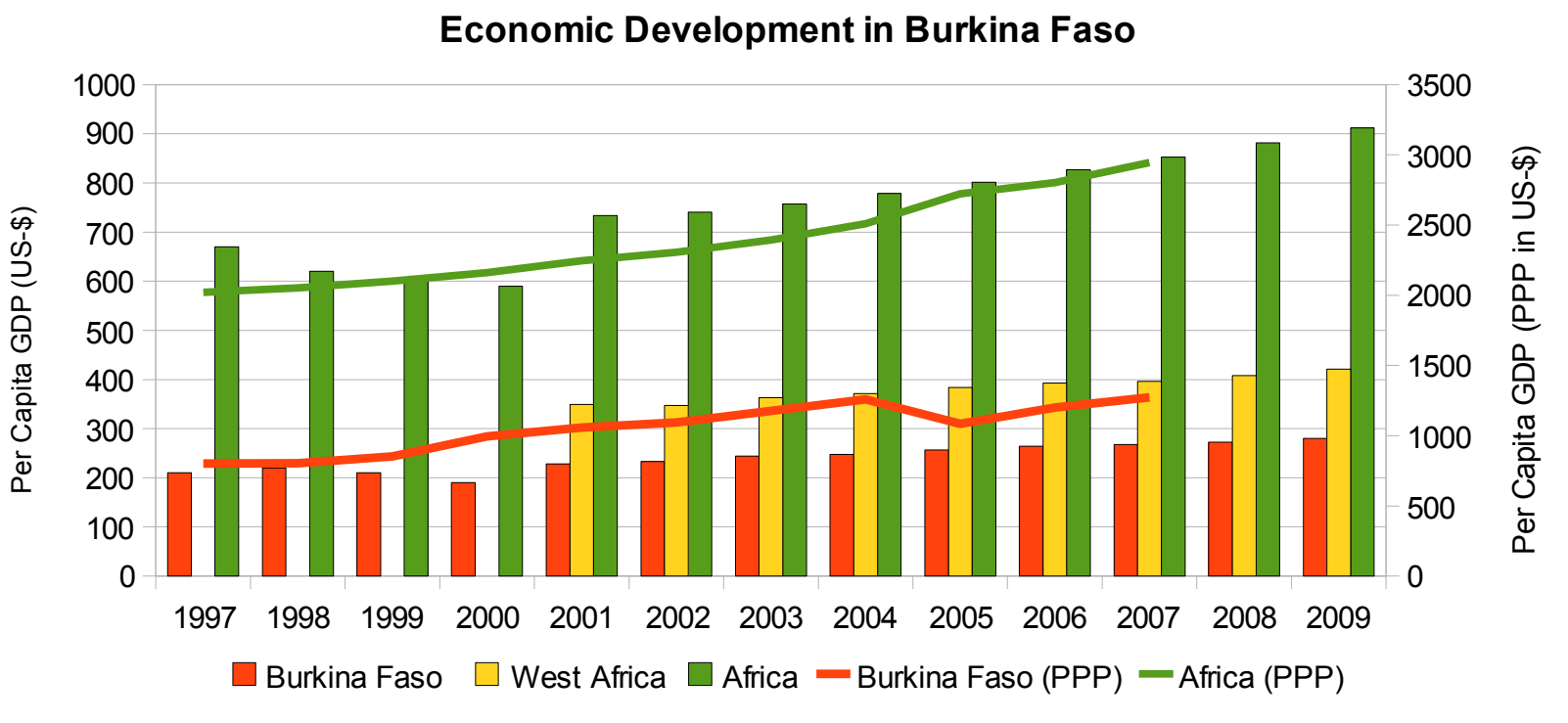

* Data for 2008/2009 are estimates / projections.

Figure 79: Economic development in Burkina Faso ${ }^{1275}$

In 2007, the industrial sector recorded a growth of $8,2 \%$ in production value, led by major construction projects in Ouagadougou and the start of commercial gold production at Taparko mine in Namentenga province. Several other mining projects are expected to become commercially operational by the end of $2009 .{ }^{1276}$

Since Burkina Faso's energy supply depends heavily on oil and gas, initiatives are currently underway to promote bio-fuels. ${ }^{1277}$ Their implementation could have a major impact on land use and the agricultural sector and increase the demand for irrigation, which would then be linked to vector-borne disease transmission.

1273 OeCD \& African Development Bank (2008), p. 167.

1274 EINEDER, F. (2009), p. 8.

1275 Compiled from OECD \& African Development Bank (2002 to 2008).

1276 OeCD \& African Development Bank (2008), pp. $168 \mathrm{f}$.

1277 OeCD \& African Development Bank (2008), p. 174. 
One major hurdle for development is the poor state of Burkina Faso's transport infrastructure. Most roads outside the urban centers are unpaved and a railway system is almost nonexistent. The road system still largely follows the pattern drafted during the colonial era, which means that most larger roads connect the sites of resource excavation or production with the urban centers of Ouagadougou and Bobo-Dioulasso, but that there are otherwise few inter- and intraregional connections. ${ }^{1278}$ The poor state of Burkina Faso's transport infrastructure does not only impede economic development but also limits access to health care.

The government revenue has grown considerably in recent years, from $11,9 \%$ of the GDP in $1995^{1279}$ to $18 \%$ of the GDP in $2007^{1280}$. However, government finances depend heavily on international grants. The economy's main sector, agriculture, is largely outside the tax system. Burkina Faso's trade deficit, which is due to a weak export sector, also contributes to the shortage of government funds. ${ }^{1281}$

Burkina Faso's national government identified improvements in the health and education sectors as the key prerequisites for poverty reduction in the near future. ${ }^{1282}$

\subsubsection{Land Use and Agriculture}

As in neighboring countries, Burkina Faso's agriculture is characterized mostly by subsistence farming. Agricultural land use is most pronounced in the central part of the country where much of the savanna is either cultivated or used for animal grazing (see figure 80).

The agro-ecological potential of the Sahelo-Sudanian zone is largely determined by the region's climate and soils. Cultivable soils are often subject to a fallow rotation system, with bush fires being laid annually to increase soil fertility. ${ }^{1283}$ Due to the massive evaporation, soils desiccate rapidly and salination may occur. For the growth of rainfed cultures, the temporal distribution of rainfall is often more important than the annual amount of precipitation. About $200 \mathrm{~mm}$ to $250 \mathrm{~mm}$ of rain suffice if they fall during a period of about two months, whereas higher amounts may be insufficient if they fall over longer, partially dry intervals. ${ }^{1284}$

1278 HAMMER, T. (2005), p. 106.

1279 OeCD \& African Development Bank (2002), p. 65.

1280 OeCD \& African Development Bank (2008), p. 170.

1281 OeCD \& African Development Bank (2002), pp. $65 \mathrm{f}$.

1282 Direction Générale de l'Information et des Statistiques Sanitaires $\left(2009^{1}\right)$, p. 1.

1283 Devineau, J.L. \& Fournier, A. (2007), p. 352.

1284 Hammer, T. (2005), p. 19. 
Most of Burkina Faso's cereal production is grown under rain-fed conditions, and both at the national level and in Kossi Province, sorghum and millet are the main crops. ${ }^{1285}$ Adverse effects of climate variability on agriculture are exacerbated by relatively wetter or drier periods that may persist for several years or decades. ${ }^{1286}$ However, vulnerable rainfed agriculture is not an abnormality but rather a characteristic of the Sahel and Sahelo-Sudanian zone. ${ }^{1287}$

\section{Burkina Faso: Land Use Types}

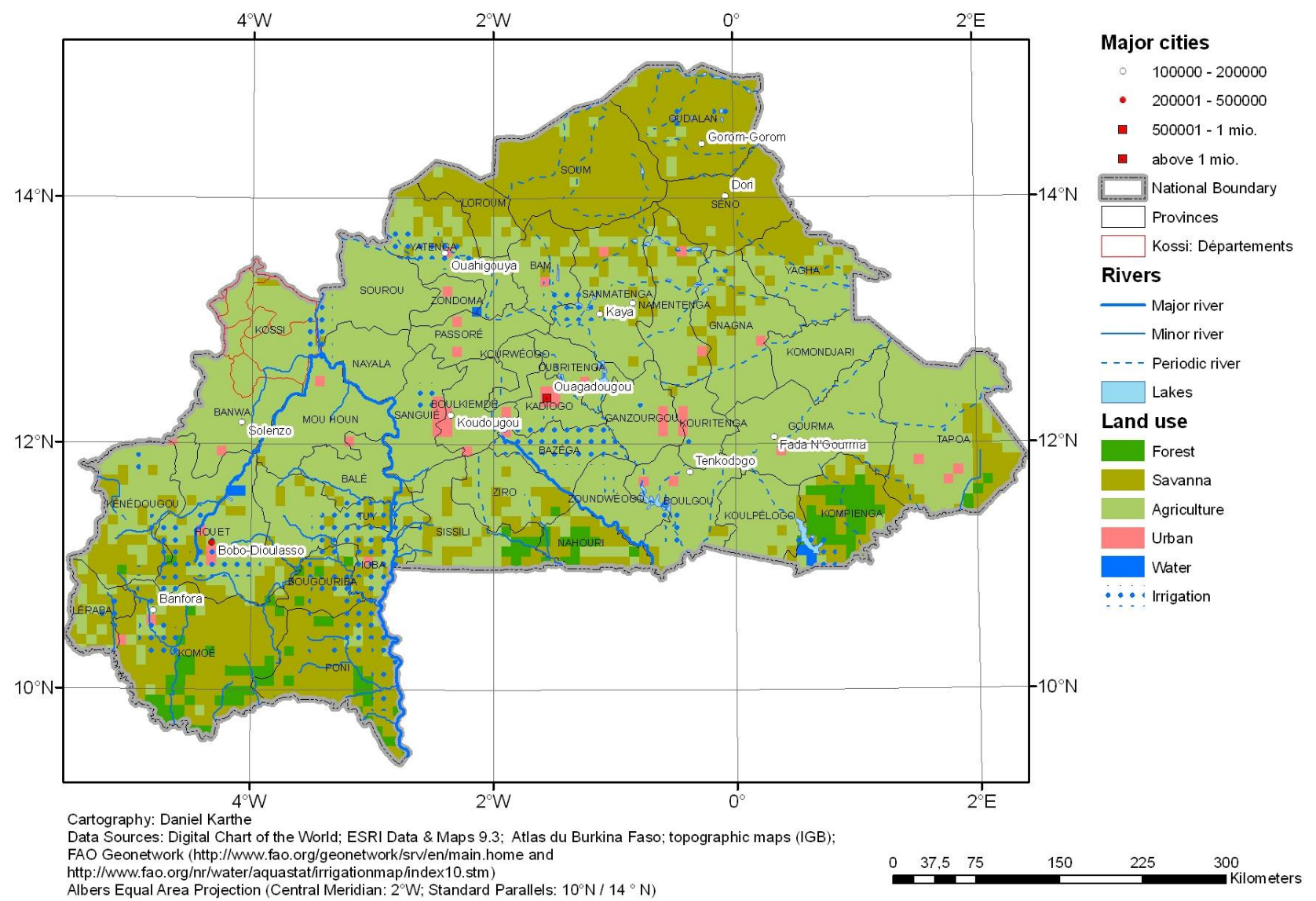

Figure 80: Land use types in Burkina Faso ${ }^{1288}$

1285 Ingram, K.T.; Roncoli, M.C. \& Kirshen, P.H. (2002), pp. 331;333.

1286 Korodjouma, O.; Badiori, O.; Ayemou, A. \& Michel, S.P. (2006), p. 218.

1287 HAMMER, T. (2005), p. 34.

1288 Based on FAO Geonetwork; Digital Chart of the World;

YAHMED, D.B. (2005); ESRI Data \& Maps 9.3 and topographic maps (IGB). 
One frequent feature of cultivated fields in Burkina Faso is that large parts of the woody vegetation are left standing or even protected by local farmers. The resulting anthropogenic landscapes are referred to as parkland savannas. ${ }^{1289}$

The farming systems of Burkina Faso traditionally include fallow periods destined to regenerate the fertility of cultivated soils. With increasing population pressure, this traditional

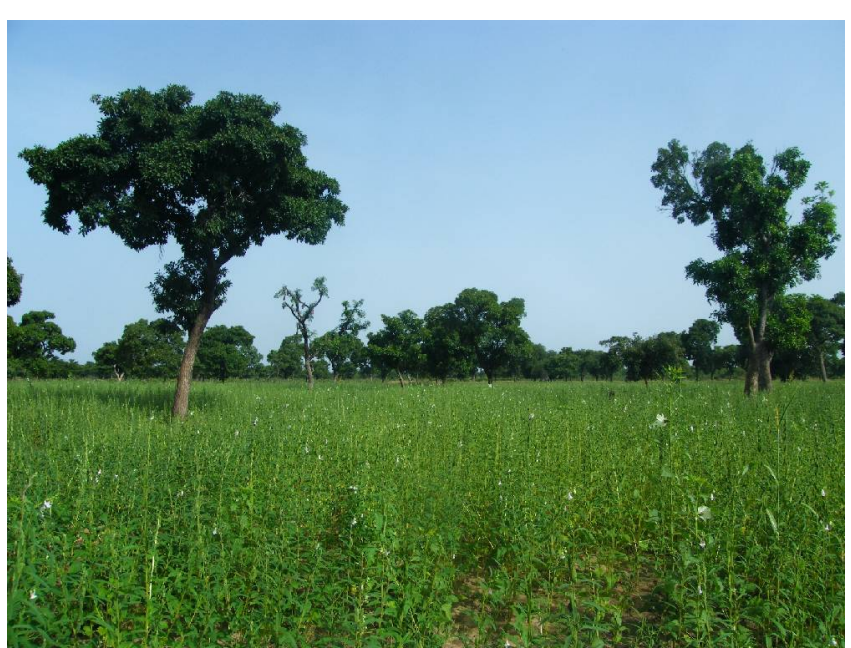

Figure 81: Parkland savanna with sesame field practice is disappearing. ${ }^{1290}$

Even though irrigation projects are limited, currently covering only around 32.000 hectares of cropland, they already account for an estimated $65 \%^{1291}$ to $86 \%{ }^{1292}$ of the freshwater use in Burkina Faso. Since larger projects typically involve the building of dams and water reservoirs, they often bring about environmental changes favoring the transmission of malaria by providing mosquito habitats and breeding sites. Since agricultural activities are Burkina Faso's key user of water, their type and local pattern are also among the most important anthropogenic determinants of malaria.

Pastoralism, in the northern sections of the country in nomadic and seminomadic form, is the dominant form of primary sector activities in regions unsuited for crop cultivation, but is also practiced to a substantial degree in the the center and south of the country where it is mixed with agriculture (agropastoralism). In this regard, Burkina Faso is very typical of the nations falling into the Sahel and the adjoining Sahelo-Sudanian zone. ${ }^{1293}$

1289 Maranz, S. \& Wiesmann, Z. (2003), p. 1506.

1290 OUADBA, J.M. (1991), p. 331.

1291 Barbier, B.; Dembelé, Y. \& Comparoré, L. (2006), p. 23.

1292 WORLD BANK (2007), p. 324.

1293 http://www.fao.org/nr/lada/images/stories/LUSMAPV1_june09/lus ssa.jpg, accessed $30 / 07 / 2009$ 


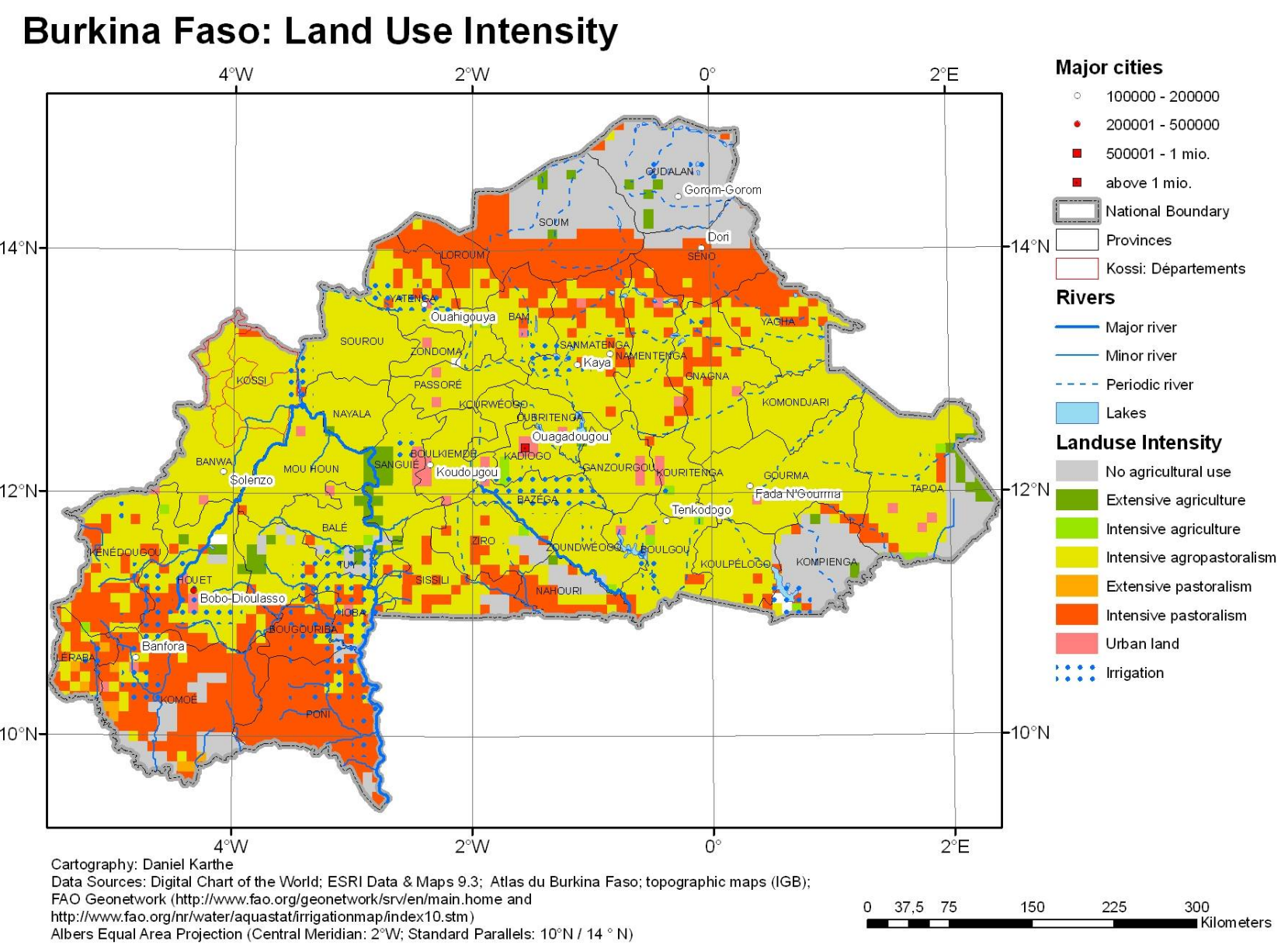

Figure 82: Land use intensity in Burkina Faso ${ }^{1294}$

Within Burkina Faso, Kossi province falls into a belt characterized by intensive agro-pastoralism (see figures 80 and 82 ), with rainfed agriculture and/or herding of cattle and goats being practiced around most settlements. The northeastern part of Kossi, along the Sourou river, is home to a major irrigation project that extends from Illa on the Kossi side of the river to Niassan and Di in Sourou province. In this region, water is diverted from the Sourou river to produce cash crops as diverse as rice, sugarcane, tomatoes and bananas. Small-scale irrigation "projects", often consisting of a single motor pump providing water for a few small fields, have recently been started along the Mouhoun.

1294 Based on FAO Geonetwork; Digital Chart of the World;

YAHMED, D.B. (2005); ESRI Data \& Maps 9.3 and topographic maps (IGB). 


\subsubsection{Sorghum and Millet: Traditional Subsistence Crops}

Sorghum (Sorghum bicolor, see figure 83 and Sorghum guinea) is planted in large parts of Burkina Faso's central plateau region on moderately dry soils. Pearl millet (Pennisetum glaucum, figure 84) is more drought-tolerant than sorghum, but it also yields less than sorghum under normal rainfall conditions. ${ }^{1295}$ Sorghum and millet together cover about $65 \%$ to $85 \%$ of the cultivated area in most provinces. ${ }^{1296}$ During the past 15 years or so, farmers have shifted from long-duration (120 to 150 days) to short duration (70 to 90 days) varieties of sorghum. ${ }^{1297}$

Sorghum is less susceptible to water logging than millet, so more sorghum is planted when above normal rains are forecast. ${ }^{1298}$ Both crops are currently grown on more than a million hectares each (table 57). Within the study region, they are grown within and around practically all human settlements and are indicators of local soil and climate conditions: 


\begin{tabular}{|c|c|c|}
\hline Environment & Sorghum & Millet \\
\hline Temperature & $\begin{array}{l}\text { - } 13^{\circ} \mathrm{C} \text { required for } \\
\text { germination; } \\
\text { - optimum for seedling } \\
\text { growth: } 33^{\circ} \mathrm{C} \text {; } \\
\text { - soil temperatures }>40^{\circ} \mathrm{C} \\
\text { lethal }{ }^{1299}\end{array}$ & $\begin{array}{l}16 \text { to } 32^{\circ} \mathrm{C} \text { required for } \\
\text { growth }\end{array}$ \\
\hline Precipitation & $\begin{array}{l}\text { - } 600 \text { to } 1000 \mathrm{~mm} \text { annually }{ }^{1301} \\
\text { - tolerates desiccation }{ }^{1302}\end{array}$ & $\begin{array}{l}\text { - } 200 \text { to } 800 \mathrm{~mm} \text { annually }{ }^{1303} \\
\text { - relatively high yields even at } \\
\text { low humidity }{ }^{1304}\end{array}$ \\
\hline Soil & $\begin{array}{l}\text { - In West Africa usually grown } \\
\text { on vertisols and alfisols }{ }^{1305} \\
\text { - tolerates waterlogging }\end{array}$ & $\begin{array}{l}\text { - in West Africa usually found } \\
\text { on aridisols, alfisols and } \\
\text { entisols } \\
\text { - preferred to sorghum on } \\
\text { sandy soils } \\
\text { - grows best on light loams } \\
\text { - does not tolerate } \\
\text { waterlogging }{ }^{1306}\end{array}$ \\
\hline
\end{tabular}

Table 56: Environmental prerequisites for sorghum and millet production

Except for occasional pools of water forming during the rainy season, rainfed sorghum and millet fields are usually not suited as anopheline breeding sites. Since sorghum is more tolerant to temporal water logging than millet, regions around sorghum fields may represent zones of higher malaria risk than around millet fields.

1299 Norman, M.J.T.; Pearson, C.J. \& Searle, P.G.E. (1995), p. 149.

1300 Sys, C.; van Ranst, E.; Debaveye, J. \& Beernaert, F. (1993), p. 89.

1301 Norman, M.J.T.; Pearson, C.J. \& Searle, P.G.E. (1995), p. 161.

1302 Eineder, F. (2009), p. 26.

1303 Norman, M.J.T.; Pearson, C.J. \& Searle, P.g.E. (1995), p. 165.

1304 EINEDER, F. (2009), p. 24.

1305 Norman, M.J.T.; Pearson, C.J. \& Searle, P.G.E. (1995), p. 156.

1306 Norman, M.J.T.; Pearson, C.J. \& Searle, P.G.E. (1995), p. 173. 


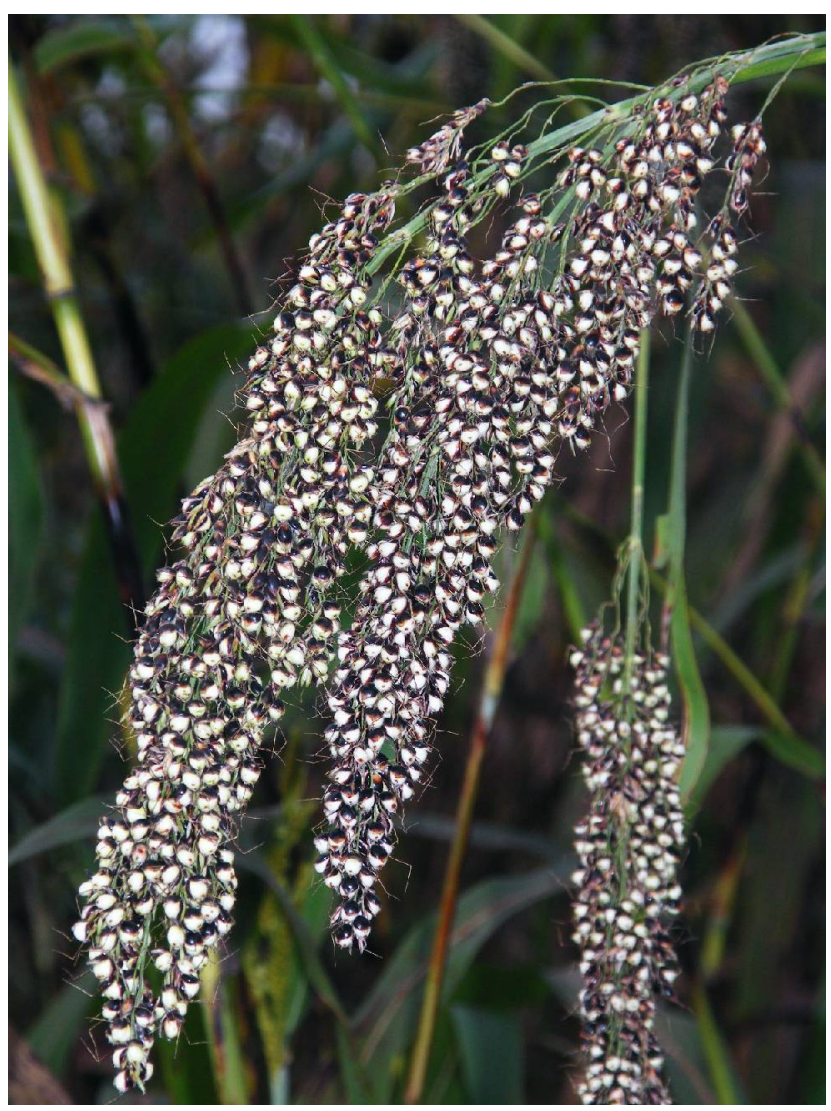

Figure 83: Sorghum bicolor

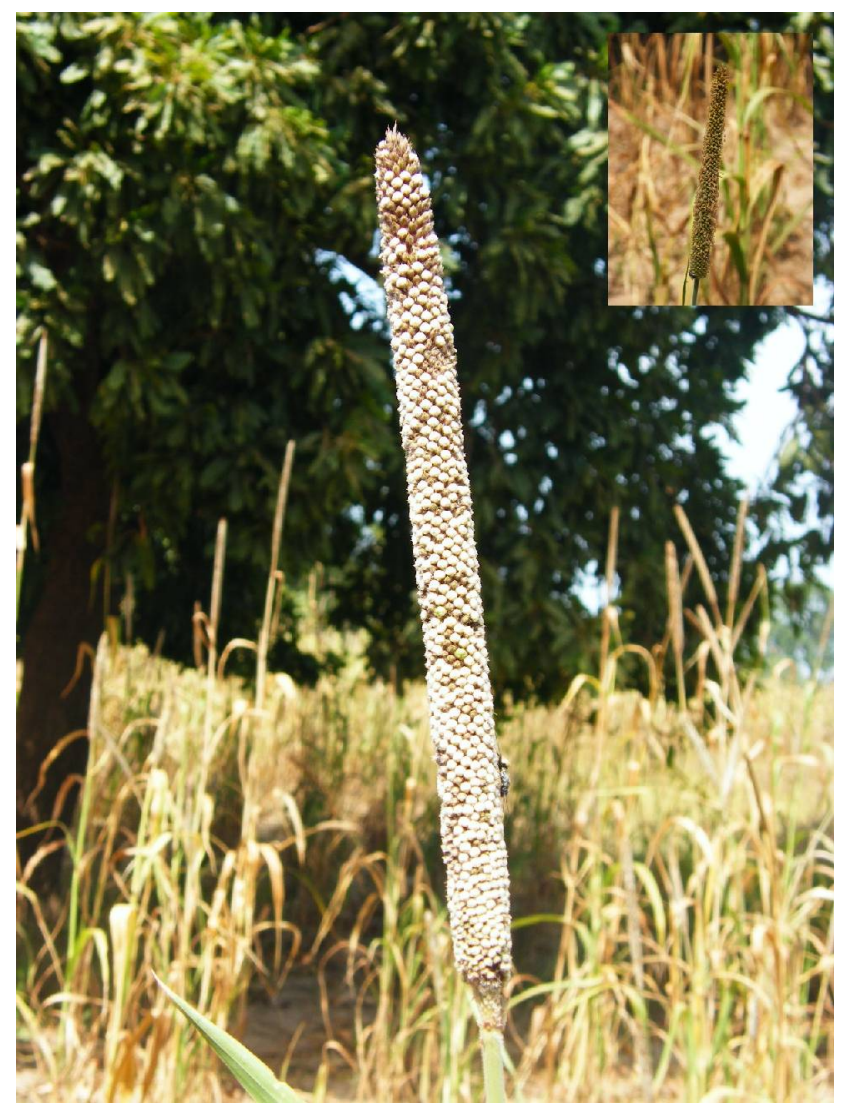

Figure 84: Pennisetum glaucum

\subsubsection{Market Crops and Irrigated Agriculture}

Maize (Zea mays) and rice (Oryza sativa) are two crops of moderate but increasing importance (see figure 86 ). Maize covers about $12 \%$ of the area used for cereal production in Burkina Faso but is of little importance in Kossi province. It is, however, grown on the eastern bank of the Sourou (in Sourou province). Maize requires more water than sorghum or millet. Under rainfed conditions, it grows best in areas that receive an annual precipitation between 1000 and $1500 \mathrm{~mm}$ per year. It is more sensitive to drought and waterlogging than sorghum and millet. ${ }^{1307}$

Rice cultivation, on the other hand, is found in southern Burkina Faso and around perennial rivers, where it is often connected with irrigation projects. Under rainfed conditions, the optimum precipitation for rice is around $1600 \mathrm{~mm} \cdot{ }^{1308}$ Since the demand for rice has been growing by $12 \%$ annually in recent years, there are plans to increase the domestic

1307 Sys, C.; van Ranst, E.; Debaveye, J. \& Beernaer 1308 Sys, C.; van Ranst, E.; Debaveye, J. \& Beernaer 
production. The Sourou Valley is the most important rice-growing region in northern Burkina Faso; more than 2/3 of the irrigated area of around 5000 ha are used for rice. ${ }^{1309}$ Even though irrigated agriculture is still the exception in Burkina Faso, at least $65 \%$ of the consumed surface water is used for irrigation purposes. ${ }^{1310}$

The area cultivated with maize has more than doubled within the last decade (see figure 86). With a growing period of 70 days, maize is well adapted to short rainy seasons but needs regular rainfall, careful weeding and good soil fertility. In Burkina Faso, it is usually planted in small manured plots. ${ }^{1311}$

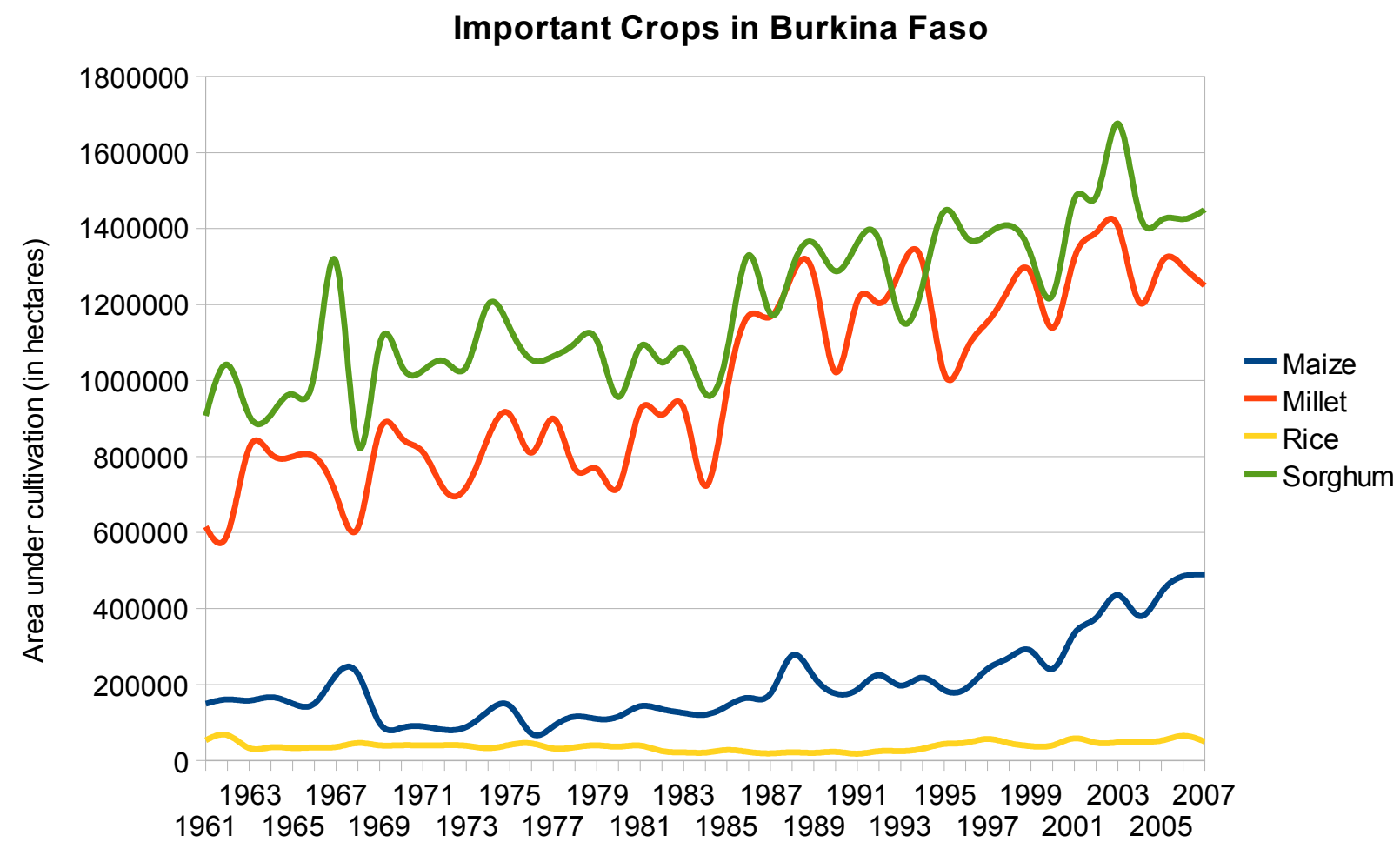

Figure 86: Area under cultivation with important cereal crops ${ }^{1312}$ 


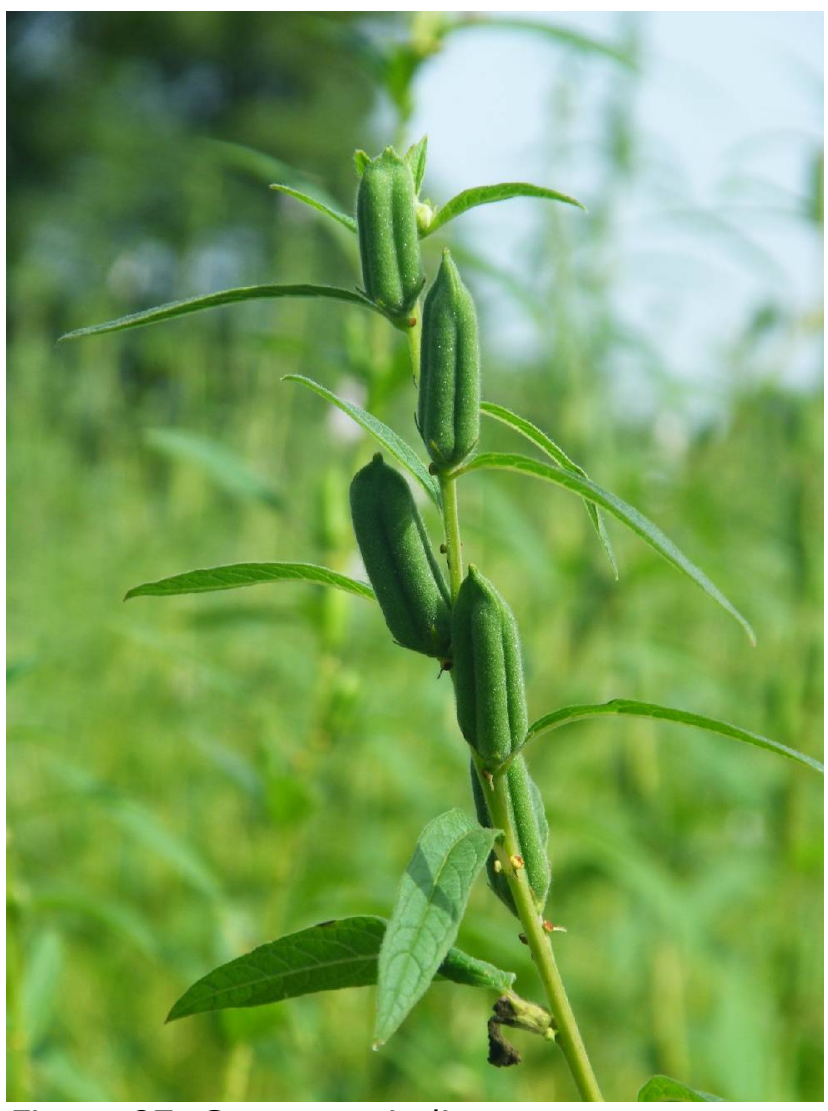

Figure 87: Sesamum indicum

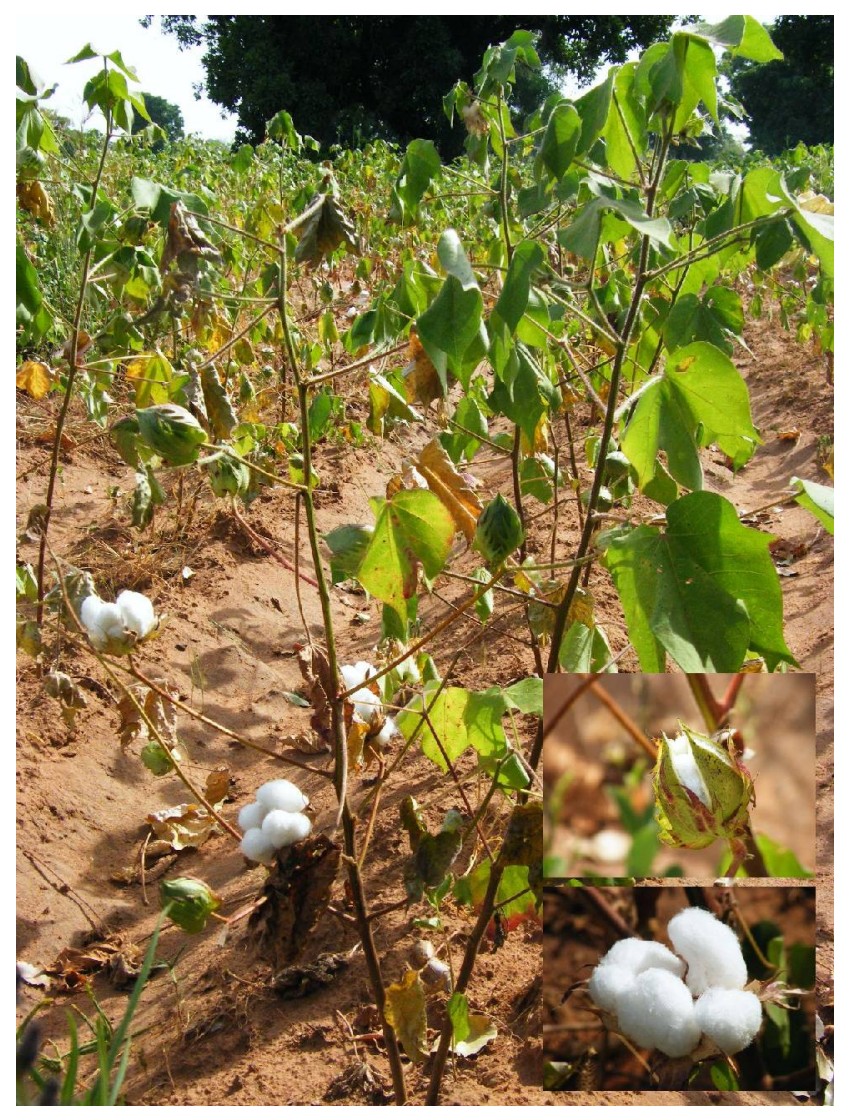

Figure 88: Gossypium hirsutum

Cotton (Gossypium hirsutum) is Burkina Faso's principal cash crop and is grown in Southern Kossi province (see figure 88 ). Cotton production provides an income for around 2 million Burkinabès and the area under cultivation has been extended continuously in recent years (see figure 89). ${ }^{1313}$ Structural reforms, especially towards a greater involvement of farmers in the stateowned cotton-processing company Sofitex greatly boosted the national cotton production..$^{1314}$ While cotton grows at temperatures ranging between $18^{\circ} \mathrm{C}$ and $40^{\circ} \mathrm{C}$, an annual precipitation of at least $500 \mathrm{~mm}$ is required. Optimum conditions are between 900 to $1200 \mathrm{~mm}$. Cotton prefers well-drained alluvial soils but vertisols are suitable unless waterlogging occurs. ${ }^{1315}$

Other important cash crops grown in Burkina Faso include peanuts, sesame (see figure 87), shea nuts (karité, see pages 210 and 218) and sugarcane. Peanuts (Arachis hypogaea) are grown in the western part of Kossi province. They grow at temperatures between $10^{\circ} \mathrm{C}$ and $38^{\circ} \mathrm{C}$ but a yield decrease is observed above $30^{\circ} \mathrm{C}$. $300 \mathrm{~mm}$ of rainfall during the growing cycle (90 to 140 days) are required but $400 \mathrm{~mm}$ to $1100 \mathrm{~mm}$ are preferable. Sandy soils such

1313 YAHMED, D.B. (2005), p. 96.

1314 OeCD \& African Development Bank (2002), p. 62.

1315 Sys, C.; van Ranst, E.; Debaveye, J. \& Beernaert, F. (1993), p. 63. 
as sandy loams are well suited since peanuts cannot stand flooding. ${ }^{1316}$ Sesame (Sesamum indicum) is a crop of growing importance in both Burkina Faso and Kossi province and is primarily grown as a cash crop. ${ }^{1317}$ Since it requires relatively little attention, it is often grown in the parkland savanna between villages. The optimal temperature range is between 25 and $29^{\circ} \mathrm{C}$ and 300 to $800 \mathrm{~mm}$ of precipitation during the growing cycle (70 to 100 days) are suitable. Sesame prefers loamy soils and is intolerant to waterlogging. ${ }^{1318}$ Sugarcane (Saccharum officinarum) is grown in the Sourou Valley irrigation zone, but it occupies only a minor part of the area under cultivation. Under rainfed conditions, it requires a precipitation of at least $1300 \mathrm{~mm}$ per growing period (at least 270 days). ${ }^{1319}$

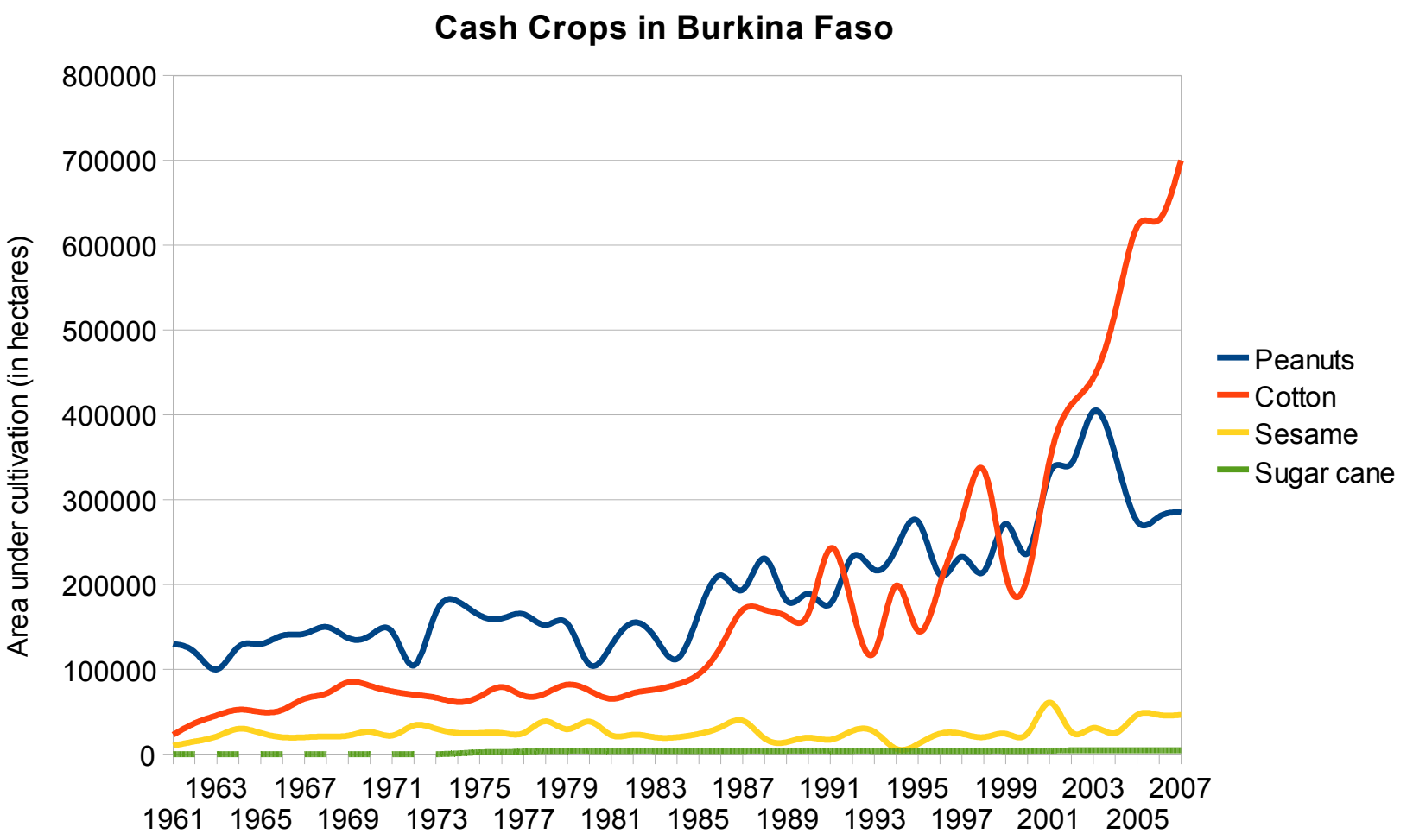

Figure 89: Area cultivated with cash crops $^{1320}$

Vegetables -mainly onions, tomatoes and green beans- are cultivated on several small plots around villages in Kossi province. As compared to major food crops such as sorghum or millet, they are only cultivated on a tiny fraction of the arable land. In 2007, the combined area covered by sorghum and millet was nearly 1000 times the area covered by the three vegetables together.

1316 Sys, C.; van Ranst, E.; Debaveye, J. \& Beernaert, F. (1993), p. 74.

1317 YAHMED, D.B. (2005), p. 96.

1318 Sys, C.; van Ranst, E.; Debaveye, J. \& Beernaert, F. (1993), p. 136.

1319 Sys, C.; van Ranst, E.; Debaveye, J. \& Beernaert, F. (1993), p. 147.

1320 http://faostat.fao.org/site/567/default.aspx, accessed 15/05/2009. 
Cassava (Manihot esculenta), also referred to as manioc and yam (Dioscorea rotundata) are of somewhat greater importance. Cassava has a temperature range for growth between 12 and $35^{\circ} \mathrm{C}$ and requires at least $500 \mathrm{~mm}$ of rainfall for growth; the optimum is between 1400 and $1800 \mathrm{~mm}$. Cassava is sensitive to waterlogging, so no flooding should occur. The plant therefore prefers sandy loams and can grow on poor soils. On very fertile soils, vegetative growth takes place at the expense of the roots. ${ }^{1321}$ Cassava can be grown on soils of low fertility where other crops fail ${ }^{1322}$ and is tolerant to prolonged periods of drought during the growing season. ${ }^{1323}$ Yam grows best in regions with a short dry season of up to 4 months and at least $1150 \mathrm{~mm}$ of precipitation during the growing season. The dry margins of the 'yam zone' are characterized by an annual rainfall of around $400 \mathrm{~mm}$. Yam requires soils of high fertility; in West Africa, it is therefore traditionally grown as the first crop after clearing. Yam is intolerant of waterlogging; nevertheless, it is in West Africa confined to river flood plains where the soils are deep and preferably sandy loams. ${ }^{1324}$

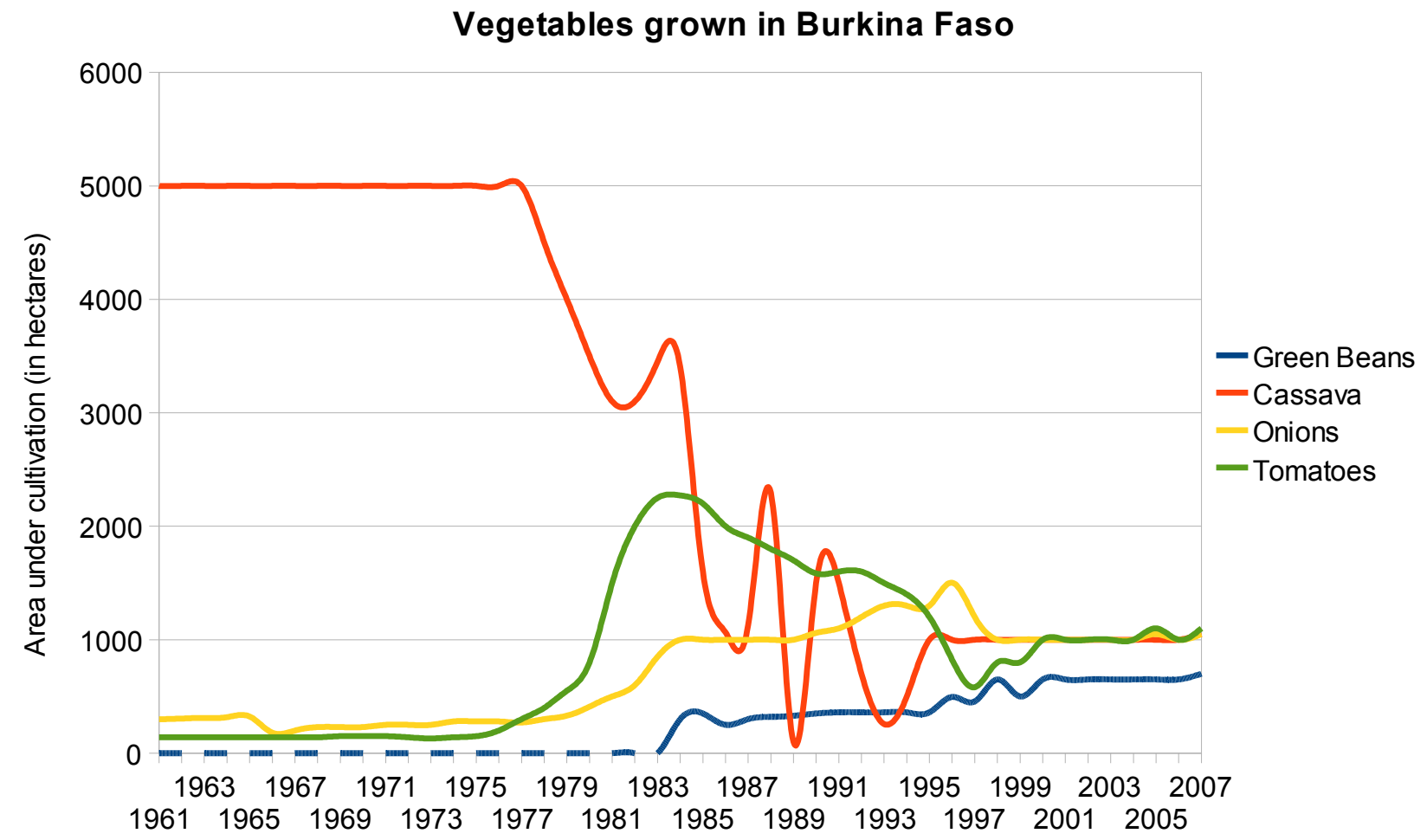

Figure 90: Area cultivated with vegetables ${ }^{1325}$

The relative importance of Burkina Faso's major crops is illustrated by table 57 (seperata data for Kossi are not available):

1321 Sys, C.; van Ranst, E.; Debaveye, J. \& Beernaert, F. (1993), p. 42.

1322 Norman, M.J.T.; Pearson, C.J. \& Searle, P.G.E. (1995), p. 278.

1323 Sys, C.; van Ranst, E.; Debaveye, J. \& Beernaert, F. (1993), p. 42.

1324 Norman, M.J.T.; Pearson, C.J. \& Searle, P.G.E. (1995), pp. 308; 312.

1325 http://faostat.fao.org/site/567/default.aspx, accessed 15/05/2009. 


\begin{tabular}{|l|r|r|r|r|r|}
\hline \multicolumn{7}{|c|}{ Area under cultivation (in 1000 hectares) } \\
\hline & 1967 & 1977 & 1987 & 1997 & \multicolumn{1}{|c|}{2007} \\
\hline $\begin{array}{l}\text { Sorghum } \\
\text { Sorghum bicolor }\end{array}$ & 1312 & 1064 & 1176 & 1386 & $1450^{*}$ \\
\hline $\begin{array}{l}\text { Millet } \\
\text { Pennisetum glaucum }\end{array}$ & 700 & 900 & 1168 & 1155 & $1250^{*}$ \\
\hline $\begin{array}{l}\text { Maize } \\
\text { Zea mays }\end{array}$ & 225 & 90 & 176 & 241 & 490 \\
\hline $\begin{array}{l}\text { Peanuts } \\
\text { Arachis hypogaea }\end{array}$ & 142 & $165^{*}$ & 193.9 & 232.7 & $285^{*}$ \\
\hline $\begin{array}{l}\text { Rice } \\
\text { Oryza sativa }\end{array}$ & 35,8 & 31,9 & 18,9 & 56,8 & $50,0^{*}$ \\
\hline $\begin{array}{l}\text { Sesame } \\
\text { Sesamum indicum }\end{array}$ & $20,0^{*}$ & $25,0^{*}$ & 39,6 & 24 & 46,4 \\
\hline $\begin{array}{l}\text { Yam } \\
\text { Dioscorea rotundata }\end{array}$ & $6,0^{*}$ & 5,5 & 10,9 & $5,3^{*}$ & $2,6^{*}$ \\
\hline $\begin{array}{l}\text { Cassava (=manioc; tapioka) } \\
\text { Manihot esculenta }\end{array}$ & $5,0^{*}$ & $5,0^{*}$ & 1,1 & 1 & 1,1 \\
\hline * FAO estimate & & & & & \\
\hline
\end{tabular}

Table 57: Area cultivated by important crops (Burkina Faso) ${ }^{1326}$

The different ecological settings typically chosen for certain agricultural crops can be indicators whether a certain locality is a suitable mosquito habitat. Moreover, within Kossi province some of the crops require irrigation, since the region's average annual rainfall is insufficient for their rainfed cultivation. Depending on the irrigation techniques used, vector habitats may be created in regions previously devoid of them. Therefore, the spatial pattern of crop cultivation and changes therein may be one of the drivers of malaria transmission.

1326 http://faostat.fao.org/, accessed 02/10/08. 
Geographic Determinants of Malaria Transmission

\begin{tabular}{|c|c|}
\hline Agro-ecological conditions & Crops indicating the condition \\
\hline \multicolumn{2}{|c|}{ Conditions unsuitable for mosquito breeding / survival } \\
\hline $\begin{array}{l}\text { Permeable (e.g. sandy) soils } \\
\text { preventing waterlogging }\end{array}$ & $\begin{array}{l}\text { Millet, cotton, peanuts, sesame, } \\
\text { cassava, yam }\end{array}$ \\
\hline Drought-prone regions & Cassava \\
\hline \multicolumn{2}{|c|}{ Conditions favoring mosquito breeding and/or survival } \\
\hline Irrigation & Maize, rice, [cotton], sugarcane, yam \\
\hline Soils prone to waterlogging & Sorghum, rice \\
\hline
\end{tabular}

Table 58: Important crops and agro-ecological conditions for their cultivation in Kossi

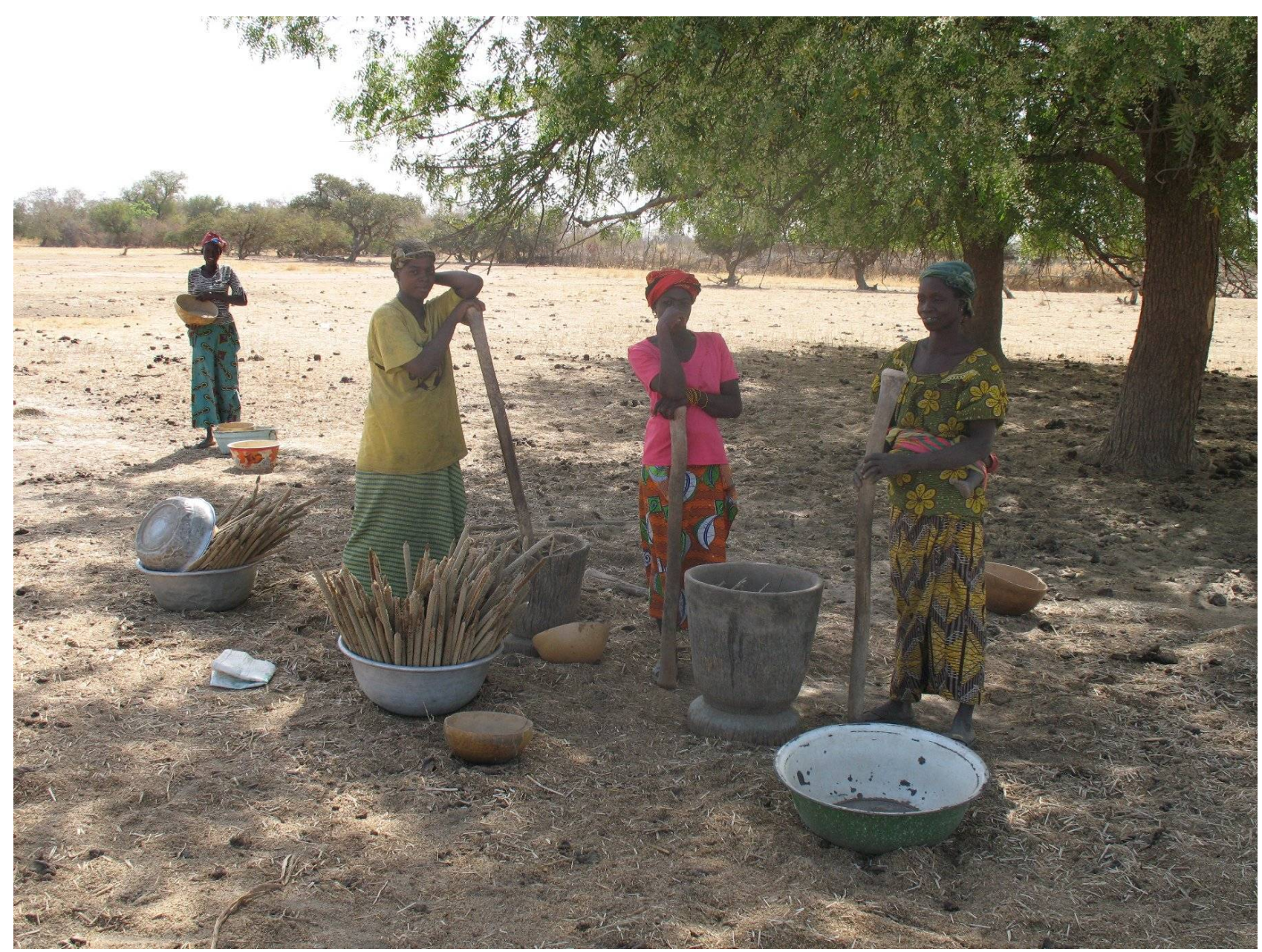

Figure 91: Women grinding pearl millet (Pennisetum glaucum) 


\subsubsection{Animal Husbandry and Agro-Pastoralism}

Animal husbandry accounts for $12 \%$ of Burkina Faso's GDP, and is the key activity for some of the country's ethnic groups, such as the Peulh in Kossi. Kossi province falls into a zone of relatively intensive agro-pastoralism (see figure 80 ) where both animal husbandry and farming are important sources of income. By international standards, animal densities are low and the landuse is of an extensive type, but Burkina Faso's national government supports an intensification particularly of dairy cattle rearing. The region's cattle density is between 15 and 20 animals $/ \mathrm{km}^{2}$, as opposed to higher densities found towards the northeast. ${ }^{1327}$ Pigs, goats and chickens are often kept by villagers, and their numbers have increased considerably in recent decades (for national data, see table 59). The animal density -particularly in comparison with the population density- may be an important factor influencing the frequency of infective contacts between the human population and potential vectors of disease such as anopheline mosquitoes. In areas of higher animal densities - such as villages primarily inhabited by cattle herders - there may be a lower risk of malarial infections than in villages with few animals. Unless the local mosquito population is strongly anthropophilic, reared animals may function as alternative sources of blood, thereby diverting anophelines from humans and reducing the risk of malarial infections.

\begin{tabular}{|l|r|r|r|r|r|}
\hline Livestock & Number in thousands & & \\
\hline Camels & 1967 & 1977 & 1987 & 1997 & 2007 \\
\hline Cattle & $12,0^{*}$ & $11,0^{*}$ & 12 & 13,5 & $15,8^{*}$ \\
\hline Chickens & 2400 & 2600 & 3711 & $5561^{*}$ & $8764^{*}$ \\
\hline Goats & $9750^{*}$ & 10400 & 16052 & $20733^{*}$ & $27150^{*}$ \\
\hline Pigs & $2200^{+}$ & 2556 & 5889 & $8259^{*}$ & $11427^{*}$ \\
\hline Sheep & 130 & 159 & 459 & $1064^{*}$ & $2763^{*}$ \\
\hline${ }^{*}=$ FAO estimate; ${ }^{+}=$unofficial figure & $1500^{*}$ & 2300 & 4619 & $5861^{*}$ & $7321^{*}$ \\
\hline
\end{tabular}

Table 59: Development of livestock in Burkina Faso ${ }^{1328}$

Cattle and goats are found widely in Kossi Province and typically exploit the environment in an extensive and complimentary way. ${ }^{1329}$

1327 YAHMED, D.B. (2005), p. $98 \mathrm{f}$.

1328 http://faostat.fao.org/site/573/default.aspx, accessed 30/07/2009.

1329 Devineau, J.L. \& Fournier, A. (2007), p. 352. 


\subsubsection{Agricultural Production and Nutrition}

The per capita food production in Burkina Faso is currently growing at about $1.3 \%$ per year. ${ }^{1330}$ However, the country's agricultural production does still not meet the demand needed for a balanced supply. Despite the high proportion of the work force employed in agriculture, the per capita production -particularly of meat, vegetables and fruits- falls considerably behind the levels of industrialized nations:

\begin{tabular}{|l|l|l|l|l|}
\hline & $\begin{array}{l}\text { Annual } \\
\text { cereal } \\
\text { production } \\
\text { (per capita) }\end{array}$ & $\begin{array}{l}\text { Annual meat } \\
\text { production } \\
\text { (per capita) }\end{array}$ & $\begin{array}{l}\text { Annual fruit } \\
\text { and } \\
\text { vegetable } \\
\text { production } \\
\text { (per } \\
\text { capita) }\end{array}$ & $\begin{array}{l}\text { Population } \\
\text { engaged in } \\
\text { agriculture }\end{array}$ \\
\hline Burkina Faso & $263 \mathrm{~kg}$ & $17 \mathrm{~kg}$ & $24 \mathrm{~kg}$ & $92.0 \% \%^{1332}$ \\
\hline Germany & $551 \mathrm{~kg}$ & $85 \mathrm{~kg}$ & $63 \mathrm{~kg}$ & $2.4 \%^{1333}$ \\
\hline
\end{tabular}

Table 60: Per capita agricultural production of Burkina Faso and Germany

To avoid food shortages in the future, Burkina Faso's government propagates an intensification of agriculture in the form of irrigation projects. ${ }^{1334}$ Currently, only $0.5 \%$ of the arable land in Burkina Faso are irrigated, but between 1990 and 2003 , this area has grown by about $1.5 \%$ annually. ${ }^{1335}$

Between 2006 and 2007, Burkina Faso's cotton production fell drastically from 649.400 to 434.000 tonnes. This was due to unsuitable weather conditions, a fall of market prices (from 210 FCFA in 2005 to 165 FCFA in 2006 and 145 FCFA per $\mathrm{kg}$ in 2007) and price increases for inputs. Given the importance of cotton as a cash crop, this shortfall in production caused a reduced economic growth in 2007. ${ }^{1336}$

1330 WORLD BANK (2007), p. 326.

1331 WORLD BANK (2007), p. 326.

1332 HAMmer, T. (2005), p. 107.

1333 WORLD BANK (2007), p. 320.

1334 YAHMED, D.B. (2005), p. 96.

1335 WORLD BANK (2007), p. 324.

1336 OeCD \& African Development Bank (2008), pp. 167f;

OeCD \& African Development Bank (2006), p. 151. 


\subsubsection{Education}

Despite great progress in the recent past, Burkina Faso's literacy rate and educational indicators are still among the world's poorest. This also affects the public health situation in the country: people are often unaware of the ways of disease transmission, with 'traditional' concepts of disease sometimes given preference over explanations offered by school medicine.

\subsubsection{Primary, Secondary and Tertiary Education in Burkina Faso}

In spite of a state education system, there is a high illiteracy in Burkina Faso that is estimated to be between $73 \%{ }^{1337}$ and $87 \%{ }^{1338}$. In Kossi province, the illiteracy rate is slightly above the officially declared national average; about $65 \%$ of men and $86.6 \%$ of the women are illiterate. ${ }^{1339}$ About $75 \%$ of the population have no formal education; just around $5 \%$ have attended secondary schools, and less than $0.5 \%$ universities. ${ }^{1340}$ However, the net rates of schooling have been increasing in recent years and are currently around $40 \%$ for primary schooling and between $10 \%^{1341}$ and $20 \%^{1342}$ for secondary schooling.

The provision of technical education is concentrated in Ouagadougou and BoboDioulasso. In other regions, vocational training is essentially poorly organized and essentially provided by vocational training centers (CFPs, Centres pour la formation professionelle). Other institutions tend to have very high registration fees (FCFA $150.000^{1343}$ to FCFA $500.000^{1344}$ per year), making them prohibitively expensive for most Burkinabès. ${ }^{1345}$

In 2007, Burkina Faso's government passed an education act which aims at introducing free schooling up to the age of 16. A test-run began in one département in each province at the start of the 2007/08 academic year; the goal is to create one secondary school in every department of the country. ${ }^{1346}$ In October 2005, the University of Koudougou was opened as the third public university in the country. ${ }^{1347}$

1337 WÜRTHWEIN, R. (2002), p. 117.

1338 OeCD \& African Development Bank (2007), p. 158.

1339 WÜRTHWEIN, R. (2002), pp. 140; 142.

1340 WürthWEIN, R. (2002), p. 144.

1341 OeCD \& African Development Bank (2007), p. 159.

1342 Direction Générale de l'Information et des Statistiques Sanitaires $\left(2009^{1}\right)$, p. 9.

1343 FCFA $150.000 \approx € 228$

1344 FCFA $500.000 \approx € 762$

1345 OeCD \& African Development Bank (2008), p. 175.

1346 OeCD \& African Development Bank (2008), p 178.

1347 OeCD \& African Development Bank (2008), p 178. 


\subsubsection{Health Education and Awareness}

In West Africa, malaria is often not perceived as a vector-borne infectious disease but attributed to direct environmental influences, including rain, cool weather or sunshine. ${ }^{1348}$ Instead of a biomedical explanation, several disease concepts that often represent malarial infections are distinguished in rural Burkina Faso. ${ }^{1349}$

\begin{tabular}{|c|c|c|c|}
\hline Disease concept & Symptoms & $\begin{array}{l}\text { Perceived } \\
\text { causes }\end{array}$ & Usual treatment \\
\hline $\begin{array}{l}\text { Sumaya } \\
\text { "Illness of the } \\
\text { cold" } \\
\text { ( } \approx \text { uncomplicated } \\
\text { malaria) }\end{array}$ & $\begin{array}{l}\text { Fever, weakness, } \\
\text { cold, loss of } \\
\text { appetite, pain, } \\
\text { diarrhea, vomiting }\end{array}$ & $\begin{array}{l}\text { Dirty environment, } \\
\text { coldness, wind; } \\
\text { certain foods (e.g. } \\
\text { too much sugar) }\end{array}$ & $\begin{array}{l}\text { Home treatment } \\
\text { using herbal } \\
\text { drinks/baths, } \\
\text { modern drugs } \\
\text { (paracetamol, } \\
\text { chloroquine) }\end{array}$ \\
\hline $\begin{array}{l}\text { Dusukun yelema } \\
\text { "Displaced heart" }\end{array}$ & $\begin{array}{l}\text { Respiratory } \\
\text { difficulties; } \\
\text { vomiting; } \\
\text { diarrhea; fever; } \\
\text { loss of appetite }\end{array}$ & $\begin{array}{l}\text { Other diseases; } \\
\text { supernatural } \\
\text { factors (which } \\
\text { cause the heart to } \\
\text { shift to an } \\
\text { abnormal position) }\end{array}$ & $\begin{array}{l}\text { Massage and } \\
\text { herbal treatments } \\
\text { by traditional } \\
\text { healers }\end{array}$ \\
\hline $\begin{array}{l}\text { Kono } \\
\text { "Bird illness" } \\
(\approx \text { cerebral } \\
\text { malaria })\end{array}$ & Convulsions, coma & $\begin{array}{l}\text { Birds flying over } \\
\text { sleeping persons }\end{array}$ & $\begin{array}{l}\text { Herbal treatments, } \\
\text { spiritual } \\
\text { incantations, } \\
\text { wearing of feather } \\
\text { chains, massages }\end{array}$ \\
\hline $\begin{array}{l}\text { Djoliban } \\
\text { "Blood is finished" } \\
\text { ( } \approx \text { severe anemia) }\end{array}$ & $\begin{array}{l}\text { Paleness, fever, } \\
\text { vomiting, loss of } \\
\text { appetite }\end{array}$ & $\begin{array}{l}\text { Other illnesses, } \\
\text { over-exertion, } \\
\text { insufficient food }\end{array}$ & $\begin{array}{l}\text { Treatment usually } \\
\text { at formal health } \\
\text { institutions (no } \\
\text { traditional } \\
\text { remedies) }\end{array}$ \\
\hline
\end{tabular}

Table 61: Local concepts of (potential) malarial infections ${ }^{1350}$

Since malaria is often not attributed to mosquito bites, the necessity of antivectorial measures may not be recognized and proper methods of individual prophylaxis not be taken by local populations.

1348 Einterz, E.M. (2003), p. 51.

1349 Beiersmann, C.; Sanou, A.; Wladarsch, E. et al. (2007), doi:10.1186/1475-2875-6-106.

1350 Beiersmann, C.; Sanou, A.; Wladarsch, E. et al. (2007), doi:10.1186/1475-2875-6-106. 


\subsubsection{Public Health Situation}

Despite all progress in recent years, the public health situation in Burkina Faso is still poor, with high and currently rising prevalence rates of malaria being one of the key problems.

\subsubsection{General Public Health Indicators}

There are conflicting estimates regarding Burkina Faso's life expectancy, ranging from 45 years (UN estimate) to 54 years (Institut National de la Statistique et de la Démographie). ${ }^{1351}$

Childhood mortality (i.e. the death rate for children below the age of five years) went down from $219 \%$ to $151 \%$ between 2000 and 2006. A reduction in the mortality rate has been observed for many infectious diseases, and even the prevalence of HIV/AIDS has gone down over the past ten years. In spite of all these advances, however, Burkina Faso will not be able to reach the Millennium Development Goals without a redoubling of the efforts. ${ }^{1352}$ Indeed, many health indicators are still worrying, including the infant mortality rate (i.e. the death rate for children below the age of one year; see figure 92). Governmental health spending in Burkina Faso is in the order of only 9 US-\$ per capita per year. ${ }^{1353}$ However, in recent years Burkina Faso's government has increased the health budget at a rate of nearly $8 \%$ annually. ${ }^{1354}$ 


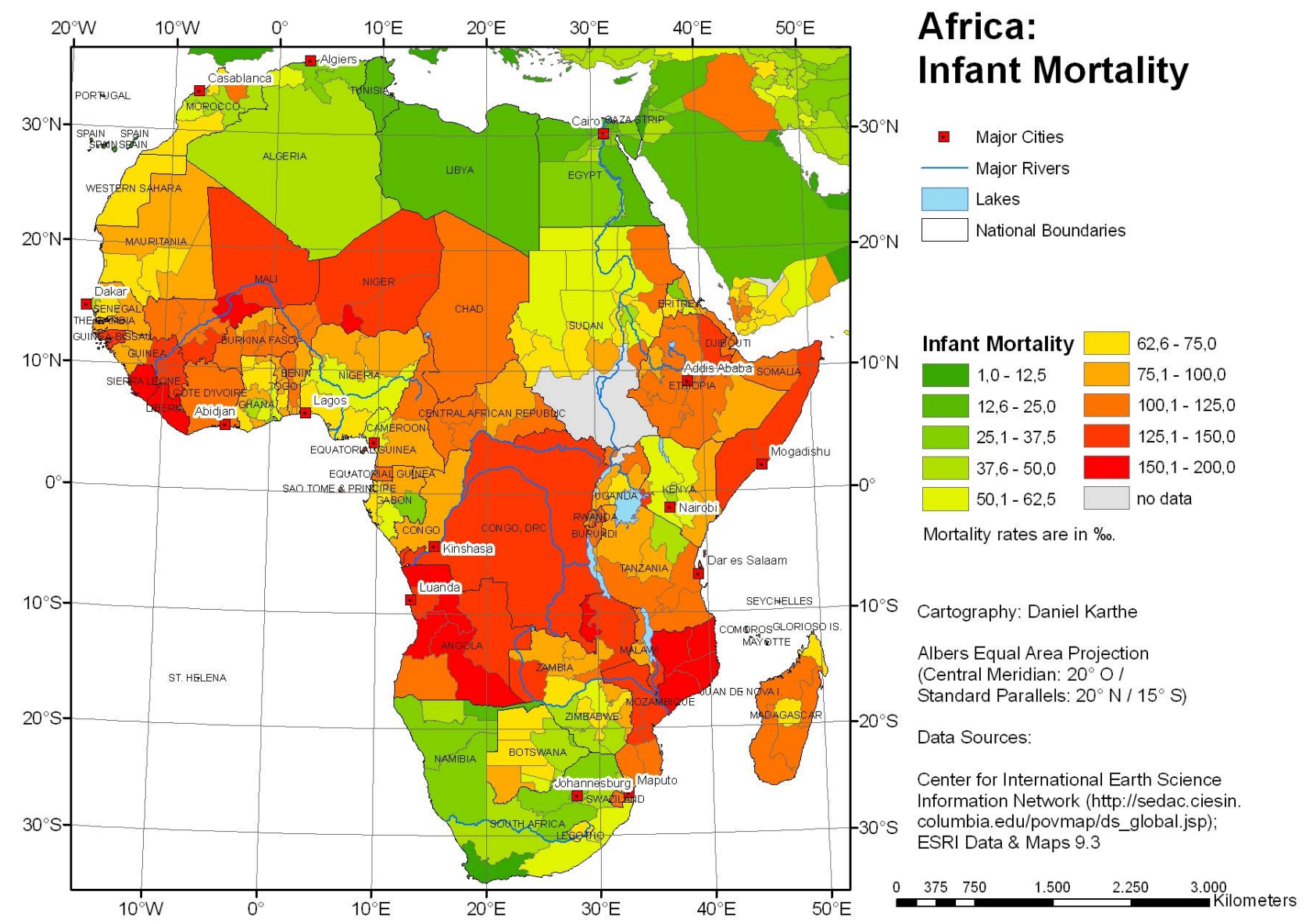

Figure 92: Infant mortality in Africa ${ }^{1355}$

Currently, around $36 \%$ of Burkina Faso's population have access to public health care. Burkina Faso's government defines access to health as the ability to reach any sort of health center within 30 minutes using a mode of transport that is usually available to a locality's residents. However, there are enormous regional disparities with regard to health care: $69,7 \%$ of the urban but only $28,8 \%$ of the rural population have access to health ${ }^{1356}$; around $40 \%$ of all medical professionals are found in the national capital region that is home to only $12 \%$ of Burkina Faso's population. ${ }^{1357}$ Within Burkina Faso, the provinces that form the Boucle du Mouhoun are one of the more disadvantaged peripheral regions (see table 62).

1355 Based on International Earth Science Information Network and ESRI Data \& Maps 9.3.

1356 Direction Générale de L'Information et des Statistiques Sanitaires $\left(2009^{2}\right)$, p. 8.

1357 Direction Générale de L'Information et des Statistiques Sanitaires $\left(2009^{1}\right)$, p. 50. 


\begin{tabular}{|l|l|l|l|}
\hline & Burkina Faso & $\begin{array}{l}\text { Centre } \\
\text { (National capital } \\
\text { region) }\end{array}$ & $\begin{array}{l}\text { Boucle du } \\
\text { Mouhoun }\end{array}$ \\
\hline $\begin{array}{l}\text { Inhabitants per } \\
\text { physician }\end{array}$ & 31144 & 9358 & 65923 \\
\hline $\begin{array}{l}\text { Inhabitants per } \\
\text { nurse }\end{array}$ & 6413 & 3211 & 7513 \\
\hline
\end{tabular}

Table 62: Medical coverage in Burkina Faso: regional disparities ${ }^{1358}$

\subsubsection{Major Public Health Concerns}

Major public health problems include malnutrition, a limited access to safe sanitation and drinking water and a high prevalence of vector-borne infectious diseases, particularly malaria. The relative importance of these problems varies both regionally and in the way they affect different age groups (see figure 93). ${ }^{1359}$

\section{Leading Causes of Death in Nouna DSS (1999-2003)}

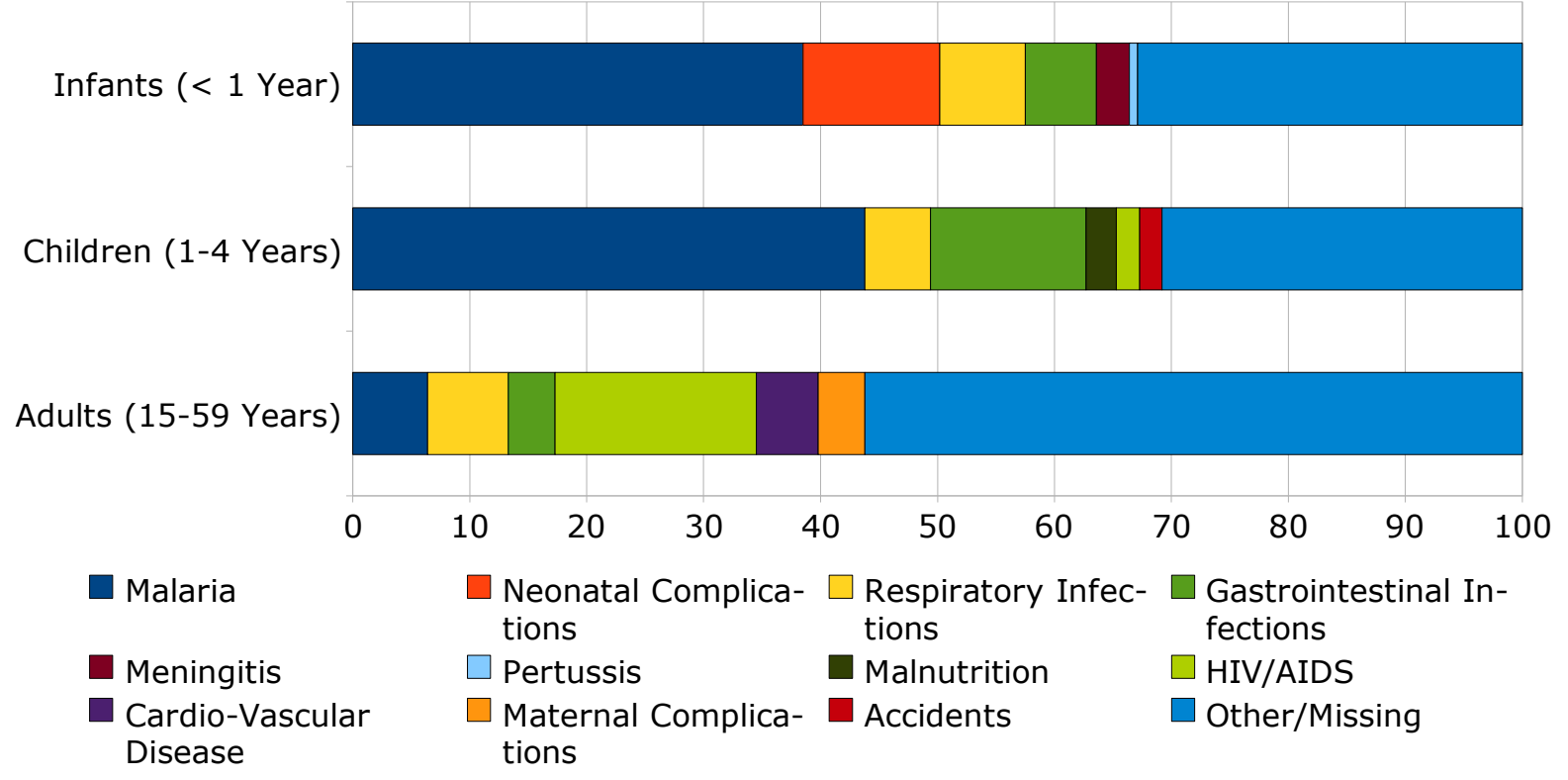

Figure 93: Leading Causes of Death in Nouna DSS (1999-2003) $)^{1360}$

1358 Direction Générale de l'Information et des Statistiques Sanitaires $\left(2009^{1}\right)$, pp. 60-63.

1359 Becher, H.; Kynast-Wolf, G.; Sié, A. et al. (2008), p. 106.

1360 Becher, H.; Kynast-Wolf, G.; Sié, A. et al. (2008), p. 108. 
While the FAO considers around $10 \%$ of Burkina Faso's population to be malnourished ${ }^{1361}$, the situation in the country is considered more alarming by several aid agencies ${ }^{1362}$ and Burkina Faso's Health Ministry. ${ }^{1363}$ It is estimated that half of the deaths in children under the age of five are due to malnutrition, including micro-nutritional deficiencies. $19 \%$ of all children below the age of five suffer from acute malnutrition (emaciation) and $39 \%$ from chronic malnutrition (growth retardation). This situation has been deteriorating during the past ten years. ${ }^{1364}$ In a national survey carried out in $2007,49,5 \%$ of all one year-old children showed signs of growth retardation. ${ }^{1365}$ In Kossi Province, malnutrition is a major determinant of childhood mortality. ${ }^{1366}$ One particular problem is anemia which affects $91 \%$ of all children below the age of five and more than half of all reproductive-age women. ${ }^{1367}$ In 2004/05, a shortfall in the food-crop harvests created a national crisis which lasted until September 2005 when above-normal rains resulted in good crop yields. Particularly badly hit were the Sahel region provinces (Soum, Seno, Oudalan) which had already been hit by a locust invasion in $2004 .{ }^{1368}$

Even though Burkina Faso's government adopted an action plan for drinking water and sanitation in 2003, improvements have been largely restricted to urban areas, where $88.5 \%$ of all households have access to running water. In rural areas, the respective figure is $4 \%$. Another $78 \%$ have access to water from wells (down from $92 \%$ in 1999), whereas the proportion of people using surface water has risen to more than $17 \%$. Less than half of the rural population has a water source located within a 15 minute walk of their home, and $85 \%$ have no access to toilets of any description. This situation increases the spread of infectious diseases. ${ }^{1369}$

1361 http://www.fao.org/es/ess/faostat/foodsecurity/, accessed 29.05.2009.

1362 EINEDER, F. (2009), p. 10.

1363 Direction Générale de l'Information et des Statistiques Sanitaires $\left(2009^{1}\right)$, p. 4.

1364 OeCD \& African Development Bank (2007), p. 159.

1365 Direction Générale de l'Information et des Statistiques Sanitaires $\left(2009^{2}\right)$, p. 6.

1366 Hammer, G.P.; Somé, F.; Müller, O. et al. (2006), doi:10.1186/1475-2875-5-47.

1367 OeCD \& African Development Bank (2007), p. 159.

1368 OeCD \& African Development Bank (2006), pp. $151 \mathrm{f}$.

1369 OeCD \& African Development Bank (2007), p. 157. 


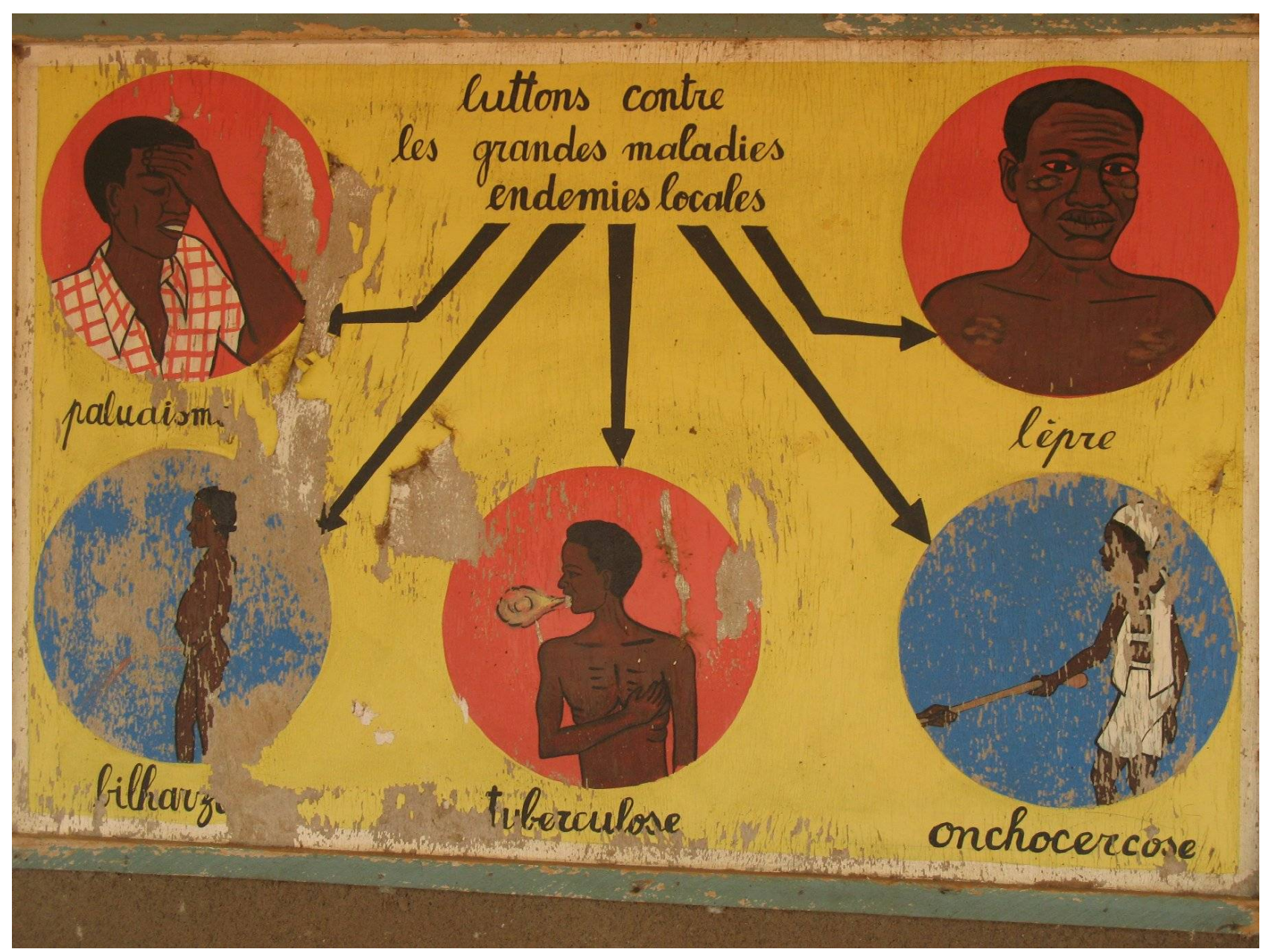

Figure 94: Signboard at Nouna District Hospital

Several infectious diseases are endemic in the study area (see figure 94), including malaria, schistosomiasis (bilharziosis), tuberculosis, onchocerciasis and leprosy (the latter two being rare). Out of these five diseases, three are vector-borne. Their relative importance is illustrated by table 63, which gives an overview of the disease burdens of major infectious diseases in Burkina Faso and Sub-Saharan Africa. 


\begin{tabular}{|c|c|c|c|c|}
\hline \multirow[t]{2}{*}{ Disease } & \multicolumn{2}{|c|}{ Burkina Faso } & \multicolumn{2}{|c|}{ Sub-Sahara Africa } \\
\hline & $\begin{array}{l}\text { Annual } \\
\text { number of } \\
\text { deaths }^{1370}\end{array}$ & $\begin{array}{l}\text { Quality of } \\
\text { life lost, } \\
\text { expressed } \\
\text { in } \\
\text { DALYs }{ }^{1371}\end{array}$ & $\begin{array}{l}\text { Annual } \\
\text { number of } \\
\text { deaths }^{1372}\end{array}$ & $\begin{array}{l}\text { Quality of } \\
\text { life lost, } \\
\text { expressed } \\
\text { in } \\
\text { DALYs }{ }^{1373}\end{array}$ \\
\hline \multicolumn{5}{|c|}{ Vector-borne infectious diseases } \\
\hline Malaria & 25.700 & 927.000 & 1.093 .000 & 35,447 mio. \\
\hline Schistosomiasis & 800 & 36.000 & 2.000 & 1,184 mio. \\
\hline Filariasis & 200 & 49.000 & 0 & 1,656 mio. \\
\hline Trypanosomiasis & 500 & 17.000 & 48.000 & 1,310 mio. \\
\hline Leishmaniasis & N/A & 3.000 & 8.000 & 0,312 mio. \\
\hline \multicolumn{5}{|c|}{ Other communicable diseases } \\
\hline HIV/AIDS & 31.900 & 953.000 & 2.058 .000 & 56,820 mio. \\
\hline Tuberculosis & 3.800 & 105.000 & 317.000 & 8,084 mio. \\
\hline Diarrheal diseases & 21.800 & 717.000 & 712.000 & 22,046 mio. \\
\hline $\begin{array}{l}\text { "Childhood" diseases } \\
\text { (e.g. measles, pertussis) }\end{array}$ & 7.100 & 263.000 & 745.000 & 23,198 mio. \\
\hline Meningitis & 2.000 & 97.000 & 23.000 & 0,941 mio. \\
\hline Hepatitis B \& C & 1.100 & 35.000 & 29.000 & 0,753 mio. \\
\hline $\begin{array}{l}\text { Helminthic infections } \\
\text { (ascariasis, hookworm } \\
\text { infections) }\end{array}$ & 100 & 40.000 & 4.000 & 0,905 mio. \\
\hline
\end{tabular}

Table 63: Mortality and morbidity burden of important infectious diseases in Burkina Faso

Besides HIV/AIDS and gastro-intestinal infections, malaria is the main cause of morbidity and mortality in Burkina Faso. In Nouna Health District (i.e. Kossi Province), malaria is the most important cause of mortality for children below the age of five years (see figure 95 ). ${ }^{1374}$

1370 http://www.who.int/evidence/bod; accessed 22/11/2007.

1371 http://www.who.int/evidence/bod; accessed 22/11/2007.

1372 Lopez, A.; Mathers, C.D.; Ezzati, M. et al. (ed.) (2006), p. 162.

1373 Lopez, A.; Mathers, C.D.; Ezzati, M. et al. (ed.) (2006), p. 216.

1374 Tipke, M.; Diallo, S.; Coulibaly, B. et al. (2008), doi:10.1186/1475-2875-7-95. 


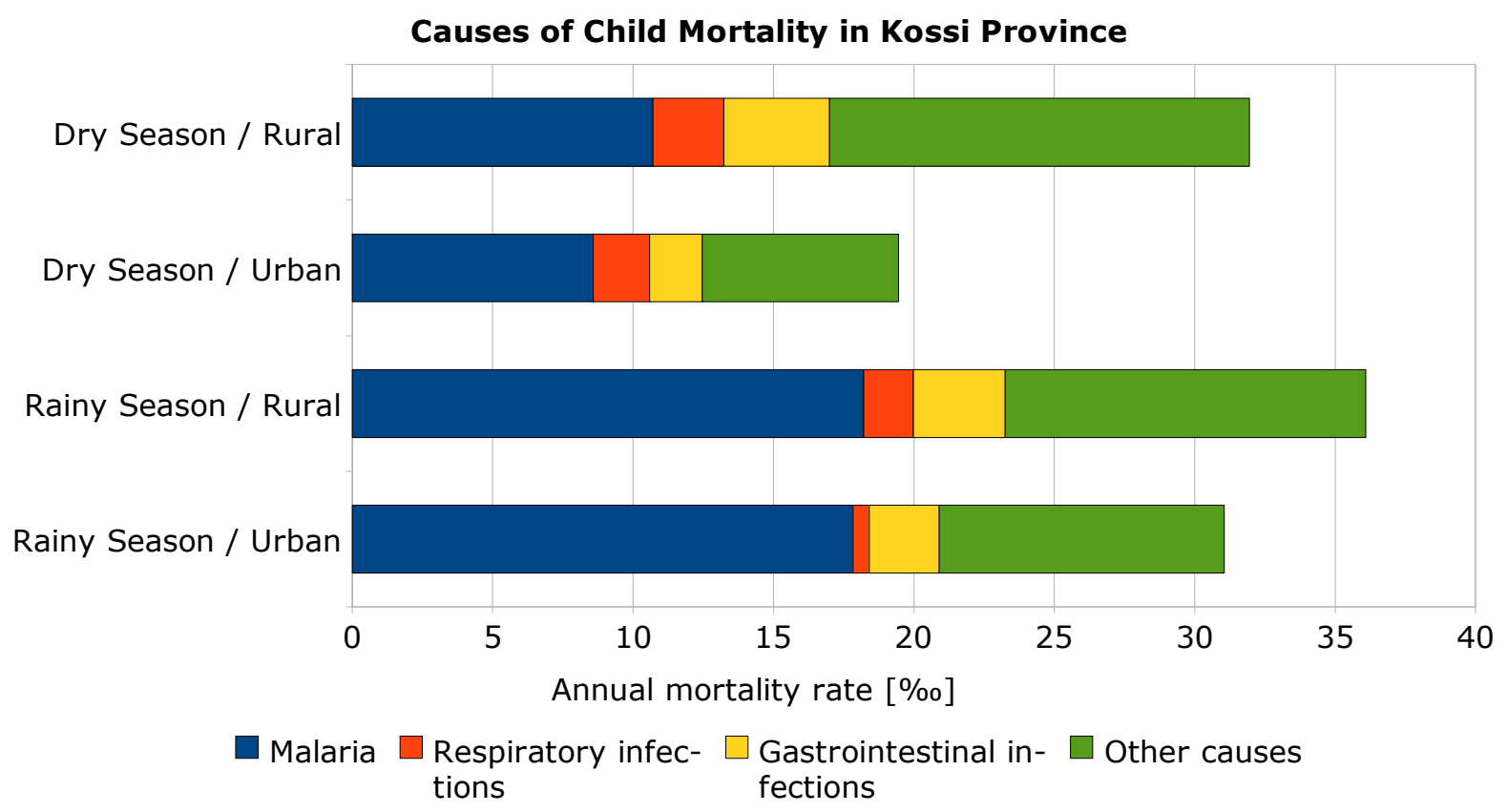

Figure 95: Causes of mortality among children under five (Nouna DSS, 1999-2003) 1375 $^{1375}$

According to the health practitioner in charge of Wèrèbèrè CSPS, the three diseases which caused the highest frequencies of consultation are malaria $(31,56 \%)$, respiratory infections (excluding tuberculosis, $26,43 \%)$ and diarrhea $(6,17 \%)$.

\subsubsection{Public Health Infrastructure}

Burkina Faso is divided into 13 administrative health regions, which comprise 63 health districts overall ${ }^{1376}$, each covering a population of 200 to 300 thousand individuals. At least one health care facility in each district is a hospital with surgery capacities ${ }^{1377}$ (CMA or "Centre médical avec antenne chirurgicale"). The country has a total of 3 teaching hospitals, 11 regional hospitals (CHR or "Centre hospitalier régional") and 55 district hospitals. While the regional hospitals are staffed by specialist physicians, district hospitals are usually run by nurses supervised by a few physicians. Four research centers in the country focus on malaria: the Centre National de Recherche et de Formation sur le Paludisme (CNRFP) and the Institut de Recherche en Sciences de la Santé (IRSS) in Ouagadougou; the Centre Muraz in Bobo-Dioulasso and

1375 Hammer, G.P.; Somé, F.; Müller, O. et al. (2006), doi:10.1186/1475-2875-5-47.

1376 Direction Générale de L'Information et des Statistiques Sanitaires $\left(2009^{1}\right)$, p. 10.

1377 WürthWEin, R. (2002), p. 96. 
the Centre de Recherche en Santé de Nouna (CRSN) in Nouna. ${ }^{1378}$ Privatesector health care facilities are concentrated in Ouagadougou and BoboDioulasso. Moreover, traditional health practitioners are officially recognized by Burkina Faso's Health Ministry. ${ }^{1379}$

Burkina Faso's health districts themselves are subdivided into smaller areas organized around a hospital or a so-called Centre de Santé et de Promotion Sociale (CSPS), the basic health care facility in the Burkinian health system. ${ }^{1380}$ The number of CSPS has nearly doubled since 1998, indicating that great progress has been made in the field of access to basic health care. ${ }^{1381}$ In 2008, one CSPS covered an average population of 9692 persons. ${ }^{1382}$ The areas served by the CSPS of Lékuy, Wèrèbèrè and Toni are presented as examples for which both the actual malaria transmission situation and risk potentials will be described in the following sections.

Lékuy CSPS is located in the southeast of Kossi, close to the main road connecting Nouna with Dédougou and Ouagadougou. While much of the region can be characterized as dry savanna, the Mouhoun forms its eastern border. The center serves the smallest population of the three CSPS presented here (about 4864 persons in 2008), but the seven villages falling into the zone of its responsibility are spread over a fairly large area. In a region where public transport is scarce and the private ownership of motorized vehicles virtually non-existent, this situation means that access of villagers to the CSPS is rather difficult. Among the villages served by the CSPS of Lékuy, Kodougou will be dealt with in more detail.

1378 Kouyaté, B.; Sié, A.; Yé, M. et al. (2007), p. 998.

1379 Direction Générale de L'Information et des Statistiques Sanitaires $\left(2009^{1}\right)$, pp. $10 f$.

1380 WürthWEIN, R. (2002), p. 96.

1381 Direction Générale de l'Information et des Statistiques Sanitaires $\left(2009^{2}\right)$, p. 4.

1382 Direction Générale de l'Information et des Statistiques Sanitaires $\left(2009^{1}\right)$, p. 6. 
Chapter 3 - Case Study: Malaria in Kossi Province

\begin{tabular}{|l|l|r|r|r|}
\hline Village & $\begin{array}{l}\text { Distance } \\
\text { from Lékuy }\end{array}$ & $\begin{array}{l}\text { Population } \\
\mathbf{2 0 0 6}\end{array}$ & $\begin{array}{l}\text { Population } \\
\mathbf{2 0 0 7}\end{array}$ & $\begin{array}{l}\text { Population } \\
\mathbf{2 0 0 8}\end{array}$ \\
\hline Lékuy & & 924 & 948 & 973 \\
\hline Borakuy & $12 \mathrm{~km}$ & 404 & 415 & 426 \\
\hline $\begin{array}{l}\text { Kodougou } \\
\text { Mossi }\end{array}$ & $26 \mathrm{~km}$ & 1003 & 1029 & 1056 \\
\hline $\begin{array}{l}\text { Kodougou } \\
\text { Bobo }\end{array}$ & $25 \mathrm{~km}$ & 449 & 461 & 473 \\
\hline Nokuy Mossi & $25 \mathrm{~km}$ & 683 & 701 & 719 \\
\hline Nokuy Badala & $29 \mathrm{~km}$ & 696 & 714 & 733 \\
\hline Biron Badala & $27 \mathrm{~km}$ & 460 & 472 & 484 \\
\hline Total & & $\mathbf{4 6 2 0}$ & $\mathbf{4 7 4 0}$ & $\mathbf{4 8 6 4}$ \\
\hline
\end{tabular}

Table 64: Villages covered by CSPS Lékuy ${ }^{1383}$

Wèrèbèrè CSPS is located in the far northeast of Kossi, fairly close to the border between Burkina Faso and Mali. The five villages served by the CSPS have a total population of around 6749 (in mid-2008), with the case study village of Illa being the northeastern region's major settlement. Many 'roads' in northeastern Kossi become impassable during the rainy season, making access to the CSPS considerably more difficult than in other parts of the province. Moreover, Illa is located in close proximity to the Sourou and one of its tributaries, with swampy conditions and irrigated agriculture found around their banks.

\begin{tabular}{|l|l|r|r|r|}
\hline Village & $\begin{array}{l}\text { Distance } \\
\text { from } \\
\text { Wèrèbèrè }\end{array}$ & $\begin{array}{l}\text { Population } \\
\text { 2006 }\end{array}$ & $\begin{array}{l}\text { Population } \\
\mathbf{2 0 0 7}\end{array}$ & $\begin{array}{l}\text { Population } \\
\mathbf{2 0 0 8}\end{array}$ \\
\hline Wèrèbèrè & & 1204 & 1235 & 1267 \\
\hline Koubé & $8 \mathrm{~km}$ & 746 & 766 & 786 \\
\hline Weresse & $18 \mathrm{~km}$ & 1440 & 1478 & 1517 \\
\hline Kinséré & $22 \mathrm{~km}$ & 1086 & 1114 & 1143 \\
\hline Illa & $15 \mathrm{~km}$ & 1934 & 1984 & 2036 \\
\hline Total & & $\mathbf{6 4 1 0}$ & $\mathbf{6 5 7 7}$ & $\mathbf{6 7 4 9}$ \\
\hline
\end{tabular}

Table 65: Villages covered by CSPS Wèrèbèrè ${ }^{1384}$

1383 Data from internal records of CSPS Lékuy.

1384 Data from internal records of CSPS Wèrèbèrè. 
Due to limited access to Wèrèbèrè CSPS during the rainy season, some patients may resort to the CSPS at Bomborokuy or Barani; others may not visit any CSPS due to flooded roads.

In the southwestern part of Kossi, Toni CSPS is located in an environment that is characteristic for much of the province: dry savanna and the complete absence of permanent bodies of water. The CSPS covers a larger population within a more compact area than the CSPS at Lékuy and Wèrèbèrè. Toni itself is the study village and similar in size to two other settlements in the region, namely Kamadena and Kermena.

\begin{tabular}{|l|l|r|r|r|r|}
\hline Village & $\begin{array}{l}\text { Distance } \\
\text { from Toni }\end{array}$ & $\begin{array}{l}\text { Population } \\
\mathbf{2 0 0 6}\end{array}$ & $\begin{array}{l}\text { Population } \\
\mathbf{2 0 0 7}\end{array}$ & $\begin{array}{l}\text { Population } \\
\mathbf{2 0 0 8}\end{array}$ \\
\hline Toni & & 2162 & 2218 & 2276 \\
\hline Dembèlela & $6 \mathrm{~km}$ & 451 & 463 & 475 \\
\hline Kermena & $6 \mathrm{~km}$ & 2407 & 2470 & 2534 \\
\hline Kamadena & $6 \mathrm{~km}$ & 2140 & 2196 & 2253 \\
\hline Doukoura & $9 \mathrm{~km}$ & 405 & 415 & 426 \\
\hline Total & & $\mathbf{7 5 6 5}$ & $\mathbf{7 7 6 2}$ & $\mathbf{7 9 6 4}$ \\
\hline
\end{tabular}

Table 66: Villages covered by CSPS Toni ${ }^{1385}$
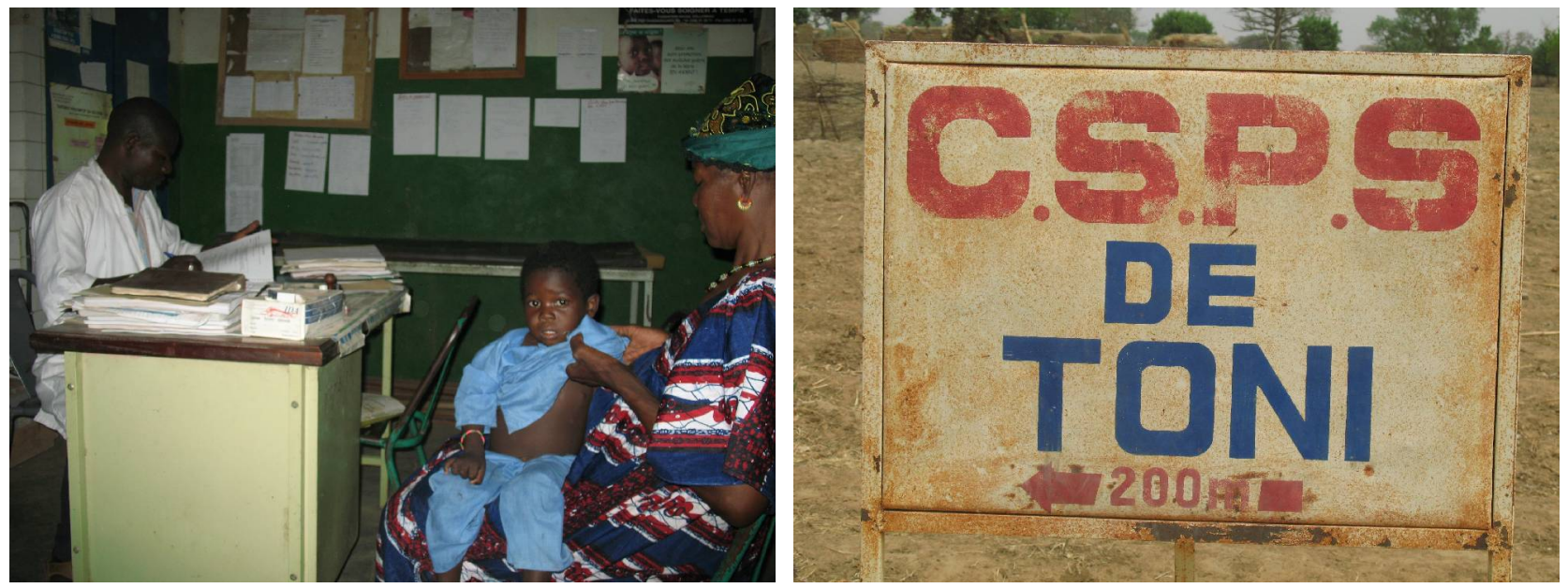

Figure 96: Toni CSPS

Nouna Health District (NHD), which is by its spatial extent identical to Kossi Province, covers a population of just above 295.000 people (2008) living in 274 villages served by 32 rural health centers and the district hospital (CMA) in Nouna. ${ }^{1386}$ The district has a size of $7464 \mathrm{~km}^{2}{ }^{1387}$ The rural health centers (CSPS) typically serve 7 to 10 villages and are usually staffed by two nurses 1385 Data from internal records of CSPS Wèrèbèrè. 
and one obstetrician. ${ }^{1388}$ In Nouna Health District, a Demographic Surveillance System (DSS) has been implemented, surveying the population of four CSPS with a study population of 31.280 inhabitants. ${ }^{1389}$ The research zone of the Centre de Recherche en Santé de Nouna (CRSN) covers a rural area (41 villages, 30.000 people) and an urban area (Nouna town, 25.000 inhabitants)..$^{1390}$

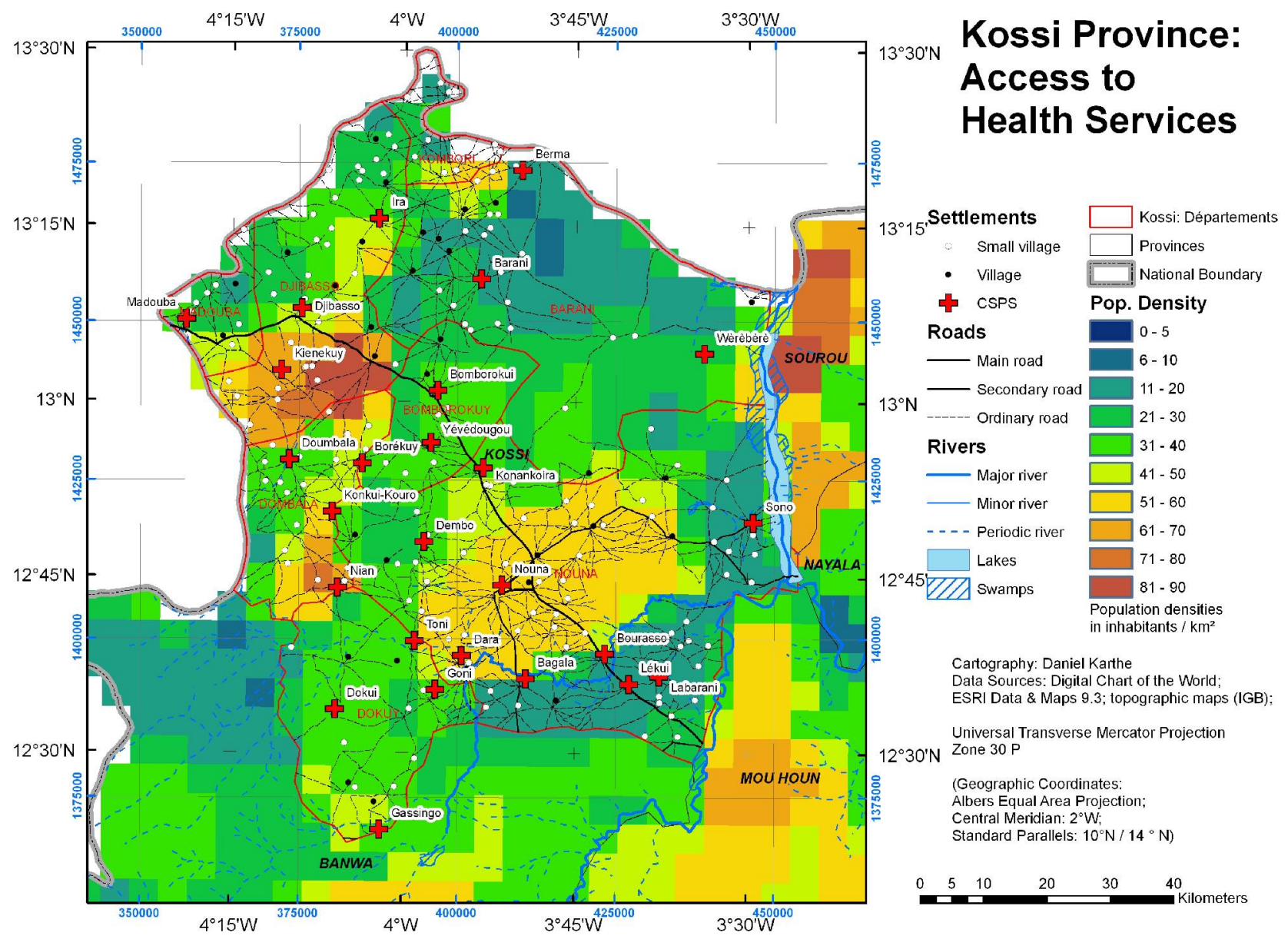

Figure 97: Health services in Kossi Province ${ }^{1391}$

1386 Direction Générale de l'Information et des Statistiques Sanitaires $\left(2009^{2}\right)$, p. 22;

KouYATÉ, B.; SiÉ, A.; Ý́, M. et al. (2007), p. 998.

1387 Pfeiffer, K.; Somé, F.; Müller, O. (2008), p. 419.

1388 Kouyaté, B.; Somé, F.; JaHn, A. et al. (2008), doi:10.1186/1475-2875-7-50.

1389 WüRTHWEIn, R. (2002), p. 96.

1390 Hammer, G.P.; Somé, F.; Müller, O. et al. (2006), doi:10.1186/1475-2875-5-47.

1391 Based on Digital Chart of the World; ESRI Data \& Maps 9.3; topographic maps (IGB) and information provided by CRSN Nouna. 
In rural Burkina Faso, diagnosed cases of malaria can often not be confirmed in the laboratory. Therefore, the national guidelines of the Ministry of Health are usually applied for malaria diagnosis: Uncomplicated malaria is defined as lowgrade fever of $37.5^{\circ} \mathrm{C}$ or more, either alone or combined with other symptoms (headache, back pain, shivering, sweating, muscle pain, nausea and vomiting). At the same time, assessment of patients for other common febrile diseases such as meningitis or respiratory tract infections is recommended. ${ }^{1392}$ However, considerable over-diagnosis, as described for several other African countries, has not been observed by a study in Nouna Health District, and most patients with fever or other symptoms of malaria were actually found to be parasitized. ${ }^{1393}$

Populations affected by malaria sometimes have no access to public health services. In Kossi, only a minority of malaria patients are treated at formal health services. ${ }^{1394}$ Nearly $90 \%$ of the chloroquine treatment for young children is given outside the formal health sector. ${ }^{1395}$ The great majority of children who die from malaria had not visited formal health services. ${ }^{1396}$ Treatment often takes place at home ${ }^{1397}$, and traditional health practitioners (guérriseurs) may be consulted by the local population. These work either as spiritualist healers or as herbalists and their treatment techniques range from the use of plant, animal and mineral products to body massages and spiritual incantations. ${ }^{1398}$

1392 Pfeiffer, K.; Somé, F.; Müller, O. (2008), pp. $418 \mathrm{f}$.

1393 Pfeiffer, K.; Somé, F.; Müller, O. (2008), p. 424.

1394 KouYaté, B.; SiÉ, A.; Ý́, M. et al. (2007), p. 998.

1395 Pfeiffer, K.; Somé, F.; Müller, O. (2008), p. 423.

1396 KouYaté, B.; SiÉ, A.; Yé, M. et al. (2007), p. 998.

1397 KouYaté, B.; Somé, F.; JaHn, A. et al. (2008), doi:10.1186/1475-2875-7-50.

1398 Beiersmann, C.; Sanou, A.; Wladarsch, E. et al. (2007), doi:10.1186/1475-2875-6-106. 


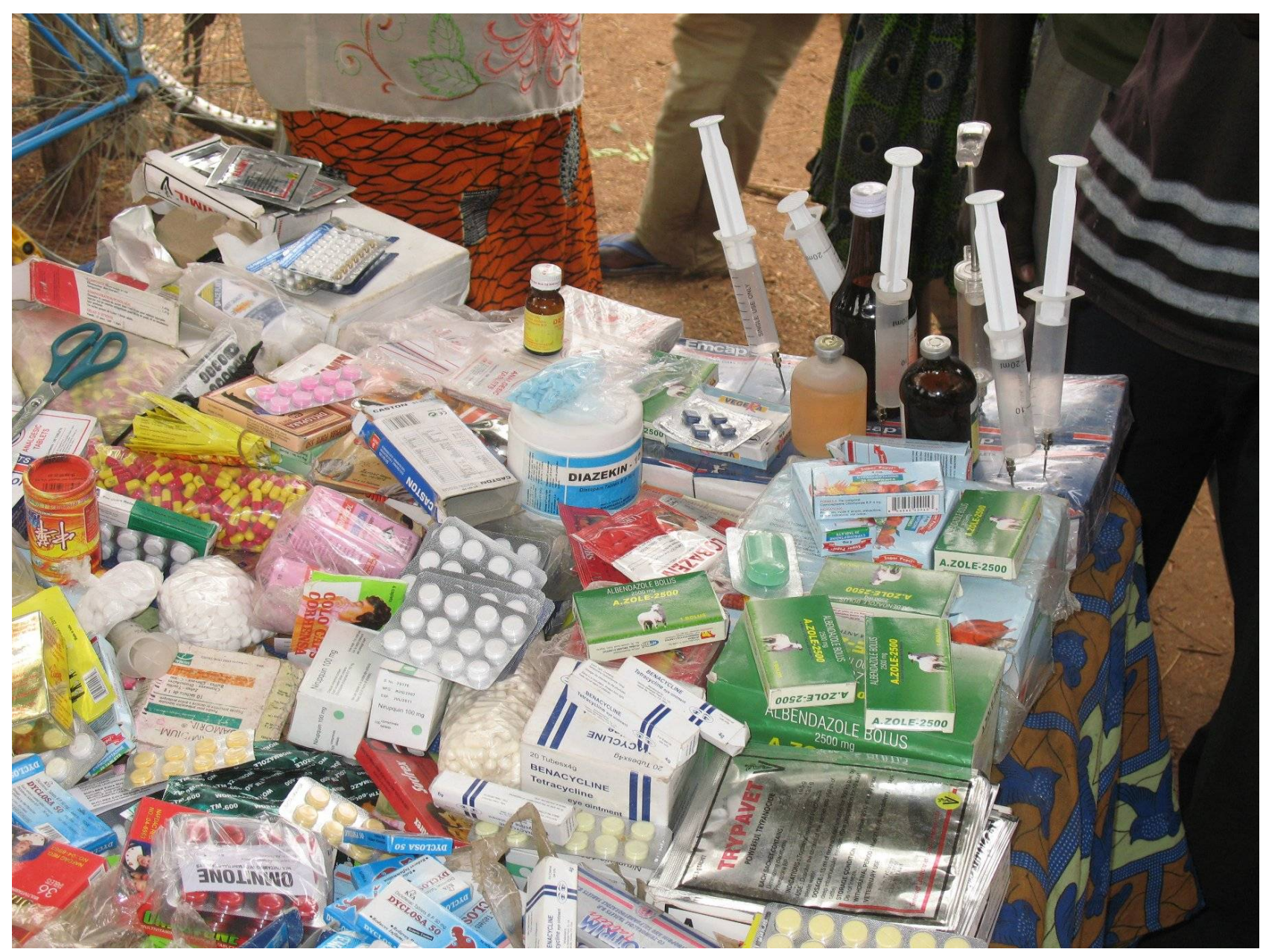

Figure 98: Sale of medicine at a market stall in Djibasso

Antimalarials are available both from formal institutions (public health facilities, private pharmacies) and unlicensed sources (e.g. markets, street vendors; see figure 98). A recent study in Nouna Health District revealed that around $90 \%$ of the drugs available at such informal sources are of substandard quality. ${ }^{1399}$

\subsubsection{Local Malaria Burden}

In Burkina Faso, malaria is by far the most important cause for medical consultations. In $2008,44.5 \%$ of the people seeking medical advice in district hospitals did so because of malaria, as compared to $14.1 \%$ for the second most common reason, infections of the upper respiratory tract. Malaria figures even more prominently among the causes of hospitalizations: in $2008,72.1 \%$

1399 Tipke, M.; Diallo, S.; Coulibaly, B. et al. (2008), doi:10.1186/1475-2875-7-95. 
of all hospital treatments at the district level were because of malaria. ${ }^{1400}$ Moreover, at the national level the malaria burden has risen considerably recent years (see table 67):

\begin{tabular}{|l|l|l|l|l|}
\hline Malaria Incidence & $\mathbf{2 0 0 5}$ & $\mathbf{2 0 0 6}$ & $\mathbf{2 0 0 7}$ & $\mathbf{2 0 0 8}$ \\
\hline Total Cases (B.F.) & 1861158 & 2337550 & 2947011 & 3441982 \\
\hline
\end{tabular}

Table 67: Malaria cases in Burkina Faso, 2005 to $2008^{1401}$

Based on a population of 14,73 millions covered, the average malaria incidence rate in 2008 was around $233,6 \%$. However, this figure varied widely between $133,1 \%$ in Soum Province and $360,9 \%$ in Boulgou Province. In general, malaria incidence rates in 2008 tended to be lower in Burkina Faso's northern provinces while high rates were recorded in southern and southeastern Burkina Faso. The proportion of malaria cases diagnosed as "severe malaria" also varied considerably, ranging from around $2 \%$ to $20 \% .{ }^{1402}$ Figure 99 shows the spatial pattern of malaria distribution in Burkina Faso in 2008.

1400 Direction Générale de l'Information et des Statistiques Sanitaires $\left(2009^{1}\right)$, pp. $17 f$.

1401 Direction Générale de l'Information et des Statistiques Sanitaires $\left(2009^{2}\right)$, p. 2.

1402 Calculated from Direction Générale de L'Information et des Statistiques Sanitaires $\left(2009^{2}\right)$, pp. 29f. \& 133-136. 


\section{Burkina Faso: Malaria Incidence in 2008}

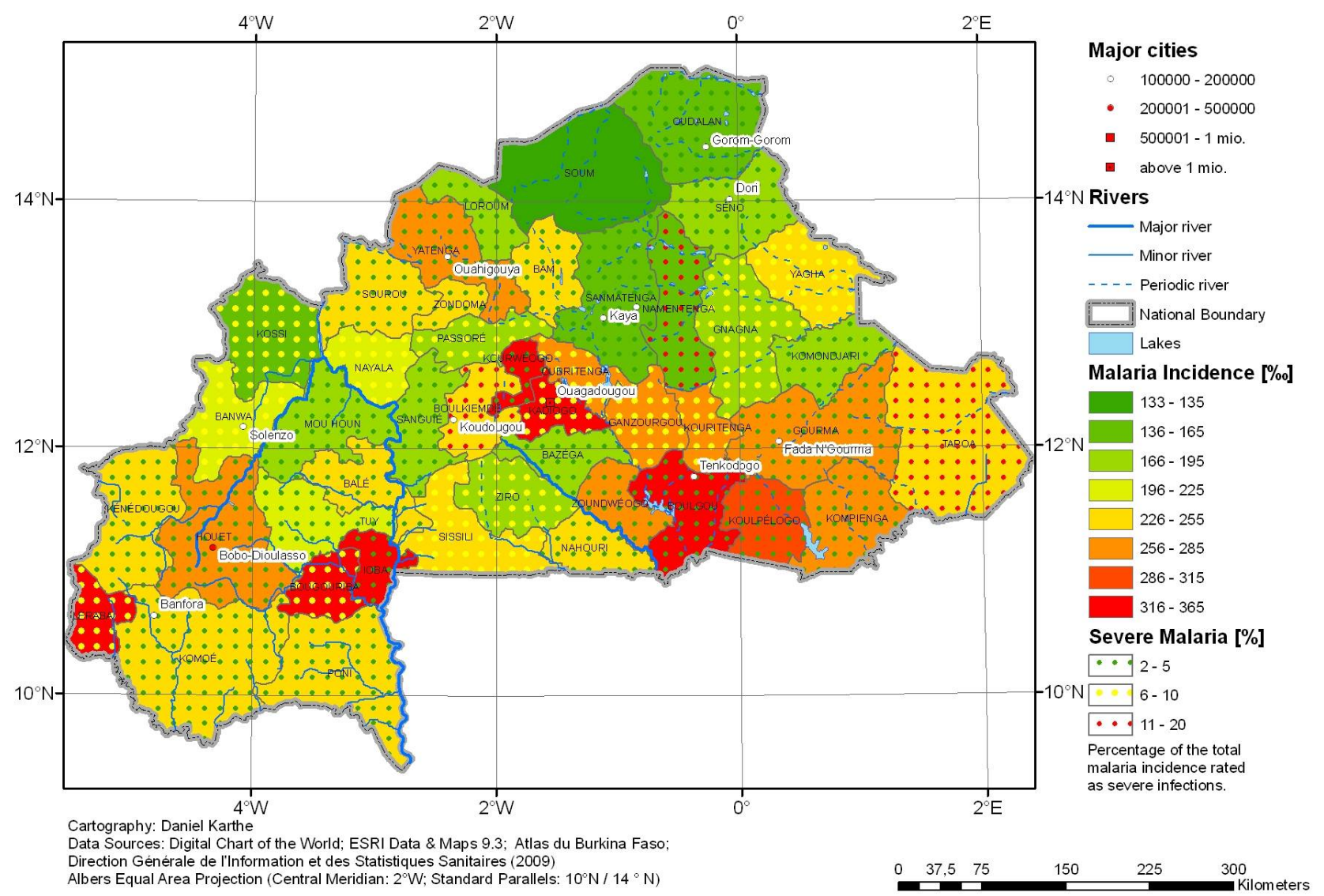

Figure 99: Spatial pattern of malaria incidence in Burkina Faso ${ }^{1403}$

Kossi Province recorded a mean malaria incidence of $137,6 \%$ in 2008 , of which just above $5 \%$ were severe cases. ${ }^{1404}$ Malaria accounts for about $27.7 \%$ of the total burden of disease and $25.9 \%$ of all deaths ${ }^{1405}$, more than any other illness. This is particularly true for the youngest and oldest age groups (see table 68):

\begin{tabular}{|l|c|c|c|c|c|c|c|}
\hline \multicolumn{1}{|c|}{ Age Group } & $\mathbf{0 - 4}$ & $\mathbf{5 - 1 4}$ & $\mathbf{1 5 - 2 9}$ & $\mathbf{3 0 - 4 4}$ & $\mathbf{4 5 - 5 9}$ & $\mathbf{6 0 - 6 9}$ & $\mathbf{7 0 +}$ \\
\hline $\begin{array}{l}\text { \% of all deaths } \\
\text { caused by malaria }\end{array}$ & $29,2 \%$ & $34,0 \%$ & $34,4 \%$ & $18,2 \%$ & $7,5 \%$ & $23,0 \%$ & $26,7 \%$ \\
\hline
\end{tabular}

Table 68: Malaria - the leasing cause of death in most age groups in Nouna ${ }^{1406}$

1403 Based on Direction Générale de l'Information et des Statistiques Sanitaires (2009²);

Digital Chart of the World; ESRI Data \& Maps 9.3 and topographic maps (IGB).

1404 Calculated from Direction Générale de L'Information et des Statistiques Sanitaires $\left(2009^{2}\right)$, pp. 29f. \& 133-136.

1405 WÜRTHWEIN, R. (2002), pp. $100 \& 103$.

1406 WürTHWEIN, R. (2002), p. 110. 
The malaria mortality burden is not only unevenly distributed between age groups, but the percentage of deaths caused by malaria also varies seasonally (see figure 100). At the height of the rainy season, malaria is responsible for more than $70 \%$ of all infant and childhood mortality, a figure that reduces to around $40 \%$ to $50 \%$ during the dry season. For adults, by contrast, malaria is a much less important cause of death.

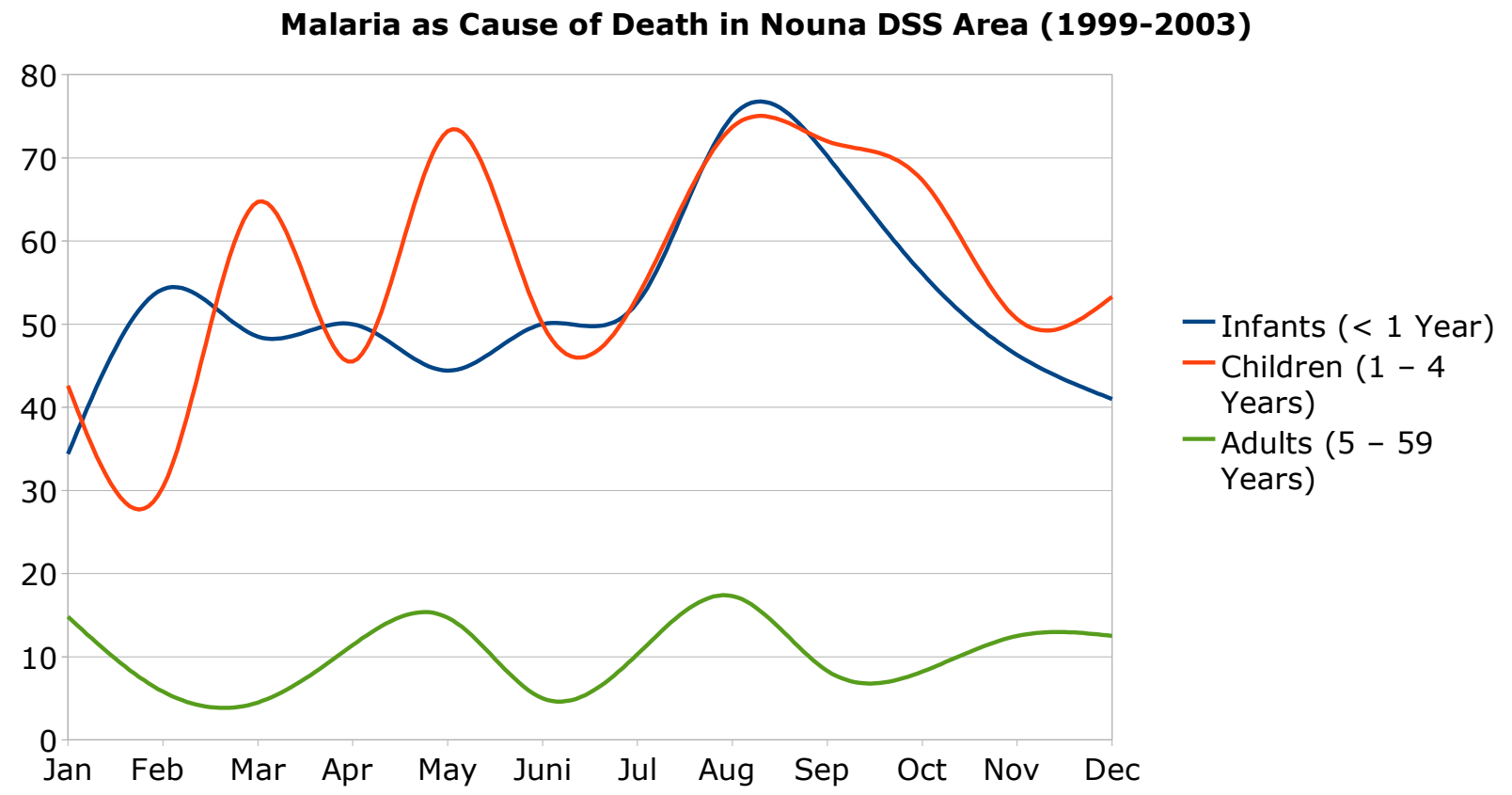

Figure 100: Malaria as cause of death in Nouna DSS Area (1999-2003) ${ }^{1407}$

Malaria is a major economic problem: households in Nouna Health District spend around US\$ 2 to treat one fever episode. Considering that the average child suffers about 6 fever episodes per year, costs for presumptive malaria treatment can easily become prohibitive for local families. The officially recommended ACT therapy even comes at a price of US\$ 6.50 for one course of treatment. ${ }^{1408}$ Moreover, chloroquine resistance was first reported in Burkina Faso in 1988 but has increased rapidly since then. ${ }^{1409}$ In Kossi Province, treatment failure rates of chloroquine, until recently the official first-line treatment for uncomplicated malaria, have recently reached alarming levels (53\% in Nouna town). ${ }^{1410}$ Nevertheless, chloroquine remains the de facto first line treatment. ${ }^{1411}$

1407 Becher, H.; Kynast-Wolf, G.; Sié, A. et al. (2008), p. 108.

1408 KouYaté, B.; Sié, A.; Yé, M. et al. (2007), p. 998.

1409 KouYaté, B.; Sié, A.; Yé, M. et al. (2007), p. 997.

1410 Hammer, G.P.; Somé, F.; Müller, O. et al. (2006), doi:10.1186/1475-2875-5-47.

1411 KouYaté, B.; Sié, A.; Yé, M. et al. (2007), p. 999. 


\subsection{Geographic Pattern of Malaria Incidence and Risk}

Kossi Province is a region of holoendemic malaria and the transmission intensity typically ranges between 100 and 900 to 1000 infective bites per person and year. ${ }^{1412}$ Most of the malaria burden falls on children, who at preschool age experience around two malaria episodes per year. ${ }^{1413}$ However, the dynamics of malaria transmission are both spatially and temporally heterogeneous.

\subsubsection{Spatial Distribution Pattern}

Even though malaria occurs throughout Kossi Province, there are marked differences between different areas. While over longer distances, malaria incidence rates decrease from south to north, this general trend is overlaid by more local pattern, with particularly high case numbers observed close to the Sourou.

\subsubsection{Malaria Transmission in Kossi Province}

In Burkina Faso -as in the whole of West Africa- the latitudinal gradient in precipitation is reflected by the length of the malaria transmission season (see figure 101): whereas malaria tends to be highly seasonal to epidemic in the north, it is highly endemic in large parts of the country falling into the SaheloSudanese and Sudanese ecological zones. 


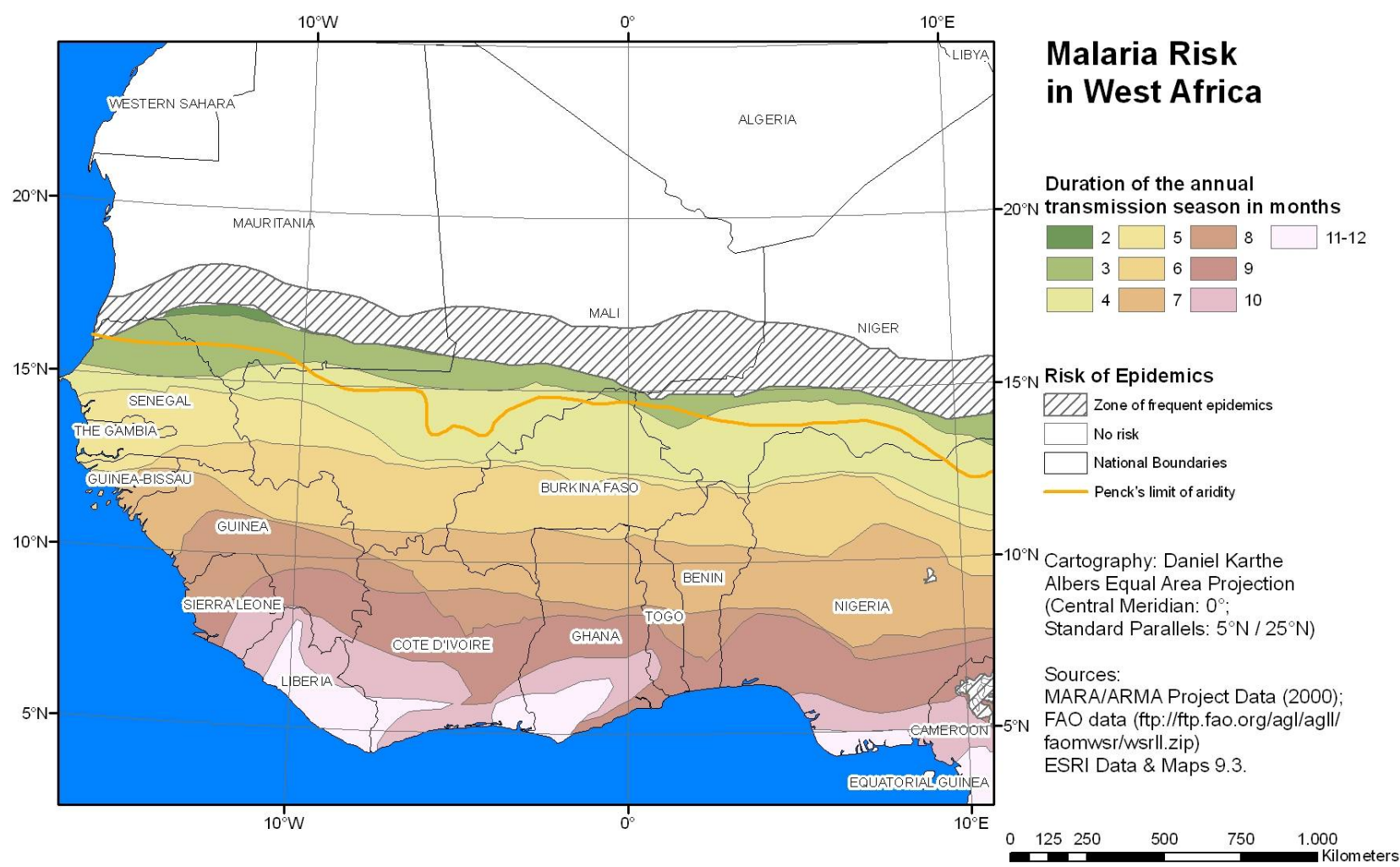

Figure 101: Endemic and epidemic malaria in West Africa ${ }^{1414}$

However, the length of the transmission season may vary considerably within a certain zone, particularly when permanent natural water bodies or irrigation projects provide breeding sites outside the rainy season. Moreover, the mere length of the transmission season does not reflect the actual transmission intensity during this period.

Kossi Province falls into a zone where the length of the transmission season typically ranges from 4 to 5 months, a situation that is quite typical for much of northern Burkina Faso. In 2008, the average malaria incidence rate in Kossi was $137,6 \%{ }^{1415}$ Annual totals from all 26 CSPS operating in Kossi from January to December 2008 have been used to prepare a map depicting malaria incidence pattern in the province (see figure 102). An analysis of the underlying temporal pattern is presented in chapter 3.3.2.

1414 Based on MARA/ARMA (2000); FAO Soil map

(ftp://ftp.fao.org/agl/agll/faomwsr/wsrll.zip) and ESRI Data and Maps 9.3.

1415 Calculated from Direction Générale de l'Information et des Statistiques Sanitaires $\left(2009^{2}\right)$, pp. 29f. \& 133-136. 


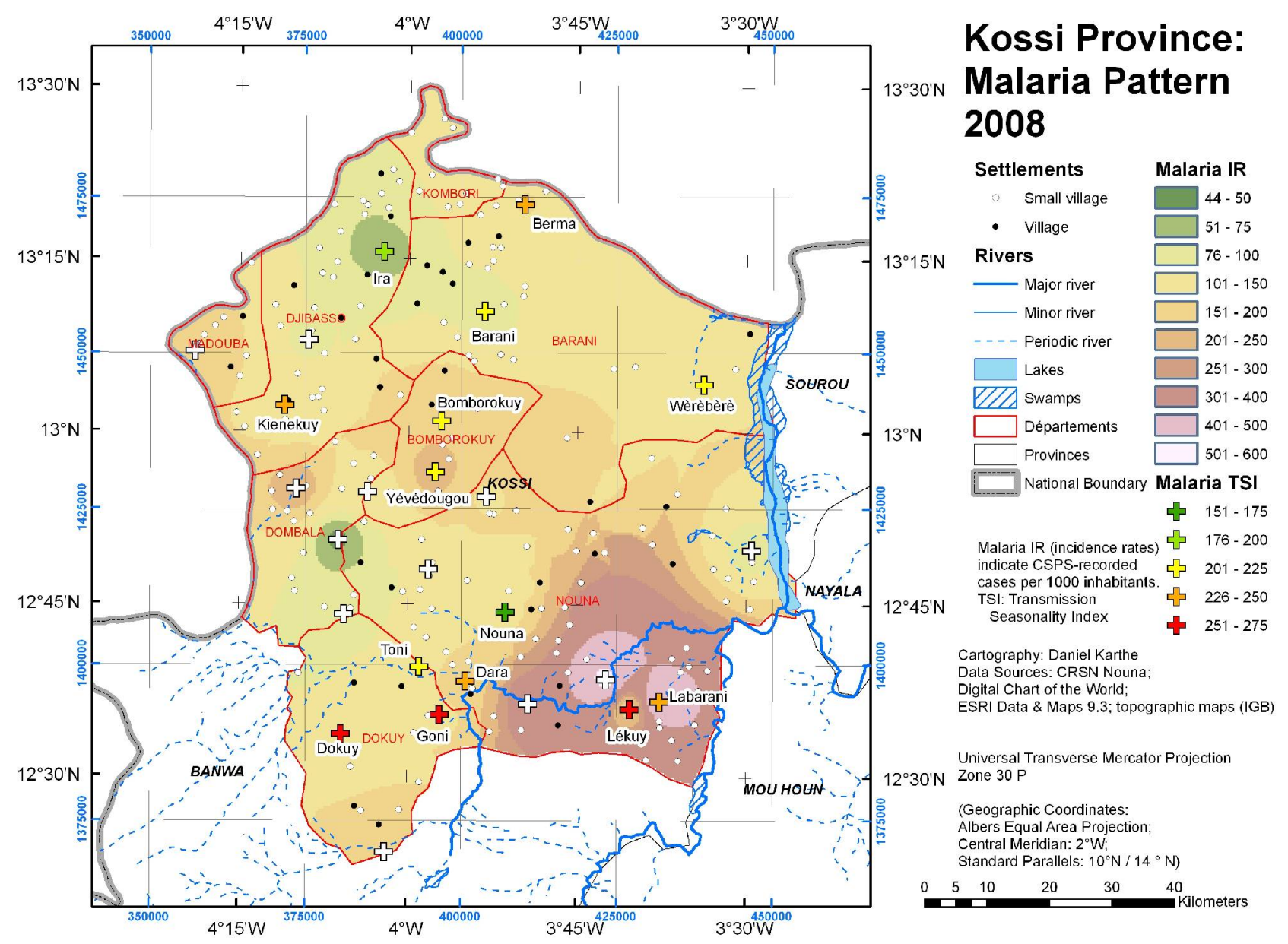

Figure 102: Geographic distribution of malaria in Kossi $(2008)^{1416}$

Malaria incidence varied considerably within Kossi Province. Incidence rates were lowest in the north and west of the region and highest in the southeast. In 2008, the lowest incidence rates were recorded at Konkui-Kouro CSPS in Doumbala District (44 cases / 1000 inhabitants) and Ira CSPS in Djibasso District (67 cases / 1000 inhabitants), and the highest incidence rate at Bourasso CSPS in Nouna District (524 cases / 1000 inhabitants).

The highest rates are found around the near $180^{\circ}$ turn of the Mouhoun River (formerly "Black Volta"). This region differs from other parts of Kossi province in several ways: annual average rainfall is about $100 \mathrm{~mm}$ more than in the northern districts; surface water (including small periodic streams and slowmoving or stagnating water on the banks of the Mouhoun) is found more widely; gallery forests line the Mouhoun, creating a microclimate and ecological niches entirely different from the dry savanna regions dominating the province;

1416 Based on CRSN Nouna; Digital Chart of the World; ESRI Data \& Maps 9.3 and topographic maps (IGB). 
and small-scale irrigation has increased considerably in recent years. Moreover, a higher concentration of CSPS in the south of Kossi than in the north mean that these health centers are more accessible; consequently, the percentage of actual infections that are officially recorded may differ regionally.

\subsubsection{Identifying Potential Zones of Transmission}

Malaria transmission intensity can be expected to vary not only at the regional scale (here defined as intra-provincial variation), but also at the local scale (for the purpose of this study defined as variations within villages). The relatively short flight range of anopheline mosquitoes, usually assumed to be around two kilometers, means that transmission risks can vary even within settlements, particularly if mosquito breeding sites are unevenly distributed.

For several reasons, it is difficult to assess local pattern of malaria transmission empirically. Malaria incidence data provided by rural health centers (CSPSs) more or less accurately reflect the situation in the area covered by the CSPS (people may consult traditional healers instead of the government-run CSPS or another CSPS which may be more convenient to access). However, since each health center covers several communities in its vicinity, statistics of malaria incidence are an average for the CSPS's zone of "responsibility" that usually consists of villages which are several kilometers apart; statistics are not routinely available for individual villages or even parts thereof. Moreover, the location where an infection is first noted is not necessarily the location where transmission took place.

Because of these difficulties and the absence of spatio-temporal data on other predictors such as entomological inoculation rates, this study identified potential zones of transmission based on mosquito habitat availability and vector-to-host contact. Potential zones of transmission are here regarded as two kilometer zones around any water body that is likely to function as a breeding site for at least some time of the year. In the study region, potential breeding sites include both natural water bodies such as perennial rivers, stream-bed pools in periodic streams, swamps, and (temporary) lakes like mares, and larval habitats of anthropogenic origin such as irrigated fields, water reservoirs, irrigation/drainage channels and excavation sites for mud bricks.

Three villages have been chosen for the mapping of potential zones of malaria transmission: Illa located in the northeast of Kossi, Kodougou in the southeast and Toni in the southwest (see table 69, figures 97 and 102). 


\begin{tabular}{|l|l|l|l|}
\hline Village & $\begin{array}{l}\text { Population } \\
(\mathbf{2 0 0 8})^{\mathbf{1 4 1 7}}\end{array}$ & Environment & $\begin{array}{l}\text { Nearest } \\
\text { CSPS }\end{array}$ \\
\hline Illa & 2036 & $\begin{array}{l}\text { Proximity to Sourou 'river' and } \\
\text { swamp; several nearby irrigation } \\
\text { projects (large-scale) }\end{array}$ & Wèrèbèrè \\
\hline Kodougou & $1529^{1418}$ & $\begin{array}{l}\text { Proximity to Mouhoun and gallery } \\
\text { forest; small-scale irrigation }\end{array}$ & Lékuy \\
\hline Toni & 2276 & Located amid dry savanna & Toni \\
\hline
\end{tabular}

Table 69: Characteristics of study villages

Illa is the largest village under the responsibility of Wèrèbèrè CSPS and located on the western bank of the Sourou, just south of the Burkina Faso - Mali border. To the north of Illa, and roughly along the international border, lies a zone of swampy character that is used by locals for washing animals and laundry and plucking the bulbs of lotus flowers (see figure 135). Moreover, since the beginning of 2008, irrigated agriculture has been introduced on the verge of this swampy zone (see figures 103 and 114). This comes in addition to the major irrigation scheme found on the eastern bank of the Sourou.

Based on IKONOS satellite imagery acquired towards the height of the dry season of 2006 and field campaigns in 2007 and 2008, major mosquito breeding sites in the region

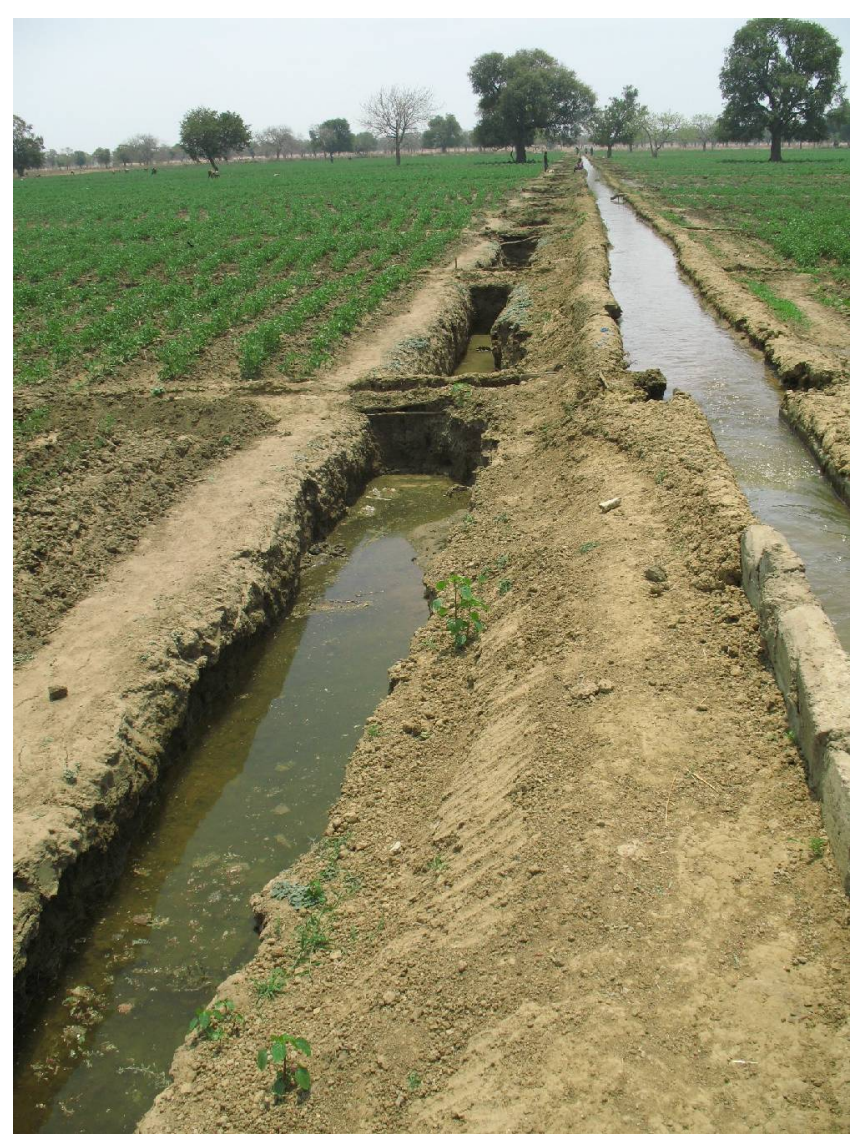

Figure 103: Newly dug irrigation and drainage channels, Illa surrounding Illa were identified. Assuming an average mosquito flight range of $2 \mathrm{~km}$, figure 104 shows the zone of potential malaria transmission in this part of the study region.

1417 Data from internal records of the CSPS at Wèrèbèrè, Lékuy and Toni. 1418 Kodougou Mossi: 1056, Kodougou Bobo: 473. 


\section{Illa Region: Zone of Potential Malaria Transmission}

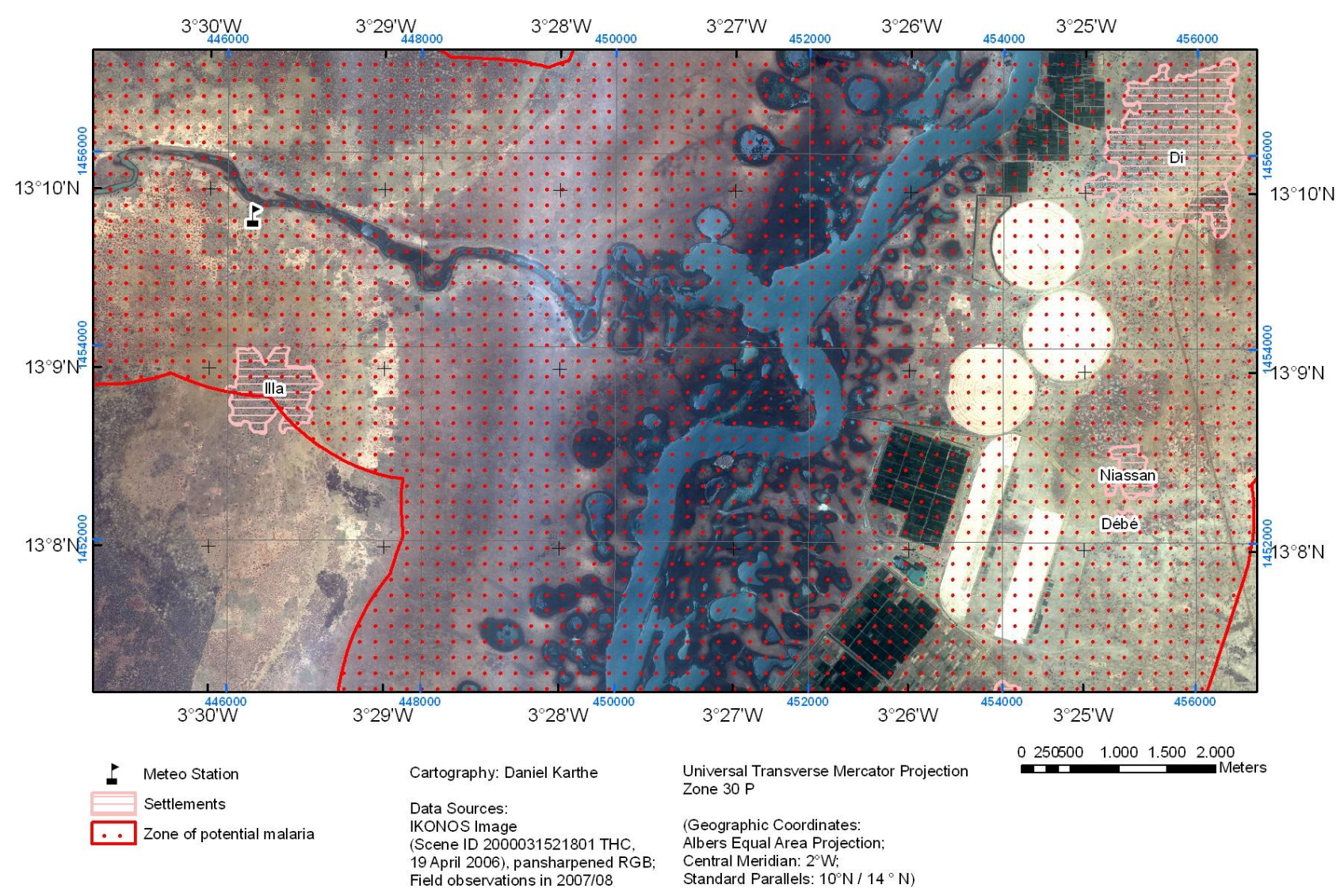

Figure 104: Zone of potential malaria transmission around Illa ${ }^{1419}$

The presence of large amounts of practically stagnating water on two sides of the village mean that much of Illa falls into a zone of potential malaria transmission. Moreover, because of agricultural, domestic (and sometimes recreational) activities, people regularly enter mosquito habitats, thus facilitating the contact between vectors and hosts.

Even though the zone of potential malaria transmission figure 104 is only an estimate based on expected anopheline flight ranges, it offers an approximate identification of the zone of highest transmission risk in the absence of empirical data on mosquito presence, entomological inoculation rates or actual case data representing intra-village variations. It should be noted that minor breeding sites, particularly those present only during or shortly after the rainy season, have not been included for the production of this map. Nevertheless, the map offers several insights: Currently, about $73 \%$ of Illa fall into the zone of potential transmission within the $2 \mathrm{~km}$ radius around major and quasipermanent breeding sites. Much of the farmland used for rainfed agriculture

1419 Based on IKONOS Image (ID 2000031521801 THC; data of acquisition: 19 April 2006) and field surveys in 2007/08. 
around Illa also falls into the zone of potential transmission, as do all human activities that are linked to surface water. Finally, an extension of large-scale irrigated agriculture into Kossi (as it has been observed in Sourou province over the past three decades) is likely to create a situation under which even larger parts of the village would become zones of high infection risk.

Kodougou's location resembles Illa's by its proximity to a major water body, which is in this case the Mouhoun (see figure 105). However, irrigation in this region only occurs at a small scale, and in contrast to the Sourou, the Mouhoun is surrounded by a gallery forest (see figure 116). Since high resolution satellite imagery was unavailable for the Kodougou region, mapping of the zone of potential transmission is in this case based on the IGB's topographic map (scale: 1:200.000), digital map data of the CRSN (village limits) and field campaigns carried out during the dry and wet seasons of 2007/2008.

\section{Topographic Map of Kodougou Region}

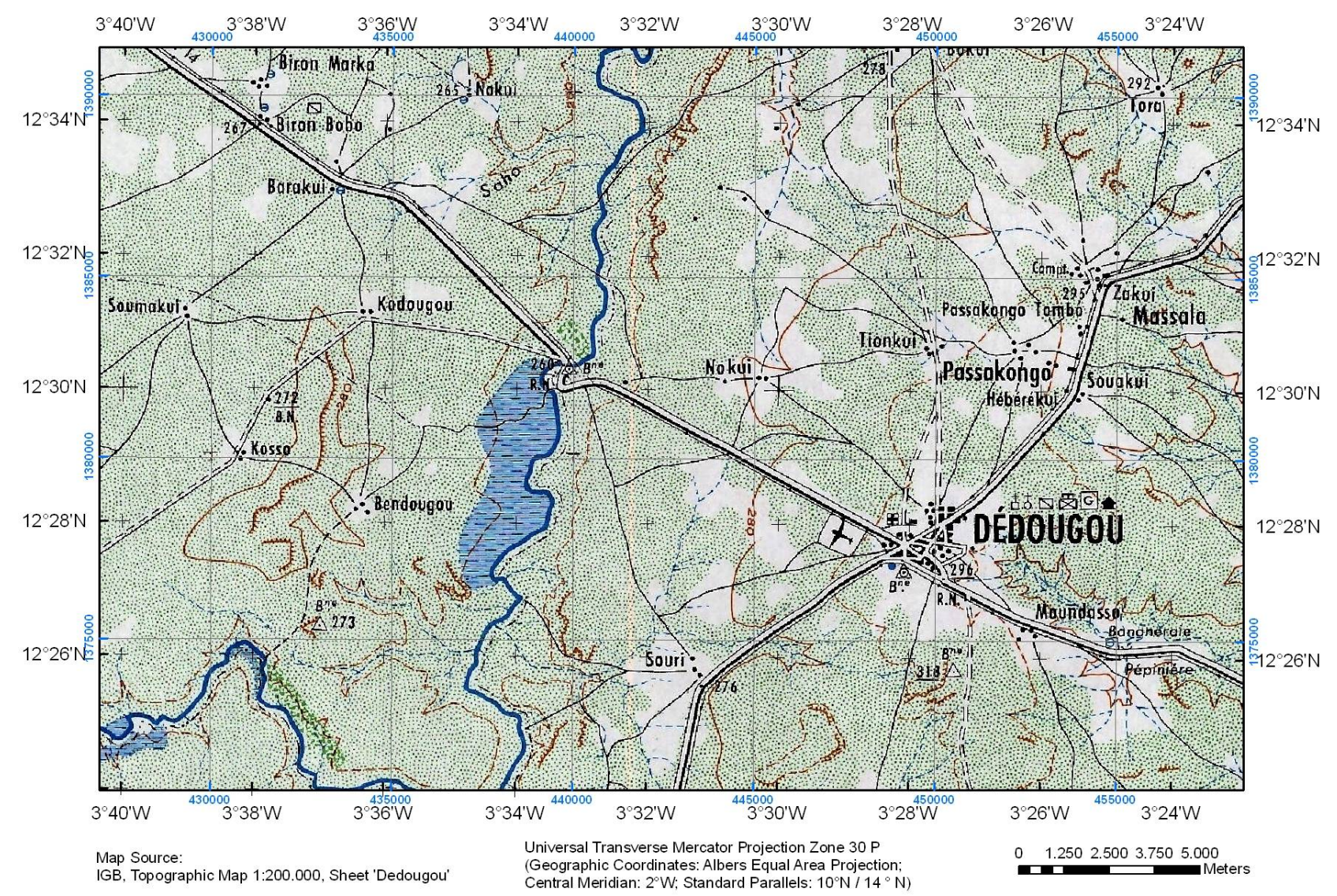

Figure 105: Topographic map of Kodougou region ${ }^{1420}$

1420 Based on Institut Géographique du Burkina (1971), 1:200.000 topographic map, sheet 'Dédougou'. 
Kodougou, which is located along the main road from Dédougou to Kossi's provincial capital Nouna, is divided into two parts: the larger Kodougou Mossi which is located only a few hundred meters west of the Mouhoun, and the much smaller Kodougou Bobo, that is located about $3.5 \mathrm{~km}$ to the west of Kodougou Mossi. There are several major differences between the two parts of Kodougou - apart from the ethnic majorities indicated in their names.

Due to its location, a few houses in Kodougou Mossi are located very close to the bank of the Mouhoun. Even though this is still the exception, irrigated agriculture is practiced along the river, in and around the zone that may become inundated during the rainy season. So far, agricultural activities in Kodougou Mossi include, besides the ubiquitous cultivation of sorghum and millet, the production of several types of vegetables, fruits and cassava. In Kodougou Bobo, agriculture is always rainfed and limited to the cultivation of sorghum and millet.

Several large depressions are found close to the road from Kodougou Mossi towards Kodougou Bobo. Some of them are caused by excavation of mud that is used for the production of bricks, while others may be natural depressions. At an average depth of around three meters, these depressions fill up with water during the rainy season, creating larval habitats in very close proximity to houses in Kodougou Mossi. Moreover, local children come to these pools to swim, whereas farmers bring their animals for washing and drinking purposes (see figure 106). 


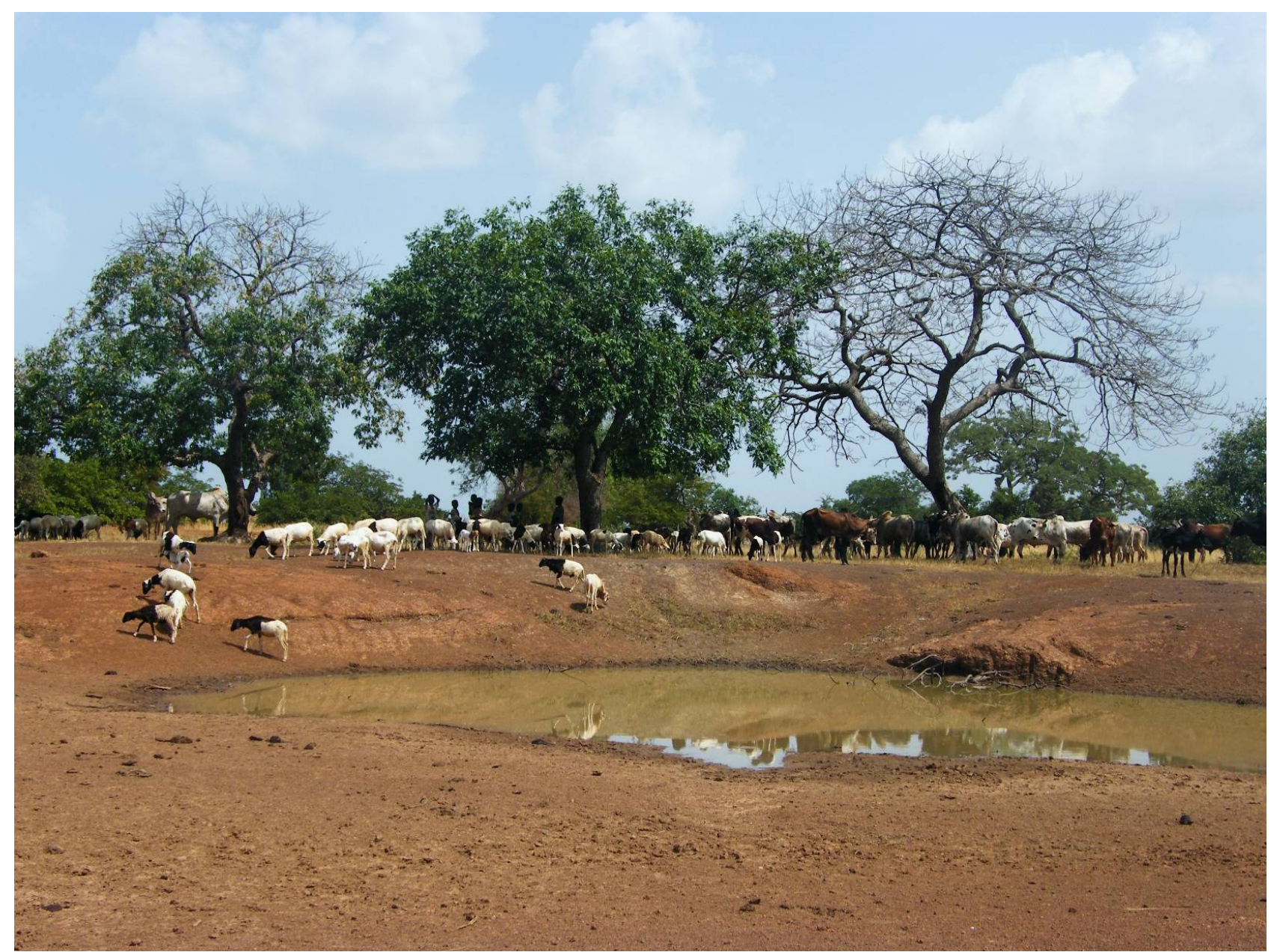

Figure 106: Depression near Kodougou Mossi at the beginning of the dry season

Finally, several 'traditional' wells (see figure 107) can be found in Kodougou Mossi. Since the 'modern' well has been dysfunctional, they form the only backbone of the villagers' water supply. Due to their 'open' construction, they may at the same time be potential mosquito breeding sites. Because of their small size and the known preference of Anopheles gambiae for warm and sunlit bodies of water, they are -if at all- habitats of minor importance. However their location in central areas of the village, the presence of water even during the dry season and the absence of empirical evidence (e.g. larval density counts, information on adult emergence rates), mean that they are at least a potential source of vector mosquitoes. 


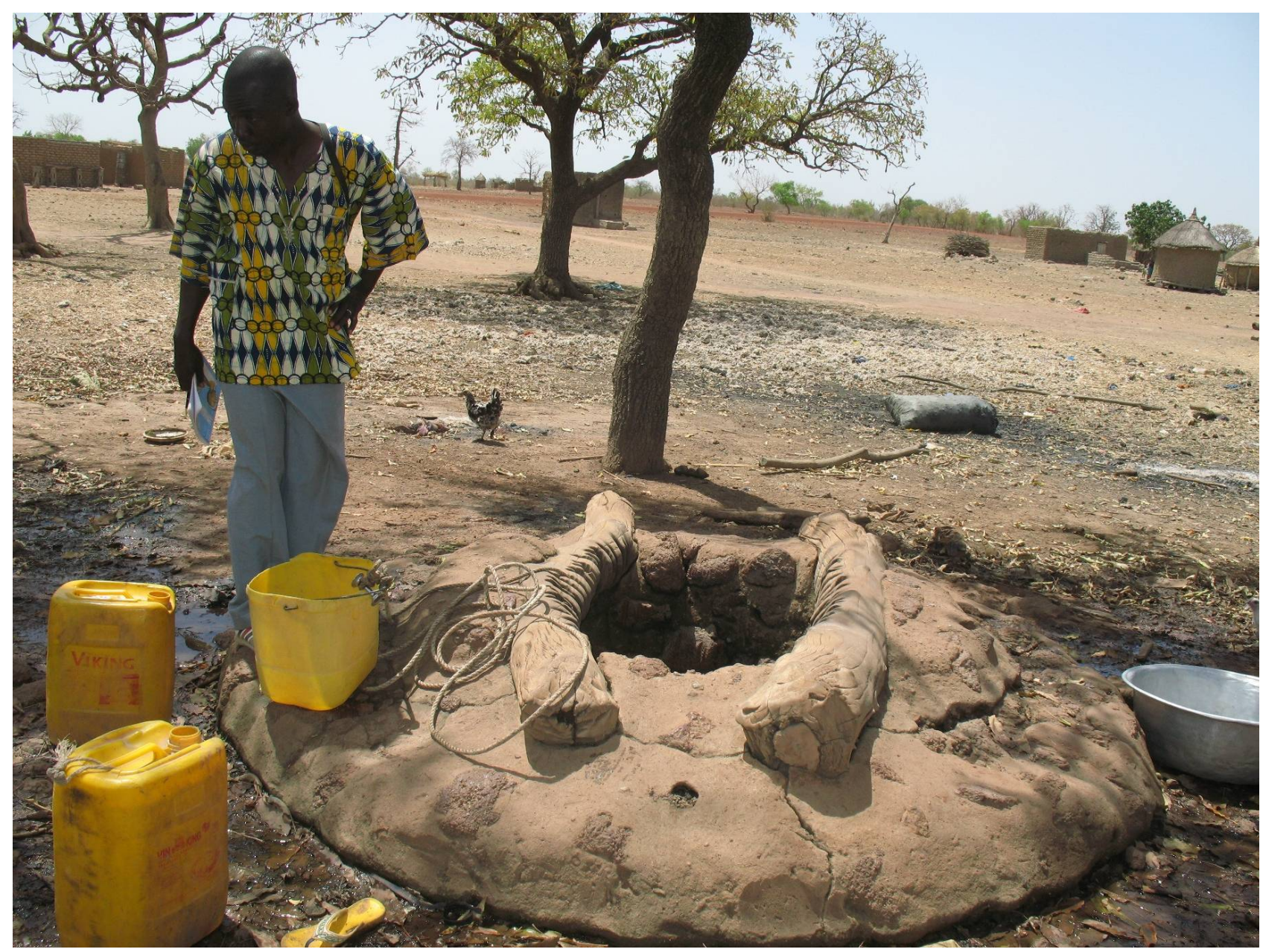

Figure 107: 'Traditional' well in Kodougou

In contrast to Kodougou Bobo, the area of Kodougou Mossi falls entirely into the zone of potential malaria transmission. A multitude of mosquito habitats distributed around the settlement increase the likelihood of vectors being present even if some of their potential habitats are not realized (see figure 108). In and around Kodougou, both permanent and temporary larval habitats are found, resulting in somewhat different realizations of high risk zones. 


\section{Kodougou Region: Zone of Potential Malaria Transmission}

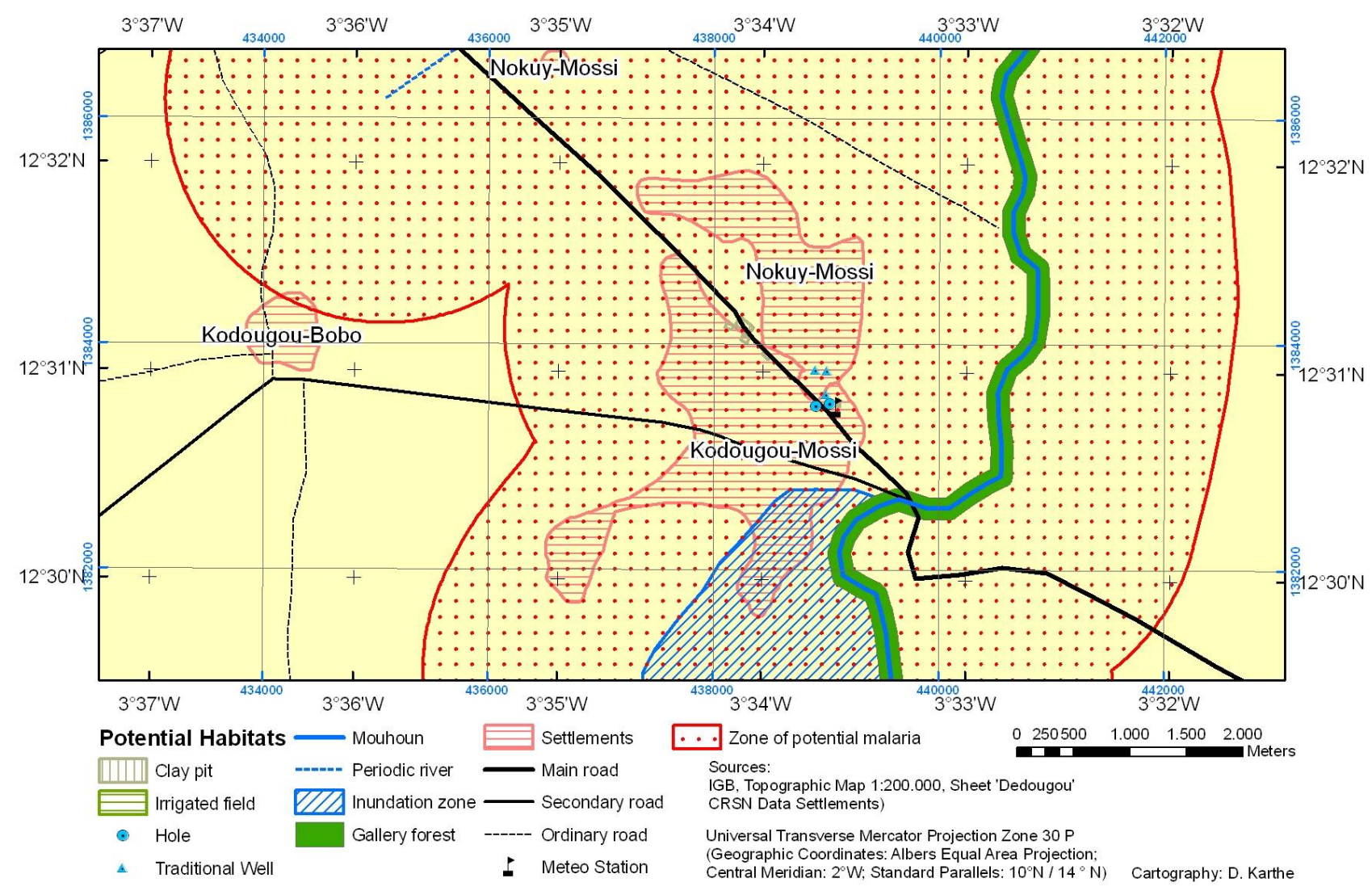

Figure 108: Kodougou region: zone of potential malaria transmission ${ }^{1421}$

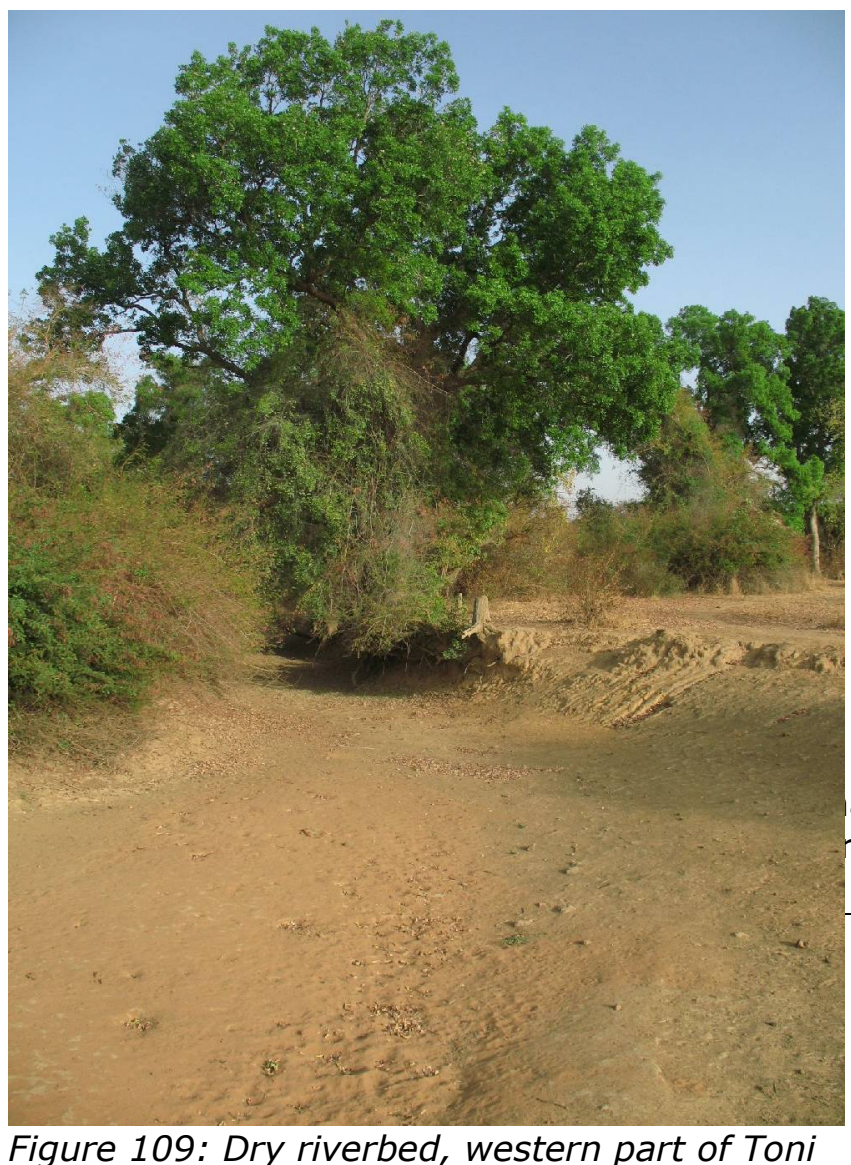

Toni's location differs from Illa's and Kodougou's in one important way: the village is surrounded by dry savanna but there is no permanent water body within its vicinity. A temporary stream flows through Toni's western section but falls completely dry by around February (see figure 109). The same is true for the ponds found in Toni and the neighboring village of Kamadena. Between the two villages, about $2 \mathrm{~km}$

a (1971), 1:200.000 topographic map, veys in $2007 / 2008$. 
to the south of Toni, lies one of Kossi's few patches of dry forest.

The production of bricks is a major activity in both villages, and consequently, large depressions have been created (see figure 110) These clay pits are of varying shape and size but typically have a depth of around $2 \mathrm{~m}$. They fill with water during the rainy season, forming temporary but relatively long-lasting larval habitats. Moreover, their relatively central locations mean that these breeding sites significantly contribute to contacts between hosts and vectors. Moreover, a number of smaller holes has been dug for the storage of animal fodder, food items or waste disposal (see figure 111).

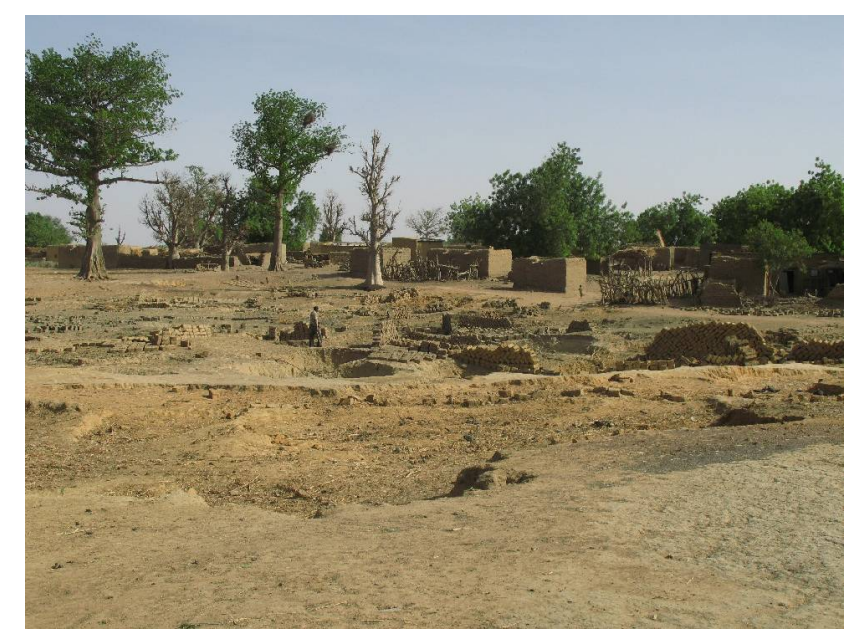

Figure 110: Clay pits in Toni

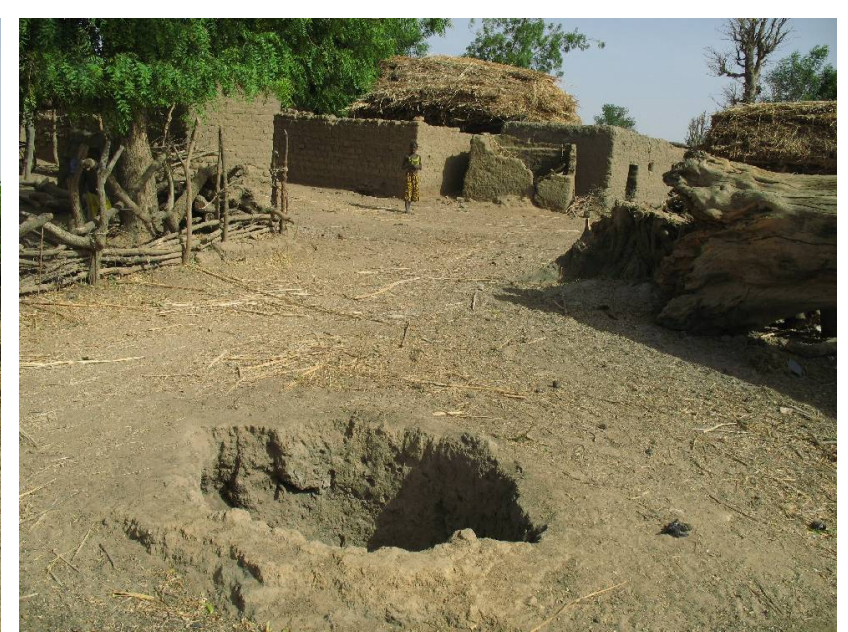

Figure 111: Hole for storage of karité nuts

Even though these holes are usually not in use during the rainy season, they tend to fill up with water, creating larval habitats right next to houses.

For the Toni region, QuickBird imagery showing the situation in December 2003 formed the basis for the preparation of a malaria risk map. For this part of Kossi, ground truthing and field studies were carried out during the dry seasons of 2007 and 2008. Due to the many potential breeding sites, Toni and Kamadena fall entirely into the zone of high malaria risk (see figure 112), which is in this case highest during the rainy season and the period thereafter. Within two to four months of the end of the rainy season, most of these breeding sites fall completely dry. 


\section{Toni Region: Zone of Potential Malaria Transmission}

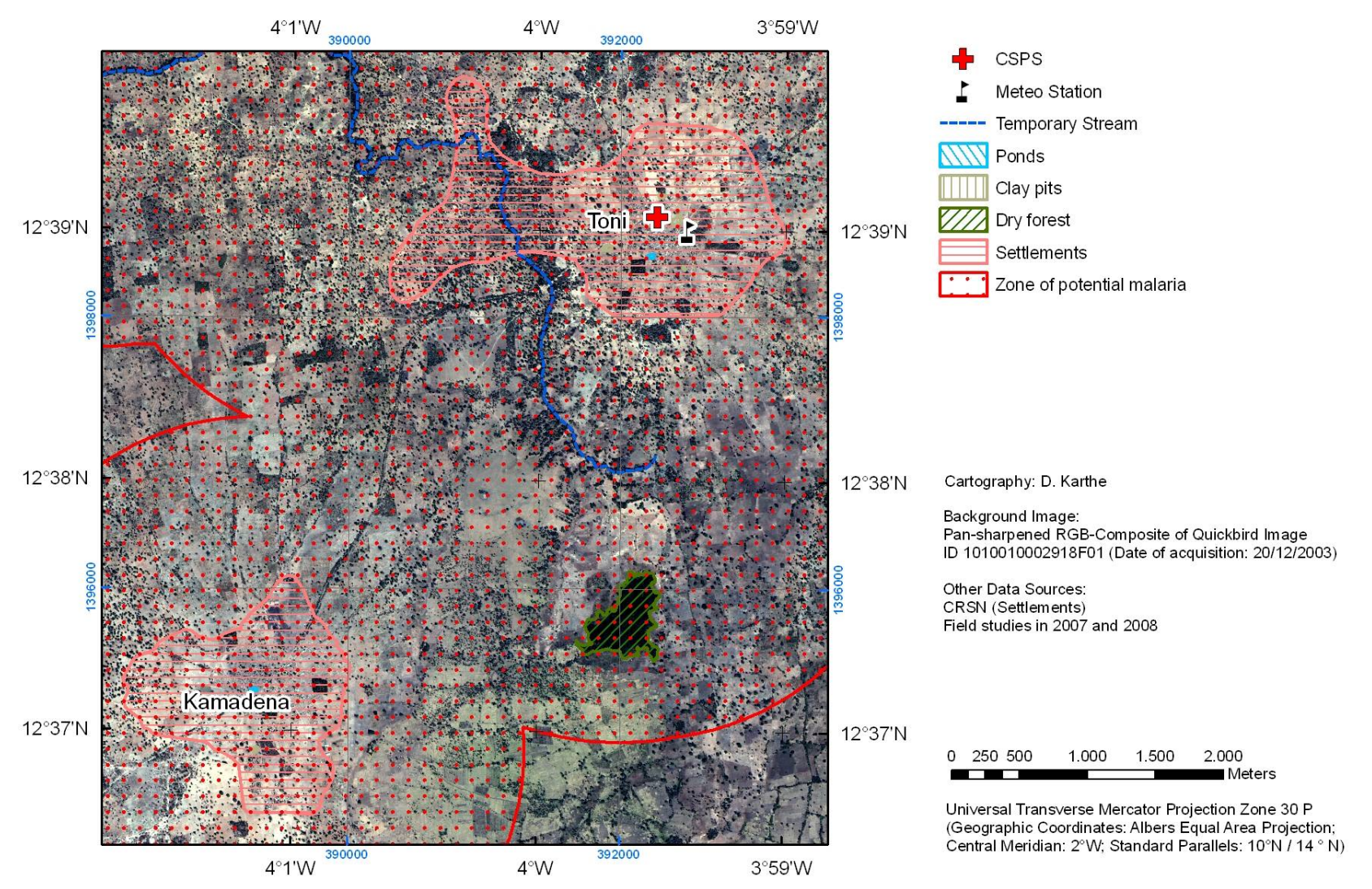

Figure 112: Toni region: zone of potential malaria transmission ${ }^{1422}$

All of the three study villages fall largely into zones of elevated malaria risk due to their proximity to mosquito breeding sites. Whereas some of these larval habitats are large, permanent and more or less natural bodies of water such as the Mouhoun and Sourou, others are smaller, temporary and sometimes of anthropogenic origin. For the framework of this study, all breeding sites have been treated equally even though their productivity may be very different. Moreover, the presence of larval breeding sites is just one prerequisite of malaria transmission.

1422 Map based on QuickBird image (ID 1010010002918F01; data of acquisition: 20/12/2003); CRSN data and field surveys in 2007/2008. 


\subsubsection{Microclimatic Variations}

Breeding site availability is a prominent but not the sole factor ruling the spatial pattern of malaria transmission in semi-arid regions like Kossi province. While the climate is a major driver of temporal transmission pattern, microclimatic variations are potentially relevant for the spatial distribution of malaria.

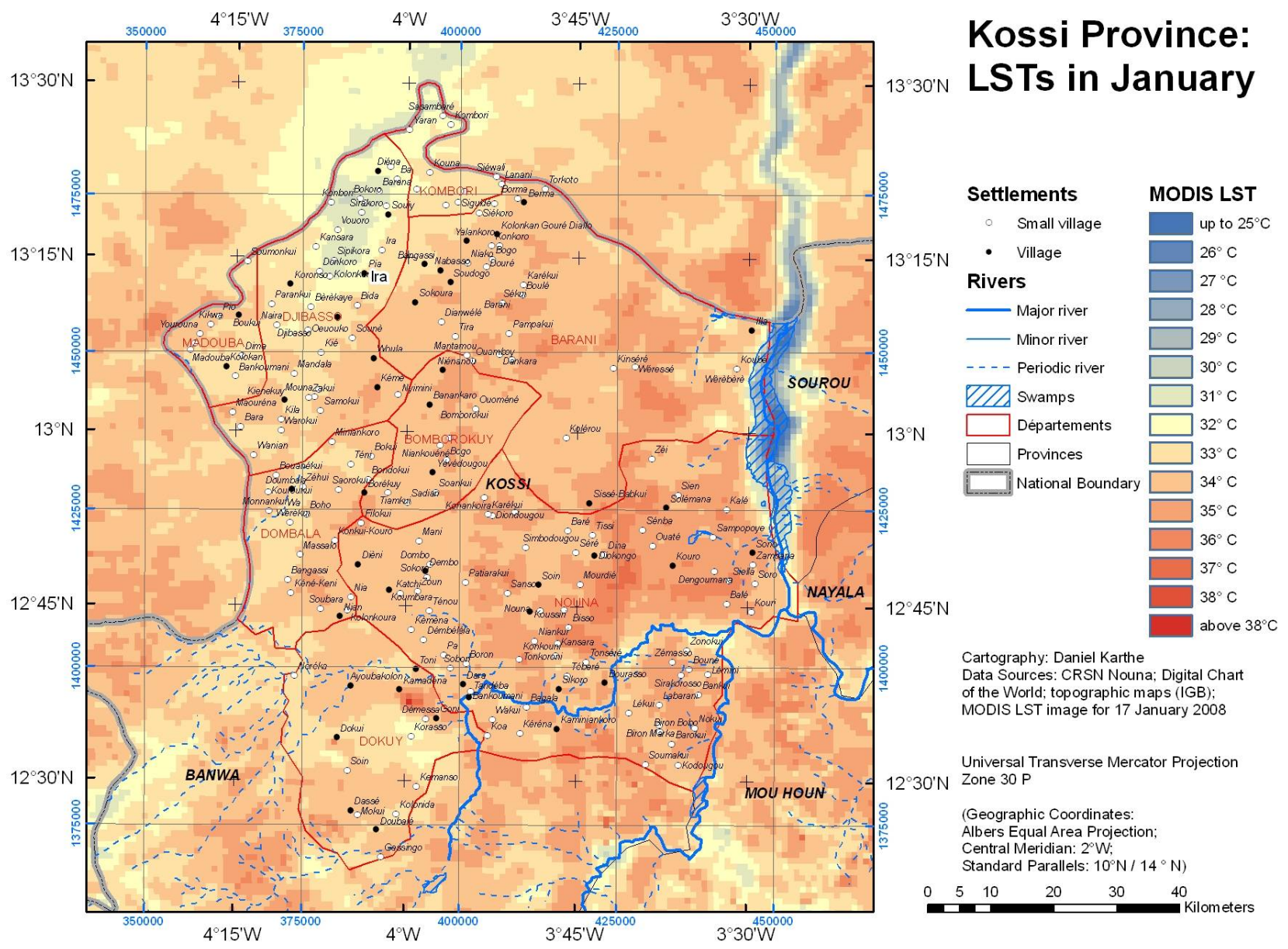

Figure 113: Land surface temperatures in Kossi Province, 17 January $2008^{1423}$

The daytime land surface temperature (LST) pattern in Kossi around midJanuary 2008 is illustrated by figure 113 . Not surprisingly, the lowest surface temperatures were found around the Sourou, which is by its surface the region's largest water body. LSTs of around $25^{\circ} \mathrm{C}$ in this area compared to an average LST of around $30^{\circ} \mathrm{C}$ for Kossi Province and a maximum value of $42^{\circ} \mathrm{C}$. While differences in air temperatures may be smaller due to permanent energy fluxes, the presence of relatively warmer or cooler surfaces certainly has an impact on ambient temperatures, particularly in face of a surface temperature

1423 Based on Terra MODIS LST (MOD11A1) image, date of acquisition: 17/01/08; CRSN Nouna; Digital Chart of the World and topographic maps (IGB). 
gradient of $17 \mathrm{~K}$. Other areas of comparatively low surface temperatures include the forested tracts around the Mouhoun and Vounhou, and the more elevated regions in northwestern Kossi.

Since mosquito breeding sites and habitats are closely linked with surface water, often being surrounded by vegetation, it can be concluded that they typically fall into regions of relatively lower temperatures which are caused by the influence of relatively cool water masses, evaporative cooling and shade provided by plants. At the same time, humidity in such locations may be considerably higher than in other parts of the dry savanna.

In this context, the very limited availability of meteorological data, which are often recorded at sites that may not at all be representative for the situation found in mosquito habitats, appears to be particularly problematic. The examples of Illa and Kodougou have been chosen for a comparison of the microclimate observed at meteorological station sites and their vicinity. For the region around Toni, microclimatic pattern are expected to be of lesser relevance for malaria transmission, even though an investigation of the pattern found in the dry forest south of the village should be undertaken.

In Illa, a meteorological station was set up in 2004 about $1.2 \mathrm{~km}$ north of the village. It is surrounded by bushes in the north and west, and dry savanna and mostly rainfed fields towards the south and east. In 2007/08, a large irrigation project was begun east of the station, where formerly rainfed fields and savanna land have been transformed into irrigated cropland used predominantly for the cultivation of vegetables. About $300 \mathrm{~m}$ north of the meteo station is an area of partially inundated grassland (see figure 114). During the wet season, this swampy land becomes an affluent of the Sourou river; during the dry season, a belt of pools remains. As stated before, these pools are used by locals for washing purposes, for watering livestock, and for plucking the bulbs of lotus flowers (which are used for consumption). At the same time, this zone is of major importance for mosquito breeding. 


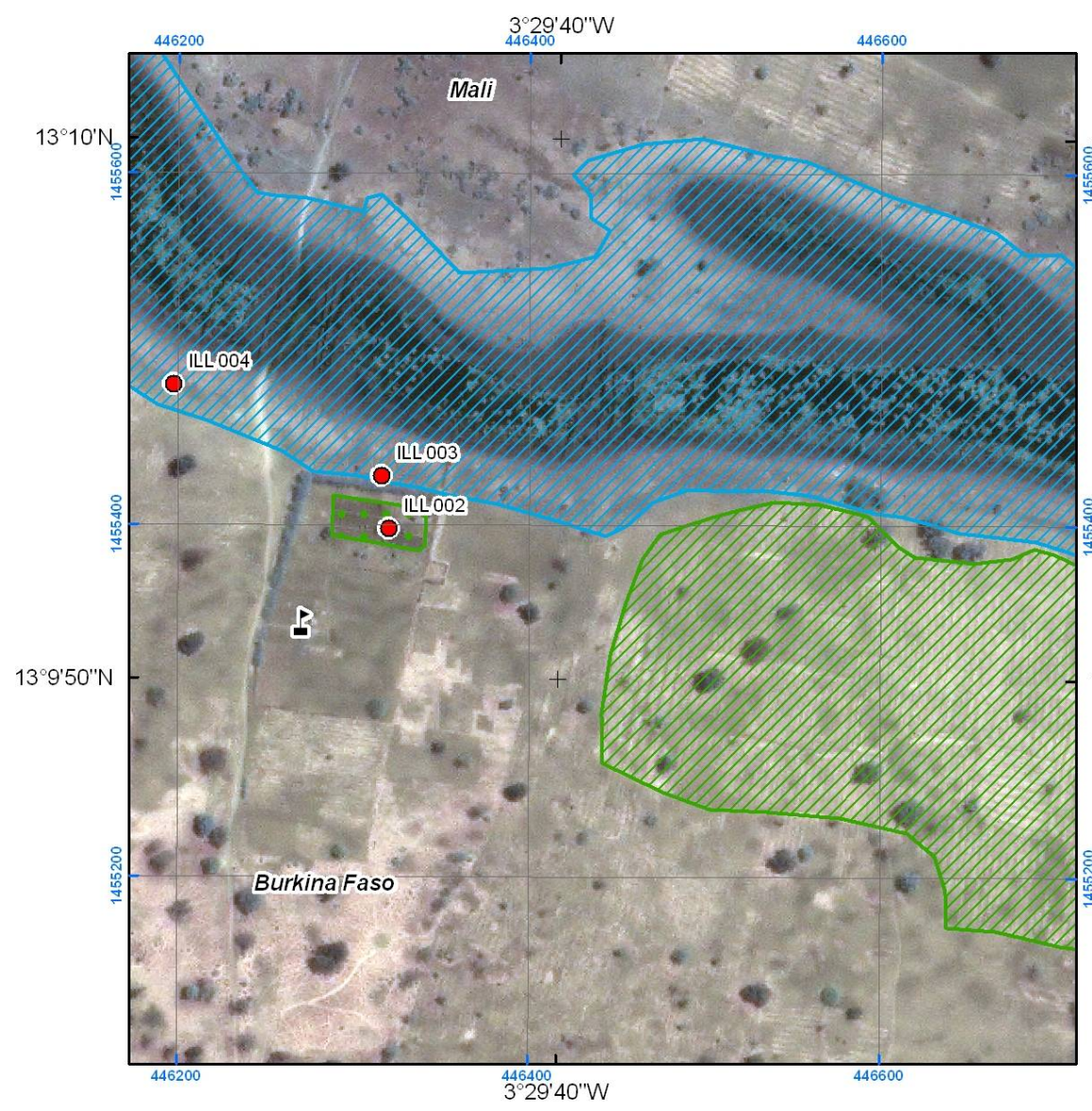

\section{Mosquito Habitats North of IIla}

$13^{\circ} 10^{\prime} \mathrm{N}$

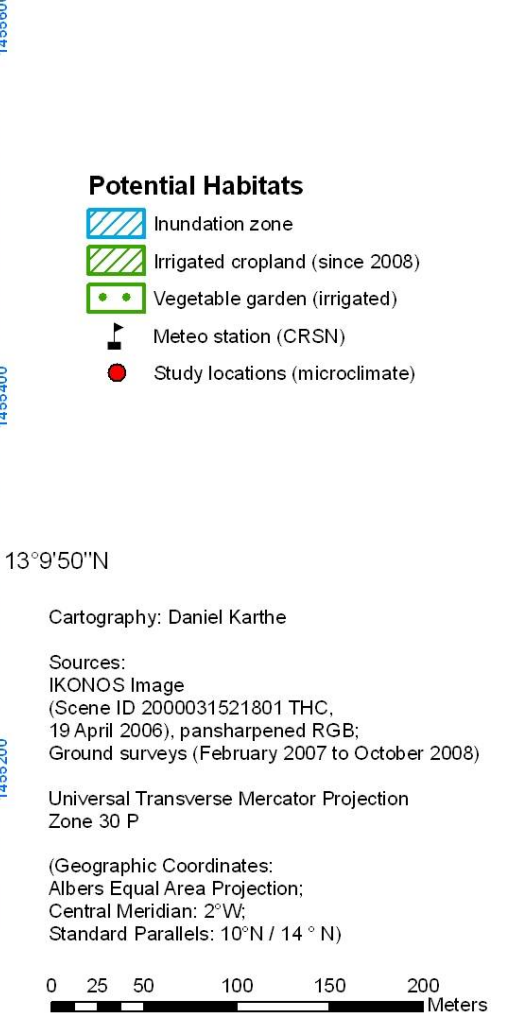

Figure 114: Mosquito larval habitats north of Illa ${ }^{1424}$

Even though only limited observation data for field studies carried out during the dry seasons of 2007 and 2008 are available, these indicate that the meteorological station of Illa pretty much reflects the conditions in the village, while the dry season moisture is considerably higher in the inundation zone north of Illa. At the same time, midday temperatures were found to be $0.6 \mathrm{~K}$ to $3.9 \mathrm{~K}$ below meteo station records. While the differences may at first sight seem small, they are of considerable malariologic relevance. While an increase in humidity more or less directly favors mosquito survival at the low levels observed during the dry season, this effect is supported by the concurrent reduction in temperature close to water. In fact, while conditions in the open savanna may already be unfavorable for parasite development in the mosquito (outside temperatures $>40^{\circ} \mathrm{C}$ ), moist zones offer a refuge for mosquitoes which does not only prolong their own lifespan but which also favors parasite development. The situations recorded on two typical dry season days around noon is presented in table 70 .

1424 Based on IKONOS Image (ID 2000031521801 THC; data of acquisition: 19 April 2006) and field surveys in 2007/08. 
Chapter 3 - Case Study: Malaria in Kossi Province

\begin{tabular}{|c|c|c|c|c|c|}
\hline $\begin{array}{l}\text { Date } \\
\text { Time }\end{array}$ & $\begin{array}{l}\text { / Meteo } \\
\text { station }\end{array}$ & ILL 002 & ILL 003 & ILL 004 & Water ${ }^{1425}$ \\
\hline $18 / 03 / 08$ & $\begin{array}{l}38.2^{\circ} \mathrm{C} \\
9.7 \%\end{array}$ & $\begin{array}{l}38.4{ }^{\circ} \mathrm{C} \\
9.6 \%\end{array}$ & $\begin{array}{l}36.7^{\circ} \mathrm{C} \\
15.8 \%\end{array}$ & $\begin{array}{l}37.6^{\circ} \mathrm{C} \\
16.3^{\circ}\end{array}$ & $26.8^{\circ} \mathrm{C}$ \\
\hline $07 / 02 / 07$ & $\begin{array}{l}40.5^{\circ} \mathrm{C} \\
8.5 \%\end{array}$ & $\begin{array}{l}41^{\circ} \mathrm{C} \\
11.1 \%\end{array}$ & $\begin{array}{l}39.4{ }^{\circ} \mathrm{C} \\
16.0 \%\end{array}$ & $\begin{array}{l}36.6^{\circ} \mathrm{C} \\
21.5 \%\end{array}$ & $32.9^{\circ} \mathrm{C}$ \\
\hline \multicolumn{6}{|c|}{$\begin{array}{l}\text { ILL 002: Vegetable field north of Illa meteo station } \\
\text { ILL 003: Fringe of inundation zone, north of Illa } \\
\text { ILL 004: Just inside inundation zone north of Illa }\end{array}$} \\
\hline
\end{tabular}

Table 70: Field survey of microclimatic conditions close to Illa ${ }^{1426}$

Like Illa, Kodougou is located in the proximity of water, but both the natural character of the river (with a perennial flow of water) and its surrounding gallery forest are markedly different from the situation in Illa. Moreover, Kodougou falls into the southern part of Kossi, resulting in an annual rainfall that is about $100 \mathrm{~mm}$ more than in Illa (see figure 51).

In Kodougou Mossi, a meteorological station was installed amidst millet fields at the southern fringe of the village in 2004. Dry savanna and millet fields dominate the surrounding landscape. One notable exception is the area on both sides of the Mouhoun or Black Volta, where gallery forest extends for about $25 \mathrm{~m}$ from the dry-season shoreline. In recent years, irrigated agriculture has been introduced on the western bank of the river. Moreover, some of the land close to the river may become inundated during the wet season.

1425 The values indicated here are averages for 5 measurements at a depth of about $1 \mathrm{~cm}$. 1426 Based on field studies in $2007 / 2008$. 


\section{Kodougou Region: Microclimate (Location of Study Sites)}

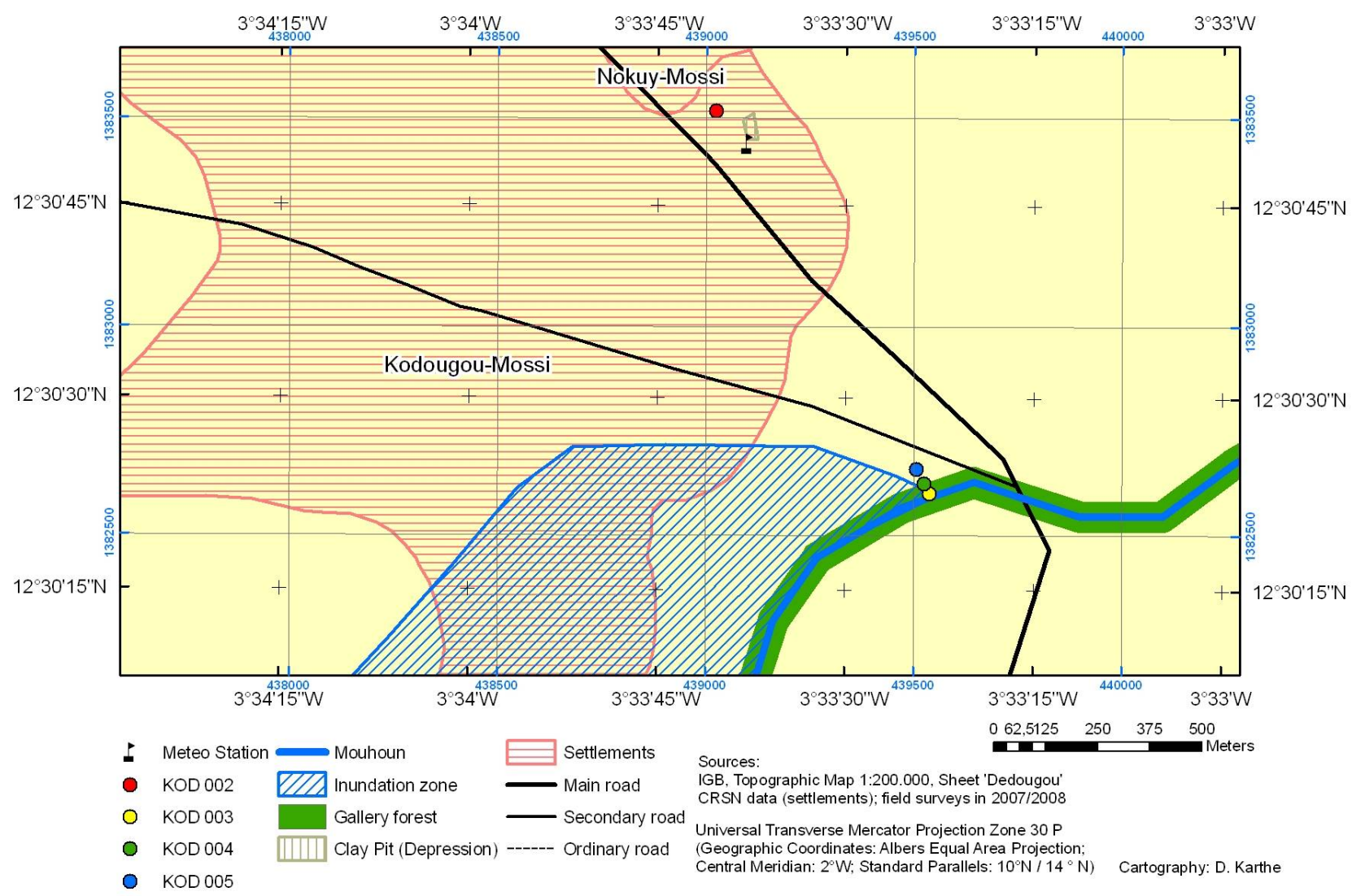

Figure 115: Location of Kodougou meteo station and test sites ${ }^{1427}$

The riverine gallery forest has a microclimate that differs sharply from the situation found in the open savanna or the village. This is illustrated by data from a typical dry-season day. Since only very little variation in temperature and humidity were recorded in the open savanna, three test sites close to the river bank were chosen for a comparison of the situation in and around the gallery forest and the savanna. One additional test site (KOD 002) is located amidst typical mud-brick houses in Kodougou Mossi.

1427 Based on Institut Géographique du Burkina (1971), 1:200.000 topographic map, sheet 'Dédougou', CRSN data and field surveys in 2007/2008. 


\begin{tabular}{|l|l|}
\hline Test site & Description \\
\hline KOD 001 & $\begin{array}{l}\text { Kodougou meteorological station, located amidst millet fields and a } \\
\text { clay pit }\end{array}$ \\
\hline KOD 002 & Open space between mud-brick houses in Kodougou Mossi \\
\hline KOD 003 & Bank of the Mouhoun river (adjacent to water; within gallery forest) \\
\hline KOD 004 & Edge of the gallery forest, about $25 \mathrm{~m}$ from the river \\
\hline KOD 005 & Bare field, just outside the gallery forest \\
\hline
\end{tabular}

Table 71: Study sites in Kodougou

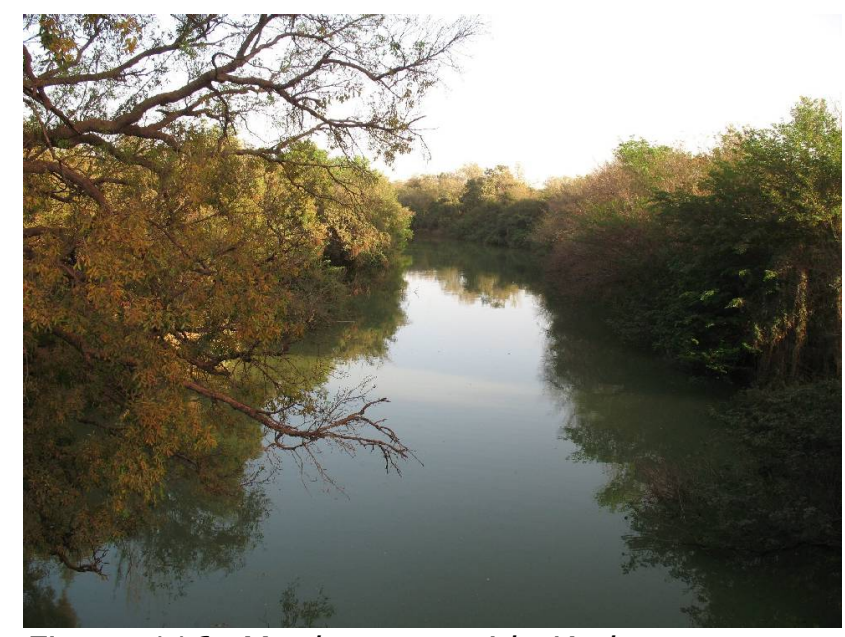

Figure 116: Mouhoun outside Kodougou

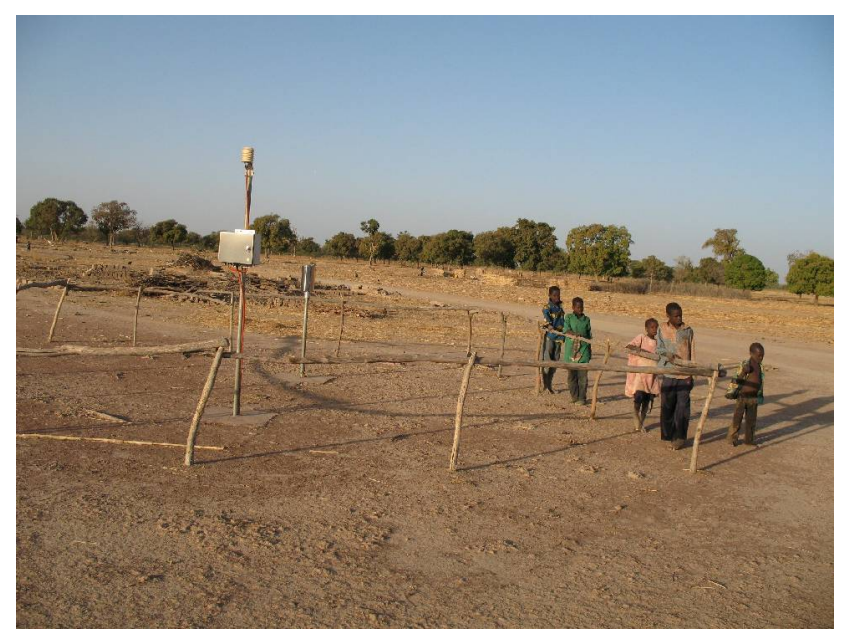

Figure 117: Kodougou meteo station

Two tests were carried out in the Kodougou region: in February 2007, the temperature and humidity were followed up at hourly intervals for one day using a hand-held thermo-hygrometer, and in March 2008, a data-logger registering temperature and humidity was installed in the gallery forest between KOD 002 and KOD 003 test sites (its measurements could not be meaningfully used because of non-availability of meteorological station data). Even though the short sampling periods during field visits mean that the results may not be representative, they indicate that further research into the microclimate of (potential) vector breeding sites and habitats is required. 


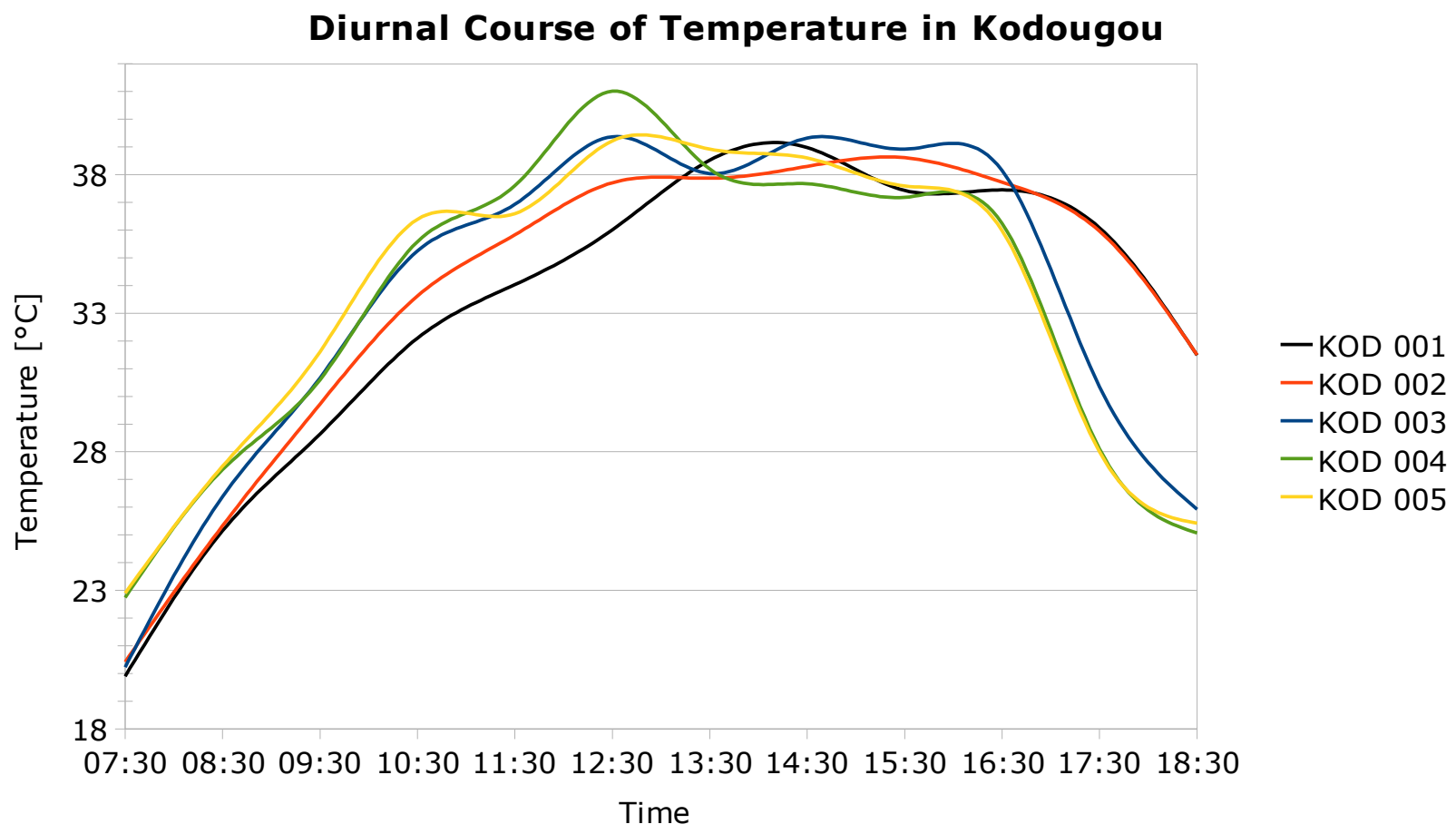

Figure 118: Diurnal course of temperature in Kodougou (08 February 2007) ${ }^{1428}$

Whereas the measurements in February 2007 revealed that there is only little difference in the average temperature for the five test sites $\left(33.0^{\circ} \mathrm{C}\right.$ at $\mathrm{KOD}$ 001 to $33.6^{\circ} \mathrm{C}$ at KOD 002), the observed differences in the diurnal course of temperature were far greater. While afternoon temperatures were relatively uniform (not more than $\pm 1 \mathrm{~K}$ deviation from the mean of the test sites), with the lowest values being observed in the gallery forest, there were major differences during the morning and early evening, when the temperature difference between the gallery forest and the village peaked at more than $8 \mathrm{~K}$ (see figure 118). Similar differences could be seen with regard to humidity. Low humidities of around $10 \%$ were recorded during the hot daytime hours, with slightly higher values occurring in the gallery forest. During the early morning and the evening hours, significantly higher humidities were recorded close to the river and within the gallery forest and adjoining areas (see figure 119).

1428 Based on field study in 2007. 


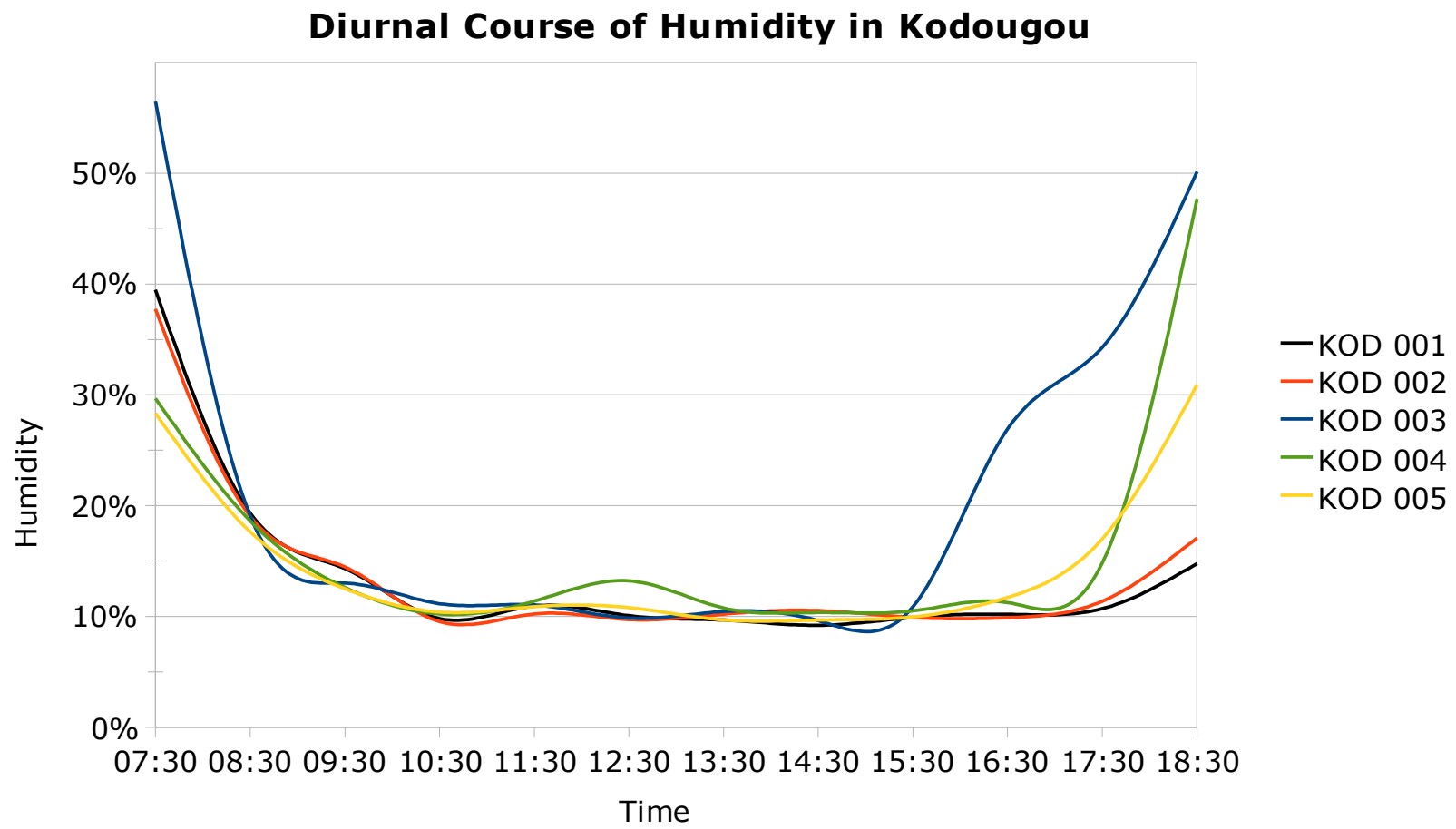

Figure 119: Diurnal course of humidity in Kodougou (08 February 2007) ${ }^{1429}$

The combined effects of slightly lower temperatures and higher humidities and the concomitant differences in in other factors (e.g. availability of shade, presence and state of vegetation) mean that a microclimate conducive to vector survival may be present in Kodougou's proximity even at times when the local meteorological station indicates unfavorable conditions.

1429 Based on field study in 2007. 


\subsubsection{Temporal Distribution Pattern}

Malaria incidence in Kossi Province follows a basic seasonal pattern with an increase in case numbers following shortly after the onset of the rainy season. However, the degree of seasonality and annual course of malaria incidence varies both from one year to another and between different locations.

\subsubsection{Transmission Seasonality}

For practically all study locations and years, malaria incidence in Kossi follows a clear temporal pattern: Case numbers tend to rise in the second half of the year, around 2 to 4 weeks after the onset of the rainy season and slowly decline after its end in October.

To characterize the degree of transmission seasonality, a transmission seasonality index (TSI) was calculated (for symbols see table 72):

TSI $=\left(\left[2 C_{2} / C_{t}\right]+\left[C_{\max }-C_{\min }\right] /\left[C_{\max }+C_{\min }\right]\right) * 100$

This index takes into account both the difference of accumulated transmission in the high transmission season (here defined as the second half of the year) and the low transmission season, and the difference between the months recording the highest and lowest case numbers. An index of 100 would represent an equal distribution of malaria; higher values indicate higher degrees of seasonal variation. 
Chapter 3 - Case Study: Malaria in Kossi Province

\begin{tabular}{|c|c|c|c|c|c|c|}
\hline \multirow[t]{2}{*}{ CSPS } & \multicolumn{3}{|c|}{ Malaria Cases in 2008} & \multicolumn{2}{|c|}{ Monthly Cases } & \multirow[t]{2}{*}{ TSI } \\
\hline & $\begin{array}{l}\text { Total } \\
\left(C_{t}\right)\end{array}$ & $\begin{array}{l}\text { Jul - Dec } \\
\left(C_{2}\right)\end{array}$ & $\begin{array}{l}\text { Jan - Jun } \\
\left(C_{1}\right)\end{array}$ & $\begin{array}{l}\text { Minimum } \\
\left(\mathrm{C}_{\min }\right)\end{array}$ & $\begin{array}{l}\text { Maximum } \\
\left(C_{\max }\right)\end{array}$ & \\
\hline Barani & 2095 & 1543 & 552 & 79 & 418 & 215,51 \\
\hline Berma & 2114 & 1627 & 487 & 64 & 397 & 226,16 \\
\hline Bomborokuy & 2020 & 1518 & 502 & 64 & 315 & 216,52 \\
\hline Dara & 1487 & 1128 & 359 & 32 & 235 & 227,74 \\
\hline Dokuy & 1485 & 1243 & 242 & 29 & 368 & 252,8 \\
\hline Goni & 790 & 677 & 113 & 13 & 161 & 256,45 \\
\hline Ira & 1867 & 1215 & 652 & 73 & 305 & 191,53 \\
\hline Kienekuy & 1110 & 867 & 243 & 21 & 217 & 238,57 \\
\hline Labarani & 1259 & 1009 & 250 & 29 & 272 & 241,02 \\
\hline Lékuy & 814 & 717 & 97 & 6 & 213 & 270,69 \\
\hline Nouna & 1341 & 471 & 870 & 15 & 194 & 155,89 \\
\hline Toni & 932 & 672 & 260 & 23 & 200 & 223,58 \\
\hline Wèrèbèrè & 930 & 648 & 282 & 33 & 183 & 208,8 \\
\hline Yévédougou & 948 & 653 & 295 & 22 & 187 & 216,71 \\
\hline All CSPS & 19192 & $\begin{array}{l}13988 \\
(72,9 \%)\end{array}$ & $\begin{array}{l}5204 \\
(27,1 \%)\end{array}$ & & & 218,5 \\
\hline \multicolumn{7}{|c|}{$\begin{array}{l}\mathrm{C}_{1}: \text { cases during January to June period } \\
\mathrm{C}_{2}: \text { cases during July to December period } \\
\mathrm{C}_{\mathrm{t}} \text { : annual cases (total) } \\
\mathrm{C}_{\min } \text { : cases during the month of lowest incidence } \\
\mathrm{C}_{\max } \text { : cases during the month of highest incidence }\end{array}$} \\
\hline
\end{tabular}

Table 72: Malaria seasonality in Kossi province ${ }^{1430}$

Malaria transmission in Kossi Province is highly seasonal, with nearly three fourths of all cases recorded during the second half of the year (see table 72). Even though transmission never really ceases, case numbers decline substantially as drier conditions begin to prevail. Monthly malaria incidence then rises by a factor of around 5 to more than 30 towards the month of highest incidence.

1430 Based on data collected from individual CSPSs and CRSN Nouna. 
Figure 120 illustrates the spatial pattern of malaria seasonality which varies considerably within Kossi Province. In general, transmission seasonality is higher in the southern part of Kossi with the exception of the capital region around Nouna and decreases towards the North. It should be noted, however, that the map is based on data from only 14 CSPS.

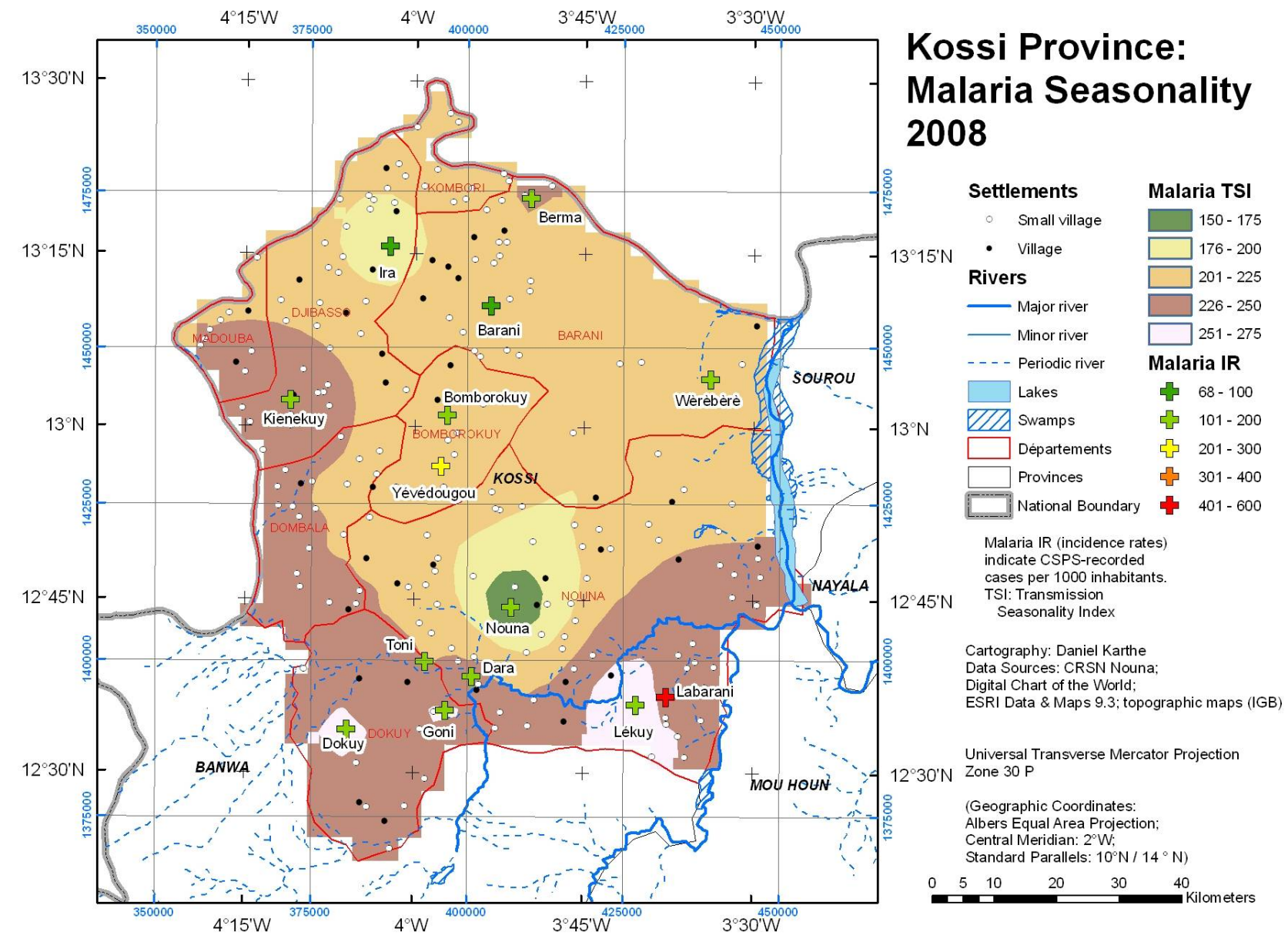

Figure 120: Spatial pattern of malaria seasonality in $2008^{1431}$

The lowest degree of seasonality was recorded in Kossi's capital region (Nouna), the only urban area in the province. The greatest variation was recorded at Lékuy CSPS, where more than $88 \%$ of all cases were recorded during the high transmission season, and monthly malaria-related consultations ranged from 6 in May to 213 in August. In general, malaria incidence tended to be lowest in June at an average 4,43 cases / 1000 inhabitants and then increased rapidly to nearly the 7 fold level in August (30,93 cases / 1000 inhabitants). The respective total case numbers were 562 for June and 3560 for August for the survey region. Even though the seasonality in case numbers follows similar pattern for practically all CSPS, there is an enormous spatial variation of monthly incidence (see figure 121),

1431 Based on data collected from individual CSPSs and CRSN Nouna; ESRI Data \& Maps 9.3; Digital Chart of the World and topographic maps (IGB). 
with rates ranging from $0,45 \%$ o (Nouna) to $11,42 \%$ (Labarani) in June and $3,96 \%$ o (Nouna) to $103,58 \%$ in Labarani. The dry season risk of contracting malaria was thus higher in the Labarani region than the rainy season risk in the Nouna region. A distance of only around $28,5 \mathrm{~km}$ between the CSPS illustrates the highly local pattern of malaria transmission.

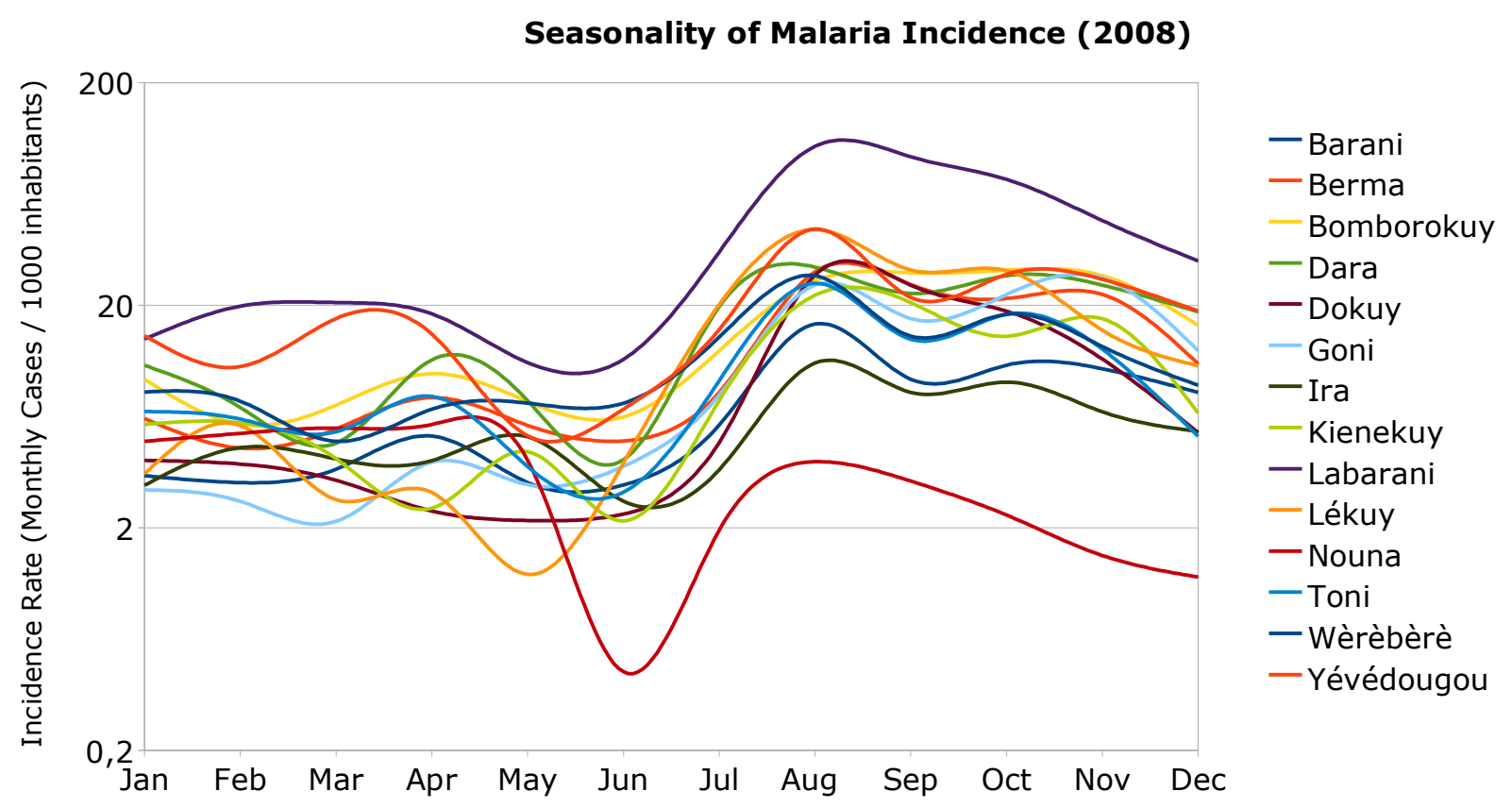

Figure 121: Seasonality of malaria incidence in Kossi (2008) $)^{1432}$

Table 73 shows the mean malaria incidence for three selected CSPS for the period from 2005 to 2008. August is clearly the month with the highest incidence, with case numbers more than doubling at all three CSPS as compared to July. Malaria incidence rates then remain on a relatively high level in September and October, followed by a decline from November to March, then undulating somewhat at relatively low levels. May and June tend to be the months of lowest malaria incidence.

In the 2005 to 2008 average, the rise in case numbers between June and July was less pronounced for Wèrèbèrè CSPS than for Lékuy and Toni. Plausible explanations are the later onset of the rainy season and lower precipitation totals in the northernmost region of Kossi as compared to the other two CSPS representing southern Kossi.

1432 Based on data collected from individual CSPSs and CRSN Nouna. 
Geographic Determinants of Malaria Transmission

\begin{tabular}{|l|c|c|c|c|}
\hline Month / Year & Lékuy & Wèrèbèrè & Toni & Average \\
\hline January & 8,33 & 8,86 & 9,4 & 8,86 \\
\hline February & 7,59 & 9,12 & 9,72 & 8,81 \\
\hline March & 5,07 & 8,09 & 7,99 & 7,05 \\
\hline April & 5,18 & 10,9 & 6,52 & 7,53 \\
\hline May & 3,79 & 5,74 & 4,47 & 4,67 \\
\hline June & 5,56 & 6,24 & 3,72 & 5,17 \\
\hline July & 12,55 & 8,78 & 7,01 & 9,45 \\
\hline August & 28,53 & 19,1 & 15,3 & 20,97 \\
\hline September & 18,86 & 11,59 & 10,44 & 13,63 \\
\hline October & 21,05 & 13,09 & 11,52 & 15,22 \\
\hline November & 14 & 11,55 & 9,17 & 11,57 \\
\hline December & 9,4 & 7,2 & 5,74 & 7,45 \\
\hline Total & $\mathbf{1 3 9 , 9}$ & $\mathbf{1 2 0 , 2 4}$ & $\mathbf{1 0 1}$ & $\mathbf{1 2 0 , 3 8}$ \\
\hline
\end{tabular}

Table 73: Average malaria incidence rates (2005 to 2008) recorded at three CSPS in Kossi ${ }^{1433}$

One remarkable feature of the seasonality pattern is a notable decline in observed infections from August to September, followed by another increase between September and October. This pattern showed for not only in the average incidence for the three CSPS and each CSPS individually (four year means) but was also discernible for other CSPS investigated in 2008 (see figure 121 and table 74).

\begin{tabular}{|l|c|c|c|c|c|}
\hline Month & $\mathbf{2 0 0 5}$ & $\mathbf{2 0 0 6}$ & $\mathbf{2 0 0 7}$ & $\mathbf{2 0 0 8}$ & Mean \\
\hline August & 301 & 238 & 364 & 596 & 374,75 \\
\hline September & 154 & 165 & 305 & 350 & 243,5 \\
\hline October & 197 & 155 & 382 & 407 & 285,25 \\
\hline
\end{tabular}

Table 74: Combined malaria cases recorded at Lékuy, Wèrèbèrè and Toni CSPS

Potential but hypothetical explanations include that this phenomenon is linked to poorer breeding conditions due to excessive rainfall and/or a more difficult access to health centers. Another hypothesis - a drop in malaria following an interruption in the rainy period - is rather unlikely since there is no indication for this phenomenon in the rainfall data from Dédougou (which may not correctly represent the situation in Kossi), and since this effect was noted in all years.

1433 Incidence rates calculated from case data obtained from the three CSPS. 


\subsubsection{Interannual Variations in Malaria Incidence}

Malaria incidence does not only vary seasonally, but marked differences in cumulative annual morbidity and mortality are observable in the study region. The interannual variations observed at three CSPS - Wèrèbèrè, Lékuy and Toni - show a marked increase in malaria transmission in 2008 as compared to preceding years and a relatively disparate situation and general trend at the three CSPS between 2005 and 2007.

At Lékuy CSPS, the annual malaria incidence ranged between above 100 and 167 cases per 1000 inhabitants (see table 75). However, annual incidence rates for 2005 and 2006 must be treated with some caution since data for a total of one and two months, respectively, were not available. Since these months did not fall into the high transmission season, the general trend probably remains unaffected, with 2006 being the year of the lowest and 2008 of the highest malaria incidence. The data for 2006 show an unusual seasonal pattern with a notable peak in December, i.e. outside the transmission season.

\begin{tabular}{|c|c|c|c|c|c|}
\hline $\begin{array}{l}\text { Month / } \\
\text { Year }\end{array}$ & 2004 & 2005 & 2006 & 2007 & 2008 \\
\hline January & N/A & 89 & 28 & 22 & 17 \\
\hline February & 34 & 30 & 65 & 19 & 28 \\
\hline March & 8 & 49 & N/A & 33 & 13 \\
\hline April & 19 & 41 & 23 & 19 & 14 \\
\hline May & 11 & 20 & 11 & 34 & 6 \\
\hline June & 16 & 24 & 25 & 36 & 19 \\
\hline July & 24 & 42 & 37 & 58 & 98 \\
\hline August & N/A & 110 & 79 & 132 & 213 \\
\hline September & 69 & 44 & 38 & 131 & 140 \\
\hline October & 201 & 77 & 53 & 125 & 139 \\
\hline November & 70 & 112 & $\mathrm{~N} / \mathrm{A}$ & 75 & 75 \\
\hline December & 70 & N/A & 106 & 18 & 52 \\
\hline Total $^{*}$ & 522 & 638 & 465 & 702 & 814 \\
\hline Population & 4389 & 4503 & 4620 & 4740 & 4864 \\
\hline Incidence* & $118,93 \%$ & $141,68 \%$ & $100,65 \%$ & $148,1 \%$ & $167,35 \%$ \\
\hline
\end{tabular}

Table 75: Malaria cases recorded at Lékuy CSPS ${ }^{1434}$

1434 Case data obtained from Lékuy CSPS. 
At Wèrèbèrè CSPS, the highest incidence rate was observed in 2004 , followed by a massive drop in 2005. Since then, a constant increase in malaria incidence could be noted (see table 76). Despite a rise from around 103 to 138 cases per 1000 inhabitants, malaria incidence remained at a lower level than at Lékuy CSPS. Like for Lékuy, 2006 was the most unusual year with respect to malaria seasonality. However, the common feature is the relatively low rainy season incidence but not the month of highest incidence which for Wèrèbèrè was clearly April 2006.

\begin{tabular}{|l|c|c|c|c|c|}
\hline $\begin{array}{l}\text { Month / } \\
\text { Year }\end{array}$ & $\mathbf{2 0 0 4}$ & $\mathbf{2 0 0 5}$ & $\mathbf{2 0 0 6}$ & $\mathbf{2 0 0 7}$ & $\mathbf{2 0 0 8}$ \\
\hline January & 58 & 83 & 52 & 40 & 55 \\
\hline February & 38 & 96 & 54 & 37 & 50 \\
\hline March & 160 & 66 & 49 & 62 & 33 \\
\hline April & 103 & 67 & 117 & 53 & 46 \\
\hline May & 89 & 15 & 40 & 45 & 49 \\
\hline June & 64 & 30 & 34 & 49 & 49 \\
\hline July & 45 & 30 & 43 & 58 & 97 \\
\hline August & 90 & 85 & 77 & 151 & 183 \\
\hline September & 87 & 49 & 57 & 97 & 98 \\
\hline October & 84 & 44 & 48 & 125 & 123 \\
\hline November & 88 & 46 & 85 & 81 & 88 \\
\hline December & 79 & 34 & 42 & 52 & 59 \\
\hline Total & $\mathbf{9 8 5}$ & $\mathbf{6 4 5}$ & $\mathbf{6 9 8}$ & $\mathbf{8 5 0}$ & $\mathbf{9 3 0}$ \\
\hline Population & $\mathbf{6 0 9 0}$ & $\mathbf{6 2 4 8}$ & $\mathbf{6 4 1 0}$ & $\mathbf{6 5 7 7}$ & $\mathbf{6 7 4 9}$ \\
\hline Incidence & $\mathbf{1 6 1 , 7 4} \% \mathbf{1 0 3 , 2 3} \%$ & $\mathbf{1 0 8 , 8 9} \%$ & $\mathbf{1 2 9 , 2 4} \%$ & $\mathbf{1 3 7 , 8} \%$ \\
\hline
\end{tabular}

Table 76: Malaria cases recorded at Wèrèbèrè CSPS ${ }^{1435}$

Toni CSPS recorded the lowest annual incidence rates which ranged from around $76 \%$ in 2007 to $117 \%$ in 2008 (see table 77). Once again, the pattern of malaria incidence differed from the usual seasonality in 2006, this time with a peak in January and February. In Toni, the level of malaria in 2008 remained at the level observed in 2005, but there was a relatively sharp contrast between 2007 and 2008 as the years of lowest and highest incidence.

1435 Case data obtained from Wèrèbèrè CSPS. 
Chapter 3 - Case Study: Malaria in Kossi Province

\begin{tabular}{|c|c|c|c|c|}
\hline Month / Year & 2005 & 2006 & 2007 & 2008 \\
\hline January & 96 & 104 & 35 & 53 \\
\hline February & 107 & 89 & 53 & 49 \\
\hline March & 81 & 77 & 44 & 43 \\
\hline April & 55 & 39 & 44 & 62 \\
\hline May & 41 & 47 & 19 & 30 \\
\hline June & 39 & 30 & 22 & 23 \\
\hline July & 69 & 35 & 38 & 73 \\
\hline August & 106 & 82 & 81 & 200 \\
\hline September & 61 & 70 & 77 & 112 \\
\hline October & 76 & 54 & 78 & 145 \\
\hline November & 60 & 61 & 59 & 101 \\
\hline December & 61 & 35 & 39 & 41 \\
\hline Total & 852 & 723 & 589 & 932 \\
\hline Population & 7373 & 7565 & 7762 & 7965 \\
\hline Incidence & $115,56 \%$ & $95,57 \%$ & $75,88 \%$ & $117,01 \%$ \\
\hline
\end{tabular}

Table 77: Malaria cases recorded at Toni CSPS ${ }^{1436}$

The variation of the cumulative annual malaria incidence situations between 2005 and 2008 is summarized in table 78 and figure 122 . While 2006 was the year with the lowest allover incidence of malaria, case numbers in 2008 were about $40 \%$ higher. In 2006 , the spatial distribution of malaria was relatively uniform, whereas in other years, large discrepancies can be observed between the CSPS recording the highest and lowest incidence rates.

\begin{tabular}{|c|c|c|c|c|c|}
\hline Year & 2005 & 2006 & 2007 & 2008 & $\begin{array}{c}\text { Population } \\
\text { (2008) }\end{array}$ \\
\hline Lékuy & $141,68 \%$ & $100,65 \%$ & $148,1 \%$ & $167,35 \%$ & 4864 \\
\hline Wèrèbèrè & $103,23 \%$ & $108,89 \%$ & $129,24 \%$ & $137,8 \%$ & 6749 \\
\hline Toni & $115,56 \%$ & $95,57 \%$ \% & 75,88 \%о & $117,01 \%$ & 7965 \\
\hline Mean 1437 & $120,16 \%$ & $101,70 \%$ & $115,41 \%$ & $140,72 \%$ & $\begin{array}{l}\text { Total: } \\
19578\end{array}$ \\
\hline
\end{tabular}

Table 78: Malaria incidence rates at three CSPSs in Kossi (2005-2008) ${ }^{1438}$

1436 Case data obtained from Toni CSPS; data for 2004 could not be obtained.

1437 Average weighted by the population of the CSPS.

1438 Incidence rates calculated from case data obtained from the three CSPS. 
2008 , the year with the highest allover incidence of malaria, actually started with below-average case rates that were among the lowest of the 2005 to 2008 period. The situation remained relatively stable until June. However, malaria incidence then roughly tripled from June to July, reaching a level that normally occurs one month later. While the peak in August is normal, this was the month when malaria cases significantly exceeded the levels observed from 2005 to 2007. Until October, the course of malaria incidence showed the typical pattern (a fall in September and slight rise in October), but remained at an unusual high level. By November, the situation normalized and was comparable to preceding years. In 2006, a year of comparably low levels of malaria transmission, the annual course of observed incidences remained much more stable, undulating between monthly incidence rates of $5 \%$ to $13 \%$.

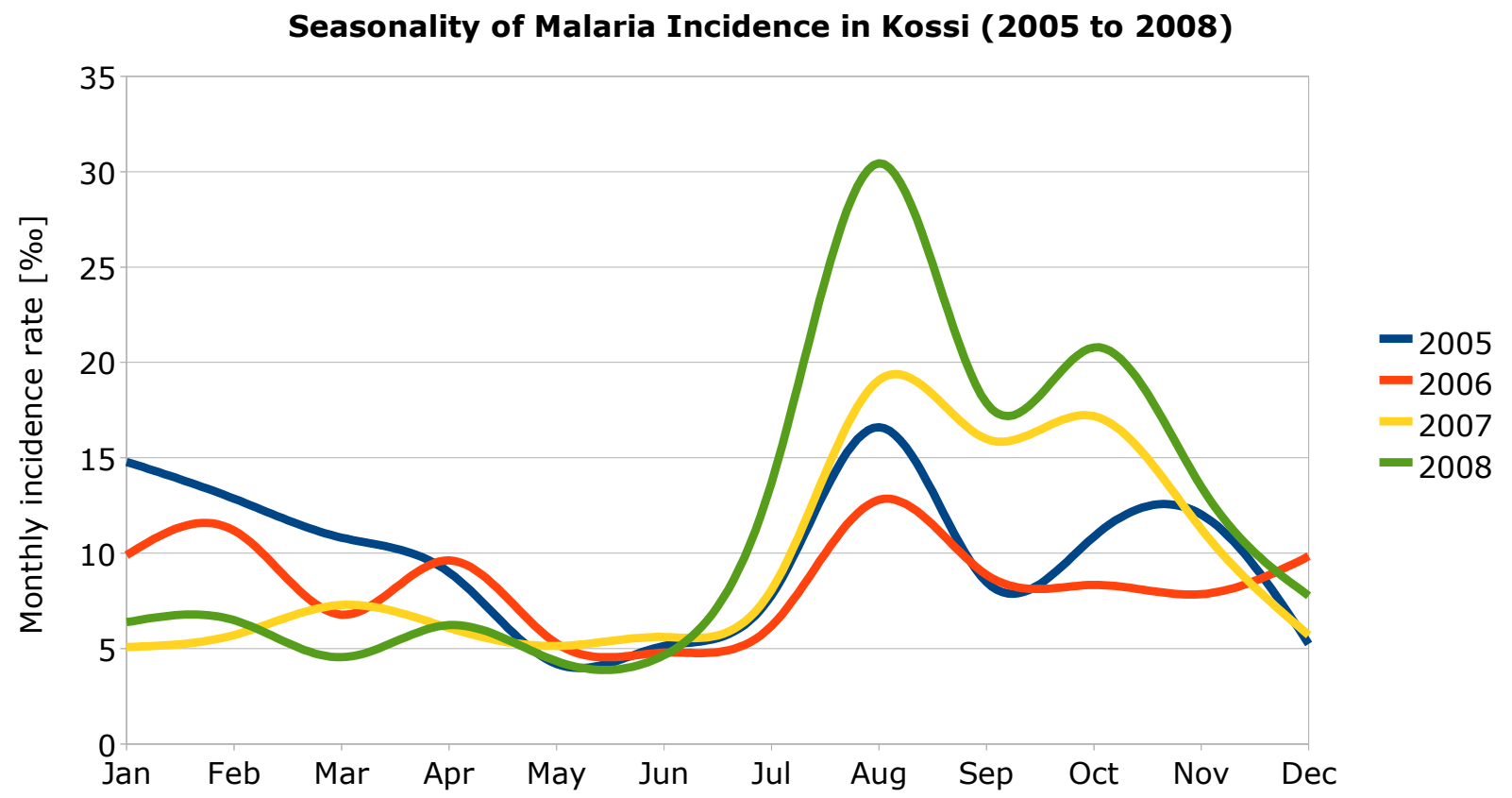

Figure 122: Seasonality of malaria incidence in Kossi, 2005 to $2008^{1439}$

2006 was the most unusual year, characterized by low incidence rates, a low level of seasonality and very different pattern at the three study sites: Toni CSPS recorded the highest incidence in January, Wèrèbèrè in April and Lékuy CSPS in December. Moreover, the characteristic drop and rise in September/October did not occur in 2006.

1439 Incidence rates calculated from case data obtained from Toni, Lékuy and Wèrèbèrè CSPS. 
One likely explanation for the very different situations observed in 2006 and other years is rainfall variability, both with respect to precipitation totals and the time of onset of the rainy season: In years of high malaria incidence such as 2008, the greatest deviation from average incidence levels can be observed during the rainy season.

\subsection{Determinants of Malaria in Kossi Province}

The spatial and temporal pattern of malaria incidence in Kossi Province are determined both by environmental factors regulating the reproduction, distribution and activity of vector mosquitoes and socioeconomic factors that influence vector-to-host contact.

\subsubsection{Malaria Vectors and Their Distribution}

The presence of vector breeding sites and suitable environmental conditions within mosquito habitats are the prerequisite for the establishment of anopheline populations capable of transmitting malaria. Within Kossi Province, there are marked differences in both habitat availability and vector distribution.

\subsubsection{Vector Population}

Various mosquito species are present in western Burkina Faso, but the composition of the mosquito population varies widely. Besides anophelines, mosquitoes of the genera Culex, Aedes and Mansonia occur. ${ }^{1440}$ Within Burkina Faso, Kossi Province falls into a zone of co-occurrence of Anopheles gambiae and Anopheles arabiensis (see figure 123), two of the most potent vectors of malaria. The principal genotype of Anopheles gambiae is the Mopti form which is well adapted to man-made habitats.

1440 Internal records of CRSN Nouna (personal communication with Mr.Saïdou Ouédraogo and Mr. François d'Assise Gonro). 


\section{Burkina Faso: Distribution of Malaria Vectors}

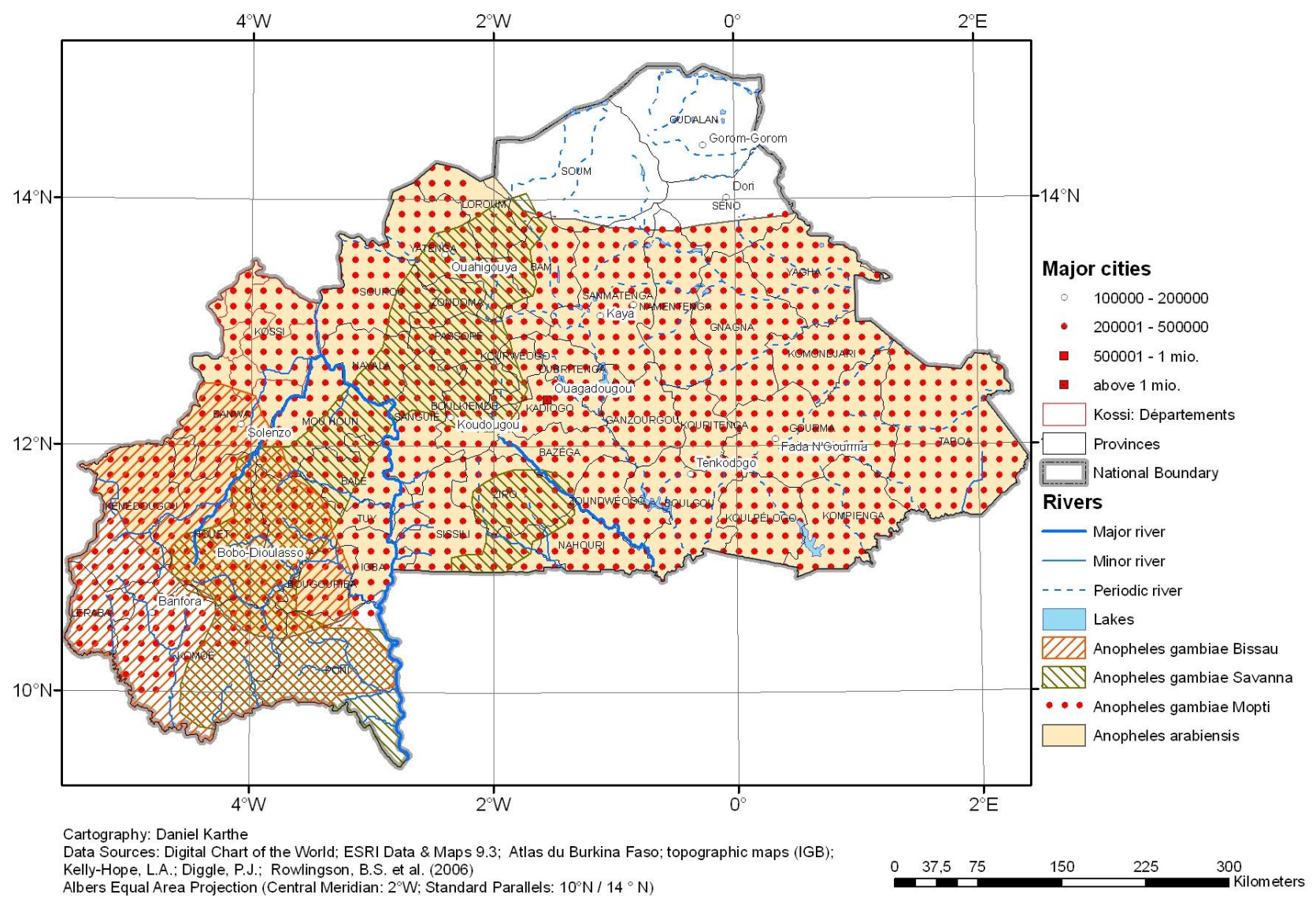

Figure 123: Distribution of important malaria vectors in Burkina Faso ${ }^{1441}$

So far, there has only been a limited number of systematic entomological surveys in Kossi province. Most of them were carried out by light trap capture (LTC, see figure 124) while some were counter-checked using the human landing catch (HLC) and pyrethrum spray capture (PSC) methods.

Recent mosquito survey data were available for two of the study villages, but it should be noted that the sampling periods did not coincide with the core period of the malaria survey carried out in the framework of this study. Table 79 provides an overview of entomological survey results at Kodougou. Different surveys revealed extremely different compositions of the mosquito population. Therefore, the following generalizations should be treated with some care:

1441 Based on Kelly-Hope, L.A.; Diggle, P.J.; Rowlingson, B.S. et al. (2006); Digital Chart of the World; ESRI Data \& Maps 9.3 and topographic maps (IGB). 
- Anophelines made up large but variable parts of the mosquito population; they tended to predominate towards the end of the dry season.

- The LTC method underestimated the proportion of anophelines entering houses or bloodfeeding on humans.

- Anopheles gambiae was overproportionately represented in indoor catches, outlining the comparatively high level of endophagy of this species.

- Anopheles funestus appeared to outnumber Anopheles gambiae (only) during the dry season.

Since the exact locations of the mosquito trap placements were unknown and since survey results are from different years, there may, however, also be different

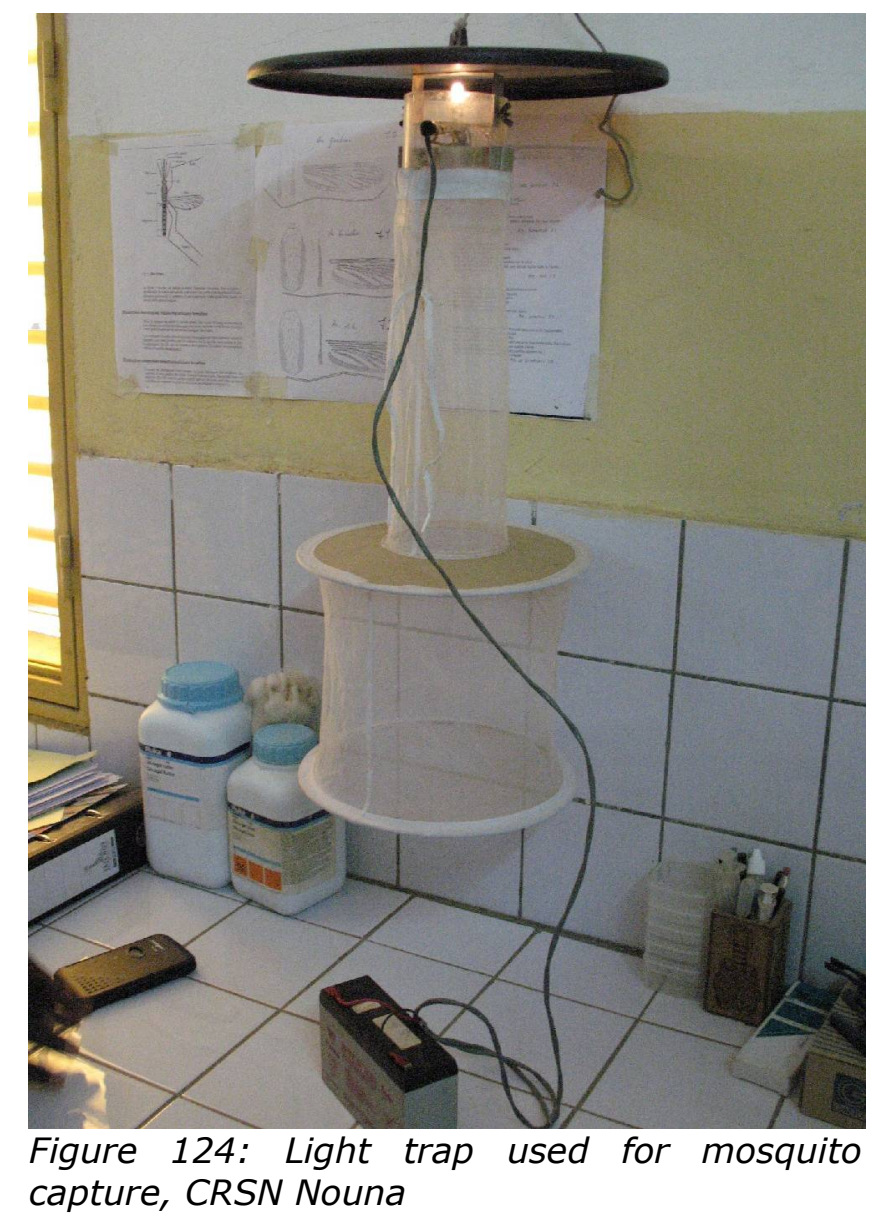
explanations for the observations. An "average" for all surveys has been calculated and while these percentages may be more representative of the allover situation, it should be kept in mind that they refer to surveys using different (and partially undocumented) sample sizes and methods. 


\begin{tabular}{|l|l|l|l|l|l|}
\hline $\begin{array}{l}\text { Location } \\
\text { Date } \\
\text { Method }\end{array}$ & $\begin{array}{l}\text { Composition of anopheline } \\
\text { population * }\end{array}$ & $\begin{array}{l}\text { Anopheles } \\
\text { gambiae }\end{array}$ & $\begin{array}{l}\text { Anopheles } \\
\text { funestus }\end{array}$ & $\begin{array}{l}\text { Anopheles } \\
\text { nili }\end{array}$ & $\begin{array}{l}\text { Composition of entire } \\
\text { mosquito population } \\
\text { spp. }\end{array}$ \\
\hline $\begin{array}{l}\text { Kodougou } \\
\text { Mar 2001 } \\
\text { LTC }\end{array}$ & $2 \%$ & $86 \%$ & $?$ & $93 \%$ & $\begin{array}{l}\text { Mansonia, } \\
\text { Culex and } \\
\text { Aedes spp. }\end{array}$ \\
\hline $\begin{array}{l}\text { Kodougou } \\
\text { Dec 2003 to } \\
\text { Nov 2004 } \\
\text { LTC }\end{array}$ & $79 \%$ & $20 \%$ & $1 \%$ & $27 \%$ & $73 \%$ \\
\hline $\begin{array}{l}\text { Kodougou } \\
\text { Dec 2003 to } \\
\text { Nov 2004 } \\
\text { HLC }\end{array}$ & $67 \%$ & $10 \%$ & $23 \%$ & $37 \%$ & $63 \%$ \\
\hline $\begin{array}{l}\text { Kodougou } \\
\text { Dec 2003 to } \\
\text { Nov 2004 } \\
\text { PSC }\end{array}$ & $97 \%$ & $3 \%$ & $0 \%$ & $64 \%$ & $36 \%$ \\
\hline Average & $\mathbf{6 1 \%}$ & $\mathbf{3 0 \%}$ & $\geq \mathbf{6 \%}$ & $\mathbf{5 5 \%}$ & \\
\hline * Percentages do not add up to 100\% when additional species were identified. \\
\hline
\end{tabular}

Table 79: Results of mosquito surveys in the Lékuy CSPS area ${ }^{1442}$

The results of the mosquito surveys conducted in villages around Toni CSPS are presented in table 80 . The surveys showed a dominance of anophelines, particularly during the cool season, when Anopheles gambiae appeared to be less dominant than at other times of the year. However, the same caveats mentioned in the discussion of the data from Kodougou also apply here.

1442 Data sources: CRSN Nouna (personal communication with Mr. Saïdou Ouédraogo); YÉ, Y. (2006), p. 93-95. 


\begin{tabular}{|c|c|c|c|c|c|}
\hline \multirow{2}{*}{$\begin{array}{l}\text { Location } \\
\text { Date } \\
\text { Method }\end{array}$} & \multicolumn{3}{|c|}{$\begin{array}{l}\text { Composition of anopheline } \\
\text { population * }\end{array}$} & \multicolumn{2}{|c|}{$\begin{array}{l}\text { Composition of entire } \\
\text { mosquito population }\end{array}$} \\
\hline & $\begin{array}{l}\text { Anopheles } \\
\text { gambiae }\end{array}$ & $\begin{array}{l}\text { Anopheles } \\
\text { funestus }\end{array}$ & $\begin{array}{l}\text { Anopheles } \\
\text { nili }\end{array}$ & $\begin{array}{l}\text { Anopheles } \\
\text { spp. }\end{array}$ & $\begin{array}{l}\text { Mansonia, } \\
\text { Culex and } \\
\text { Aedes spp. }\end{array}$ \\
\hline $\begin{array}{l}\text { Goni } \\
\text { Dec } 2003 \text { to } \\
\text { Nov } 2004 \\
\text { LTC }\end{array}$ & $80 \%$ & $19 \%$ & $1 \%$ & $44 \%$ & $56 \%$ \\
\hline $\begin{array}{l}\text { Goni } \\
\text { Dec } 2003 \text { to } \\
\text { Nov } 2004 \\
\text { HLC }\end{array}$ & $94 \%$ & $6 \%$ & $0 \%$ & $33 \%$ & $67 \%$ \\
\hline $\begin{array}{l}\text { Goni } \\
\text { Dec } 2003 \text { to } \\
\text { Nov } 2004 \\
\text { PSC }\end{array}$ & $99 \%$ & $1 \%$ & $0 \%$ & $65 \%$ & $35 \%$ \\
\hline $\begin{array}{l}\text { Kamadena } \\
\text { Dec } 2003 \\
\text { LTC }\end{array}$ & $55 \%$ & $27 \%$ & $11 \%$ & $96 \%$ & $4 \%$ \\
\hline $\begin{array}{l}\text { Dembèlela } \\
\text { Dec } 2003 \\
\text { LTC }\end{array}$ & $56 \%$ & $42 \%$ & $0 \%$ & $92 \%$ & $8 \%$ \\
\hline Average & $77 \%$ & $19 \%$ & $2 \%$ & $66 \%$ & $34 \%$ \\
\hline
\end{tabular}

Table 80: Results of mosquito surveys in the Toni CSPS area ${ }^{1443}$

The surveys carried out in Kodougou and various villages in the Toni CSPS area indicate some differences between the two locations/areas. The villages in the zone of Toni CSPS fall into a dry savanna region without nearby permanent water bodies. Both the percentage of anophelines in general and of Anopheles gambiae in particular were greater here than at Kodougou, the village in proximity to the Mouhoun river. By contrast, Anopheles funestus was found in greater proportion in Kodougou, which is not surprising since this vector readily breeds in streams and prefers shaded locations for oviposition as are found in the gallery forest surrounding the Mouhoun.

1443 Data sources: CRSN Nouna (personal communication with Mr. Saïdou Ouédraogo); YÉ, Y. (2006), p. 93-95. 


\subsubsection{Vector Habitats}

Numerous types of vector habitats are found in Kossi Province. Perennial habitats include slowmoving streams like the Sourou and Mouhoun which are partially flanked by gallery forests and man-made reservoirs used for irrigation (see figure 125). Temporary habitats include relatively long-lasting mares as well as small transient pools, irrigated fields and man-made excavations usually created either for storage purposes or linked to the manufacture of mud-bricks. Their malariologic importance is based upon their location within or just outside villages. Moreover, population growth results in the continued demand for bricks, thus resulting in both new excavations and the extension of existing ones. While small collections of water forming due to animal footprints or tire tracks sometimes allow the breeding of anopheline larvae, they have not been investigated in this

Figure 125: Reservoir on the Sourou's bank
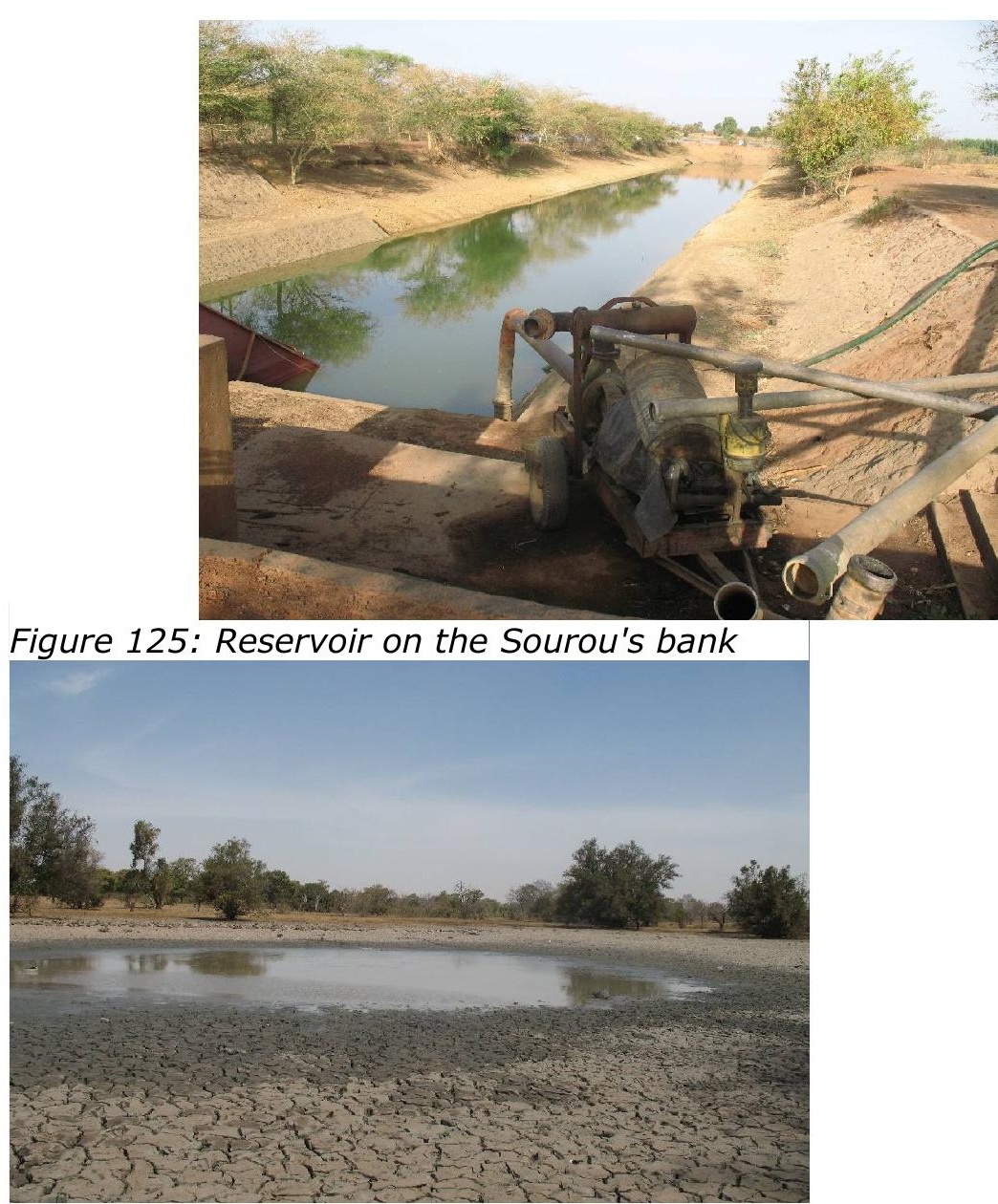

Figure 126: Mare study due to their high numbers, short-lived nature and the difficulty to assess whether they actually contribute to mosquito reproduction. In fact, even the observation of larvae and pupae is insufficient to determine the productiveness of such habitats since desiccation may occur prior to adult emergence.

Different types and numbers of vector habitats were found in the study villages. Probably the most important difference is that villages close to the Mouhoun and Sourou fall into a zone where mosquito breeding may be perennial and where gallery forests, temporal inundation zones and swampland represent potential refugia for mosquito populations during the height of the dry season due to both lower temperatures and higher levels of air humidity. By contrast, villages not located in proximity to the two rivers typically feature transient breeding sites. These begin to fill up with water shortly after the onset of the rainy season and persist from anywhere between a few days and several months. 
One important insight from the study is that relatively limited numbers of breeding sites create relatively large zones of potential malaria transmission. However, neither the duration of inundation in different habitats types nor their productivity under variable meteorological conditions have been systematically assessed for the study region yet.

\subsubsection{Geographic Determinants of Malaria Transmission}

Several geographic factors have an influence on the spatio-temporal pattern of malaria transmission in Kossi. Climate is an important determinant of malaria transmission dynamics, an vegetation indices appear to be a good predictor of malaria risks. Higher elevations tend to be connected to lower risks, but this is most likely due to the absence of permanent bodies of surface water. Personal protection was found to vary considerably and can be expected to have an impact disease incidence, too, as may other sociogeographic factors.

\subsubsection{Climate}

In areas where anophelines breed in temporary bodies of water, surface water must be available for a sufficiently long period so that the complete process from oviposition to larval and finally adult mosquito emergence can take place. In Kossi Province, temperatures during the rainy season are around $26^{\circ} \mathrm{C}$ to $27^{\circ} \mathrm{C}$. In regions where permanent bodies of water do not exist, this means that temporary larval breeding sites must persist for around 10 days or more for emergence of adults.

Malaria incidence in Kossi Province is closely related to precipitation. Figure 127 depicts the annual course of precipitation, vector density and malaria incidence recorded in Cissé during 2004. Both the vector density and the incidence of clinical malaria peaked during the second month of substantial rainfall. However, even at very low vector densities, malaria transmission appears to continue. 
Cissé: Precipitation, Vector Density and Clinical Malaria (2004)

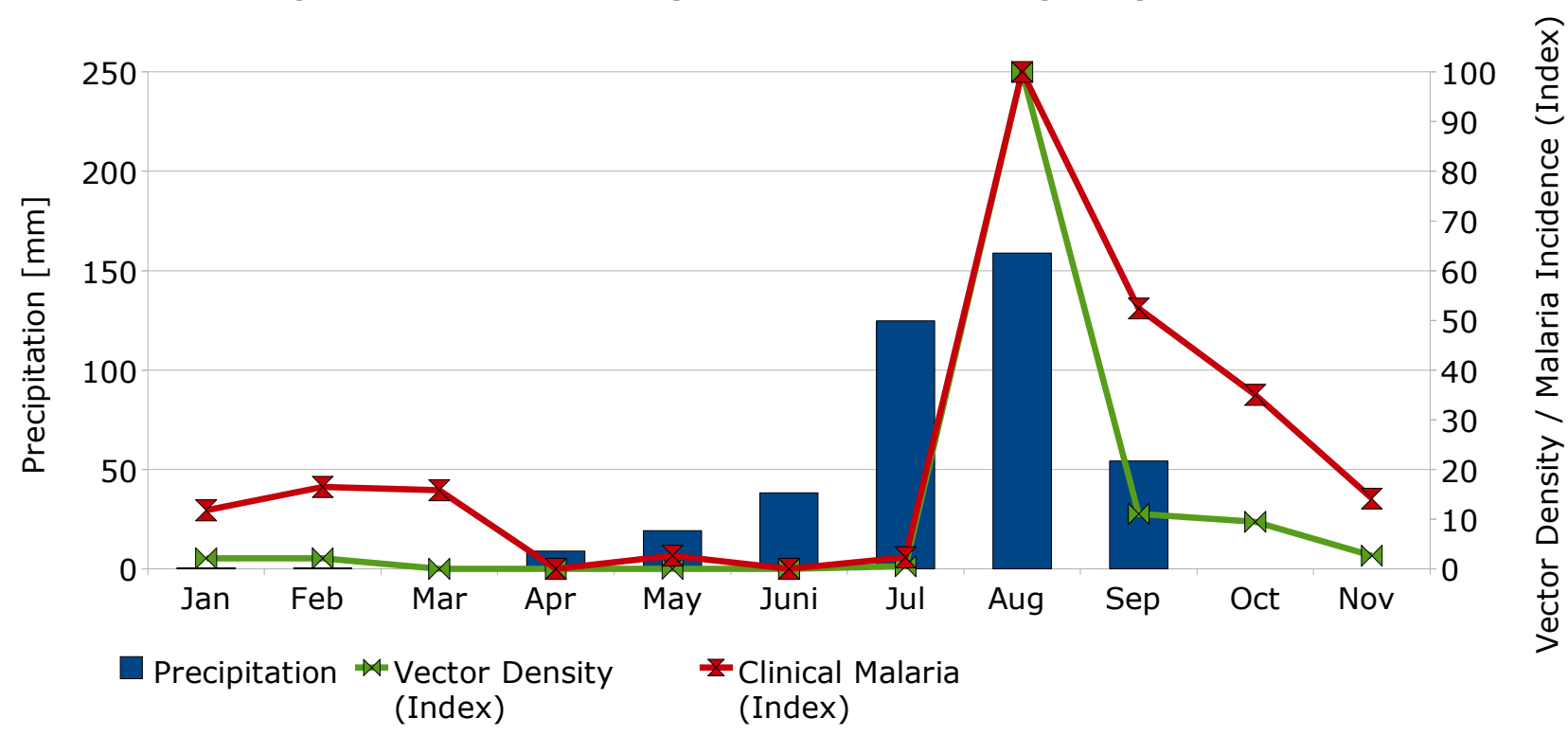

Figure 127: Precipitation, vector density and clinical malaria in Cissé1444

Figure 128 shows the malaria incidence rates in children under five and average rainfall recorded by meteorological stations located in Cissé, Toni (near Goni) and Nouna in 2004. Averaging was used as a gap-filling strategy which was necessary due to incomplete datasets. However, since meteo stations do not represent the situation in mosquito habitats anyways, a three station average may in fact just as well reflect the situation there.

1444 Based on Yé, Y. (2005), pp. 79 \& 98 and climate data provided by CRSN. 


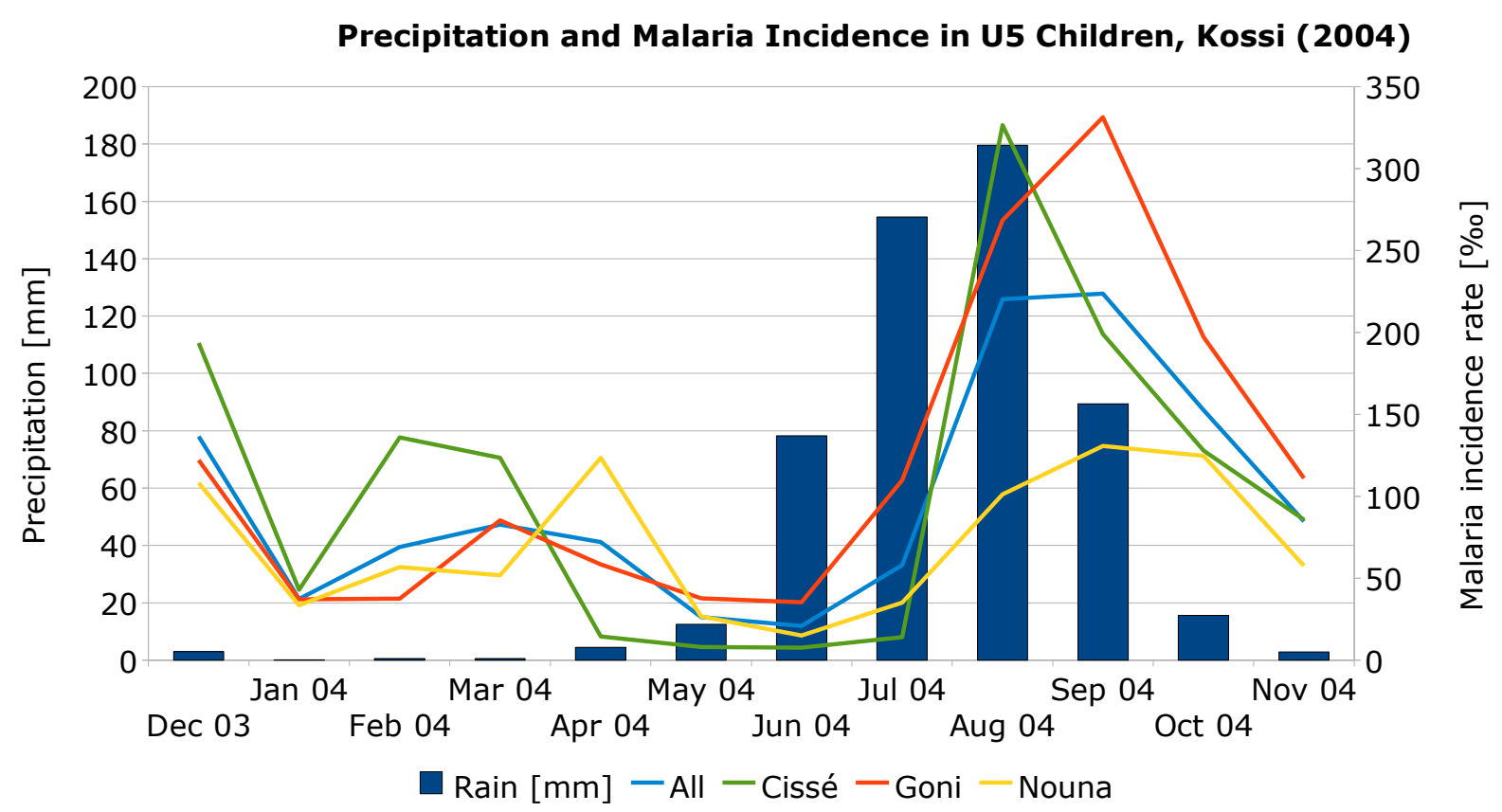

Figure 128: Precipitation and malaria incidence in U5 children in Kossi (2004) 1445

During the dry season, malaria incidence remained at a relatively low but variable level and rose sharply around one to two months after the onset of the rainy season. Around and shortly after the peak of the rainy season, malaria incidence rose to about three times the dry season level. Rates decreased relatively uniformly between September and November; this drop occurred more gradually than the rapid increase in July/August. In the urban environment represented by Nouna, the moisture-related increase was both less marked and slower.

Figure 129 shows a longer time series for the temporal variation of malaria incidence and precipitation. Malaria incidence here refers to an average for Wèrèbèrè, Toni and Lékuy CSPSs, while precipitation data were recorded at Dédougou. The period from 2005 to 2008 encompassed years of comparably lower and higher precipitation: 2005 was the driest year $(591 \mathrm{~mm}), 2007$ the wettest year $(702 \mathrm{~mm})$. Rainfall in 2006 and 2008 was close to the long-term mean of the 1984 to 2008 period, but despite similar rainfall totals, the distribution of rains was very different in these two years: while 2006 saw a relatively long-lasting rainy season of moderate intensity, rainfall in 2008 was more intensive but concentrated.

1445 Based on Yé, Y.; Louis, V.R.; Simboro, S. \& Sauerborn, R. (2007), doi:10.1186/1471-2458-7-101, and climate data provided by CRSN. 


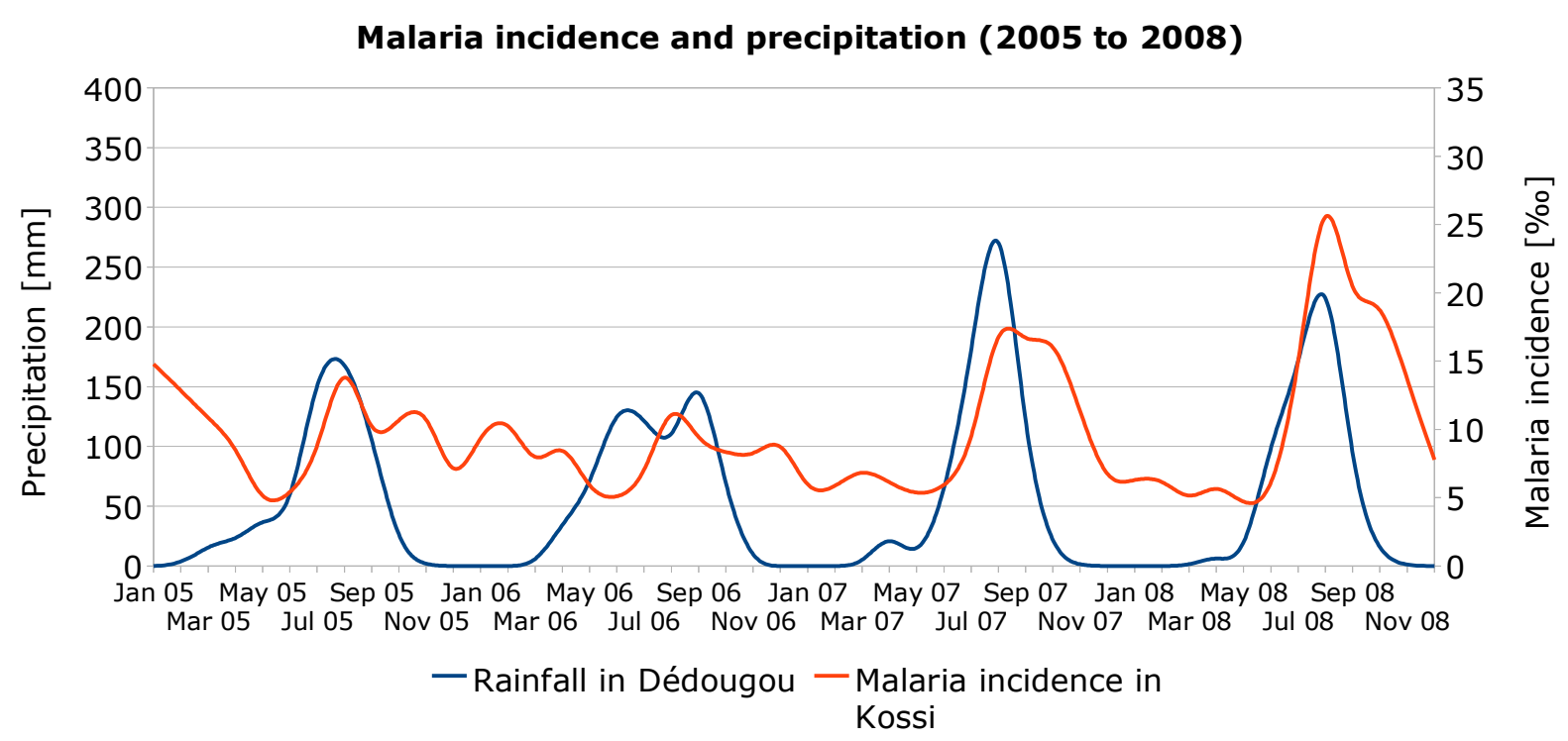

Figure 129: Malaria incidence and precipitation in Kossi (2005 to 2008) ${ }^{1446}$

In years of low rainfall (e.g., 2005) or moderate but relatively well-distributed rainfall (e.g., 2006), malaria incidence followed a relatively a-seasonal pattern. In years of relatively abundant and highly concentrated rainfall (e.g., 2007 and 2008), a marked rise in malaria incidence followed around one month after the onset of intensive rains. Malaria incidence then remains at relatively high levels for a period of around 2 months following rains.

Yé et al. (2007) noted that rainfall had an observable effect on clinical malaria in Kossi only for monthly quantities of more than $100 \mathrm{~mm}^{1447}$, a fact apparently linked to massive evaporation and water infiltration into the soil at the beginning of the rainy season. This claim is supported by the observations from 2005 to 2008; in fact, a clear link between rainfall and malaria incidence only becomes discernible at monthly quantities of $150 \mathrm{~mm}$.

Figure 130 illustrates the links between precipitation, vector density and malaria incidence in and around Kodougou. In fact, the density of the malaria vector population appears to be more sensitive to rainfall seasonality than malaria case numbers (see figure 130): while observed vector numbers (the index is proportional to vector mosquito catches using light traps) were close to zero during the dry season, their numbers increased in August, the month following the "full" onset of the rainy season. The incidence of clinical malaria roughly but not exactly followed the same pattern, indicating that other parameters play a role, too.

1446 Based on data provided by the CSPSs in Lékuy, Toni and Wèrèbèrè, and meteorological data from Dédogou.

1447 Yé, Y.; Louis, V.R.; Simboro, S. \& Sauerborn, R. (2007), doi:10.1186/1471-2458-7-101. 


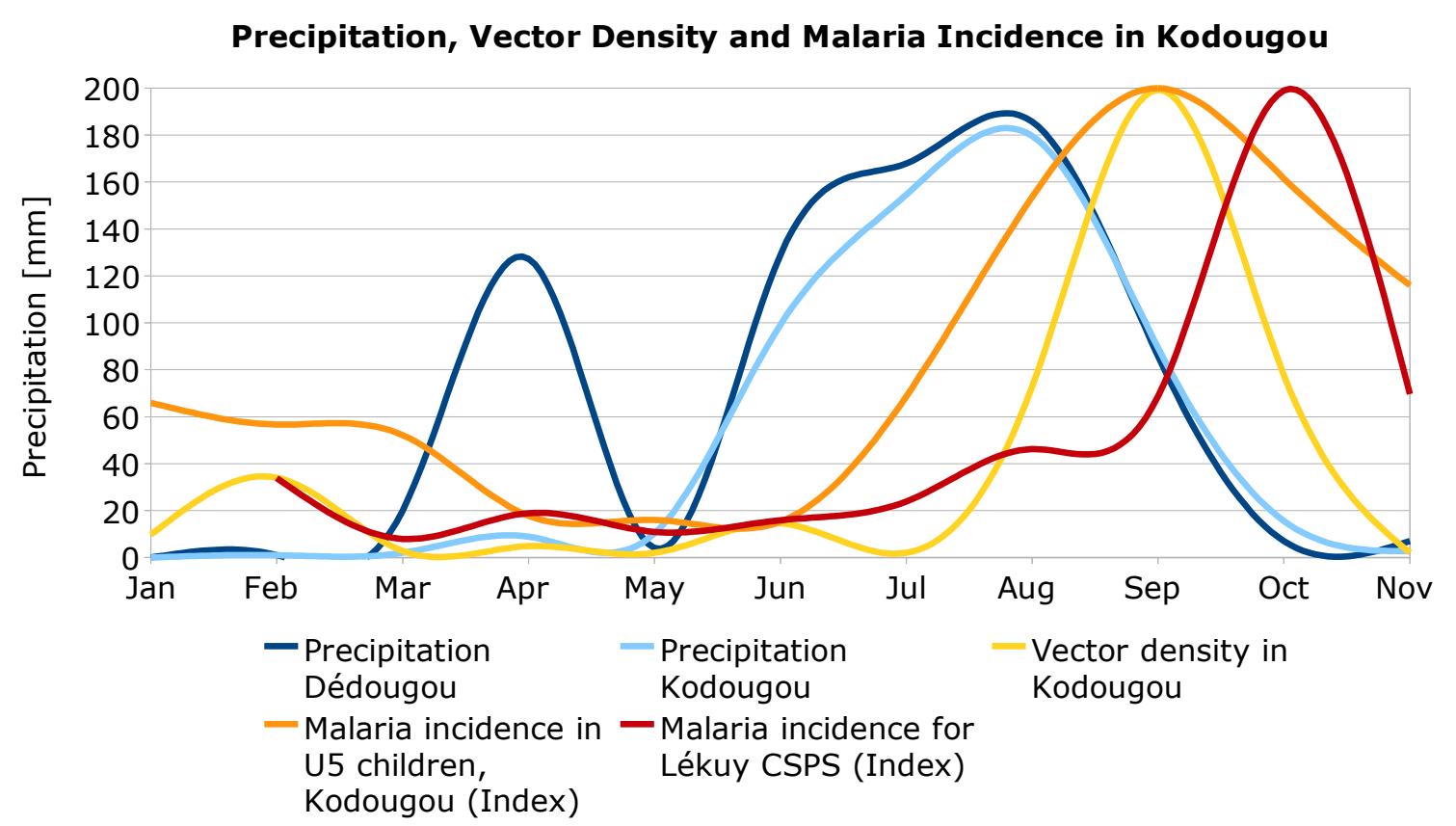

Figure 130: Precipitation, vector density and clinical malaria in Kodougou (2004) ${ }^{1448}$

Temperature also plays a role in determining malaria incidence. YÉ et al. (2007) found temperature to be the best meteorological predictor of malaria incidence in Kossi. ${ }^{1449}$ A sudden drop in the numbers of mosquitoes between December and January is probably a result of temperatures falling to below $16^{\circ} \mathrm{C}$, the threshold for the larval development of Anopheles gambiae. Despite a decline in vector numbers during the cool season (December to February) and hot and dry phase (March to May), adult mosquitoes can be found in the study region throughout the year. Research into the age structure of the mosquito population has indicated that there is not simply an aging mosquito population but that there is perennial breeding. ${ }^{1450}$

Data for the year 2004 in Nouna show that temperature alone is not a good predictor of malaria transmission (see figure 131). Observations here are based on vector numbers captured by the LTC method (with light traps being stationed in Nouna), weather data from Nouna's meteorological station and Terra MODIS land surface temperatures ( 8 day MVCs for midday). While a rise in vector numbers coincided with a prior decrease in temperatures in between July and October, similar temperatures during the "winter" months were not associated with comparable increases in vector numbers, most probably

1448 Based on Yé, Y. (2005), pp. 81 \& 98; malaria case data provided by CSPS Lékuy and climate data provided by CRSN Nouna.

1449 Yé, Y.; Louis, V.R.; Simboro, S. \& SaUerborn, R. (2007), doi:10.1186/1471-2458-7-101.

1450 Yé, Y.; Sauerborn, R.; Séraphin, S. \& Hoshen, M. (2007), p. 381. 
because of the absence of suitable breeding sites. Moreover, declining temperatures during the rainy season may in fact be little more than the cooling effect brought by monsoonal rains.

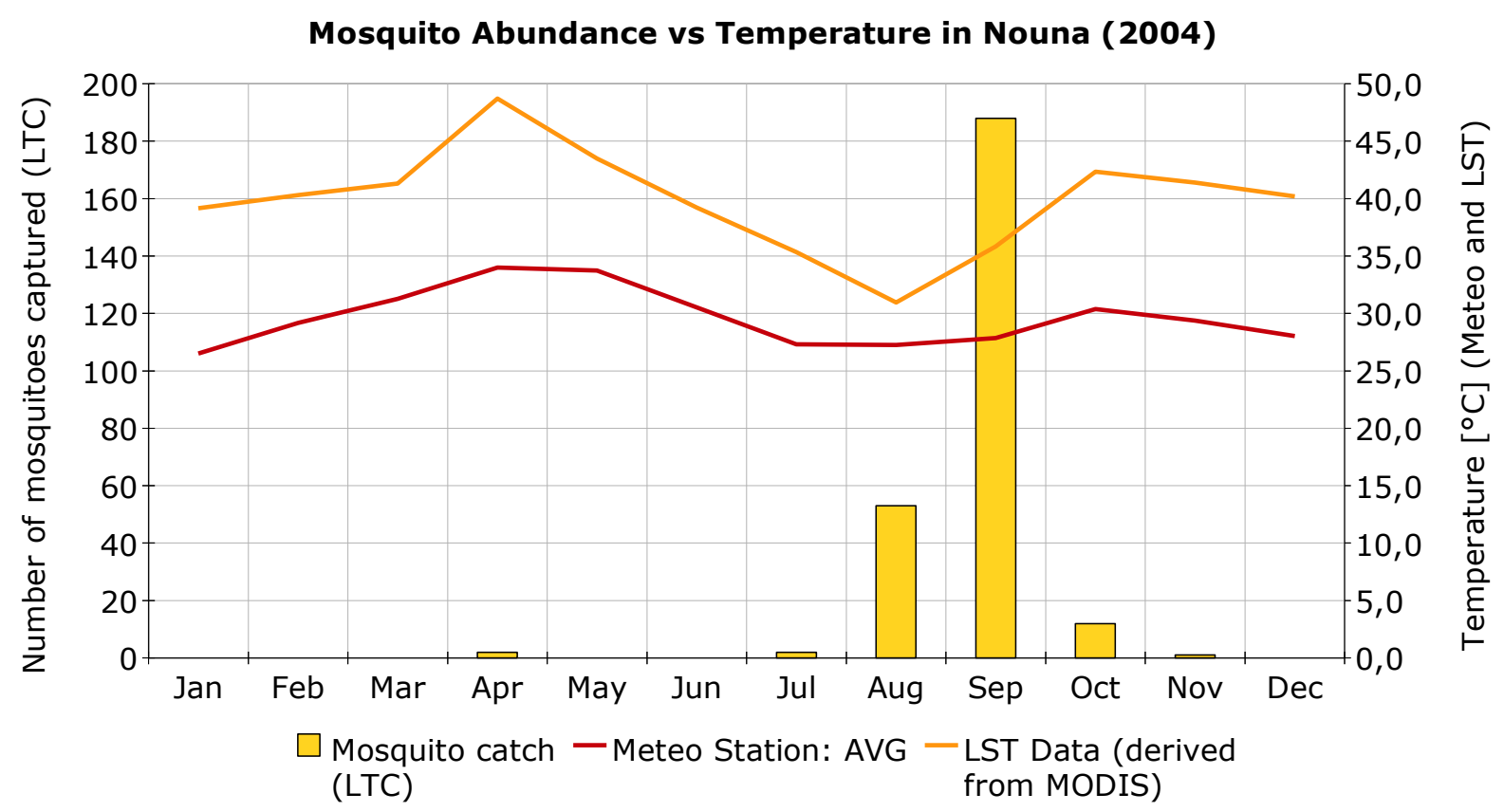

Figure 131: Anopheles gambiae abundance vs. temperature in Nouna (2004) ${ }^{1451}$

Figure 131 also illustrates that LSTs and air temperatures followed a similar pattern, with an apparent reduction in their difference during the rainy season. The relatively strong overestimation air temperatures by LSTs is due to several factors, including not only the more intensive heating of the ground during the dry season but also different measuring periods (average temperatures recorded by Nouna meteo station as opposed to midday measurements by the satellite) and the MVC compositing technique utilized during LST data preprocessing.

\subsubsection{Other Environmental Factors}

Climate is an important but not the only environmental determinant of the spatial and temporal pattern of malaria transmission dynamics in Kossi.

The relief and hydrography are closely related in Kossi province. While the relief is flat enough to be of minor importance regarding temperature and rainfall and elevations are far from the limit of malaria transmission, higher altitudes in Kossi tend to be related to lower malaria incidence. The cause of

1451 Data sources: CRSN Nouna (temperature data); calculated monthly mean LST temperatures (Terra MODIS, MOD11A1 product); Yé, Y. (2005), p. 98 (LTC catches). 
this effect may not lie in the relief itself but in hydrographic pattern which are closely linked to it: the high-incidence areas fall into the low-lying zones close to the Mouhoun and Sourou rivers, while considerably lower incidences are found in higher-lying northwestern Kossi.

Figure 132 illustrates the relation between elevation and malaria incidence. Overall, elevation is negatively correlated with malaria incidence $(r=-0,35)$, with the highest rates observed in the regions of lowest altitude. In fact, low altitude appears to be a prerequisite for very high levels of malaria. Other than this restriction of very high malaria incidence to the lowest lying regions, elevation is clearly not a good overall predictor of malaria risk, with levels of around $100 \%$ o to $200 \%$ o occurring anywhere between $260 \mathrm{~m}$ and $350 \mathrm{~m}$.

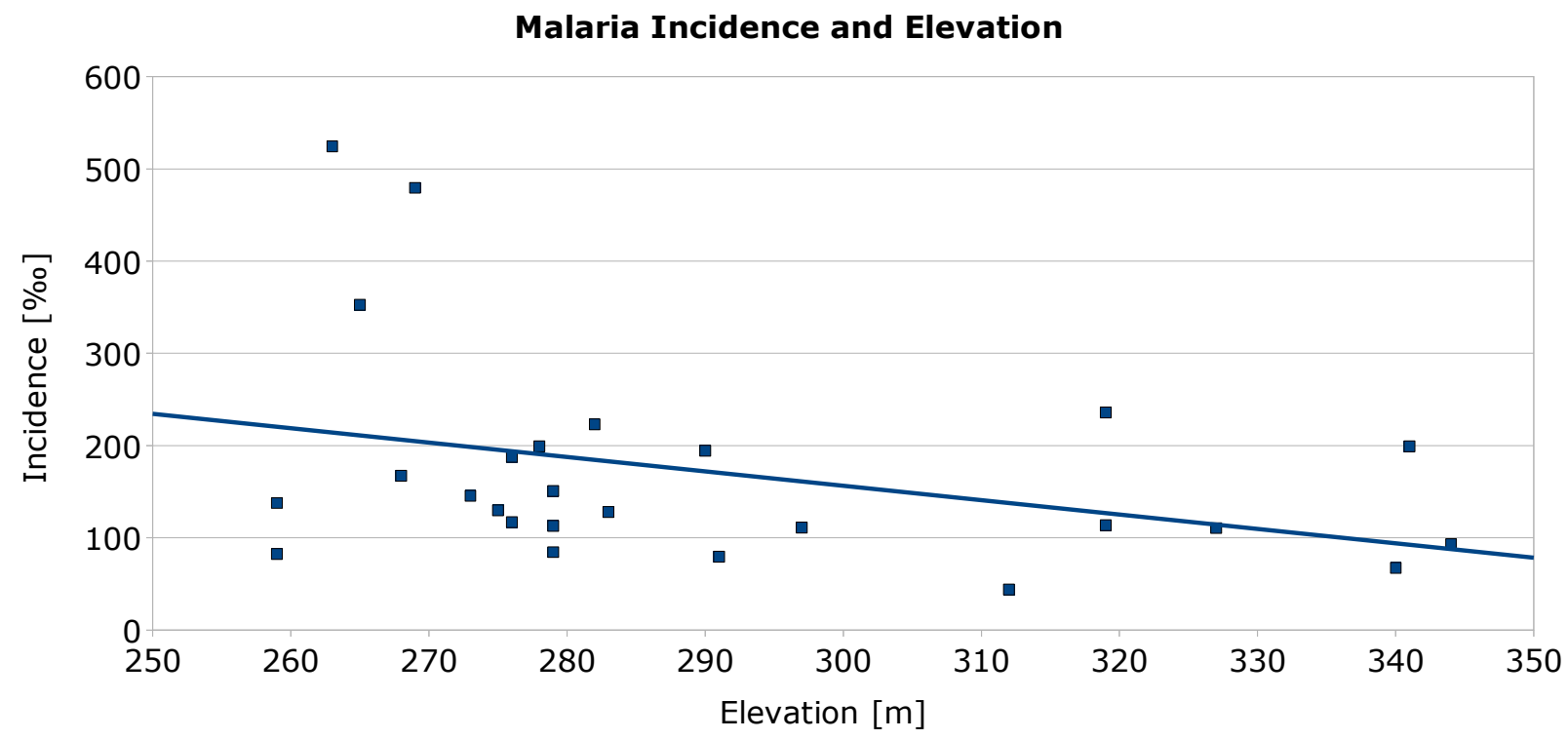

Figure 132: Malaria incidence and elevation in Kossi ${ }^{1452}$

In fact, the hydrological situation appears to play a greater role here (see table 81):

1452 Based on malaria incidence rates of 26 CSPSs in Kossi and their altitude derived from SRTM maps. 


\begin{tabular}{|c|c|c|c|}
\hline $\begin{array}{l}\text { Hydrological } \\
\text { situation }\end{array}$ & CSPS & $\begin{array}{l}\text { Malaria } \\
\text { incidence } \\
\text { range }\end{array}$ & $\begin{array}{l}\text { Average } \\
\text { malaria } \\
\text { incidence }\end{array}$ \\
\hline $\begin{array}{l}\text { Regions without } \\
\text { major bodies of } \\
\text { surface water }\end{array}$ & $\begin{array}{l}\text { Barani, Berma, Bomborokuy, } \\
\text { Borekuy, Dembo, Djibasso, } \\
\text { Dokuy, Doumbala, Ira, } \\
\text { Kienekuy, Konankoira, } \\
\text { Konkui-Kouro, Madouba, } \\
\text { Nian, Nouna, Yévédougou }\end{array}$ & $\begin{array}{l}44 \% \text { o to } \\
236 \% \text { o }\end{array}$ & $131 \%$ \\
\hline $\begin{array}{l}\text { Regions close to } \\
\text { temporary } \\
\text { streams }\end{array}$ & Gassinko, Goni, Toni & $\begin{array}{l}117 \% \text { o to } \\
195 \% \text { o }\end{array}$ & $147 \%$ \\
\hline $\begin{array}{l}\text { Regions close to } \\
\text { perennial rivers }\end{array}$ & $\begin{array}{l}\text { Bagala, Bourasso, Dara, } \\
\text { Labarani, Lékuy, Sono, } \\
\text { Wèrèbèrè }\end{array}$ & $\begin{array}{l}82 \% \text { to } \\
524 \% \text { o }\end{array}$ & $276 \%$ \\
\hline
\end{tabular}

Table 81: Hydrological situation and malaria incidence

All CSPS that are close to major perennial rivers recorded higher malaria incidences in 2008 than those without any major surface water bodies within their zone. The average altitude of the CSPS close to the Sourou and Mouhoun was $266 \mathrm{~m}$, while the average altitude of those in the without surface water bodies was $303 \mathrm{~m}$. Relief and hydrography are thus closely linked in Kossi, with hydrography probably being the more important factor with regard to malaria transmission.

In dry savanna areas, vegetation indices such as the NDVI or EVI have been found to be well suited to assess pattern of rainfall and thus moisture distribution. Figure 133 illustrates that the NDVI is a relatively good proxy for malaria risk. 


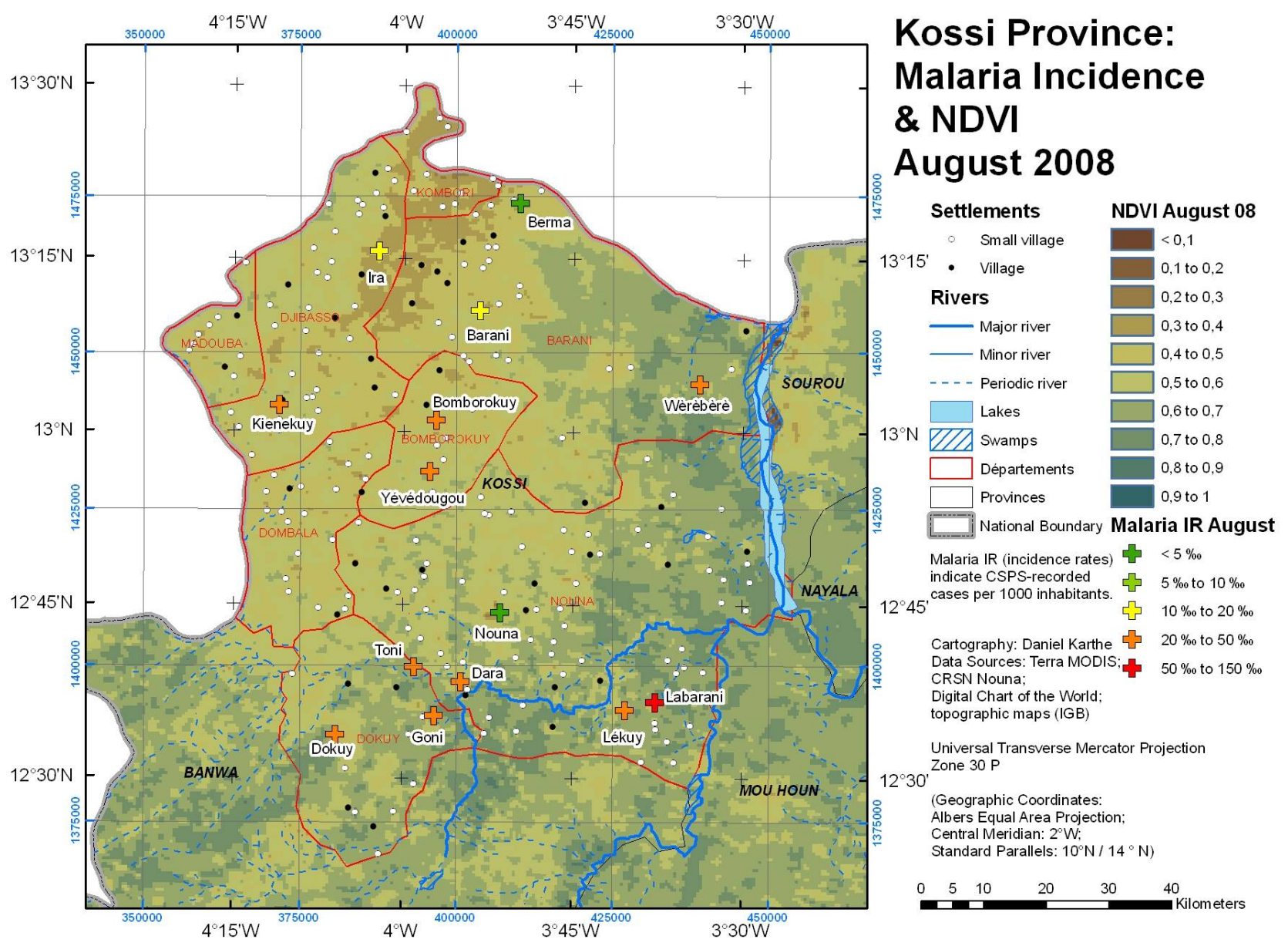

Figure 133: Malaria incidence and NDVI in Kossi (August 2008) ${ }^{1453}$

In August 2008, NDVIs observed over Kossi Province ranged from around 0,3 to 0,8 . Malaria incidence rates tended to be higher in areas of higher NDVIs (see table 82). In fact, the regions of highest malaria incidence coincided with the regions of highest NDVIs whereas the regions of lowest malaria incidence coincided with the lowest NDVIs observed.

\begin{tabular}{|l|l|l|l|l|}
\multirow{2}{*}{ CSPS } & \multicolumn{3}{|c|}{5 km Buffer Zone around CSPS } & Malaria \\
\cline { 2 - 5 } & Mean NDVI & Min NDVI & Max NDVI & Incidence \\
\hline Ira & 0,42 & 0,31 & 0,57 & $11,00 \%$ \\
\hline Nouna & 0,52 & 0,27 & 0,67 & $3,96 \% 0$ \\
\hline Lékuy & 0,63 & 0,43 & 0,74 & $43,93 \% 0$ \\
\hline Labarani & 0,68 & 0,45 & 0,8 & $103,57 \% 0$ \\
\hline
\end{tabular}

Table 82: Malaria incidence and NDVI (August 2008)

1453 Based on Terra MODIS NDVI (MVC of MOD13A1) images; data provided by individual CSPSs \& CRSN Nouna; Digital Chart of the World and topographic maps (IGB). 
At the regional scale, vegetation indices are thus relatively good predictors of malaria transmission risk. Moreover, they do not only quite well reflect precipitation (see figure 134): the response time of the vegetation to rainfall is around one month, roughly equaling the time lag between rainfall and an increase in mosquito abundance. This explained why NDVIs showed a higher degree of correlation with mosquito abundance than rainfall $\left(r^{2}=0,74\right.$ vs. $\left.r^{2}=0,48\right)$.

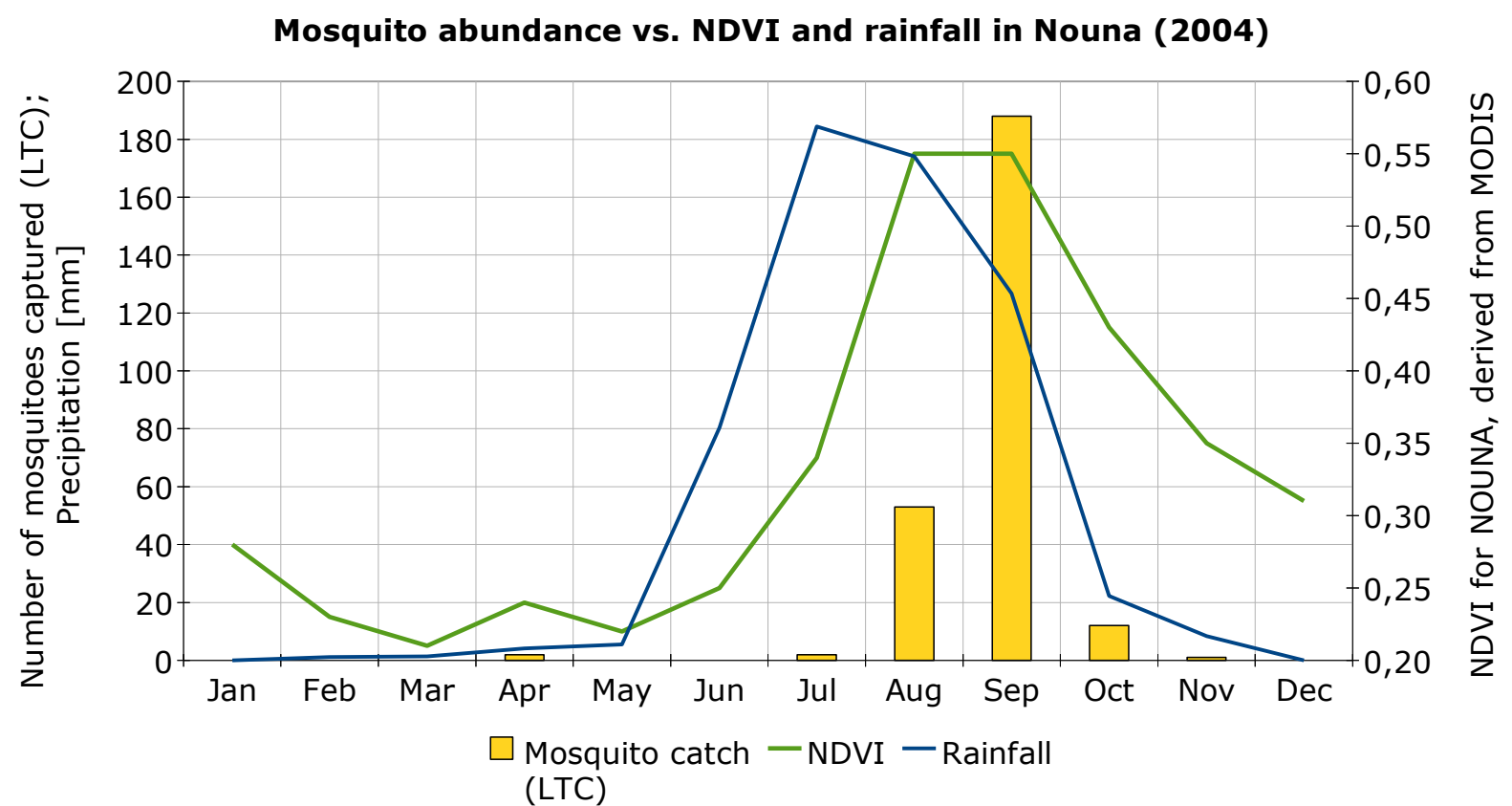

Figure 134: Mosquito abundance vs. NDVI and rainfall in Nouna (2004) ${ }^{1454}$

During the dry season, NDVIs in the region do not fall below a value of around 0,2 . Values between this base level and around 0,4 tend to be associated with low vector densities and malaria incidences (see figure 134 and table 82). NDVIs exceeding 0,4 and reaching levels of around 0,8 in some parts of the province were found to be associated with higher mosquito abundances and malaria incidences.

1454 Data sources: CRSN Nouna (rainfall data); calculated monthly mean NDVI data (Terra MODIS, MOD13A1 product); YÉ, Y. (2005), p. 98 (LTC catches). 


\subsubsection{Socio-Economic and Socio-Cultural Determinants}

Socio-economic and socio-cultural determinants of malaria have not been the main focus of this study, but their role is briefly discussed here as they may interfere with environmental determinants of malaria transmission. Besides land use changes, important anthropogenic determinants of malaria transmission include occupational and leisure activities and measures of personal protection such as bednet use.

Occupational and leisure activities increase the risk of malaria transmission when they cause people to spend time in or close to mosquito habitats. Irrigated rice cultivation, which has not yet arrived at the large scale in Kossi Province (but which is found in neighboring Sourou Province and may soon be introduced along the Sourou's western bank) is a major risk factor, as are other forms of irrigated agriculture which are currently found close to Illa and Kodougou in the study region.

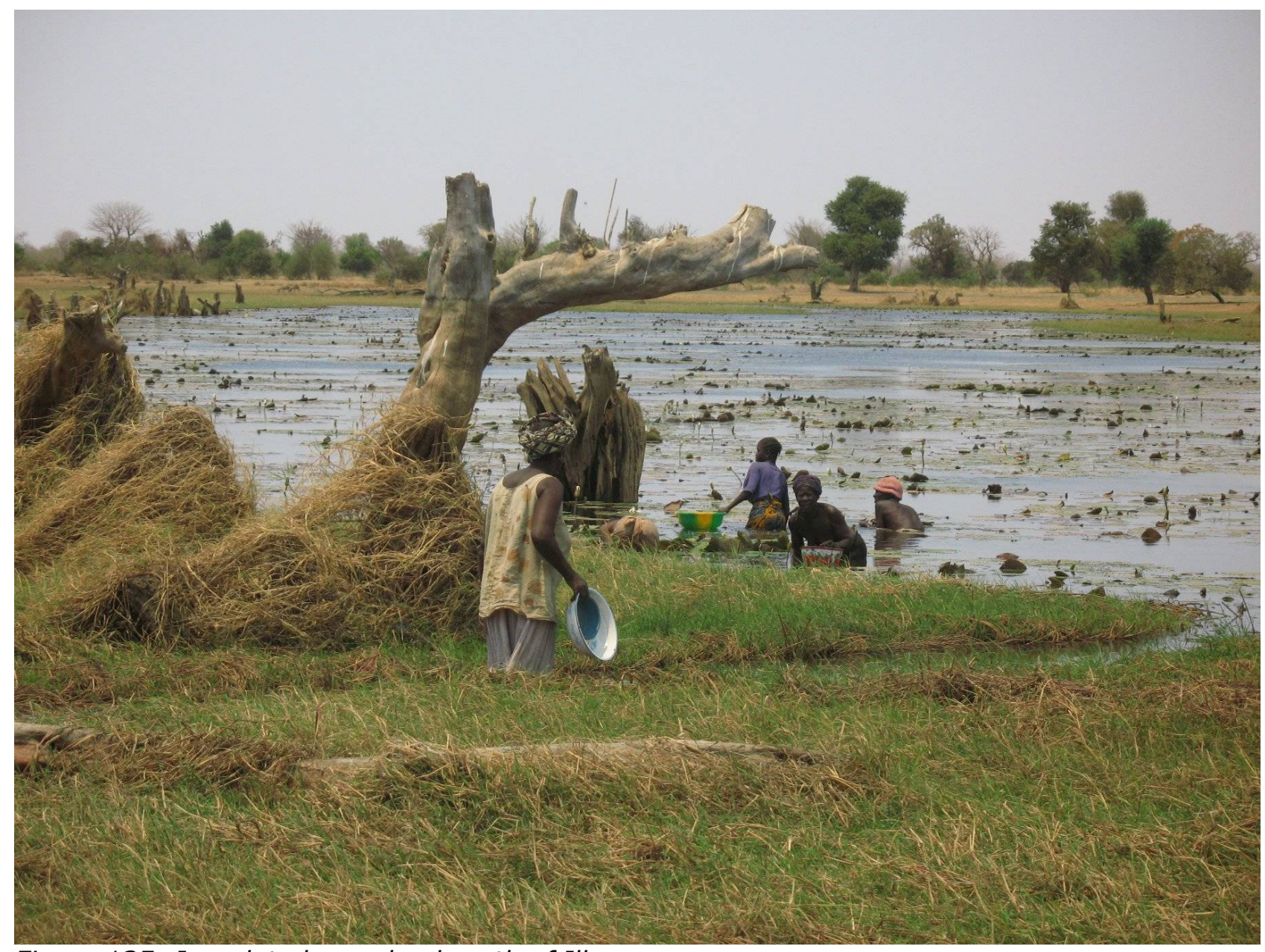

Figure 135: Inundated grassland north of Illa 
The pools in the inundated zone to the north of Illa and the Sourou are used by playing children, women washing dishes and laundry as well as fishermen and people plucking the bulbs of lotus flowers (see figure 135). All of these activities, particularly when carried out around dawn, increase the individual's risk of contracting malaria.

Ethnicity and economic status may have significant effects on malaria incidence which are related to factors such as immunology, housing conditions and occupational activities. One ethnic group present in the study area, the Peulh, is normally considered to be genetically less susceptible to malaria than other ethnic groups. However, Yé et al. (2007) did not confirm this finding. ${ }^{1455}$ Links between ethnicity, occupation and malaria risk in Kossi were not investigated within the case study, and in the absence of other studies on this topic, it can only be speculated that Mossi farmers practicing irrigation run a higher risk of malarial infection than Peulh cattle herders (who may even benefit from zooprophylaxis). An investigation into the role of housing conditions by the CRSN found that structures inhabited by poorer sections of the population that typically had mud or grass roofs were at greater risk of invasion by malaria vectors than homes having iron roofs. ${ }^{1456}$

Various studies have shown considerable reductions in malaria incidence resulting from the use of bednets, particularly ITNs. Two random surveys were carried out in Illa and Toni to assess mosquito net usage and other protective measures. For selected houses, geographic positions were mapped and at least one household member was interviewed and asked about the number of household members, the number of persons currently sleeping under bednets and other forms of protection being practiced.

In Illa, information about prophylactic measures practiced by 151 inhabitants $(7,4 \%$ of the total population) living in the eastern part of the village was obtained. The members of all households except for one belonged to the ethnic group of the Dafing. Due to its proximity to the Sourou and adjacent irrigation projects, the area surveyed falls completely into the zone of high malaria risk. bednet use was the most common form of personal protection, being practiced by $47,7 \%$ of the population. All households possessed at least one bednet, and levels of bednet usage ranged between $13 \%$ and $100 \%$. Insecticides were applied in the form of spray and mosquito coils, most commonly as an additional form of protection, by $24,5 \%$ of the surveyed population. $11,3 \%$ of the population used both mosquito nets and indoor insecticides, while $41,1 \%$ did not use any form of protection. The survey results at individual household level are presented in table 83. Figure 136 provides an overview of the location of the households surveyed, including information on exposure prophylaxis practiced by their inhabitants.

1455 Yé, Y.; Kyobutungi, C.; Louis, V.R. \& Sauerborn, R. (2007), doi:10.1186/1475-2875-6-46. 1456 Yé, Y.; Hoshen, M.; Louis, V. et al. (2006), doi:10.1186/1475-2875-5-8. 
Chapter 3 - Case Study: Malaria in Kossi Province

\begin{tabular}{|l|l|l|l|l|}
\hline $\begin{array}{l}\text { House } \\
\text { no. }\end{array}$ & $\begin{array}{l}\text { No. of } \\
\text { residents }\end{array}$ & Ethnicity & $\begin{array}{l}\text { No. of residents using } \\
\text { mosquito nets }\end{array}$ & $\begin{array}{l}\text { Use of } \\
\text { insecticides }\end{array}$ \\
\hline 1 & 7 & Dafing & 1 & No \\
\hline 2 & 4 & Dafing & 2 & No \\
\hline 3 & 18 & Dafing & 12 & No \\
\hline 4 & 6 & Dafing & 4 & No \\
\hline 5 & 11 & Dafing & 4 & No \\
\hline 6 & 7 & Dafing & 7 & Yes (spray) \\
\hline 7 & 12 & Dafing & 6 & No \\
\hline 8 & 8 & Dafing & 5 & No \\
\hline 9 & 12 & Dafing & 3 & No \\
\hline 10 & 5 & Dafing & 5 & No \\
\hline 11 & 8 & Dafing & 1 & Yes (coils) \\
\hline 12 & 30 & Dafing & 10 & No \\
\hline 13 & 4 & Samo & 2 & No \\
\hline 14 & 15 & Dafing & 6 & No \\
\hline 15 & 4 & Dafing & 4 & No \\
\hline
\end{tabular}

Table 83: Survey on bednet use and personal protection in Illa 


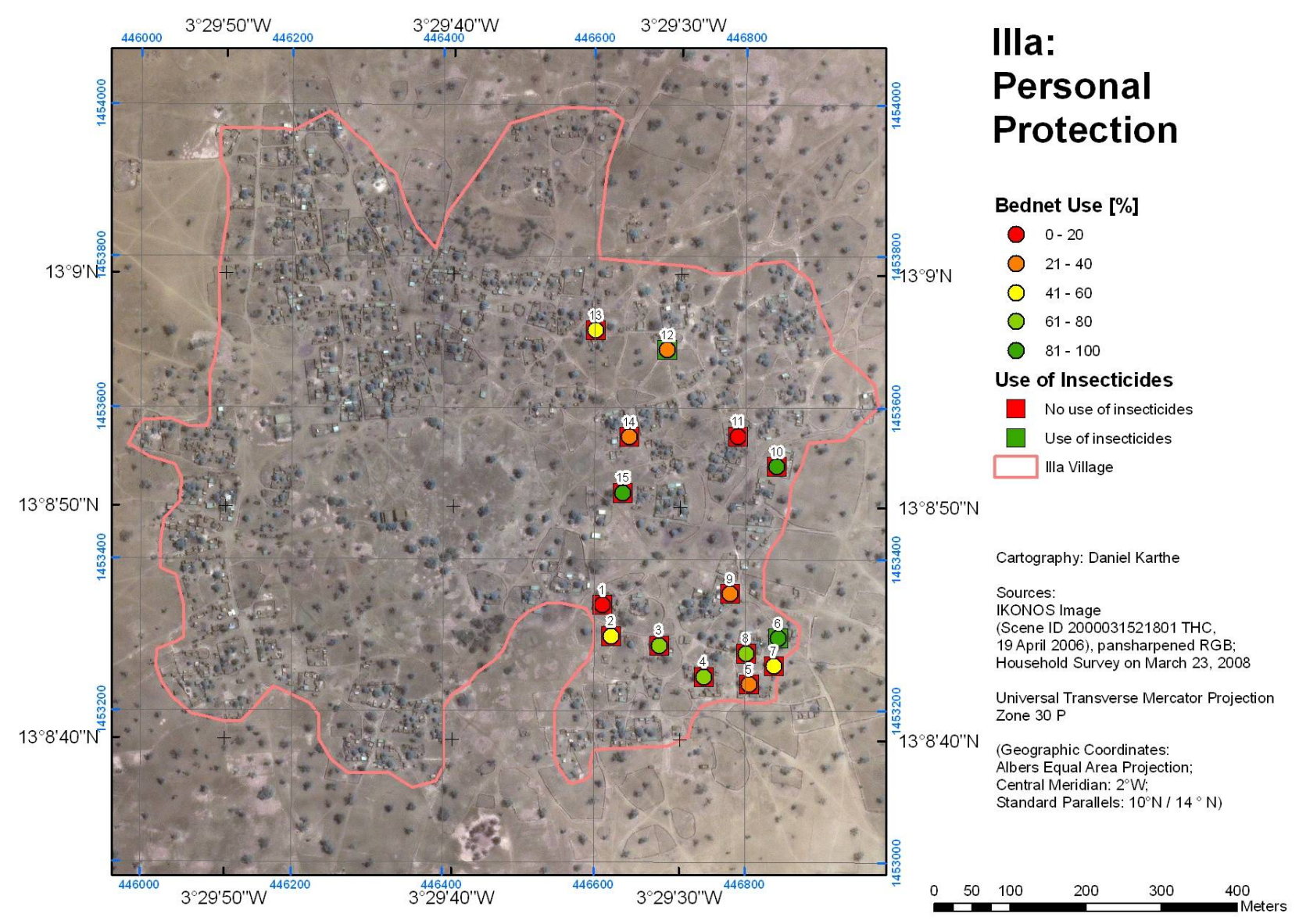

Figure 136: Survey on bednet use and personal protection in Illa ${ }^{1457}$

In Toni, the levels of personal protection were assessed among a study group of 100 residents ( $4,4 \%$ of the total village population). In contrast to Illa, there are no large-scale and permanent breeding sites nearby, and the population sample was an ethnic mix of Mossi, Samo, Peulh, Bobo and Bwaba. Only $9 \%$ of the study group were found to use mosquito nets. Not a single household possessed enough mosquito nets to protect all household members. At the time of research, impregnated mosquito-nets were given to pregnant women free of cost; according to the practitioner in charge of the CSPS, acceptance was around $70 \% .{ }^{1458}$ The use of insecticides was the more common strategy of protection in Toni and used in the houses of $80 \%$ of the study group. Mosquito coils were the dominant form, being used by $76 \%$, while sprays were used by the remaining $4 \%$. The results at household level are presented in table 84,

1457 Based on IKONOS Image (ID 2000031521801 THC; data of acquisition: 19 April 2006) and household survey (oral interview) on 23 March 2008.

1458 Personal communication with staff of Toni CSPS. 
while figure 137 shows the location of the households surveyed, including an overview of the measures of personal protection practiced by their residents.

\begin{tabular}{|l|l|l|l|l|}
$\begin{array}{l}\text { House } \\
\text { no. }\end{array}$ & $\begin{array}{l}\text { No. of } \\
\text { residents }\end{array}$ & Ethnicity & $\begin{array}{l}\text { No. of residents using } \\
\text { mosquito nets }\end{array}$ & $\begin{array}{l}\text { Use of } \\
\text { insecticides }\end{array}$ \\
\hline 1 & 6 & Mossi & 0 & Yes (coils) \\
\hline 2 & 6 & Mossi & 0 & Yes (coils) \\
\hline 3 & 8 & Mossi & 0 & Yes (coils) \\
\hline 4 & 8 & Mossi & 1 & Yes (coils) \\
\hline 5 & 6 & Samo & 1 & Yes (coils) \\
\hline 6 & 27 & Samo & 3 & Yes (coils) \\
\hline 7 & 7 & Peulh & 0 & Yes (coils) \\
\hline 8 & 4 & Peulh & 0 & No \\
\hline 9 & 8 & Bobo & 1 & No \\
\hline 10 & 1 & Bobo & 0 & No \\
\hline 11 & 5 & Mossi & 0 & Yes (coils) \\
\hline 12 & 2 & Bwaba & 1 & No \\
\hline 13 & 5 & Bwaba & 0 & No \\
\hline 14 & 3 & Bwaba & 0 & Yes (coils) \\
\hline 15 & 4 & Mossi & 2 & Yes (spray) \\
\hline
\end{tabular}

Table 84: Survey on bednet use and personal protection in Toni 


\section{Toni: Personal Protection}

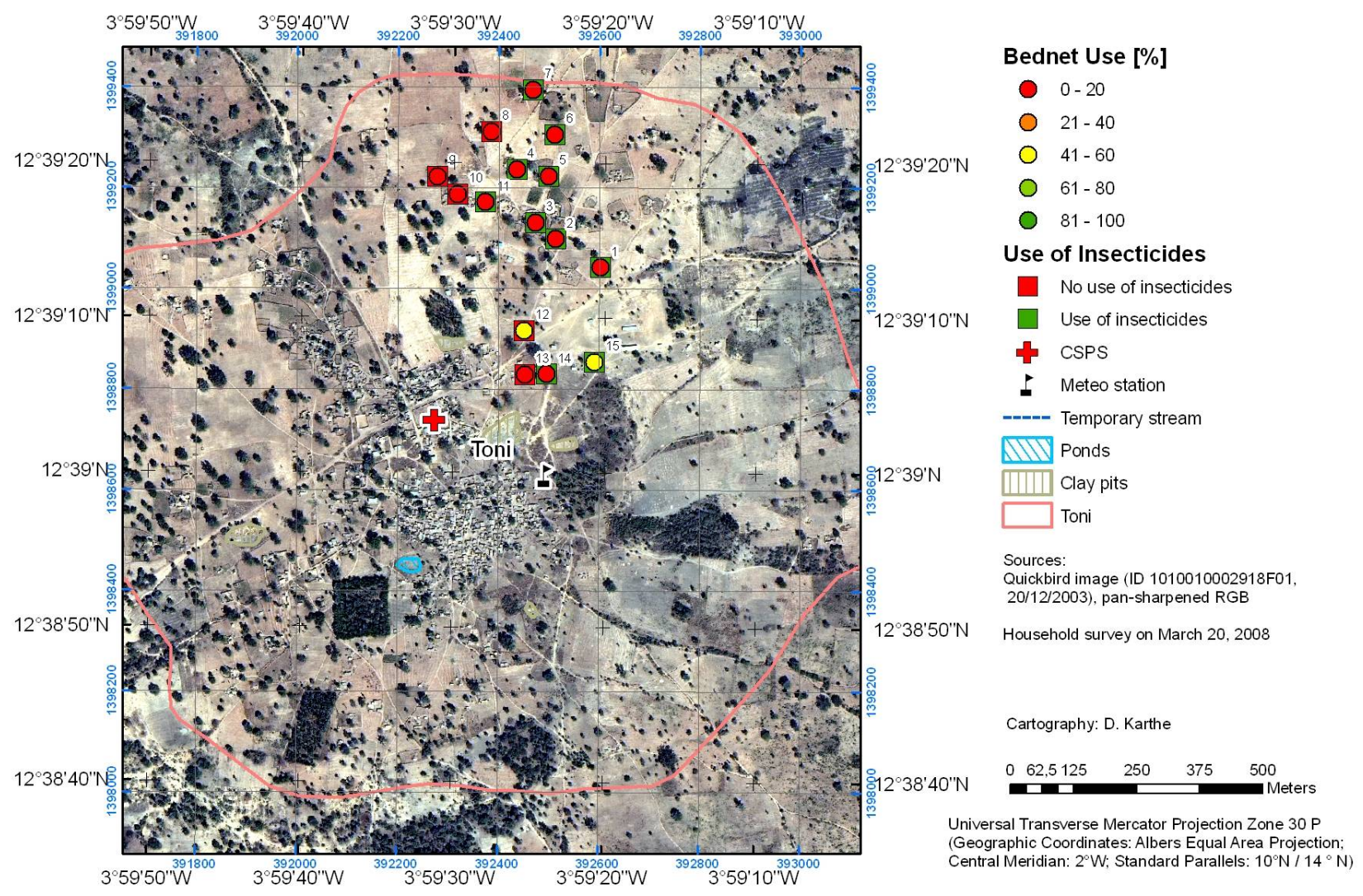

Figure 137: Survey on bednet use and personal protection in Toni ${ }^{1459}$

While the surveys carried out in Illa and Toni may not be representative for their villages or Kossi Province as a whole, they nevertheless indicate that there are large discrepancies with regard to bednet usage and other measures of personal protection at both village and household level. While mosquito nets were the principal means of protection used by villagers in Illa, residents surveyed in Toni used insecticides much more frequently (see table 85).

\begin{tabular}{|l|l|l|l|l|}
\hline Village & \multicolumn{4}{|c|}{ Percentage of the village population } \\
\cline { 2 - 5 } & using \\
bednets & $\begin{array}{l}\text { using } \\
\text { insecticides }\end{array}$ & $\begin{array}{l}\text { using } \\
\text { bednets and } \\
\text { insecticides }\end{array}$ & $\begin{array}{l}\text { using neither } \\
\text { bednets nor } \\
\text { insecticides }\end{array}$ \\
\hline Illa & $47,7 \%$ & $24,5 \%$ & $11,3 \%$ & $41,1 \%$ \\
\hline Toni & $9,0 \%$ & $80,0 \%$ & $6,0 \%$ & $18,0 \%$ \\
\hline
\end{tabular}

Table 85: Personal protection in Illa and Toni

1459 Based on QuickBird image (ID 1010010002918F01; data of acquisition: 20/12/2003) and household survey (oral interview) on 20 March 2008. 
For Illa CSPS, the average malaria incidence rate was around $20 \%$ higher than for Toni CSPS. This may not only be a consequence of ecologically rather different environments but also different levels and forms of exposure prophylaxis (see table 82 ).

However, it should be noted that it is difficult to assess and compare the efficacy of the various forms of exposure prophylaxis mentioned as they are not as uniform as they may appear at first sight. For example, the state and impregnation of bednets probably play a role as do the quality and frequency of application of the insecticides used. Neither of these factors has been assessed in the present study, and surveys by other authors have come to conflicting results. Yé et al. (2007), for example, did not find an association between self-reported use of bednets and individual malaria risks in Nouna Health District. ${ }^{1460}$

1460 Yé, Y.; Kyobutungi, C.; Louis, V.R. \& SAuerborn, R. (2007), doi:10.1186/1475-2875-6-46. 


\section{Perspectives}

Based on both the review (chapter 2) and the case study (chapter 3 ), this final chapter discusses major findings in the context of current research programs. Global developments such as climate change or population growth may have a substantial impact on the future pattern of malaria incidence (and other vectorborne diseases). More research on the links between spatial processes and vector-borne disease transmission is therefore indicated.

\subsection{Discussion of Results}

This case study showed that malaria transmission has a clearly geographic dimension. Various physio- and sociogeographic factors govern both spatial and temporal pattern of malaria incidence. Both the complexity of the environments in which disease transmission occurs and imperfect data representations of the many processes involved mean that exact quantitative models remain difficult. Nevertheless, some environmental indicators and geographic appear to be well-suited for malaria risk prediction.

\subsubsection{Synopsis}

Kossi Province is an area of holoendemic malaria transmission which in 2008 experienced an average incidence rate of 137,6 malaria cases per 1000 inhabitants. This regional average varied considerably in both time and space:

- The recorded malaria incidence ranged from 44 to 524 cases per 1000 inhabitants between different health centers in 2008, i.e. a twelve-fold difference between low- and high-incidence settings.

- Malaria incidence was generally seasonal, but with marked differences between regions of high and low seasonality. Incidence rates multiplied by factors ranging from 4 to more than 35 between the months of lowest and highest case numbers.

- There was a considerable variation in malaria incidence in the 2005 to 2008 period.

In general, malaria incidence was highest in southeastern Kossi and lowest in northwestern Kossi. The environmental factors which best explain this difference are climate (in particular the decrease in rainfall towards the north) and surface hydrology (i.e. the presence of major and permanent surface water bodies in the south and east of Kossi as opposed to a low density of minor temporary water bodies in the north and east of the Province). Moreover, 
regions of higher malaria incidence tend to be characterized by comparatively low elevations and a flat relief with minor depressions.

Malaria incidence generally followed a seasonal pattern with relatively low levels in May and June, a considerable rise in July and marked peak in disease incidence between August and October. The seasonality pattern of malaria was closely related to the seasonality pattern of precipitation, with a time lag of around one month. However, the degree of seasonality varied considerably within the province. Therefore, a transmission seasonality index was proposed. The lowest degree of seasonality was observed in Nouna, the only urban site, and the highest rates in the southeastern part of Kossi, close to the confluence of the Mouhoun and Sourou rivers.

In the years from 2005 to 2008 , marked differences in malaria incidence could be noted. In general, malaria incidence was on the increase during this period, which was too short to speak of a "trend" though. Despite the short observation period, substantial interannual variations in malaria incidence could be noted. Malaria incidence was highest and most seasonal in years of relatively high precipitation occurring during a marked rainy season, and lowest in relatively dry years without a clearly defined rainy season.

Entomological survey data helped to link environmental parameters to malaria cases via mosquito populations. Meteorological station data were expected to be of value for the prediction of conditions suitable for mosquito reproduction and malaria transmission, but the poor functioning of the equipment reduced the number of useful stations to just one, which was in fact located in Dédougou, just outside the study region. While field measurements indicated that microclimatic differences are large enough to be meaningful for vector population dynamics, such surveys are not feasible for longer-term monitoring of larger areas. Remote sensing products, on the other hand, were found to provide useful proxies for air temperature and precipitation (e.g. land surface temperature, rainfall estimates and vegetation indices).

Since data on malaria incidence were only available at the level of CSPSs (health centers) typically encompassing around 5 villages, empirical risk assessments at the village level were not possible. Therefore, vector habitats were mapped using both high-resolution satellite imagery and field surveys. Based on vector flight ranges, local risk maps could be produced. Despite a relatively limited number of major mosquito breeding sites, considerable parts of the study villages fell into zones of high transmission risks.

Large variations in anthropogenic determinants of malaria transmission, such as land use modifications or exposure prophylaxis, were noted. Even though it was not possible to quantify their importance, observed differences were large enough to assume that they significantly contribute to the malaria risk encountered by local residents. While irrigated agriculture tends to increase 
(and along with it the risk of malaria), the use of impregnated bednets or indoor insecticides may contribute to a reduction of malaria incidence.

Geographic information systems and remote sensing were found to be valuable tools for mapping and analyzing malaria incidence pattern at the regional level. A large variety of remote sensing products, ranging from elevation models to land use maps, vegetation indices and thermal infrared imagery, and from high spatial but low temporal resolution to moderate spatial but high temporal resolution datasets were found to be useful for provincial-scale assessments of the determinants of malaria transmission under dry savanna conditions in Burkina Faso.

Presently ongoing developments in both the natural and socioeconomic environment (e.g., climate and land use change, migration, population growth, economic development) mean that conditions for malaria transmission are changing. At the same time, there is no indication that in the short to medium term, these changes would result in a reduction of malaria incidence. Given the enormous dimension of the malaria burden in both its global and local context, intervention programs are urgently needed. One important prerequisite for such programs is a sound knowledge of local transmission situations and the implications of expected environmental changes, including impacts of malaria control operations. This thesis tried to provide both a comprehensive overview of malaria epidemiology and its geographic determinants and a case study focusing on geographic contributions to malaria research at the local to regional scale. The data collected and maps prepared within its framework will certainly be a good basis for future investigations going beyond the analyses presented here, and are hoped to be of use for local capacity building. 


\subsubsection{Discussion}

The present study utilized various field and RS-based methods which in their multitude reflect the complex system of factors determining the transmission dynamics of malaria at the local to regional scale.

As expected and proved by other studies, meteorological parameters, particularly rainfall, are linked to the temporal malaria pattern in dry savanna areas like Kossi Province. However, it was found that not only absolute quantities of individual factors (e.g. precipitation totals) must be taken into account but also their dynamics and constellations with other ecological and sociogeographic parameters. In regions with scarce meteorological station networks (and frequent failures of instruments), remote sensing-based products are valuable sources of additional information, particularly with regard to spatial pattern. It must be understood though that RS is not a complete alternative to meteorological station and field measurements since RS can at present not directly quantify the variables of interest (e.g., air temperature, precipitation); the use of proxies such as LSTs and vegetation indices (or rainfall estimates) always requires terrestrial data for calibration and validation.

Several other environmental and sociogeographic factors were found to be of potential importance for regional and local scale transmission pattern, but at present, their effects can only partially be quantified. Contradicting evidence found by other researchers mean that some links (e.g. the role of water turbidity for habitat productivity or the role of bednets and insecticides for exposure reduction) are still too poorly understood for inclusion into risk prediction systems. Nevertheless, remotely sensed imagery combined with ground truthing was found to be a valuable resource for several aspects of local-scale malaria risk mapping.

\subsubsection{Methodologic Approach}

The idea of relating environmental variables to the occurrence of malaria is nothing new: Ever since the discovery of the malaria transmission process, scientists have tried to investigate the factors which determine the dynamics of malaria transmission. Numerous case studies have been conducted in various parts of the world, including the African continent. However, the majority of these studies looked at only one single factor and its impact on malaria transmission dynamics, frequently in form of laboratory-based or closely controlled field studies. In reality, though, malaria transmission takes place in settings characterized by a complex interaction of environmental and anthropogenic factors which may vary simultaneously. Despite the inherent 
difficulties of measuring a multitude of parameters and their variations in time and space, the present study captured more about the complexity of situations under which malaria transmission takes place then studies investigating the role of a single parameter.

Several types of data were found to be of relevance for investigations into the dynamics of malaria transmission and its determinants. Among these, information on surface properties and meteorology on the one hand and entomology and malaria epidemiology on the other hand are most important. The pros and contras of their utilization for geomedical studies in the West African context are summarized by the following tables. It should be understood, however, that the advantages and disadvantages mentioned are relative to the alternatives presented and based on specific experiences in rural dry savanna region in Burkina Faso.

The most basic prerequisite for research into the spatial dimension of malaria epidemiology are maps. While regional maps of a 1:200.000 scale were obtained from the Institut Géographique du Burkina (IGB), the non-availability of more detailed maps of the research zones meant that both high resolution RS data and field surveys were needed for the production of local maps (see table 86). 


\begin{tabular}{|c|c|}
\hline Data type and acquisition & $\begin{array}{l}\text { Advantages (+) } \\
\text { Disadvantages (-) }\end{array}$ \\
\hline Topographic maps (IGB) & $\begin{array}{l}+ \text { low cost } \\
+1: 200.000 \text { scale available for the entire } \\
\text { country } \\
+ \text { all maps produced according to identical } \\
\text { cartographic standards } \\
\text { - seriously outdated (some map sheets from } \\
\text { 1950s/60s!) } \\
\text { - low level of detail; } 1: 50.000 \text { scale } \\
\text { unavailable for much of Burkina Faso }\end{array}$ \\
\hline $\begin{array}{l}\text { High resolution RS imagery } \\
\text { (e.g. IKONOS, QuickBird) }\end{array}$ & $\begin{array}{l}\text { + up to date information } \\
+ \text { high spatial resolution ( }<1 \mathrm{~m} \text { in } \\
\text { panchromatic channel) } \\
+ \text { suitable for time series analysis } \\
\text { - requires ground truthing } \\
\text { - (very) high cost of imagery }\end{array}$ \\
\hline Field survey data & $\begin{array}{l}\text { + information verified in situ } \\
+ \text { flexibility in the choice of relevant } \\
\text { information } \\
\text { - time-consuming, high cost } \\
\text { - only suitable for small areas }\end{array}$ \\
\hline
\end{tabular}

Table 86: Useful topographic data sources

In dry savanna regions such as Kossi, climate is a major determinant of malaria transmission since it affects various factors of malariologic relevance ranging from mosquito reproduction to sporogony and mosquito biting behavior. In the framework of the present study, climate data of three sources were used: meteorological station data, field measurements and satellite imagery (see table 87). 


\begin{tabular}{|c|c|}
\hline Data type and acquisition & $\begin{array}{l}\text { Advantages (+) } \\
\text { Disadvantages (-) }\end{array}$ \\
\hline Meteorological station data & $\begin{array}{l}\text { + long time series for some stations } \\
+ \text { precise measurements } \\
\text { - data of non-WMO stations difficult to obtain } \\
\text { - low density of the station network in Africa } \\
\text { - data often not representative for situation } \\
\text { in vector habitats } \\
\text { - serious data gaps }\end{array}$ \\
\hline Field measurements & $\begin{array}{l}\text { + flexibility: measurements at locations of } \\
\text { interest } \\
+ \text { ideal for profiles (e.g. transects from } \\
\text { habitats to meteo stations) } \\
\text { - short time series } \\
\text { - time-consuming and costly }\end{array}$ \\
\hline RS data: LST, RFE, VIs & $\begin{array}{l}\text { + spatially contiguous data } \\
\text { - limited temporal resolution } \\
\text { - limited time series } \\
\text { - data gaps, e.g. due atmospheric disturbances } \\
\text { (particularly for LST) } \\
\text { - indirect measurements/estimates of the } \\
\text { parameters of interest }\end{array}$ \\
\hline
\end{tabular}

Table 87: Evaluation of climate data used

All entomological and epidemiological data used in this study were from external sources, based primarily on the records of local health centers and research activities of the CRSN Nouna and its partners. Incidence rates presented were derived from routinely collected passive case detection data collected by local CSPSs, and active case detection data from a recent field study carried out by Dr. YAzoumé Yé and a team of CRSN scientists (see table 88). 


\begin{tabular}{|l|l|}
\hline Data type and acquisition & $\begin{array}{l}\text { Advantages (+) } \\
\text { Disadvantages (-) }\end{array}$ \\
\hline $\begin{array}{l}\text { Passive case detection data } \\
\text { from CSPSs }\end{array}$ & $\begin{array}{l}\text { + collected routinely: uninterrupted time } \\
\text { series, no extra costs } \\
+ \text { available for all CSPS } \\
+ \text { data already in statistical form } \\
\text { - risk of over- or underestimation: usually no } \\
\text { laboratory confirmation, but only self- } \\
\text { reported cases } \\
\text { - undifferentiated data for zone of CSPS, } \\
\text { potentially including outside visitors } \\
\text { - only monthly totals }\end{array}$ \\
\hline $\begin{array}{l}\text { Active case detection data } \\
\text { (field survey) }\end{array}$ & $\begin{array}{l}\text { best estimate of actual malaria incidence } \\
\text { if conducted at household level: highest } \\
\text { possible spatial/temporal resolution } \\
+ \text { may be carried out precisely in region of } \\
\text { interest } \\
- \text { not routinely available: additional costs, } \\
\text { longer time series unavailable } \\
- \text { different studies may not be comparable }\end{array}$ \\
\hline
\end{tabular}

Table 88: Techniques for estimating malaria incidence

Since malaria incidence is only indirectly related to the physio- and sociogeographic environment via the distribution and density of vector mosquitoes and their infective contacts with human host populations, a precise knowledge of these factors is desirable (see table 89). However, virtually none the relevant parameters are routinely monitored. This study tried to evaluate spatio-temporal pattern of malaria risks based on breeding site surveys, and documented and locally available information on vector populations, most frequently based on mosquito captures using light traps. While the compilation of data allowed general insights into the composition and dynamics of vector populations, vector densities could not be calculated; this made comparisons between different sites difficult. Interviews with locals were carried out in order to get an impression of personal protective measures, but limited coverage means that they should be considered a pilot study rather than an empirically significant representation of reality. 


\begin{tabular}{|c|c|c|}
\hline \multicolumn{2}{|c|}{ Data type and acquisition } & $\begin{array}{l}\text { Advantages (+) } \\
\text { Disadvantages (-) }\end{array}$ \\
\hline \multicolumn{2}{|c|}{$\begin{array}{l}\text { Breeding site surveys } \\
\text { (based on RS and field study) }\end{array}$} & $\begin{array}{l}\text { + provide information on spatial pattern } \\
\text { of (potentially productive) vector habitats } \\
+ \text { RS allows monitoring of large areas } \\
\text { - without information of habitat productivity, } \\
\text { surveys are a poor predictor of vector } \\
\text { density and distribution }\end{array}$ \\
\hline \multirow[t]{4}{*}{ Mosquito capture } & \multirow[b]{2}{*}{ LTC } & $\begin{array}{l}\text { + direct characterization of vector population } \\
\text { (e.g. species, age, sporozoite rate) } \\
\text { - only suited for exemplary investigations } \\
\text { (point data) } \\
\text { - comparisons between different studies } \\
\text { difficult }\end{array}$ \\
\hline & & $\begin{array}{l}\text { + flexible, cost-effective and safe } \\
\text { - positioning of traps has implications for } \\
\text { results } \\
\text { - anthropophilic vectors underrepresented } \\
\text { unless positioned close to (sleeping) hosts }\end{array}$ \\
\hline & PSC & $\begin{array}{l}\text { + sampling of endophagic mosquitoes } \\
\text { - results may depend on housing structures }\end{array}$ \\
\hline & HLC & $\begin{array}{l}\text { + best measure for host-to-vector contact } \\
\text { - high risk for hosts; subjective; } \\
\text { labor-intensive }\end{array}$ \\
\hline \multicolumn{2}{|l|}{$\begin{array}{l}\text { Survey on protective } \\
\text { measures }\end{array}$} & $\begin{array}{l}\text { + takes into account the decisive step of } \\
\text { vector-to-host contact } \\
\text { - data difficult to interpret without exact } \\
\text { knowledge on the efficacy of different } \\
\text { techniques }\end{array}$ \\
\hline
\end{tabular}

Table 89: Techniques for assessing malaria transmission risks

The limitations of individual techniques may be compensated for by combining data from multiple sources. Moreover, the use of such multi-source data may in fact be necessary because not all of the datasets mentioned are available for any location and time. Geographic information systems are well-suited for the integration of multi-source and multi-format (e.g. point occurrence vs. contiguous) data. 


\subsubsection{Scientific Context and Contribution}

This case study was unique in providing a comprehensive overview of the geographical factors determining malaria transmission at the local to regional scale, thereby integrating the geographic and medical perspectives. Even though many of the aspects presented here are not totally new, the holistic view of this investigation was new: the dynamics of malaria incidence were not seen as the result of a single determinant but as a consequence of the interplay of physio- and sociogeographic parameters.

The expectation that malaria incidence would be considerably higher in regions close to major water bodies was confirmed as it had already been by previous research. ${ }^{1461}$ Moreover, the finding of LACAUX et al. (2007) that a relatively small number of breeding sites results in large risk zones ${ }^{1462}$ was supported both theoretically (the production of risk maps assuming a $2 \mathrm{~km}$ flight radius of anophelines around their breeding sites) and empirically (the observation of vectors and the actual transmission of malaria throughout much of the study area).

Meteorological data were found to be related with both the seasonality and interannual variations of malaria incidence, and both temperature and precipitation were found to play a role. The statement of Yé et al. (2007), that temperature was the best meteorological predictor of malaria incidence ${ }^{1463}$, could not fully be confirmed; temperature alone appeared to be of little value for malaria prediction. As a single factor, precipitation was found to explain more but not all of the monthly and interannual variations in malaria incidence, a finding supported by most other studies carried out in similar environments. ${ }^{1464}$ However, not only absolute quantities but also the distribution of rainfall appeared to matter. Whereas most existing field studies on the effects of weather on malaria transmission relied on meteorological station data, it is argued here that microclimatic effects are underrepresented by such data but may play a major role since mosquito habitats often differ substantially from the locations characterized by meteorological stations.

Two "malaria early warning systems" that have recently become operative were found to be little suited for the prediction of malaria in the study region. While one of the systems only covers regions of epidemic malaria, the MEWS system operated by the International Research Institute for Climate and

1461 Hay, S.I.; Omumbo, J.A.; Craig, M.H. \& Snow R.W. (2000), p. 201;

Mushinzimana, E.; Munga, S.; Minakawa, N. et al. (2006), doi:10.1186/1475-2875-5-13.

1462 Lacaux, J.P.; TourRe, Y.M.; Vignolles, C. et al. (2007), p. 73.

1463 Yé, Y.; Louis, V.R.; Simboro, S. \& Sauerborn, R. (2007), doi:10.1186/1471-2458-7-101.

1464 Fillinger, U.; Sombroek, H.; Majambere, S. et al. (2009), doi:10.1186/1475-2875-8-62. 
Society in fact uses rainfall estimates. While the rainfall estimates can indeed give some idea of malaria risks, such estimates tend to be relatively imprecise $\mathrm{e}^{1465}$ and are too undifferentiated to account for regional-scale differences (see figure 138, where Kossi Province entirely falls into one zone).

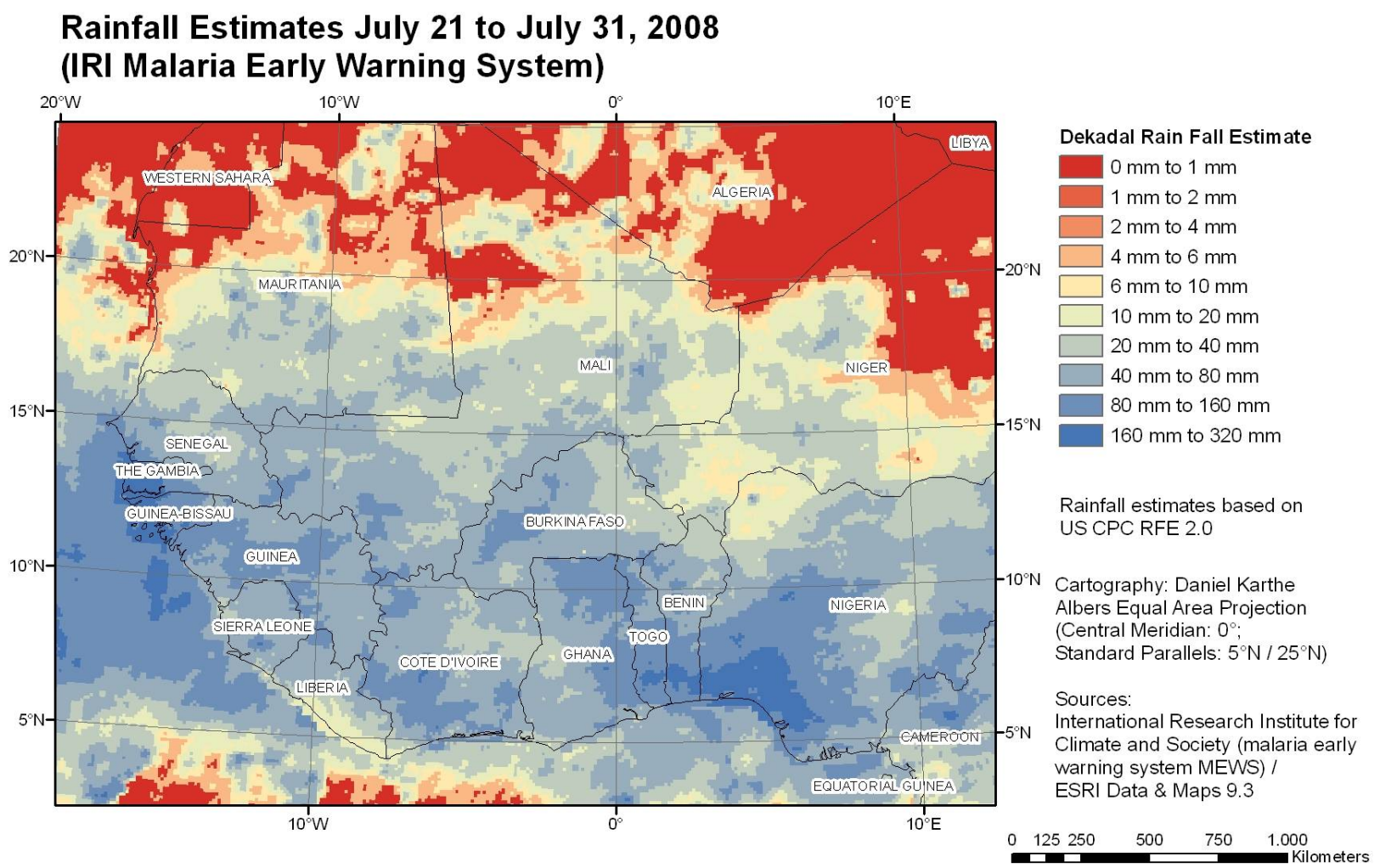

Figure 138: Rainfall estimates for West Africa (21 to 31 July 2008) (1466 $^{14}$

Vegetation indices such as the NDVI were found to be well-correlated with malaria incidence. In arid regions, they reflect regional moisture pattern ${ }^{1467}$ much better than satellite-based rainfall estimates. Even though they are not directly measures of precipitation, the response pattern of dryland vegetation to changes in water availability are very similar to those of malaria incidence. In fact, vegetation indices may even be the ecologically more meaningful indicators since they reflect the actual availability of moisture, which may be of other origins than rainfall and which is also influenced by terrain characteristics such as relief and soils.

The relatively short period of observation mean that the present study does not allow any statements about the consequences of man-made land use and cover changes. However, such changes were found to take place in the study region and have been linked to substantial alterations in malaria transmission

1465 Dinku, T.; Chidzambwa, S.; Ceccato, P. et al. (2008), p. 4097.

1466 Based on Malaria Early Warning System (MEWS) of the International Research Institute for Climate and Society and ESRI Data \& Maps 9.3.

1467 Li, J.; LeWIS, J.; RowLAND, J. et al. (2004), pp. $464 f$. 
dynamics in other regions. ${ }^{1468}$ Moreover, differences in transmission intensity could be observed in regions with and without irrigation, even though a paucity of long-term data mean that it is at present difficult to distinguish between impacts of irrigation and natural differences in surface water availability.

Other anthropogenic factors such as personal protection are believed to be of similar importance. Even though systematic investigations were not carried out, personal interviews conducted in the villages of Toni and Illa showed that local populations use different techniques to avoid mosquito bites, most notably insecticide sprays and bednets. Substantial intra- and inter-village differences were observed, but in the absence of reliable data on their effectivity and a wider coverage of the study region, it can currently only be speculated that they have a considerable impact.

The perhaps most important conclusion from this study is that even though single factors may partially explain differences in malaria transmission dynamics, combinations of physio- and sociogeographic parameters and processes determine the actual pattern of disease.

\subsubsection{Limitations of the Present Study}

The attempt to provide a comprehensive overview of the geographic pattern and determinants of malaria transmission at the provincial level means that none of them could be treated in complete detail, and the numerous sources of data used mean that both their quality and availability varied considerably.

For logistic reasons, only major larval habitats were identified in the case study. High spatial resolution RS imagery (IKONOS, QuickBird) was found to be a suitable tool for mapping the distribution of major surface water bodies but not feasible for the mapping of habitats covering less than a few square meters. Moreover, the enormous numbers and limited stability of small pools meant that they could not be mapped and monitored for productivity during the field study. Since at least two of the anopheline species present in Kossi Province, Anopheles gambiae and Anopheles arabiensis, frequently oviposit in small water pools ${ }^{1469}$, the extent of the zones of potential malaria transmission may have been underestimated. However, the findings of MinakaWA et al. (2005) that a fast desiccation of pools of less than $1 \mathrm{~m}^{3}$ can prevent adult emergence despite very high larval densities ${ }^{1470}$ could indicate that this "limitation" actually resulted in the omission of many unproductive habitats and thus a more realistic assessment of the extent of risk zones.

1468 VAn der Hoek, W. (2004), p. 95.

1469 Huang, J.; Walker, E.D.; Giroux, P.Y. et al. (2005), p. 443;

Mutuku, F.M.; Bayoh, M.N.; Gimnig, J.E. et al. (2006), p. 54.

1470 Minakawa, N.; Sonye, G. \& Yan, G. (2005), p. 298. 
Habitat productivity was not investigated but may have a major impact on the spatial distribution of vectors and disease transmission. However, contradictory results of existing studies regarding the role of physical characteristics such as turbidity result in the necessity of direct surveys (e.g. in the form of pupal density) in addition to the assessment of habitat characteristics.

While meteorological conditions were found to be the most important determinant of temporal incidence pattern of malaria, data gaps in the time series made data of some stations practically useless. This meant on the one hand that remote sensing based proxies were practically the only way of assessing spatial pattern of temperature and precipitation. Since gaps were also present in the RS time series (particularly LST), proper validation was very difficult.

Due to a severe shortage of data on the anopheline population and transmission intensity, the present study largely relied on geographic data on the one side and malaria case data on the other. While this approach has the advantage that it relates environmental information directly to disease, it must be understood that the observed links are statistical and indirect. The malaria case data used relied on passive case detection, thus potentially concealing differences in the health-seeking behavior ${ }^{1471}$ of local populations. Imperfect data on malaria incidence and little empirical information on the link between the environment, mosquito populations and malaria transmission pressure mean that exact numerical cause-effect relationships could not be identified. Since the only recent entomological data available were collected using different and partially undocumented approaches, values for different periods and locations were not comparable.

One particular limitation of this case study involved the choice of the study villages. Their selection was based on the presence of meteorological stations, which were later found to have produced considerable data gaps, making them unsuitable for definite comparisons of meteorologic variables. Moreover, malaria case data were available only at the CSPS level, making statements about individual villages difficult. Nevertheless, since geographic data indicated that intra- and inter-village differences in transmission were likely, active case detection programs in individual villages could greatly enhance the linkability of environmental and malaria case data.

The short duration of the time series available (up to 5 years of malaria case data at the maximum) mean that it was difficult to analyze interannual variations. Their nature and underlying causes would certainly be worthwhile investigating.

1471 Lautze, J.; McCartney, M.; Kirshen, P. et al. (2007), p. 987. 
Despite all limitations and problems regarding exact quantifications, the study helped to identify spatio-temporal pattern of malaria incidence and geographic parameters of malariologic relevance for regional-scale investigations, forming a basis for more extensive future research.

\subsection{The Prospects: Malaria in The Future}

It has been estimated that in the 1950s the annual incidence of malaria was around 250 million cases, with 2.5 million people dying of malaria every year. The spatial extent of malaria endemicity then decreased as a result of eradication and control programs. ${ }^{1472}$ According to conservative (!) estimates, the number of infection cases has remained on a similar level, and malaria continues to kill at least a million people each year. ${ }^{1473}$

Many global-scale developments of the $21^{\text {st }}$ century are of relevance for malaria epidemiology: global climate change will have an effect on the distribution and local population dynamics of malaria vectors and parasites; land use changes may both limit and extend mosquito habitats and breeding spaces; a growing world population results in a greater population living in malarious areas; and urbanization leads to both a concentration of people and fundamental alteration of vector habitat ecologies. Even though these changes may be considered global due to their transcontinental scale, all of them show considerable variations at the continental and subcontinental scale.

\subsubsection{Malaria and Climate Change}

According to the Intergovernmental Panel on Climate Change (IPCC), climate change will bring some health benefits (e.g., a reduction of cold-related mortality), but these will most likely be outweighed by negative effects such as increased rates of infectious diseases and malnutrition in developing countries. ${ }^{1474}$ While some health impacts of climate change can be estimated by relatively simple cause-effect models (e.g. changes in the number of thermal related deaths), others involve more complex eco-epidemiologic links (e.g. vector-borne diseases). ${ }^{1475}$ Malaria transmission is more susceptible to climate changes than many other tropical diseases. ${ }^{1476}$ The manifold links between temperature and anopheline vectors on the one hand and malaria parasites on the other hand indicate that global climate change may have a major impact on malaria epidemiology.

1472 Gilles, H.M. (1993²), p. 124.

1473 WHO (2005), p. 11 ; WHO (2008), p. 1.

1474 IPCC $\left(2007^{2}\right)$, p. 404.

1475 Martens, W.J.M. (1998), pp. $242 \mathrm{f}$.

1476 Martens, P. (1998), p. 62. 
The transmission potential of vector-borne diseases such as malaria is very sensitive to climate changes on the periphery of the present endemic areas and at higher altitudes within these areas. Changes in regional temperature and precipitation have an impact on vectors' reproduction, development rate and longevity, and changes in wind pattern may influence vector dispersal. A rise in sea-level and resultant coastal flooding may result in the establishment of lagunae and proliferation of brackish water and thus potential habitats for some mosquito species. ${ }^{1477}$

Global climate change will result in alterations of malaria transmission intensity and the spatial distribution of the disease. The number of people living in malaria risk areas is predicted to increase by $5 \%$ to $15 \%$ by $2050 .{ }^{1478}$ In malarious areas of Africa, South and Central America and South East Asia, the epidemic potential of Plasmodium vivax malaria is predicted to increase by 12 to $23 \%$. The incidence of Plasmodium falciparum malaria is predicted to rise by $15 \%$ to $27 \%$, with local increases of up to $74 \%$ by $2050 .{ }^{1479}$

\subsubsection{Climate Change in Africa}

During the $20^{\text {th }}$ century, the average temperature on the African continent has risen by an average $0.5 \mathrm{~K}$, a rate of warming that is similar to the global average. This trend appears to be accelerating, as undermined by the observation of $0.2 \mathrm{~K}$ to $0.3 \mathrm{~K}$ temperature rise between the 1961 to 1990 normal period and the 1990s. Moreover, 1998 was the warmest year of the century. ${ }^{1480}$ In the future, warming in Africa is likely to be larger than the global annual mean warming, with arid regions warming more than the moist tropics. ${ }^{1481}$ At the same time, Africa is likely to suffer from climate change more gravely than other continents since many African societies have a very limited capacity to adapt to the coming shifts in climate $^{1482}$ :

«Africa is one of the most vulnerable continents to climate change and climate variability, a situation aggravated by the interaction of 'multiple stresses', occurring at various levels, and low adaptive capacity. ${ }^{1483}$

1477 Martens, W.J.M.; Jetten, T.H.; Rotmans, J. \& Niessen, L.W. (1995), pp. $195 f$.

1478 MARTENS, P. (1998), p. 53.

1479 MARTENS, P. (1998), p. 52.

1480 Hulme, M.; Doherty, R.; Ngara, T. \& New, M. (2005), p. 30.

$1481 \operatorname{IPCC}\left(2007^{1}\right)$, p. 866.

1482 Ramin, B.M. \& McMichael, A.J. (2009), doi: 10.1007/s10393-009-0222-4.

$1483 \operatorname{IPCC}\left(2007^{2}\right)$, p. 435. 
Individual models predict large, but disparate, changes in the Sahel. Whereas the GFDL CM2.1 model (coupled climate model 2.1 of the NOAA Geophysical Fluid Dynamics Laboratory) predicts a very strong drying in the Sahel, other models such as the MIROC3.2midres (Model for Interdisciplinary Research on Climate) predict a very strong trend towards increased rainfall in the region. ${ }^{1484}$ The CM2.0 model assumes a moistening of the Sahel in the first half of the $21^{\text {st }}$ century but a rainfall reduction by the end of the century. ${ }^{1485}$ This is supported by the observation of a partial amelioration of the Sahel drought since the 1990s, which might be explained by a greenhouse-gas driven increase in rainfall. ${ }^{1486}$ However, it remains unclear whether the positive rainfall trend due to warmer SSTs will be limited to the Guinea coast or extend into the Sahel. ${ }^{1487}$

\begin{tabular}{|l|l|l|l|l|l|l|}
\hline \multirow{2}{*}{ Region } & \multicolumn{4}{|c|}{ Annual Mean Temperature } & \multicolumn{3}{l|}{ Annual Precipitation } \\
\cline { 2 - 7 } & Min & Mean & Max & Min & Mean & Max \\
\hline $\begin{array}{l}\text { West } \\
\text { Africa }\end{array}$ & $1.8 \mathrm{~K}$ & $3.3 \mathrm{~K}$ & $4.7 \mathrm{~K}$ & $-9 \%$ & $+2 \%$ & $+13 \%$ \\
\hline $\begin{array}{l}\text { East } \\
\text { Africa }\end{array}$ & $1.8 \mathrm{~K}$ & $3.2 \mathrm{~K}$ & $4.3 \mathrm{~K}$ & $-3 \%$ & $+7 \%$ & $+25 \%$ \\
\hline $\begin{array}{l}\text { South } \\
\text { Africa }\end{array}$ & $1.9 \mathrm{~K}$ & $3.4 \mathrm{~K}$ & $4.8 \mathrm{~K}$ & $-12 \%$ & $-4 \%$ & $+6 \%$ \\
\hline Sahara & $2.6 \mathrm{~K}$ & $3.6 \mathrm{~K}$ & $5.4 \mathrm{~K}$ & $-44 \%$ & $+6 \%$ & $+57 \%$ \\
\hline
\end{tabular}

Table 90: Regional projections for temperature and precipitation changes in Africa, A1B scenario $^{1488}$

In general, global circulation models predict a further increase in drought frequency and temperatures for the Sahel for the next century ${ }^{1489}$, but most of these models have not been specifically designed for the region:

\footnotetext{
«Few regional downscaling studies have been performed in West Africa so far. Most climate projections for West Africa were performed either by considering GCM output directly or with statistical downscaling approaches. $\gg^{1490}$
}

$1484 \operatorname{IPCC}\left(2007^{1}\right)$, p. 869.

1485 Held, I.M.; DelWorth, T.L.; Lu, J. et al. (2005), pp. 17892-17894.

$1486 \operatorname{IPCC}\left(2007^{1}\right)$, p. 870.

1487 Paeth, H.; Hense, A. (2004), p. 204.

1488 IPCC $\left(2007^{1}\right)$, p. 854.

1489 Ingram, K.T.; Roncoli, M.C. \& Kirshen, P.H. (2002), p. 338.

1490 Jung, G. (2006), p. 27. 
There is a controversial discussion whether and to what degree anthropogenic factors such as deforestation and changing land use systems contribute to climatic changes in the Sahel. ${ }^{1491}$ Changes in land cover are expected to impact the local climate through feedback mechanisms and exchanges in the landatmosphere boundary layer. ${ }^{1492}$ Some researchers even warn of a possible collapse of the West African monsoon system if deforestation in the coastal regions of West Africa continues ${ }^{1493}$ while others see indications for a "greening" of the Sahel ${ }^{1494}$.

In a region where meteorological station networks are sparse and where large interannual variations are the rule rather than an exception, climate predictions are fraught with numerous difficulties:

- The general reason for model uncertainties which applies for any region in the world is the imperfect representation of physical processes, numerical approximations of physical equations and simplifications in parametrizations. ${ }^{1495}$

- Potential measurement errors of meteorological stations and the sparsity of the observation network in West Africa mean that spatial interpolations may have introduced errors into climate models of the region. ${ }^{1496}$

- Observations of climate changes in the Sahel are complicated by the fact that years with considerable precipitation surplus or deficits (of up to $50 \%$ of the long-term mean) are not anomalies but very much the rule. ${ }^{1497}$

\subsubsection{Malariological Impacts of Climate Change}

The transmission potential of vector-borne diseases is very sensitive to climate changes on the periphery of the present endemic areas and at higher altitudes within these areas. ${ }^{1498}$ Changes in regional temperature and precipitation have an impact on vectors' reproduction, development rate and longevity, and changes in wind pattern may influence vector dispersal. ${ }^{1499}$ Some flying insects can be dispersed several kilometers from their original breeding area by winds. ${ }^{1500} \mathrm{~A}$ rise in sea-level and resultant coastal flooding may result in the establishment of lagunae and proliferation of brackish water and thus potential

1491 HAMMER, T. (2005), p. 20.

1492 Oguntunde, P.G.; Friesen, J.; van de Giesen, N. \& Savenije, H.H.g. (2006), p. 1185.

1493 JUNG, G. (2006), p. 19.

1494 Hountondj, Y.-C.; SokPon, N. \& Ozer, P. (2008), pp. 875f;

OLSSON, L.; EKLUNDH, L. \& ARDÖ, J. (2005), p. 564.

1495 Jung, G. (2006), p. 91.

1496 Jung, G. (2006), p. 90.

1497 HAMMER, T. (2005), p. 21.

1498 Martens, W.J.M.; Jetten, T.H.; Rotmans, J. \& Niessen, L.W. (1995), p. 195.

1499 Martens, W.J.M.; Jetten, T.H.; Rotmans, J. \& Niessen, L.W. (1995), p. 196.

1500 Curto de Casas, S.I. \& Carcavallo, R.U. (1995), p. 1437. 
habitats for some mosquito species. ${ }^{1501}$ In case of malaria, temperature changes also have an impact on the parasite's life cycle. ${ }^{1502}$ Warming temperatures tend to shorten the extrinsic incubation period which will increase the basic reproduction number. ${ }^{1503}$

Mosquitoes are extremely sensitive to climate changes. Their feeding activity, reproduction and mortality rates are directly linked to ambient temperatures. ${ }^{1504}$ In general, increasing temperatures accelerate metabolic processes. One consequence is the necessity of a larger amount of (blood) meals. ${ }^{1505} \mathrm{~A}$ moderate rise in temperatures would therefore result in higher biting rates and shorter extrinsic incubation periods in many malarious areas. Particularly in areas of low-level endemic malaria, malaria incidence rates could be increased by $50 \%$ to $100 \% .{ }^{1506}$ Excessive heat kills mosquitoes, but within their survivable range, warmer temperatures increase their reproduction and biting activity and the rate at which pathogens mature in them. Warm nights and winters favor insect survival. Fossils from the last ice age demonstrate that rapid poleward shifts of insects accompanied warming. ${ }^{1507}$

Climate change will be associated both with geographical expansions and contractions of the areas suitable for Plasmodium falciparum transmission. ${ }^{1508}$ Rising temperatures are anticipated to expand the distribution of vector-borne pathogens in both time and space, thereby exposing host populations to a longer transmission season and immunologically naïve populations to newly introduced pathogens. Vector-borne pathogens are especially effected by climate change because they spend much of their life cycle within an invertebrate host whose temperature remains similar to ambient conditions. ${ }^{1509}$

One direct effect of global warming that falls in this context is that insects move to higher altitudes. In cool highlands, where temperatures frequently fall below the threshold for parasite development in mosquitoes, small increases in temperature can disproportionately enhance the transmission potential of diseases like malaria. This is particularly problematic since people in such fringe regions have little or no immunity. In Western Kenya, for example, malaria outbreaks have occurred at altitudes of $2000 \mathrm{~m}$ when mean monthly temperatures exceeded $18^{\circ} \mathrm{C}$ and rainfall reached more than $150 \mathrm{~mm}$ per month. ${ }^{1510}$

1501 Martens, W.J.M.; Jetten, T.H.; Rotmans, J. \& Niessen, L.W. (1995), p. 196.

1502 Hay, S.I.; Omumbo, J.A.; Craig, M.H. \& Snow R.W. (2000), p. 180; Gilles, H.M. $\left(1993^{1}\right)$, p. 27.

1503 Ruan, S.; XiAO, D. \& Beier, J.C. (2008), p. 1108.

1504 HarRus, S. \& Baneth, G. (2005), p. 1313.

1505 Curto de Casas, S.I. \& Carcavallo, R.U. (1995), p. 1437.

1506 MaRTens, P. (1998), p. 96.

1507 Epstein, P.R. (2001), p. 748.

1508 IPCC $\left(2007^{2}\right)$, p. 408.

1509 PATZ, J.A. \& Reisen, W.K. (2001), p. 171.

1510 Patz, J.A. \& Reisen, W.K. (2001), p. 171. 
Higher temperatures may, in some regions, limit the survival of disease vectors. ${ }^{1511}$ However, adverse effects of climate change on vector survival may be temperated in domestic environments. ${ }^{1512}$ Mosquitoes may, for example, seek cooler habitats in their vicinity. ${ }^{1513}$ Finally, there is a temperature at which a certain mosquito species attains maximum longevity. Inter-individual variations in the mosquito population mean that some individuals have a higher life expectancy at higher temperatures than others. In the course of time, this means that the temperature optimum for a certain species may rise due to adaption and natural selection (i.e. an over-proportionate increase in heattolerant individuals) $)^{1514}$

Increasing temperatures have also been observed in the oceans up to a depth of about $3 \mathrm{~km}$. The result is a measurable change in the hydrological cycle, including a general trend towards more water vapor in the atmosphere. ${ }^{1515}$ For each $1 \mathrm{~K}$ warming, the atmosphere can hold about $6 \%$ more water vapor. ${ }^{1516}$

Vectors with cycles in aquatic environments are directly affected by such changes. ${ }^{1517}$ The length of the rainy and dry seasons and the interval between them affects larvae and adult vector development and abundance. Transmission of many parasitic diseases is confined to the rainy season. ${ }^{1518}$ High relative humidity stimulates metabolic processes of vectors and low relative humidity obliges them to feed blood more frequently to compensate the dehydration. ${ }^{1519}$

In different parts of the world, unusually severe malaria outbreaks occurred during El Niño years. Torrential rains in parts of East Africa and the southwestern Ugandan highlands during the 1997/98 El Niño led to large malaria epidemics. It is possible, though, that in some areas, exceptionally heavy rains may wash larvae from their breeding sites, resulting in reduced malaria. ${ }^{1520}$

The frequency and intensity of extreme weather events, including prolonged droughts and heavy rain events, has been increasing in the recent past, often creating conditions conducive to clusters of water- and insect-borne diseases ${ }^{1521}$ :

1511 EPSTEIN, P.R. (2001), p. 752.

1512 Curto de Casas, S.I. \& Carcavallo, R.U. (1995), p. 1437.

1513 MaRTens, P. (1998), p. 88.

1514 Martens, P. (1998), p. 88.

1515 EPSTEin, P.R. (2001), p. 747.

1516 EPSTEIn, P.R. (2001), p. 749.

1517 Curto de Casas, S.I. \& Carcavallo, R.U. (1995), p. 1437.

1518 Patz, J.A.; Graczyk, T.K.; Geller, N. \& Vittor, A. (2000), p. 1398.

1519 Curto de Casas, S.I. \& Carcavallo, R.U. (1995), p. 1437.

1520 Patz, J.A.; Graczyk, T.K.; Geller, N. \& Vittor, A. (2000), p. 1400.

1521 EPstein, P.R. (2001), p. 747. 
«Shifts of usual seasonal rhythms can alter synchronies among predators, competitors and prey, releasing opportunists from natural biological controls. ${ }^{1522}$

In Mozambique, severe floods in coincided with a $50 \%$ to $100 \%$ rise in malaria incidence in 2000. ${ }^{1523}$

\subsubsection{Malaria and Climate Change in Africa}

TANSER et al. (2003) examined the impact of climate changes predicted by three IPCC scenarios on malaria transmission in Africa for the $21^{\text {st }}$ century. Under the conditions of the B1 scenario (i.e. low greenhouse gas emissions), the exposed population in Africa would grow by $13 \%$ by the end of the century, and the allover risk of exposure by $15.5 \%$ (increase in the exposed population plus increased risk of transmission). However, large differences between different countries can be expected - in South Africa, for example, the exposed population is predicted to grow by $124.3 \%$, whereas a decrease of $44 \%$ is expected for Mauritania. Under the A1FI scenario (high greenhouse gas emissions), these changes would be more marked, ranging from a severely increased population at risk in South Africa $(+247 \%)$ to an enormous reduction in Mauritania $(-86,6 \%) .{ }^{1524}$ Figure 139 illustrates the predicted effects of climate change on the allover risk of malaria transmission in Africa during the $21^{\text {st }}$ century under different scenarios.

1522 EPSTEIn, P.R. (2001), p. 749.

1523 Ramin, B.M. \& McMichael, A.J. (2009), doi: 10.1007/s10393-009-0222-4.

1524 Tanser, F.C.; Sharp, B. \& le Sueur, D. (2003), p. 1793-1796. 
Malaria Transmission Risk and Climate Change in Africa

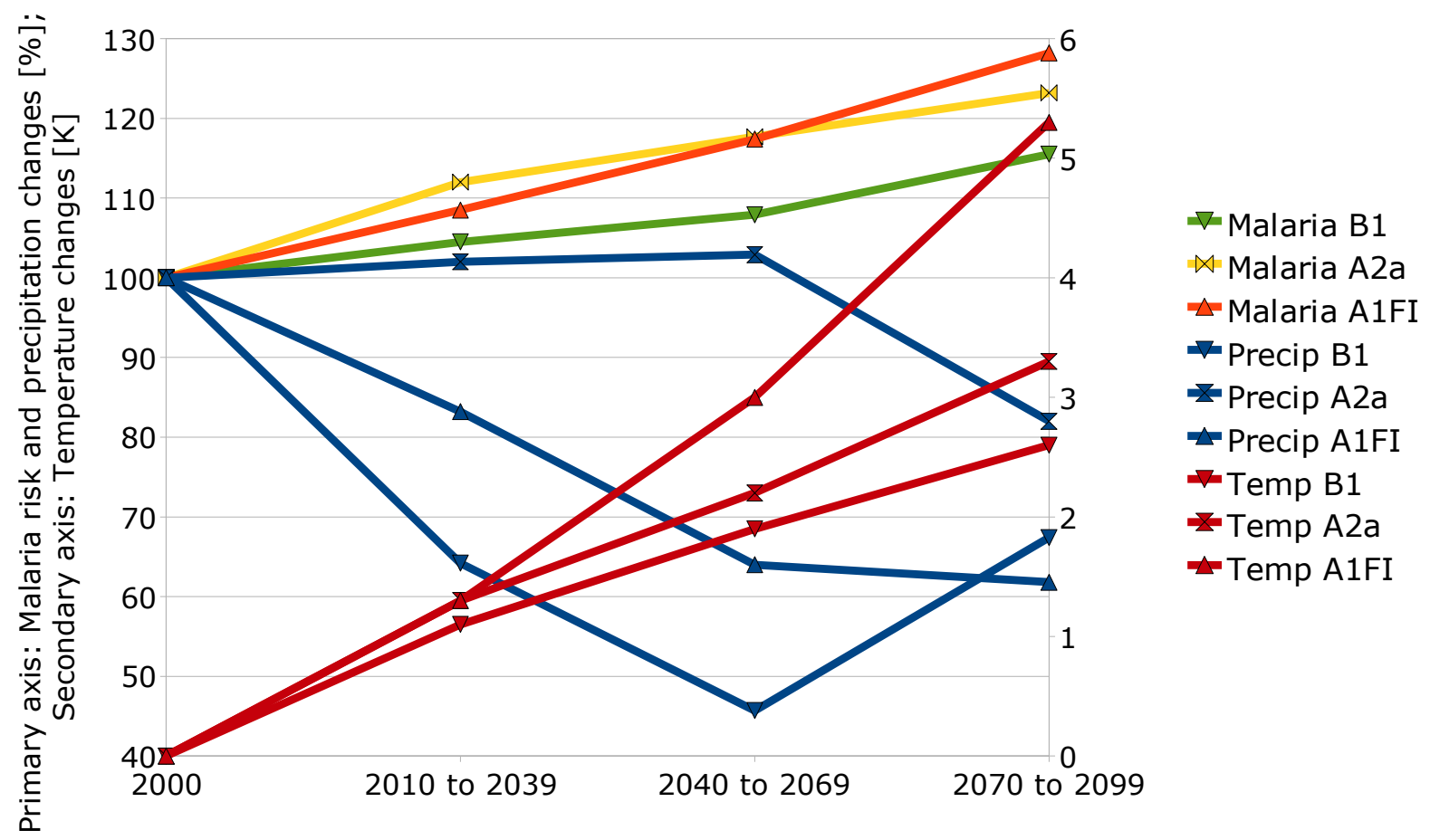

Figure 139: Malaria transmission risk and climate change in Africa ${ }^{1525}$

The potential effect of climate change in areas of existing transmission is noticeable, with a $28 \%$ to $42 \%$ increase in exposure in areas presently suitable for disease transmission by the end of the $21^{\text {st }}$ century. For the near future, the highest increase is predicted for the A2a scenario due to the combined effects of a slight increase in rainfall and a relative large rise in temperature. The A1FI scenario, in which a $5.3 \mathrm{~K}$ rise in temperature is coupled with a moderate reduction in rainfall, is believed to cause the greatest increase in malaria incidence by $2100 .^{1526}$

Some countries in West Africa (e.g. Mali, Ghana and Burkina Faso) are projected by some scenarios to show a fall in exposure to malaria transmission. ${ }^{1527}$ In the western Sahel, maximum temperatures may in the future exceed the upper threshold for mosquito survival ${ }^{1528}$, potentially making this region unsuitable for malaria transmission by $2050 .{ }^{1529}$ Since shorter

1525 Tanser, F.C.; Sharp, B. \& le Sueur, D. (2003), p. 1795.

1526 Tanser, F.C.; Sharp, B. \& le Sueur, D. (2003), p. 1797.

1527 Tanser, F.C.; Sharp, B. \& le Sueur, D. (2003), p. 1797.

1528 Thomas, C.J.; Davies, G. \& Dunn, C.E. (2004), p. 218.

1529 IPCC $\left(2007^{2}\right)$, p. 446. 
periods of exposure are linked with a reduced immunity, occasional malaria endemics may undermine a reduction in morbidity or mortality. ${ }^{1530}$ The expected impacts of climate change on malaria in West Africa during the $21^{\text {st }}$ century are illustrated in figure 140 .

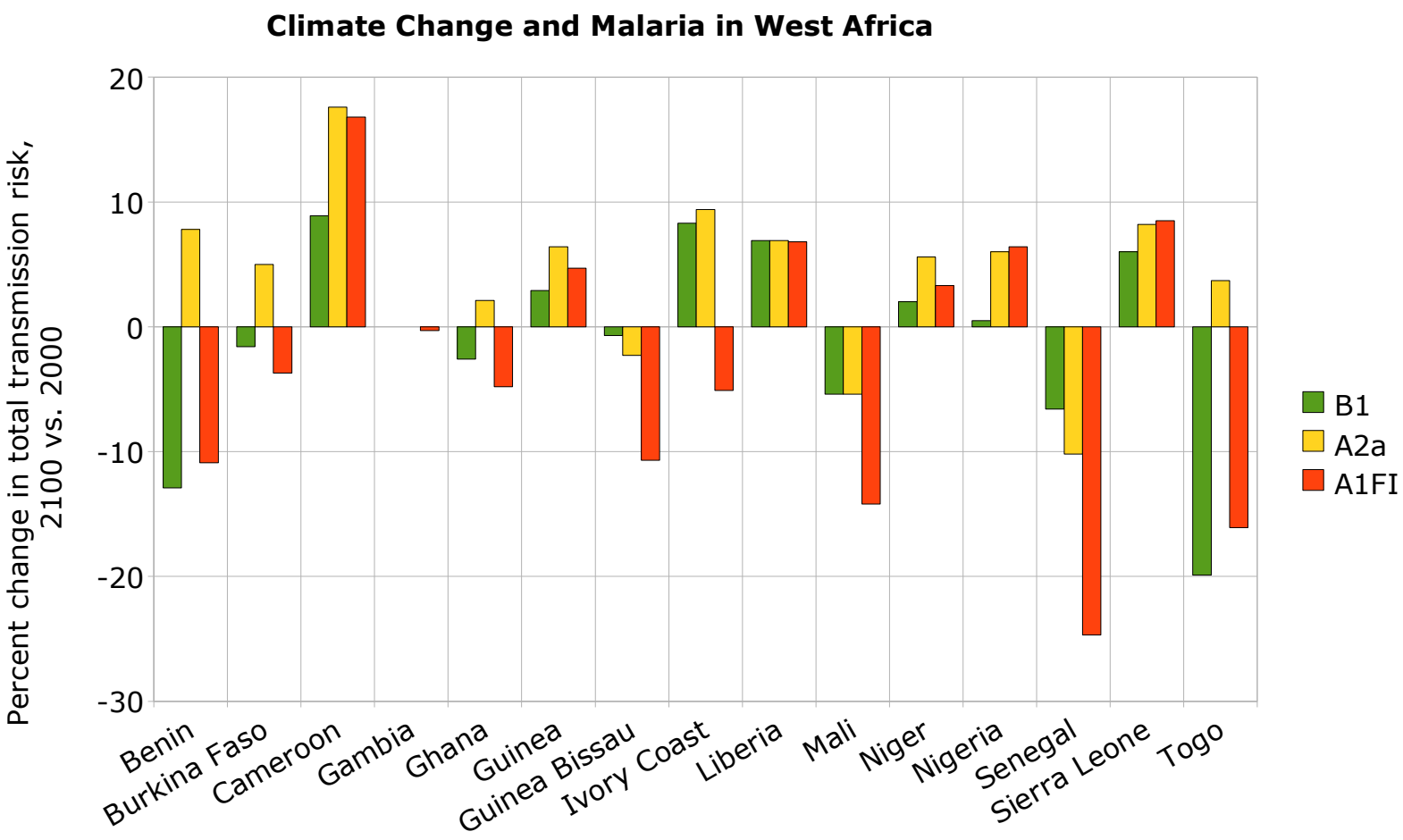

Figure 140: Effects of climate change on malaria transmission risk in West Africa ${ }^{1531}$

For Burkina Faso, most climate scenarios predict only small changes, whereas incidence in other parts of West Africa is expected to change more significantly, ranging from increases (e.g. in Cameroon) to decreases (e.g. in Senegal) by around 10 to $20 \%$. The predicted stability of transmission risks in countries like Burkina Faso may, however, mask regional changes in different geo-ecological zones.

Thомаs et al. (2004) stated that the likely effects of climate change on malaria zones in Africa would not only be spatially heterogeneous, but that range contractions are more likely than expansions. ${ }^{1532}$ It is important to note that even under a scenario of range contractions, the actual incidence of malaria 
could rise in case of increased risks in regions of high population densities. MARTENS et al. (1995), for example, expected the number of people at risk of contracting malaria to increase by $2.9 \%$ (ECHAM1-A global circulation model) to $10.1 \%$ (UKMO circulation model) by $2050 .{ }^{1533}$

\begin{tabular}{|l|l|l|}
$\begin{array}{l}\text { Future Malaria Risks } \\
\text { (climate model) }\end{array}$ & $\begin{array}{l}\mathbf{2 0 5 0} \\
\text { (ECHAM1-A) }\end{array}$ & $\begin{array}{l}\mathbf{2 1 0 0} \\
\text { (UKMO) }\end{array}$ \\
\hline $\begin{array}{l}\text { Population at risk of contracting } \\
\text { malaria }\end{array}$ & 2.800 million & 3200 million \\
\hline
\end{tabular}

Table 91: Predicted future population at risk of malaria ${ }^{1534}$

Climate change does not only occur as a global warming process but also in form of micro-climatic changes. One important source of micro-climatic change in Africa is land reclamation for agricultural purposes. ${ }^{1535}$ When such changes occur in mosquito habitats, they may be locally much more important than global change phenomena.

\subsubsection{Land Use Changes}

Local vector habitats frequently change due to deforestation, agricultural development and man-made alterations in water bodies. These activities frequently create new niches for vectors. ${ }^{1536}$ Land use change is also a potential contributor to climate change in the $21^{\text {st }}$ century. ${ }^{1537}$

Deforestation is one of the most disruptive changes affecting parasitic vector populations. Changes in the types and amounts of vegetation provide altered ecological niches and conditions for proliferation of newly arriving or adaptive existing vectors and their parasites. Whereas the ground in primary forests tends to be shaded and covered with organic matter which absorbs water and renders it quite acidic, cleared lands are generally more sunlit and prone to the formation of puddles with more neutral $\mathrm{pH}$, favoring for example the development of certain anopheline larvae. ${ }^{1538}$ In the Kenyan Highlands, deforestation has coincided with a $0,5 \mathrm{~K}$ average increase in local temperatures. ${ }^{1539}$

1533 Martens, W.J.M.; Jetten, T.H.; Rotmans, J. \& Niessen, L.W. (1995), p. 208.

1534 Martens, W.J.M.; Jetten, T.H.; Rotmans, J. \& Niessen, L.W. (1995), p. 208.

1535 IPCC $\left(2007^{2}\right)$, p. 439.

1536 HaRRus, S. \& Baneth, G. (2005), p. 1312.

1537 IPCC $\left(2007^{1}\right)$, p. 871.

1538 Patz, J.A.; GraczYK, T.K.; Geller, N. \& Vittor, A. (2000), p. 1396.

1539 IPCC $\left(2007^{2}\right)$, p. 439. 
Hydrological modifications are also closely linked with vector-borne disease transmission. In the tropics, during construction of dams and canals, excavation pits provide breeding sites for mosquitoes. ${ }^{1540}$ Additional breeding sites are provided by creation of reservoirs, irrigation canals and irrigated rice fields. Rising ground water levels in the proximity of reservoirs and water seepage below dams have been linked to the creation of water puddles colonized by anophelines. ${ }^{1541}$ Irrigated land provides a suitable breeding ground for a number of disease vectors ${ }^{1542}$ including Anopheles mosquitoes. ${ }^{1543}$

Upsurges of malaria have been coincident with irrigation projects and changes in land-use and human settlement subsequent to deforestation in western Africa. ${ }^{1544}$ Moreover, the introduction of large-scale irrigation schemes often reduced the significance of local rainfall in vector-borne disease epidemiology. ${ }^{1545}$

\subsubsection{Population Growth, Migration and Mobility}

Human population growth, migration and urbanization can lead to a concentration of susceptible parasite hosts, often including infected individuals. Assuming a $1.16 \mathrm{~K}$ temperature rise and a world population of 8.6 billions by 2050, Martens (1998) predicted the number of people at risk of malaria to increase by 720 millions. ${ }^{1546}$ Burkina Faso's current growth rate of $3.2 \%$ would theoretically result in a doubling of the population within 22 years ${ }^{1547}$. Under such conditions, not global climate change but population growth and associated land use modifications could be the key drivers of malaria risk in the next few decades.

Africa is the world's most rapidly urbanizing continent ${ }^{1548}$, with urbanization rates typically ranging between $2 \%$ and $6 \%$ in the least developed countries. ${ }^{1549}$ By 2030 , the proportion of Africans living in cities is projected to reach at least $50 \% .{ }^{1550}$

1540 Martens, W.J.M.; Jetten, T.H.; Rotmans, J. \& Niessen, L.W. (1995), p. 195.

1541 Lautze, J.; McCartney, M.; Kirshen, P. et al. (2007), p. 985.

1542 Martens, W.J.M.; Jetten, T.H.; Rotmans, J. \& Niessen, L.W. (1995), p. 195.

1543 Sissoko, M. S; Dicko, A.; Briët, O.J.T. et al. (2004), p. 162.

1544 Patz, J.A.; GraczYk, T.K.; Geller, N. \& Vittor, A. (2000), p. 1399.

1545 Martens, W.J.M.; Jetten, T.H.; Rotmans, J. \& Niessen, L.W. (1995), p. 202.

1546 MaRTens, P. (1998), p. 53.

1547 Deutsche Stiftung Weltbevölkerung (2009), p. 6.

1548 Hay, S.I.; Guerra, C.A.; TAtem, A.J. et al. (2005), p. 81.

1549 Keiser, J.; Utzinger, J.; Caldas de Castro, M. et al. (2004), p. 118.

1550 Siri, J.G.; Lindblade, K.A.; Rosen, D.H. (2008); doi:10.1186/1475-2875-7-34. 
«Especially affected will be West Africa, where the urban population annual growth rate of $6.3 \%$ is more than twice the rate of the total population growth. [... By 2025,] two out of three West Africans will live in urban centers. ${ }^{1551}$

One phenomenon that is frequently linked to urbanization is the immigration of people from rural regions. Newcomers to an area may not be immune to the locally endemic diseases, and may at the same time introduce new pathogens. ${ }^{1552}$ Migrants may also lack familiarity with self-protective habits that would limit their availability as feed sources to vectors, e.g. using bednets or avoiding outdoor activities during peak biting hours. ${ }^{1553}$

Within West Africa, there are great disparities regarding urbanization ratios. Moreover, there is no universally accepted definition of "urban" areas in Africa ${ }^{1554}$, and "urban" regions do often not fit functional definitions: rice fields and vegetable gardens may exist in the heart of cities, and livestock may be herded through central business districts. ${ }^{1555}$ In developing countries, urban agriculture may create additional opportunities for mosquito multiplication. ${ }^{1556}$

Historically, economic development and urbanization have tended to go hand in hand. One notable exception to this rule, however, is sub-Saharan Africa. The region's growth rate of urbanization has been extraordinary by international standards (and even 2,5 times higher than that of the rest of the developing world), but does not appear "to have been coupled with an improvement in economic wealth". ${ }^{1557}$ Unplanned and uncontrolled urbanization in developing countries usually results in the deterioration of public health infrastructure, sanitation, water, sewage and waste management systems, sometimes producing ideal conditions for the transmission of vector-borne diseases to large populations. ${ }^{1558}$ In adapting to changed environments, including reduced animal and increased human population, some vectors display conversion from a primarily zoophilic to a primarily anthropophilic orientation. ${ }^{1559}$ The massive aggregations of people found in the growing megacities of the developing world often present ideal conditions for the emergence of infectious agents. ${ }^{1560}$ If suitable vector breeding sites are present, the high density of human reservoirs

1551 Donnelly, M.J.; McCall, P.J.; Lengeler, C. (2005), doi:10.1186/1475-2875-4-12.

1552 Harrus, S. \& Baneth, G. (2005), p. 1313.

1553 Patz, J.A.; Graczyk, T.K.; Geller, N. \& Vittor, A. (2000), p. 1396.

1554 Keiser, J.; Utzinger, J.; Caldas de Castro, M. et al. (2004), p. 119.

1555 Robert, V.; Macintyre, K.; Keating, J. et al. (2003), p. 169.

1556 Moore, M.; Gould, P. \& Keary, B.S. (2003), p. 273.

1557 Barrios, S.; Bertinelli, L. \& Strobl, E. (2006), pp. $357 f$.

1558 HaRRUS, S. \& BanETH, G. (2005), p. 1313.

1559 Patz, J.A.; Graczyk, T.K.; Geller, N. \& Vittor, A. (2000), p. 1395.

1560 MAY, R.M. (2007), p. 502. 
of parasites in cities may lead to increased opportunities for vector-host contact and thus exchange and transmission of parasites. ${ }^{1561}$ Satellite data have shown that urban and industrial air pollution may alter pattern of rainfall and therefore vector habitats. ${ }^{1562}$

Waste dumps frequently serve as breeding sites for vectors of disease, including mosquitoes and sandflies. Items facilitating small collections of water, such as tires or cans, can serve as breeding places for mosquitoes such as Aedes spp. and Anopheles stephensi, thus contributing to the spread of dengue fever, yellow fever and malaria. ${ }^{1563}$

Aggregated data for cities as a whole can be extremely misleading since the population's health status is often unevenly distributed within cities. A lack of intra-urban differentiation in health data may therefore mask problems within urban sub-populations. ${ }^{1564}$

In the past, several major malaria epidemics were caused by the introduction of malaria vectors by passenger or freight ships and planes. In 1866, Anopheles gambiae was "accidentally" introduced into Mauritius where it caused an epidemic resulting in unusually high mortality rates. When the species was introduced into Brazil in the 1930s, it caused "a malaria epidemic that rivaled the worst outbreaks described in the literature of this disease". ${ }^{1565}$ In the 1970s, the term "airport malaria" was coined for malaria cases caused by the introduction of infectious vectors by means of air traffic or the autochthonous transmission of malaria resulting from imported cases and locally present vectors. Increasing levels of mobility and global transports mean that malaria vectors and parasitized hosts may reach almost any part of the world within just one day. ${ }^{1566}$

\subsubsection{Limitations of Future Predictions}

Even though models have been developed to predict malaria risks or individual determinants of malaria in the future, a lack of long time series mean that a validation of such predictions is still difficult. Since there are uncertainties related to many of the model inputs, predictions about future transmission intensities and spatial expansions or contractions of malarious areas are fraught with uncertainty. ${ }^{1567}$

1561 Patz, J.A.; Graczyk, T.K.; Geller, N. \& Vittor, A. (2000), p. 1396.

1562 HaRrus, S. \& Baneth, G. (2005), p. 1313.

1563 Moore, M.; Gould, P. \& Keary, B.S. (2003), p. 273.

1564 Moore, M.; Gould, P. \& Keary, B.S. (2003), p. 271.

1565 Lounibos, L.P. (2002), p. 236.

1566 ISAACSON, M. (1989), p. 740.

1567 MarTens, P. (1998), p. 66. 
Most studies on the future risks posed by vector-borne infections are based on climate scenarios and models of pathogen transmission. Many of these statistical models were developed at regional scales and may not be valid for predictions at continental or global scales. ${ }^{1568}$ Moreover, despite intensive research efforts, many aspects of future climate changes are still unpredictable, particularly at the regional level. ${ }^{1569}$ This is further complicated by the fact that climate changes do not occur in isolation but embedded in simultaneous developments such as population growth and urbanization:

\begin{abstract}
«Despite the known causal links between climate and malaria transmission dynamics, there is still much uncertainty about the potential impact of climate change on malaria at local and global scales because of the paucity of concurrent detailed historical observations of climate and malaria, the complexity of malaria disease dynamics, and the importance of non-climatic factors, including socio-economic development, immunity and drug resistance. $\gg^{1570}$
\end{abstract}

In highly endemic regions, increases in the malaria transmission potential may be counteracted by a boost in immunity, whereas the impact may be completely different in populations with initially low levels of immunity. Indeed, the highest increases in disease incidence are expected in regions of immunologically naïve populations. ${ }^{1571}$

Predictions of future malaria risks are thus complicated by the fact that malaria transmission depends on a complex set of both environmental and sociogeographic determinants. The links and interrelations of these determinants and malaria transmission are still not completely understood. At the same time, predictions regarding single factors such as temperature or population growth are fraught with uncertainties, and models taking into account several factors may result in predictions of future malaria risks for which those uncertainties add up. MaRTENS (1998) observed that the predicted range of global Plasmodium falciparum incidence would vary between 1.8 and 2.5 times the 1990 level if only climate scenarios and their impact on vectorial capacity were taken into account but that this range would extend to 1.4 to 4.8 if all uncertainties along the link chain between climate change, vectorial capacity and malaria prevalence were added up. ${ }^{1572}$

1568 Tanser, F.C.; Sharp, B. \& le Sueur, D. (2003), p. 1792.

1569 Sutherst, R.W.; IngRam, J.S.I. \& Scherm, H. (1998), p. 297.

$1570 \operatorname{IPCC}\left(2007^{2}\right)$, p. 404.

1571 MarTENS, W.J.M. (1998), pp. $242 f$ \& 248.

1572 Martens, W.J.M. (1998), pp. $248 f$. 


\subsubsection{Observable Trends}

While predictions based on models are fraught with numerous uncertainties, empirical data from recent years indicate that malaria in the study region is currently on the rise. The upsurge in malaria incidence in Kossi between 2005 and 2008 was paralleled by an even more marked rise at the national level in Burkina Faso (see table 92):

\begin{tabular}{|l|l|l|l|l|}
\hline Malaria Incidence & 2005 & 2006 & 2007 & 2008 \\
\hline Burkina Faso ${ }^{1573}$ & $146 \%$ o & $169 \%$ o & $194 \%$ o & $257 \%$ o \\
\hline Kossi: study villages $^{1574}$ & $120 \%$ o & $102 \%$ o & $115 \%$ o & $141 \%$ o \\
\hline
\end{tabular}

Table 92: Malaria incidence in Burkina Faso and the study villages

These findings illustrate that in Burkina Faso, malaria is a problem that is still far from being solved; in fact, the rising incidence of malaria contrasts sharply with a general improvement in the public health situation. Since the turn of the century, government health spending has increased ${ }^{1575}$, leading not only to an extension and upgrading of the public health infrastructure ${ }^{1576}$ but also actual progress such as a declining childhood mortality. ${ }^{1577}$ The concurrent increase in malaria incidence, is in fact one of the key threats to the nation's attainment of the Millennium Development Goals and thus a public health issue that requires urgent attention. In fact, the achievement of the 2010 and 2015 goals as formulated by the Roll Back Malaria initiative would require annual reductions of around $7 \%$ to $12 \% .{ }^{1578}$ The situation found in Burkina Faso is representative for that of many nations in Sub-Saharan Africa, where the current malaria burden may be the highest ever experienced. ${ }^{1579}$

The currently observable trends in vector-borne disease incidence, and the expected developments in fields such as climate, land use and population growth which may have an impact on their transmission in the future mean that vector-borne diseases and their spatio-temporal pattern will have to remain on the research agenda for years to come. Only then, experiences from the past and advances in VBD transmission modeling may lead to improved risk prediction systems and intervention campaigns.

1573 Direction Générale de l'Information et des Statistiques Sanitaires $\left(2009^{1}\right)$, p. 35.

1574 Average for the CSPS at Toni, Lékuy and Wèrèbèrè (data from internal records of respective CSPSS).

1575 Direction Générale de l'Information et des Statistiques Sanitaires $\left(2009^{1}\right)$, p. 54.

1576 Direction Générale de l'Information et des Statistiques Sanitaires $\left(2009^{1}\right)$, p. 6.

1577 OeCD \& African Development Bank (2008), p 178.

1578 Roll Back Malaria Partnership (2005), p. 2.

1579Hay, S.I.; Guerra, C.A.; Tatem, A.J. et al. (2005), p. 81. 


\subsection{Research Perspectives}

The high burdens caused by vector-borne diseases in general and malaria in particular and a potential rise in their incidence due to ongoing and expected changes of the physical and sociogeographic environment mean that future research is necessary in order to better understand the links between vectorborne disease transmission and the settings in which they occur. This way, the impact of man-made environmental changes could in the future be better predicted, for example before irrigation projects are implemented. Such predictions would not only be useful for project planning and the implementation of counter-measures, but also for informing local communities and health authorities in order to increase their preparedness for changing transmission pattern. The development of up-to-date risk maps and early warning systems, including their assessment and enhancement as planning tools could finally make malariologic research "pay off" in form of more efficient intervention programs and reductions of the malaria burden.

\subsubsection{Methodologic Approach}

Even though the present study tried to provide a comprehensive overview of the factors involved in the determination of malaria transmission dynamics, it is still far from presenting a complete picture, and in many ways was just a first step into assessing the importance of individual predictors of malaria for rural dry savanna regions like Kossi Province.

Advances in medical geography (and particularly malaria epidemiology and remote sensing) on the one hand and the experiences of this study on the other hand mean that several new research foci have become evident. In the future, this may lead to multidisciplinary studies systematically investigating the links between environmental determinants, mosquito vectors and malaria and their variation in time and space. Indeed, the development of a research concept for a comprehensive investigation of local to regional scale determinants of malaria transmission may be an equally important outcome as the case study results.

\subsubsection{Technical Advances}

Studies on malaria transmission are typically multidisciplinary, with advances occurring simultaneously in many related fields. From the geographic perspective, two current developments may be of particular relevance for geomedical investigations. 
The emerging field of landscape epidemiology deals with the exploration of spatial pattern of disease transmission and their links to landscape ecology. This promising, highly integrative approach takes into account the full complexity of ecologic niche determinants ${ }^{1580}$ :

\begin{abstract}
«ENM [Ecological Niche Modeling] is in the early stages of being explored for its potential for illuminating unknown phenomena in the world of disease transmission. The extensive explorations of ENM in the biodiversity field, however, serve as a benchmark of quality and acceptance for the technique. $\gg^{1581}$
\end{abstract}

Applications of ENM to disease transmission systems have been scarce but are promising since each species involved is distributed according to its ecological potentials and constraints, resulting in links between landscape ecology and the geography disease transmission. ${ }^{1582}$

Developments in satellite sensor and computing technology have been particularly dynamic, widening the potentials for their application in medical geography and landscape epidemiology. While higher spatial resolutions mean that ever smaller habitats can be detected, higher temporal resolutions could be useful for capturing transient habitats and hyperspectral data improve the characterization of mosquito habitats. Even a dedicated satellite for geomedical purposes (MEDSAT) had been proposed in the $1990 \mathrm{~s}^{1583}$, but this project has not yet been realized.

New very high spatial resolution sensors include those on the WorldView and GeoEye family of satellites (see table 93). At the time of its launch in September 2008, the GeoEye-1 satellite was offered the highest sensor solution commercially available ${ }^{1584}$. The new WorldView-2 satellite is scheduled for launch in fall of 2009. Among the very high spatial resolution satellites, its sensors will feature a previously unparalleled spectral resolution and the shortest revisit times in its category. ${ }^{1585}$

1580 Peterson, A.T. (2006), p. 1822.

1581 Peterson, A.T. (2006), p. 1825.

1582 Peterson, A.T. (2007), pp. 393-396.

1583 Epstein, P.R.; Rogers, D.J. \& Slooff, R. (1993), p. 1406.

1584 http://www.geoeye.com/CorpSite/products/imagery-sources/Default.aspx\#geoeye1, accessed 21/06/09.

1585 http://worldview2. digitalglobe.com/about/, accessed 21/06/09. 


\begin{tabular}{|c|c|c|}
\hline Sensor Characteristics & GeoEye-1 1586 & WorldView-2 $2^{1587}$ \\
\hline Spatial resolution & $\begin{array}{l}\text { Panchromatic: 0,41 m } \\
\text { Multispectral: 0,65 m }\end{array}$ & $\begin{array}{l}\text { Panchromatic: 0,46 m } \\
\text { Multispectral: } 1,84 \mathrm{~m}\end{array}$ \\
\hline Spectral resolution & $\begin{array}{l}\text { Blue: } 450-510 \mathrm{~nm} \\
\text { Green: } 510-580 \mathrm{~nm} \\
\text { Red: } 655-690 \mathrm{~nm} \\
\text { Near-IR: } 780-920 \mathrm{~nm}\end{array}$ & $\begin{array}{l}\text { Coastal: } 400-450 \mathrm{~nm} \\
\text { Blue: } 450-510 \mathrm{~nm} \\
\text { Green: } 510-580 \mathrm{~nm} \\
\text { Yellow: } 585-625 \mathrm{~nm} \\
\text { Red: } 630-690 \mathrm{~nm} \\
\text { Red Edge: } 705-745 \mathrm{~nm} \\
\text { NIR: } 770-895 \mathrm{~nm} \\
\text { NIR 2: } 860-1045 \mathrm{~nm}\end{array}$ \\
\hline Revisit time & $<3$ days & about 1.1 days \\
\hline Radiometric resolution & 11 bit & 11 bit \\
\hline
\end{tabular}

Table 93: Characteristics of the GeoEye-1 and WorldView-2 satellites

Since radar systems can penetrate clouds ${ }^{1588}$ and are well-suited for surface water detection ${ }^{1589}$, they are promising tools for the mapping of mosquito larval habitats during the rainy season. The launch of the German TerraSAR-X satellite in September 2007 marked a considerable increase in spatial resolution for radar satellites. At a repetition rate of 11 days, spatial resolutions ranging from $18 \mathrm{~m}$ ("ScanSAR" mode) to $1 \mathrm{~m}$ ("SpotLight" mode) can be achieved. The TanDEM-X is a second, very similar satellite scheduled to be launched in 2009. The aspired twin satellite constellation offers the additional benefit of digital elevation model generation at "an unprecedented accuracy". 1590

The development of new satellites and sensors is no longer restricted to a few highly industrialized nations of the West. Data from Indian Remote Sensing (IRS) satellites, for example, have already been used locally for mosquito mapping and control activities. ${ }^{1591}$ However, satellites and sensors produced by and for the indigenous needs of African nations are still non-existent.

1586 http://www.geoeye.com/CorpSite/products/imagery-sources/Default.aspx\#geoeye1, accessed 21/06/09.

1587 http://worldview2. digitalglobe.com/about/, accessed 21/06/09.

1588 De Lange, N. (2006), p. 389.

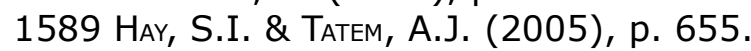

1590 http://www.infoterra.de/terrasar-x/terrasar-x-satellite-mission.html, accessed 22/07/09.

1591 Hay, S.I.; Snow, R.W. \& Rogers, D.J. (1998), p. 306; Dhiman, R.C. (2000), p. 123. 


\subsubsection{Recommended Research Foci}

Even though the links between different environmental pattern and anopheline population dynamics and behavior have been studied in the laboratory, there is still a scarcity of systematic studies under field conditions.

One very little studied question is that of exact ecological characterizations of vector habitats under natural conditions. Numerous relatively general descriptions of important vector species' habitats exist, but systematic investigations into the ecological niches occupied by these mosquitoes have been rare. Several parameters and links appear to be of particular interest in this respect. The microclimatic conditions in mosquito habitats and potential dry season refugia were found to differ substantially from conditions around human habitats or non-irrigated fields, the "typical" locations for meteorological stations in the study region. Therefore, long-term measurements around different types of habitats are necessary for their exact characterization. Not only spatial variations of mean temperature or total rainfall but also differences in their timing (e.g. the onset and duration of rains) should be investigated with regard to their importance for the geography of malaria transmission dynamics.

Whereas the influence of water temperatures on larval development has been studied in the laboratory, not much is known about thermal profiles of vector breeding sites under field conditions. Since water temperatures have a major impact on adult mosquito emergence and thus vector population densities, investigations into the links between insolation, air temperatures, habitat types and water temperatures are necessary. At the same time, basic water quality parameters, including turbidity, contents of dissolved and suspended particles, acidity should be monitored.

At the same time, entomological investigations at breeding sites are necessary to determine their productivity. So far, little is known about adult emergence rates from different larval habitats under field conditions. A systematic investigation into the productivity of aquatic habitats and its determinants on the one side, and into the links between breeding site typologies and spatial pattern of adult vector distribution could considerably improve the quality of local-scale malaria risk maps. Moreover, such research could help to identify additional determinants of vector dispersal and allow a risk stratification. Both standardized measurements of vector density and estimates of inoculation rates would be required to causally link ecological information to vector populations and finally disease occurrence data. 
Investigations into the geographic determinants malaria may also be models for research on other vector-borne diseases, including emerging diseases like CHIKUNGUNYA AND dengue fever.

\subsubsection{Project Integration}

The tremendous burden that malaria causes in West Africa on the one side and an incomplete knowledge about the geographic determinants of transmission dynamics on the other side would be reason enough for more research in this field. The co-occurrence of malaria and various other vector-borne diseases in many malaria-endemic regions, and the links between malaria and environmental and sociogeographic changes mean that there is considerable scope for project integration.

\subsubsection{Integrated Projects on VBDs}

Numerous vector-borne disease occur on the African continent, most of them being transmitted by mosquitoes. The anopheline vectors transmitting malaria, for example, are also responsible for filariasis ${ }^{1592}$ and O'nyong-nyong fever ${ }^{1593}$. Mapping of anopheline habitats, or vector control measures are therefore not only of malariologic interest.

Some of the vector borne diseases that once were important threats for human health in Africa are close to eradication (e.g., dracunculiasis ${ }^{1594}$ ), while the incidence of others has increased considerably in recent years. Dengue fever is currently regarded the most rapidly advancing vector-borne disease of the world. ${ }^{1595}$

Even though vector-borne diseases differ both in regard to the types of vectors spreading the infections and the pathogens causing them, the life cycles of many vectors - and even some parasites - are comparable to those of anopheline mosquitoes and Plasmodia and in most cases governed by the same factors.

Table 94 presents diseases with a transmission process that relatively closely resembles that of malaria. Common features of this group are vectors of the order Diptera (two-winged flies) and protozoan or helminthic parasites. The transmission process of these diseases follows the same basic pattern as that of malaria for two reasons: they are transmitted by flying insects, and the parasites have to pass an incubation period and undergo physical

1592 Otranto, D.; Stevens, J.R.; Cantacessi, C. \& Gasser, R.B. (2007), p. 117.

1593 TESH, R. B. (1982), p. 33.

1594 MulleR, R. (2005), p. 521.

1595 Spiegel, J.M.; Bonet, M.; Ibarra, A.-M. et al. (2007), p. 503. 
developments in their vectors before further transmission can occur. However, different vectors mean that diseases may be transmitted under ecological conditions differing from the zones of malaria transmission.

\begin{tabular}{|c|c|c|c|}
\hline Disease & Causative pathogen & $\begin{array}{l}\text { Important } \\
\text { Vectors }\end{array}$ & References \\
\hline $\begin{array}{l}\text { African } \\
\text { trypanosomiasis } \\
\text { (Sleeping sickness) }\end{array}$ & $\begin{array}{l}\text { Trypanosoma brucei } \\
\text { rhodesiense, } \\
\text { Trypanosoma brucei } \\
\text { gambiense }\end{array}$ & Glossina spp. & $\begin{array}{l}1596,1597,1598 \\
1599,1600^{\prime}\end{array}$ \\
\hline Filariasis & $\begin{array}{l}\text { Wuchereria bancrofti, } \\
\text { Brugia malayi, Brugia } \\
\text { timori, Dirofilaria } \\
\text { repens, Dirofilaria } \\
\text { immitis }\end{array}$ & $\begin{array}{l}\text { Aedes spp. } \\
\text { Culex spp. } \\
\text { Anopheles spp. }\end{array}$ & $1601,1602,1603$ \\
\hline Loiasis & Loa Loa & Chrysops spp. & 1604,1605 \\
\hline Leishmaniasis & Leishmania spp. & Phlebotomus spp. & 1606 \\
\hline $\begin{array}{l}\text { Onchocerciasis } \\
\text { (river blindness) }\end{array}$ & Onchocerca volvulus & Simulium spp. & 1607 \\
\hline
\end{tabular}

Table 94: Vector-borne diseases with a transmission cycle closely resembling malaria

A second group of vector-borne diseases is also transmitted by vectors of the order Diptera, but caused by viral pathogens (see table 95). One major implication is that transmission may take place directly after infection of vectors. One of these diseases, dengue fever, is considered to be a growing public health problem throughout the tropics.

1596 MolyneuX, D.H. (1998), p. 929.

1597 Otranto, D.; Stevens, J.R.; Cantacessi, C. \& Gasser, R.B. (2007), p. 117.

1598 Garcia, A.; Jamonneau, V.; Sané, B. et al. (2002), p. 429.

1599 SteRnBerg, J.M. (2004), p. 469.

1600 Belete, H.; Tikubet, G.; Petros, B. (2004), p. 710.

1601 Otranto, D.; Stevens, J.R.; Cantacessi, C. \& Gasser, R.B. (2007), p. 117.

1602 Gyapong, J.O.; Kyelem, D.; Kleinschmidt, I. et al. (2002), pp. 701.

1603 Molyneux, D.H. \& Zagaria, N. (2002), p. 24.

1604 Diggle, P.J.; Thomson, M.C.; Christensen, O.F. et al. (2007), pp. 503.

1605 Boussinesq, M. (2006), p. 718; Thomson, M.C.; Obsomer, V.; Kamgno, J. et al. (2004), p. 2.

1606 Molyneux, D.H. (1998), p. 929.

1607 MolyneuX, D.H. (1998), p. 929. 
Geographic Determinants of Malaria Transmission

\begin{tabular}{|l|l|l|l|}
\hline Disease & Causative pathogen & $\begin{array}{l}\text { Important } \\
\text { Vectors }\end{array}$ & References \\
\hline Dengue Fever & $\begin{array}{l}\text { Flaviviridae } \\
\text { (DENV1 to DENV4) }\end{array}$ & Aedes spp. & 1608,1609 \\
\hline Yellow Fever & Flaviviridae & Aedes spp. & $1610,1611,1612$ \\
\hline Chikungunya Fever & $\begin{array}{l}\text { Togaviridae } \\
\text { (CHIKV) }\end{array}$ & Aedes spp. & 1613,1614 \\
\hline $\begin{array}{l}\text { O'nyong-nyong } \\
\text { Fever }\end{array}$ & Togaviridae & $\begin{array}{l}\text { Anopheles spp. } \\
\text { Mansonia spp. }\end{array}$ & $1615,1616,1617$ \\
\hline West Nile Fever & Flaviviridae & Culex spp. & 1618,1619 \\
\hline Rift Valley Fever & Bunyaviridae & $\begin{array}{l}\text { Culex spp., } \\
\text { Aedes spp., } \\
\text { Culicoides spp., } \\
\text { Mansonia spp., } \\
\text { Amblyomma spp. }\end{array}$ & 1620,1621 \\
\hline
\end{tabular}

Table 95: Viral diseases transmitted by mosquitoes and other flying insects

Some vector-borne diseases have transmission cycles that differ considerably from that of malaria, particularly because their vectors are unable to fly. This group of diseases is quite diverse since its vectors range from freshwater snails to ticks, fleas and lice (see table 96). Therefore, transmission is restricted to certain localities, sometimes requiring physical contact with water (schistosomiasis) or the ingestion of contaminated water (dracunculiasis).

1608 Spiegel, J.M.; Bonet, M.; Ibarra, A.-M. et al. (2007), p. 503.

1609 Wichmann, O.; Jelinek, T. (2004), p. 161.

1610 Otranto, D.; Stevens, J.R.; Cantacessi, C. \& Gasser, R.B. (2007), p. 117.

1611 Monath, T.P. (2007), p. 2222.

1612 Barrett, A.D.T.; Higgs, S. (2007), p. 211.

1613 Powers, A. \& Logue, C. (2007), p. 2363.

1614 Peyrefitte, C. N.; Rousset, D.; Pastorino, B. et al. (2007), p. 768.

1615 Lanciotti, R.S.; Ludwig, M.L. \& Rwaguma, E.B. (1998), p. 258.

1616 Lutwama, J.J.; AYondo, J.; Savage, H. M. et al. (1999), p. 159.

1617 TESH, R. B. (1982), p. 33.

1618 Otranto, D.; Stevens, J.R.; Cantacessi, C. \& Gasser, R.B. (2007), p. 117.

1619 PAULI, G. (2004), p. 655.

1620 Clements, A. ; Pfeiffer, D.U.; Martin, V. et al. (2007), p. 203.

1621 WHO (2008), p. 17. 


\begin{tabular}{|l|l|l|l|}
\hline Disease & Causative pathogen & $\begin{array}{l}\text { Important } \\
\text { Vectors }\end{array}$ & References \\
\hline Schistosomiasis & $\begin{array}{l}\text { Schistosoma } \\
\text { haematobium, } \\
\text { Schistosoma mansoni }\end{array}$ & $\begin{array}{l}\text { Bulinus spp., } \\
\text { Biomphalaria spp. }\end{array}$ & 1622,1623 \\
\hline $\begin{array}{l}\text { Dracunculiasis } \\
\text { (Guinea worm } \\
\text { infection) }\end{array}$ & $\begin{array}{l}\text { Dracunculus } \\
\text { mediensis }\end{array}$ & $\begin{array}{l}\text { Thermocyclos spp., } \\
\text { Mesocyclops spp. }\end{array}$ & 1624 \\
\hline $\begin{array}{l}\text { Tick-borne } \\
\text { relapsing fever } \\
\text { (TBRF) }\end{array}$ & Borrellia crocidurae & $\begin{array}{l}\text { Ornithodoros } \\
\text { sonrai }\end{array}$ & 1625 \\
\hline $\begin{array}{l}\text { Tick-, louse and } \\
\text { flea-borne spotted } \\
\text { fever }\end{array}$ & Rickettsia spp. & Rhipicephalus spp. & 1626 \\
\hline
\end{tabular}

Table 96: Diseases transmitted by vectors not belonging to the order Diptera

Despite considerable differences between the vectors and pathogens involved, the transmission of all vector-borne diseases shares one similarity: it is determined by the ecological requirements of the organisms involved, and by human activities/behavior. For example, suitable temperature ranges and the presence of water or certain degrees of moisture are the prerequisites of all vector-borne diseases. In fact, even very different organisms such as the freshwater snails transmitting schistosomiasis and anopheline larvae may share the same habitats. Therefore, integrated approaches to transmission modeling or vector control have the promise of being particularly effective.

\subsubsection{Other Forms of Project Integration}

Many of the data required for investigations into the geographic pattern of malaria transmission are not only useful for projects dealing with other vectorborne diseases which may co-occur in the same locality but are fundamental for several other disciplines as well.

In regions where food security is a concern and malnutrition a public health problem, agro-meteorological data are frequently collected in order to better time agricultural activities. In dryland areas, the onset, duration and intensity of rains is equally important for the growth of crops and the reproduction of disease vectors. The FAO's famine early warning system (FEWS) is -despite its

1622 Conlon, P.C. (2005), p. 64; Hotez, P. J. \& FerRis, M. T. (2006) p. 5787.

1623 Brown, D. S. (1981) p. 910; Poda, J. N. ; Traoré, A. ; Sondo, B. K. (2004) p. 49.

1624 MolyneuX, D.H. (1998), p. 929.

1625 Vial, L.; DiaTtA, G.; TALL, A. et al. (2006), p. 37.

1626 Rutherford, J.S.; Macaluso, K.R.; Sмith, N. et al. (2004), pp. $910 f$. 
limitations- one example how agro-meteorological data collected are already used for malaria risk prediction. However, one common problem for both agrometeorological and vector-borne disease research is the poor availability of climate data in affected regions. Improvements in the meteorological station network would thus yield multiple benefits. At the microlevel, such information would be of interest both to local farmers choosing crops and local health centers who could use such information not only for their own preparation but also for timing information campaigns.

Since the positive effects of hydrological modifications, such as improvements in food security, generation of hydroelectric power and flood mitigation, are often undermined by adverse health effects including rises in the incidence of vector-borne diseases like malaria, a cooperation of the water and health sectors for the realization of such projects seems advisable. ${ }^{1627}$

Finally, the results of research into vector-borne transmission should also have practical results. Besides providing baseline data for the effective implementation of intervention programs, the importance local population's awareness cannot be under-emphasized. Indeed, the need for capacity building in African countries is widely recognized, particularly in the fields of global change impacts, vulnerability and adaptation. ${ }^{1628}$ Since major public health issues such as malaria are of direct relevance for virtually all inhabitants of disease-endemic regions, such capacity building should not only be directed at the training of local researchers but also the educational sector.

1627 Lautze, J.; McCartney, M.; Kirshen, P. et al. (2007), pp. 985-987.

1628 IPCC $\left(2007^{2}\right)$, p. 435. 
The following glossary contains a list of all acronyms and abbreviations used in the text. All of them are also explained at their first occurrence. Moreover, a second section defines important terms related to malaria and geographic techniques relevant for its mapping and risk prediction. All of these terms can also be found in the alphabetical index.

\section{Abbreviations Used}

ACT Artemisinin-based combination therapy

AEJ African Easterly Jet

ARMA Atlas du Risque de la Malaria en Afrique

ARTEMIS African Real Time Environmental Monitoring using Imaging Satellites

ARVI Atmospherically resistant vegetation index

ASTER Advanced Spaceborne Thermal Emission and Reflection Radiometer

AVHRR Advanced Very High Resolution Radiometer

AWD African wave disturbance

CCD Cold Cloud Duration

CFP Centre pour la formation professionelle

CGIAR Consultative Group on International Agricultural Research

CHR Centre hospitalier régional

CILSS Comité permanent Inter-États de Lutte contre la Sécheresse dans le Sahel

CMA Centre médical avec antenne chirurgicale

CONAGESE Conseil National pour la Gestion de I'Environnement

CRSN Centre de Recherche en Santé de Nouna

CSPS Centre de Santé et de Promotion Sociale

DALY Disability-adjusted life years

DDT Dichloro-diphenyl-trichloroethane

DEM Digital elevation model

DMSP Defense Meteorological Satellite Program

DSS Demographic Surveillance System

EIR Entomological inoculation rate 


\begin{tabular}{|c|c|}
\hline ENM & Ecologic niche model(ing) \\
\hline EOS & Earth Observing System (of NASA) \\
\hline ERS & European Remote Sensing Satellite \\
\hline ESA & European Space Agency \\
\hline ETM & Enhanced thematic mapper (aboard Landsat) \\
\hline EVI & Enhanced Vegetation Index \\
\hline FAO & Food and Agriculture Organization (of the United Nations) \\
\hline FCFA & Franc de la Communauté Financière Africaine $(1 €=656$ FCFA $)$ \\
\hline FEWS & Famine early warning system (of the FAO) \\
\hline FIR & Far infrared $(15 \mu \mathrm{m}-30 \mu \mathrm{m})$ \\
\hline GAC & Global Area Coverage \\
\hline GARP & Genetic Algorithm for Rule-set Predicition \\
\hline GCM & Global circulation model \\
\hline GDP & Gross domestic product \\
\hline GIS & Geographic Information System \\
\hline GLC2000 & Global land cover 2000 \\
\hline GMDPP & Global Monitoring and Disease Prediction Program \\
\hline GPCP & Global Precipitation Climatology Project \\
\hline HBI & Human Blood Index \\
\hline HLA & Human leucocyte antigen \\
\hline HLC & Human landing catch \\
\hline IGBP & International Geosphere Biosphere Project \\
\hline InSAR & Interferometric synthetic aperture radar \\
\hline INSD & Institut National de la Statistique et de la Démographie \\
\hline IPCC & Intergovernmental Panel on Climate Change \\
\hline IRS & Indoor residual spraying \\
\hline ITCZ & Intertropical convergence zone \\
\hline ITF & Intertropical front \\
\hline ITN & Insecticide-treated net \\
\hline JERS & Japanese Earth Resources Satellite \\
\hline LAC & Local area coverage \\
\hline LAI & Leaf Area Index \\
\hline LCCS & Land cover classification system \\
\hline LLIN & Long-lasting insecticidal nets \\
\hline
\end{tabular}




\begin{tabular}{|c|c|}
\hline LSE & Land surface emissivity \\
\hline LST & Land surface temperature \\
\hline LTC & Light trap capture \\
\hline LWIR & Long wavelength infrared ( $7 \mu \mathrm{m}-15 \mu \mathrm{m})$ \\
\hline MARA & Mapping Malaria Risk in Africa \\
\hline MDGs & Millennium Development Goals \\
\hline MEWS & Malaria Early Warning System \\
\hline MODIS & Moderate Resolution Imaging Spectroradiometer \\
\hline MSAVI & Modified soil-adjusted vegetation index \\
\hline MVC & Maximum value composite \\
\hline MWIR & Medium wavelength infrared ( $3 \mu \mathrm{m}-7 \mu \mathrm{m})$ \\
\hline NASA & $\begin{array}{l}\text { National Aeronautics and Space Administration (of the United } \\
\text { States) }\end{array}$ \\
\hline NDPI & Normalized difference pond index \\
\hline NDTI & Normalized difference turbidity index \\
\hline NDVI & Normalized difference vegetation index \\
\hline NHD & Nouna Health District \\
\hline NIR & Near infrared $(700 \mathrm{~nm}-1.1 \mu \mathrm{m})$ \\
\hline NOAA & $\begin{array}{l}\text { National Oceanic and Atmospheric Administration (of the United } \\
\text { States) }\end{array}$ \\
\hline NTD & Neglected tropical disease \\
\hline ORSTOM & Office de la Recherche Scientifique et Technique d'Outre-Mer \\
\hline PERSIANN & $\begin{array}{l}\text { Precipitation Estimation from Remotely Sensed Information using } \\
\text { Artificial Neural Networks }\end{array}$ \\
\hline PSC & Pyrethrum spray capture \\
\hline QC & Quality control \\
\hline RADAR & Radio detection and ranging \\
\hline RBM & Roll Back Malaria \\
\hline RFE & Rainfall estimate \\
\hline RS & Remote Sensing \\
\hline SAR & Synthetic aperture radar \\
\hline SARVI & Soil and atmospherically resistant vegetation index \\
\hline SAVI & Soil-adjusted vegetation index \\
\hline SIMA & System-wide Initiative on Malaria and Agriculture (of the CGIAR) \\
\hline SIT & Sterile insect technique \\
\hline
\end{tabular}




\begin{tabular}{ll} 
S.I. & sensu lato \\
SLC & Scan-line corrector \\
\hline SRI & Simple Ratio Index \\
\hline SPOT & Satellite Pour l'Observation de la Terre \\
\hline SRTM & Shuttle Radar Topography Mission \\
\hline S.S. & sensu stricto \\
\hline SSA & Sub-Saharan Afrika \\
\hline SST & Sea surface temperature \\
\hline SVI & Spectral vegetation index \\
\hline SWIR & Short wavelength infrared (1.1 $\mu$ m - 3 $\mu$ m) \\
\hline SWS & Soil water storage index \\
\hline TDR & Tropical Disease Research (program of the WHO) \\
TEJ & Tropical Easterly Jet \\
\hline TFR & Total fertility rate \\
\hline TIR & Thermal infrared \\
\hline TRMM & Tropical Rainfall Monitoring Mission \\
\hline TSI & Transmission seasonality index \\
\hline TVX & Temperature-vegetation index \\
U5 & Under five year old children \\
\hline UNEP & United Nations Environment Program \\
UNICEF & United Nations International Children's Emergency Fund \\
\hline USGS & United States Geological Survey \\
VBD & Vector-borne disease \\
\hline VI & Vegetation index \\
WHO & World Health Organization \\
ZPOM & Zone potentially occupied by mosquitoes \\
\hline
\end{tabular}




\section{Important Terms}

Abundance: a measure for a species' incidence in a particular ecosystem

Active case detection: a type of (malaria) survey in which a study population is routinely (regularly) tested for symptoms of disease

Active remote sensing: use of sensors which emit energy in order to scan objects

Advection: the motion of an air mass into a region where the air has different properties (e.g. temperature, moisture)

Aestivation: the fact that some mosquitoes become inactive during the dry season and take shelter in cool, damp places

Anopheles gambiae: the most important vector of human malaria on the African continent (the term may refer to the Anopheles gambiae complex, i.e. a group of morphologically similar malaria vectors)

Anthropophily: a vector species' preference for bloodfeeding on human hosts

Asymptomatic period: the time between the injection of sporozoites and the first clinical signs of malaria infection

Band: a part of the electromagnetic spectrum (treated as one entity by satellite sensors)

Bas fond: large, flat depression in West Africa, also referred to as "bassin versant"; typical location for swampland

Basic reproduction rate: the number of secondary infections divided by the number of primary infections (which gave rise to the secondary infections)

Channel: Processed band signals of a satellite sensor

Child mortality rate: the death rate for children below the age of five years

Competent host: a host that can be infected by a certain parasite and contributes to its transmission

Centre de Santé et de Promotion Sociale (CSPS): the basic health care facility in Burkina Faso's public health system; typically staffed by trained nurses and covering a population of around 5000 to 8000 people

Convection: a thermically induced rising of air masses

Culicidae: a family of flying insects that is characterized by two wings, the habit of bloodfeeding ("mosquitoes")

Digital elevation model (DEM): a digital representation of the surface topography 
Diptera: two-winged flies, a taxonomic order in the class of insects

Disability-adjusted life year (DALY): a measure for the burden of disease, combining years of life lost due to premature mortality and years of life lost due to time lived in states of less than full health

Ecologic niche: the set of environmental conditions under which a species can maintain its population without immigration of individuals from other areas

El Niño: a sea surface warming in the tropical Eastern Pacific Ocean

Emissivity: the radiant flux of a certain object divided by the radiant flux of a black body of the same kinetic temperature

Endemic malaria: the relatively constant incidence of malaria over long periods of time

Endophagy: a vector species' habit of bloodfeeding indoors

Endophily: a vector species' habit of passing much of the gonotrophic cycle indoors

Entomological inoculation rate (EIR): the number of infective mosquito bites per human per unit time

Ephemeral stream: a channel that flows only for hours or days following rainfall

Epidemic malaria: periodic or occasional sharp increase in malaria incidence

Epidemiology: the study of factors affecting the health and illness of populations, including their biologic and geographic determinants

Erythrocyte: a red blood cell, the principal carrier of oxygen in the human body

Erythrocytic cycle: the development of the malaria parasite that takes place within red blood cells

Exflagellation: the release of microgametes (motile male forms of the malaria parasite) from microgametocytes (intraerythrocytic male form of the malaria parasite)

Exo-erythrocytic cycle: the development of the malaria parasite that directly follows the infection of a human host and which precedes the erythrocytic cycle

Exophagy: a vector species' habit of bloodfeeding outdoors

Exophily: a vector species' habit of passing much of the gonotrophic cycle outdoors

Extrinsic incubation period: the duration of the sporogonic cycle, i.e. the development of the malaria parasite within the vector

Fatality rate: the proportion of disease cases resulting in death

Fecundity: an organism's ability to reproduce (as opposed to fertility) 
Fertility: a measure for the actual reproduction of an organism

Field capacity: the amount of water a soil can hold (excluding excess water that cannot be stored)

Gametocyte: the gender-specific form of the malaria parasite (intraerythrocytic stage)

Genetic Algorithm for Rule-set Predicition (GARP): an algorithm relating a species' occurrence to geoecological characteristics of its habitat

Global Monitoring and Disease Predicition Program (GMDPP): a NASA program using remote sensing data for public health purposes

Gonotrophic cycle: the period from one oviposition to the next (typically 2 to 4 days in tropical anophelines)

Gonotrophic dissociation: hibernation of anophelines during cool or dry periods

Hadley circulation: the mean meridional circulation of the lower latitudes (= trade wind circulation)

Harmattan: a dry wind coming from the Sahara which is most pronounced during the winter when it extends up to the Gulf of Guinea

Heliophily: the habit of being attracted to sunlight; an important trait of many malaria vectors, including Anopheles gambiae

Hematophagy: the habit of feeding on blood

Hibernation: a period of physiological inactivity (a survival strategy of anophelines during unsuitable environmental conditions)

Holoendemic malaria: the perennial transmission of malaria which results in a considerable degree of immunity in the local population

Human blood index (HBI): the proportion of blood meals taken from human hosts

Hydromorphic soils: soils that form under wetland conditions

Hyperendemic malaria: the intense but seasonal transmission of malaria which does not allow the development of immunity in the local population

Hypoendemic malaria: low-level but almost continuous occurence of malaria transmission

Incidence: cases of disease commencing during a certain period of time

Infant mortality rate: the death rate for children below the age of one year

Intermittent stream: a stream that ceases flowing for weeks or months each year

Intertropical Convergence Zone (ITCZ): the region where the North-East and South-East trade winds converge

Intertropical Front (ITF): here: the confluence line between moist 
southwesterly monsoonal air and the dry air masses of the northeasterly harmattan

Isohyet: a contour line connecting points of equal precipitation

Kinetic temperature (=thermodynamic temperature): an object's temperature based on its kinetic energy content

Kossi Province: a province in western Burkina Faso (around $13^{\circ} \mathrm{N}$ and $4^{\circ} \mathrm{W}$; 272.000 inhabitants) where malaria is holoendemic

K-strategist: a species that is adapted to stable habitats and thus occurs at relatively constant population densities

La Niña: a sea surface cooling in the tropical Eastern Pacific Ocean

Landscape epidemiology: an emerging discipline investigating the complex links between landscape ecology and disease transmission systems

Land surface temperature (LST): here: radiometric measurement of the earth's surface temperature (up to a depth of around $12 \mu \mathrm{m}$ ) using thermal infrared imagery

Lateritic crust: a surface forming on rocks or soils in tropical areas which is rich in iron and aluminum

Ligne de grains: an intensive belt of thunderstorms crossing Western Africa during the summer months

Lithosol: a soil that lacks horizon development due to either steep slopes or unsuitable parent materials

Macrogamete: the female form of the malaria parasite

Malaria control: a program that aims at keeping malaria transmission below a certain level

Malaria eradication: a program that aims at the complete cessation of malaria transmission

Malariology: the scientific study of malaria that includes fields as diverse as parasitology, entomology, medical sciences and medical geography

Mare: a denomination for lakes in francophone West Africa, which are usually not perennial

Medical Geography: the study of spatio-temporal pattern of disease occurrence, including investigations into the geographic determinants of disease

Merozoite: the form of malaria parasite which is the result of asexual reproduction (schizogony) and which infects and destroys red blood cells

Mesoendemic malaria: the occurrence of malaria transmission at varying intensity

Microgamete: the male form of the malaria parasite

Millennium Development Goals (MDGs): a set of eight international development goals formulated by the United Nations and due to be 
achieved by 2015

Morbidity rate: the proportion of a population living a state of disease or disability (within a certain period of time)

Mortality rate: the proportion of a population dying (within a certain period of time)

Neglected Tropical Diseases (NTDs): A group of infectious diseases that are important causes of morbidity and mortality in developing countries but not in the industrialized world

Non-competent host: an organism that cannot be infected with a parasite

Oocyst: a form of the malaria parasite that is encapsulated into a mosquito's stomach wall and which produces sporozoites

Ookinete: a form of the malaria parasite that is the result of a fusion of macro- and microgametes and which marks the beginning of the sporogonic cycle

Pan(chromatic) sharpening: a synthetic increase in the resolution of multispectral imagery using higher resolution panchromatic imagery

Parasitoid: an organism that spends much of its life attached to or within a single host organism which it ultimately kills

Passive case detection: a type of (malaria) survey relying on case detections in patients consulting health services

Passive remote sensing: use of sensors that detect natural radiation that is emitted or reflected by the objects under investigation

Perennial river: a river that has continuous flow throughout the year

Phagocyte: a type of white blood cell (an essential component of the human immune system)

Plasmodium falciparum: a human malaria parasite which is confined to tropical and subtropical areas and causes severe to fatal infections

Plasmodium knowlesi: a newly discovered human malaria parasite

Plasmodium malariae: a human malaria parasite that occurs mainly in East and West Africa and that may cause outbreaks years after the initial infection

Plasmodium ovale: the least common malaria parasite affecting humans; restricted to tropical West Africa

Plasmodium vivax: the most widespread human malaria parasite, occuring between the temperate zones and the tropics

Pre-patent period: the interval between the date of infection and the presence of malaria parasites in the peripheral blood

Prevalence: cases of disease existing at a given point of time

Radar: a system that uses electromagnetic waves (radio or microwaves) to identify objects; in remote sensing used for various purposes including 
measurements of elevation and meteorology

Radiant temperature: a temperature measurement based on the radiometric detection of the electromagnetic radiation exiting an object

Remote sensing (RS): techniques to infer information about atmospheric and/or surface characteristics from a distance

Resolution: characteristics related to the detection quality of satellite sensors, including spatial, temporal, spectral and radiometric resolution

R-strategist: a species that reproduces rapidly and readily exploits new habitats

Sahel: the transition zone between the Sahara and the dry savannas of subSaharan Africa, characterized by about $200 \mathrm{~mm}$ to $600 \mathrm{~mm}$ of rainfall

Sahelo-Sudanian zone: the dry savanna zone of sub-Saharan Africa that is characterized by about 600 to $900 \mathrm{~mm}$ of rainfall

Schizogony: the asexual reproduction of the malaria parasite in the human liver or blood

Schizont: a form of the malaria parasite that occurs either in the liver or in the bloodstream and which produces merozoites

Sensor: the device on a remote sensing satellite that measures electromagnetic radiation; also referred to as radiometer

Sensu lato (s.l.): in the wider sense; here mainly used in the context of Anopheles gambiae s.l., i.e. the entire Anopheles gambiae complex

Sensu stricto (s.s.): in the stricter sense; here used mainly in the context of Anopheles gambiae s.s. as opposed to other members of the Anopheles gambiae complex

Sickle-cell anemia: a disorder involving a mutation of a hemoglobin gene that is relatively frequent in Africa and protects from malaria

Spectral signature: the combination of reflected and absorbed electromagnetic (EM) radiation at varying wavelengths which can be used to identify an object by remote sensing

Split-window algorithm: a method used to retrieve remotely sensed surface temperatures based on the differential water vapor absorption in two adjacent infrared channels

Sporogonic cycle: the development of the malaria parasite that takes place in the mosquito

Sporozoite: the form of the malaria parasite that marks the end of the sporogonic cycle and which is infective to human hosts

Sporozoite rate: the percentage of female Anopheles mosquitoes which carry sporozoites in their salivary glands

Sudanian zone: the moist savanna region in sub-Saharan Africa 
Thermal infrared: a part of the electromagnetic spectrum ( $3 \mu \mathrm{m}$ to $15 \mu \mathrm{m}$ ) that satellite sensors use to detect radiometric surface temperatures

Trophozoite: malaria parasites which have invaded red blood cells in their host and ingest hemoglobin

Vector-borne disease (VBD): a disease that is transmitted from one host to another by some living organism, usually an insect

Vector competence: a measure for the capability of a vector species to transmit a parasite

Vectorial capacity: the total number of (potentially) infective bites on man by a local vector population

Vegetation index: a dimensionless quantifier of the presence of green biomass

Verbal autopsy: a technique used particularly in developing countries that tries to infer the circumstances leading to death by postmortem interviews with relatives

Vertisol: a soil rich in expansive clay minerals that forms deep cracks during drought; self-mulching occurs as the result of alternate shrinking and swelling

Walker circulation: the mean zonal circulation along the meteorological equator

Zoophily: a vector species' preference for bloodfeeding on animal hosts

Zygote: the first stage of a new organism that consists of a single cell; here: a fertilized macrogamete 


\section{Bibliography}

\section{Literature}

Afrane, Y.A.; Klinkenberg, E.; Drechsel, P. et al. (2004): Does irrigated urban agriculture influence the transmission of malaria in the city of Kumasi, Ghana. Acta Tropica, Vol. 89, No. 2 (January 2004), pp. 125-134.

Alano, P. (2007): Plasmodium falciparum gametocytes: still many secrets of a hidden life. Molecular Microbiology, Vol. 66, No. 2, pp. 291-302.

Alvar, J.; Yactayo, S. \& Bern, C. (2006): Leishmaniasis and poverty. Trends in Parasitology, Vol. 22, No. 12, pp. 552-557.

Амісі, R.R. (2001): The history of Italian parasitology. Veterinary Parasitology, Vol. 98, No. 1, pp. 3-30.

ANDERSON, R.P.; LEW, D. \& PETERSON, A.T. (2003): Evaluating predictive models of species' distributions: criteria for selecting optimal models. Ecological Modelling, Vol. 162, No. 3, pp. 211-232.

AnHuF, D. \& FRANKENBERG, P. (1991): Die naturnahen Vegetationszonen Westafrikas. Die Erde, Vol. 122, p. 243-265.

Anyamba, A.; Chretien, J-P.; Small, J. et al. (2006): Developing global climate anomalies suggest potential disease risks for 2006 - 2007 . International Journal of Health Geographics, Vol. 5, No. 60, doi: 10.1186./1476-072X-5-60.

AnyAmba, A. \& TUCKer, C.J. (2005): Analysis of Sahelian vegetation dynamics using NOAA-AVHRR NDVI data from 1981-2003. Journal of Arid Environments, Vol. 63, No. 33, pp. 596-614.

A'Rahman, S.H.; Mohamedani, A.A.; Mirgani, E.M. \& Ibrahim, A.M. (1996): Gender Aspects and Women's Participation in the Control and Management of Malaria in Central Sudan. Social Science \& Medicine, Vol. 42, No. 10, pp. 1433-1446.

Barbier, B.; Dembelé, Y. \& Compaoré, L. (2006): L'eau au Burkina Faso: usages actuels et perspectives. Sud Sciences \& Technologies, No. 14, pp. 20-29.

Barrett, A.D.T. \& Higgs, S. (2007): Yellow Fever: A Disease that Has Yet to be Conquered. In: Annual Review of Entomology, Vol. 52, No. 1, pp. 209-229. 
BARRETT, E.C. (1993): Precipitation measurement by satellites: Towards community algorithms. Advances in Space Research, Vol. 13, No. 5, pp. 119-136.

Barrios, S.; Bertinelli, L. \& Strobl, E. (2006): Climatic change and ruralurban migration: The case of sub-Saharan Africa. Journal of Urban Economics, Vol. 60, No. 3, pp. 357-371.

BAYOH, M.N. \& LINDSAY, S.W. (2003): Effect of temperature on the development of the aquatic stages of Anopheles gambiae sensu strictu (Diptera: Culicidae). Bulletin of Entomological Research, Vol. 93, No. 5, pp. 375-381.

BAYOH, M.N. \& LINDSAY, S.W. (2004): Temperature-related duration of aquatic stages of the Afrotropical malaria vector mosquito Anopheles gambiae in the laboratory. In: Medical and Veterinary Entomology, Vol. 18, No. 2, pp. 174-179.

BAYOH, M.N.; THOMAS, C.J. \& LindSAY, S.W. (2001): Mapping the distributions of chromosomal forms of Anopheles gambiae in West Africa using climate data. Medical and Veterinary Entomology, Vol. 15, No. 3, pp. 267-274.

Becher, H.; Kynast-Wolf, G.; Sié, A. et al. (2008): Patterns of malaria: causespecific and all-cause mortality in a malaria-endemic area of west Africa. American Journal of Tropical Medicine \& Hygiene, Vol. 78, No. 1, pp. 106-113.

BeIER, J.C. (1998): Malaria parasite development in mosquitoes. Annual Review of Entomology, Vol. 43, pp. 519-543.

Beier, J.C.; Killeen, G.F. \& Githure, J.I. (1999): Short report: entomologic inoculation rates and Plasmodium falciparum malaria prevalence in Africa. American Journal of Tropical Medicine and Hygiene Vol. 61, No. 1, pp. 109-113.

Beiersmann, C.; Sanou, A.; Wladarsch, E. et al. (2007): Malaria in rural Burkina Faso: local illness concepts, patterns of traditional treatment and influence on health-seeking behaviour. Malaria Journal, Vol. 6, No. 106, doi:10.1186/1475-2875-6-106.

Belete, H.; Tikubet, G.; Petros, B. et al. (2004): Control of human African trypanosomiasis: trap and odour preferences of tsetse flies (Glossina morsitans submorsitans) in the upper Didessa river valley of Ethiopia. Tropical Medicine and International Health, Vol. 9, No. 6, pp. 710-714.

Berié, E. \& Kobert, H. (2005): Der Fischer Weltalmanach 2006. Frankfurt/Main: Fischer Taschenbuch Verlag. 
Bethemont, J.; Faggi, P.; Zounagrana, T.P. (2003): La Vallée du Sourou (Burkina Faso): Genèse d'un territoire hydraulique dans I'Afrique soudanosahélienne. Paris: Harmattan.

Beyrer, C.; Villar, J.C.; Sumanvanichiij, V. et al. (2007): Neglected diseases, civil conflicts, and the right to health. The Lancet, Vol. 370, No. 9587, pp. 619-626.

Bharati, L.; Rodgers, C.; ERdenberger, T. et al. (2008): Integration of economic and hydrologic models: Exploring conjunctive irrigation water use strategies in the Volta Basin. Agricultural Water Management, Vol. 95, No. 8, pp. 925-936.

Bonnet, S.; Gouagna, L.C.; PaUL, R.E. et al. (2003): Estimation of malaria transmission from humans to mosquitoes in two neighbouring villages in south Cameroon: evaluation and comparison of several indices. Transactions of the Royal Society of Tropical Medicine and Hygiene, Vol. 97, No. 1, pp. 53-59.

Boudet, G. \& Lebrun, J.P. (1986): Catalogue des Plantes Vasculaires du Mali. Maisons Alfort: Etudes et Synthèses de l'Institut d'Elevage et de Médecine Véterinaire des Pays Tropicaux.

Bradley, C.A. \& Altizer, S. (2005): Parasites hinder monarch butterfly flight: implications for disease spread in migratory hosts, Ecology Letters, Vol. 8, No. 3, pp. 290-300.

BREMAN, J.G. (2001): The ears of the hippopotamus: manifestations, determinants, and estimates of the malaria burden. American Journal of Tropical Medicine and Hygiene Vol. 64, No. 1-2 (supplement), pp. 1-11.

Breman, J.G. (2009): Eradicating malaria. Science Progress, Vol. 92, No. 1, pp. 1-38.

Breman, J.G., Alilio, M.S. \& White, N. (2007): Defining and Defeating the Intolerable Burden of Malaria: Progess and Perspectives. American Journal of Tropical Medicine and Hygiene, Vol. 71, No. 6 (Supplement), p. vi-xi.

Brengues, J. \& Coz, J. (1973): Quelques aspects fondamentaux de la biologie d'Anopheles Gambiae Giles (Sp. A.) et d'Anopheles Funestus Giles en zone de savane humide d'Afrique de l'Ouest. Cahiers ORSTOM, Série Entomologie Medicale et Parasitologie, Vol. 11, No. 2, pp. 107-126.

BRIËT, O.J.T.; Dossou-Yovo, J.; AKodo, E. et al. (2003): The relationship between Anopheles gambiae density and rice cultivation in the savannah zone and forest zone of Côte d'Ivoire. Tropical Medicine and International Health, Vol. 8, No. 5, pp. 439-448. 
BROOKER, S.; HAY, S.I. \& BUNDY, D.A.P. (2002): Tools from ecology: useful for evaluating infection risk models? Trends in Parasitology, Vol. 18, No. 2, pp. 70-74.

BRown, D.S. (1981): Generic nomenclature of freshwater snails commonly classified in the genus Bulinus (Mollusca: Basommatophora). Journal of Natural History, Vol. 15, No. 6, pp. 909-915.

Budde, M.E.; Tappan, G.; Rowland, J. et al. (2004): Assessing land cover performance in Senegal, West Africa using $1 \mathrm{~km}$ integrated NDVI and local variance analysis. Journal of Arid Environments, Vol. 59, No. 3, pp. 481-498.

Bureau Central du Recensement (2007): Resultats Préliminaires du Recensement Général de la Population et de l'Habitation de 2006. Ouagadougou.

Carnevale, P. \& Mouchet, J. (1987): Prospects for Malaria Control. International Journal of Parasitology, Vol. 17, No. 1, pp. 181-187.

CARTER, R. \& MEndis, K. (2006): Measuring malaria. American Journal of Tropical Medicine and Hygiene, Vol. 74, No. 2, pp. $187 \mathrm{f}$.

CAULFIELD, L.E.; RichARD, S. \& BLACK, R. (2004): Undernutrition of an Underlying Cause of Malaria Morbidity and Mortality in Children Less than Five Years Old. American Journal of Tropical Medicine and Hygiene, Vol. 71, No. 2 (supplement), pp. 55-63.

Charlwood, J.D.; Vij, R. \& Billingsley, P.F. (2000): Dry Season Refugia of Malaria-Transmitting Mosquitoes in a Dry Savanna Zone of East Africa. American Journal of Tropical Medicine and Hygiene, Vol. 62, No. 6, pp. 726732.

Chimbari, M.J.; Chirebvu, E. \& Ndlela, B. (2004): Malaria and Schistosomiasis risks associated with surface and sprinkler irrigation systems in Zimbabwe. Acta Tropica, Vol. 89, No. 2, pp. 205-213.

Chiyaka, C.; Tchuenche, J.M.; Garira, W. \& Dube, S. (2008): A mathematical analysis of the effects of control strategies on the transmission dynamics of malaria. Applied Mathematics and Computation, Vol. 195, No. 2, pp. 641-662.

CHRISTOPHIDES, G.K. (2005): Transgenic mosquitoes and malaria transmission. Cellular Microbiology, Vol. 7, No. 3, pp. 325-333.

Clarke, S.E.; Bøgh, C.; Brown, R. et al. (2001): Do untreated bednets protect against malaria? Transactions of the Royal Society of Tropical Medicine and Hygiene, Vol. 95, No. 5, pp. 457-462. 
Clements, A.C.A.; Pfeiffer, D.U., Martin, V. et al. (2007): Spatial Risk Assessment of Rift Valley Fever in Senegal. Vector-Borne and Zoonotic Diseases. Vol. 7, No. 2, pp. 203-216.

Coetzee, Maureen (2004): Distribution of the African Malaria Vectors of the Anopheles Gambiae Complex. American Journal of Tropical Medicine and Hygiene, Vol. 70, No. 2, pp. $103 \mathrm{f}$.

Coleman, P.G. \& Alphey, L. (2004): Genetic control of vector populations: an imminent prospect. Tropical Medicine and International Health. Vol. 9, No. 4, pp. 433-437.

Coll, C.; Caselles, V.; Galve, J.M. et al. (2005): Ground measurements for the validation of land surface temperatues derived from AATSR and MODIS data. Remote Sensing of Environment, Vol. 97, No. 3, pp. 288-300.

Coluzzi, M. (1992): Malaria Vector Analysis and Control. Parasitology Today, Vol. 8, No. 4, pp. 113-118.

Conlon, P.C. (2005): Schistosomiasis. Medicine, Vol. 33, No. 8, pp. 64-67.

Coulter, J.B.S. (2002): Global importance of parasitic disease. Current Paediatrics, Vol. 12, No. 3, pp. 523-533.

Craig M.H., Snow R.W. \& Le SUeUR D. (1999) A climate-based distribution model of malaria transmission in sub-Saharan Africa. Parasitology Today, Vol. 15, No. 3, pp.105-111.

Curran, P.J.; Atkinson, P.M.; Foody, G.M. \& Milton, E.J. (2000): Linking Remote Sensing, Land Cover and Disease. In: HAY, S.I. et al. (Hrsg.) (2000): Remote sensing and geographical information systems in epidemiology, S. 37-80. [Tropeninstitut Heidelberg, ZS 3::47]

Curto de Casas, S.I. \& Carcavallo, R.U. (1995): Climate Change and Vectorborne diseases distribution. Social Sciences \& Medicine, Vol. 40, No. 11, pp. 1437-1440.

$D_{A B A}$, S. (1999): Note on effects of soil surface crust on the grain yield of sorghum (Sorghum bicolor) in the Sahel. Field Crops Research, Vol. 61, No. 3, pp. 193-199.

Daly, H.W.; Doyen, J.T. \& PuRCELL, A.H. (1998): Introduction to Insect Biology and Diversity. Oxford \& New York: Oxford University Press.

DASH, PRASANJit (2004): Land Surface Temperature and Emmisivity Retrieval from Satellite Measurements. Karlsruhe \{Dissertation\} 
De Lange, N. (2006): Geoinformatik in Theorie und Praxis. Berlin, Heidelberg: Springer.

Depinay, J.M.O.; Mbogo, C.M.; Killeen, G. et al. (2004): A simulation model of African Anopheles ecology and population dynamics for the analysis of malaria transmission. Malaria Journal, Vol. 3, No. 29, doi:10.1186/14752875-3-29.

De Plaen, R.; Seka, M.-L. \& Koutoua, A. (2004): The paddy, the vector and the caregiver: lessons from an ecosystem approach to irrigation and malaria in Northern Côte d'Ivoire. Acta Tropica, Vol. 89, No. 2, pp. 135146.

Dettner, K. \& Peters, W. (Ed.) (2002): Lehrbuch der Entomologie. München: Elsevier.

Deutsche Stiftung Weltbevölkerung (Ed.) (2009): DSW Datenreport 2009. Soziale und demographische Daten zur Weltbevölkerung. Hannover: DSW.

DEVINEAU, J.L. \& FOURNIER, A. (2007): Integrating environmental and sociological approaches to assess the ecology and diversity of herbaceous species in a Sudan-type savanna (Bondoukuy, western Burkina Faso). Flora - Morphology, Distribution, Functional Ecology of Plants, Vol. 202, No. 5, pp. 350-370.

De Wit, A.J.W,; Boogaard, H.L. \& van Diepen, C.A. (2004): Using NOAA-AVHRR estimates of land surface temperature for regional agrometeorological modelling. International Journal of Applied Earth Observation and Geoinformation, Vol. 5, No. 3, pp. 187-204.

Dhiman, R.C. (2000): Remote Sensing: A Visionary Tool in Malaria Epidemiology. Indian Council of Medical Research Bulletin, Vol. 30, No. 11, p. 123-129.

Diesfeld, H.J. (1995): Klima und Gesundheit im Spiegel der Zeit. In: Fricke, W. \& SchweikarT, J. (Ed.) (1995): Krankheit und Raum, pp. 35-54. Stuttgart: Franz Steiner Verlag.

Dimopoulos, G. (2003): Insect immunity and its implication in mosquitomalaria interactions. Cellular Microbiology, Vol. 5, No. 1, pp. 3-14.

Dinku, T.; Chidzambwa, S.; Ceccato, P. et al. (2008): Validation of highresolution satellite rainfall products over complex terrain. International Journal of Remote Sensing, Vol. 29, No. 14, pp. $4097-4110$.

Direction Générale de l'Information et des Statistiques Sanitaires $\left(2009^{1}\right)$ : Tableau de 
Bord Santé 2008. Ouagadougou.

Direction Générale de l'Information et des Statistiques Sanitaires $\left(2009^{2}\right)$ : Annuaire statistique 2008. Ouagadougou.

Direction Générale de l'Institut National de la Statistique et de la Démographie (2005): Analyse des Déterminants de la Pauvreté dans la Boucle du Mouhoun. Ouagadougou.

Diuk-Wasser, M.A.; Toure, M.B.; Dolo, G. et al. (2005): Vector abundance and malaria transmission in rice-growing villages in Mali. American Journal of Tropical Medicine and Hygiene, Vol. 72 No. 6, pp. 725-731.

Dolo, G.; BRIËT, O.J.T.; DAO, A. et al. (2004): Malaria transmission in relation to rice cultivation in the irrigated Sahel of Mali. Acta Tropica, Vol. 89 No. 2, pp. 99-108.

Donnelly, M.J.; McCall, P.J.; Lengeler, C. et al. (2005): Malaria and urbanization in sub-Saharan Africa. Malaria Journal, Vol. 4, No. 12, doi: $10.1186 / 1475-2875-4-12$.

Doolan, D.L.; Dobaño, C. \& BAIRD, J.K. (2009): Acquired Immunity to Malaria. Clinical Microbiology Reviews, Vol. 22, No. 1, pp. 13-36.

EdILL, F.E.; TRIPÉT, F.; TourÉ, Y.T. et al. (2005): Water quality and immatures of the $M$ and $S$ forms of Anopheles gambiae s.s. and An. arabiensis in a Malian village. Malaria Journal, Vol. 5, No. 35, doi:10.1186/1475-2875-5-35.

EINEDER, F. (2009): Klimawandel und Ernährungssicherung an Fallbeispielen Afrikas. Göttingen. \{Thesis\}

Einterz, E.M. (2003): Perceptions of malaria transmission, presentation and management in Northern Cameroon. In: Transactions of the Royal Society of Tropical Medicine and Hygiene, Vol. 97, No. 1, pp. 51-59.

Epstein, P.R.; Rogers, D. \& Slooff, R. (1993): Satellite imaging and vectorborne diseases. The Lancet, Vol. 341, No.8857, pp. 1404-1406.

EPSTEIn, P.R. (2001): Climate change and emerging infectious diseases. Microbes and Infection, Vol. 3, No. 9, pp. 747-754.

Erasmi, S.; Kappas, M.; Twele, A. \& Ardiansyah, M. (2007): From global to regional scale: Remote Sensing based concepts and methods for mapping land-cover and land-cover change in tropical regions. In: Tscharntke, T.; Leuschner, C.; Zeller, M. et al. (eds.) (2007): Stability of tropical rainforest margins: Linking ecological, economic and social constraints, pp. 437-462. Heidelberg: Springer-Verlag. 
Fahsi, A.; TSegaye, T.; TAdesse, W. \& Coleman, T. (2000): Incorporation of digital elevation models with Landsat-TM data to improve land cover classification accuracy. Forest Ecology and Management, Vol. 128, No. 1-2, pp. 57-64.

FERGUSON, H.M. \& READ, A.F. (2002): Why is the effect of malaria parasites on mosquito survival still unresolved? Trends in Parasitology, Vol. 18, No. 6, pp. 256-261.

Fillinger, U.; Sombroek, H.; Majambere, S. et al. (2009): Identifying the most productive breeding sites for malaria mosquitoes in The Gambia. Malaria Journal, Vol. 8, No. 62, doi:10.1186/1475-2875-8-62.

Ouadba, J.M. (1991): Note sur les caratéristiques de la végétation ligneuse et harbacée d'une jachère protégée en zone soudanienne dégradée. In: Floret, C. \& Serpantié, G. (1991): La Jachère en Afrique de I'Ouest, pp. 331-340. Paris: Éditions de L'ORSTOM.

Foley, D.H. \& ToRres, E.P. (2006): Population structure of an island malaria vector. Medical and Veterinary Entomology, Vol. 20, No. 4, pp. 393-401.

Fox, A.N.; Pitts, R.J.; Robertson, H.M. et al. (2001): Candidate odorant receptors from the malaria vector mosquito Anopheles gambiae and evidence of down-regulation in response to blood feeding. Proceedings of the National Academy of Sciences of the United States of America, Vol. 98, No. 25, pp. 14693-14697.

Garcia, A.; Jamonneau, V.; Sané, B. et al. (2002): Host age and time of exposure in Trypansoma brucei gambiense Human African Trypanosomiasis. Tropical Medicine and International Health, Vol. 7, No. 5, pp. $429-434$.

GARCIA, R. \& HUFFAKER, C.B. (1979): Ecosystem Management for Suppression of Vectors of Human Malaria and Schistosomiasis. Agro-Ecosystems, Vol. 5, pp. 295-315.

GaRCIA, R. (1983): Mosquito Management: Ecological Approaches. Environmental Management, Vol. 7, No. 1, pp. 73-78.

Garros, C.; van Bortel, W.; Trung, H.D. et al. (2006): Review of the Minimus Complex of Anopheles, main malaria vector in Southeast Asia: from taxonomic issues to vector control strategies. Tropical Medicine and International Health, Vol. 11, No. 1, pp 102-114.

Geerken, R. \& ILAiwi, M. (2004): Assessment of rangeland degradation and development of a strategy for rehabilitation. Remote Sensing of Environment, Vol. 90, No. 4, pp. 490-504. 
Geerling, C. (1982): Guide de Terrain des Ligneux Sahéliens et SoudanoGuinéens. Wageningen (The Netherlands): Veenman \& Zonen B.V.

GeERLING, C. (1985): The status of the woody species of the Sudan and Sahel zones of West Africa. Forest Ecology and Management, Vol. 13, No. 34, pp. 247-255.

Gemperli, A.; Sogoba, N.; Fondjo, E. et al. (2006): Mapping malaria transmission in West and Central Africa. Tropical Medicine and International Health, Vol. 11, No. 7, pp. 1032-1046.

Gemperli, A.; Vounatsou, P.; Kleinschmidt, I. et al. (2004): Spatial Patterns of Infant Mortality in Mali: The Effect of Malaria Endemicity. American Journal of Epidemiology, Vol. 159, No. 1, pp. 64-72.

Gilles, H.M. \& WarRelL, D.A. (Ed.) (1993): Bruce-Chwatt's Essential Malariology. London, Boston, Melbourne, Auckland: Edward Arnold.

Gilles, H.M. $\left(1993^{1}\right)$ : The malaria parasites. In: Gilles, H.M. \& Warrell, D.A. (Ed.) (1993): Bruce-Chwatt's Essential Malariology, pp. 12-27. London, Boston, Melbourne, Auckland: Edward Arnold.

Gilles, H.M. (19932): Epidemiology of malaria. In: Gilles, H.M. \& WarReLL, D.A. (Ed.) (1993): Bruce-Chwatt's Essential Malariology, pp. 124-163. London, Boston, Melbourne, Auckland: Edward Arnold.

Glass, G.E. (2000): Update: Spatial Aspects of Epidemiology: The Interface with Medical Geography. Epidemiological Reviews, Vol. 22, No. 1, pp. 136-139.

Govella, N.J.; Chaki, P.P.; Geissbuhler, Y. et al. (2009): A new tent trap for sampling exophagic and endophagic members of the Anopheles gambiae complex. Malaria Journal, Vol. 8, No. 157, doi:10.1186/1475-28758-157.

Green, R. M. \& HAY, S.I. (2002): The potential of Pathfinder AVHRR data for providing surrogate climatic variables across Africa and Europe for epidemiological applications. In: Remote Sensing of Environment, No. 79, No. 2-3, pp. 166-175.

Greenwood, B.M. (1997): Malaria Transmission and Vector Control. Parasitology Today, Vol. 13, No. 2, pp. 90-92.

GReEnwood, B. \& TARGet, G. (2009): Do we still need a malaria vaccine? 
Parasite Immunology, Vol. 31, No. 9, pp. 582-586.

Griffiths, J.F. (1972): Climates of Africa. Amsterdam, London, New York: Elsevier Publishing Company.

Grover-Kopec, E.; Kawano, M.; Klaver, R.W. et al. (2005): An online operational rainfall-monitoring resource for epidemic malaria early warning systems in Africa. Malaria Journal, Vol. 4, No. 6, doi:10.1186/1475-2875-46.

GuerRa, C.A; SnOw, R.W. \& HAY, S.I. (2006): Mapping the global extent of malaria in 2005. Trends in Parasitology, Vol. 22, No. 8, pp. 353-358.

Gu, W.; Killeen, G.F.; Mbogo, C.M. et al. (2003): An individual-based model of Plasmodium falciparum malaria transmission on the coast of Kenya. In: Transactions of the Royal Society of Tropical Medicine and Hygiene, Vol. 97 No. 1, pp. 43-50.

GuYAT, H.L. \& Snow, R. (2002): The cost of not treating bednets. Trends in Parasitology, Vol. 18, No. 1, pp. 12-16.

GyApong, J.O.; Kyelem, D.; Kleinschmidt, I. et al. (2002): The use of spatial analysis in mapping the distribution of bancroftian filariasis in four West African countries. Annals of Tropical Medicine and Parasitology, Vol. 96, No. 7, pp. 695-705.

Hammer, G.P.; Somé, F.; Müller, O. et al. (2006): Pattern of cause-specific childhood mortality in a malaria endemic area of Burkina Faso. Malaria Journal Vol. 5, No.47, doi:10.1186/1475-2875-5-47.

Hammer, T. (2005): Sahel. Gotha \& Stuttgart: Klett Perthes.

HaRrus, S. \& BANETH, G. (2005): Drivers for the emergence and reemergence of vector-borne protozoal and bacterial diseases. International Journal for Parasitology, Vol. 35, No. 11-12, pp. 1309-1318.

$\mathrm{H}_{\mathrm{AY}}$, S.I. (2000): An Overview of Remote Sensing and Geodesy for Epidemiology and Public Health Application. In: HAY, S.I.; RANDOLPH, S.E.; Rogers, D.F. et al. (Ed.) (2000): Remote sensing and geographical information systems in epidemiology, p.. 1-35.

Hay, S.I.; Guerra, C.A.; Gething, P.W. et al. (2009): A World Malaria Map: Plasmodium falciparum Endemicity in 2007. PLOS Medicine, Vol. 6, No. 3, pp. 286-293.

HAY, S.I.; GuerRA, C.A.; TATEM, A.J. et al. (2005): Urbanization, malaria transmission and disease burden in Africa. Nature Reviews Microbiology, Vol. 3, No. 1, pp. 81-90. 
HAY, S.I. \& LenNon, J.J. (1999): Deriving metereological variables across Africa for the study and control of vector-borne disease: a comparison of remote sensing and spatial interpolation of climate. Tropical Medicine and International Health, Vol. 4, No. 1, pp. 58-71.

Hay, S.I.; Omumbo, J.A.; Craig, M.H. \& Snow R.W. (2000): Earth observation, geographic information systems and Plasmodium falciparum malaria in Sub-Saharan Africa. In: HAY, S.I.; RANDOLPH, S.E.; RogerS, D.F. et al. (Ed.) (2000): Remote sensing and geographical information systems in epidemiology, pp. 173-215.

HaY, S.I.; Snow, R.W. \& Rogers, D.J. (1998): From Predicting Mosquito Habitat to Malaria Seasons Using Remotely Sensed Data: Practice, Problems and Perspectives. Parasitology Today, Vol. 14, No. 8, pp. 306-313.

$\mathrm{H}_{\mathrm{AY}}$, S.I. \& TATEM, A.J. (2005): Letters to the Editor: Remote sensing of malaria in urban areas: two scales, two problems. American Journal of Tropical Medicine and Hygiene, Vol. 72 No. 6, pp. 655 f.

Held, I.M.; Delworth, T.L.; Lu, J. et al. (2005): Simulation of Sahel drought in the 20th and 21st centuries. Proceedings of the National Academy of Sciences of the United States of America, Vol. 102, No. 50, pp.17891-17896.

Hemingway, J.; Beaty, B.J.; Rowland, M. et al. (2006): The Innovative Vector Control Consortium: improved control of mosquito-borne diseases. Trends in Parasitology, Vol. 22, No. 7, pp. 308-312.

Herrmann, S.M. \& Hutchinson, C.F. (2005): The changing contexts of the desertification debate. Journal of Arid Environments, Vol. 63, No. 3, pp. 538-555.

Holding, P.A. \& KitsAo-WeKulo, P.K. (2004): Describing The Burden of Malaria on Child Development: What Should We Be Measuring and How Should We Be Measuring It? American Journal of Tropical Medicine and Hygiene, Vol. 71, No. 2 (Supplement), pp. 71-79.

HOSHEN, M.B. \& MORSE, A.P. (2004): A weather-driven model of malaria transmission. Malaria Journal Vol. 3, No. 32, doi:10.1186/1475-2875-3-32.

Hotez, P.J. \& FerRis, M.T. (2006): The antipoverty vaccines. Vaccine, Vol. 24, No. $31 / 32$, pp. 5787-5799.

Hotez, P.J.; Molyneux, D.H.; Fenwick, A. et al. (2006): Incorporating a RapidImpact Package for Neglected Tropical Diseases with Programs for HIV/AIDS, Tuberculosis, and Malaria. PloS Medicine, Vol. 3, No. 5, pp. 576-584. 
Hottin, O.F. \& OuÉDRAOGO, F. (1976): Carte Géologique de la République de Haute Volta. Ouagadougou: Direction de la Géologie et des Mines. \{Map, scale $1: 1.000 .000\}$

Hougard, J.M.; Fontenille, D.; Chandre, F. et al. (2002): Combating malaria vectors in Africa: current directions of research. Trends in Parasitology, Vol. 18 , No. 7, pp. 283-286.

Hountondi, Y.-C.; Sokpon, N. \& OzeR, P. (2006): Analysis of the vegetation trends using low resolution remote sensing data in Burkina Faso (1982-1999) for the monitoring of desertification. International Journal of Remote Sensing, Vol. 27, No. 5, pp. 871-884.

Huang, J.; WalkeR, E.D.; Giroux, P.Y. et al. (2005): Ovipositional site selection by Anopheles gambiae: influence of substrate moisture and texture. In: Medical and Veterinary Entomology, Vol. 19, No. 4, pp. 442-450.

Huang, J.; Walker, E.D.; Otienoburu, P.E. et al. (2006): Laboratory tests of oviposition by the African malaria mosquito, Anopheles gambiae, on dark soil as influenced by presence or absence of vegetation. In: Malaria Journal, Vol. 5, No. 88, doi:10.1186/1475-2875-5-88.

Huang, J.; Walker, E.D.; Vulule, J. \& Miller, J.R. (2006): Daily temperature profiles in and around Western Kenyan larval habitats of Anopheles gambiae as related to egg mortality. Malaria Journal, Vol. 5, No. 87, doi: $10.1186 / 1475-2875-5-87$.

Huete, A.R.; Justice, C. \& van Leeuwen, W. (1999): MODIS Vegetation Index MOD 13) Algorithm Theoretical Basis Document. http://modis.gsfc.nasa.gov/data/atbd/atbd mod13.pdf

Huete, A.R., Liu, H.Q., Batchily, K. \& van Leeuwen, W. (1997): A comparison of vegetation indices over a global set of TM images for EOS-MODIS. Remote Sensing of Environment, Vol. 59, No. 3, pp. 440-451.

Hughes, D.A. (2005): Comparison of satellite rainfall data with observations from gauging station networks. Journal of Hydrology, Vol. 327, No. 3-4, pp. 399-410.

Hulme, M. (2001): Climatic perspectives on Sahelian desiccation: 1973 1998. Global Environmental Change, Vol. 11, No. 1, pp. 19-29. 
Hulme, M.; Doherty, R.; Ngara, T. \& New, M. (2005): Global warming and African climate change: a reassessment. In: Low, P.S. (Ed.) (2005): Climate Change and Africa. Cambridge, New York, Melbourne et al.: Cambridge University Press.

HYDE, J.E. (2007): Drug-resistant malaria - an insight. FEBS Journal, Vol. 274 , No. 18 , pp. $4688-4698$.

IJUMBA J.N. \& LindSAY, S.W. (2001): Impact of irrigation on malaria in Africa: paddies paradox. Medical and Veterinary Entomology, Vol. 15, No. 1, pp. 111.

Ingram, K.T.; Roncoli, M.C. \& Kirshen,P.H. (2002): Opportunities and constraints for farmers of west Africa to use seasonal precipitation forecasts with Burkina Faso as a case study. Agricultural Systems, Vol. 74, No. 3, pp. 331-349.

IPCC (2007 $)$ : Climate Change 2007 - The Physical Science Basis. Contribution of Working Group $I$ to the Fourth Assessment Report of the Intergovernmental Panel on Climate Change. Cambridge \& New York: Cambridge University Press.

IPCC $\left(2007^{2}\right)$ : Climate Change 2007 - Impacts, Adaptation and Vulnerability. Contribution of Working Group II to the Fourth Assessment Report of the Intergovernmental Panel on Climate Change. Cambridge \& New York: Cambridge University Press.

ISAACSON, M. (1989): Airport malaria: a review. Bulletin of the World Health Organization, Vol. 67, No. 6, pp. 737-743.

Islam, M.; Thenkabail, T.S.; Kulawardhana, R.W. et al. (2008): Semi-automated methods for mapping wetlands using Landsat ETM+ and SRTM data. International Journal of Remote Sensing, Vol. 29, No. 24, pp. 7077-7106.

IUSS WoRking GRoup WRB (2006): World reference base for soil resources 2006. World Soil Resources Reports No. 103. Rome: FAO.

JACOB, B.G.; MUtURI, E.J.; FUNES, J.E. et al. (2006): A grid-based infrastructure for ecological forecasting of rice land Anopheles arabiensis aquatic larval habitats. Malaria Journal, Vol. 5, No. 91, doi: 10.1186/1475-2875-591.

JacoB, B.G; Muturi, E.J.; Mwangangi, J.M. et al. (2007): Remote and field level quantification of vegetation covariates for malaria mapping in three rice agro-village complexes in Central Kenya. International Journal of Health Geographics, Vol. 6, No. 21, doi:10.1186/1476-072X-6-21. 
Jensen, J.R. (2000): Remote Sensing of the Environment. Upper Saddle River, NJ: Prentice Hall.

Jensen, J.R. (2005): Introductory Digital Image Processing. Upper Saddle River, NJ: Prentice Hall.

Jung, G. (2006): Regional Climate Change and the Impact on Hydrology in the Volta Basin of West Africa. Augsburg \& Karlsruhe: Berichte des Forschungszentrums Karlsruhe, FZKA 7240. \{Dissertation\}

Justice, C.O.; Townshend, J.R.G.; Vermote, E.F. et al. (2002): An overview of MODIS Land data processing and product status. Remote Sensing of Environment, Vol. 83, No. 1-2, pp. 3-15.

KAPPAS, M. (2006): Naturraumpotential und Landnutzung im Oudalan. Stuttgart: ibidem Verlag.

KaPpaS, M. (2009): Klimatologie: Klimaforschung im 21. Jahrhundert Herausforderung für Natur- und Sozialwissenschaften. Heidelberg: Spektrum Akademischer Verlag.

KARTHE, D. \& KAPAA, M. (2007): Modelling Malaria Transmission in a Rural Region in West Africa: A Case Study of Nouna District, Burkina Faso. In: Kappas, M.; Kleinn, C. \& Sloboda, B. (2007): Global Change Issues in Developing and Emerging Countries. Proceedings of the $2^{\text {nd }}$ Göttingen GIS and Remote Sensing Days 2006. Göttingen: Universitätsverlag Göttingen.

Kamanishia, T.; Kuroinaa, H.; Kojima, M. et al. (2000): TRMM Precipitation Radar. Advances in Space Research, Vol. 25, No. 5, pp. 969-972.

Kebede, A.; McCann, J.C.; Kiszewski, A.E. et al. (2005): New evidence of the effects of agro-ecologic change on malaria transmission. American Journal of Tropical Medicine and Hygiene, Vol. 73 No. 4 (April 2005), pp. 676680.

Keiser, J.; Utzinger, J.; Caldas de Castro, M. et al. (2004): Urbanization in subsaharan Africa and implication for malaria control. American Journal of Tropical Medicine and Hygiene, Vol. 71, No. 2 (supplement), pp. 118-127.

Kelly-Hope, L.A.; Diggle, P.J.; Rowlingson, B.S. et al. (2006): Negative spatial association between Iymphatic filariasis and malaria in West Africa. Tropical Medicine and International Health, Vol. 11, No. 2, pp. 129-135.

KESSELS, O. (2006): Qualitätsanalyse verschiedener digitaler Geländemodelle und deren Eignung für die Prozessierung von Satellitenbilddaten in den Tropen. Stuttgart: ibidem Verlag. 
Killeen, G.F.; Seyoum, A. \& Knols, B.G. (2004): Rationalizing Historical Successes of Malaria Control in Africa in Terms of Mosquito Resource Availability Management. American Journal of Tropical Medicine and Hygiene, Vol. 71, No. 2 (Supplement), pp. 87-93.

Killeen, G.F.; McKenzie, F.E.; Foy, B.D. et al. (2000): A simplified model for predicting malaria entomologic inoculation rates based on entomologic and parasitologic parameters relevant to control. American Journal of Tropical Medicine and Hygiene, Vol. 62, No. 5, pp. 535-544.

Kiszewski, A. \& Teklehaimanot, A. (2004): A review of the clinical and epidemiologic burdens of epidemic malaria. American Journal of Tropical Medicine and Hygiene, Vol. 71, No. 2 (supplement), pp. 128-135.

Kleinschmidt, I.; Bagayoko, M.; Clarke, G.P.Y. et al. (2000): A spatial statistical approach to malaria mapping. International Journal of Epidemiology, Vol. 29, No. 2 , pp. 355-361.

Kleinschmidt, I.; Oмumbo, J.; BRIËt, O. et al. (2001): An empirical malaria distribution map for West Africa. Tropical Medicine and International Health, Vol. 6, No. 10, pp. 779-786.

Klotchkoff, J.C. \& Devey, M. (2004): Le Burkina Faso Aujourd'hui. Paris: Les Éditions du Jaguar.

KLOWDEN, M.J. (2007): Making generalizations about vectors: Is there a physiology of "the mosquito". Entomological Research, Vo. 37, No. 1, pp. 1-13.

Koenraadt, C.J.M.; Paajumans, K.P.; Githeko, A.K. et al. (2003): Egg hatching, larval movement and larval survival of the malaria vector Anopheles gambiae in desiccating habitats. Malaria Journal, Vol. 2, No. 20, doi: 10.1186/1475-2875-2-20.

Kolonialpolttisches Amt der NSDAP (Ed.) (1942): Die wichtigsten menschlichen Tropenkrankheiten in Afrika. Munich, Germany. \{Map, Scale 1:15.000.000\}

Konradesen, F.; van der Hoek, W.; Amerasinghe, F.P. et al. (2004): Engineering and malaria control: learning from the past 100 years. Acta Tropica, Vol. 89 No. 2 (January 2004), pp. 99-108.

Korodjouma, O.; Badiori, O.; Ayemou, A. \& Michel, S.P. (2006): Long-term effect of ploughing, and organic matter input on soil moisture characteristics of a Ferric Lixisol in Burkina Faso. Soil \& Tillage Research, Vol. 88, No. 1-2, pp. 217-224. 
KouYATÉ, B.; SIÉ, A.; YÉ, M. et al. (2007): The Great Failure of Malaria Control in Africa: A District Perspective from Burkina Faso. PLoS Medicine, Vol. 4, No. 6, pp. 997-1000.

KouYATÉ, B.; Somé, F.; JaHN, A. et al. (2008): Process and effects of a community intervention on malaria in rural Burkina Faso: randomized controlled trial. Malaria Journal, Vol. 7, No. 50, doi:10.1186/1475-2875-750.

Kumar, L.; Rietkerk, M.; v. Langevelde, F. et al. (2002): Relationship between vegetation growth rates at the onset of the wet season and soil type in the Sahel of Burkina Faso: implications for resource utilisation at large scales. Ecological Modelling, Vol. 149, No. 1, pp. 143-152.

Lacaux, J.P; Tourre, Y.M.; Vignolles, C. et al. (2007): Classification of ponds from high-spatial resolution remote sensing: Application to Rift Valley Fever epidemics in Senegal. Remote Sensing of Environment, Vol. 106, No 1 , pp. 66-74.

Laclavère, G. (Ed.) (2004): Atlas du Burkina Faso. Paris: Les Éditions Jeune Afrique.

Lanciotti, R.S.; Ludwig, M.L.; Rwaguma, E.B. et al. (1998): Emergence of Epidemic O'nyong-nyong Fever in Uganda after a 35-Year Absence: genetic Characterization of the Virus. Virology, Vol. 252, No. 1, pp. 258268.

Langhorne, J.; Noungu, F.M.; SponaAs, A.M. \& Marsh, K. (2008): Immunity to malaria: more questions than answers. Nature Immunology, Vol. 9, No. 7, pp. 725-732.

Lautze, J.; McCartney, M.; Kirshen, P. et al. (2007): Effect of a large dam on malaria risk: the Koka reservoir in Ethiopia. Tropical Medicine and International Health, Vol. 12, No. 8, pp. 982-989.

Laux, P.; Kunstmann, H. \& Bárdossy, A. (2008): Predicting the regional onset of the rainy season in West Africa. International Journal of Climatology, Vol. 28, No. 3, pp. 329-342.

Levine, R.S.; Peterson, T. \& Benedict, M.Q. (2004 $\left.{ }^{1}\right)$ : Geographic and Ecologic Distributions of the Anopheles Gambiae Complex predicted using a Genetic Algorithm. American Journal of Tropical Medicine and Hygiene, Vol. 70, No. 2, pp. 105-109.

Levine, R.S.; Peterson, T. \& Benedict, M.Q. $\left(2004^{2}\right)$ : Distribution of Members of Anopheles quadrimaculatus Say s.l. (Diptera: Culicidae) and Implications for Their Roles in Malaria Transmission in the United States. Journal of Medical Entomology, Vol. 41, No. 4, pp. 607-613. 
LI, J.; LeWIS, J.; RoWLAND, J. et al. (2004): Evaluation of land performance in Senegal using multitemporal NDVI and rainfall series. Journal of Arid Environments, Vol. 59, No. 3, pp. 463-480.

Lindsay, S.W.; Jawara, M.; Paine, K. et al. (2003): Changes in house design reduce exposure to malaria mosquitoes. Tropical Medicine and International Health, Vol. 8, No. 6, pp. 512-517.

LINDSAY, S.W. \& KIRBY, M.J. (2004): Responses of adult mosquitoes of two sibling species, Anopheles arabiensis and Anopheles gambiae s.s. (Diptera: Culicidae), to high temperatures. Bulletin of Entomological Research, Vol. 94, No. 5, pp. 441-448.

Lopez, A.; Mathers, C.D.; Ezzati, M. et al. (Ed.) (2006): Global Burden of Disease and Risk Factors. New York: Oxford University Press.

Lounibos, L.P. (2002): Invasions by Insect Vectors of Human Disease. Annual Review of Entomology, Vol. 47, pp. 233-266.

LUDWIG, R. \& SCHNEIDER, P. (2006): Validation of digital elevation models from SRTM X-SAR for applications in hydrologic modeling. ISPRS Journal of Photogrammetry \& Remote Sensing, Vol. 60, No. 5, pp. 339-358.

LunetTA R.S.; KNight, J.F.; EdiriwickRema, J. et al. (2006): Land-cover change detection using multi-temporal MODIS NDVI data. Remote Sensing of Environment, Vol. 105, No. 2, pp. 142-154.

Lutwama, J.J.; Kayondo, J.; Savage, H. M. et al. (1999): Epidemic O'nyongnyong Fever In Southcentral Uganda, 1996-1997: Entomologic Studies In Bbaale Village, Rakai District. Annual Journal of Tropical Medicine. Vol. 61 , No. 1 , pp. $156-162$

Mabaso, M.L.H; Craig, M.; Ross, A. \& SMITH, T. (2007): Environmental predictors of the seasonality of malaria transmission in Africa: the challenge. American Journal of Tropical Medicine and Hygiene, Vol. 76, No. 1, pp. 33-38.

MarAnz, S. \& Wiesmann, Z. (2003): Evidence for indigenous selection and distribution of the shea tree, Vitellaria paradoxa, and its potential significance to prevailing parkland savanna tree patterns in subSaharan Africa north of the equator. Journal of Biogeography, Vol. 30, No. 10, pp. $1505-1516$.

MarRama, L.; Jambou, R.; RAKOtoRivony, I. et al. (2004): Malaria transmission in Southern Madagascar: influence of the environment and hydroagricultural works in sub-arid and humid regions. Part 1: Entomological investigations. Acta Tropica, Vol. 89 No. 2, pp. 193-203. 
MARSH, K. (1993): Immunology of human malaria. In: GILLES, H.M. \& WARRELL, D.A. (Ed.) (1993): Bruce-Chwatt's Essential Malariology, pp. 60-77. London, Boston, Melbourne, Auckland: Edward Arnold.

Martens, P. (1998): Health and Climate Change. London: Earthscan Publications.

MARTENS, W.J.M. (1998): Health Impacts of Climate Change and Ozone Depletion: An Ecoepidemiologic Modeling Approach. Environmental Health Perspectives, Vol. 106, supplement 1, pp. 241-251.

Martens, W.J.M.; Jetten, T.H.; Rotmans, J. \& Niessen, L.W. (1995): Climate change and vector-borne diseases. Global Environmental Change, Vol. 5, No. 3, pp. 195-209.

Martin, C.; Curtis, B.; Fraser, C. \& Sharp, B. (2002): The use of a GIS-based malaria information system for malaria research and control in South Africa. Health \& Place, Vol. 8, No. 4, pp. 227-236.

Mateos, J.; Vega, M.; Molina, R. \& Katsaggelos, A.K. (2008): Pansharpening of multispectral images using a TV-based super-resolution algorithm. Journal of Physics, Vol. 139, doi:10.1088/1742-6596/139/1/012022.

Mathenge, E.M.; Killeen, G.F.; Oulo, D.O. et al. (2002): Development of an exposure-free bednet trap for sampling Afrotropical malaria vectors. Medical and Veterinary Entomology, Vol. 16, No. 1, pp. 67-74.

Matuschewski, K. \& Mueller, A.K. (2007): Vaccines against malaria - an update. FEBS Journal, Vol. 274, No. 18, pp. 4680-4687.

MAY, R.M. (2007): Parasites, people and policy: infectious dieseases and the Development Goals. Trends in Ecology and Evolution, Vol. 22, No. 10, pp. 497-503.

Mayaux, P.; Bartholome, E.; Fritz, S. \& Belward, A. (2004): A new land-cover map of Africa for the year 2000. Journal of Biogeography Vol. 31, No. 6, pp. 861-877.

McCallum, I.; Obersteiner, M.; Nilsson, S. \& Shvidenko, A. (2006): A spatial comparison of four satellite derived $1 \mathrm{~km}$ global land cover datasets. International Journal of Applied Earth Observation and Geoinformation, Vol. 8, No. 4 , pp. 246-255.

McKenzie, F.E. (2000): Why model malaria? Parasitology Today, Vol. 16, No. 
12, pp. 511-516.

McKenzie, F.E. \& Bossert, W.H. (1997): Mixed-species Plasmodium infections of humans. Journal of Parasitology, Vol. 83, No. 4, pp. 593-600.

McKenZie, F.E. \& SAMBA, E.M. (2004): The Role of Mathematical Modelling in Evidence-Based Malaria Control. American Journal of Tropical Medicine and Hygiene, Vol. 71, No. 2 (Supplement), pp. 94-96.

Michel, A.P.; Guelbeogo, W.M.; Grushko, O. et al. (2005): Molecular differentiation between chromosomally defined incipient species of Anopheles funestus. Insect Molecular Biology, Vol. 14, No. 4, pp. 375-387.

Miliaresis, G.C. \& Argialasb, D.P. (1999): Segmentation of physiographic features from the global digital elevation model/GTOPO30. Computers \& Geosciences, Vol. 25, No. 7, pp. 715-728.

Minakawa, N.; Munga, S.; Atieli, F. et al. (2005): Spatial distribution of anopheline larval habitats in Western Kenyan highlands: effects of land cover types and topography. American Journal of Tropical Medicine and Hygiene, Vol. 73, No. 1, pp. 157-165.

Minakawa, N. \& Sonye, G. (2004): Habitat characteristics of Anopheles gambiae s.s. larvae in a Kenyan highland. In: Medical and Veterinary Entomology, Vol. 18 (2004), pp. 301-305.

Minakawa, N.; Sonye, G.; Yan, G. (2005): Relationships Between Occurrence of Anopheles gambiae s.I. (Diptera: Culicidae) and Size and Stability of Larval Habitats. Journal of Medical Entomology, Vol. 42, No. 3, pp. 295-300.

Molineaux, L. (1985): The pros and cons of malaria modelling. Transactions of the Royal Society of Tropical Medicine and Hygiene, Vol. 79, No. 6, pp. 743747.

Molineaux, L. \& Gramaccia, G. (1980): The Garki Project: Research on the Epidemiology and Control of Malaria in the Sudan Savanna of West Africa. Geneva: World Health Organization.

Molyneux, D.H. (1998): Vector-borne parasitic diseases - an overview of recent changes. International Journal for Parasitology, Vol. 28, pp. 927-934.

MolYneuX, D.H. \& ZAGARIA, N. (2002): Lymphatic filariasis elimination: progress in global programme development. Annals of Tropical Medicine and Parasitology, Vol. 96, No. 2, pp. 15-40.

Monath, T. P. (2007): Dengue and Yellow fever - Challenges for the Development and Use of Vaccines. In: The New England Journal of Medicine. Vol. 357, No. 22, pp. 2222-2225. 
MOORE, D.A. \& CARPENTER, T.E. (1999): Spatial analytical methods and geographic information systems: use in health research and epidemiology. Epidemiological Reviews, Vol. 21, No. 2, pp. 143-161.

MOORE, M.; Gould, P. \& KeARY, B.S. (2003): Global urbanization and impact on health. International Journal of Hygiene and Environmental Health, Vol. 206, No. 4-5, pp. 269-278.

MulleR, R. (2005): Guinea worm disease - the final chapter? Trends in Parasitology, Vol. 21, No. 11, pp. 521-524.

Mung'ala-Odera, V.; Snow, R.W. \& Newton, C.R.J. (2004): The Burden of the Neurocognitive Impairment Associated With Plasmodium Falciparum Malaria in Sub-Saharan Africa. American Journal of Tropical Medicine and Hygiene, Vol. 71, No. 2 (Supplement), pp. 64-70.

Mushinzimana, E.; Munga, S.; Minakawa, N. et al. (2006): Landscape determinants and remote sensing of anopheline mosquito larval habitats in the western Kenya highlands. Malaria Journal, Vol. 5, No. 13, doi:10.1186/1475-2875-5-13.

Mutero, C.M.; Amerasinghe, F.; Boelee, E. et al. (2005): Systemwide Initiative on Malaria and Agriculture: An Innovative Framework for Research and Capacity Building. EcoHealth, Vol. 2, No. 1, pp. 11-16.

Mutero, C.M.; Blank, H.; Konradsen, F. \& van der Hoek, W. (2000): Water management for controlling the breeding of Anopheles mosquitoes in rice irrigation schemes in Kenya. Acta Tropica, Vol. 76, No. 3, pp. 253-263.

Mutero, C.M.; NG'Ang'A, P.N.; Wekoyela, P. et al. (2004): Ammonium sulphate fertiliser increases larval populations of Anopheles arabiensis and culicine mosquitoes in rice fields. Acta Tropica, Vol. 89 No. 2, pp. 187-192.

Mutuku, F.M.; Bayoh, M.N.; Gimnig, J.E. et al. (2006): Pupal habitat productivity of Anopheles gambiae complex mosquitoes in a rural village in Western Kenya. American Journal of Tropical Medicine and Hygiene, Vol. 74 No. 1, pp. 54-61.

Mutuku, F.M.; BAYOH, M.N.; Hightower, A.W. et al. (2009): A supervised land cover classification of a western Kenya lowland endemic for human malaria: associations of land cover with larval Anopheles habitats. International Journal of Health Geographics, Vol. 8, No. 19, doi:10.1186/1476072X-8-19.

Muturi, E.J.; Shililu, J.I.; Gu, W. et al. (2007): Larval habitat dynamics and diversity of Culex mosquitoes in rice agro-ecosystem in Mwea, Kenya. American Journal of Tropical Medicine and Hygiene, Vol. 76, No. 1, pp. 95-102. 
Nathan, S.S; Kalaivani, K. \& Murugan, K. (2005): Effects of neem limonoids on the malaria vector Anopheles stephensi Liston (Diptera: Culicidae). Acta Tropica, Vol. 96, No. 1, pp. 47-55.

Nelson, G.C. \& R. D. Robertson, R.D. (2007): Comparing the GLC2000 and GeoCover LC land cover datasets for use in economic modelling of land use. International Journal of Remote Sensing, Vol. 28, No. 19, pp. 4243-4262.

Nicholson, S. (2005): On the question of the "recovery" of the rains in the Westnext term African Sahel. Journal of Arid Environments, Vol. 63, No. 3, pp. 615-641.

Noor, A.M.; Mutheu, J.J.; TAtem, A.J. et al. (2009): Insecticide-treated net coverage in Africa: mapping progress in 2000-07. The Lancet, Vol. 373, No. 1 , pp. 58-67.

Norman, M.J.T.; Pearson, C.J. \& Searle, P.G.E. (1995): The ecology of tropical food crops. Cambridge, New York \& Melbourne: Cambridge University Press.

OeCD \& African Development Bank (2002): African Economic Outlook 2002. Paris: OECD Publications.

OecD \& African Development Bank (2006): African Economic Outlook 2006. Paris: OECD Publications.

OeCD \& African Development Bank (2007): African Economic Outlook 2007. Paris: OECD Publications.

OeCD \& African Development Bank (2008): African Economic Outlook 2008. Paris: OECD Publications.

Oguntunde, P.G.; Friesen, J.; van de Giesen, N. \& Savenije H. (2006): Hydroclimatology of the Volta River Basin in West Africa: Trends and variability from 1901 to 2002. Physics and Chemistry of the Earth, Vol. 31, No. 18 , pp. $1180-1188$.

OKECH, B.A.; GOUAGNA, L.C.; YAN, G. et al. (2007): Larval habitats of Anopheles gambiae s.s. (Diptera: Culicidae) influences vector competence to Plasmodium falciparum parasites. Malaria Journal, Vol. 6, No. 50, doi:10.1186/1475-2875-6-50.

OlsSON, L.; EKLUNDH, L. \& ARDÖB, J. (2005): A recent greening of the Saheltrends, patterns and potential causes. Journal of Arid Environments, Vol. 63 , No. 3, pp. 556-566. 
Омимво, J.A.; OumA, J.; RepuodA, B. et al. (1998): Mapping malaria transmission intensity using geographical informationm systems (GIS): an example from Kenya. Annals of Tropical Medicine and Parasitology, Vol. 92, No. 1, pp. 7-21.

Onori, E., Beales, P.F. \& Gilles, H.M. (1993): From malaria eradication to malaria control: the past, the present and the future. In: GILLES, H.M. \& WARRELL, D.A. (Ed.) (1993): Bruce-Chwatt's Essential Malariology, pp. 267282. London, Boston, Melbourne, Auckland: Edward Arnold.

Organisation Mondiale de la Santé (Ed.) (1995): Lutte contre les vecteurs du paludisme et autres maladies transmises par les moustiques. Geneva: OMS, Série de Rapports techniques 857.

ORSTOM (1973): Carte Pédologique de Reconaissance de la République de Haute-Volta. Ouest-Nord. Paris \& Dakar: Insitut Géographique National / Office de la Recherche Scientifique et Technique d'Outre-Mer. \{Map, scale $1: 500.000\}$

Otranto, D.; Stevens, J.R.; Cantacessi, C. \& Gasser, R.B. (2007): Parasite transmission by insects: a female affair? Trends in Parasitology, Vol. 24, No. 3, pp. 116-119.

Ouattara, Y.; Sanon, S.; Traoré, Y. et al. (2006): Antimalarial activity of Swartzia madagascariensis desv. (Leguminosae), Combretum glutinosum guill. \& perr. (Combretaceae) and Tinospora bakis miers. (menispermaceae), Burkina Faso medicinal plants. African Journal of Traditional, Complementary and Alternative Medicines, Vol. 3, No. 1, pp. 75-81.

OVergati, H. (2001): Spatial and Temporal Distribution of Malaria Mosquitoes: Associations with Landscape Structure and Vegetation in Northern Thailand. Uppsala: Acta Universitatis Agriculturae Sueciae. \{Dissertation\}

OVERGARD, H. (2007): Effect of plant structure on oviposition behavior of Anopheles minimus s.l. Journal of Vector Ecology, Vol. 32, No. 2, pp. 193197.

Paajmans, K.P.; Takken, W.; Githeko, A.K. \& Jacobs, A.F.G. (2008): The effect of water turbidity on the near-surface water temperature of larval habitats of the malaria mosquito Anopheles gambiae. International Journal of Biometeorology, Vol. 52, No. 8, pp.747-753.

Paajumans, K.P; Wandago, M.O.; Githeko, A.K.; TakKen, W. (2007): Unexpected High Losses of Anopheles gambiae Larvae Due to Rainfall. PLOS ONE Vol. 2, No. 11, doi:10.1371/journal. pone.0001146. 
Paeth, H. \& Hense, A. (2004): SST Versus Climate Change Signals in West African Rainfall: 20th-Century Variations and Future Projections. Climate Change, Vol. 65, No. 1-2, pp. 179-208.

Panjarathinam, R. (1990): Textbook of Medical Parasitology. Hyderabad: Orient Longman.

Pates, H. \& Curtis, C. (2005): Mosquito Behavior and Vector Control. Annual Review of Entomology, Vol. 50, pp. 53-70.

Paturel, J.E.; Boubacar, I. \& l'Aour, A. (2004): Evolution de la Pluviométrie Annuelle en Afrique de I'Ouest et Centrale au Xxème Siècle. Sud Sciences \& Technologies, No. 13, pp. 40-46.

PATZ, J.A.; GraczYK, T.K.; Geller, N. \& V VittoR, Y. (2000): effects of environmental change on emerging parasitic diseases. International Journal for Parasitology, Vol. 30, No.12-13, pp. 1395-1405.

PATZ, J.A. \& REISEN, W.K. (2001): Immunology, climate change and vectorborne diseases. Trends in Immunology, Vol. 22, No. 4, pp. 171-172.

Patz, J.A.; Strzepek K.; Lele, S. et al. (1998): Predicting key malaria transmission factors, biting and entomological inoculation rates, using modeled soil moisture in Kenya. Tropical Medicine and International Health, Vol. 3 No. 10, pp. 818-827.

Pauli, G. (2004): West-Nil-Virus. Prävalenz und Bedeutung als Zoonosenerreger. Bundesgesundheitsblatt. Vol. 47, No. 7, p. 653-660.

Peterson, A.T. \& Cohoon, K.P. (1999): Sensitivity of distributional prediction algorithms next term to geographic data completeness. Ecological Modelling, Vol. 117, No. 1, pp. 159-164.

Peterson, A. T. (2001): Predicting Species' Geographic Distributions Based on Ecological Niche Modelling. The Condor, Vol. 103, No. 3, pp. 599-605.

Peterson, A.T. (2006): Ecologic Niche Modeling and Spatial Patterns of Disease Transmission. Emerging Infectious Diseases, Vol. 12, No. 12, pp. 1822-1826.

Peterson, A.T. (2007): Ecologic Niche Modeling and Understanding the Geography of Disease Transmission. Veterinaria Italiana, Vol. 43, No. 3, pp. 393-400.

Peters, W. (2002): Medizinische Entomologie. In: Dettner, K. \& Peters, W. (Ed.) (2002): Lehrbuch der Entomologie, pp. 635-670. München: Elsevier. 
Peyrefitte, C. N.; Rousset, D.; Pastorino, B. et al. (2007): Chikungunya Virus, Cameroon, 2006. Emerging Infectious Diseases. Vol. 13, No. 5, pp. 768-771.

Pfeiffer, K.; Somé, F.; Müller, O. et al. (2008): Clinical diagnosis of malaria and the risk of chloroquine self-medication in rural health centres in Burkina Faso. Tropical Medicine and International Health, Vol. 13, No. 3, pp. 418-26.

Pinheiro, A.C.T.; Descloitres, J.; Privette, J.L. et al. (2007): Near-real time retrievals of land surface temperature within the MODIS Rapid Response System. In: Remote Sensing of Environment, Vol. 106, No. 3, pp. 326-336.

Pinheiro, A.C.T.; Mahoney, R.; Privette, J.L. \& Tucker, C.J. (2006): Development of a long termn record of NOAA-14 AVHRR land surface temperature over Africa. In: Remote Sensing of Environment, Vol. 103, No. 2, pp. 153164.

PodA, J.N.; TRAORÉ, A. \& SONDO B.K. (2004): Schistosomiasis endemic in Burkina Faso. Bulletin de la Société de pathologie exotique, Vol. 97, No. 1, pp. 47-52.

Population Reference Bureau (2008): 2008 World Population Data Sheet. Washington D.C.

POWERS, A. \& LOGUE, C. (2007): Changing patterns of chikungunya virus: reemergence of a zoonotic arbovirus. Journal of General Virology. Vol. 88, No. 9, pp. 2363-2377.

PRinodko, L. \& GowaRd, S. (1997): Estimation of Air Temperature from Remotely Sensed Surface Observations. In: Remote Sensing of the Environment, Vol. 60, No. 3, pp. 335-346.

QI, J., Chehbouni, A., Huete, A.R. et al. (1994): A modified soil adjusted vegetation index. Remote Sensing of Environment, Vol. 48, No. 2, pp. 119126.

Qunhua, L.; Xin, K.; Chnagzhi, C. et al. (2004): New irrigation methods sustain malaria control in Sichuan Province, China. Acta Tropica, Vol. 89 No. 2, pp. 241-247.

Rabus, B.; Eineder, M.; Roth, A. \& Bamler, R. (2003): The shuttle radar topography mission-a new class of digital elevation models acquired by spaceborne radar. ISPRS Journal of Photogrammetry \& Remote Sensing, Vol. 57, No. 4, pp. 241- 262. 
Ramasamy, M.S.; Srikrishnaraj, K.A.; Hadirin, N. et al. (2000): Physiological aspects of multiple blood feeding in the malaria vector Anopheles tessellatus. Journal of Insect Physiology, Vol. 46, No. 6, pp. 1051-1059.

Ramin, B.M. \& McMichael, A.J. (2009): Climate Change and Health in SubSaharan Africa: A Case-Based Perspective. EcoHealth, doi: 10.1007/ s10393-009-0222-4.

Rasmussen, K.; Fog, B. \& MAdsen, J.E. (2001): Desertification in reverse? Observations from northern Burkina Faso. Global Environmental Change, Vol. 11 , No. 4, pp. 271-282.

Reij, C.; Tappan, G. \& Belemvire, A. (2005): Changing land management practices and vegetation on the Central Plateau of Burkina Faso (1968-2002). Journal of Arid Environments, Vol. 63, No. 3, pp. 642-659.

Remme, J.H.F.; Blas, E.; Chitsulo, L. et al. (2002): Strategic emphases for tropical diseases research: a TDR perspective. Trends in Microbiology, Vol. 10, No. 10 , pp. $435-440$.

Reuter, H. I.; Nelson, A. \& J Jevis, A. (2007): An evaluation of void filling interpolation methods for SRTM data. International Journal of Geographical Information Science, Vol. 21, No. 9, pp. 983-1008.

RIECKMANN, K.H. (2006): The chequered history of malaria control: are new and better tools the ultimate answer? Annals of Tropical Medicine and Parasitology, Vol. 100, No. 8, pp. 647-662.

Rivero, A. \& Ferguson, H.M. (2003): The energetic budget of Anopheles stephensi infected with Plasmodium chabaudi: is energy depletion a mechanism for virulence? Proceedings of the Royal Society London: Biological Sciences, Vol. 270, No. 1522, pp. 1365-1371.

Robert, V., Awono-Ambene, H.P. \& Thioulouse, J. (1998): Ecology of larval mosquitoes, with special reference to Anopheles arabiensis (Diptera: Culcidae) in market-garden welles in urban Dakar, Senegal. Journal of Medical Entomology, Vol. 35, No. 6, pp. 948-955.

Robert, V.; Macintyre, K.; Keating, J. et al. (2003): Malaria transmission in urban sub-Saharan Africa. American Journal of Tropical Medicine and Hygiene, Vol. 68, No. 2, pp. 169-176.

Rogers, D.J.; Rudolph, S.E.; SNOW, R.W.; HAY, S.I. (2002): Satellite imagery in the study and forecast of malaria. Nature, Vol. 415, No. 6872, pp. 710715 . 
Roll Back Malaria Partnership (2005): Global Strategic Plan - Roll Back Malaria 2005-2015. Geneva.

Roll Back Malaria Partnership (2008): The Global Malaria Action Plan. For a malaria-free world. Geneva.

Ruan, S.; XIAO, D. \& BeIER, J.C. (2008): On the Delayed Ross-Macdonald Model for Malaria Transmission. Bulletin of Mathematical Biology, Vol. 70, No. 4, pp. 1098-1114.

Rudloff, W. (1981): World Climates. Stuttgart: Wissenschaftliche Verlagsgesellschaft.

Rutherford, J.S.; Macaluso, K.R.; Smith, N. et al. (2004): Fatal Spotted Fever Rickettiosis, Kenya. Emerging Infectious Diseases, Vol. 10, No. 5, pp. 910913.

SAMBA, E.M. (2004): Bridging the Gap: Linking Research, Training and Service Delivery to Reduce the Malaria Burden in Africa. American Journal of Tropical Medicine and Hygiene, Vol. 71, No. 2 (Supplement), pp. ii-iii.

SANDERS, B.F. (2007): Evaluation of on-line DEMs for flood inundation modeling. Advances in Water Resources, Vol. 30, No. 8, pp. 1831-1843.

Scharlemann, J.P.W.; Benz, D.; Hay, S.I. et al. (2008): Global Data for Ecology and Epidemiology: A Novel Algorithm for Temporal Fourier Processing MODIS Data. PLOS ONE. Vol. 3, No. 1, pp. 1-13.

Schütt, P.; Weisgerber, H.; Schuck, H.J. et al. (2006): Bäume der Tropen. Hamburg: Nikol Verlagsgesellschaft.

Serfling, R.E. (1952): Entomological Survey Methods. Public Health Reports, Vol. 67, No. 10. pp. 1020-1025.

Service, M.W. (1993): The Anopheles Vector. In: Gilles, H.M. \& Warrell, D.A. (Ed.) (1993): Bruce-Chwatt's Essential Malariology, pp. 96-123. London, Boston, Melbourne, Auckland: Edward Arnold.

Simard, F.; Lehmann, T.; Lemasson, J.-J. et al. (2000): Persistence of Anopheles arabiensis during the severe dry season conditions in Senegal: an indirect approach using microsatellite loci. Insect Molecular Biology, Vol. 9, No. 5, 2000, pp. 467-479.

Sipe, N.G. \& Dale, P. (2003): Challenges in using geographic information systems (GIS) to understand and control malaria in Indonesia. Malaria Journal, Vol. 2, No. 36, doi:10.1186/1475-2875-2-36. 
Siri, J.G.; Lindblade, K.A.; Rosen, D.H. et al. (2008): Quantitative urban classification for malaria epidemiology in sub-Saharan Africa. Malaria Journal, Vol. 7, No. 34, doi:10.1186/1475-2875-7-34.

SIssoko, M. S; Dicko, A.; BRIËT, O.J.T. et al. (2004): Malaria incidence in relation to rice cultivation in the irrigated Sahel of Mali. Acta Tropica, Vol. 89, No. 2 , pp. 161-170.

Smith, T.; Killeen, G.; Lengeler, C. \& Tanner, M. (2004): Relationships Between the Outcome of Plasmodium Falciparum Infection and the Intensity of Transmission in Africa. American Journal of Tropical Medicine and Hygiene, Vol. 71, No. 2 (Supplement), pp. 80-86.

Smith, T.; Maire, N.; Dietz, K. et al. (2006): Relationship between the entomologic inoculation rate and the force of infection for Plasmodium falciparum malaria. American Journal of Tropical Medicine and Hygiene, Vol. 75, No. 2 (Supplement), pp. 11-18.

SNOW, R.W. \& HAY, S.I. (2006): Comparing methods of estimating the global morbidity burden from Plasmodium falciparum malaria. American Journal of Tropical Medicine and Hygiene, Vol. 74 No. 2, pp. $189 f$.

SNow, R.W. \& MARSH, K. (1998): New insights into the epidemiology of malaria relevant for disease control. British Medical Bulletin, Vol. 54, No. 2, pp. 293-309.

Spiegel, J.M.; Bonet, M.; IbarRa, A.-M. et al. (2007): Social and environmental determinats of Aedes aegypti infestation in Central Havana: results of a case-control study nested in an integrated dengue surveillance programme in Cuba. Tropical Medicine and International Health. Vol. 12, No. 4, pp. 503-510.

Stern, R.D.; Dennett, M.D. \& Garbutt, D.J. (1981): The start of the rains in West Africa. International Journal of Climatology, Vol. 1, No. 1, pp. $59-68$.

Sternberg, J.M. (2004): Human African Trypanosomiasis: clinical presentation and immune response. Parasite Immunology, Vol. 26, No. 11/12, pp. 469-476.

Stisen, S. Sandholt, I.; NørgaArd, A. et al. (2007): Estimation of diurnal air temperature using MSG SEVIRI data in West Africa. Remote Sensing of Environment, Vol. 110, No. 2, pp. 262-274.

StockWeLL, D.R.B. \& Peterson, A.T. (2002): Effects of sample size on accuracy of species distribution models. Ecological Modelling, Vol. 148, No. 1, pp. 113. 
Sultan, B. \& Janicot, S. (2003): The West African Monsoon Dynamics. Part II: The "Preonset" and "Onset" of the Summer Monsoon. Journal of Climate, Vol. 16, No. 21, pp. 3407-3427.

Sutherst, R.W.; Ingram, J.S.I. \& Scherm, H. (1998): Immunology, climate change and vector-borne diseases. Parasitology Today, Vol. 14, No. 8, pp. 297-299.

Sys, C.; van Ranst, E.; Debaveye, J. \& Beernaert, F. (1993): Land Evaluation Part III: Crop Requirements. Ghent: Publications of the International Training Centre for Post-Graduate Soil Scientists.

TAKKEN, W. \& KNOLS, B.G.J. (1999): Odor-mediated behavior of afrotropical malaria mosquitoes. Annual Review of Entomology, Vol. 44, pp. 131-157.

TANG, B:; BI, Y.; LI, Z.-L. \& XIA, J. (2008): Generalized Split-Window Algorithm for Estimate of Land Surface Temperature from Chinese Geostationary FengYun Meteorological Satellite (FY-2C) Data. Sensors, Vol. 8, No. 2, pp. 933-951.

TANSER, F.C. \& LE SUEUR, D. (2002): The application of geographical information systems to important public health problems in Africa. International Journal of Health Geographics, Vol. 1, No. 4, doi: 10.1186/1476072X-1-4.

Tanser, F.C.; Sharp, B. \& le Sueur, D. (2003): Potential effect of climate change on malaria transmission in Africa. The Lancet, Vol. 362, No. 9398, pp. 1792-1798.

Tatem, A.J., Goetz, S.J. \& Hay, S.I. (2006): Terra and Aqua: new data for epidemiology and public health. International Journal of Applied Earth Observation and Geoinformation, Vol. 6, pp. 33-46.

Teklehaimanot, H.D.; Lipsitch, M.; Teklehaimanot, A. \& Schwartz, J. (2004): Weather-based prediction of Plasmodium falciparum malaria in epidemic-prone regions of Ethiopia I. Patterns of lagged weather effects reflect biological mechanisms. Malaria Journal, Vol. 3, No. 41, doi:10.1186/1475-2875-3-41.

Teklehaimanot, H.D.; Schwartz, J.; Teklehaimanot, A. \& Lipsitch, M. (2004): Weather-based prediction of Plasmodium falciparum malaria in epidemic-prone regions of Ethiopia II. Weather-based prediction systems perform comparably to early detection systems in identifying times for interventions. Malaria Journal, Vol. 3, No. 44, doi:10.1186/14752875-3-44.

The Global Fund to Fight AidS, Tuberculosis and Malaria (2007): The Global Fund: Who We Are, What We Do. Geneva. 
Thomas, C.J.; Davies, G. \& Dunn, C.E. (2004): Mixed picture for changes in stable malaria distribution with future climate in Africa. Trends in Parasitology, Vol. 20, No. 5, pp. 216-220.

Ter Kuile, F.O.; Parise, M.E.; Verhoeff F.H. et al. (2004): The Burden of Coinfection with Human Immunodeficiency Virus Type 1 and Malaria in Pregnant Women in Sub-Saharan Africa. American Journal of Tropical Medicine and Hygiene, Vol. 71, No.2 (Supplement), pp. 41-54.

TESH, R.B. (1982): Arthritides Caused by Mosquito-Borne Viruses. Annual Reviews of Medicine. Vol. 33, No. 1, pp. 31-40.

ThIES, E. (1995): Principaux Ligneux Agro-Forestiers de la Guinée. Roßdorf: TZ-Verlag.

Thomson, M.C.; Connor, S.J.; Milligan, P. \& Flasse, S.P. (1997): Mapping Malaria Risk in Africa: What can Satellite Data Contribute? Parasitology Today, Vol. 13, No. 8, pp. 313-318.

Thomson, M.C.; D'Alessandro, U.; Bennetr, S. et al. (1994): Malaria prevalence is inversely related to vector density in The Gambia, West Africa. Transactions of the Royal Society of Tropical Medicine and Hygiene, Vol. 88, No. 6, pp. 638-643.

Tipke, M.; Diallo, S., Coulibaly, B. et al. (2008): Substandard anti-malarial drugs in Burkina Faso. Malaria Journal, Vol. 7, No. 95, doi:10.1186/14752875-7-95.

TODRY, S. \& BEJON, P. (2009): Malaria vaccine development: Lessons from the field. European Journal of Immunology, Vol. 39, No. 8, pp. 2007-2010.

TOURÉ, Y.T. (1989): The current state of studies of malaria vectors and the antivectorial campaign in West Africa. Iransactions of the Royal Society of Tropical Medicine and Hygiene, Vol. 83, Supplement, pp. 39-41.

Touré, Y.T.; Oduola, A.M.J. \& Morel, M. (2004): The Anopheles gambiae genome: next steps for malaria vector control. Trends in Parasitology, Vol. 20, No. 3, pp. 142-149.

TREITZ, P. \& RogAN, J. (2004): Remote sensing for mapping and monitoring land-cover and land-use change-an introduction. Progress in Planning, Vol. 61, No. 4, pp. 269-279.

Trouiller, P.; Olliaro, P.; Torneele, E. et al. (2002): Drug development for neglected diseases: a deficient market and public-health policy failure. The Lancet, Vol. 359, No. 9324, pp. 2188-2194. 
TUtejA, R. (2007): Malaria - an overview. FEBS Journal, Vol. 274, No. 18, pp. 4670-4679.

United Nations Environment Programme (2008): Africa: Atlas of Our Changing Environment. London: Earthprint.

VAN DER HOEK, W. (2004): How can better farming methods reduce malaria? Acta Tropica, Vol. 89 No. 2 (January 2004), pp. 95-97.

VAN ZYL, J.J. (2001): The Shuttle Radar Topography Mission (SRTM): a breakthrough in remote sensing of topography. Acta Astronautica, Vol. 48 , No. 5, pp. 559-565.

VARro, M.T. (36 B.C.): Rerum Rusticarum De Agri Cultura. Liber Primus, XII.

VIAL, L.; DIATTA, G.; TALL, A. et al. (2006): Incidence of tick-borne relapsing fever in West Africa: longitudinal study. The Lancet, Vol. 368, No. 9529, pp. 37-43.

VittoR, A.Y.; GiLmAn, R.H.; Tielsch, J. et al. (2006): The effect of deforestation on the human-biting rate of Anopheles darlingi, the primary vector of falciparum malaria in the Peruvian Amazon. American Journal of Tropical Medicine and Hygiene, Vol. 74, No. 1, pp. 3-11.

von MaydelL, H.J. (1990): Arbres et arbustes du Sahel. Eschborn: Gesellschaft für Technische Zusammenarbeit.

WAN, Z. (1999): MODIS Land-Surface Temperature Algorithm Theoretical Basis Document (LST ATBD).

http://modis.gsfc.nasa.gov/data/atbd/atbd mod11.pdf

WAN, Z.; LI, Z.-L. (2008): Radiance-based validation of the V5 MODIS land-surface temperature product. International Journal of Remote Sensing, Vol. 29, No. 17-18, pp. 5373 - 5395.

WAN, Z.; ZHANG, Y.; ZHANG, Q. et al. (2002): Validation of the land-surface temperature products retrieved from Terra Moderate Resolution Imaging Spectroradiometer data. Remote Sensing of Environment, Vol. 83 (2002), pp. 163-180.

Weischet, W. \& Endicher, W. (2000): Regionale Klimatologie: Die Alte Welt. Stuttgart \& Leipzig: Teubner.

Whiте, G.B. (1982): Malaria Vector Ecology and Genetics. British Medical Bulletin, Vol. 38, No. 2, pp. 207-212.

Wichmann, O. \& Jelinek, T. (2004): Dengue in Travelers: a Review. Journal of Travel Medicine. Vol. 11, No. 3, pp. 161-170. 
Winch, P.J.; Lloyd, L.S.; Hoemeke, L. \& Leontsini, E. (1993): Vector Control at the household level: an analysis of its impact on women. Acta Tropica, Vol. 56, No. 4, pp. 327-339.

World BANK (2007): World Development Report 2008: Agriculture for Development. Washington, D.C.: Quebecor Press.

World Health Organization (Ed.) (2005): World Malaria Report 2005. Geneva: WHO.

World Health Organization (Ed.) (2008): World Malaria Report 2008. Geneva: WHO.

Wrighley, R. (2000): Pathological Topographies and Cultural Itineraries: mapping malaria in $18^{\text {th }}$ and $19^{\text {th }}$ century Rome. In: WRIGHLEY, R. \& REVILL, G. (Ed.) (2000): Pathologies of Travel, pp. 207-228. Amsterdam \& Atlanta, GA: Rodopi.

WürthWEIN, RALPH (2002): Measuring the Burden of Disease, the Structure of Income, and Returns to Education in Rural West Africa. Heidelberg. \{Dissertation\} [http://www.ub.uni-heidelberg.de/archiv/3218]

YAHMED, D.B. (2005): Atlas de I'Afrique - Burkina Faso. Paris: Les Éditions Jeune Afrique.

Ye-Ebiyo, Y.; Pollack, R.J,; Kiszewski, A. \& Spielman, J. (2003): Enhancement of development of larval Anopheles arabiensis by proximity to flowering maize (zea mays) in turbid water and when crowded. American Journal of Tropical Medicine and Hygiene, Vol. 68, No. 6, pp. 748-752.

Yé, Y. (2005): Incorporating environmental factors in modelling malaria transmission in under five children in rural Burkina Faso. Heidelberg. \{Dissertation\}

Yé, Y.; Hoshen, M.; Louis, V. et al. (2006): Housing conditions and Plasmodium falciparum infection: protective effect of iron-sheet roofed houses. Malaria Journal, Vol. 5, No. 8, doi:10.1186/1475-2875-5-8.

Yé, Y.; Kyobutungi, C.; Louis, V.R. \& Sauerborn, R. (2007): Micro-epidemiology of Plasmodium falciparum malaria: Is there any difference in transmission risk between neighbouring villages? Malaria Journal, Vol. 6, No. 46, doi:10.1186/1475-2875-6-46. 
Yé, Y.; Louis, V.R.; Simboro, S. \& SAUerborn, R. (2007): Effect of meteorological factors on clinical malaria risk among children: an assessment using village-based meteorological stations and community-based parasitological survey. Malaria Journal, Vol. 7, No. 101, doi:10.1186/14712458-7-101.

Yé, Y. , Sauerborn, R., Séraphin, S. \& Hoshen, M. (2007): Using modelling to assess the risk of malarial infection during the dry season, on a local scale in an endemic area of rural Burkina Faso. Annals of Tropical Medicine \& Parasitology, Vol. 101, No. 5, pp. 375-389.

ZaVALtA, J.O. \& Rossignol, P.A. (2004): Community-level analysis of risk of vector-borne disease. In: Transactions of the Royal Society of Tropical Medicine and Hygiene, Vol. 98 No. 10, pp. 610-618.

ZhanG, C.; LI, W. \& Travis, D. (2007): Gaps-fill of SLC-off Landsat ETM+ satellite image using a geostatistical approach. International Journal of Remote Sensing, Vol. 28, No. 22, pp. 5103 - 5122.

ZwÖLfeR, H. (2002): Regulation der Populationsdichte. In: DETTNER, K. \& Peters, W. (Ed.) (2002): Lehrbuch der Entomologie, pp. 701-720. München: Elsevier. 
CIA World Fact https://www.cia.gov/library/publications/the-worldBook factbook/

Digital Chart of the http://www.maproom.psu.edu/dcw/ World

ESRI Data and Maps

FAO Africover data

FAO ClimNET

FAO Land Degradation Assessment in Drylands

FAO Map of World http://www.fao.org/ag/agl/agll/wrb/soilres.stm Soil Resources

FAO Stat

MARA initiative

MEWS (Malaria Early Warning System, IRI)

Roll Back Malaria http://www.rollbackmalaria.org/ partnership

Seed leaflets of Copenhagen University

SRTM data
ESRI (2008): Data \& Maps 9.3. Redlands, CA. \{DVD\} http://www.africover.org/system/africover data.php http://geonetwork3.fao.org/climpag/agroclimdb en.php

http://www.fao.org/nr/lada/

http://faostat.fao.org/

www.mara.org.za

http://ingrid.Ideo.columbia.edu/maproom/.Health/.Regi onal/.Africa/.Malaria/.MEWS/

http://en.sl.life.ku.dk/Publikationer/Udgivelser.aspx? katid=all

http://srtm.csi.cgiar.org/ 
Stop Malaria Now http://www.stopmalarianow.org/ initiative

Tu Tiempo climate http://www.tutiempo.net/clima/Dedougou/655050.htm data for Dédougou

UN Millennium http://www.un.org/millenniumgoals/

Development

Goals

UN World

http://esa.un.org/unpp/index.asp

Population

Database

US Census Bureau http://www.census.gov/ipc/www/idb/country.php

International

Database

US Center for

Disease Control

(malaria)

US Center for

Disease Control

(Public Health Image Library)

USGS GSTOPO 30 dataset

WHO Global Burden of Disease information http://www.cdc.gov/malaria

http://phil.cdc.gov/

http://edc.usgs.gov/products/elevation/gtopo30/gtopo3 $\underline{0 . h t m l}$

http://www.who.int/healthinfo/global_burden disease 


\section{Alphabetical Index}

A

A1FI scenario..........................344f.

Abundance.................................32

Acacia albida...........................214

Acacia ataxacantha....................214

Acacia dudgeoni........................215

Acacia ehrenbergiana................215

Acacia gourmaensis...................215

Acacia laeta............................215

Acacia macrostachya............213, 215

Acacia nilotica...........................215

Acacia pennata.........................215

Acacia raddiana.........................215

Acacia senegal...................215, 226

Acacia seyal.....................210, 214

Acacia spp..............................214

Acquired immunity...................54f.

ACT ..............................157, 271

Active case detection..................85

Active remote sensing.................119

Acute infection...........................53

Acute malnutrition.....................259

Adansonia digitata........210, 220, 222

Adaption.................................343

Adult emergence.....36, 71f., 280, 308

Advection..........................191, 193

Aedes spp...............302, 350, $358 f$.

AEJ.............................194, 198

Aestivation................................38

African Easterly Jet.............193f., 198

African locust bean tree...............210

African trypanosomiasis..........9, 358

African Wave Disturbance.............193

Africover.............................128

Agro-pastoralism..........131, 241, 252

Air temperature.................136, 139

Aldrovandi, Ulisse......................26

Alluvial soil......................215, 247

Alphonse Laveran...................15, 26

Amblyomma spp........................359

American trypanosomiasis...........160

Anemia................................ 19ff.

Animal husbandry.....................252

Anopheles.....16, 27f., 32, 36, 48, 359
Anopheles albimanus...................35

Anopheles arabiensis.....7, 35, 37, 41, 43f., 64, 67, 70, 72, 77, 102, 111, $144,155,302$

Anopheles bwambae...................44

Anopheles coustani....................45

Anopheles culcifacies....................35

Anopheles darlingi...................... 79

Anopheles flavirostris...................41

Anopheles funestus 7, 36f., 41, 43, 44, 75, 77, 102, 304ff.

Anopheles gambiae....7, 32, 35ff., 41, 43f., 45, 61, 63, 66ff., 71f., 75, 77, 79, 102, 280, 304ff.

Anopheles gambiae ....................302

Anopheles gambiae Bissau............46 46

Anopheles gambiae Complex..........43

Anopheles gambiae Mopti........45, 82

Anopheles gambiae s.l....... 73, 75, 82

Anopheles gambiae s.s..........70, 111

Anopheles gambiae Savanna..........45

Anopheles gambiae sensu lato.......43

Anopheles gambiae sensu stricto....43

Anopheles maculatus..................151

Anopheles melas......................43ff.

Anopheles merus.........................44

Anopheles minimus.....................76

Anopheles minimus s.l................41

Anopheles minimus species E.........41

Anopheles moucheti.....................45

Anopheles nili...................445, 305f.

Anopheles pharaoensis.................45

Anopheles quadriannulatus. . .43f., 111

Anopheles spp.........................358

Anopheles stephensi............41, 350

Anopheline larvae.......................33

Anopheline mosquitoes..................32

Anophelism without malaria...........82

Anthropophily................35, 44, 105

Anti-anopheline measures.............34

Anti-disease immunity.................. 55

Antibodies against sporozoites........56

Antiparasitic immunity.................55

Antiprotozoals.........................163

Antitoxic immunity....................55 
Aqua (satellite) 131

Aquaculture....

Aquatic habitats. 151

Arachis hypogaea. 247,250

Arenosol. .177

Aridisol. 176

Aristida mutabilis. 225

ARMA 12,93

Artemisinin-based combination therapy..... 157,271 ARVI 130,144

Asexual reproduction .48

ASTER. 137

Asymptomatic period .48

Atlas du Risque de la Malaria en Afrique. .93

Atmospheric window....

123

Atmospherically resistant vegetation

index. 130

Atovaquone. 156

Autochthonous malaria .29

AVHRR.... 127

AWD 193

Azadirachta indica. $150,210 f$.

Azadirachtin 150

\section{B}

Bacillus sphaericus. 153

Bacillus thuringiensis ... 153

Balanites aegyptiaca.....210, 215, 224 Band. 121 Baobab. 210,222

Barani. ... 232

Barani CSPS 265,294

Basic reproduction number...........342 Basic reproduction rate. .98, 100, 118, 164

Bednet.......84, 145, 147, 154, $319 \mathrm{ff}$. Berma CSPS........................294

Bilharziosis. ....3

Biogeography. 110

Biomphalaria spp. 360

Biron Badala 264

Biting behavior. 34,104

Black Volta 172

Blood meal.... $25,33,35,37$ Bobo .229f., $321 \mathrm{f}$.
Bomborokuy CSPS.............265, 294

Borakuy.............................264

Borrellia crocidurae..................360

Boulgou Province....................269

Bourasso...........................232

Bourasso CSPS.......................274

Bouts of fever........................48

Breeding site......40, 43, 66ff., 70, 73, 76ff., 82f., 112, 124, 126, 143, 145, 148f., 152, 166, 241, 273, 275ff., 280, 285f., 290, 343, 348f.

Brugia malayi, .......................358

Brugia timori, ........................ 358

Bulinus spp............................ 360

Bunyaviridae........................359

Burkina Faso............45, 54, 82, 345

Bushfire..............................225

Butter tree..........................210

Butyrospermum parkii...............218

Bwaba.......................229f., $321 f$.

C

C-band.........................121, 124

Carotinoid...........................129

Cassava...............................249ff.

Causal models........................13

CCD.............................67, 140

Cenchrus biflorus....................225

Central nervous system...............20

Centre de Recherche en Santé de Nouna.......................263, 266

Centre de Santé et de Promotion Sociale...............................263

Centre hospitalier régional..........262

Centre médical avec antenne chirurgicale........................262

Centre Muraz.......................262

Centre National de Recherche et de Formation sur le Paludisme.......262 Cerebral malaria.......................21 CGIAR.......................... 81, 162

Chagas' disease................... 9, 160

Channel...............................121

Chemoprophylaxis..................156f.

Chikungunya fever..............357, 359

CHIKV................................359 
Childhood mortality.....2, 7, 9, 11, 21, 256, 271, 352

Chlorophyll.... 129

Chloroquine.... $6,156,267,271$

Chloroquine resistance.................6

Chloroquine-resistant malaria.......156

CHR. 262

Chronic infection .53

Chronic malnutrition. 259

Chrysops spp. 358

CILSS 196

Cissé. 309

Classic Models..... .99

Clay pit. 283

Climate change. 339

Cloud cover. 184

CM2.1 model. 340

CMA. 262

CNRFP. 262

Co-infections. ..4

Coastal areas. .43

Coelomomyces.... 153

Coinfections $9,11,20 f$.

Cold cloud duration. 67,140

Combretaceae. 212,226

Combretum aculateum 212 Combretum glutinosum......210, $212 \mathrm{f}$. Combretum micranthum.....210, $212 \mathrm{f}$. Combretum micranthum,...$\ldots \ldots \ldots .215$ Combretum nigricans. $212 f$.

Combretum paniculatum.............212 Comité permanent Inter-États de Lutte contre la Sécheresse dans le Sahel. 196

Common guppy. .... 152

Communicable infectious diseases....2 Comoé basin. 172

Comorbid condition......................20

Complete immunity....................56

CONAGESE. 196

Conseil National pour la Gestion de I'Environnement....................196 Consultative Group on International Agricultural Research.........81, 162 Contact rate. 97,118 Convection 192,194 Copper acetoarsenite 149

Côte d'Ivoire. .41
Cotton..................237, 247, 251

Critical vectorial capacity............106 CRSN. . 263,266

CSPS 263

Culex.................................. 302

Culex quinquefasciatus...............72

Culex spp...............................

Culicidae.............................. 32

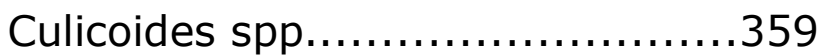

Cumulonimbus....................... 193

Cyprinodon macularius..............152

\section{D}

Dactyloctenium aegypticum........225 Dafing.........................229f., 320 Daily survival probability.............103 DALYs................................... 4 Dara CSPS..........................294

DDT.............................149f., 159

De animalibus insectis................26

Dédougou....................184, 237

Defense Meteorological Satellite Program..............................127

Deforestation...............79, 341, 347

Deltamethrine..........................154

DEM............................... $124 \mathrm{f}$.

Dembèlela....................265, 306

Demographic Surveillance System. 266

Dengue fever......3 , 9, 350, 357, 359

DENV1............................. 359

DENV4............................... 359

Desert date...................210, 215

Desert pupfish.......................152

Desiccation........33, 61, 68, 181, 244

Di .................................... 242

Diagnosing malaria.................... 85

Diapause................................ 37

Diarrhea.............................262

Diarrheal diseases......................4

Dichloro-diphenyl-trichloroethane.149, 159

Dieldrin...............................150

Dietary components....................55

Digital elevation model.........121, 124

Dioscorea rotundata................249f.

Diptera.........................32, 357

Dirofilaria immitis....................358 
Dirofilaria repens.

Disability-adjusted life years.

Dispersion.

DMSP.

Dokuy.

Dokuy CSPS

Dormant stage.

Doukoura.

Dracunculiasis.

Dracunculus mediensis

Drought..........34, 37, 195, 243, 343

Drought monitoring.

.131

Drug resistance.............6, 156, 271

Dry forest..........204, 207, 215f., 226

Dry savanna.204, 225, 263, 265, 282, $286,306,315,327 f ., 330,353$

DSS.

266

\section{E}

Early warning system.

ECHAM1-A model.

Ecological niche. 347

Ecological niche model.

Economic development. 110

Egg

238

Egg dormancy...

EIR.

El Niño.

$.78,106$

Elevation 198,343

Embryogenesis. 314

Emergence. .61

Emissivity. .63

Endemic malaria. $.135 f$.

Endemicity $7,22,31$

Endemism $24,55,101$

Endogenous cycle.

Endogenous stage. .207

Endogenous Stage. .53

Endophagy

Endophily

ENM.

Entomological inoculation rate....106, 118

Entomological survey.

Environmental management.

EOS. 150

Ephemeral stream.
.131

174
Epidemic malaria. 30

Epidemic potential.......................24

Epidemics...............................163

Epidemiology of malaria...............25

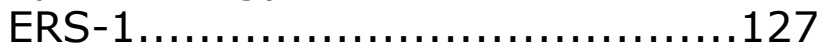

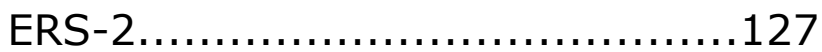

Erythrocyte...........................48

Erythrocytic cycle......................5 52

Erythrocytic schizogony.............51f.

Erythrocytic stage........29, 48, 51, 56

ESA.................................... 127

Ethiopia...........................80, 141

Ethnicity...............................319

Evapo-transpiration...................173

Evaporation.............................239

EVI..............................130, 315

Exflagellation..........................49

Exo-erythrocytic schizogony...........51

Exo-erythrocytic stage.................29

Exogenous cycle.........................50

Exogenous stage.....................47f.

Exophagy.................................35

Exophily.................................. 35

Exposure prophylaxis.................147

Extrinsic incubation period 48, 60, 342

$\mathbf{F}$

Faidherbia albida....................214

Fallow.................................. 241

Famine Early Warning System......143

FAO.......................................128

FAO Land Cover Classification System

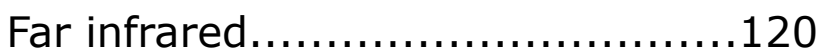

Fatality rate............................ 87

Fecundity................................ 37

Feeding cycle...........................113

Ferralitic soil..........................180

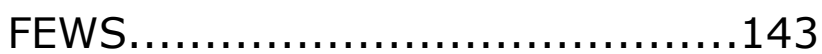

Field capacity........................182

Filariasis................ 9, 32, 261, 357f.

FIR........................................120

Firewood harvesting..................227

Flaviviridae.............................359

Flight range........................34, 40

Fluvisol..................................177

Forêt sèche...........................226 
Fulani. 230

Fulbe.

230

Fumigation mats

150

Fundamental ecological niche....

Fuzzy classification

\section{G}

Gallery forest......215, 220, 276, 278, 288, 306

Gambusia affinis. $151 \mathrm{f}$

Gametocyte. .28f., 48, 50, 52, 60, 157 Garki model........65, 102, 104f., 164 Garki study....................102, 150 GARP. $110 \mathrm{f}$. Gasterosteus aculeatus...............152 Genetic Algorithm for Rule-set Prediction 110

Genetic control..... 165

GeoCover LC. 127

GeoEye. 354

GeoEye-1.... $354 f$

Geographic information system....14, 110,143

Georeferencing.... 123

Georegistering .... 123

George Macdonald.........97, 100, 164 Geostationary satellite..............122f. Gestation period......................113 GFDL model............................ 340 Ghana. 54,345

Giovanni Maria Lancisi. .26

GIS $14,110,143$

GLC 2000 127,207

Gleysol... 177

Global Fund to Fight AIDS,

Tuberculosis and Malaria... 162

Global Land Cover 2000.

Global Monitoring and Disease

Prediction Program. 126

Global Precipitation Climatology

Project.

Global warming..... 339

Glossina spp.... 358

GMDPP 126

Goni 306

Goni CSPS 294

Gonotrophic cycle. $37,112 f ., 117$
Gonotrophic dissociation.

Gossypium hirsutum.

GPCP. 247

Gramineae. 141

Grass and thorn savanna............204

GTOPO30. 124

Guérriseur.

267

Guiera senegalensis....

Guinea worm infection 360

Gulf of Guinea. 191,198

H

Habitat productivity. $70 \mathrm{ff}$.

Hadley circulation. 191,193

Harmattan 192

Health information system...........163

Hematophagy.........................48

Hemoglobin C......................... 54

Hemoglobin E.......................... 54

Hemoglobin S........................ 54

Hepatic stage.....................29, 48

Hepatitis B............................ 3

Hibernation............................ $37 f$.

Histosol................................ 177

HIV/AIDS..........3f., 9, 19ff., 256, 261

HLA ..................................... 55

HLC..................... $88,303,305 f$.

Holoendemic malaria..................58

Holoendemicity..............21, 31, 89

Host................................27

Host seeking..............................

House-spraying...................... 149

Human blood index......................35

Human landing catch............88, 303

Human leucocyte antigens.............55

Humidity.................................66

Hydroelectric energy.................173

Hydrography.....................314, 325

Hydromorphic soil...................... 180

Hyperendemicity..................31, 89

Hyperspectral system...............122

Hypnozoite.............................. 51

Hypoendemicity................... 31,89

\section{I}

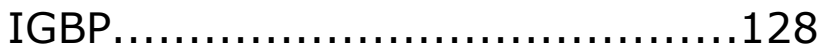

IKONOS.......132, 145, 276, 287, 321 
Illa...200, 242, 264, 276f., 286, 318f., 321

Illa CSPS 324

Immune response................... 52

Immune system..................... 51

Immunity........................ 30f., 52

Immunity to erythrocytic stages.....56

Immunity to sexual stages............57

Immunosuppression...................19

Imported malaria......................29

Incidence.............................. 86

Incubation period..................... 105

Indigenous malaria..................29

Infant mortality.................256, 271

Infectedness..................... 41,60

Infectious tropical diseases.............

Infectiveness.......................41, 60

Infectivity ........................... 48

Infiltration....................116, 173

Innate resistance...................... 54

Inoculation rate...................... 102

InSAR.........................121, 124

Insecticide.....83, 149, 151, 163, 320, $322 f$.

Insolation.

184

Institut de Recherche en Sciences de la Santé.

262

Integrated ecological model.........112

Interferometric radar.................121

Interferometric synthetic aperture radar...............................124 Intergovernmental Panel on Climate Change. 338

Intermittent irrigation................. 83 International Geosphere Biosphere Project. 128 Intertropical Convergence Zone....191

Intertropical front. 193

Introduced malaria 29

Inundation. 79,218

IPCC 338,344

Ira CSPS $274,294,316$

Irrigation $81,173 f ., 241 f ., 276$

Irrigation malaria..................40, 81

IRS. 355

IRSS 262

ITCZ $191 f$.

ITF. 193
ITN.

\section{J}

Japanese encephalitis................... 3

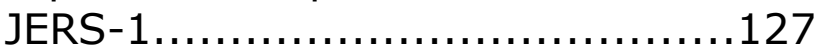

John Macculoch........................26

Jujube tree..........................210

$\mathbf{K}$

K-strategist.......................... 33

Kamadena......................265, 306

Karité..................210, 218, 283

Kenya. 69, 73, 75f., 79, 131, 145, 342

Kermena...............................265

Kienekuy CSPS......................294

Kinetic heat......................... 134

Kinetic temperature................... 135

Kinkeliba..............................210

Kinséré..............................264

Kodougou....200, 276, 278, 288, 303, 305,318

Kodougou Bobo...........264, 279, 281

Kodougou Mossi.......264, 279ff., $288 \mathrm{f}$.

Konkui-Kouro CSPS..................274

Kossi.....................205f., 210, 285

Kossi Province.................272, 325

Koubé..................................264

Koudougou...........................237

Kwashiorkor.......................... 55

$\mathbf{L}$

La Niña..............................198

Labarani CSPS...........294, 296, 316

LAI.................................... 129

Lake Volta............................. 172

Lancisi, Giovanni Maria.................26

Land cover...............75, 126f., 129

Land cover classification.............124

Land degradation......................131

Land surface emissivity.............136

Land surface temperature....134, 285, $312 f$.

Land use..............................116

Land use and cover change..........127

Landsat 7 ETM +........128f., 132, 144

Landscape ecology....................354 
Landscape epidemiology

Lannea microcarpa...

.354

Large-scale irrigation

$210 \mathrm{ff}$.

Larvae. 32,44

Larval density.

71,280

Larval habitat. .145

Larval mortality

Larval survival. .63

Larvicide. 149,164

Laterite crust. 169

Laveran, Alphonse.

15,26

LCCS. 127

Leaf area index 129

Leishmania spp. 358

Leishmaniasis.

Lékuy $9,20,261,358$

CSPS 263, 276, 294ff., 300, 316

Leprosy.........................3, 9, 160

Leptosol. 177

Léri... 174

Life cycle

Light trap capture. 303,312

Lignes de grains. 194

Limit of rainfed agriculture. Lithosol. 204

Lixisol. 180

Loa Loa. 177

Lobi. 358

Loiasis. $229 f$.

Long wavelength infrared 358

Long wavelength ultraviolet.

Longevity. 120

Loss of imm

LSE

LST

LTC.

LUCC.

LWIR. .120

Lymphatic filariasis

$38,44,66$

31,58

$\ldots 134,136,285,312 f$.

\section{M}

Macculoch, John... .26

Macdonald, George..............100, 164

Macrogamete. .49

Macrogametocyte.

Maize $80,245 f, 250 f$.

Malaria..3, 9, 160, 261, 342, 347, 350
Malaria burden...........................2

Malaria control....................149, 159 Malaria early warning system 143, 334 Malaria epidemic........................30 Malaria eradication..........12, 159, 162 Malaria eradication program.........159 Malaria in Sub-Saharan Africa.......... 5 Malaria mapping...................12, 85

Malaria modeling.........................85

Malaria monitoring......................85

Malaria morbidity...................2, 161

Malaria mortality...................2, 161

Malaria parasite..........................46

Malaria risk areas........................2

Malaria survey..........................85

Malaria transmission cycle.............28

Malaria transmission models..........13

Malaria vectors..........................43

Malarone................................. 156

Mali.....................77, 84, 276, 345

Malnutrition..............................258

Man-biting habit.......................103

Man-biting rate........................117

Mandé...................................230

Mangifera indica................210, 220

Mango tree.......................210, 220

Manihot esculenta.....................249f.

Manioc.....................................249f.

Mansonia spp.....................302, 359

Mapping Malaria Risk in Africa........93 93

MARA/ARMA initiative.............12, 93

Marasmus...................................55

Marcus Terentius Varro................25

Mare..............................215, 307

Marka...............................229f.

Mate seeking...............................39

Mating..................................... 37

Maximum value composite...123, 312, 316

MDGs..............................10, 146

Medical geography......................16 Medium wavelength infrared.........120 Medium wavelength ultraviolet......120 MEDSAT..................................354 Mefloquine.............................156

Meningitis.................................. 3 Merozoite.................29, 48, 51f., 54 Mesocyclops spp.......................360 
Mesoendemicity...................31, 89

Mesophyll...............................129

Mesostoma.............................153

MEWS...........................143, 334

Miasma...................................26

Microgamete............................49

Microgametocyte.......................48

Microhabitat.............................38

Microwave........................120, 141

Midinfrared...............................134

Millennium Development Goals....10f., 146,256

Millet. 243, $250 f$.

Ministry of Health......................267

MIR...................................134

MIROC3.2midres.......................340

Mixed pixel...............................122

MOD11 L2............................ 137

MOD11A1 ............................... 138

MOD11A2 .............................138

Moderate Resolution Imaging

Spectrometer.

.131

MODIS. $126 \mathrm{f} ., 130 \mathrm{f} ., 136 \mathrm{ff}$.

MODIS LST.............137, 285, 312f. MODIS NDVI.........................

MODIS Rapid Response System....138

Moist savanna............................ 204

Moisture index...........................67 67

Monkey bread tree....................210

Monsoonal air..........................193

Morbidity...................................86

Mortality............................87, 118

Mosquito.................................32

Mosquito coils.......................150

Mosquito fish.........................151f.

Mosquito population......................38

Mossi.................228ff., 319, 321f.

Mouhoun...172ff., 180, 263, 274, 276, 278, 284, 288, 306, 315

Mouhoun subbasin....................173

Mozambique......................69, 150

MSAVI...................................130

Multispectral system..................122

MVC.......................123, 312, 316

MWIR............................120, 134

\section{$\mathbf{N}$}

Nakambé. 172

Natural selection........................343

NDPI................................... 133

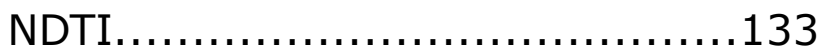

NDVI........75, 116, 129ff., 144, 315f.

Near infrared...........................120

Nectar seeking.............................39

Neem tree.............................210f.

Neglected tropical diseases.....8f., 160

NHD.....................................265

Niassan...................................242

Niger basin.............................172

NIR .................................... 120

Nokuy Badala...........................264

Nokuy Mossi............................. 264

Nomadism.........................204, 241

Normalized Difference Pond Index. 133 Normalized Difference Turbidity Index

Normalized Difference Vegetation Index................................. 129

Northern Sudan region................187

Nouna....................192, 232, 309

Nouna CMA......................296, 316

Nouna CSPS............................294

Nouna Health District. 261, 265, 267f., 324

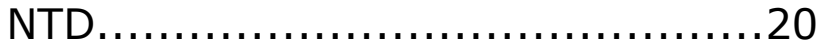

NTDs.......................................

$\mathbf{0}$

O'nyong-nyong fever......32, 357, 359

Office de la Recherche Scientifique et

Technique d'Outre-Mer.............177

Onchocerca volvulus...................358

Onchocerciasis...............9, 160, 358

Onset of rainy season...................197

Oocyst......................28, 41, 48ff.

Ookinete........................28, 49f.

Oreochromis spilurus.................152

Ornithodoros sonrai.....................360

ORSTOM.................................177

Oryza sativa......................245, 250

Oubritenga.............................230

Overgrazing.....................131, 226

Oviposition....33, 37, 44, 48, 66, 69f., 73ff., 113, 151, 308 
Oviposition rate.

\section{P}

Pan-sharpening. 133

Panicum laetum. 225

Parasitation. .41

Parasite rate.

Parasite reservoir.... .99

Parasitemia. 46,52

Parasitoid. ... 34

Paris Green. 149

Parkia biglobosa...

210

Parkland savanna.... 241

Passive case detection. .85

Passive immunity. 102

Passive remote sensing. 119

Pastoralism. 241

Peanut. $247,250 f$.

Pearl millet.... 243,251

Penck's limit of aridity. 204

Pendjari. 172

Pennisetum glaucum.........243, 250f.

Perennial habitat......................307

Perennial river..........................172

Perennial transmission..................31

Permethrine...........................154

PERSIANN........................... 141

Peulh............229ff., 252, 319, 321f.

Phagocyte............................. 51

Phlebotomus spp......................358

Piliostigma thonningii................225

Plasmodia...................27f., 44, 46

Plasmodium cynomolgi................41

Plasmodium falciparum. 5, 7, 18, 21ff., $25,32,38,46 f f ., 51 f ., 57,59,66$,

$69,79,87,99,156,342$

Plasmodium falciparum malaria......65

Plasmodium knowlesi.................47

Plasmodium malariae....29, 46ff., 51f., 59

Plasmodium ovale....29, 46ff., 51f., 59

Plasmodium vivax...29, 46ff., 51f., 59, 99, 156

Poecilia reticulata.

Polar-orbiting satellite. $122 \mathrm{f}$.

Polyparasitism. ..9

Population density. $.76,118,231$
Population dynamics 32, 34, 134, 197, 326,338

Potential evaporation..................195

Poverty...............................238

Poverty line.........................236

Pre-erythrocytic immunity............56

Pre-erythrocytic schizogony.........51f.

Pre-onset of rainy season...........193

Pre-patent period........................51

Precambrian shield.................. 168

Precipitation. 66ff., 75, 116, 140f., 186 Precipitation Estimation from

Remotely Sensed Information using

Artificial Neural Networks..........141

Precipitation index...................140

Predation.............................63

Predator34, 68, 70, 72, 151, 153, 344

Predatory mosquito..................153

Pregnancy.............................. 58

Prevalence...................65, 86, 118

Primaquine...........................156

Process-based model...............110

Proguanil...............................156

Protozoa.................................46

PSC.......................88, 303, 305f.

Pseudo-urbanity......................77

Pseudogley.......................... 180

Pupae..................................... 32

Pupal habitat........................... 71

Pupal productivity...................... 71

Pyrethrum spray capture........88, 303

Pyrimethamine......................156

$\mathbf{Q}$

QuickBird................132, 283, 323

Quinine............................. 156

\section{$\mathbf{R}$}

R-strategist.......................... 33

Radar.............................. 141

Radar remote sensing...............121

Radiant temperature.................134

Radiometer..........................121

Radiometric resolution................122

Radiometric temperature............135

Rain forest...........................222

Rainfall................66ff., 131, $140 f$. 
Rainfall concentration index

Rainfall events.

.116

Rainfall index

Rainfall prediction estimate.

140

Rainfall prediction estimate...........141

Rainfed agriculture. 239,277

Rainforest. 201,205

Rainy season.

.116

RBM 161

Real aperture radar. 121

Realized ecological niche 111

Recovery rate. $97,102,118$

Rectification. .123

Red blood cell membrane. 54

Reflected infrared. 120

Regosol 177

Relief. 116

Remote sensing.........................14 Rerum Rusticarum De Agri Cultura. . 25 Residual insecticide. 149

Resistance. 151,163

Resting places. ..35

Retreatment... 154

RFE2. 141

Rhipicephalus spp 360

Rice. $81,242,245,250 f$.

Rickettsia spp. 360

Rift Valley fever 359

River blindness. 358

Roll Back Malaria. 161

Roman fever. .26

Ronald Ross. $27,97,147,158$

Ross, Ronald. $27,97,147,158$

\section{$\mathbf{S}$}

Saccharum officinarum 248 Sahel.....82, 84, 141, 187, 194, 198, $201,206,222,229,233,259,345$

Sahelo-Sudanian zone 183f., 187, 205, $233,239,241$

Salination .239

Samo 230f., $321 \mathrm{f}$

SAR. 121,127

SARVI. 130

SAVI. 130,144

Scan-line corrector. 129

Schistosoma haematobium..........360

Schistosoma mansoni.
Schistosomiasis. $9,20,261,360$

Schizogony..............................47

Schizont............................29, 51

Schoenefeldia gracilis.................225

Sclerocarya birrea.....................225

Sea surface temperature............136

Seasonal transmission....................31

Selection..................................343

Semi-desert............................201

Seminomadism.........................241

Senegal..............................44, 64

Sensor...................................121

Sesame........................248, $250 f$.

Sesamum indicum..............248, 250

Sexual cycle..............................48

Sexual differentiation...................48

Sexual stage..............................57

Sexually transmitted diseases..........2

Seyal acacia...............................210

Shea butter..............................219

Short wavelength infrared.............120

Short wavelength ultraviolet..........120

Shuttle Radar Topography Mission...15, 124

Sickle cell anemia......................54

SIMA.................................81, 162

Simian Plasmodia........................29

Simple ratio index....................129

Simulium spp..........................358

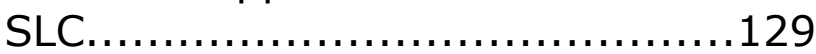

SLC-off imagery........................129

Sleeping sickness.......................358

Sofitex....................................247

Soft classification....................122

Soil and atmospherically resistant vegetation index.....................130

Soil moisture................73, 75, 136

Soil water storage index.............116

Soil-adjusted vegetation index......130

Sols minéraux bruts....................177

Sols peu évolués.......................177

Sorghum.......................243, $250 f$.

Sorghum bicolor.................243, 250

Sorghum guinea......................243

Soum Province..........................269

Soumbara...............................217

Sourou.....173f., 242, 245, 276, 284f., 307,315 
Sourou Depression..............174, 180

Sourou Province.........................318

Sourou Valley....................246, 248

South Africa............................. 150

Southern Sudan region................188

Spatial behavior....................34, 39

Spatial resolution.......................122

Species A...............................43

Species B...........................43

Species C................................43

Species D...............................44

Specific immunity......................55

Spectral resolution....................122

Spectral signature....................121

Spectral unmixing.....................122

Spectral vegetation index.............129

Spleen rate...............................87

Split-window LST......................137

Sporogonic cycle...60f., 103, 105, 117

Sporogonic development..............38

Sporogony..........28, 47, 49, 61, 101

Sporozoite..........28, 41, 46, 48ff., 56

Sporozoite rate........................41

SPOT-4............................... 127

SPOT-5............................. 133

Spotted fever..........................360

Sprinkler irrigation......................83

SRI.....................................129f.

SRTM........................... 15, $124 f$.

SST .................................... 136

SST anomaly...........................198

Statistical models..................13, 114

Stefan-Boltzmann law................120

Sterile insect technique..............165

Stickleback.............................. 152

Strain-specific theory..................56

Streambed..................................70

Sub-Saharan Africa..... 7, 21, 23, 43f., 163

Subsistence farming...........236, 239

Subsistence income...................237

Sudan.......................................229

Sudan savanna.......................... 102

Sudanian zone...................188, 205

Sugarcane.................242, 248, 251

Supervised classification..............126

Surface runoff.....................116, 173

Surveillance............................. 148
SVI....................................... 129

Swamp................................276

Swaziland................................150

SWIR.....................................120

SWS...................................116

Symptoms of malaria..................85

Synthetic aperture radar..............121

System-wide Initiative on Malaria and

Agriculture....................81, 162

\section{$\mathbf{T}$}

Tamarindus indica...............210, 219

TanDEM-X...............................355

Tanzania..........................113, 150

TBRF.....................................360

TDR............................... 8, 160

TDR portfolio.............................9

TEJ..............................193, 198

Temperature59ff., 116, 120, 134, 184, 307f., 312f., 326

Temperature-vegetation index......136

Temporal resolution.....................122

Temporary habitat.......................307

Terra.................................... 126

Terra (satellite).....................131

TerraSAR-X..............................355

Terrestrial infrared radiation..........134

TFR......................................233

Thalassemia............................ 54

The Gambia........45, 67, 71, 84, 154

Thermal infrared..........120, 128, 134

Thermocyclos spp.......................360

Tick-borne relapsing fever ...........360

Tilapia...................................152

Times of biting .......................... 35

TIR........................................ 134

Togaviridae...............................359

Toni....200, 265, 276, 283, 309, 321, 323

Toni CSPS. 265, 276, 294, 296f., 299f., 305,324

Topographic index.....................116

Total fertility rate......................233

Toxin release............................48

Toxorynchites..........................153

Trade wind inversion...................193

Transmission...............................55 
Transmission cycle.....................29

Transmission pressure.................. 15

Transmission process..............25, 27

Transmission risk.......................34

Transmission seasonality index.....293

Transport infrastructure.............239

Transvaal..............................222

TRMM................................ 141

Trophozoite........................... 52

Tropical Disease Research........8, 160

Tropical diseases........................

Tropical Easterly Jet............193, 198

Tropical Rainfall Measuring Mission 141

Trypanosoma brucei gambiense....358

Trypanosoma brucei rhodesiense. .358

Trypanosomiasis........9, 20, 261, 358

TSI...................................293

Tuberculosis....................3f., 9, 261

Turbidity........................72f., 133

TVX.........................136, 139

\section{$\mathbf{U}$}

Uganda. 30,343

UKMO model.............................347

Ulisse Aldrovandi.......................26

Underemployment....................236

Unemployment......................236

Unsupervised classification...........126

Urban malaria............................ 77

UV-A................................. 120

UV-B................................

UV-C ...............................

V

Varro, Marcus Terentius................25

Vector.............................. 27, 32

Vector competence...........34, 42, 50

Vector control..................147, 149

Vector density.............71, 103, 117

Vector dispersal.................339, 341

Vector life expectancy...............104

Vector longevity........34, 38, 64, 117

Vector mortality....................... 34

Vector population.....................103

Vector-borne disease.............32, 258

Vector-borne infections.................

Vectorial capacity. .98, 103f., 106, 117
Vectors..................................43

Vegetation index................75, 129

Verbal autopsy....................... 86

Vertical accuracy...................125

Vertisol.............177, 180, 215, 247

Virga.................................193

Vitellaria paradoxa..............210, 218

Volta Basin......................172, 195

\section{$\mathbf{W}$}

Walker circulation..............191, 198

Water scarcity........................ 172

Water stress......................... 172

Water turbidity ......................... 72

Waterborne diseases................... 3

Wèrèbèrè......................264, 276

Wèrèbèrè CSPS. .264, 276, 294, 296f., $299 f$.

Weresse...............................264

West Africa.................. 7, 25, 43f., 54

West African monsoon..........192, 341

West Nile fever...........................359

White Volta........................... 172

Wild grape tree................210, 212

Wilting point........................... 182

Winter.............................. 192

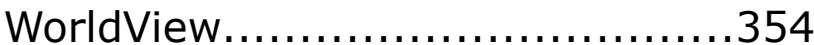

WorldView-2 ......................

Wuchereria bancrofti.................358

\section{$\mathbf{x}$}

X-band. $.121,124$

$\mathbf{Y}$

Yam $249 \mathrm{ff}$

Yatenga...............................230

Yellow fever....................350, 359

Yévédougou CSPS..................294

$\mathbf{Z}$

Zea mays.................... $80,245,250$

Zimbabwe.......................83, 141

Ziziphus mauritania......210, 218, 224

Zone guinéenne......................205

Zone of potential malaria transmission

277,282 
Zone potentially occupied by mosquitoes.

Zoophily

$.35,105$

Zone sahélienne.

143

Zooprophylaxis.....

154,319

Zone soudanienne..............188, 205

Zone soudano-sahélienne. .187

143

Zygote....... 


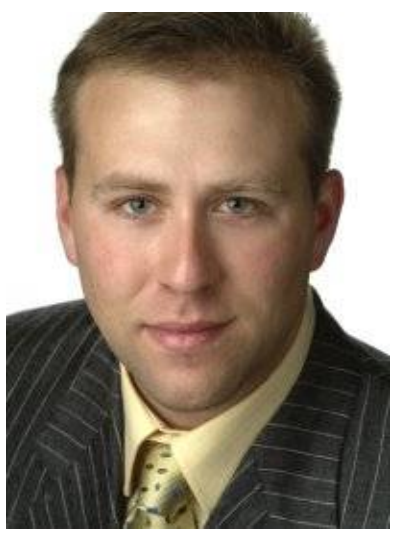

Date of Birth: $\quad 10.11 .75$

Place of Birth: Mannheim, Germany

Parents:

Manfred and Angelika Karthe (* Kehrer)

Nationality: German

Confession: Protestant

Marital Status: Unmarried

\section{Schooling and Academic Education}

Schooling: 1982 until 1995, graduation (Abitur) in June 1995

- 1982 until 1986: student of Rheinau-Grundschule (elementary school) in Mannheim;

- 1986 until 1995: student of Moll-Gymnasium (high school) in Mannheim

- participation in student and youth exchange programs with France, Israel, Japan and the USA; acquisition of a US High School Diploma and the Presidential Academic Fitness Award in Gold

University and Teachers' Academy: completed with the first and second state examination for teachers (high school); currently doctoral candidate

- 1996 until 2002: student of Geography and English (State Examination) at Mannheim University; 2006 to 2009: doctoral candidate at Göttingen University

- recipient of a DAAD (German Academic Exchange Service) scholarship and 13 months' stay at Presidency College, Calcutta, India

- September 2002 until July 2004: professsional course at the Teachers' Academy Heilbronn ("Seminar für Schulpädagogik - Gymnasien"); certificate for bilingual teaching

\section{Professional Experience}

\section{Academic and teaching experience:}

- 1997 until 2002: student research assistent in the departments of Physical Geography and English Linguistics, Mannheim University

- since 2002: high school teacher (English and Geography); currently: Otto-HahnGymnasium, Göttingen

- since 2006: assistant researcher and lecturer, Cartography, GIS and Remote Sensing Section, Department of Geography, Göttingen University

\section{Additional experiences:}

- since 2008: project advisor, Westermann publishing company, Braunschweig

- please refer to separate list for thesis papers, publication and conference lectures 


\section{Articles Published in Journals}

Siegmund, A. \& Karthe, D. (2004): Die Böden der Kapverden. Eine Bestandsaufnahme auf den Inseln Fogo, Boavista und Santo Antao. Geoöko, Vol. 39, No. 6, pp. 20-25.

KARTHE, D. (2002): Trinkwasserversorgung in Megastädten. Das Beispiel Calcutta, Indien. Geographische Rundschau, Vol. 54, No. 7-8, pp. 33-37.

Karthe, D. \& Stähle, M. (2008): Water Supply and Health in India. Geographische Rundschau International Edition, Vol. 4, No. 2, pp. 10-15.

Karthe, D.; Reeh, T. \& Augustin, J. (2009): Tourism and Health in Egypt: A Geomedical Perspective. Geographische Rundschau International Edition, Vol. 5, No. 3, pp. 4-11.

KARTHE, D. \& TRAORÉ, I. (2009): Geographic Pattern of Malaria Transmission: A Case Study from Kossi Province, Burkina Faso. Geoöko, Vol. 30, No. 1-2, pp. 43-63.

\section{Conference Papers}

KARTHE, D. (2001): Drinking Water Contamination in Calcutta. In: PICKFORD, Jонм (Ed.) (2001): Water, Sanitation and Hygiene: Challenges of the . Proceedings of the 26th WEDC Conference, Dhaka, pp. 224-226. Loughborough, UK.

KARTHE, D. \& KAPAA, M. (2007): Modelling Malaria Transmission in a Rural Region in West Africa: A Case Study of Nouna District, Burkina Faso. In: Kappas, M.; Kleinn, C. \& SlOBODA, B. (2007): Global Change Issues in Developing and Emerging Countries. Proceedings of the $2^{\text {nd }}$ Göttingen GIS and Remote Sensing Days 2006. Göttingen: Universitätsverlag Göttingen.

\section{Monographs and Contributions to Books}

KARTHE, D. \& KAPAAS, M. (2009): Malariaübertragung in Westafrika: Die Rolle natürlicher und anthropogener Determinanten. In: GLASER, R.; KREMB, K. \& Drescher, A. (Hrsg.) (2009): Afrika, pp. 76-87. Darmstadt: Wissenschaftliche Buchgesellschaft.

KARTHE, D. (2006): Trinkwasser in Calcutta: Versorgungsproblematik einer indischen Megastadt. Stuttgart: ibidem Verlag. 
«Our vision is a world free from the burden of malaria.

By 2015, the malaria-specific Millennium Development Goal (MDG) is achieved, and malaria is no longer a major cause of mortality and no longer a barrier to social and economic development and growth anywhere in the world.

Beyond 2015, all countries and partners sustain their political and financial commitment to malaria control efforts. The burden of malaria never rises above the 2015 level, ensuring that malaria does not re-emerge as a global threat.

In the long term, global malaria eradication is achieved. There is no malaria infection in any country. Malaria control efforts can be stopped. ${ }^{1629}$

Vision of the Roll Back Malaria Partnership, 2008

1629 Roll Back Malaria Partnership (2008), p. 25. 